\author{
Universidade De SÃo PAUlo \\ Instituto de Astronomia, Geofísica e Ciências Atmosféricas \\ Departamento de Astronomia
}

Carla Martinez Canelo

\title{
DistRIBUIÇÃO CÓSMICA DE AROMÁTICOS E OUTRAS ESPÉCIES DE INTERESSE BIÓTICO
}

SÃO PAULO 



\section{Carla Martinez Canelo}

\section{DisTRIBUiÇÃo CÓSMICA DE AROMÁTICOS E OUTRAS ESPÉCIES DE INTERESSE BIÓTICO}

Tese apresentada ao Departamento de Astronomia do Instituto de Astronomia, Geofísica e Ciências Atmosféricas da Universidade de São Paulo como requisito parcial para a obtenção do título de Doutor em Ciências.

Área de Concentração: Astronomia Orientador(a): Prof. Dr. Amâncio César Santos Friaça

Versão Corrigida. O original encontra-se disponível na Unidade.

SÃO PAULO 

Aos meus pais e irmã que sempre estiveram do meu lado me apoiando e incentivando. Às próximas gerações, que lutem pelos seus sonhos e pela Ciência. 



\section{Agradecimentos}

À minha mãe Silvana, meu pai Claudio e minha irmã Carolina, por sempre me apoiarem e cuidarem de mim nos momentos mais difíceis;

Ao Prof. Dr. Amâncio César Santos Friaça por continuar a me orientar no Doutorado;

Aos meus colaboradores Professora Dra. Dinalva Aires de Sales e Dr. Edgar Mendoza, por toda a ajuda e experiência;

À Professora Dra. Vera Jatenco Silva Pereira por ter acompanhado todo este trabalho e por sua atenção;

Aos amigos que cruzaram meu caminho e dividiram a convivência acadêmica comigo.

Ao CNPq, pelo apoio financeiro, sob o projeto $\mathrm{n}^{o}$ : 141714/2016-6;

Ao Instituto de Astronomia, Geofísica e Ciências Atmosféricas - IAG/USP - juntamente com sua equipe atenciosa da Secretaria e da Informática.

Esta tese/dissertação foi escrita em IATEX com a classe IAGTESE, para teses e dissertações do IAG. 

"[...] humanity will reach maturity and wisdom on the day that it begins not just to tolerate, but take a special delight in differences in ideas and differences in life forms. [...] If we cannot learn to actually enjoy those small differences, to take a positive delight in those small differences between our own kind, here on this planet, then we do not deserve to go out into space and meet the diversity that is almost certainly out there." 



\section{Resumo}

Esta tese apresenta um estudo astrobiológico e astroquímico da complexidade molecular no Universo, através de faixas espectrais distintas. Os hidrocarbonetos policíclicos aromáticos (PAHs) e os heterociclos policíclicos aromáticos nitrogenados (PANHs), que possuem um potencial para formar moléculas prebióticas, foram estudados em uma amostra de 126 a 155 galáxias com emissão dominada por starburst, extraídas do projeto Spitzer/IRS ATLAS. Esses objetos tiveram suas bandas de PAH do infravermelho médio de 6.2, 7.7 e $8.6 \mu \mathrm{m}$ ajustadas e distribuídas nas classes A, B e C de Peeters. Pela primeira vez na literatura, um estudo de PAHs e das classes de Peeters é realizado com estatística robusta para um grande número de galáxias. O desvio para o azul da banda em $6.2 \mu \mathrm{m}$, típico de um objeto classe A, foi atribuído a moléculas de PANH e parece dominar essa emissão espectral. Análises dessas bandas também indicam que a distribuição das variações de perfil de $\mathrm{PAH}$ ao longo do redshift das galáxias pode sugerir uma possível escala de tempo evolutiva dos PAHs. Ademais, precursores de PAHs e PANHs como $\mathrm{HNCO}, \mathrm{HC}_{3} \mathrm{~N}$ e $\mathrm{NH}_{2} \mathrm{CHO}$, podem ser observados em comprimentos de onda de rádio. Levantamentos de linhas espectrais revelaram ricos reservatórios moleculares em G331.512-0.103, um núcleo quente molecular no centro de um energético outflow. As observações foram feitas com a antena APEX no intervalo de frequência de 160-355 GHz. Em particular, foram detectadas 42 transições de $\mathrm{HNCO}$, com uma temperatura de excitação de cerca de $60 \mathrm{~K}$. A abundância e evolução química do HNCO foram modeladas com o código astroquímico Nautilus, que simula reações em grãos e fase gasosa em núcleos moleculares. Por fim, o código também foi usado para simular a abundância de ${ }^{13} \mathrm{CS}$, OCS, $\mathrm{CH}_{3} \mathrm{CHO}$ e $\mathrm{CH}_{3} \mathrm{OCHO}$ no núcleo frio infravermelho chamado de IRDC-C9 Main. 



\section{Abstract}

This thesis presents an astrobiological and astrochemical study of the molecular complexity in the Universe, through distinct spectral regions. Polycyclic aromatic hydrocarbons (PAHs) and polycyclic aromatic nitrogen heterocycles (PANHs), which have the potential to form prebiotic molecules, were studied for a sample of 126 to 155 starburst-dominated galaxies, extracted from the Spitzer/IRS ATLAS project. These objects have their 6.2, 7.7 and $8.6 \mu \mathrm{m}$ mid-infrared (MIR) PAH bands fitted and separated into the Peeters' A, $\mathrm{B}$ and $\mathrm{C}$ classes. For the first time in literature, a study of PAHs and Peeters classes is performed with robust statistics for a large number of galaxies. The blueshift of the $6.2 \mu \mathrm{m}$ band, typical for a class A object, was attributed to PANH molecules and seems to dominate this spectral feature. Analyses of these bands also indicate that the distribution of the PAH profile variations along the redshift of the galaxies could suggest a possible PAH evolutionary timescale. In addition, precursors of PAHs and PANHs such as HNCO, $\mathrm{HC}_{3} \mathrm{~N}$ and $\mathrm{NH}_{2} \mathrm{CHO}$, can be observed at radio wavelengths. Spectral line surveys revealed rich molecular reservoirs in G331.512-0.103, a compact radio source in the center of an energetic molecular outflow. The observations were carried out with the APEX antenna in the interval frequency of 160-355 GHz. In particular, 42 transitions of HNCO were detected with a excitation temperature about $60 \mathrm{~K}$. The abundance and chemical evolution of HNCO were modeled with the astrochemical code NAUTILUS, which simulates grain and gas phase reactions in molecular cores. Finally, the code was also used to simulate the abundances of ${ }^{13} \mathrm{CS}$, OCS, $\mathrm{CH}_{3} \mathrm{CHO}$ and $\mathrm{CH}_{3} \mathrm{OCHO}$ in the infrared cold core called as IRDC-C9 Main. 



\section{Lista de Figuras}

1.1 Estrutura molecular do benzeno, pirimidina, uracil, timina e citosina. . . . 20

1.2 Exemplos de classificação da estrutura de PAHs. . . . . . . . . . . . . . . . 22

1.3 Evolução de moléculas carbonáceas e PAHs. . . . . . . . . . . . . . . . . 23

1.4 Visão geral das possíveis diferenças no perfil das bandas de 6.2, 7.7 e $8.6 \mu \mathrm{m}$,

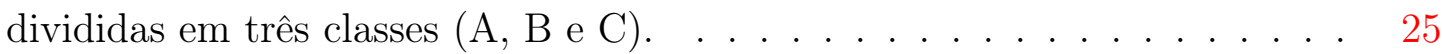

1.5 Representação simples do ciclo de vida estelar sobreposto à evolução dos

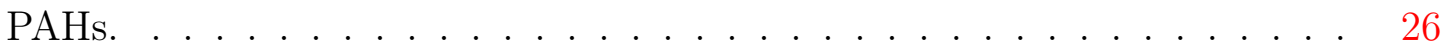

1.6 Esquema do modelo unificado de AGNs. . . . . . . . . . . . . . . . . . . . 29

1.7 Rotas sintéticas para a construção de moléculas aromáticas monocíclicas e bicíclicas contendo nitrogênio. . . . . . . . . . . . . . . . . . . . . 32

1.8 Esquema do HNCO como um portador simples de ligações peptídicas. . . . 33

1.9 Comparação de abundâncias moleculares para $\mathrm{NH}_{2} \mathrm{CHO}$ e $\mathrm{HNCO}$ em uma amostra de fontes galácticas. . . . . . . . . . . . . . . . 35

1.10 Mecanismo bicíclico de reações entre $\mathrm{NH}_{2} \mathrm{CHO}, \mathrm{H}_{2} \mathrm{NCO}$ e HNCO . . . . . . 36

3.2 Esboço do modelo simples proposto para explicar a emissão de moléculas detectadas em G331. . . . . . . . . . . . . . . . . . . . 90

3.3 Mapa do contínuo de IRDC-C9 Main, mostrando a emissão de um núcleo com uma cauda nas redondezas. . . . . . . . . . . . . . . . . . . . . . . . 91

3.4 Espectros do núcleo (superior) e cauda (inferior) de IRDC-C9 Main mostrando a identificação das linhas mais intensas. . . . . . . . . . . . . . . 92

3.5 Uma das bandas espectrais observadas do objeto G331 com frequência de 347.8 a $351.8 \mathrm{GHz} \ldots \ldots \ldots \ldots$ 
3.6 Ajuste Gaussiano das linhas de HNCO com frequências em 219798.274 e $351633.257 \mathrm{MHz} . \ldots \ldots \ldots \ldots 117$

3.7 Linhas de emissão do HNCO para $K_{a}=0$ com uma resolução de $1 \mathrm{~km} / \mathrm{s}$. . . 119

3.8 Linhas de emissão do HNCO para $K_{a}=1$ com uma resolução de $1 \mathrm{~km} / \mathrm{s}$. . . 120

3.9 Linhas de emissão do HNCO para $K_{a}=2$ com uma resolução de $1 \mathrm{~km} / \mathrm{s}$. . . 121

3.10 Emissões de HNCO em 175189.027 e 330848.569 MHz com seus respectivos ajustes Gaussianos. . . . . . . . . . . . . . . . . . . . 122

3.11 Emissão de HNCO $\left(13_{3,10}-12_{3,9}\right)$ em 285541.575 MHz. . . . . . . . . . . . 123

3.12 Diagramas rotacionas de HNCO para uma correção da diluição do feixe de 5 arcsec. . . . . . . . . . . . . . . . . . . . 125

3.13 Histogramas das densidades de coluna de HNCO e suas abundâncias relativas ao $\mathrm{H}_{2}$ em diversos objetos . . . . . . . . . . . . . . . . . . . 127

3.14 Simulações químicas realizadas para o HNCO em diferentes densidades e temperaturas. . . . . . . . . . . . . . . . . 130

3.15 Melhores ajustes da evolução temporal das abundâncias de HNCO simuladas com NAUtilus. . . . . . . . . . . . . . . . . . . . . . . 132

3.16 Evolução temporal das abundâncias simuladas com o NAUTILUs e distância do desacordo. . . . . . . . . . . . . . . . . . . . 135

3.17 Rede química de CS e OCS para um modelo com temperatura de $15 \mathrm{~K}$. . 136

3.18 Evolução temporal das densidade de coluna moleculares simuladas com NAutilus com a rede química completa . . . . . . . . . . . . . 139

3.19 Evolução temporal das densidade de coluna moleculares simuladas com NAUTILUS com a rede química alterada. . . . . . . . . . . . . . . . . 141

3.20 Evolução temporal das densidade de coluna moleculares simuladas com NAutilus para uma temperatura de 450 K. . . . . . . . . . . . . . . . . . 143

3.21 Principal rede química com os caminhos de formação e destruição de $\mathrm{CH}_{3} \mathrm{CN}$.144 


\section{Lista de Tabelas}

1.1 Modos de vibração de algumas bandas de PAHs. . . . . . . . . . . . . . . 24

1.2 Comprimentos de onda centrais das classes de Peeters. . . . . . . . . . . . 24

3.1 Visão geral das propriedades globais de alguns ambientes de nuvens mole-

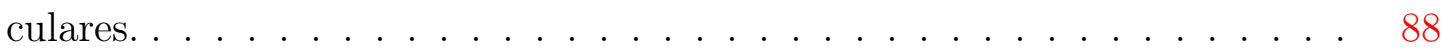

3.2 Abundâncias iniciais assumidas para o modelo do NAutiLus. . . . . . . . . 93

3.3 Análise espectral preliminar das linhas de emissão observadas em G331 com

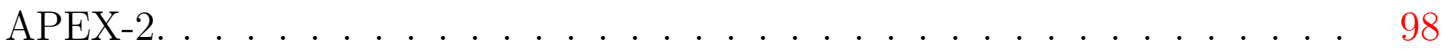

3.4 Linhas de HNCO detectadas em G331. . . . . . . . . . . . . . . . 118

3.5 Abundâncias moleculares relativas a $\mathrm{n}\left(\mathrm{H}_{2}\right)$ derivadas para IRDC-C9 MAIN (Beaklini et al., 2020). . . . . . . . . . . . . . . . . . . 134

A.1 Imagens dos ajustes do contínuo e banda de $6.2 \mu \mathrm{m}$ das 29 galáxias com emissão dominada por starburst. . . . . . . . . . . . . . . . 167

A.2 Imagens dos ajustes do contínuo e bandas de 6.2, 7.7 e $8.6 \mu \mathrm{m}$ das 126 galáxias com emissão dominada por starburst. . . . . . . . . . . . . . . . . 175 



\section{Sumário}

1. Introdução . . . . . . . . . . . . . . . . . . . . . . . . . . . . . . 19

1.1 Moléculas aromáticas no meio interestelar . . . . . . . . . . . . . . . . 21

1.1.1 Variações nos perfis de PAH no infravermelho . . . . . . . . . . . . 23

1.2 Moléculas aromáticas em galáxias . . . . . . . . . . . . . . . 27

1.2.1 Galáxias Starburst . . . . . . . . . . . . . . . . 28

1.2.2 Galáxias Ativas . . . . . . . . . . . . . . . . . . . . . . . 28

1.2.3 PAHs em objetos extragalácticos . . . . . . . . . . . . . . . 29

1.3 Moléculas prebióticas no meio interestelar . . . . . . . . . . . . . . . 31

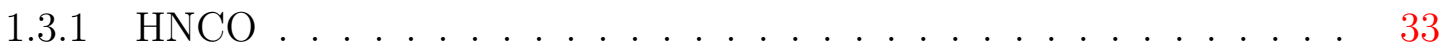

1.4 Organização da tese e objetivos . . . . . . . . . . . . . . . 36

2. PARTE I - Aromáticos no infravermelho médio . . . . . . . . . . . . . . . . . . 39

2.1 Artigo publicado sobre a banda de $6.2 \mu \mathrm{m}$ em galáxias dominadas por star-

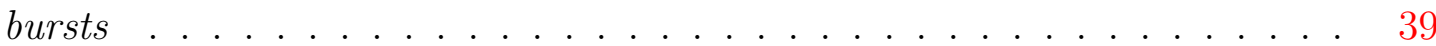

2.2 Artigo submetido sobre a região de 6 a $9 \mu \mathrm{m}$ em galáxias dominadas por

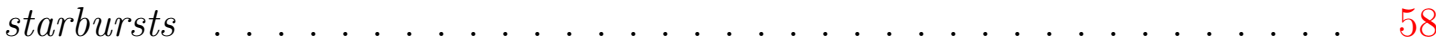

3. PARTE II - Moléculas de interesse biótico no sub/milimétrico . . . . . . . . . . 87

3.1 Objetos galácticos estudados e o código NAUTILUS . . . . . . . . . . . 87

3.1.1 O núcleo molecular quente G331.512-0.103 . . . . . . . . . . . . 88

3.1.2 A nuvem fria infravermelha IRDC-C9 Main . . . . . . . . . . . . . 89

3.1.3 Modelagem química com Nautilus . . . . . . . . . . . . . . . . . . 92

3.1.3.1 Distância de desacordo . . . . . . . . . . . . . . . . . 94

3.2 Análises Observacionais de G331.512-0.103 . . . . . . . . . . . . . . . 94 
3.2.1 HNCO em G331: Deteç̧ão e Modelagem Química . . . . . . . . . 117

3.2.1.1 Diagrama rotacional e propriedades derivadas . . . . . . 124

3.2.1.2 Simulações químicas com Nautilus ........ 129

3.3 Simulações químicas com NAutilus para IRDC-C9 Main . . . . . . . . . 133

3.3.1 Outra abordagem com Nautilus . . . . . . . . . . . . 134

3.3.2 Simulação para $\mathrm{T}=450 \mathrm{~K}$. . . . . . . . . . . . . . . . . 142

4. Conclusão . . . . . . . . . . . . . . . . . . . . . . . 145

4.1 Próximas pesquisas e perspectivas . . . . . . . . . . . . 147

4.1.1 PAHs em fontes extragalácticas . . . . . . . . . . . . . . . . 147

4.1 .2 Observação e modelagem de COMs . . . . . . . . . . . . . . . 148

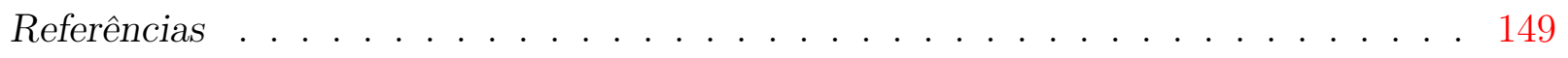

$\begin{array}{ll}\text { Apêndice } & 165\end{array}$

A. Figuras dos ajustes das bandas de PAH . . . . . . . . . . . . . . . . . . 167 
Capítulo 1

\section{Introdução}

A Astrobiologia pode ser definida como o estudo das origens, evolução, distribuição e futuro da vida na Terra e no Universo (Blumberg, 2003). Sendo uma ciência multi e interdisciplinar, ela abrange diversas outras áreas científicas para pesquisar a vida em um contexto cósmico, desde a vida na Terra e sua relação dinâmica com o planeta ao longo do tempo até a procura por outros planetas e quais as possíveis condições necessárias (geológicas, biológicas e astrofísicas, por exemplo) para a existência de vida. Dentre as possibilidades de se abordar esse tema, a Astroquímica - estudo da formação, destruição e excitação de moléculas em ambientes astrofísicos e sua influência na dinâmica, estrutura e evolução de objetos astronômicos (Dalgarno, 2008) - fornece ferramentas para estudar moléculas orgânicas complexas (COMs, sigla em inglês) e prebióticas em ambientes astrofísicos.

Moléculas prebióticas podem ser definidas como moléculas que possivelmente estariam envolvidas nos processos que levam à origem da vida (Herbst e van Dishoeck, 2009). A presença interestelar dessas moléculas suporta a ideia de que o material orgânico necessário para o surgimento da vida na Terra também poderia ter tido origens extraterrestres, assim como pode indicar uma probabilidade da existência de vida ${ }^{1}$ em outros planetas. Nesse sentido, conhecer a distribuição dessas espécies moleculares no Universo é mais um passo para melhor compreender quão provável a vida pode ser, além dos mecanismos de formação, destruição e transporte desse material para planetas ou luas rochosas que possam permitir uma química prebiótica que leve às origens de vida.

Os blocos construtores da vida contêm, principalmente, os elementos $\mathrm{H}, \mathrm{C}, \mathrm{O}, \mathrm{N}$ e, em menor quantidade, P e S. Normalmente referidos como CHONPS, também estão entre os

\footnotetext{
${ }^{1}$ Vida similar à presente na Terra, baseada nos mesmos compostos químicos.
} 
elementos mais abundantes do Universo, com exceção dos últimos dois e o hélio. Por esse motivo, é de se esperar uma alta variedade de moléculas compostas por esses elementos em diversos ambientes astrofísicos. De fato, dentre as espécies já observadas no meio interestelar (ISM) e circunstelar, a maioria é de natureza orgânica, principalmente no caso de moléculas com seis ou mais átomos (Herbst e van Dishoeck, 2009).

Dentro da classe de macromoléculas, os hidrocarbonetos policíclicos aromáticos (PAHs, sigla em inglês) representam um arranjo efetivo para acumular carbono no Universo, sendo o material orgânico dominante no espaço e podendo conter cerca de $15 \%$ do carbono no ISM (Ehrenfreund et al., 2006). Devido à sua robustez, eles estão entre as mais abundantes espécies moleculares que devem ter sido transportadas quase intactas aos planetas, tanto por impactos de asteroides e cometas como por deposição de poeira interplanetária (Ehrenfreund et al., 2002).

Os PAHs podem constituir uma etapa de canais de produção de moléculas heterocíclicas nitrogenadas (N-heterociclos), especialmente quando seus átomos de hidrogênio ou carbono são substituídos por nitrogênio, dando origem aos PANHs (sigla em inglês para heterociclos policíclicos aromáticos nitrogenados). PAHs, PANHs e N-heterociclos são de grande interesse astroquímico e astrobiológico por poderem ter desempenhado um papel prebiótico nas ori-<smiles>c1ccccc1</smiles>

Benzene Pyrimidine

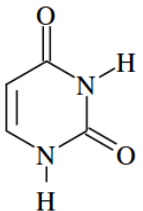

Uracil<smiles>Cc1c[nH]c(=O)[nH]c1=O</smiles>

Thymine<smiles>c1cncnc1</smiles>

Figura 1.1: Estrutura molecular do benzeno, pirimidina e as nucleobases uracil, timina e citosina. Imagem extraída de Mendoza et al. (2013). gens da vida em estágios antecessores do mundo RNA/DNA² (Ehrenfreund et al., 2006), tanto na Terra como em outros ambientes astrofísicos.

Além de serem processados em moléculas mais simples, enriquecendo a química do ISM, essas macromoléculas também podem estar ligadas à formação de nucleobases (Figura 1.1). Nucleobases são estruturas aromáticas compostas por moléculas monocíclicas ou bicíclicas que compõem os blocos básicos dos nucleotídios do RNA e DNA (Parker et al., 2015).

Além disso, algumas moléculas prebióticas mais simples também podem produzir não

\footnotetext{
${ }^{2}$ Estágio anterior às origens da vida, em que moléculas de RNA e, posteriormente, DNA, desempenharam o papel de catalisadores e moléculas genéticas na bioquímica terrestre (e.g. Joyce, 2002).
} 
só nucleobases como outro compostos essenciais à vida na Terra, como aminoácidos. A formamida $\left(\mathrm{NH}_{2} \mathrm{CHO}\right)$, por exemplo, tem se mostrado uma das moléculas prebióticas mais importantes que já foram detectadas no ISM. Sua primeira deteç̧ão ocorreu em 1971 na nuvem molecular gigante de Sgr B2 (Rubin et al., 1971). Estudos recentes mostraram a alta probabilidade de formação de moléculas prebióticas em asteroides por meio da irradiação da formamida por prótons (Saladino e et al., 2015).

Coelho (2018) simulou a abundância de algumas nucleobases em regiões de fotodissociação (PDRs, da sigla em inglês) através do código Meudon PDR. As reações de formação dessas moléculas a partir da formamida foram extraídas de Ferus et al. (2015). De acordo com as simulações, uracila e a citosina estariam dentro da faixa de possível detecção com abundâncias maiores que $10^{-13}$ em relação ao $\mathrm{H}_{2}$.

Neste cenário, a análise da composição química do ISM também pode revelar a formação, distribuição e complexidade de moléculas prebióticas em diferentes ambientes astrofísicos. Núcleos quentes moleculares, por exemplo, são objetos de estudos que podem fornecer tais informações, uma vez que possuem alta abundância de COMs em suas regiões internas (Bisschop et al., 2013).

\subsection{Moléculas aromáticas no meio interestelar}

PAHs são, basicamente, anéis de carbono aromáticos hexagonais com átomos de hidrogênio ligados na periferia (Andrews et al., 2015). Desde o naftaleno com somente dois anéis de benzeno até PAHs com mais de cem átomos de carbono, essas moléculas podem estar dispostas em várias estruturas (Figura 1.2). Dentre elas, os PAHs pericondensados e os simétricos estão entre os mais estáveis do ponto de vista da reatividade química e da fragmentação (Andrews et al., 2015).

Fundamental para o entendimento da região espectral do infravermelho médio (MIR, na sigla em inglês), essa classe de moléculas domina a emissão em banda nesses comprimentos de onda com as AIBs (sigla em inglês para Bandas Aromáticas no Infravermelho, Joblin et al., 1992). Ao serem expostos a fótons de alta energia de até $13.6 \mathrm{eV}$, os PAHs ficam eletronicamente excitados, alcançando temperaturas de até $1000 \mathrm{~K}$, e rapidamente redistribuem a energia absorvida entre todos os estados vibracionais disponíveis, podendo fragmentar ou emitir fótons no MIR através de um processo de fluorescência (Andrews 

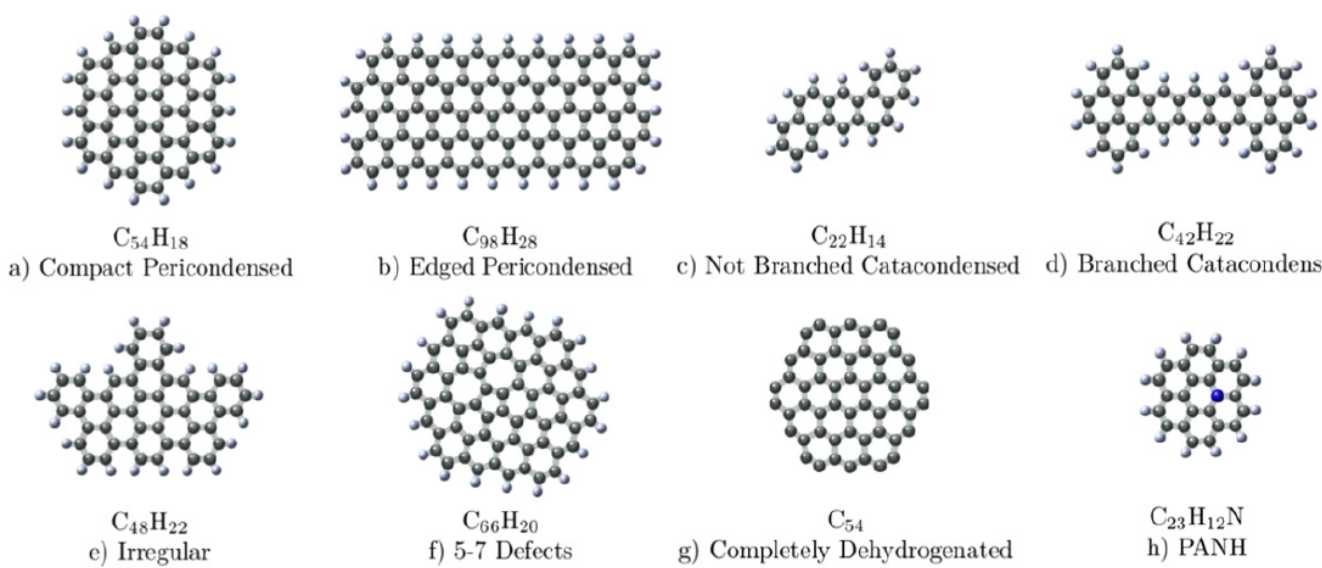

$\mathrm{C}_{22} \mathrm{H}_{14}$

$\mathrm{C}_{42} \mathrm{H}_{22}$

b) Edged Pericondensed

c) Not Branched Catacondensed

d) Branched Catacondensed

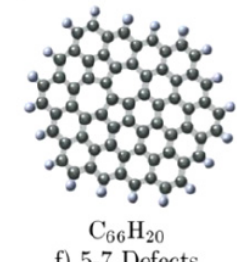

f) 5-7 Defects

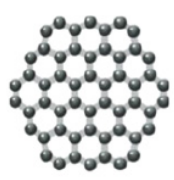

$\mathrm{C}_{54}$

g) Completely Dehydrogenated

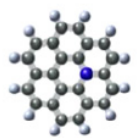

$\mathrm{C}_{23} \mathrm{H}_{12} \mathrm{~N}$

h) PANH

Figura 1.2: Exemplos de classificação da estrutura de PAHs. (a) e (b) - PAHs pericondensados: núcleos compactos de carbono. (c) e (d) - PAHs catacondensados: PAHs lineares. (e) - PAHs cuja forma não se assemelha às demais classificações. (f) - PAHs com 5 a 7 defeitos de anéis fundidos. (g) - PAHs completamente desidrogenados: todos os hidrogênios foram removidos. (h) - PAHs nitrogenados: o nitrogênio substituindo um átomo de carbono do núcleo do PAH. Imagem retirada de Andrews et al. (2015).

et al., 2015). Até $50 \%$ da luminosidade emitida nessa região espectral pode ser devida aos PAHs, com bandas de emissão mais intensas em 3.3, 6.2, 7.7, 8.6, 11.3 e $12.7 \mu \mathrm{m}$ (Li, 2004). Existem também bandas mais fracas atribuídas a essas moléculas, com comprimentos de onda em $3.4,3.5,5.25,5.75,6.0,6.9,7.5,10.5,11.0,13.5,14.2,17.4$ e $18.9 \mu \mathrm{m}$.

PAHs são abundantes e onipresentes em quase todos os ambientes astrofísicos relacionados com gás, poeira e iluminação por fótons ultravioleta (UV), como regiões HII, nebulosas de reflexão, estrelas jovens e AGBs (sigla em inglês para ramo assimptótico das gigantes), superfícies de nuvens escuras, entre outros (Tielens, 2008). Em especial, eles dominam o aquecimento de gás neutro e o balanço de ionização em nuvens moleculares e são importantes traçadores de regiões de formação estelar.

Por serem um material dominante e estável, espera-se que PAHs e macromoléculas carbonáceas exerçam um papel essencial na física e química do ISM (Berné et al., 2015). Um diagrama da evolução química dessas moléculas em diferentes ambientes astrofísicos, de acordo com a intensidade da radiação em cada um, pode ser visto na Figura 1.3. Em nuvens mais densas, grãos de poeira conseguem bloquear boa parte da radiação UV de estrelas próximas e há uma maior complexidade molecular. Quanto mais perto das bordas da nuvem, a intensidade do campo de radiação aumenta e passa a fragmentar os grãos em outras espécies moleculares menores.

No limite entre a nuvem molecular e a PDR, PAHs começam a ser altamente processa- 


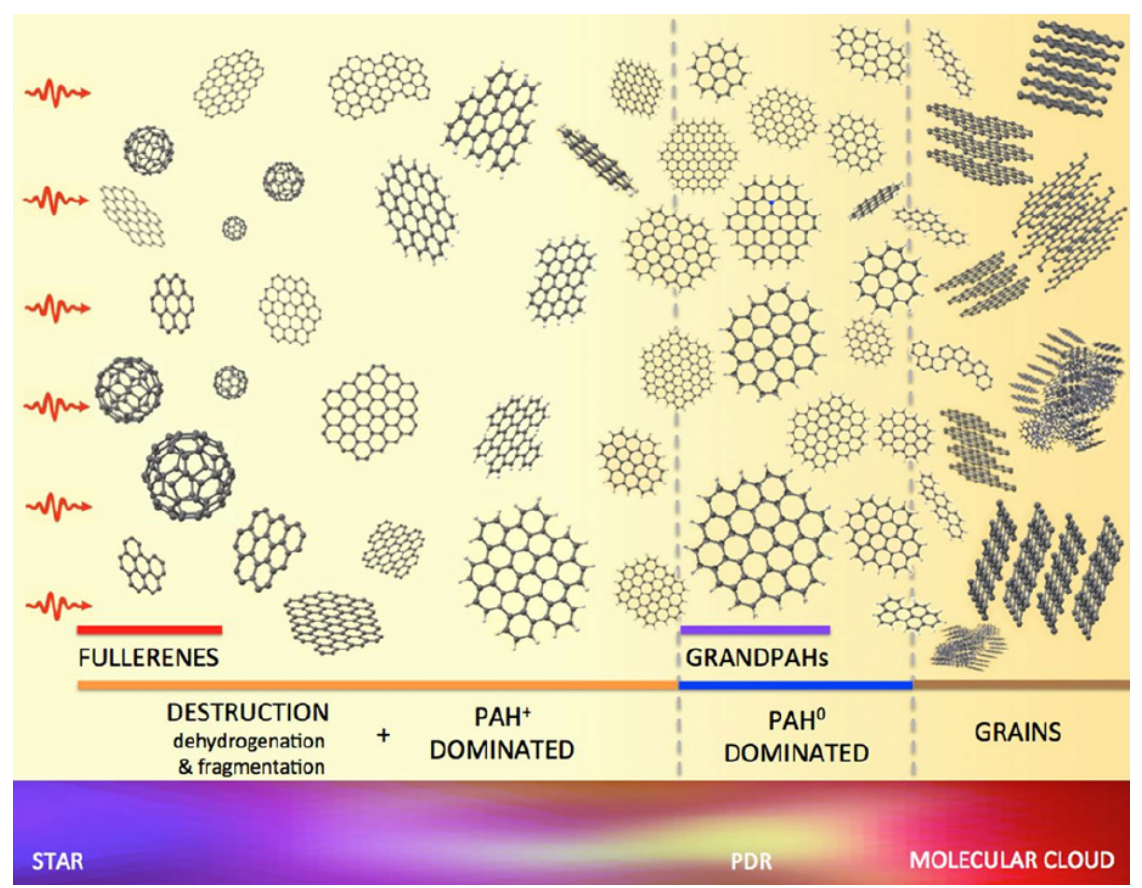

Figura 1.3: Evolução proposta de moléculas carbonáceas e PAHs mediante radiação UV em diferentes ambientes astrofísicos. Figura retirada de Andrews et al. (2015).

dos, formando uma população com as estruturas mais estáveis (grandPAH, Andrews et al., 2015). Conforme maior a proximidade da estrela, mais os PAHs são ionizados e destruídos, gerando moléculas cada vez menores. Dentre esses subprodutos dos PAHs, vale ressaltar o fulereno $\left(\mathrm{C}_{60}\right)$, formado a partir da isomerização de PAHs (Tielens, 2013). Apesar de ser uma molécula relativamente grande, sua estrutura em formato de bola de futebol permite que ele sobreviva em ambientes com radiação de altas energias.

\subsubsection{Variações nos perfis de PAH no infravermelho}

Uma análise mais aprofundada das observações das emissões de PAHs em diferentes ambientes astrofísicos revelou variações nos seus perfis de emissão. No geral, essas variações espectrais observacionais podem ser divididas em variações na intensidade relativa de diferentes bandas de emissão no MIR e variações na posição do pico e no perfil dessas bandas (Tielens, 2008).

As emissões de PAHs se originam, basicamente, dos modos de vibração C-C e C-H dessas moléculas. A Tabela 1.1 mostra algumas das bandas com os respectivos modos vibracionais a que estão ligadas. Enquanto os perfis referentes aos modos C-C podem ser muito mutáveis na região espectral de 5 a $9 \mu \mathrm{m}$, aqueles oriundos dos modos C-H 
sofrem pouca variação. Os modos C-H e C-C não estão necessariamente interligados (van Diedenhoven et al., 2004), mas já se está bem estabelecida na literatura a correlação entre as bandas de 3.3 e $11.2 \mu \mathrm{m}$, assim como a correlação ainda mais intensa entre as bandas de 6.2, 7.7 e $8.6 \mu \mathrm{m}$ (Peeters et al., 2017). Mais detalhes sobre as demais bandas e seus respectivos modos vibracionais e população emissora de PAHs podem ser encontrados em Peeters et al. (2017).

Tabela 1.1 - Modos de vibração de algumas bandas de PAHs (Hudgins e Allamandola, 1999; Tielens, 2005).

\begin{tabular}{cc}
\hline Intervalos $(\mu \mathrm{m})$ & Modo de vibração \\
\hline $3.2-3.4$ & $C$-H stretching \\
$6.1-6.5$ & $C$-C stretching \\
$6.5-8.5$ & $C$-C stretching $+C C$ in-plane bending \\
$8.3-8.9$ & $C$ - $C$ in-plane wagging \\
$11.0-15.0$ & $C$ - $H$ out-of-plane bending \\
\hline
\end{tabular}

As variações nos perfis podem estar relacionadas às condições físicas locais dos objetos e/ou às características da população de PAHs. No primeiro caso, pode ser apontado o efeito acumulado de processamento nas regiões onde a emissão originou-se; no segundo, destacam-se a composição da família de PAHs, tamanho molecular, carga, geometria e heterogeneidade (Sales et al., 2013).

Para melhor compreender essas variações, Peeters et al. (2002) sugeriu a divisão sistemática das bandas de PAHs de 6.2, 7.7 e $11.2 \mu \mathrm{m}$ em três classes - A, B e C - dependendo da interpretação da variação da posição do pico dos perfis. Posteriormente, essa classificação foi estendida para outras bandas por van Diedenhoven et al. (2004). A Tabela 1.2 resume o sistema de classificação de Peeters para as três bandas que serão estudadas nesse trabalho. As diferenças esperadas nos perfis de cada classe estão exemplificadas na Figura 1.4 .

Tabela 1.2 - Comprimentos de onda centrais das classes de Peeters (Peeters et al., 2002).

\begin{tabular}{cccc}
\hline Class & $6.2 \mu \mathrm{m}$ & $7.7 \mu \mathrm{m}$ & $8.6 \mu \mathrm{m}$ \\
\hline $\mathrm{A}$ & $<6.23$ & $\sim 7.6$ & $<8.6$ \\
\hline $\mathrm{B}$ & $6.23<\lambda<6.29$ & $\sim 7.8$ & $>8.6$ \\
\hline $\mathrm{C}$ & $>6.29$ & $\sim 8.22$ & \\
\hline
\end{tabular}



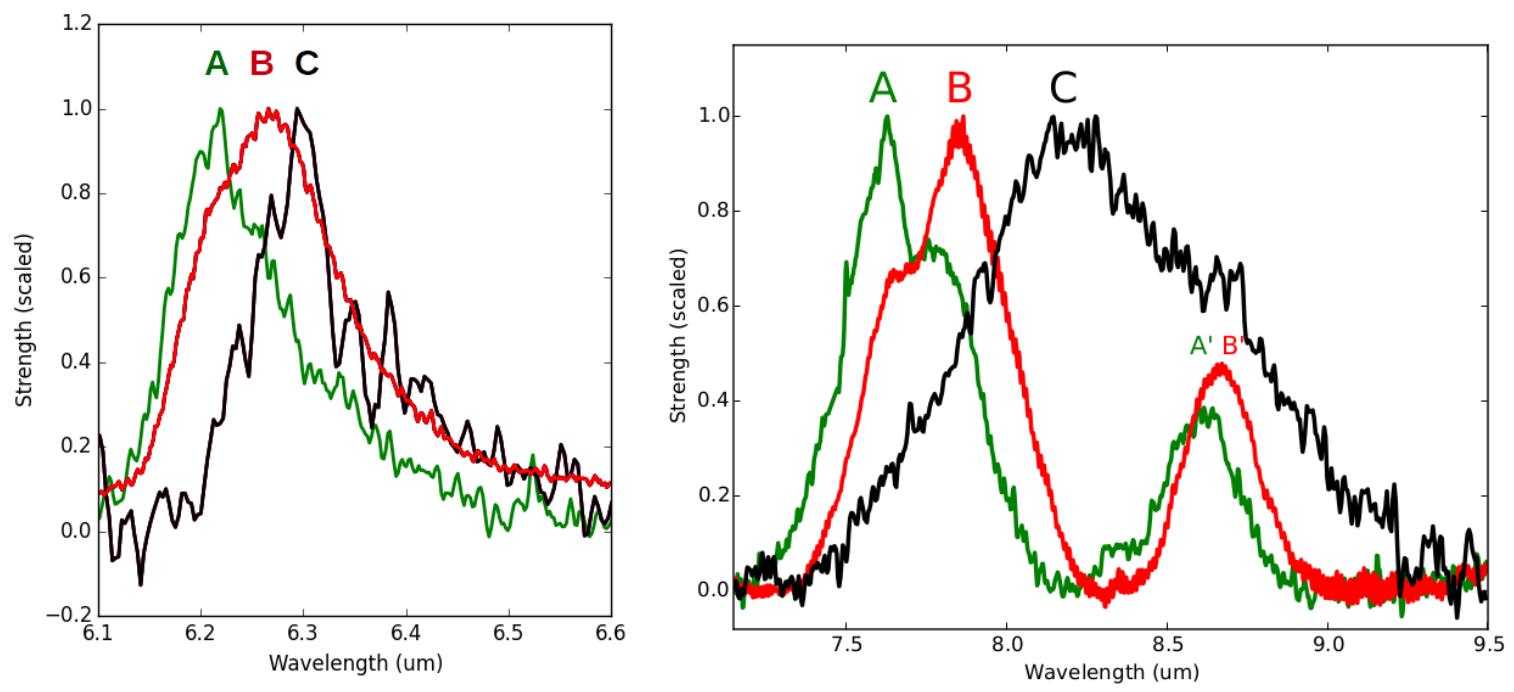

Figura 1.4: Visão geral das possíveis diferenças no perfil das bandas 6.2, 7.7 e $8.6 \mu \mathrm{m}$, divididas em três classes (A, B e C) e ilustradas pelos espectros normalizados dos objetos: A - região HII IRAS 23133+6050; B - estrela Pós-AGB HD 44179; e C - estrela Pós-AGB IRAS 13416 - 6243. Espectros normalizados disponibilizados por Peeters et al. (2002); van Diedenhoven et al. (2004).

É importante notar que a banda (ou complexo) de $7.7 \mu \mathrm{m}$ contém dois perfis distintos em 7.6 e $7.8 \mu \mathrm{m}$, ambos presentes em objetos classes A e B. Nessa situação, a relação de intensidade entre os dois pode ser usada para determinar a qual das classes o objeto pertence (por exemplo, Peeters et al., 2002, 2017). No caso da classe C, os dois perfis e a banda de $8.6 \mu \mathrm{m}$ são representados por um único e largo bump, que pode estar principalmente relacionado com grãos de poeira ou aglomerados de PAHs (Peeters et al., 2017).

Como mencionado, as diferentes classes também podem estar relacionadas aos ambientes astrofísicos em que os PAHs estão inseridos, que selecionam as principais populações emissoras. Normalmente, objetos classe A estão conectados a material interestelar iluminado por estrelas, como regiões HII, nebulosas de reflexão e meio interestelar em geral (da Galáxia e de objetos extragalácticos). Já a classe B está mais relacionada com material circunstelar, nebulosas planetárias, diversos objetos pós-AGB e estrelas Herbig AeBe. Por fim, a classe C tem sido atribuída a uns poucos objetos pós-AGB extremamente enriquecidos por carbono (Peeters et al., 2002; Tielens, 2008).

Ademais, Shannon e Boersma (2019) sugeriram um ciclo evolutivo aromático dependendo conjuntamente do ciclo de vida de estrelas de baixa e média massa. De acordo com o artigo, a classificação do complexo de $7.7 \mu \mathrm{m}$ está vinculada ao tipo de objeto: ambientes interestelares expostos mostram perfis da classe A enquanto perfis de classe B são observados principalmente em ambientes circunstelares, como previsto. Ambos os ciclos 
podem ser vistos esquematizados na Figura 1.5.

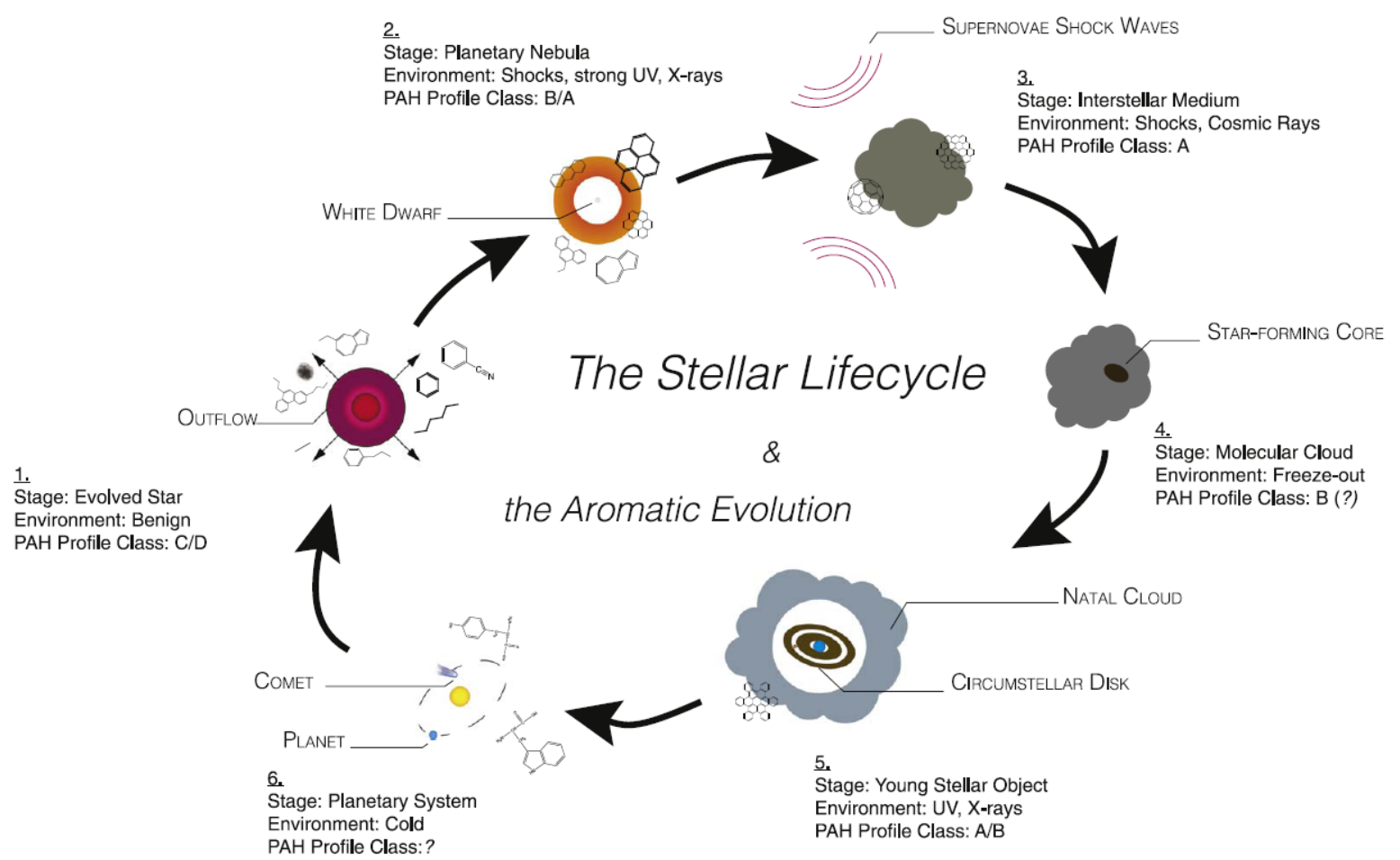

Figura 1.5: Representação simples e idealizada do ciclo de vida estelar de estrelas de baixa e média massa, sobrepostas à evolução dos PAHs e espécies relacionadas. Os materiais aromáticos são formados na ejeção de estrelas evoluídas, processados no ISM e incorporados aos sistemas estelares jovens. Imagem retirada de Shannon e Boersma (2019).

Como mencionado, outra causa para a variação nos perfis pode ser a população emissora de PAHs. Em muitos casos, PAHs ionizados ou neutros são usados para se explicar determinada classe (cátions, mais especificamente, estão mais relacionados com a classe A). Em outros, tais variações são utilizadas para se tentar inferir qual o intervalo de tamanho dos PAHs responsáveis pelas emissões (Ricca et al., 2018, por exemplo). Porém, outras condições também podem ser consideradas, como a heterogeneização.

Esse é o caso da banda de $6.2 \mu \mathrm{m}$ em objetos classe A, que apresenta um desvio da posição do pico para o azul, com comprimentos de onda menores que $6.22 \mu \mathrm{m}$. De acordo com os experimentos realizados por Hudgins et al. (2005), esse desvio não consegue ser esclarecido somente pelo aumento no tamanho do PAH. Da mesma forma, a substituição de átomos de carbono dos anéis aromáticos por Si, a substituição periférica de átomos de O, a complexação com átomos de metais e a diminuição da simetria molecular não são suficientes para reproduzir a posição dessa banda interestelar observada na classe A. 
Ainda segundo Hudgins et al. (2005), apenas a substituição dos átomos mais internos de carbono por nitrogênio (ou seja, PANHs) conseguiu reproduzir as observações e satisfazer as restrições astrofísicas envolvidas nessa questão simultaneamente. Esses resultados também possibilitaram estimar o grau de substituição de N na população de PAH e inferir um limite mínimo de 1 a $2 \%$ do nitrogênio cósmico capturado em PANHs.

Neste sentido, a identificação da classe da banda de $6.2 \mu \mathrm{m}$ pode ser um meio de mostrar se PANHs estão presentes e quão importantes podem ser para essa emissão. Tal estudo pode ampliar o censo do nitrogênio no Universo, uma vez que PANHs representariam um reservatório adicional desse elemento, em condições de densidade e temperaturas distintas daquelas da fase gasosa e em gelos.

Além da banda de $6.2 \mu \mathrm{m}$, PANHs também podem influenciar as bandas de 3.3, 7.7 e $11.2 \mu \mathrm{m}$ (van Diedenhoven et al., 2004). Especificamente, a banda de $7.7 \mu \mathrm{m}$ poderia indicar a presença de PANHs mesmo que a de $6.2 \mu \mathrm{m}$ não tenha sido observada, uma vez que ambas estão diretamente relacionadas devido ao mesmo modo vibracional (Tielens, 2005). Também foi constatado que, em alguns casos, as classes estão correlacionadas ao longo do espectro, principalmente a classe A.

A partir do que foi apresentado, pode-se perceber que o estudo dessas bandas de PAH, das classes de Peeters e de suas correlações é de extrema importância para o melhor entendimento da química e física do ISM.

\subsection{Moléculas aromáticas em galáxias}

Embora a proximidade dos objetos do ISM da Galáxia permita uma melhor resolução, incluindo resolução espacial para identificar emissões moleculares específicas de determinadas regiões em uma fonte, o estudo da química de objetos extragalácticos é bastante relevante. Além de fornecer um comparativo com nossa Galáxia e identificar as diferenças entre ambientes em galáxias distintas, ele também possibilita uma visão do passado e da evolução química do Universo. Antes de se discutir as observações e estudos dos PAHs do ponto de vista extragaláctico, será feita uma breve introdução sobre galáxias starburst e ativas, que serão mencionadas ao longo da tese. 


\subsubsection{Galáxias Starburst}

As galáxias que recebem essa nomenclatura apresentam taxas de formação estelar consideravelmente maiores do que o esperado em galáxias espirais como a nossa, ou seja, elas estão sofrendo um surto de formação estelar e podem ter uma taxa de 10 a $300 \mathrm{M}_{\odot} /$ ano $\left(\mathrm{M}_{\odot}=\right.$ massas solares $)$. Como comparação, a Via Láctea produz cerca de $2 \mathrm{M}_{\odot} /$ ano (Schneider, 2006). Normalmente, essa alta formação estelar ocorre nas regiões centrais das galáxias, onde a radiação UV liberada das novas estrelas é absorvida pela poeira e reemitida como radiação térmica no IR. A luminosidade dessas galáxias pode estar quase inteiramente concentrada no IR, como é o caso das Ultra Luminous Infra-Red Galaxies (ULIRGs).

ULIRGs são galáxias com as luminosidades infravermelhas mais intensas. Uma hipótese é que elas representariam uma etapa da fusão de duas galáxias espirais ricas em gás, normalmente chamadas de mergers. Quando esse gás molecular direciona-se para as regiões centrais do merger, ele inicia um surto de formação estelar e, em alguns casos, atividade AGN (e.e. Sanders et al., 1988; Guimarães, 2006), que será discutida a seguir. Também pode-se citar as Lyman Break Galaxies, que são galáxias com formação estelar intensa a altos redshifts.

\subsubsection{Galáxias Ativas}

Galáxias ativas são aquelas que possuem uma fonte de energia luminosa muito intensa nos seus centros, referida como núcleo galáctico ativo (AGN, da sigla em inglês). O AGN consiste em um buraco negro supermassivo e ativo que consome matéria de um disco de acreção, e se situa no centro da galáxia em questão. Além de emitirem radiação em todo o espectro eletromagnético, AGNs costumam ser tão brilhantes que ofuscam suas galáxias hospedeiras. Eles estão entre os objetos mais brilhantes e distantes já observados - os quasares (Schneider, 2006).

O modelo unificado de AGNs permite explicar a grande variedade de tipos de AGN observados ao se considerarem diferentes ângulos de observação da estrutura dessas galáxias. Na Figura 1.6, o buraco negro e o disco de acreção são mostrados no centro da galáxia, envolvidos por um toroide espesso de gás e poeira que absorve parte da radiação emitida pelo núcleo galáctico. A observação dessa região nuclear pode ser obstruída em proporções 
variadas de acordo com a linha de visada do observador em relação à orientação do toro. Por esse motivo, são observadas diferenças no brilho e espectros de diversos tipos de AGN.

Se houver a observação de jatos de partículas relativísticas gerados na vizinhança do buraco negro, os AGNs podem ser considerados como radio-loud (alto volume de rádio). Caso contrário, se jatos não forem observados, eles são chamados de radio-quiet (referidos como ruído silencioso). Exemplos do segundo caso são as galáxias Seyfert 1 e 2. Enquanto as Seyferts 1 apresentam tanto linhas de emissão estreitas (larguras em torno de $10000 \mathrm{~km} / \mathrm{s}$ ) como largas (larguras de vários 100 km/s) no óptico, Seyferts 2 só apresentam linhas estreitas (Schneider, 2006).

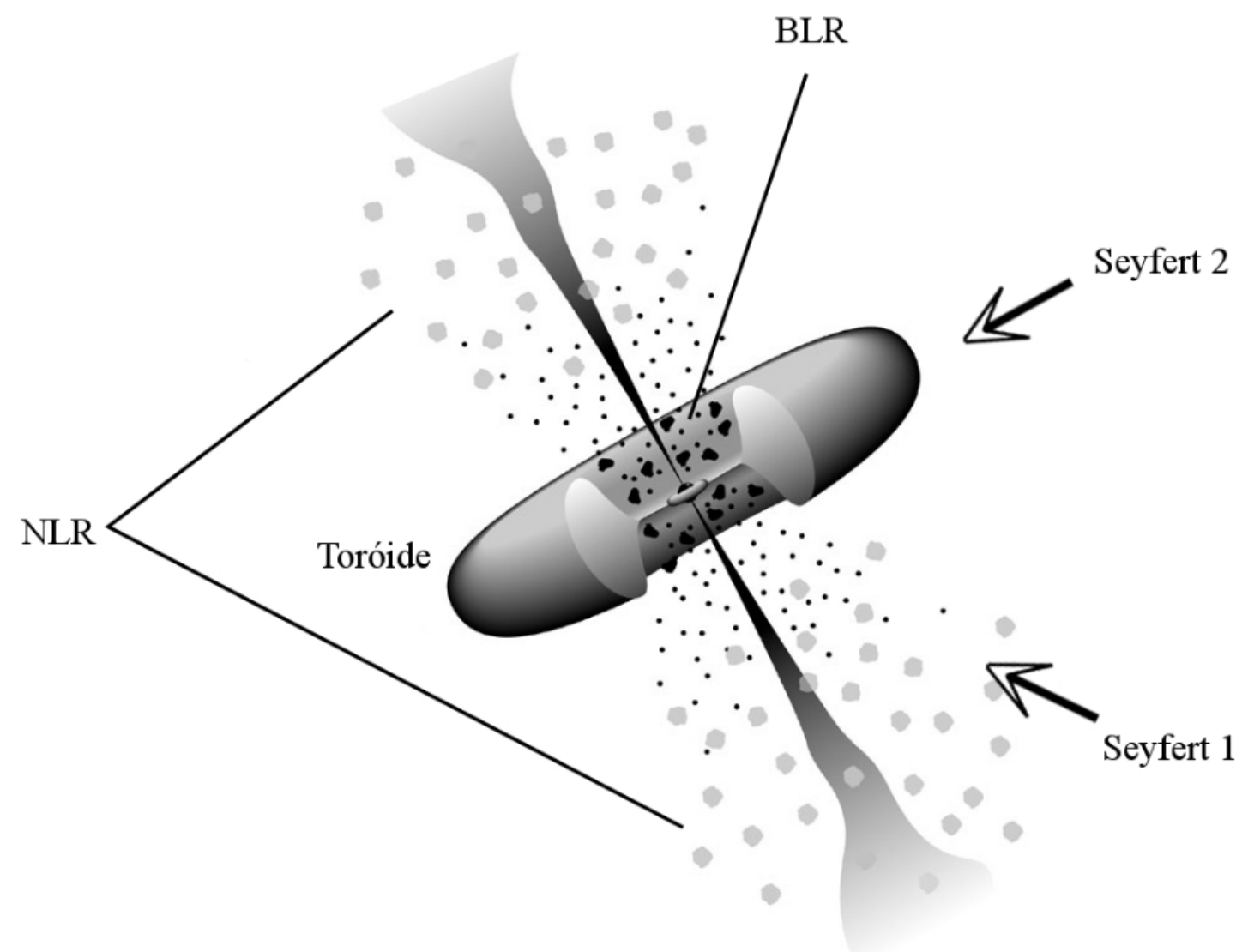

Figura 1.6: Esquema do modelo unificado de AGNs, no qual o tipo de objeto observado depende do ângulo de visada, como indicado pelas setas. Imagem retirada de Dutra (2015), adaptada de Urry e Padovani (1995). BLR - Broad Line Region; NLRGs - Narrow Line Region.

\subsubsection{PAHs em objetos extragalácticos}

Embora o estudo de PAHs tem se concentrado principalmente em objetos galácticos, suas emissões já foram detectadas em diversos objetos extragalácticos. Por causa da alta 
luminosidade das bandas de PAHs, elas podem ser visíveis até em objetos a alto redshift, onde dominariam o espectro no infravermelho (Papovich et al., 2006; Teplitz et al., 2007). Até 2009, o redshift mais elevado para o qual bandas dos PAHs foram detectadas era de $\mathrm{z}=$ 3.074 para a galáxia Cosmic Eye, uma Lyman Break Galaxy com forte lente gravitacional (Siana et al., 2009). Posteriormente, a banda de $6.2 \mu \mathrm{m}$ foi observada em $\mathrm{z}=4.055$ na galáxia submilimétrica GN20 (Riechers et al., 2014).

Bandas de PAH já foram utilizadas para estimar distâncias de galáxias através do cálculo do desvio para o vermelho (redshift) de seus comprimentos de onda. Dados de arquivo do instrumento IRS (Houck et al., 2004) a bordo do telescópio Spitzer (Werner et al., 2004), cuja faixa espectral concentra as mais fortes bandas de emissão dos PAHs, já foram usados com o objetivo de obter os redshifts de objetos a partir das bandas de PAHs identificadas (por exemplo, Yan et al., 2005, 2007).

Um tipo de objeto com muitas emissões de PAHs é o starburst, uma vez que está embebido em altas densidades de energia e quantidades significativas de gás e poeira. Dessa maneira, galáxias com emissão predominantemente de starbursts apresentam intensa emissão de PAH, como já estudado por Canelo (2016). Com uma amostra ampla desse tipo de galáxias, Canelo (2016) analisou e classificou a banda de $6.2 \mu \mathrm{m}$ de 169 objetos. Infelizmente, a utilização de dois métodos distintos para o ajuste do perfil não permitiu uma comparação com resultados da literatura. Ainda assim, uma dominância da classe A para esta banda, sugerindo uma forte contribuição de PANHs para a emissão, já pôde ser notada.

Mesmo sendo extremamente resistentes a altos níveis de radiação UV, emissões de PAH tendem a diminuir e se modificar em starburts altamente energéticos e chegam a desaparecer em galáxias AGN (Yan et al., 2007). Apesar disso, PAHs já foram detectados em regiões nucleares de galáxias de até uma dúzia de parsecs do AGN. Esse fato sugere a presença de um material empoeirado, como um toro nuclear ou disco, que permite a sobrevivência de moléculas de PAH em ambientes tão próximos a AGNs (Sales et al., 2010, 2013; Ruschel-Dutra et al., 2014; Alonso-Herrero et al., 2014, 2016; Monfredini et al., 2019). Os perfis de PAHs desses objetos, quando presentes, poderiam ser compatíveis com a classe $\mathrm{C}$ de Peeters, que apresenta um bump entre 7 e $9 \mu \mathrm{m}$.

A pesquisa de PAHs em fontes extragalácticas é fundamental para se estudar a evolução de galáxias e, consequentemente, do Universo. Além de possibilitar a comparação entre 
diferentes ambientes e fontes de radiação (estelar ou AGN), ela permite explorar galáxias a alto redshift que são observadas no IR. Essa é uma área que deve crescer nos próximos anos com o lançamento do James Webb Space Telescope (JWST ${ }^{3}$ ), que vai possibilitar a observação de galáxias com até z 10 (Stiavelli et al., 2009).

\subsection{Moléculas prebióticas no meio interestelar}

A partir das informações coletadas com as bandas de PAHs no MIR, não é possível especificar quais moléculas são responsáveis pelos perfis de emissão. Entretanto, N-heterociclos possuem elevado momento de dipolo, permitindo a medição de suas transições rotacionais em laboratório e, consequentemente, possibilitando suas detecções em comprimentos de onda de rádio no ISM (Charnley et al., 2005).

A busca por essas moléculas e seus precursores já ocorre desde 1973 com cianeto de vinila $\left(\mathrm{C}_{2} \mathrm{H}_{3} \mathrm{CN}\right)$, piridina $\left(\mathrm{C}_{5} \mathrm{H}_{5} \mathrm{~N}\right)$ e pirimidina $\left(\mathrm{C}_{4} \mathrm{H}_{4} \mathrm{~N}_{2}\right)$ (Simon e Simon, 1973). Evidências das mesmas moléculas em meteoritos e núcleos moleculares quentes (Kuan et al., 2003) estimulam novas explorações de suas emissões interestelares como, por exemplo, em envelopes de estrelas ricas em carbono. Infelizmente, N-heterociclos ainda não foram observados no ISM, provavelmente por causa das baixas abundâncias e o forte processamento por fotodissociação que dificultam as observações (Peeters et al., 2005; Schwell et al., 2008).

Adicionar mais moléculas no inventário astroquímico é essencial para testar teorias de formação, determinar suas capacidades de sobrevivência em diversos ambientes astrofísicos e restringir a mistura de compostos orgânicos disponível para a química pré-biótica (Charnley et al., 2005). Neste sentido, pequenas unidades de N-heterociclos tais como pirrol $\left(\mathrm{C}_{4} \mathrm{H}_{5} \mathrm{~N}\right)$, piridina e pirimidina são importantes alvos que devem ter suas assinaturas espectrais procuradas no ISM, uma vez que são fundamentais para a bioquímica terrestre e devem ter sido cruciais para as origens da vida na Terra.

A descoberta de uma das moléculas aromáticas mais simples com nitrogênio no ISM impulsiona a continuação dessa pesquisa. Transições hiperfinas resolvidas da benzonitrila $\left(\mathrm{c}-\mathrm{C}_{6} \mathrm{H}_{5} \mathrm{CN}\right)$ foram detectadas na nuvem molecular TMC-1 (McGuire et al., 2018). A benzonitrila também pode ser percursora da formação de PAHs mais complexos. Pirrol e piridina também foram procurados nessa fonte, mas não foram identificados.

\footnotetext{
3 https://www.jwst.nasa.gov/
} 


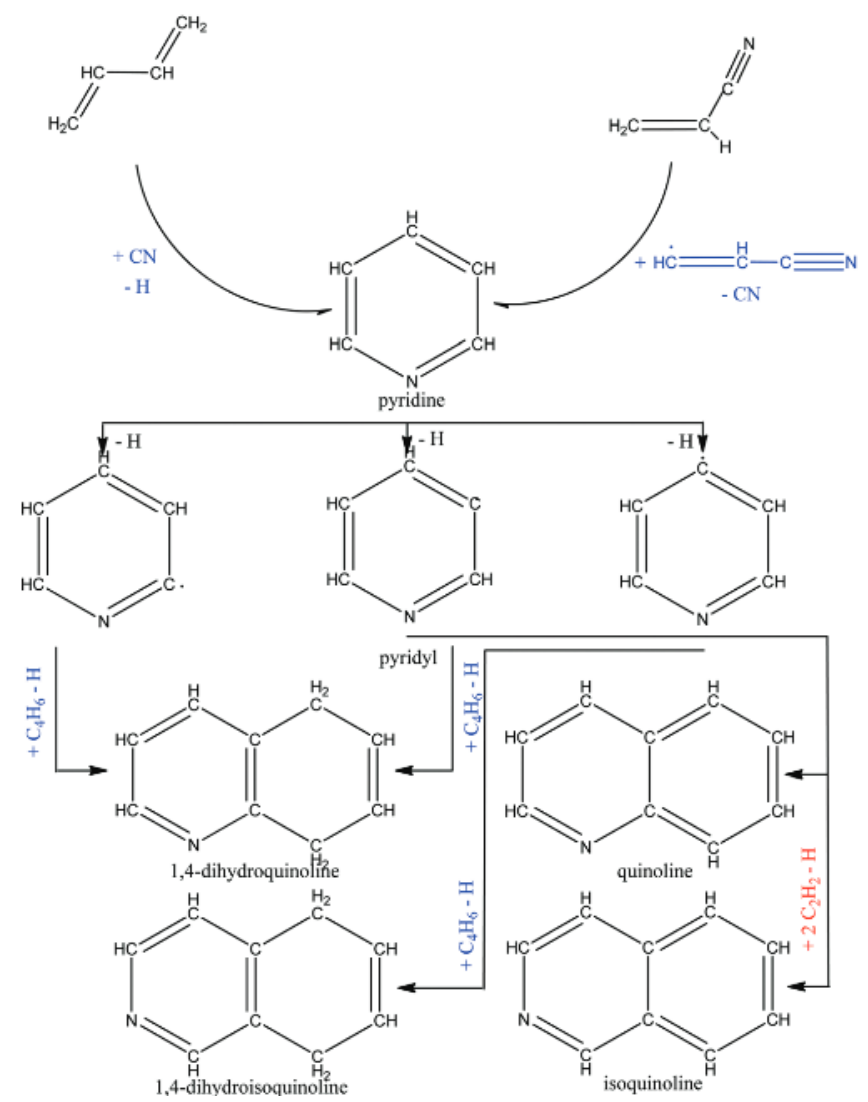

Figura 1.7: Rotas sintéticas para a construção de moléculas aromáticas monocíclicas e bicíclicas contendo nitrogênio. Figura extraída de Parker e Kaiser (2017).

Devido à dificuldade de observação dessas moléculas, uma outra abordagem é buscar emissões dos precursores destes N-heterociclos. Estudar suas rotas de formação e mesmo derivar os limites superiores de suas densidades de coluna (e.g. Charnley et al., 2005) podem fornecer um conhecimento maior de suas abundâncias e condições físico-químicas no ISM. A Figura 1.7 mostra rotas de formação de alguns N-heterociclos a partir do cianeto de vinila, evidenciando seu papel prebiótico. O cianeto de vinila não só foi detectado no ISM como também na lua de Saturno, Titã, pelo radiotelescópio Atacama Large Millimiter/submillimiter Array (ALMA $\left.{ }^{4}\right)$ (Palmer et al., 2017). Simulações anteriores sugeriam que essa molécula, no ambiente de Titã, pode ser a melhor candidata para a formação de hipotéticas membranas celulares (azotosomes, Stevenson et al., 2015). Contudo, Sandström e Rahm (2020) avaliaram a viabilidade termodinâmica da formação dessa membrana e, de acordo com os cálculos quânticos, elas não são candidatas viáveis à automontagem, semelhantes às bicamadas lipídicas em água líquida.

A formamida, como já mencionado, pode estar envolvida na formação de nucleobases, aminoácidos e açúcares e foi observada em ambientes astrofísicos como cometas (e.g Biver et al., 2014) e protoestrelas (e.g. Coutens et al., 2016). Além dela, alguns dos precursores de N-heterociclos mais simples e abundantes no ISM são $\mathrm{HNCO}, \mathrm{HC}_{3} \mathrm{~N}, \mathrm{C}_{3} \mathrm{H}$ e $\mathrm{HC}_{3} \mathrm{NH}^{+}$. Eles estão envolvidos em reações químicas propostas no ISM (tanto na fase gasosa como em gelo) cujos produtos finais incluem as nucleobases timina e uracil (Majumdar et al., 2015).

\footnotetext{
${ }^{4}$ www.almaobservatory.org/
} 


\subsubsection{HNCO}

Nesse trabalho, será dada uma ênfase maior ao ácido isociânico (HNCO). Ele é a molécula mais simples que contém quatro dos seis elementos biogênicos abreviados como CHONPS, presentes em todos os seres vivos. Além desse motivo, também pode ser considerado como uma molécula prebiótica por poder ser incluído como uma ligação peptídica [-(H)N-C(O)-] entre dois aminoácidos (Fedoseev et al., 2015), assim como a formamida (Ligterink et al., 2018). De fato, HNCO e formamida são as moléculas mais abundantes do ISM que possuem essa estrutura tipo amida.

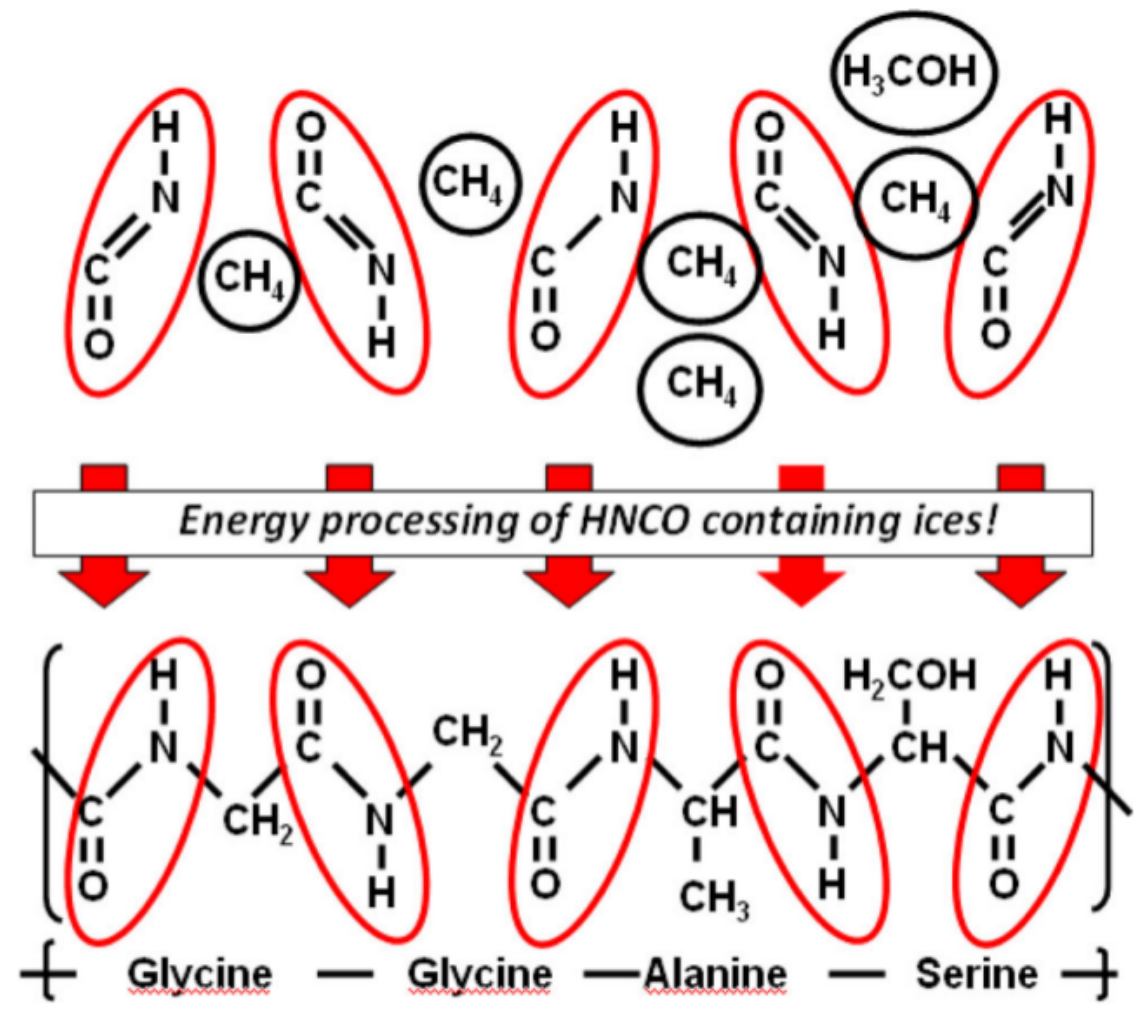

Figura 1.8: Esquema ilustrando a importância potencial do HNCO como um portador simples de ligações peptídicas para a produção de aminoácidos em gelos interestelares. Imagem retirada de Fedoseev et al. (2015).

A Figura 1.8 mostra um esquema da possível atuação do HNCO para formar aminoácidos, incluindo o mais simples deles - a glicina. A glicina, que é um bloco construtor de proteínas, já foi detectada na coma de $67 \mathrm{P} /$ Churyumov-Gerasimenko pelo espectrômetro de massa ROSINA (Rosetta Orbiter Spectrometer for Ion and Neutral Analysis), a bordo da sonda Rosetta (Altwegg et al., 2016). De acordo com os experimentos recentes, o processo de formação de moléculas com ligações peptídicas pode ocorrer a partir do HNCO e $\mathrm{CH}_{4}$ no estado sólido sob irradiação de fótons do UV distante (Ligterink et al., 2018). 
Tais condições são interessantes do ponto de vista astroquímico por serem semelhantes aos grãos de gelo interestelares.

Desde a primeira deteç̧ão de HNCO em 1972 no complexo de nuvens moleculares Sgr B2 (Snyder e Buhl, 1972), a presença do HNCO tem sido discutida em vários ambientes astrofísicos, incluindo outras nuvens moleculares, regiões protoestelares, cometas, atmosferas planetárias e até objetos extragalácticos. O HNCO interestelar foi proposto tanto como um traçador de gás denso, por já ter sido observado em regiões de alta densidade, como um traçador de choques, pois sua abundância parece ser elevada em regiões de choque de gás (Hernández-Gómez et al., 2019, e referências ali contidas).

Essa molécula também poderia estar presente na atmosfera superior de Titã, com abundâncias relativamente altas em relação aos muitos outros compostos que já foram identificados em espectros da Cassini/INMS (Dobrijevic et al., 2014). Por outro lado, as abundâncias interestelares do HNCO são relativamente baixas em nuvens ou regiões de formação estelar. De acordo com Altwegg et al. (2020), a evidência de sais de amônia no cometa 67P/Churyumov-Gerasimenko pode sugerir que espécies como haletos e HNCO estão na forma de sal, apresentando altas temperaturas de sublimação. Nessa situação, o HNCO precisaria de uma temperatura muito alta para ser evaporado dos grãos de gelo no ISM, o que dificultaria sua detecção na fase gasosa.

Considerando a formação do HNCO, reações na fase gasosa não são suficientes para explicar as abundâncias observadas. Pelo contrário, reações em superfícies de grãos também precisam ser levadas em conta para modelar as abundâncias do HNCO e de outras moléculas de interesse astrobiológico (Hernández-Gómez et al., 2019). Dentre elas, a reação $\mathrm{NH}+\mathrm{CO} \rightarrow \mathrm{HNCO}$, normalmente proposta em estudos espectroscópicos para explicar a formação de HNCO em misturas análogas de gelo interestelar processados por próton ou radiação UV, deve ser a principal via de formação dessa molécula (Fedoseev et al., 2015).

Outra questão significativa sobre o HNCO é sua provável relação química com a formamida, já apontada com uma importante molécula prebiótica. Evidências observacionais apontam para uma correlação entre as duas espécies, que apresentam uma razão de abundância molecular de $[\mathrm{HNCO}] /\left[\mathrm{NH}_{2} \mathrm{CHO}\right]$ 3.0 para várias fontes galácticas (Figura 1.9, Mendoza et al., 2014). Segundo Bisschop et al. (2007), as duas espécies podem compartilhar um esquema comum de formação no estado sólido (grãos de gelos no ISM), incluindo condições similares durante a formação e/ou equilíbrio entre os processos formação e des- 
truição. Elas também podem estar conectadas por causa de seus precursores em comum (López-Sepulcre et al., 2019).

Ademais, foi sugerida a produção de formamida diretamente do HNCO a partir da adição de átomos de hidrogênio (hidrogenação) em superfícies de grãos. Contudo, experimentos demostraram que esse processo possui uma barreira de ativação e é ineficiente (Fedoseev et al., 2015; Noble et al., 2015; Ligterink et al., 2018). Entretanto, Haupa et al. (2019) mostraram recentemente a possível ligação entre $\mathrm{HNCO}$ e formamida na fase sólida usando uma matriz de $\mathrm{H}_{2}$ a $3 \mathrm{~K}$ como substrato, na presença de átomos de H. Nessas condições, as duas espécies são ligadas por meio de um ciclo du-

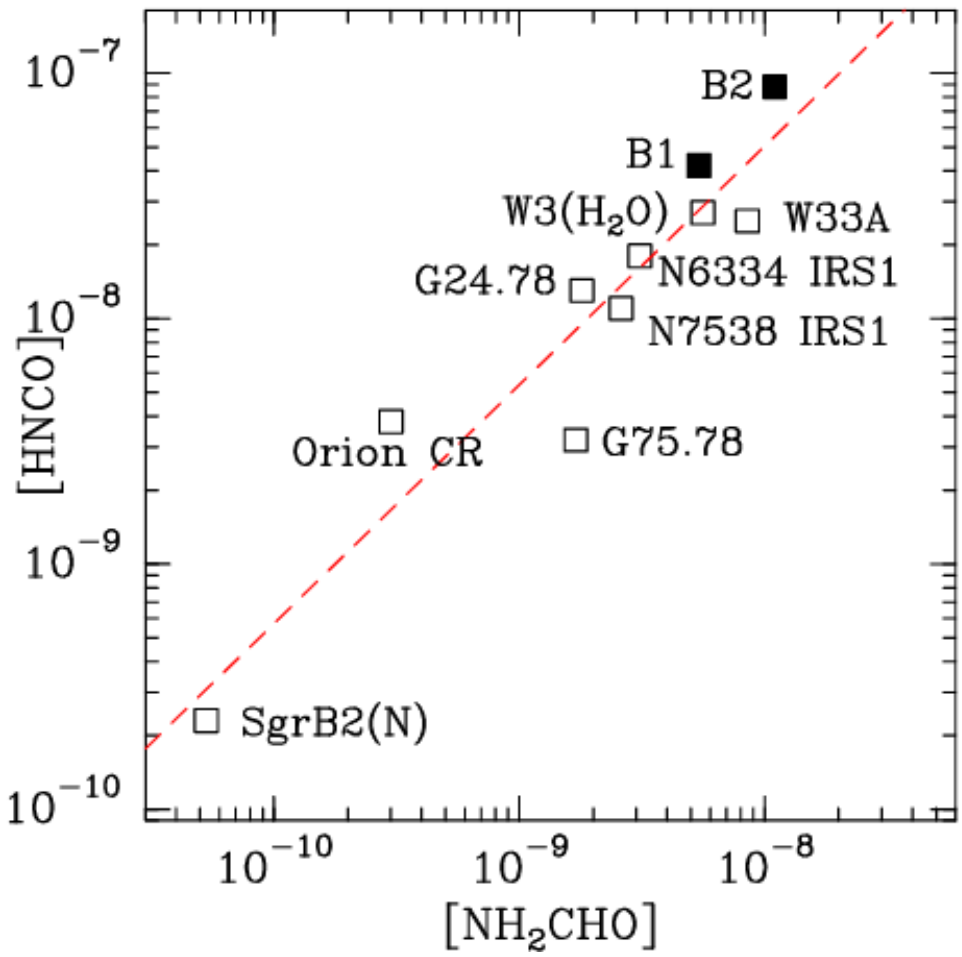

Figura 1.9: Comparação de abundâncias moleculares para $\mathrm{NH}_{2} \mathrm{CHO}$ e $\mathrm{HNCO}$ em uma amostra de fontes galácticas. A linha tracejada representa o melhor ajuste de potência $[\mathrm{HNCO}]=3.0 \times\left[\mathrm{NH}_{2} \mathrm{CHO}\right]^{0.97}$. Gráfico extraído de Mendoza et al. (2014). plo que consiste em abstração e adição de H (Figura 1.10).

Também foi demonstrado que as moléculas de formamida são principalmente convertidas em HNCO na presença de uma grande quantidade de átomos de $\mathrm{H}$, por causa da reação 3 da Figura 1.10, que possui uma pequena barreira (Haupa et al., 2019). Além de reproduzir as observações astronômicas, esse fato permite explicar por que o HNCO é sistematicamente mais abundante que a formamida, mesmo não sendo sua molécula-mãe (López-Sepulcre et al., 2019). 

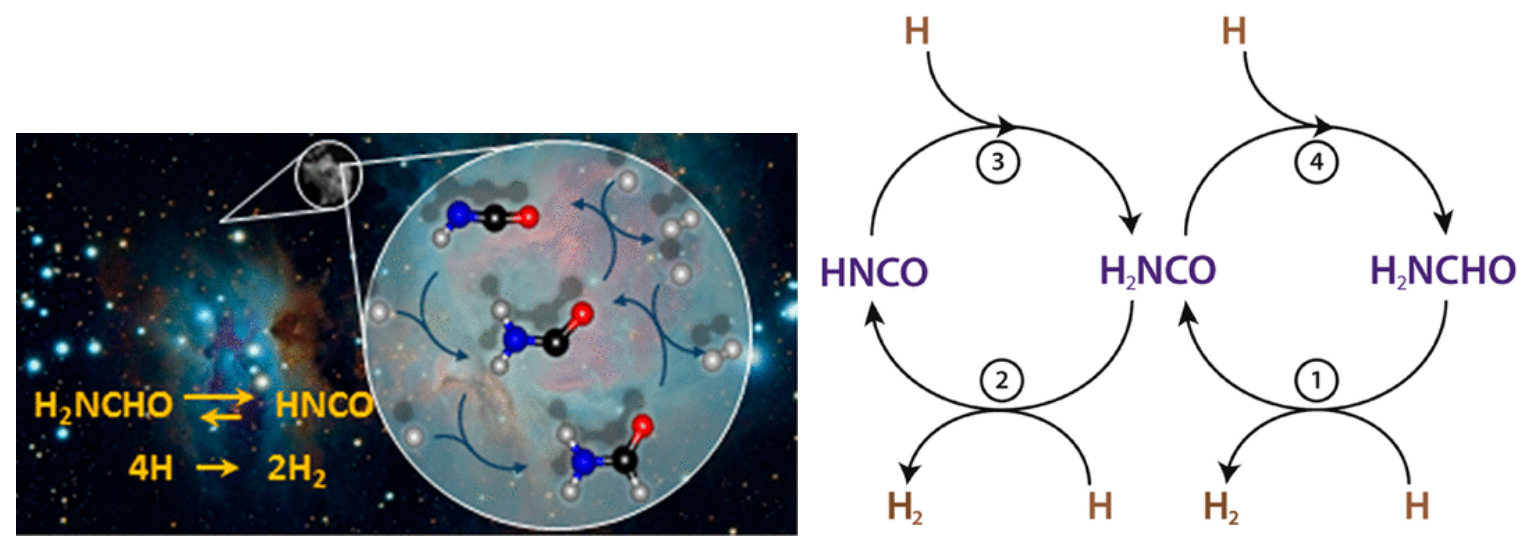

Figura 1.10: Duas representações do mecanismo bicíclico de reações de abstração e adição de $\mathrm{H}$ que correlacionam $\mathrm{NH}_{2} \mathrm{CHO}, \mathrm{H}_{2} \mathrm{NCO}$ e $\mathrm{HNCO}$ em grãos de gelo interestelares. De acordo com esse mecanismo, a formação catalítica de $\mathrm{H}_{2}$ a partir de átomos de $\mathrm{H}$ também pode ocorrer. Figuras retiradas de Haupa et al. (2019).

\subsection{Organização da tese e objetivos}

As análises e resultados da tese estão divididos em duas grandes partes nos respectivos Capítulos 2 e 3. A primeira parte abrange o estudo de moléculas aromáticas no MIR. O objetivo principal desse estudo é analisar emissões dessa classe de moléculas para uma amostra de galáxias, classificar as bandas de PAH de acordo com o método de Peeters e analisar as relações entre as diferentes bandas e suas respectivas classes. Também se pretende interpretar a relação entre as bandas e os tipos de galáxias observados, especialmente ao longo do redshift das fontes. Por fim, serão discutidas possíveis identificações de populações emissoras de PAHs nestes ambientes, como a contribuição de PANHs para a banda de $6.2 \mu \mathrm{m}$. A discussão é dada através de dois artigos. O primeiro (Canelo et al., 2018, seção 2.1), publicado no jornal Monthly Notices of the Royal Astronomical Society (MNRAS) pela Oxford University Press em nome da Royal Astronomical Society, discute justamente a banda de PAH de $6.2 \mu \mathrm{m}$. Além de ajustá-la para uma amostra de galáxias com emissão dominada por starburst, a interpretação da classificação de Peeters é sugerida como uma possível maneira de identificação da emissão de PANHs. A amostra estudada é a mesma de Canelo (2016). Contudo, Canelo (2016) usou a ferramenta PAHFIT para ajustar os contínuos das galáxias e o perfil de Lorentz para ajustar a banda de duas maneiras diferentes. Canelo et al. (2018), por outro lado, utiliza splines para o contínuo e Gaussiana para o perfil da banda. A metodologia é padronizada em relação aos trabalhos 
anteriores e é discutida na seção 2.1 .

O segundo artigo (Canelo et al., submitted, seção 2.2), submetido ao MNRAS, expande o estudo anterior para as bandas de 7.7 e $8.6 \mu \mathrm{m}$ e também usa os resultados de Canelo et al. (2018) para comparar as três bandas de PAH e suas correlações. Diversos testes foram realizados ao longo do doutorado para os ajustes dessas duas bandas de PAH. Foi considerada a contribuição de emissões mais tênues de outros PAHs e também a composição de diferentes perfis a serem ajustados dependendo da banda - perfil de Drude, de Lorentz e/ou Gaussiana. Finalmente, optou-se por continuar a metodologia de Canelo et al. (2018) para se manter a padronização do trabalho. Foram utilizadas somente três Gaussianas (duas para o complexo de $7.7 \mu \mathrm{m}$ ) e manteve-se o spline local ${ }^{5}$ para o contínuo.

O Capítulo 3, por sua vez, foca em moléculas menores do que os PAHs, as COMs. Nessa segunda parte, o trabalho é feito na região do sub/milimétrico em que transições rotacionais podem ser observadas. O principal objetivo é observar e estudar precursores de N-heterociclos e outras COMs de interesse prebiótico. Também são estudadas as abundâncias e rotas de formação de algumas COMs através de simulação química de algumas fontes do ISM da Galáxia. Em suma, esse capítulo é uma abordagem complementar a do anterior, mostrando outras possibilidades de pesquisa em Astroquímica e Astrobiologia.

A seção 3.2 discute o núcleo molecular quente G331.512-0.103, que já possui diversas campanhas de observação. Dentre elas, são mostradas possíveis identificações de várias linhas de emissão de COMs. Em seguida, a seção 3.2.1 se concentra nas observações do HNCO nessa fonte. Uma molécula simples e prebiótica, o HNCO foi observado pela antena APEX (Atacama Pathfinder EXperiment ${ }^{6}$ ) em diversas transições. Análises dessa molécula contribuem para o melhor entendimento desse objeto, tanto de suas condições físicas como da sua complexidade química.

Modelagens e simulações químicas são ferramentas importantes para auxiliar a interpretação dos resultados observacionais. Elas também fornecem uma visão dos processos de formação e destruição de moléculas aplicada em ambientes astrofísicos. Nesse sentido, as seções 3.2.1.2 e 3.3 mostram simulações realizadas com o código NAUTiLus para duas fontes diferentes do ISM, G331.512-0.103 e o núcleo frio infravermelho IRDC-C9 Main. As

\footnotetext{
${ }^{5}$ No caso da região espectral de 7 a $9 \mu \mathrm{m}$, dois tipos de splines podem ser usados para o ajuste do contínuo. Ambos foram testados e o spline local foi escolhido. Essa discussão é feita na seção 2.2.

6 http://www.apex-telescope.org/
} 
moléculas simuladas variam entre as fontes porque dependem de suas respectivas observações.

Finalmente, o Capítulo 4 resume os principais resultados e as conclusões dos tópicos trabalhados ao decorrer da tese, assim como suas perspectivas. No Apêndice A, os ajustes do contínuo e das bandas de PAH, que foram discutidos no Capítulo 2, são apresentados em forma de figuras para todas as galáxias estudadas. 
Capítulo 2

PARTE I - Aromáticos no infravermelho médio

2.1 Artigo publicado sobre a banda de $6.2 \mu \mathrm{m}$ em galáxias dominadas por starbursts 


\title{
Variations in the $6.2 \mu \mathrm{m}$ emission profile in starburst-dominated galaxies: a signature of polycyclic aromatic nitrogen heterocycles (PANHs)?
}

\author{
Carla M. Canelo, ${ }^{1 \star}$ Amâncio C. S. Friaça, ${ }^{1}$ Dinalva A. Sales, ${ }^{2}$ Miriani G. Pastoriza ${ }^{3}$ \\ and Daniel Ruschel-Dutra ${ }^{4}$ \\ ${ }^{1}$ Departamento de Astronomia, Instituto de Astronomia, Geofísica e Ciências Atmosféricas, Universidade de São Paulo, 05508-090 São Paulo, Brazil \\ ${ }^{2}$ Instituto de Matemática, Estatística e Física, Universidade Federal do Rio Grande, 96201-900 Rio Grande do Sul, Brazil \\ ${ }^{3}$ Instituto de Física, Universidade Federal do Rio Grande do Sul, 91501-970 Rio Grande do Sul, Brazil \\ ${ }^{4}$ Centro de Física e Matemática, Universidade Federal de Santa Catarina, 88040-900 Santa Catarina, Brazil
}

Accepted 2017 December 27. Received 2017 December 22; in original form 2017 June 14

\begin{abstract}
Analyses of the polycyclic aromatic hydrocarbon (PAH) feature profiles, especially the $6.2 \mu \mathrm{m}$ feature, could indicate the presence of nitrogen incorporated in their aromatic rings. In this work, 155 predominantly starburst-dominated galaxies (including $\mathrm{H}$ II regions and Seyferts, for example), extracted from the Spitzer/Infrared Spectrograph ATLAS project, have their $6.2 \mu \mathrm{m}$ profiles fitted allowing their separation into the Peeters' A, B, and C classes. 67 per cent of these galaxies were classified as class A, 31 per cent were as class B, and 2 percent as class C. Currently, class A sources, corresponding to a central wavelength near $6.22 \mu \mathrm{m}$, seem only to be explained by polycyclic aromatic nitrogen heterocycles (PANHs), whereas class B may represent a mix between PAHs and PANHs emissions or different PANH structures or ionization states. Therefore, these spectra suggest a significant presence of PANHs in the interstellar medium (ISM) of these galaxies that could be related to their starburst-dominated emission. These results also suggest that PANHs constitute another reservoir of nitrogen in the Universe, in addition to the nitrogen in the gas phase and ices of the ISM.
\end{abstract}

Key words: astrobiology-astrochemistry-ISM: molecules-galaxies: ISM-infrared: galaxies.

\section{INTRODUCTION}

A considerable fraction of the carbon in the interstellar medium (ISM), 20 percent or more, is in the form of polycyclic aromatic hydrocarbons (PAHs; Joblin, Leger \& Martin 1992). In addition, the mid-infrared (MIR) emission from many objects is dominated by bands of a molecular class, which includes PAHs, sometimes referred to the Aromatic Infra-red Bands (AIBs; Joblin et al. 1992). All other classes of organics and inorganics represent only a tiny fraction of the emitting material that contributes to the AIBs (Allamandola, Hudgins \& Sandford 1999). Up to 50 per cent of the luminosity emitted in the MIR can be due to PAHs, with the most prominent bands emitting at 3.3, 6.2, 7.7, 8.6, 11.3, and $12.7 \mu \mathrm{m}(\mathrm{Li}$ 2004). Their high luminosity allows them to be observed in highredshift objects, where they may dominate the IR spectrum range (Papovich et al. 2006; Teplitz et al. 2007). Recently, the largest redshift in which PAH bands were detected was for the Cosmic Eye Galaxy with $z=3.074$, a Lyman Break Galaxy with strong gravitational lens (Siana et al. 2009). Years later, the $6.2 \mu \mathrm{m}$ band

^E-mail: camcanelo@gmail.com was observed in the submillimetre galaxy GN20, with a redshift of $z=4.055$ (Riechers et al. 2014).

Because of their stable molecular structure, PAHs are the dominant molecular organic material in space (Ehrenfreund et al. 2006) and, together with other aromatic macromolecules, they are the most abundant class of molecular species that must have been transported to the planets by comets, meteorites, and interplanetary dust deposition (Ehrenfreund et al. 2002). Produced in other parts of the Solar system or Galaxy, they have been delivered almost intact to planets such as Earth and Mars. They could also have undergone a stage of production of nitrogen heterocyclic molecules which, along with PAHs, are of a great astrobiological interest. In addition, in the PAH World model for the origins of life, they played a key role in the stages preceding the RNA World (Ehrenfreund et al. 2006), not only on Earth but in other astrophysical environments as well.

When a PAH incorporates nitrogen in place of a carbon atom, it is called a polycyclic aromatic nitrogen heterocycle (PANH). It has been suggested that a significant fraction of the nitrogen in the ISM is depleted into PANHs (Hudgins et al. 2005). Peeters et al. (2002) considered the $6-9 \mu \mathrm{m}$ spectral range of several astrophysical objects and studied the presence of profile variations among the PAH bands. They found that their sample could be separated into 
three different classes - A, B, and C-depending on the peak positions of the bands. Later, van Diedenhoven et al. (2004) extended the approach for the 3.3 and $11.2 \mu \mathrm{m}$ bands and revealed a correlation between the classification of the PAH bands and their profiles.

In general, an A classification in the 6-9 $\mu \mathrm{m}$ region also implies an $A_{3.3}$ and $A_{11.2}$, but $B_{3.3}$ and $B_{11.2}$ do not necessarily correlate with each other or with $\mathrm{B}_{6-9}$ and $\mathrm{C}_{6-9}$. Normally, the profile $\mathrm{A}$ peaks at $6.2 \mu \mathrm{m}$ while profiles $\mathrm{B}$ and $\mathrm{C}$ peak at longer wavelengths. The classes A and B differ largely in the relative strength of subcomponents at 7.6 and $7.8 \mu \mathrm{m}$, which seem to have shifted to $8.2 \mu \mathrm{m}$ for class C (Tielens 2008). Also, the classes are linked to the type of source. Class A sources are associated with interstellar material illuminated by a star, including $\mathrm{H}$ II regions, reflection nebulae, and the general ISM of the Milky Way and other galaxies. Class B objects are associated with circumstellar material and include planetary nebula, a variety of post-AGB objects and Herbig AeBe stars. Class $\mathrm{C}$ sources are limited to a few extreme carbon-rich post-AGB objects.

Thus, analyses of PAH feature profiles, especially the $6.2 \mu \mathrm{m}$ band, could indicate the presence of nitrogen incorporated into the rings. The class A $6.2 \mu \mathrm{m}$ band corresponds to a central wavelength at $6.22 \mu \mathrm{m}$ and has only been well reproduced by carbon replaced by nitrogen into the aromatic rings (Hudgins et al. 2005). These PAH features are prominent in star-forming systems, reduced and modified in high-intensity starbursts and, eventually, disappear in active galactic nuclei (AGNs) systems (Yan et al. 2007). The spectral continuum shape of starburst spectra is dominated by strong emission features from PAHs (Genzel \& Cesarsky 2000) and the 5-8 $\mu \mathrm{m}$ spectral range of starburst galaxies is not only extremely rich in atomic and molecular emission and absorption features but is also dominated by emission from the $6.2 \mu \mathrm{m}$ PAH feature and the blue wing of the $7.7 \mu \mathrm{m}$ PAH complex (Brandl et al. 2006). In fact, the emission and absorption features of dust grains predominate the MIR spectra of starburst galaxies and most ULIRGs (ultraluminous infrared galaxies; Yan et al. 2005).

Identification of the feature classes can show if PANHs may be present in the spectrum of the sources and account for this PAH band of the MIR emission. With this in mind, we here analysed and classified the $6.2 \mu \mathrm{m}$ feature of 206 galaxies observed with Spitzer according to the Peeters' classes, searching for the PANH contribution to this PAH band. This paper is structured as follows: Section 2 explains the selection of our sample and Section 3 describes the data analysis performed in the spectroscopic data. Results are discussed in Section 4 and Section 5 presents the summary and conclusion.

\section{DATA SELECTION}

The Spitzer/Infrared Spectrograph (IRS) ATLAS project (HernánCaballero \& Hatziminaoglou 2011) possesses around 750 reduced spectra of several types of extragalactic objects, such as Seyfert, radiogalaxies, and submillimetre galaxies. They were observed in low and high resolution by the IRS (Houck et al. 2004) of the Spitzer Space Telescope (Werner et al. 2004) and their reduced spectra were extracted from Post-script figures uploaded to the arxiv.org pre-print service by their authors. The ATLAS project also offers the best redshift values of the sources found in the literature and checked with NED (NASA Extragalactic Database). The data are not at the rest wavelength and, for this correction, we used values published by the authors (see Table A1). However, in the case of high redshifts, the values could have been obtained only through PAH bands themselves or through the IRS spectrum, with no other accurate measure. According to Weedman et al. (2006), the uncer-

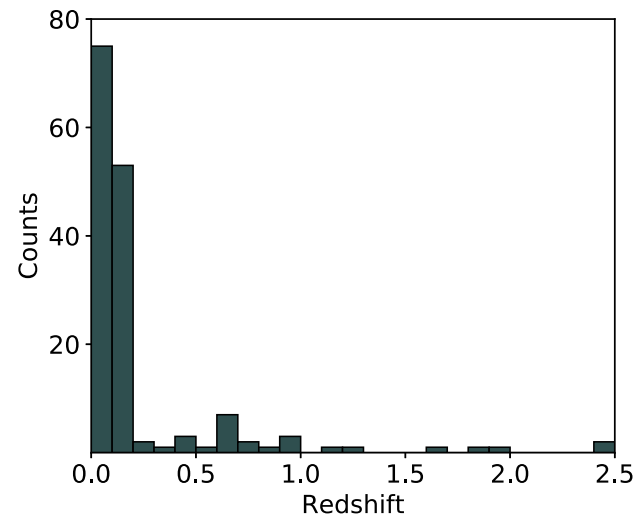

Figure 1. Histogram of the 155 redshifts of our galaxy's sample.

tainties for spectroscopic redshifts greater than 1 from IRS spectrum are typically \pm 0.2 . These sources are indicated in the table. This situation may interfere with the rest wavelengths of the spectra and compromise the original peak position of the features. For this reason, these cases must take into account a possible extra shift in the $6.2 \mu \mathrm{m}$ central wavelength for the analysis.

The spectra may have an inferior quality if compared to the fully and properly reduced spectra because, in some cases, the flux uncertainties are missing or the spectra are smoothed, for instance. The accuracy with which the original wavelength and flux values are recovered is limited by the resolution of the Post-script figure, once they were transformed from the Post-script coordinates (sets of points representing the spectra in the figures; Hernán-Caballero \& Hatziminaoglou 2011). Nevertheless, according to the authors, the resulting introduced uncertainty to the wavelength calibration is an order of magnitude smaller than the spectral resolution $(R \sim 100)$ in the low-resolution module of IRS. Therefore, its impact is negligible and statistical analysis of the sample is little, if at all, affected (Hernán-Caballero \& Hatziminaoglou 2011).

For a better understanding of the PANH distribution in the Universe, starburst galaxies are the best targets since they carry different burst of young stellar population and, consequently, present strong PAH emission in the MIR spectral wavelengths. We therefore selected objects from the ATLAS MIR starburst-dominated galaxies (MIR_SB sample), which is composed of 257 sources previously classified as starburst-dominated by Hernán-Caballero \& Hatziminaoglou (2011). The limit set between AGN- and starburstdominated sources was based on the fraction of a PDR (photodissociation region) component at $r_{\mathrm{PDR}}=0.15$, corresponding to equivalent widths $(\mathrm{EW})$ of $\mathrm{EW}_{6.2}=0.2 \mu \mathrm{m}$ or $\mathrm{EW}_{11.3}=0.2 \mu \mathrm{m}$ as an alternative boundary.

From their subsample, 219 objects have a wavelength coverage that includes the $6.2 \mu \mathrm{m}$ PAH band. However, some galaxies do not have enough data points for a trustworthy fit or the profiles were peculiar and were not used in this work. We also included two other ULIRGs observed by Yan et al. (2007) due to their strong $\mathrm{PAH}$ features. Table A1 presents information of our sample that is composed by 155 galaxies. The distribution of the redshifts can be seen in Fig. 1 .

\section{DATA ANALYSIS}

Before the $6.2 \mu \mathrm{m}$ feature profile was fitted, the spectral contributions of the silicate absorption and line emissions were subtracted 
from the spectra using PAHFIT (Smith et al. 2007). This IDL ${ }^{1}$ script was created to decompose low-resolution IRS spectra into dust features, stellar and thermal dust continuum, silicate absorption, and ionic and molecular line emission. Although PAHFIT also recovers PAH features, the central wavelengths of the bands are fixed in the code. This lack of flexibility prevents the account of the peak position variations and the fits of PAH bands obtained with this tool were disregarded in this work.

The continuum of the galaxies was fitted with a spline with anchor points at roughly 5.0, 5.4, 5.5, 5.8, 6.6, 7.0, 8.2, 9.0, 9.3, 9.9, 10.2, $10.5,10.7,11.7,12.1,13.1,13.9,14.7$, and $15.0 \mu \mathrm{m}$ according to the method utilized in Peeters et al. (2017). The inclusion of each point depended on the presence of the PAH plateaus (at 5-10 and $10-15 \mu \mathrm{m}$ ) and molecular bands (at $10.68 \mu \mathrm{m}$, for example). The spline decomposition was chosen because it allows us to isolate the $6.2 \mu \mathrm{m}$ band.

As already discussed in Peeters et al. (2017), the overall conclusions on PAH intensity correlations for a large sample of objects are independent of the chosen decomposition approach (e.g. Smith et al. 2007; Galliano et al. 2008). However, we analysed 20 galaxies of our sample - 10 with strong PAH plateaus and 10 with none or weak plateaus - in order to perceive the stability of our $6.2 \mu \mathrm{m}$ fitting according to the continuum decomposition obtained with spline and with PAHFIT. These galaxies have their $6.2 \mu \mathrm{m}$ profile fitted (as it will be discussed in the next section) and the results revealed that the band intensities and the full width at half-maximum (FWHM) showed greater discrepancies but the central wavelengths had no significant changes. Therefore, we can conclude that the presence of plateaus did not influence the results. As our work focuses on peak position of the profile, the chosen continuum decomposition applied does not interfere in the final analysis. A discussion of this comparison can be found in the Appendix B. Two examples of these decompositions can be seen in Figs 2 and 3, with and without PAH plateaus, respectively.

\subsection{The $6.2 \mu \mathrm{m}$ feature profile}

The differences among the $6.2 \mu \mathrm{m}$ PAH profiles in such astrophysical environments have been attributed, for example, to the local physical conditions and of the PAH molecules' size, charge, geometry, and heterogeneity (e.g. Draine \& Li 2001, 2007; Smith et al. 2007; Sales et al. 2012). The CC vibration modes of the 5$9 \mu \mathrm{m}$ wavelengths produce profiles (and their peak positions) highly variable, even in relatively low-resolution data (Tielens 2008). On the other hand, $\mathrm{CH}$ modes vary less and the variation may not be necessarily connected to the others (van Diedenhoven et al. 2004; Tielens 2008; Candian \& Sarre 2015).

According to Hudgins \& Allamandola (1999), PAHs with roughly 20 carbon atoms already contribute to the $6.2 \mu \mathrm{m}$ emission and the spacing among the PAH bands increases with molecular size. In general, for this band, objects grouped in class A have an asymmetric profile composed of a sharp blue rise and a red tail with central wavelength varying up to $6.23 \mu \mathrm{m}$ (Peeters et al. 2002). In classes $\mathrm{B}$ and $\mathrm{C}$, this asymmetry decreases and, for the $\mathrm{C}$, the peak position is greater than $6.29 \mu \mathrm{m}$. As already discussed, Hudgins et al. (2005) considered the blueshift of this band (peak positions $\leq 6.22 \mu \mathrm{m}$ ) which characterizes a class A object. The increase of PAH size, the substitution of carbon atoms by silicon or oxygen, the metal ion complexation $\left(\mathrm{Fe}^{+}, \mathrm{Mg}^{+}\right.$, and $\left.\mathrm{Mg}^{2+}\right)$, and the molecular symmetry

\footnotetext{
${ }^{1}$ Interactive Data Language, available at http://ittvis.com/idl
}
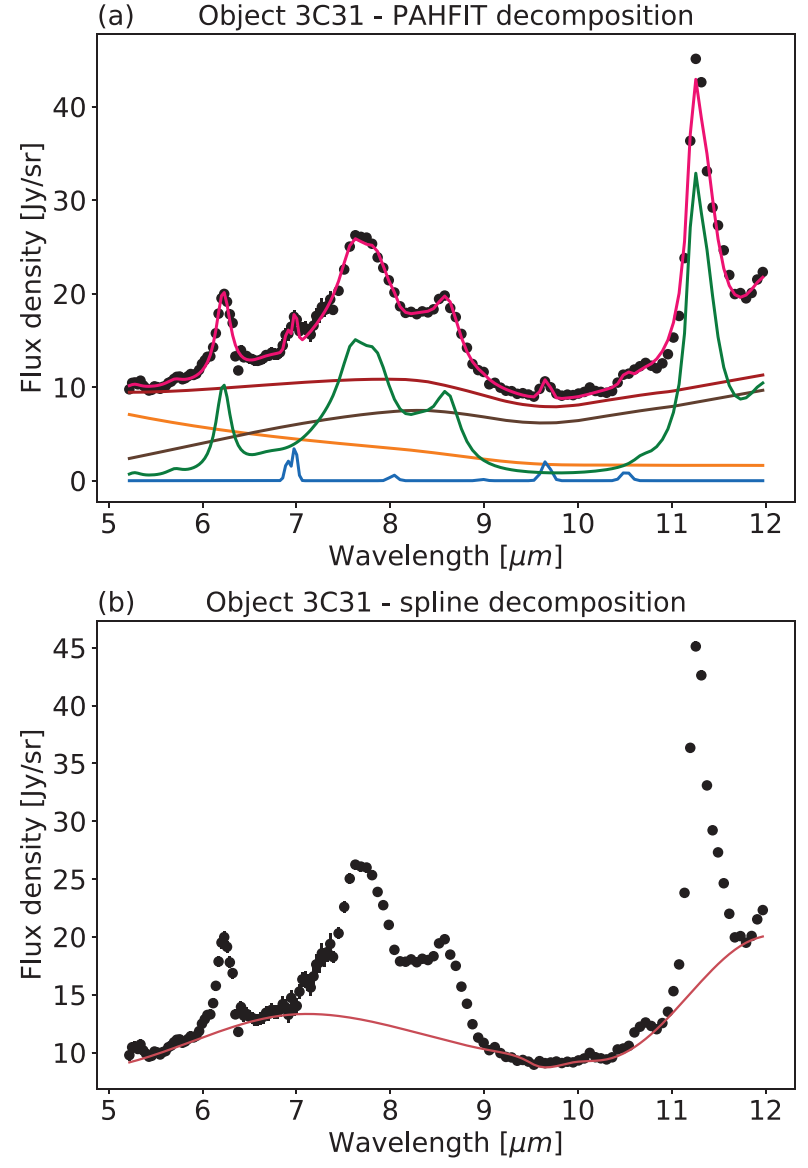

Figure 2. Comparison between the spectral decomposition with PAHFIT and with spline continuum for an object with the PAH plateaus. (a) Result of the PAHFIT decomposition of the $3 \mathrm{C} 31$ spectrum. The data points are represented by the dots with the vertical error bars as uncertainties. The pink line corresponds to the best-fitting model, the green line is the dust (AIB, $\mathrm{PAH}$ ) contribution, and blue one is the ionic and molecular lines contribution. The red line represents the total continuum contribution, and the brown and orange lines correspond to its individual thermal and stellar components, respectively. (b) The red line represents the spline decomposition of the continuum. The data points are represented by the dots with the vertical error bars as uncertainties.

variation were not able to reproduce the observed position of this interstellar band while simultaneously satisfying the astrophysical implications (for instance, the cosmic abundance of the chemically reactive elements). Apparently, of the possible substitutions they consider, nitrogen incorporated into the inner part of the rings is the only solution capable of reproducing the observed profile. The authors also estimated that a lower limit of 1-2 per cent of the cosmic nitrogen is retained in the PAH molecules located in the ISM (e.g. Hudgins, Bauschlicher \& Allamandola 2005; Boersma, Bregman \& Allamandola 2013; Boersma et al. 2014).

To accomplish this study of the $6.2 \mu \mathrm{m}$ PAH profile of starburst galaxies, we constructed a PYTHON-based script to estimate its central wavelength through the optimization algorithms from the submodule scipy.optmize.curve fit (hereafter curve fit). The data were fitted by a Gaussian profile (equation 1). The uncertainties were also derived using this tool with least-squares minimization and we used normalized root mean square (rms) deviation to evaluate the quality of the fit (equation 2). The initial guesses selected 
(a) Object NGC1365 - PAHFIT decomposition

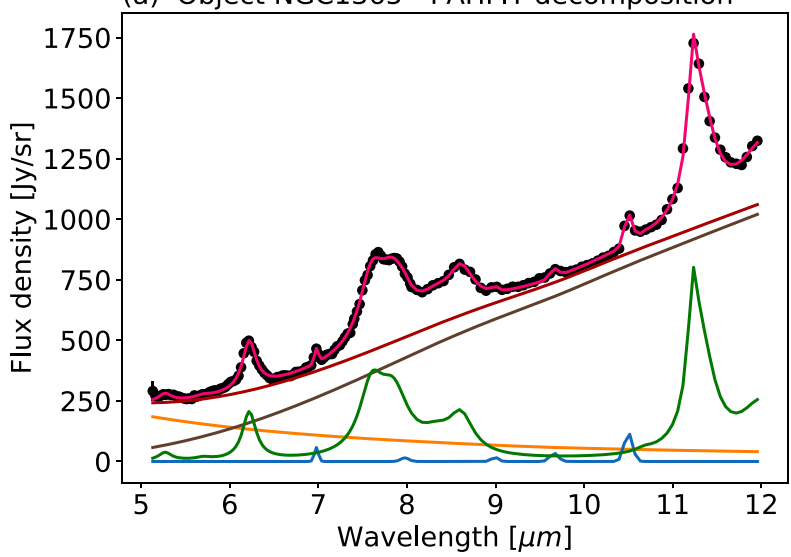

(b) Object NGC1365 - spline decomposition

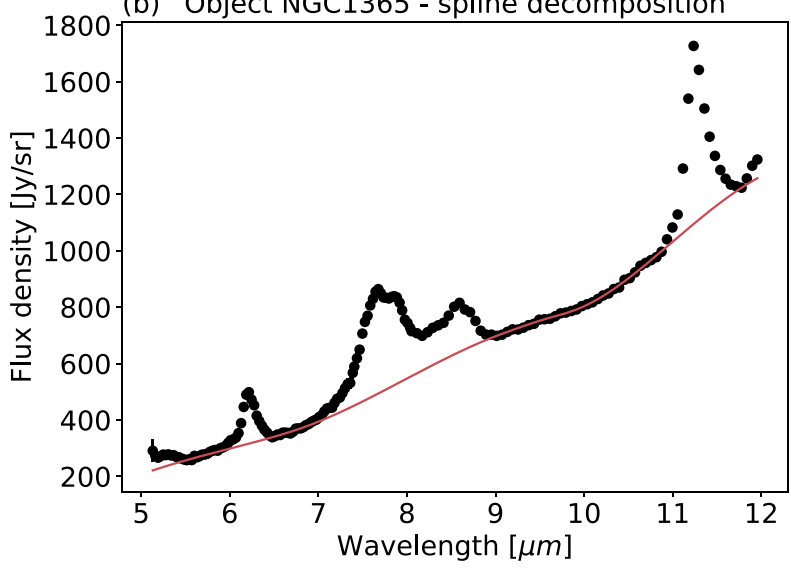

Figure 3. Comparison between the spectral decomposition with PAHFIT and with spline for an object without the PAH plateaus. (a) Result of the PAHFIT decomposition of the $3 \mathrm{C} 31$ spectrum. The data points are represented by the dots with the vertical error bars as uncertainties. The pink line corresponds to the best-fitting model, the green line is the dust (AIB, PAH) contribution, and blue one is the ionic and molecular lines contribution. The red line represents the total continuum contribution, and the brown and orange lines correspond to its individual thermal and stellar components, respectively. (b) The red line represents the spline decomposition of the continuum. The data points are represented by the dots with the vertical error bars as uncertainties.

were the values of $6.22 \mu \mathrm{m}$ for the peak position and 0.187 for the FWHM (Smith et al. 2007). An example of this fit can been seen in Fig. 4 (top),

$I_{\text {gauss }}=\frac{A}{\sigma \sqrt{2 \pi}} \exp \left(-\frac{\left(x-\lambda_{c}\right)^{2}}{2 \sigma^{2}}\right)$,

where $A$ is the amplitude, $\lambda_{\mathrm{c}}$ is the central wavelength, and the FWHM is given by FWHM $\sim 2.3548 \sigma$,

$\operatorname{rms}($ per cent $)=\frac{100}{I_{\max }} \sqrt{\frac{1}{N} \sum_{i=1}^{N} x_{i}^{2}}$,

where $x_{i}^{2}$ are the quadratic residues, $N$ the number of data, and $I_{\max }$ is the maximum flux intensity in the evaluated range. Rms values lower than 10 percent indicate an appropriate fit and values lower than 5 per cent indicate a very good fit.

The asymmetry of this specific feature caused the deviation of the fitted peak to redder wavelength and, to handle this, these objects had the data points of the profile's red tail removed from the fit
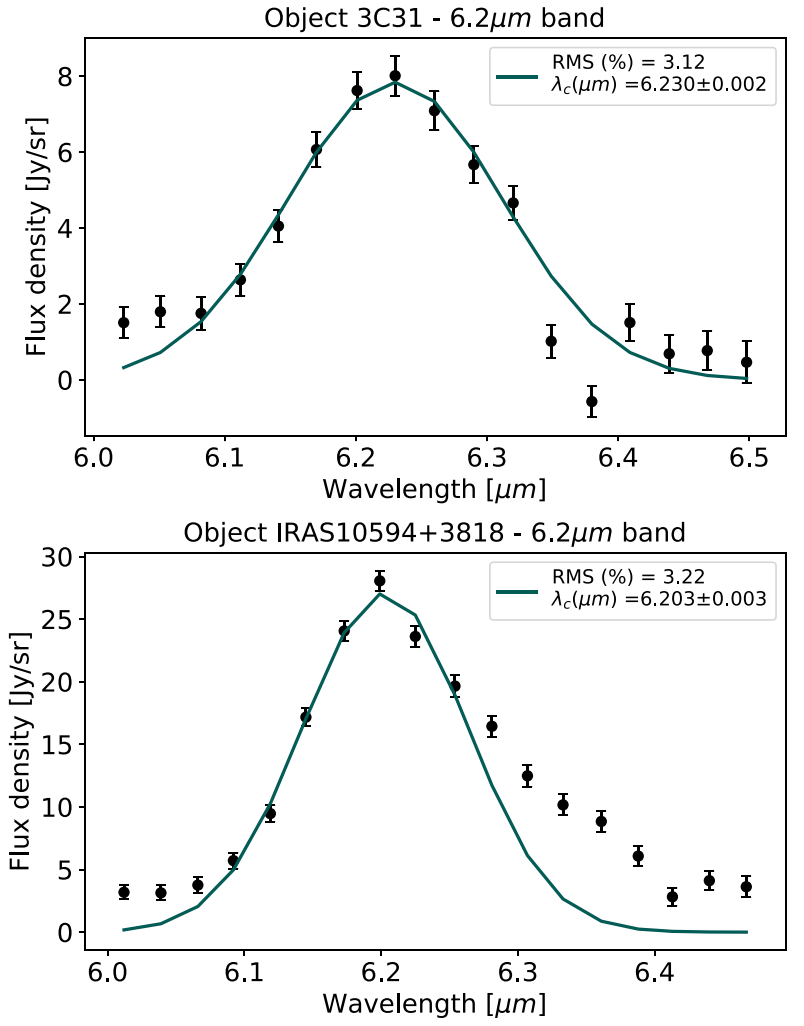

Figure 4. Profile of $6.2 \mu \mathrm{m}$ band fitted with curve_fit for the objects $3 \mathrm{C} 31$ (top) and IRAS10594+3818 ( bottom). Labels show the values of the peak position and normalized rms. In the case of IRAS10594+3818, the fitting range was reduced to exclude this more evident red tail.

following the same method of Peeters et al. (2017). One possible contributor to this red tail is anharmonic hot-band emission which is a natural consequence of the PAH model, although it is not a major player in determining the profile itself (Hudgins \& Allamandola 1999). Some galaxies of our sample required a reduction in the fitting range to better exclude the red tail and they are marked with an asterisk in Table A2. An example of this case is shown in Fig. 4 (bottom).

We also noted the presence of another emission component of the $6.2 \mu \mathrm{m}$ band near $6.35 \mu \mathrm{m}$ in some sources and performed the fitting adding a new subcomponent to the $6.22 \mu \mathrm{m}$ band (Fig. 5 , top) in order to compare the two procedures. The inclusion of the second feature seems to encompass the fit for highly asymmetric profiles by reproducing their red tail, previously underestimated in some galaxies, as for the object IRAS10594+3818 (Fig. 5, bottom). Even so, in these situations, there is no real indication of the presence of the second feature and the asymmetry could be just a characteristic of the anharmonic profile (Tielens 2008). In this work, we used only the fitting with just one Gaussian to standardize the analysis.

With peak positions obtained, we were able to group the galaxies into the three Peeters' classes (Table 1). Fig. 6 shows the class distribution.

\section{RESULTS AND DISCUSSION}

The second feature near $6.35 \mu \mathrm{m}$ has been attributed in some cases to the inherent asymmetry of the band profile. Nevertheless, its peak position varied from 6.246 to $6.471 \mu \mathrm{m}$ and there are a few possibilities that could give insight into its nature. According to 

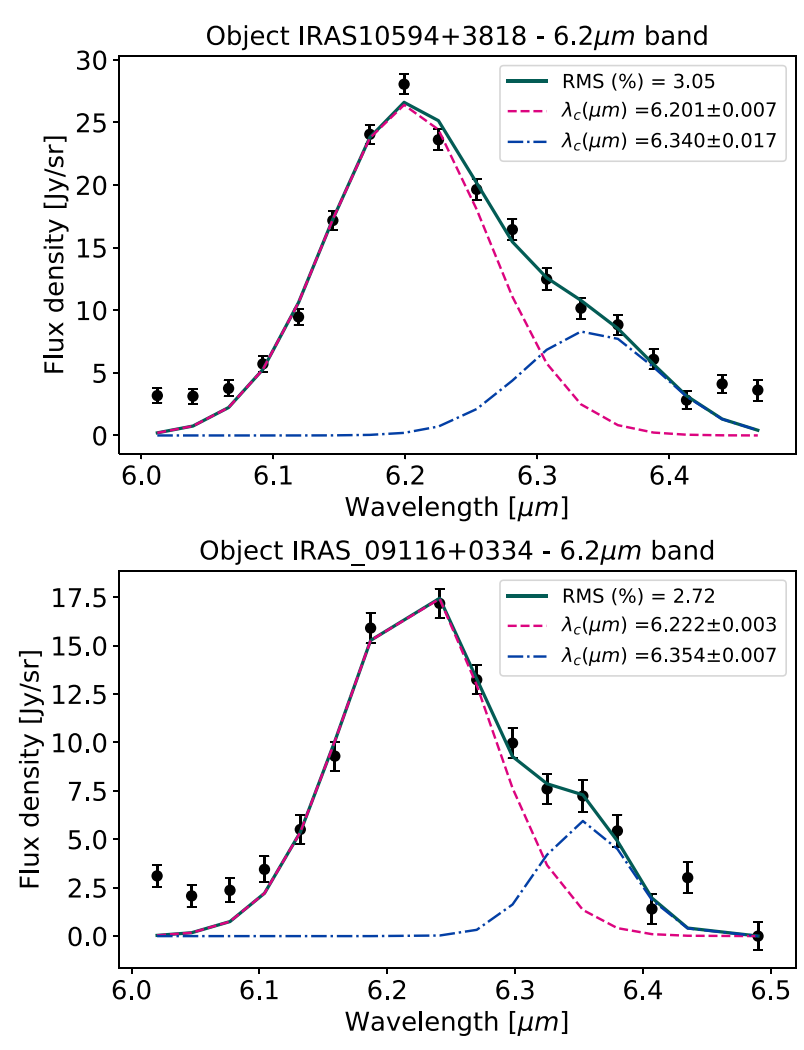

Figure 5. Profile of $6.2 \mu \mathrm{m}$ band fitted with curve_fit for the objects IRAS10594+3818 (top) and IRAS_09116+0334 (bottom) using two different Gaussian profiles. Labels show the values of the peak positions and normalized rms.

Table 1. Intervals for each Peeters' classes (Peeters et al. 2002).

\begin{tabular}{lc}
\hline Class & Interval $(\mu \mathrm{m})$ \\
\hline $\mathrm{A}$ & $<6.23$ \\
$\mathrm{~B}$ & $6.23<\lambda<6.29$ \\
$\mathrm{C}$ & $>6.29$ \\
\hline
\end{tabular}

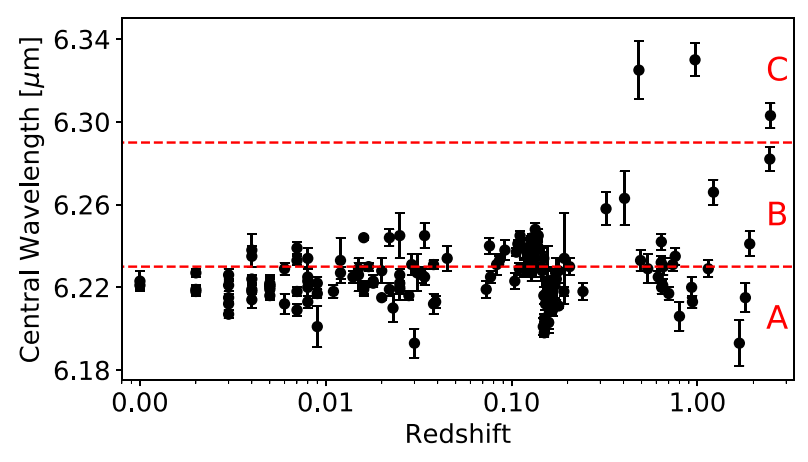

Figure 6. Distribution of the central wavelengths of the $6.2 \mu \mathrm{m}$ band for the redshift of the sources. The $x$-axis is in logarithmic scale. The dashed lines are the limits among the Peeters' classes, represented also by the letters $\mathrm{A}, \mathrm{B}$, and $\mathrm{C}$.
Table 2. Number of galaxies that fall into each Peeters' class.

\begin{tabular}{llll}
\hline Galaxies & Class A & Class B & Class C \\
\hline 155 & 67 percent & 31 percent & 2 percent \\
\hline
\end{tabular}

Pino et al. (2008), who performed experiments with PAHs in order to verify the origins of their emissions, bands near $6.3 \mu \mathrm{m}$ may be related to aliphatic features. On the other hand, the $6.4 \mu \mathrm{m}$ band observed in the reflection nebula NGC7023 was attributed to the $\mathrm{C}_{60}^{+}$(Berné, Montillaud \& Joblin 2015). PAH cations, without any carbon substituted, could also be the responsible for this emission (Hudgins et al. 2005). Finally, peaks at $\approx 6.41 \mu \mathrm{m}$ could be due to perylene-like structures (Candian, Sarre \& Tielens 2014).

Table 2 summarizes the separation of the 155 objects into Peeters' classes derived from the curve_fit fits. Details are given in Table A2. An overview of the results is shown in Fig. 6, which displays the distribution of the central wavelengths taking account of redshifts of the galaxies. There is a small predominance of class A objects over class B objects (more evident for redshifts lower than 0.05). Only three galaxies were classified as C.

Pino et al. (2008) have already noticed that class A objects are the most common in the Universe and embrace several astrophysical sources, while class $\mathrm{C}$ objects are in the minority. Our results point to the same conclusions, especially for starburst-dominated sources whose class A members are up to 67 percent in our study. This evidence is more pronounced at lower redshifts, as can be seen in Fig. 6. In addition, if we consider class $B$ as a mixture between PANH and PAH emissions, as pointed out by Peeters et al. (2002), we can verify that PANHs dominate this subclass of galaxies based on analysis of the $6.2 \mu \mathrm{m}$ PAH.

Regarding class C, just three objects do not allow us to distinguish any pattern. Apparently, they may be expected in higher redshifts, which might also imply an evolutionary time-scale of PAH molecules. Chemically young astrophysical sources might have reduced PAH abundances and PAH molecules are not as efficiently produced in low-metallicity environments because fewer carbon atoms are available in the ISM (Shivaei et al. 2017). In this case, class $\mathrm{C}$ could be represented by very small grains (VSGs). It was already noticed that VSGs may be responsible for the extended red wing and redshift of the peak position of the $11.2 \mu \mathrm{m}$ PAH band (Rosenberg et al. 2011). Besides, they may also be the carrier of the $7.8 \mu \mathrm{m}$ subcomponent of the $7.7 \mu \mathrm{m}$ PAH band and the $8.25 \mu \mathrm{m}$ component of the $8.6 \mu \mathrm{m}$ PAH band (Peeters et al. 2017), which are expected to be stronger for class $\mathrm{C}$ objects (Section 1). Nevertheless, as the higher redshift objects were extrapolated by the PAH bands themselves and were not corroborated with other spectroscopic or photometric data, any additional analysis may be misguided because the redshift errors can be as large as $0.1-0.2$ depending on how many features were used in the calculation (Yan et al. 2007).

According to Ota (2016), the substitution of three or more nitrogen atoms into PAHs does not provide molecules that describe the observed features. This could indicate that compounds of astrobiological interest such as purine and adenine may not be synthesized in the ISM. On the other hand, considering just one or two nitrogen atoms, some species were capable of reproducing the observations $\left(\mathrm{C}_{7} \mathrm{H}_{5} \mathrm{~N}_{2}-\mathrm{ab}^{3+}\right.$, for example). However, only small PANHs were considered in their study and they are easily destroyed in the ISM than larger molecules. We can expect that larger PANHs are correlated to the $6.2 \mu \mathrm{m}$ band emission since PAHs with $>20-30$ carbon atoms are thought to dominate the emitting interstellar PAHs. 


\section{(a) Starbursts}

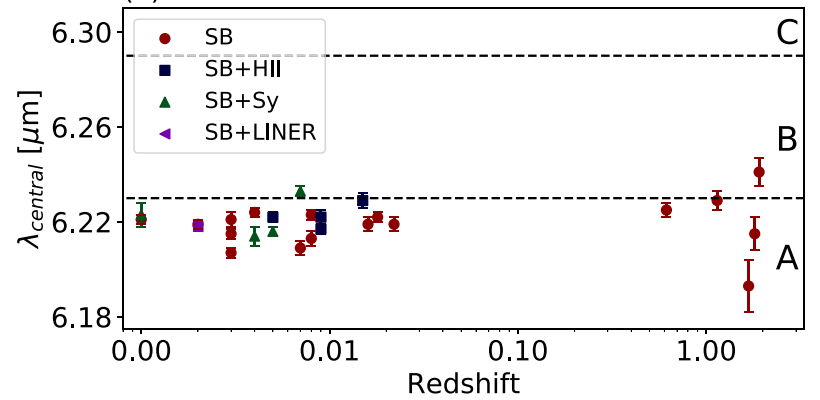

(b) Seyferts

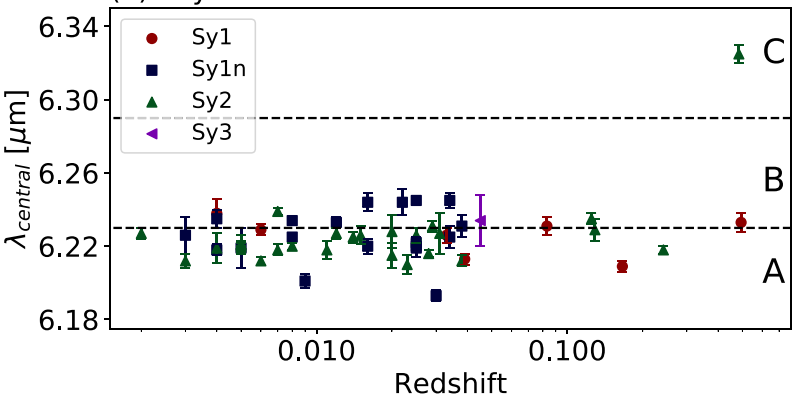

(c) ULIRGs

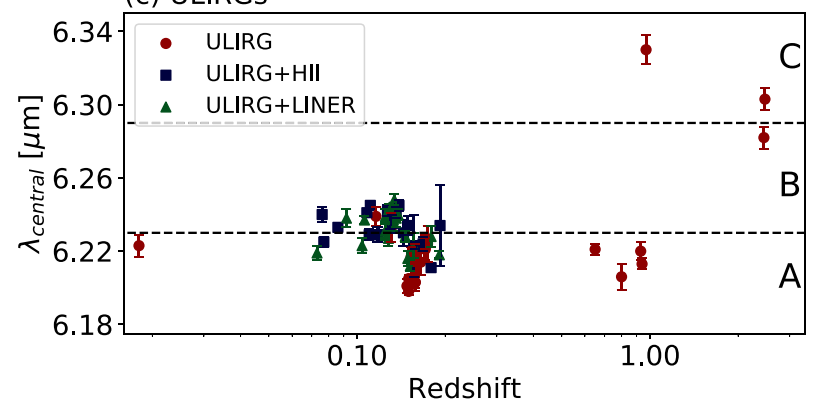

(d) Others

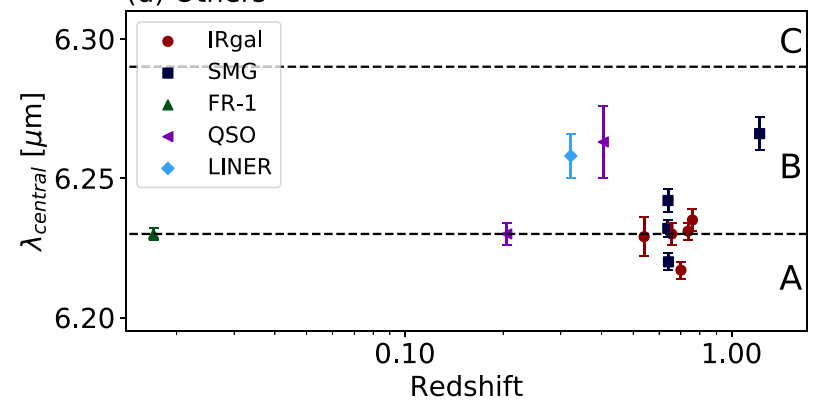

Figure 7. Distribution of the central wavelengths of the $6.2 \mu \mathrm{m}$ band according to the galaxy type - (a) Starbursts; (b) Seyferts; (c) ULIRGs; and (d) Others. The dashed lines are the limits among the Peeters' classes, indicated also by A, B, or C letter. Acronyms: AGN - active galactic nucleus, FR Fanaroff-Riley galaxy, H II-H II region, IRgal - Infrared galaxy, LINER low-ionization nuclear emission-line region, QSO - quasi-stellar object, SB - Starburst galaxy, SMG - submillimetre Galaxy, Sy - Seyfert galaxy, and ULIRG - ultraluminous infrared galaxy.

Fig. 7 illustrates the class distribution in different galaxy types for both methods applied (see Table A1). Table 3 presents the percentages of each class for the three most abundant objects of the galaxies - starbursts (17 per cent), Seyferts (34 per cent), and ULIRGs (41 per cent). The other types comprehend 8 per cent.
Table 3. Peeters' class distribution for starbursts, Seyferts, and ULIRG galaxies.

\begin{tabular}{|c|c|c|c|c|}
\hline Object & $\begin{array}{c}\text { A } \\
\text { (per cent) }\end{array}$ & $\begin{array}{c}\text { B } \\
\text { (per cent) }\end{array}$ & $\begin{array}{c}\mathrm{C} \\
\text { (per cent) }\end{array}$ & $\begin{array}{c}\text { Total of the } \\
\text { sample (per cent) }\end{array}$ \\
\hline $\begin{array}{l}\mathrm{SB} \\
\mathrm{SB}+\mathrm{H}_{\text {II }} \\
\mathrm{SB}+\mathrm{Sy}\end{array}$ & 92 & 8 & 0 & 17 \\
\hline $\begin{array}{l}\text { Sy1 } \\
\text { Sy1n } \\
\text { Sy2 } \\
\text { Sy3 }\end{array}$ & 70 & 28 & 2 & 34 \\
\hline $\begin{array}{l}\text { ULIRG } \\
\text { ULIRG + H II } \\
\text { ULIRG + LINER }\end{array}$ & 59 & 38 & 3 & 41 \\
\hline Others & 46 & 54 & 0 & 8 \\
\hline
\end{tabular}

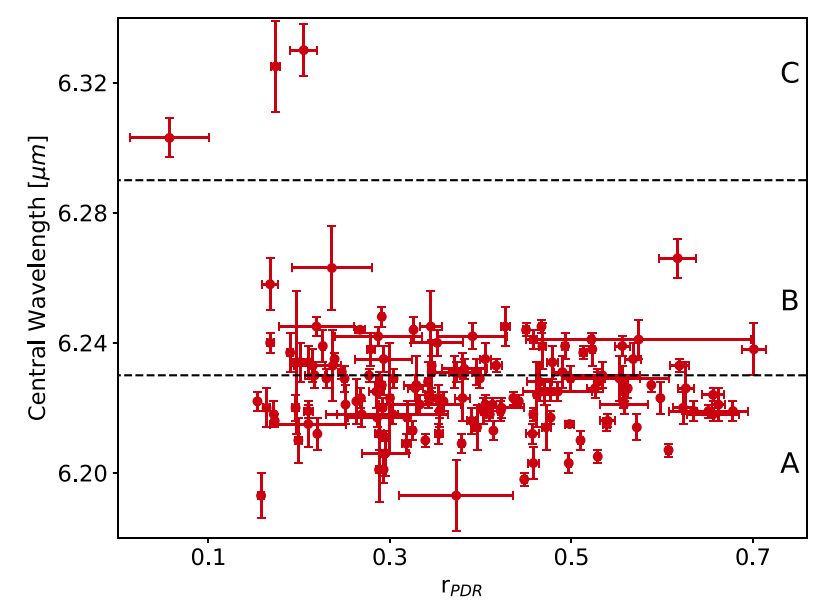

Figure 8. Distribution of the central wavelengths of the $6.2 \mu \mathrm{m}$ band for the spectral contribution of PDR component $r_{\mathrm{PDR}}$ of 152 sources whose values were available in the ATLAS project. The uncertainties are displayed as error bars when available. The dashed lines are the limits among the Peeters' classes, represented also by letters A, B, and C.

In the scenario in which class A $6.2 \mu \mathrm{m}$ band position arises from PANHs, the importance of these species is greater in starbursts. However, the fact that most of the ULIRGs and Seyferts are class A objects indicates a significant presence of PANHs also in these environments. The prevalence of class A objects in our study could be explained by the dominance of star formation contribution in all sources of our sample, or by the ubiquity of PANHs in galaxies. Studies of samples with a larger number of AGN-dominated sources could help to clarify this issue. Only in SMGs, class B dominates: three in four galaxies are classified as B. Again, more data is needed for further analysis.

Fig. 8 shows the distribution of the central wavelengths of the $6.2 \mu \mathrm{m}$ band for the spectral contribution of the PDR component $\left(r_{\mathrm{PDR}}\right)$ of the sources, as calculated by Hernán-Caballero \& Hatziminaoglou (2011). The $r_{\mathrm{PDR}}$ value is the ratio of the total integrated luminosity to the total luminosity of the PDR component in the 5-15 $\mu \mathrm{m}$ rest-frame range. In this sense, the class $C$ sources present low contribution of star formation and greater contribution of the ANG or $\mathrm{H}$ II region components, also calculated by HernánCaballero \& Hatziminaoglou (2011). Table 4 shows these respective values for the three class $\mathrm{C}$ sources. 
Table 4. Spectral contribution of the AGN, H II, and PDR components for the class C objects (Hernán-Caballero \& Hatziminaoglou 2011).

\begin{tabular}{lccc}
\hline Object & $r_{\mathrm{AGN}}$ & $r_{H \mathrm{II}}$ & $r_{\mathrm{PDR}}$ \\
\hline MIPS 180 & $0.439 \pm 0.075$ & $0.504 \pm 0.112$ & $0.057 \pm 0.044$ \\
SDSS_J00562 & $0.571 \pm 0.008$ & $0.256 \pm 0.006$ & $0.174 \pm 0.005$ \\
1.72+003 235.8 & & & \\
SWIRE4_J1036 & $0.458 \pm 0.022$ & $0.337 \pm 0.014$ & $0.205 \pm 0.015$ \\
37.18+584217.0 & & & \\
\hline
\end{tabular}

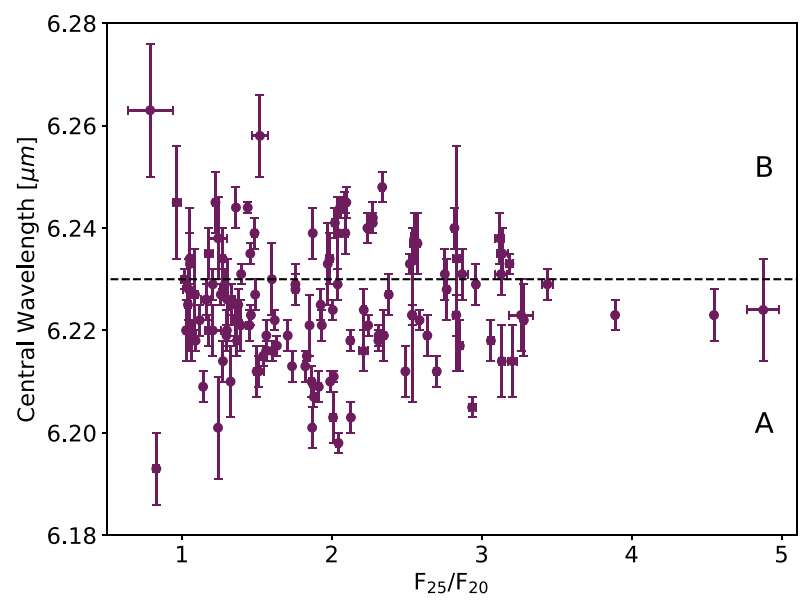

Figure 9. Distribution of the central wavelengths of the $6.2 \mu \mathrm{m}$ band for the $F_{25} / F_{20}$ ratio of 129 sources whose values were available in the ATLAS project. The uncertainties are displayed as error bars when available. The dashed lines are the limits among the Peeters' classes, represented also by letters A, B, and C.

On the other hand, if we consider the $\mathrm{F}_{25} / \mathrm{F}_{20}$ ratio (Fig. 9), we can see that class A objects are the coldest. The values of $r_{\mathrm{PDR}}, \mathrm{F}_{25}$, and $F_{20}$ together with their respective uncertainties were extracted directly from the ATLAS project when they were available. The uncertainty of the $\mathrm{F}_{25} / \mathrm{F}_{20}$ ratio was propagated from the $\mathrm{F}_{25}$ and $\mathrm{F}_{20}$ errors. The values of $r_{\mathrm{PDR}}$ and $\mathrm{F}_{25} / \mathrm{F}_{20}$ ratio can be seen in Table A3.

\section{CONCLUSIONS}

We have analysed the MIR spectra of 155 starburst-dominated galaxies, searching for the contribution of the Peeters' class A $6.2 \mu \mathrm{m}$ band to the total sample. To date, the class A position of this band can only be attributed to PANHs, PAHs containing $N$ atoms. Thus, the PAH feature profiles, especially the $6.2 \mu \mathrm{m}$ band, could indicate the presence of nitrogen incorporated to the rings.

The fitted $6.2 \mu \mathrm{m}$ profiles were classified in classes A, B, and C following Peeters et al. (2002). At 67 percent of the sample, class A profiles clearly dominate, suggesting a significant presence of PANHs in the ISM of these galaxies. Class B corresponded to a percentage of 31 per cent, indicating a significantly smaller contribution of PANHs in these sources. Only class C, with a small percentage of 2 per cent, seems not to be influenced by these molecules. These trends give support to the suggestion that class A/B $6.2 \mu \mathrm{m}$ band variations track changes in PANH ionization state or molecular structure (Bauschlicher, Peeters \& Allamandola 2009). In addition, we can see that class A objects are colder compared to class B objects.

Within the PANH scenario, the ubiquity of PANHs could indicate another reservoir of nitrogen in the Universe, with density and temperature conditions that differ from those of gas and ices phases. As shown in Fig. 7, they can be present in the ISM of starburst galaxies, ULIRGs, Seyferts, infrared, and submillimetre galaxies. Furthermore, they are responsible for an important fraction of the MIR emission, especially for the $6.2 \mu \mathrm{m}$ band. These findings also give support to the idea of their contribution to the origins of life on Earth and elsewhere, since they could form nucleobase-type structures in the ISM (Elsila et al. 2006; Parker et al. 2015).

Extension of this analysis to other types of objects also available in the ATLAS project, such as AGNs, could shed light on how the starburst-dominated emission of the sources is responsible for the majority of class A objects and could provide a broader overview of the $6.2 \mu \mathrm{m}$ band behaviour in astrophysical environments.

Moreover, it will also be possible to explore the other PAH bands in more detail, which could be of great interest to this study. Since 6.2 and $7.7 \mu \mathrm{m}$ bands are both caused by the CC stretching vibrational mode, they are connected to each other in some cases, mainly for class A (van Diedenhoven et al. 2004). This association could furnish another strategy for deriving the variations of the $6.2 \mu \mathrm{m}$ band in an indirect way.

The question why the presence of PANHs is more apparent in some galaxies but not in others could be addressed with chemical evolution models taking into account differences in metallicity, star formation history, and the nature of molecular clouds in the harbouring galaxy, e.g. the chemodynamical model in Friaça \& Barbuy (2017). In addition, further computational calculations together with laboratory measurements are needed to make more robust predictions of the role of PANHs in the profile of the PAH emission bands, mainly in the conditions prevailing in galaxies with star formation.

\section{ACKNOWLEDGEMENTS}

Special acknowledgements to CAPES (Comissão de Aperfeiçoamento de Pessoal do Nível Superior) and CNPq (Conselho Nacional de Desenvolvimento Científico e Tecnológico) for the financial support.

\section{REFERENCES}

Allamandola L. J., Hudgins D. M., Sandford S. A., 1999, ApJ, 511, L115 Bauschlicher C. W., Jr, Peeters E., Allamandola L. J., 2009, ApJ, 697, 311 Berné O., Montillaud J., Joblin C., 2015, A\&A, 577, A133

Boersma C., Bregman J. D., Allamandola L. J., 2013, ApJ, 769, 117

Boersma C. et al., 2014, ApJS, 211, 8

Brandl B. R. et al., 2006, ApJ, 653, 1129

Buchanan C. L., Gallimore J. F., O’Dea C. P., Baum S. A., Axon D. J., Robinson A., Elitzur M., Elvis M., 2006, AJ, 132, 401

Candian A., Sarre P. J., 2015, MNRAS, 448, 2960

Candian A., Sarre P. J., Tielens A. G. G. M., 2014, ApJ, 791, L10

Dasyra K. M. et al., 2009, ApJ, 701, 1123

Deo R. P., Crenshaw D. M., Kraemer S. B., Dietrich M., Elitzur M., Teplitz H., Turner T. J., 2007, ApJ, 671, 124

Draine B. T., Li A., 2001, in American Astronomical Society Meeting Abstracts. p. 1451

Draine B. T., Li A., 2007, ApJ, 657, 810

Ehrenfreund P. et al., 2002, Rep. Prog. Phys., 65, 1427

Ehrenfreund P., Rasmussen S., Cleaves J., Chen L., 2006, Astrobiology, 6, 490

Elsila J. E., Hammond M. R., Bernstein M. P., Sandford S. A., Zare R. N., 2006, Meteorit. Planet. Sci., 41, 785

Farrah D., Weedman D., Lonsdale C. J., Polletta M., Rowan-Robinson M., Houck J., Smith H. E., 2009, ApJ, 696, 2044

Friaça A. C. S., Barbuy B., 2017, A\&A, 598, A121 
Galliano F., Madden S. C., Tielens A. G. G. M., Peeters E., Jones A. P., 2008, ApJ, 679, 310

Genzel R., Cesarsky C. J., 2000, ARA\&A, 38, 761

Hernán-Caballero A., Hatziminaoglou E., 2011, MNRAS, 414, 500

Hernán-Caballero A. et al., 2009, MNRAS, 395, 1695

Hiner K. D., Canalizo G., Lacy M., Sajina A., Armus L., Ridgway S., Storrie-Lombardi L., 2009, ApJ, 706, 508

Houck J. R. et al., 2004, ApJS, 154, 18

Hudgins D. M., Allamandola L. J., 1999, ApJ, 513, L69

Hudgins D. M., Bauschlicher C. W., Jr, Allamandola L. J., 2005, ApJ, 632, 316

Imanishi M., 2009, ApJ, 694, 751

Imanishi M., Dudley C. C., Maiolino R., Maloney P. R., Nakagawa T., Risaliti G., 2007, ApJS, 171, 72

Imanishi M., Maiolino R., Nakagawa T., 2010, ApJ, 709, 801

Joblin C., Leger A., Martin P., 1992, ApJ, 393, L79

Leipski C., Antonucci R., Ogle P., Whysong D., 2009, ApJ, 701, 891

Li A., 2004, in Witt A. N., Clayton G. C., Draine B. T., eds, ASP Conf. Ser Vol. 309, Astrophysics of Dust. Astron. Soc. Pac., San Francisco, p. 417

Murphy E. J., Chary R.-R., Alexander D. M., Dickinson M., Magnelli B., Morrison G., Pope A., Teplitz H. I., 2009, ApJ, 698, 1380

Ota N., 2016, preprint (arXiv:1603.03399)

Papovich C. et al., 2006, ApJ, 640, 92

Parker D. S. N., Kaiser R. I., Kostko O., Troy T. P., Ahmed M., Mebel A. M., Tielens A. G. G. M., 2015, ApJ, 803, 53

Peeters E., Hony S., Van Kerckhoven C., Tielens A. G. G. M., Allamandola L. J., Hudgins D. M., Bauschlicher C. W., 2002, A\&A, 390, 1089

Peeters E., Bauschlicher C. W., Jr, Allamandola L. J., Tielens A. G. G. M., Ricca A., Wolfire M. G., 2017, ApJ, 836, 198
Pino T. et al., 2008, A\&A, 490, 665

Pope A. et al., 2008, ApJ, 675, 1171

Riechers D. A. et al., 2014, ApJ, 786, 31

Rosenberg M. J. F., Berné O., Boersma C., Allamandola L. J., Tielens A. G. G. M., 2011, A\&A, 532, A128

Sales D. A., Pastoriza M. G., Riffel R., Winge C., 2012, MNRAS, 429, 2634

Shi Y. et al., 2006, ApJ, 653, 127

Shivaei I. et al., 2017, ApJ, 837, 157

Siana B. et al., 2009, ApJ, 698, 1273

Smith J. D. T. et al., 2007, ApJ, 656, 770

Sturm E., Hasinger G., Lehmann I., Mainieri V., Genzel R., Lehnert M. D., Lutz D., Tacconi L. J., 2006, ApJ, 642, 81

Teplitz H. I. et al., 2007, ApJ, 659, 941

Tielens A. G. G. M., 2008, ARA\&A, 46, 289

van Diedenhoven B., Peeters E., Van Kerckhoven C., Hony S., Hudgins D.

M., Allamandola L. J., Tielens A. G. G. M., 2004, ApJ, 611, 928

Weedman D. W., Houck J. R., 2009, ApJ, 693, 370

Weedman D. W. et al., 2005, ApJ, 633, 706

Weedman D. et al., 2006, ApJ, 653, 101

Werner M. W. et al., 2004, ApJS, 154, 1

Wu Y., Charmandaris V., Huang J., Spinoglio L., Tommasin S., 2009, ApJ, 701,658

Yan L. et al., 2005, ApJ, 628, 604

Yan L. et al., 2007, ApJ, 658, 778

Zakamska N. L., Gómez L., Strauss M. A., Krolik J. H., 2008, AJ, 136, 1607

\section{APPENDIX A: SOURCES - IDENTIFICATION AND DERIVED PROPERTIES}

Table A1. Sources and their respective information extracted from the MIR_SB sample (Spitzer/IRS ATLAS, version 1.0) and Yan et al. (2007), including their ID, type, source reference, right ascension, declination, and redshift. Acronyms: AGN - active galactic nucleus, FR - Fanaroff-Riley galaxy, H II-H II region, IRgal - Infrared galaxy, LINER - low-ionization nuclear emission-line region, QSO - quasi-stellar object, SB - Starburst galaxy, SMG - submillimetre Galaxy, Sy - Seyfert galaxy, and ULIRG - ultraluminous infrared galaxy.

\begin{tabular}{|c|c|c|c|c|c|}
\hline ID & Type & Reference & RA (hms) & Dec. $(\mathrm{dms})$ & $z$ \\
\hline $3 \mathrm{C} 293$ & Sy3 & Leipski et al. (2009) & $13: 52: 17.80$ & $31: 26: 46.50$ & 0.045 \\
\hline $3 C 31$ & FR-1 & Leipski et al. (2009) & 01:07:24.90 & $32: 24: 45.20$ & 0.017 \\
\hline Arp220 & ULIRG & Imanishi et al. (2007) & $15: 34: 57.10$ & $23: 30: 11.00$ & 0.018 \\
\hline CGCG381-051 & Sy2 & Wu et al. (2009) & $23: 48: 41.70$ & $02: 14: 23.00$ & 0.031 \\
\hline E12-G21 & Sy1 & Wu et al. (2009) & $00: 40: 47.80$ & $-79: 14: 27.00$ & 0.033 \\
\hline EIRS-14 ${ }^{a}$ & SB & Hernán-Caballero et al. (2009) & $16: 35: 36.64$ & $40: 47: 53.80$ & 0.615 \\
\hline EIRS-41 & QSO & Hernán-Caballero et al. (2009) & $16: 34: 28.15$ & $41: 27: 42.60$ & 0.405 \\
\hline GN26 & SMG & Pope et al. (2008) & $12: 36: 34.51$ & $62: 12: 40.90$ & 1.219 \\
\hline IC342 & $\mathrm{SB}$ & Brandl et al. (2006) & $03: 46: 48.51$ & $68: 05: 46.00$ & 0.001 \\
\hline IRAS02021-2103 & ULIRG & Imanishi, Maiolino \& Nakagawa (2010) & 02:04:27.30 & $-20: 49: 41$ & 0.116 \\
\hline IRAS02480-3745 & ULIRG & Imanishi et al. (2010) & 02:50:01.70 & $-37: 32: 45$ & 0.165 \\
\hline IRAS03209-0806 & Sy1 & Imanishi et al. (2010) & $03: 23: 22.90$ & $-07: 56: 15$ & 0.166 \\
\hline IRAS04074-2801 & ULIRG & Imanishi et al. (2010) & 04:09:30.40 & $-27: 53: 44$ & 0.153 \\
\hline IRAS $08591+5248$ & ULIRG & Imanishi et al. (2010) & 09:02:47.50 & $52: 36: 30$ & 0.158 \\
\hline IRAS10594+3818 & ULIRG & Imanishi et al. (2010) & 11:02:14.00 & $38: 02: 35$ & 0.158 \\
\hline IRAS12447+3721 & ULIRG & Imanishi et al. (2010) & $12: 47: 07.70$ & $37: 05: 37$ & 0.158 \\
\hline IRAS13106-0922 & ULIRG & Imanishi et al. (2010) & $13: 13: 14.80$ & $-09: 38: 00$ & 0.174 \\
\hline IRAS14121-0126 & ULIRG & Imanishi et al. (2010) & $14: 14: 45.50$ & $-01: 40: 55$ & 0.150 \\
\hline IRAS14197+0813 & ULIRG & Imanishi et al. (2010) & $14: 22: 11.60$ & 07:59:28AM & 0.131 \\
\hline IRAS14202+2615 & ULIRG & Imanishi et al. (2010) & $14: 22: 31.40$ & $26: 02: 05$ & 0.159 \\
\hline IRAS14485-2434 & ULIRG & Imanishi et al. (2010) & $14: 51: 23.80$ & $-24: 46: 30$ & 0.148 \\
\hline IRAS $15043+5754$ & ULIRG & Imanishi et al. (2010) & $15: 05: 39.50$ & $57: 43: 07$ & 0.150 \\
\hline IRAS21477+0502 & ULIRG & Imanishi et al. (2010) & $21: 50: 16.30$ & 05:16:03AM & 0.171 \\
\hline IRAS22088-1831 & ULIRG & Imanishi et al. (2010) & $22: 11: 33.80$ & $-18: 17: 06$ & 0.170 \\
\hline
\end{tabular}


Table A1 - continued

\begin{tabular}{|c|c|c|c|c|c|}
\hline ID & Type & Reference & RA (hms) & Dec. $(\mathrm{dms})$ & $z$ \\
\hline IRAS_00091-0738 & ULIRG + Н II & Imanishi et al. (2007) & $00: 11: 43.30$ & $-07: 22: 07.00$ & 0.118 \\
\hline IRAS_00456-2904 & ULIRG + $\mathrm{H}_{\text {II }}$ & Imanishi et al. (2007) & 00:48:06.80 & $-28: 48: 19.00$ & 0.110 \\
\hline IRAS_01199-2307 & ULIRG + H II & Imanishi (2009) & 01:22:20.90 & $-22: 52: 07.00$ & 0.156 \\
\hline IRAS_01355-1814 & ULIRG + $\mathrm{H}_{\text {II }}$ & Imanishi (2009) & 01:37:57.40 & $-17: 59: 21.00$ & 0.192 \\
\hline IRAS_01494-1845 & ULIRG & Imanishi (2009) & 01:51:51.40 & $-18: 30: 46.00$ & 0.158 \\
\hline IRAS_02411+0353 & ULIRG + Н II & Imanishi et al. (2007) & $02: 43: 46.10$ & 04:06:37.00 & 0.144 \\
\hline IRAS_03250+1606 & ULIRG + LINER & Imanishi et al. (2007) & 03:27:49.80 & 16:17:00.00 & 0.129 \\
\hline IRAS_03521+0028 & ULIRG + LINER & Imanishi (2009) & 03:54:42.20 & 00:37:03.00 & 0.152 \\
\hline IRAS_08201+2801 & ULIRG + Н II & Imanishi (2009) & $08: 23: 12.60$ & 27:51:40.00 & 0.168 \\
\hline IRAS_09039+0503 & ULIRG + LINER & Imanishi et al. (2007) & 09:06:34.20 & 04:51:25.00 & 0.125 \\
\hline IRAS_09116+0334 & ULIRG + LINER & Imanishi et al. (2007) & 09:14:13.80 & 03:22:01.00 & 0.146 \\
\hline IRAS_09463+8141 & ULIRG + LINER & Imanishi (2009) & 09:53:00.50 & $81: 27: 28.00$ & 0.156 \\
\hline IRAS_09539+0857 & Sy2 & Imanishi et al. (2007) & 09:56:34.30 & 08:43:06.00 & 0.129 \\
\hline IRAS_10190+1322 & ULIRG + Н II & Imanishi et al. (2007) & $10: 21: 42.50$ & 13:06:54.00 & 0.077 \\
\hline IRAS_10485-1447 & ULIRG + LINER & Imanishi et al. (2007) & 10:51:03.10 & $-15: 03: 22.00$ & 0.133 \\
\hline IRAS_10494+4424 & ULIRG + LINER & Imanishi et al. (2007) & $10: 52: 23.50$ & 44:08:48.00 & 0.092 \\
\hline IRAS_11130-2659 & ULIRG + LINER & Imanishi et al. (2007) & $11: 15: 31.60$ & $-27: 16: 23.00$ & 0.136 \\
\hline IRAS_11387+4116 & ULIRG + Н II & Imanishi et al. (2007) & $11: 41: 22.00$ & 40:59:51.00 & 0.149 \\
\hline IRAS_11506+1331 & ULIRG + $\mathrm{H}_{\text {II }}$ & Imanishi et al. (2007) & $11: 53: 14.20$ & $13: 14: 28.00$ & 0.127 \\
\hline IRAS_12112+0305 & ULIRG + LINER & Imanishi et al. (2007) & $12: 13: 46.00$ & 02:48:38.00 & 0.073 \\
\hline IRAS_12359-0725 & ULIRG + LINER & Imanishi et al. (2007) & $12: 38: 31.60$ & $-07: 42: 25.00$ & 0.138 \\
\hline IRAS_13335-2612 & ULIRG + LINER & Imanishi et al. (2007) & $13: 36: 22.30$ & $-26: 27: 34.00$ & 0.125 \\
\hline IRAS_13469+5833 & ULIRG + Н ІІ & Imanishi (2009) & $13: 48: 40.20$ & 58:18:52.00 & 0.158 \\
\hline IRAS_13509+0442 & ULIRG + $\mathrm{H}_{\mathrm{II}}$ & Imanishi et al. (2007) & $13: 53: 31.60$ & 04:28:05.00 & 0.136 \\
\hline IRAS_13539+2920 & ULIRG + $\mathrm{H}_{\text {II }}$ & Imanishi et al. (2007) & 13:56:10.00 & 29:05:35.00 & 0.108 \\
\hline IRAS_14060+2919 & ULIRG + $\mathrm{H}_{\text {II }}$ & Imanishi et al. (2007) & 14:08:19.00 & 29:04:47.00 & 0.117 \\
\hline IRAS_14252-1550 & ULIRG + LINER & Imanishi et al. (2007) & $14: 28: 01.10$ & $-16: 03: 39.00$ & 0.149 \\
\hline IRAS_14348-1447 & Sy1 & Imanishi et al. (2007) & $14: 37: 38.30$ & $-15: 00: 23.00$ & 0.083 \\
\hline IRAS_15206+3342 & Sy2 & Imanishi et al. (2007) & $15: 22: 38.00$ & $33: 31: 36.00$ & 0.125 \\
\hline IRAS_15225+2350 & ULIRG + Н ІІ & Imanishi et al. (2007) & $15: 24: 43.90$ & $23: 40: 10.00$ & 0.139 \\
\hline IRAS_16090-0139 & ULIRG + LINER & Imanishi et al. (2007) & $16: 11: 40.50$ & $-01: 47: 06.00$ & 0.134 \\
\hline IRAS_16300+1558 & Sy2 & Imanishi (2009) & $16: 32: 21.40$ & $15: 51: 46.00$ & 0.242 \\
\hline IRAS_16333+4630 & ULIRG + LINER & Imanishi (2009) & $16: 34: 52.60$ & $46: 24: 53.00$ & 0.191 \\
\hline IRAS_16474+3430 & ULIRG + $\mathrm{H}_{\mathrm{II}}$ & Imanishi et al. (2007) & $16: 49: 14.20$ & $34: 25: 10.00$ & 0.111 \\
\hline IRAS_16487+5447 & ULIRG + LINER & Imanishi et al. (2007) & $16: 49: 47.00$ & $54: 42: 35.00$ & 0.104 \\
\hline IRAS_17028+5817 & ULIRG + LINER & Imanishi et al. (2007) & 17:03:41.90 & 58:13:45.00 & 0.106 \\
\hline IRAS_17068+4027 & ULIRG + Н II & Imanishi (2009) & $17: 08: 31.90$ & $40: 23: 28.00$ & 0.179 \\
\hline IRAS_20414-1651 & ULIRG + Н II & Imanishi et al. (2007) & 20:44:18.20 & $-16: 40: 16.00$ & 0.086 \\
\hline IRAS_21208-0519 & ULIRG + $\mathrm{H}_{\text {II }}$ & Imanishi et al. (2007) & 21:23:29.10 & $-05: 06: 56.00$ & 0.130 \\
\hline IRAS_21329-2346 & ULIRG + LINER & Imanishi et al. (2007) & $21: 35: 45.80$ & $-23: 32: 35.00$ & 0.125 \\
\hline IRAS_22206-2715 & ULIRG + $\mathrm{H}_{\text {II }}$ & Imanishi et al. (2007) & $22: 23: 28.90$ & $-27: 00: 04.00$ & 0.132 \\
\hline IRAS_22491-1808 & ULIRG + $\mathrm{H}_{\text {II }}$ & Imanishi et al. (2007) & $22: 51: 49.20$ & $-17: 52: 23.00$ & 0.076 \\
\hline IRAS_23129+2548 & ULIRG + LINER & Imanishi (2009) & $23: 15: 21.40$ & 26:04:32.00 & 0.179 \\
\hline IRAS_23234+0946 & ULIRG + LINER & Imanishi et al. (2007) & $23: 25: 56.20$ & 10:02:49.00 & 0.128 \\
\hline LH_H901A & QSO2 & Sturm et al. (2006) & $10: 52: 52.80$ & 57:29:00.00 & 0.205 \\
\hline M-2-40-4 & Sy1.9 & Wu et al. (2009) & $15: 48: 24.90$ & $-13: 45: 28.00$ & 0.025 \\
\hline M-5-13-17 & Sy1.5 & Wu et al. (2009) & 05:19:35.80 & $-32: 39: 28.00$ & 0.012 \\
\hline$M+0-29-23$ & Sy2 & Wu et al. (2009) & $11: 21: 12.20$ & $-02: 59: 03.00$ & 0.025 \\
\hline MIPS180 ${ }^{a}$ & ULIRG & Yan et al. (2007) & $17: 15: 43.54$ & $58: 35: 31.20$ & 2.470 \\
\hline MIPS562 & IRgal & Dasyra et al. (2009) & $17: 12: 39.60$ & 58:59:55.10 & 0.540 \\
\hline MIPS8040 & IRgal & Dasyra et al. (2009) & $17: 13: 12.00$ & 60:08:40.20 & 0.759 \\
\hline MIPS8242a & ULIRG & Yan et al. (2007) & $17: 14: 33.17$ & 59:39:11.20 & 2.450 \\
\hline MIPS15755 & IRgal & Dasyra et al. (2009) & 17:18:34.90 & 59:45:34.10 & 0.736 \\
\hline MIPS22307 & IRgal & Dasyra et al. (2009) & 17:19:51.40 & $58: 42: 22.80$ & 0.700 \\
\hline MIPS22352 & IRgal & Dasyra et al. (2009) & $17: 21: 47.70$ & 58:53:55.90 & 0.656 \\
\hline Mrk52 & SB & Brandl et al. (2006) & $12: 25: 42.67$ & 00:34:20.40 & 0.007 \\
\hline Mrk273 & Sy2 & Wu et al. (2009) & $13: 44: 42.10$ & 55:53:13.00 & 0.038 \\
\hline Mrk334 & Sy1.8 & Deo et al. (2007) & 00:03:09.60 & 21:57:37.00 & 0.022 \\
\hline Mrk471 & Sy1.8 & Deo et al. (2007) & $14: 22: 55.40$ & 32:51:03.00 & 0.034 \\
\hline Mrk609 & Sy1.8 & Deo et al. (2007) & $03: 25: 25.30$ & $-06: 08: 38.00$ & 0.034 \\
\hline Mrk622 & Sy2 & Deo et al. (2007) & 08:07:41.00 & 39:00:15.00 & 0.023 \\
\hline Mrk883 & Sy1.9 & Deo et al. (2007) & $16: 29: 52.90$ & 24:26:38.00 & 0.038 \\
\hline Mrk938 & Sy2 & Wu et al. (2009) & 00:11:06.50 & $-12: 06: 26.00$ & 0.020 \\
\hline Mrk1066 & Sy2 & Shi et al. (2006) & 02:59:58.60 & $36: 49: 14.00$ & 0.012 \\
\hline
\end{tabular}


Table A1 - continued

\begin{tabular}{|c|c|c|c|c|c|}
\hline ID & Type & Reference & RA (hms) & Dec. (dms) & $z$ \\
\hline Murphy3 & SMG & Murphy et al. (2009) & $12: 36: 03.25$ & $62: 11: 10.80$ & 0.638 \\
\hline Murphy8 & SMG & Murphy et al. (2009) & $12: 36: 22.48$ & $62: 15: 44.30$ & 0.639 \\
\hline NGC513 & Sy2 & Wu et al. (2009) & $01: 24: 26.80$ & $33: 47: 58.0$ & 0.02 \\
\hline NGC520 & $\mathrm{SB}+\mathrm{Sy} 1.8$ & Brandl et al. (2006) & $01: 24: 35.07$ & $03: 47: 32.7$ & 0.0071 \\
\hline NGC660 & $\mathrm{SB}$ & Brandl et al. (2006) & $01: 43: 02.45$ & $13: 38: 44.4$ & 0.0029 \\
\hline NGC1056 & Sy2 & Wu et al. (2009) & $02: 42: 48.30$ & $28: 34: 27.00$ & 0.005 \\
\hline NGC1097 & $\mathrm{SB}+\mathrm{Sy} 1$ & Wu et al. (2009) & 02:46:19.08 & $-30: 16: 28.00$ & 0.004 \\
\hline NGC1125 & Sy 2 & Wu et al. (2009) & $02: 51: 40.30$ & $-16: 39: 04.00$ & 0.011 \\
\hline NGC1143 & Sy2 & Wu et al. (2009) & $02: 55: 12.20$ & $-00: 11: 01.00$ & 0.029 \\
\hline NGC1222 & SB & Brandl et al. (2006) & $03: 08: 56.74$ & $-02: 57: 18.50$ & 0.008 \\
\hline NGC1365 & $\mathrm{SB}+\mathrm{Sy} 1.8$ & Brandl et al. (2006) & $03: 33: 36.37$ & $-36: 08: 25.50$ & 0.005 \\
\hline NGC1566 & Sy 1.5 & Wu et al. (2009) & 04:20:00.40 & $-54: 56: 16.00$ & 0.005 \\
\hline NGC1614 & $\mathrm{SB}+\mathrm{H}_{\text {II }}$ & Brandl et al. (2006) & $04: 33: 59.85$ & $-08: 34: 44.00$ & 0.015 \\
\hline NGC1667 & Sy2 & Wu et al. (2009) & $04: 48: 37.10$ & $-06: 19: 12.00$ & 0.015 \\
\hline NGC2273 & Sy1 & Shi et al. (2006) & 06:50:08.60 & $60: 50: 45.00$ & 0.006 \\
\hline NGC2623 & $\mathrm{SB}$ & Brandl et al. (2006) & 08:38:24.08 & $25: 45: 16.90$ & 0.018 \\
\hline NGC2992 & Sy1.9 & Wu et al. (2009) & $09: 45: 42.00$ & $-14: 19: 35.00$ & 0.008 \\
\hline NGC3079 & Sy2 & Weedman et al. (2005) & 10:01:57.80 & $55: 40: 47.00$ & 0.004 \\
\hline NGC3227 & Sy 1.5 & Wu et al. (2009) & $10: 23: 30.60$ & $19: 51: 54.00$ & 0.004 \\
\hline NGC3256 & SB & Brandl et al. (2006) & $10: 27: 51.27$ & $-43: 54: 13.80$ & 0.008 \\
\hline NGC3310 & $\mathrm{SB}+\mathrm{H}_{\text {II }}$ & Brandl et al. (2006) & $10: 38: 45.96$ & $53: 30: 12.00$ & 0.005 \\
\hline NGC3511 & Sy1 & Wu et al. (2009) & $11: 03: 23.80$ & $-23: 05: 12.00$ & 0.004 \\
\hline NGC3556 & $\mathrm{SB}$ & Brandl et al. (2006) & 11:11:30.97 & $55: 40: 26.80$ & 0.003 \\
\hline NGC3628 & $\mathrm{SB}+$ LINER & Brandl et al. (2006) & $11: 20: 17.02$ & $13: 35: 22.20$ & 0.002 \\
\hline NGC3786 & Sy 1.8 & Deo et al. (2007) & $11: 39: 42.50$ & $31: 54: 33.00$ & 0.009 \\
\hline NGC3982 & Sy1.9 & Wu et al. (2009) & $11: 56: 28.10$ & $55: 07: 31.00$ & 0.004 \\
\hline NGC4088 & SB & Brandl et al. (2006) & $12: 05: 34.19$ & $50: 32: 20.50$ & 0.003 \\
\hline NGC4194 & $\mathrm{SB}+\mathrm{H}_{\text {II }}$ & Brandl et al. (2006) & 12:14:09.64 & $54: 31: 34.60$ & 0.009 \\
\hline NGC4388 & Sy2 & Wu et al. (2009) & $12: 25: 46.70$ & $12: 39: 44.00$ & 0.008 \\
\hline NGC4945 & $\mathrm{SB}+\mathrm{Sy} 2$ & Brandl et al. (2006) & $13: 05: 27.48$ & $-49: 28: 05.60$ & 0.001 \\
\hline NGC5005 & Sy2 & Wu et al. (2009) & $13: 10: 56.20$ & $37: 03: 33.00$ & 0.003 \\
\hline NGC5033 & Sy 1.8 & Wu et al. (2009) & $13: 13: 27.50$ & $36: 35: 38.00$ & 0.003 \\
\hline NGC5135 & Sy2 & Wu et al. (2009) & $13: 25: 44.00$ & $-29: 50: 01.00$ & 0.014 \\
\hline NGC5194 & Sy2 & Wu et al. (2009) & $13: 29: 52.70$ & $47: 11: 43.00$ & 0.002 \\
\hline NGC5256 & Sy2 & Wu et al. (2009) & $13: 38: 17.50$ & $48: 16: 37.00$ & 0.028 \\
\hline NGC5674 & Sy1.9 & Shi et al. (2006) & $14: 33: 52.20$ & $05: 27: 30.00$ & 0.025 \\
\hline NGC5953 & Sy2 & Buchanan et al. (2006) & $15: 34: 32.40$ & $15: 11: 38.00$ & 0.007 \\
\hline NGC6810 & Sy2 & Wu et al. (2009) & $19: 43: 34.40$ & $-58: 39: 21.00$ & 0.007 \\
\hline NGC6890 & Sy1.9 & Wu et al. (2009) & $20: 18: 18.10$ & $-44: 48: 25.00$ & 0.008 \\
\hline NGC7130 & Sy1.9 & Buchanan et al. (2006) & $21: 48: 19.50$ & $-34: 57: 05.00$ & 0.016 \\
\hline NGC7252 & $\mathrm{SB}$ & Brandl et al. (2006) & $22: 20: 44.77$ & $-24: 40: 41.80$ & 0.016 \\
\hline NGC7469 & Sy 1.5 & Wu et al. (2009) & $23: 03: 15.60$ & $08: 52: 26.00$ & 0.016 \\
\hline NGC7496 & Sy2 & Buchanan et al. (2006) & 23:09:47.30 & $-43: 25: 41.00$ & 0.006 \\
\hline NGC7582 & Sy2 & Wu et al. (2009) & $23: 18: 23.50$ & $-42: 22: 14.00$ & 0.005 \\
\hline NGC7590 & Sy2 & Wu et al. (2009) & $23: 18: 55.00$ & $-42: 14: 17.00$ & 0.005 \\
\hline NGC7603 & Sy 1.5 & Wu et al. (2009) & $23: 18: 56.60$ & $00: 14: 38.00$ & 0.030 \\
\hline NGC7714 & $\mathrm{SB}+\mathrm{H}_{\text {II }}$ & Brandl et al. (2006) & $23: 36: 14.10$ & 02:09:18.60 & 0.009 \\
\hline SDSS_J005621.72+003235.8 & Sy2 & Zakamska et al. (2008) & $00: 56: 21.72$ & $00: 32: 35.80$ & 0.484 \\
\hline SJ103837.03+582214. $8^{a}$ & SB & Weedman et al. (2006) & $10: 38: 37.03$ & $58: 22: 14.80$ & 1.680 \\
\hline SJ104217.17+575459. $2^{a}$ & SB & Weedman et al. (2006) & $10: 42: 17.17$ & $57: 54: 59.20$ & 1.910 \\
\hline SJ104731.08+581016.1 ${ }^{a}$ & SB & Weedman et al. (2006) & $10: 47: 31.08$ & $58: 10: 16.10$ & 1.810 \\
\hline SST172458.3+591545 & Sy1 & Hiner et al. (2009) & $17: 24: 58.30$ & $59: 15: 45$ & 0.494 \\
\hline SWIRE4_J103637.18+584217.0 ${ }^{a}$ & ULIRG & Farrah et al. (2009) & $10: 36: 37.18$ & $58: 42: 17.00$ & 0.970 \\
\hline SWIRE4_J104057.84+565238.9 ${ }^{a}$ & ULIRG & Farrah et al. (2009) & $10: 40: 57.84$ & $56: 52: 38.90$ & 0.930 \\
\hline SWIRE4_J104117.93+595822.9 ${ }^{a}$ & ULIRG & Farrah et al. (2009) & $10: 41: 17.93$ & $59: 58: 22.90$ & 0.650 \\
\hline SWIRE4_J104830.58+591810.2 & ULIRG & Farrah et al. (2009) & $10: 48: 30.58$ & $59: 18: 10.20$ & 0.940 \\
\hline SWIRE4_J105943.83+572524.9 ${ }^{a}$ & ULIRG & Farrah et al. (2009) & $10: 59: 43.83$ & $57: 25: 24.90$ & 0.800 \\
\hline UGC5101 & Sy1 & Wu et al. (2009) & $09: 35: 51.60$ & $61: 21: 11.00$ & 0.039 \\
\hline UGC7064 & Sy 1.9 & Wu et al. (2009) & 12:04:43.30 & $31: 10: 38.00$ & 0.025 \\
\hline UGC12138 & Sy 1.8 & Deo et al. (2007) & $22: 40: 17.00$ & 08:03:14.00 & 0.025 \\
\hline
\end{tabular}

${ }^{a}$ Objects with redshift obtained through the IRS spectrum. 
Table A2. Best-fitting results for the $6.2 \mu \mathrm{m}$ band utilizing curve fit and equation (1) (Section 3.1). $A$ is the amplitude, $\lambda_{\mathrm{c}}$ is the central wavelength, and FWHM is the full width at half-maximum.

\begin{tabular}{|c|c|c|c|c|c|c|c|c|}
\hline Source & Class & $\begin{array}{c}A \\
\left(\mathrm{Jy} \mathrm{sr}^{-1}\right)\end{array}$ & $\begin{array}{c}\operatorname{Err} A \\
\left(\mathrm{Jy} \mathrm{sr}^{-1}\right)\end{array}$ & $\begin{array}{c}\lambda_{\mathrm{c}} \\
(\mu \mathrm{m})\end{array}$ & $\begin{array}{c}\text { Err } \lambda_{c} \\
(\mu \mathrm{m})\end{array}$ & FWHM & Err FWHM & $\begin{array}{l}\mathrm{rms} \\
(\%)\end{array}$ \\
\hline $3 \mathrm{C} 293$ & B & 0.695 & 0.049 & 6.234 & 0.006 & 0.175 & 0.015 & 7.155 \\
\hline AGN15 & $\mathrm{B}$ & 0.453 & 0.037 & 6.258 & 0.008 & 0.209 & 0.021 & 5.692 \\
\hline Arp220 & A & 35.186 & 1.446 & 6.223 & 0.003 & 0.164 & 0.009 & 4.986 \\
\hline CGCG381-051 & A & 7.201 & 0.839 & 6.227 & 0.009 & 0.149 & 0.021 & 12.127 \\
\hline EIRS-14 & A & 0.208 & 0.008 & 6.225 & 0.003 & 0.202 & 0.010 & 2.937 \\
\hline EIRS-2* & A & 0.181 & 0.011 & 6.229 & 0.004 & 0.135 & 0.011 & 6.905 \\
\hline EIRS-41 & B & 0.118 & 0.023 & 6.263 & 0.013 & 0.155 & 0.036 & 11.921 \\
\hline GN26 & $\mathrm{B}$ & 0.155 & 0.012 & 6.266 & 0.006 & 0.154 & 0.014 & 5.712 \\
\hline IC $342 *$ & A & 39.660 & 1.254 & 6.221 & 0.002 & 0.134 & 0.005 & 3.527 \\
\hline IRAS02021-2103* & B & 3.261 & 0.165 & 6.239 & 0.005 & 0.189 & 0.011 & 4.195 \\
\hline IRAS02480-3745* & A & 2.701 & 0.184 & 6.214 & 0.007 & 0.203 & 0.017 & 5.412 \\
\hline IRAS03209-0806* & A & 2.506 & 0.098 & 6.209 & 0.003 & 0.147 & 0.007 & 4.738 \\
\hline IRAS05020-2941* & A & 1.632 & 0.200 & 6.214 & 0.007 & 0.115 & 0.017 & 13.094 \\
\hline IRAS08591+5248* & A & 2.224 & 0.098 & 6.210 & 0.003 & 0.144 & 0.008 & 4.428 \\
\hline IRAS10594+3818* & A & 4.092 & 0.161 & 6.203 & 0.003 & 0.142 & 0.007 & 3.221 \\
\hline IRAS12447+3721 & A & 1.968 & 0.120 & 6.219 & 0.005 & 0.167 & 0.012 & 4.497 \\
\hline IRAS13106-0922 & A & 1.021 & 0.127 & 6.224 & 0.010 & 0.166 & 0.025 & 15.227 \\
\hline IRAS14121-0126* & A & 2.751 & 0.084 & 6.198 & 0.002 & 0.109 & 0.004 & 2.837 \\
\hline IRAS14197+0813 & $\mathrm{B}$ & 1.203 & 0.153 & 6.233 & 0.008 & 0.121 & 0.016 & 9.285 \\
\hline IRAS14202+2615 & A & 5.443 & 0.188 & 6.210 & 0.002 & 0.151 & 0.006 & 4.393 \\
\hline IRAS14485-2434* & A & 2.063 & 0.130 & 6.201 & 0.004 & 0.123 & 0.010 & 5.812 \\
\hline IRAS15043+5754* & A & 2.390 & 0.066 & 6.205 & 0.002 & 0.134 & 0.005 & 2.762 \\
\hline IRAS21477+0502 & A & 1.702 & 0.140 & 6.221 & 0.006 & 0.159 & 0.015 & 10.208 \\
\hline IRAS22088-1831 & A & 1.284 & 0.102 & 6.222 & 0.007 & 0.171 & 0.017 & 10.250 \\
\hline IRAS_00091-0738* & A & 0.812 & 0.073 & 6.229 & 0.004 & 0.083 & 0.009 & 6.277 \\
\hline IRAS_00456-2904* & A & 5.286 & 0.224 & 6.229 & 0.003 & 0.132 & 0.008 & 2.837 \\
\hline IRAS_01199-2307 & A & 1.390 & 0.339 & 6.223 & 0.017 & 0.143 & 0.042 & 12.177 \\
\hline IRAS_02411+0353* & A & 3.782 & 0.409 & 6.230 & 0.007 & 0.124 & 0.015 & 5.021 \\
\hline IRAS_03250+1606 & $\mathrm{B}$ & 2.986 & 0.095 & 6.244 & 0.002 & 0.146 & 0.006 & 2.936 \\
\hline IRAS_03521+0028* & A & 1.724 & 0.086 & 6.212 & 0.003 & 0.152 & 0.010 & 4.857 \\
\hline IRAS_08201+2801 & A & 2.126 & 0.092 & 6.224 & 0.004 & 0.191 & 0.011 & 3.149 \\
\hline IRAS_09039+0503* & $\mathrm{B}$ & 1.037 & 0.096 & 6.231 & 0.005 & 0.119 & 0.014 & 6.377 \\
\hline IRAS_09116+0334 & A & 2.959 & 0.127 & 6.228 & 0.003 & 0.162 & 0.009 & 5.452 \\
\hline IRAS_09463+8141 & A & 0.970 & 0.092 & 6.223 & 0.007 & 0.147 & 0.017 & 4.404 \\
\hline IRAS_09539+0857 & A & 1.073 & 0.054 & 6.229 & 0.003 & 0.141 & 0.009 & 5.355 \\
\hline IRAS_10190+1322* & A & 11.248 & 0.452 & 6.225 & 0.003 & 0.162 & 0.008 & 3.899 \\
\hline IRAS_10485-1447 & $\mathrm{B}$ & 1.554 & 0.120 & 6.237 & 0.006 & 0.159 & 0.014 & 7.475 \\
\hline IRAS_10494+4424 & $\mathrm{B}$ & 5.380 & 0.333 & 6.238 & 0.005 & 0.153 & 0.011 & 5.899 \\
\hline IRAS_11130-2659* & $\mathrm{B}$ & 0.785 & 0.079 & 6.238 & 0.005 & 0.107 & 0.013 & 8.459 \\
\hline IRAS_11387+4116 & $\mathrm{B}$ & 1.466 & 0.105 & 6.234 & 0.005 & 0.135 & 0.012 & 5.900 \\
\hline IRAS_11506+1331* & $\mathrm{B}$ & 3.769 & 0.150 & 6.242 & 0.003 & 0.138 & 0.007 & 2.846 \\
\hline IRAS_12112+0305* & A & 5.955 & 0.461 & 6.219 & 0.004 & 0.123 & 0.012 & 3.856 \\
\hline IRAS_12359-0725 & $\mathrm{B}$ & 1.091 & 0.059 & 6.240 & 0.003 & 0.139 & 0.010 & 5.229 \\
\hline IRAS_13335-2612* & $\mathrm{B}$ & 3.467 & 0.156 & 6.239 & 0.004 & 0.177 & 0.009 & 4.648 \\
\hline IRAS_13469+5833 & A & 1.186 & 0.036 & 6.223 & 0.002 & 0.150 & 0.005 & 2.225 \\
\hline IRAS_13509+0442* & $\mathrm{B}$ & 2.571 & 0.118 & 6.241 & 0.003 & 0.130 & 0.008 & 4.209 \\
\hline IRAS_13539+2920 & $\mathrm{B}$ & 4.008 & 0.111 & 6.241 & 0.002 & 0.142 & 0.005 & 2.954 \\
\hline IRAS_14060+2919* & A & 4.536 & 0.302 & 6.229 & 0.004 & 0.138 & 0.012 & 4.740 \\
\hline IRAS_14252-1550* & A & 1.482 & 0.092 & 6.216 & 0.004 & 0.151 & 0.012 & 5.129 \\
\hline IRAS_14348-1447 & $\mathrm{B}$ & 6.712 & 0.419 & 6.231 & 0.005 & 0.170 & 0.014 & 5.871 \\
\hline IRAS_15206+3342* & $\mathrm{B}$ & 3.895 & 0.169 & 6.235 & 0.002 & 0.117 & 0.006 & 3.030 \\
\hline IRAS_15225+2350* & $\mathrm{B}$ & 1.409 & 0.092 & 6.245 & 0.003 & 0.107 & 0.009 & 4.177 \\
\hline IRAS_16090-0139 & $\mathrm{B}$ & 3.432 & 0.139 & 6.248 & 0.003 & 0.153 & 0.008 & 3.864 \\
\hline IRAS_16300+1558 & A & 1.091 & 0.062 & 6.218 & 0.004 & 0.140 & 0.010 & 4.454 \\
\hline IRAS_16333+4630 & A & 1.812 & 0.068 & 6.218 & 0.002 & 0.122 & 0.005 & 4.118 \\
\hline IRAS_16474+3430* & $\mathrm{B}$ & 4.680 & 0.169 & 6.245 & 0.002 & 0.143 & 0.006 & 2.260 \\
\hline IRAS_16487+5447* & A & 1.361 & 0.116 & 6.223 & 0.004 & 0.102 & 0.010 & 5.914 \\
\hline
\end{tabular}


Table A2 - continued

\begin{tabular}{|c|c|c|c|c|c|c|c|c|}
\hline Source & Class & $\begin{array}{c}A \\
\left(\mathrm{Jy} \mathrm{sr}^{-1}\right)\end{array}$ & $\begin{array}{c}\operatorname{Err} A \\
\left(\mathrm{Jy} \mathrm{sr}^{-1}\right)\end{array}$ & $\begin{array}{c}\lambda_{\mathrm{c}} \\
(\mu \mathrm{m})\end{array}$ & $\begin{array}{c}\text { Err } \lambda_{c} \\
(\mu \mathrm{m})\end{array}$ & FWHM & Err FWHM & $\begin{array}{l}\mathrm{rms} \\
(\%)\end{array}$ \\
\hline IRAS_17028+5817* & $\mathrm{B}$ & 2.479 & 0.074 & 6.237 & 0.002 & 0.138 & 0.005 & 2.784 \\
\hline IRAS_20414-1651 & $\mathrm{B}$ & 2.495 & 0.086 & 6.233 & 0.002 & 0.133 & 0.006 & 3.963 \\
\hline IRAS_21208-0519* & $\mathrm{B}$ & 2.247 & 0.206 & 6.239 & 0.007 & 0.174 & 0.021 & 8.246 \\
\hline IRAS_21329-2346* & $\mathrm{B}$ & 1.100 & 0.077 & 6.231 & 0.004 & 0.117 & 0.010 & 5.661 \\
\hline IRAS_22491-1808 & $\mathrm{B}$ & 4.206 & 0.271 & 6.240 & 0.004 & 0.127 & 0.009 & 4.841 \\
\hline IRAS_23129+2548 & A & 1.533 & 0.111 & 6.228 & 0.006 & 0.173 & 0.016 & 9.015 \\
\hline IRAS_23234+0946 & A & 1.550 & 0.087 & 6.227 & 0.004 & 0.164 & 0.012 & 6.789 \\
\hline LH_H901A & A & 0.119 & 0.006 & 6.230 & 0.004 & 0.159 & 0.010 & 5.777 \\
\hline$M+0-29-23$ & A & 25.504 & 0.864 & 6.226 & 0.003 & 0.163 & 0.007 & 4.229 \\
\hline M-2-40-4 & A & 11.328 & 0.820 & 6.220 & 0.006 & 0.173 & 0.016 & 8.827 \\
\hline M-5-13-17 & $\mathrm{B}$ & 12.566 & 1.449 & 6.233 & 0.011 & 0.250 & 0.036 & 7.829 \\
\hline MIPS180* & $\mathrm{C}$ & 0.137 & 0.006 & 6.303 & 0.006 & 0.320 & 0.016 & 10.540 \\
\hline MIPS8040 & B & 0.174 & 0.010 & 6.235 & 0.004 & 0.132 & 0.009 & 5.225 \\
\hline MIPS8242* & B & 0.050 & 0.004 & 6.282 & 0.006 & 0.146 & 0.014 & 9.851 \\
\hline MIPS15755 & $\mathrm{B}$ & 0.223 & 0.010 & 6.231 & 0.003 & 0.156 & 0.009 & 4.730 \\
\hline MIPS22307* & A & 0.115 & 0.007 & 6.217 & 0.003 & 0.090 & 0.006 & 4.758 \\
\hline MIPS22352 & A & 0.324 & 0.013 & 6.230 & 0.004 & 0.225 & 0.012 & 4.023 \\
\hline Mrk52* & A & 12.490 & 0.481 & 6.209 & 0.003 & 0.146 & 0.007 & 3.900 \\
\hline Mrk273* & A & 11.823 & 0.976 & 6.212 & 0.005 & 0.130 & 0.013 & 7.593 \\
\hline Mrk334 & B & 16.966 & 0.760 & 6.244 & 0.004 & 0.156 & 0.009 & 3.191 \\
\hline Mrk471 & B & 3.212 & 0.231 & 6.245 & 0.006 & 0.181 & 0.015 & 3.970 \\
\hline Mrk609 & A & 10.671 & 0.479 & 6.225 & 0.004 & 0.164 & 0.009 & 4.127 \\
\hline Mrk622 & A & 2.439 & 0.234 & 6.210 & 0.007 & 0.141 & 0.016 & 6.779 \\
\hline Mrk883 & B & 2.445 & 0.061 & 6.231 & 0.002 & 0.174 & 0.005 & 2.178 \\
\hline Mrk938* & A & 32.861 & 0.501 & 6.215 & 0.001 & 0.134 & 0.002 & 1.422 \\
\hline Mrk1066 & A & 19.651 & 0.593 & 6.227 & 0.003 & 0.205 & 0.008 & 3.265 \\
\hline Murphy3 & B & 0.183 & 0.006 & 6.232 & 0.003 & 0.188 & 0.008 & 3.300 \\
\hline NGC513* & A & 11.852 & 1.162 & 6.228 & 0.006 & 0.179 & 0.024 & 6.312 \\
\hline NGC520* & $\mathrm{B}$ & 65.937 & 1.346 & 6.233 & 0.002 & 0.148 & 0.004 & 2.020 \\
\hline NGC660* & A & 139.918 & 4.928 & 6.221 & 0.003 & 0.154 & 0.007 & 4.166 \\
\hline NGC1056* & A & 33.577 & 2.062 & 6.221 & 0.005 & 0.155 & 0.012 & 5.681 \\
\hline NGC1097* & A & 117.594 & 5.763 & 6.214 & 0.004 & 0.163 & 0.010 & 3.869 \\
\hline NGC1125 & A & 7.325 & 0.305 & 6.218 & 0.003 & 0.139 & 0.007 & 4.936 \\
\hline NGC1143 & B & 22.696 & 1.473 & 6.231 & 0.005 & 0.160 & 0.013 & 7.458 \\
\hline NGC1222* & A & 26.367 & 0.807 & 6.223 & 0.002 & 0.153 & 0.006 & 3.244 \\
\hline NGC1365 & A & 32.675 & 1.013 & 6.216 & 0.002 & 0.169 & 0.007 & 3.541 \\
\hline NGC1566* & A & 28.350 & 1.036 & 6.219 & 0.003 & 0.147 & 0.007 & 3.827 \\
\hline NGC1614* & A & 62.998 & 2.944 & 6.229 & 0.003 & 0.141 & 0.008 & 2.908 \\
\hline NGC1667 & A & 22.479 & 2.130 & 6.226 & 0.008 & 0.177 & 0.021 & 10.439 \\
\hline NGC2146* & A & 334.740 & 10.222 & 6.224 & 0.002 & 0.151 & 0.006 & 3.538 \\
\hline NGC2273 & A & 12.619 & 0.484 & 6.229 & 0.003 & 0.198 & 0.010 & 4.383 \\
\hline NGC2623* & A & 18.147 & 0.518 & 6.222 & 0.002 & 0.138 & 0.005 & 3.274 \\
\hline NGC2992 & $\mathrm{B}$ & 21.411 & 1.301 & 6.234 & 0.005 & 0.192 & 0.015 & 6.077 \\
\hline NGC3079* & A & 87.002 & 2.532 & 6.219 & 0.002 & 0.161 & 0.006 & 3.278 \\
\hline NGC3227* & A & 24.795 & 1.318 & 6.218 & 0.004 & 0.167 & 0.011 & 4.418 \\
\hline NGC3256* & A & 120.455 & 4.367 & 6.213 & 0.003 & 0.148 & 0.007 & 3.916 \\
\hline NGC3310* & A & 39.139 & 1.175 & 6.222 & 0.002 & 0.161 & 0.006 & 3.374 \\
\hline NGC3511 & B & 25.836 & 1.976 & 6.238 & 0.008 & 0.224 & 0.020 & 5.848 \\
\hline NGC3556* & A & 21.479 & 0.657 & 6.207 & 0.002 & 0.149 & 0.006 & 3.398 \\
\hline NGC3628* & A & 75.757 & 2.304 & 6.218 & 0.002 & 0.143 & 0.005 & 2.679 \\
\hline NGC3786* & A & 4.701 & 0.695 & 6.201 & 0.010 & 0.148 & 0.028 & 7.798 \\
\hline NGC3982 & B & 20.085 & 1.241 & 6.235 & 0.005 & 0.179 & 0.013 & 6.602 \\
\hline NGC4088* & A & 13.764 & 0.410 & 6.215 & 0.002 & 0.145 & 0.005 & 2.587 \\
\hline NGC4194* & A & 83.204 & 2.671 & 6.217 & 0.002 & 0.148 & 0.006 & 3.778 \\
\hline NGC4388 & A & 18.174 & 0.864 & 6.220 & 0.004 & 0.186 & 0.011 & 4.722 \\
\hline NGC4676* & A & 11.294 & 0.465 & 6.219 & 0.003 & 0.141 & 0.007 & 3.152 \\
\hline NGC4818* & A & 45.248 & 1.499 & 6.219 & 0.002 & 0.135 & 0.005 & 2.490 \\
\hline NGC4945* & A & 111.908 & 9.806 & 6.223 & 0.005 & 0.116 & 0.012 & 7.312 \\
\hline
\end{tabular}


Table A2 - continued

\begin{tabular}{|c|c|c|c|c|c|c|c|c|}
\hline Source & Class & $\begin{array}{c}A \\
\left(\mathrm{Jy} \mathrm{sr}^{-1}\right)\end{array}$ & $\begin{array}{c}\operatorname{Err} A \\
\left(\mathrm{Jy} \mathrm{sr}^{-1}\right)\end{array}$ & $\begin{array}{c}\lambda_{\mathrm{c}} \\
(\mu \mathrm{m})\end{array}$ & $\begin{array}{c}\text { Err } \lambda_{c} \\
(\mu \mathrm{m})\end{array}$ & FWHM & Err FWHM & $\begin{array}{l}\mathrm{rms} \\
(\%)\end{array}$ \\
\hline NGC5005 & $\mathrm{A}$ & 17.236 & 0.666 & 6.212 & 0.003 & 0.157 & 0.008 & 4.646 \\
\hline NGC5135 & $\mathrm{A}$ & 45.839 & 1.783 & 6.225 & 0.003 & 0.156 & 0.007 & 4.074 \\
\hline NGC5194 & $\mathrm{A}$ & 135.664 & 3.886 & 6.227 & 0.002 & 0.176 & 0.006 & 3.395 \\
\hline NGC5256* & A & 18.107 & 0.654 & 6.216 & 0.002 & 0.138 & 0.006 & 3.635 \\
\hline NGC5674 & A & 1.939 & 0.036 & 6.219 & 0.002 & 0.256 & 0.006 & 1.332 \\
\hline NGC5953 & B & 13.449 & 0.467 & 6.239 & 0.003 & 0.165 & 0.007 & 4.183 \\
\hline NGC6810* & A & 56.167 & 1.677 & 6.218 & 0.002 & 0.146 & 0.005 & 2.894 \\
\hline NGC6890* & A & 11.585 & 0.861 & 6.225 & 0.005 & 0.173 & 0.017 & 6.735 \\
\hline NGC7130 & $\mathrm{B}$ & 18.447 & 0.331 & 6.244 & 0.001 & 0.171 & 0.004 & 2.149 \\
\hline NGC7252* & A & 23.719 & 0.900 & 6.219 & 0.003 & 0.149 & 0.007 & 3.234 \\
\hline NGC7469* & A & 58.434 & 2.659 & 6.220 & 0.003 & 0.143 & 0.008 & 4.315 \\
\hline NGC7496 & A & 13.969 & 0.896 & 6.212 & 0.005 & 0.169 & 0.013 & 8.060 \\
\hline NGC7582* & A & 88.536 & 3.987 & 6.221 & 0.003 & 0.137 & 0.007 & 4.654 \\
\hline NGC7603* & A & 8.112 & 0.852 & 6.193 & 0.007 & 0.152 & 0.021 & 9.211 \\
\hline NGC7714* & A & 28.309 & 1.152 & 6.222 & 0.003 & 0.138 & 0.006 & 2.478 \\
\hline SDSS_J005621.72+003235.8* & $\mathrm{C}$ & 0.948 & 0.096 & 6.325 & 0.014 & 0.392 & 0.053 & 10.465 \\
\hline SJ103837.03+582214.8 & A & 0.222 & 0.026 & 6.193 & 0.011 & 0.216 & 0.033 & 12.454 \\
\hline SJ104217.17+575459.2 & $\mathrm{B}$ & 0.205 & 0.013 & 6.241 & 0.006 & 0.247 & 0.019 & 4.945 \\
\hline SJ104731.08+581016.1 & A & 0.251 & 0.022 & 6.215 & 0.007 & 0.185 & 0.021 & 8.785 \\
\hline SST172458.3+591545 & $\mathrm{B}$ & 0.115 & 0.008 & 6.233 & 0.005 & 0.160 & 0.014 & 7.053 \\
\hline SWIRE4_J103637.18+584217.0* & $\mathrm{C}$ & 0.229 & 0.014 & 6.330 & 0.008 & 0.275 & 0.020 & 9.759 \\
\hline SWIRE4_J104057.84+565238.9 & A & 0.099 & 0.006 & 6.220 & 0.005 & 0.192 & 0.014 & 5.687 \\
\hline SWIRE4_J104117.93+595822.9 & A & 0.105 & 0.003 & 6.221 & 0.003 & 0.200 & 0.008 & 2.939 \\
\hline SWIRE4_J104830.58+591810.2* & A & 0.208 & 0.008 & 6.213 & 0.003 & 0.164 & 0.009 & 4.055 \\
\hline SWIRE4_J105943.83+572524.9* & A & 0.085 & 0.009 & 6.206 & 0.007 & 0.136 & 0.017 & 10.854 \\
\hline UGC5101* & A & 14.196 & 0.681 & 6.213 & 0.003 & 0.150 & 0.009 & 5.359 \\
\hline UGC7064* & B & 11.935 & 1.186 & 6.245 & 0.011 & 0.227 & 0.029 & 11.408 \\
\hline UGC12138 & $\mathrm{A}$ & 2.622 & 0.118 & 6.222 & 0.003 & 0.155 & 0.009 & 3.400 \\
\hline
\end{tabular}

* Objects with profilesle's red tail disconsidered from the fit.

Table A3. Values of $r_{\mathrm{PDR}}$ and $\mathrm{F}_{25} / \mathrm{F}_{20}$ ratio for sources from the MIR_SB sample (Spitzer/IRS ATLAS, version 1.0).

\begin{tabular}{|c|c|c|c|c|}
\hline ID & $r_{\mathrm{PDR}}$ & Err $r_{\mathrm{PDR}}$ & $\mathrm{F}_{25} / \mathrm{F}_{20}$ & $\operatorname{Err~}_{25} / \mathrm{F}_{20}$ \\
\hline $3 \mathrm{C} 293$ & 0.201 & 0.002 & 1.273 & 0.016 \\
\hline $3 \mathrm{C} 31$ & 0.277 & 0.002 & 1.017 & 0.019 \\
\hline AGN15 & 0.168 & 0.009 & 1.521 & 0.051 \\
\hline Arp220 & 0.268 & 0.001 & 3.89 & 0.009 \\
\hline CGCG381-051 & 0.329 & 0.013 & 1.088 & 0.022 \\
\hline E12-G21 & 0.328 & 0.008 & 1.162 & 0.035 \\
\hline EIRS-14 & 0.485 & 0.038 & - & - \\
\hline EIRS-2 & 0.556 & 0.019 & - & - \\
\hline EIRS-41 & 0.236 & 0.044 & 0.789 & 0.149 \\
\hline GN26 & 0.617 & 0.02 & - & - \\
\hline IC342 & 0.413 & 0.001 & 2.243 & 0.004 \\
\hline IRAS02021-2103 & 0.226 & 0.002 & 1.873 & 0.007 \\
\hline IRAS02480-3745 & 0.472 & 0.005 & 3.205 & 0.029 \\
\hline IRAS03209-0806 & 0.379 & 0.003 & 1.914 & 0.011 \\
\hline IRAS04074-2801 & 0.319 & 0.002 & 2.854 & 0.021 \\
\hline IRAS05020-2941 & 0.396 & 0.003 & 3.134 & 0.019 \\
\hline IRAS08591+5248 & 0.51 & 0.003 & 1.866 & 0.018 \\
\hline IRAS10594+3818 & 0.497 & 0.002 & 2.128 & 0.01 \\
\hline IRAS12447+3721 & 0.355 & 0.003 & 2.348 & 0.016 \\
\hline IRAS13106-0922 & 0.462 & 0.003 & 4.878 & 0.108 \\
\hline IRAS14121-0126 & 0.448 & 0.003 & 2.045 & 0.013 \\
\hline IRAS14197+0813 & 0.346 & 0.004 & 1.974 & 0.013 \\
\hline IRAS14202+2615 & 0.339 & 0.002 & 1.99 & 0.006 \\
\hline IRAS14485-2434 & 0.293 & 0.002 & 1.869 & 0.011 \\
\hline IRAS15043+5754 & 0.529 & 0.002 & 2.936 & 0.024 \\
\hline IRAS21477+0502 & 0.251 & 0.001 & 1.852 & 0.006 \\
\hline
\end{tabular}


Variations in the $6.2 \mu \mathrm{m}$ PAH band

Table A3 - continued

\begin{tabular}{|c|c|c|c|c|}
\hline ID & $r_{\mathrm{PDR}}$ & Err $r_{\mathrm{PDR}}$ & $\mathrm{F}_{25} / \mathrm{F}_{20}$ & Err $F_{25} / F_{20}$ \\
\hline IRAS22088-1831 & 0.263 & 0.001 & 3.281 & 0.013 \\
\hline IRAS_00091-0738 & 0.25 & 0.003 & 2.961 & 0.015 \\
\hline IRAS_00456-2904 & 0.529 & 0.003 & 2.039 & 0.012 \\
\hline IRAS_01199-2307 & 0.3 & 0.003 & 2.537 & 0.016 \\
\hline IRAS_01355-1814 & 0.197 & 0.004 & 2.834 & 0.024 \\
\hline IRAS_01494-1845 & 0.458 & 0.006 & 2.009 & 0.023 \\
\hline IRAS_02411+0353 & 0.38 & 0.003 & 1.601 & 0.007 \\
\hline IRAS_03250+1606 & 0.45 & 0.003 & 2.056 & 0.019 \\
\hline IRAS_03521+0028 & 0.354 & 0.004 & 2.7 & 0.018 \\
\hline IRAS_08201+2801 & 0.343 & 0.004 & 2.214 & 0.012 \\
\hline IRAS_09039+0503 & 0.371 & 0.006 & 2.87 & 0.036 \\
\hline IRAS_09116+0334 & 0.529 & 0.004 & 1.758 & 0.019 \\
\hline IRAS_09463+8141 & 0.38 & 0.008 & 3.262 & 0.077 \\
\hline IRAS_09539+0857 & 0.304 & 0.004 & 3.438 & 0.035 \\
\hline IRAS_10190+1322 & 0.559 & 0.001 & 1.926 & 0.009 \\
\hline IRAS_10485-1447 & 0.19 & 0.003 & 2.572 & 0.02 \\
\hline IRAS_10494+4424 & 0.523 & 0.003 & 2.551 & 0.02 \\
\hline IRAS_11130-2659 & 0.279 & 0.004 & 3.119 & 0.029 \\
\hline IRAS_11387+4116 & 0.479 & 0.006 & 1.986 & 0.023 \\
\hline IRAS_11506+1331 & 0.287 & 0.048 & 2.274 & 0.011 \\
\hline IRAS_12112+0305 & 0.403 & 0.003 & 2.637 & 0.014 \\
\hline IRAS_12359-0725 & 0.168 & 0.004 & 2.238 & 0.013 \\
\hline IRAS_13335-2612 & 0.493 & 0.003 & 2.091 & 0.02 \\
\hline IRAS_13469+5833 & 0.346 & 0.006 & 2.535 & 0.012 \\
\hline IRAS_13509+0442 & 0.458 & 0.004 & 2.021 & 0.018 \\
\hline IRAS_13539+2920 & 0.522 & 0.003 & 2.274 & 0.018 \\
\hline IRAS_14060+2919 & 0.499 & 0.003 & 1.757 & 0.011 \\
\hline IRAS_14252-1550 & 0.39 & 0.005 & 2.212 & 0.028 \\
\hline IRAS_14348-1447 & 0.346 & 0.003 & 2.754 & 0.014 \\
\hline IRAS_15206+3342 & 0.239 & 0.003 & 1.457 & 0.005 \\
\hline IRAS_15225+2350 & 0.219 & 0.041 & 2.097 & 0.01 \\
\hline IRAS_16090-0139 & 0.291 & 0.002 & 2.336 & 0.011 \\
\hline IRAS_16300+1558 & 0.269 & 0.04 & 3.062 & 0.02 \\
\hline IRAS_16333+4630 & 0.41 & 0.004 & 2.124 & 0.018 \\
\hline IRAS_16474+3430 & 0.467 & 0.003 & 2.084 & 0.013 \\
\hline IRAS_16487+5447 & 0.354 & 0.003 & 2.83 & 0.02 \\
\hline IRAS_17028+5817 & 0.513 & 0.004 & 2.55 & 0.027 \\
\hline IRAS_17068+4027 & 0.294 & 0.003 & 2.013 & 0.01 \\
\hline IRAS_20414-1651 & 0.417 & 0.006 & 3.185 & 0.025 \\
\hline IRAS_21208-0519 & 0.468 & 0.004 & 2.041 & 0.02 \\
\hline IRAS_21329-2346 & 0.395 & 0.005 & 3.13 & 0.038 \\
\hline IRAS_22206-2715 & 0.405 & 0.006 & 3.139 & 0.038 \\
\hline IRAS_22491-1808 & 0.352 & 0.029 & 2.818 & 0.014 \\
\hline IRAS_23129+2548 & 0.342 & 0.003 & 2.766 & 0.014 \\
\hline IRAS_23234+0946 & 0.289 & 0.005 & 2.38 & 0.018 \\
\hline LH_H901A & 0.217 & 0.011 & - & - \\
\hline$M+0-29-23$ & 0.526 & 0.009 & 1.332 & 0.023 \\
\hline M-2-40-4 & 0.164 & 0.004 & 1.029 & 0.014 \\
\hline M-5-13-17 & 0.237 & 0.009 & 1.054 & 0.021 \\
\hline MIPS 15755 & 0.373 & 0.026 & - & - \\
\hline MIPS180 & 0.057 & 0.044 & - & - \\
\hline MIPS22307 & 0.286 & 0.032 & - & - \\
\hline MIPS22352 & 0.534 & 0.041 & - & - \\
\hline MIPS562 & 0.553 & 0.054 & - & - \\
\hline MIPS8040 & 0.293 & 0.031 & - & - \\
\hline Mrk1066 & 0.291 & 0.002 & 1.489 & 0.004 \\
\hline Mrk273 & 0.22 & 0.002 & 2.491 & 0.008 \\
\hline Mrk334 & 0.326 & 0.002 & 1.361 & 0.004 \\
\hline Mrk471 & 0.427 & 0.005 & 1.224 & 0.014 \\
\hline Mrk52 & 0.318 & 0.005 & 1.142 & 0.005 \\
\hline Mrk609 & 0.47 & 0.003 & 1.33 & 0.008 \\
\hline Mrk622 & 0.199 & 0.004 & 1.325 & 0.006 \\
\hline
\end{tabular}


Table A3 - continued

\begin{tabular}{|c|c|c|c|c|}
\hline ID & $r_{\mathrm{PDR}}$ & Err $r_{\mathrm{PDR}}$ & $\mathrm{F}_{25} / \mathrm{F}_{20}$ & Err $F_{25} / F_{20}$ \\
\hline Mrk883 & 0.247 & 0.004 & 1.396 & 0.008 \\
\hline Mrk938 & 0.498 & 0.005 & 1.834 & 0.019 \\
\hline Murphy22 & 0.423 & 0.025 & - & - \\
\hline Murphy3 & 0.382 & 0.025 & - & - \\
\hline Murphy8 & 0.391 & 0.038 & - & - \\
\hline NGC1056 & 0.662 & 0.007 & 1.394 & 0.037 \\
\hline NGC1097 & 0.572 & 0.001 & 1.274 & 0.004 \\
\hline NGC1125 & 0.303 & 0.061 & 1.368 & 0.022 \\
\hline NGC1143 & 0.49 & 0.009 & - & - \\
\hline NGC1222 & 0.436 & 0.002 & 1.461 & 0.005 \\
\hline NGC1365 & 0.173 & 0.001 & 1.562 & 0.003 \\
\hline NGC1566 & 0.422 & 0.003 & 1.053 & 0.005 \\
\hline NGC1614 & 0.399 & 0.001 & 1.288 & 0.003 \\
\hline NGC1667 & 0.626 & 0.009 & 1.304 & 0.045 \\
\hline NGC2146 & 0.656 & 0.012 & 2.007 & 0.003 \\
\hline NGC2273 & 0.23 & 0.001 & 1.206 & 0.004 \\
\hline NGC2623 & 0.442 & 0.002 & 2.585 & 0.011 \\
\hline NGC2992 & 0.21 & 0.005 & 1.053 & 0.012 \\
\hline NGC3079 & 0.651 & 0.043 & 2.312 & 0.011 \\
\hline NGC3227 & 0.172 & 0.002 & 1.067 & 0.004 \\
\hline NGC3256 & 0.414 & 0.001 & 1.735 & 0.002 \\
\hline NGC3310 & 0.557 & 0.002 & 1.62 & 0.007 \\
\hline NGC3511 & 0.701 & 0.013 & 1.242 & 0.062 \\
\hline NGC3556 & 0.607 & 0.003 & 1.881 & 0.014 \\
\hline NGC3628 & 0.656 & 0.029 & 2.311 & 0.009 \\
\hline NGC3786 & 0.288 & 0.003 & 1.241 & 0.008 \\
\hline NGC3982 & 0.568 & 0.009 & 1.18 & 0.028 \\
\hline NGC4088 & 0.539 & 0.004 & 1.542 & 0.012 \\
\hline NGC4194 & 0.477 & 0.001 & 1.634 & 0.004 \\
\hline NGC4388 & 0.196 & 0.004 & 1.301 & 0.011 \\
\hline NGC4676 & 0.635 & 0.003 & 1.706 & 0.01 \\
\hline NGC4818 & 0.355 & 0.001 & 1.298 & 0.003 \\
\hline NGC4945 & 0.598 & 0.001 & 4.55 & 0.01 \\
\hline NGC5005 & 0.457 & 0.007 & 1.514 & 0.033 \\
\hline NGC5033 & 0.562 & 0.002 & 1.162 & 0.005 \\
\hline NGC5135 & 0.477 & 0.004 & 1.376 & 0.012 \\
\hline NGC513 & 0.465 & 0.014 & 1.046 & 0.037 \\
\hline NGC5194 & 0.588 & 0.001 & 1.26 & 0.004 \\
\hline NGC520 & 0.619 & 0.01 & 2.521 & 0.007 \\
\hline NGC5256 & 0.54 & 0.008 & 1.602 & 0.028 \\
\hline NGC5674 & 0.21 & 0.005 & - & - \\
\hline NGC5953 & 0.556 & 0.008 & 1.485 & 0.019 \\
\hline NGC660 & 0.558 & 0.026 & 1.934 & 0.004 \\
\hline NGC6810 & 0.458 & 0.004 & 1.092 & 0.008 \\
\hline NGC6890 & 0.285 & 0.008 & 1.041 & 0.02 \\
\hline NGC7130 & 0.267 & 0.005 & 1.44 & 0.009 \\
\hline NGC7252 & 0.678 & 0.002 & 1.564 & 0.009 \\
\hline NGC7469 & 0.292 & 0.003 & 1.181 & 0.006 \\
\hline NGC7496 & 0.288 & 0.005 & 1.499 & 0.012 \\
\hline NGC7582 & 0.405 & 0.002 & 1.451 & 0.008 \\
\hline NGC7590 & 0.623 & 0.011 & 1.206 & 0.055 \\
\hline NGC7603 & 0.158 & 0.003 & 0.83 & 0.02 \\
\hline NGC7714 & 0.359 & 0.002 & 1.361 & 0.004 \\
\hline SDSS_J005621.72+003235.8 & 0.174 & 0.005 & - & - \\
\hline SJ103837.03+582214.8 & 0.373 & 0.063 & - & - \\
\hline SJ104217.17+575459.2 & 0.574 & 0.124 & - & - \\
\hline SJ104731.08+581016.1 & 0.21 & 0.042 & - & - \\
\hline SST172458.3+591545 & 0.215 & 0.021 & - & - \\
\hline SWIRE4_J103637.18+584217.0 & 0.205 & 0.015 & - & - \\
\hline SWIRE4_J104117.93+595822.9 & 0.333 & 0.045 & - & - \\
\hline SWIRE4_J105943.83+572524.9 & 0.295 & 0.026 & - & - \\
\hline UGC12138 & 0.154 & 0.002 & 1.119 & 0.005 \\
\hline UGC5101 & 0.325 & 0.003 & 1.824 & 0.006 \\
\hline UGC7064 & 0.345 & 0.012 & 0.966 & 0.027 \\
\hline
\end{tabular}




\section{APPENDIX B: COMPARISON OF THE $6.2 \mu \mathrm{m}$ RESULTS FOR THE CONTINUUM DECOMPOSITION}

The Tables B1-B3 and the Figs B1-B3 compare the differences among the $6.2 \mu \mathrm{m}$ band fits according to the spectral continuum contribution fitted with the three methods described below. Such contribution was subtracted from the spectra before the band was fitted. 20 galaxies were selected for this comparison, 10 with strong PAH plateaus, and 10 with weak or none plateaus (see Section 3). To standardize the comparison, in all cases the band was fitted using the same method. We fitted using only one Gaussian profile and the submodule curve fit. The following methodology was applied. (i) Subtraction of the continuum and ionic and molecular contributions fitted with PAHFIT,

(ii) only the subtraction of the continuum contribution fitted with PAHFIT, and

(iii) subtraction of the ionic and molecular contributions fitted with PAHFIT and the subtraction of the continuum contribution decomposed with spline.

The central wavelengths, which are the objective of this work, presented values range within the uncertainty bar of the parameter. Therefore, the method chosen does not influence the final results in our analysis. On the other hand, the band intensity and the FWHM

Table B1. Results of the fitted amplitude $(A)$ of the band of $6.2 \mu \mathrm{m}$ for each continuum decomposition method.

\begin{tabular}{|c|c|c|c|c|}
\hline ID & Plateau & (i) $A\left(\mathrm{Jy} \mathrm{sr}^{-1}\right)$ & (ii) $A\left(\mathrm{Jy} \mathrm{sr}^{-1}\right)$ & (iii) $A\left(\mathrm{Jy} \mathrm{sr}^{-1}\right)$ \\
\hline $3 \mathrm{C} 31$ & yes & $2.422 \pm 0.078$ & $2.422 \pm 0.078$ & $1.611 \pm 0.043$ \\
\hline IRAS_00456-2904 & yes & $8.190 \pm 0.532$ & $8.190 \pm 0.532$ & $5.286 \pm 0.224$ \\
\hline IRAS_09116+0334 & yes & $4.386 \pm 0.215$ & $4.386 \pm 0.215$ & $2.959 \pm 0.127$ \\
\hline IRAS_10190+1322 & yes & $15.824 \pm 0.710$ & $15.824 \pm 0.710$ & $11.248 \pm 0.452$ \\
\hline IRAS_16474+3430 & yes & $7.327 \pm 0.449$ & $7.327 \pm 0.449$ & $4.680 \pm 0.169$ \\
\hline IRAS08591+5248 & yes & $3.620 \pm 0.201$ & $3.945 \pm 0.213$ & $2.224 \pm 0.098$ \\
\hline IRAS14202+2615 & yes & $7.619 \pm 0.272$ & $7.619 \pm 0.272$ & $5.443 \pm 0.188$ \\
\hline IRAS15043+5754 & yes & $4.105 \pm 0.207$ & $4.105 \pm 0.207$ & $2.390 \pm 0.066$ \\
\hline Mrk1066 & no & $35.947 \pm 0.476$ & $37.757 \pm 0.762$ & $19.651 \pm 0.593$ \\
\hline Mrk334 & no & $26.897 \pm 2.344$ & $26.897 \pm 2.344$ & $16.966 \pm 0.760$ \\
\hline Mrk52 & no & $15.240 \pm 0.640$ & $15.240 \pm 0.640$ & $12.490 \pm 0.481$ \\
\hline Mrk609 & no & $15.649 \pm 0.807$ & $15.649 \pm 0.807$ & $10.671 \pm 0.479$ \\
\hline Mrk622 & no & $3.440 \pm 0.331$ & $3.945 \pm 0.199$ & $2.439 \pm 0.479$ \\
\hline Mrk883 & no & $2.994 \pm 0.089$ & $2.994 \pm 0.089$ & $2.445 \pm 0.061$ \\
\hline NGC1097 & yes & $191.185 \pm 8.974$ & $193.840 \pm 7.407$ & $117.594 \pm 5.763$ \\
\hline NGC1222 & no & $39.608 \pm 1.380$ & $39.608 \pm 1.380$ & $26.367 \pm 0.807$ \\
\hline NGC1365 & no & $40.451 \pm 1.171$ & $40.451 \pm 1.171$ & $32.675 \pm 1.013$ \\
\hline NGC2273 & no & $22.736 \pm 0.326$ & $23.609 \pm 0.496$ & $12.619 \pm 0.484$ \\
\hline NGC2992 & no & $30.599 \pm 1.694$ & $30.599 \pm 1.694$ & $21.411 \pm 1.301$ \\
\hline NGC3556 & yes & $35.212 \pm 1.338$ & $35.212 \pm 1.338$ & $21.479 \pm 0.657$ \\
\hline
\end{tabular}

Table B2. Results of the fitted central wavelength $\left(\lambda_{\mathrm{c}}\right)$ of the band of $6.2 \mu \mathrm{m}$ for each continuum decomposition method.

\begin{tabular}{|c|c|c|c|c|}
\hline ID & Plateau & (i) $\lambda_{\mathrm{c}}(\mu \mathrm{m})$ & (ii) $\lambda_{\mathrm{c}}(\mu \mathrm{m})$ & (iii) $\lambda_{\mathrm{c}}(\mu \mathrm{m})$ \\
\hline $3 C 31$ & yes & $6.233 \pm 0.003$ & $6.233 \pm 0.003$ & $6.230 \pm 0.002$ \\
\hline IRAS_00456-2904 & yes & $6.238 \pm 0.007$ & $6.238 \pm 0.007$ & $6.229 \pm 0.003$ \\
\hline IRAS_09116+0334 & yes & $6.231 \pm 0.005$ & $6.231 \pm 0.005$ & $6.228 \pm 0.003$ \\
\hline IRAS_10190+1322 & yes & $6.233 \pm 0.005$ & $6.233 \pm 0.005$ & $6.225 \pm 0.003$ \\
\hline IRAS_16474+3430 & yes & $6.251 \pm 0.006$ & $6.251 \pm 0.006$ & $6.245 \pm 0.002$ \\
\hline IRAS08591+5248 & yes & $6.216 \pm 0.005$ & $6.203 \pm 0.004$ & $6.210 \pm 0.003$ \\
\hline IRAS14202+2615 & yes & $6.212 \pm 0.003$ & $6.212 \pm 0.003$ & $6.210 \pm 0.002$ \\
\hline IRAS15043+5754 & yes & $6.214 \pm 0.005$ & $6.214 \pm 0.005$ & $6.205 \pm 0.002$ \\
\hline Mrk1066 & no & $6.231 \pm 0.001$ & $6.230 \pm 0.002$ & $6.227 \pm 0.003$ \\
\hline Mrk334 & no & $6.245 \pm 0.009$ & $6.245 \pm 0.009$ & $6.244 \pm 0.004$ \\
\hline Mrk52 & no & $6.214 \pm 0.003$ & $6.214 \pm 0.003$ & $6.209 \pm 0.003$ \\
\hline Mrk609 & no & $6.225 \pm 0.005$ & $6.225 \pm 0.005$ & $6.225 \pm 0.004$ \\
\hline Mrk622 & no & $6.209 \pm 0.008$ & $6.197 \pm 0.004$ & $6.210 \pm 0.007$ \\
\hline Mrk883 & no & $6.229 \pm 0.003$ & $6.229 \pm 0.003$ & $6.231 \pm 0.002$ \\
\hline NGC1097 & yes & $6.217 \pm 0.004$ & $6.213 \pm 0.003$ & $6.214 \pm 0.004$ \\
\hline NGC1222 & no & $6.227 \pm 0.003$ & $6.227 \pm 0.003$ & $6.223 \pm 0.002$ \\
\hline NGC1365 & no & $6.216 \pm 0.002$ & $6.216 \pm 0.002$ & $6.216 \pm 0.002$ \\
\hline NGC2273 & no & $6.232 \pm 0.001$ & $6.232 \pm 0.001$ & $6.229 \pm 0.003$ \\
\hline NGC2992 & no & $6.237 \pm 0.005$ & $6.237 \pm 0.005$ & $6.234 \pm 0.005$ \\
\hline NGC3556 & yes & $6.213 \pm 0.003$ & $6.213 \pm 0.003$ & $6.207 \pm 0.002$ \\
\hline
\end{tabular}


Table B3. Results of the fitted FWHM of the band of $6.2 \mu \mathrm{m}$ for each continuum decomposition method.

\begin{tabular}{lcccr}
\hline ID & Plateau & (i) FWHM & (ii) FWHM & (iii) FWHM \\
\hline 3C31 & yes & $0.233 \pm 0.010$ & $0.233 \pm 0.010$ & $0.197 \pm 0.007$ \\
IRAS_00456-2904 & yes & $0.186 \pm 0.016$ & $0.186 \pm 0.016$ & $0.132 \pm 0.008$ \\
IRAS_09116+0334 & yes & $0.202 \pm 0.014$ & $0.202 \pm 0.014$ & $0.162 \pm 0.009$ \\
IRAS_10190+1322 & yes & $0.201 \pm 0.011$ & $0.201 \pm 0.011$ & $0.162 \pm 0.008$ \\
IRAS_16474+3430 & yes & $0.192 \pm 0.014$ & $0.195 \pm 0.014$ & $0.143 \pm 0.006$ \\
IRAS08591+5248 & yes & $0.178 \pm 0.008$ & $0.181 \pm 0.013$ & $0.144 \pm 0.008$ \\
IRAS14202+2615 & yes & $0.196 \pm 0.013$ & $0.178 \pm 0.008$ & $0.151 \pm 0.006$ \\
IRAS15043+5754 & yes & $0.195 \pm 0.014$ & $0.196 \pm 0.013$ & $0.134 \pm 0.005$ \\
Mrk1066 & no & $0.269 \pm 0.004$ & $0.286 \pm 0.007$ & $0.205 \pm 0.008$ \\
Mrk334 & no & $0.209 \pm 0.024$ & $0.209 \pm 0.024$ & $0.156 \pm 0.009$ \\
Mrk52 & no & $0.168 \pm 0.009$ & $0.168 \pm 0.009$ & $0.146 \pm 0.007$ \\
Mrk609 & no & $0.203 \pm 0.013$ & $0.203 \pm 0.013$ & $0.164 \pm 0.009$ \\
Mrk622 & no & $0.170 \pm 0.022$ & $0.184 \pm 0.013$ & $0.141 \pm 0.016$ \\
Mrk883 & no & $0.196 \pm 0.007$ & $0.196 \pm 0.007$ & $0.174 \pm 0.005$ \\
NGC1097 & yes & $0.212 \pm 0.013$ & $0.207 \pm 0.010$ & $0.163 \pm 0.010$ \\
NGC1222 & no & $0.195 \pm 0.009$ & $0.195 \pm 0.009$ & $0.153 \pm 0.006$ \\
NGC1365 & no & $0.186 \pm 0.007$ & $0.186 \pm 0.007$ & $0.169 \pm 0.007$ \\
NGC2273 & no & $0.262 \pm 0.005$ & $0.275 \pm 0.007$ & $0.198 \pm 0.010$ \\
NGC2992 & no & $0.223 \pm 0.015$ & $0.198 \pm 0.010$ & $0.192 \pm 0.015$ \\
NGC3556 & yes & $0.198 \pm 0.010$ & $0.149 \pm 0.006$ \\
\hline
\end{tabular}

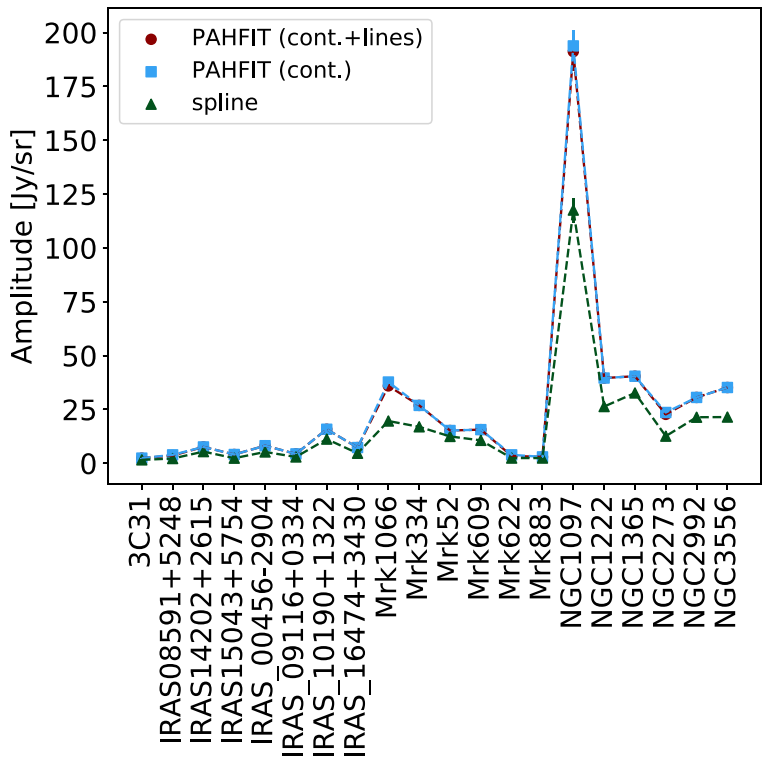

Figure B1. Results for the $6.2 \mu \mathrm{m}$ profile amplitude depending on each decomposition method - (i) circle; (ii) square; and (iii) triangle.

even doubled their values depending on whether the fit of the continuum was performed by PAHFIT or by the spline.

The methods (i) and (ii) resulted, in general, in the same values. The only difference between both is whether the ionic and molecular lines contributions were subtracted or not from the spectra before the band analysis. As no large blending of these lines is known, this similarity in the results was already expected, revealing that the contribution of these lines is insignificant for the final results.

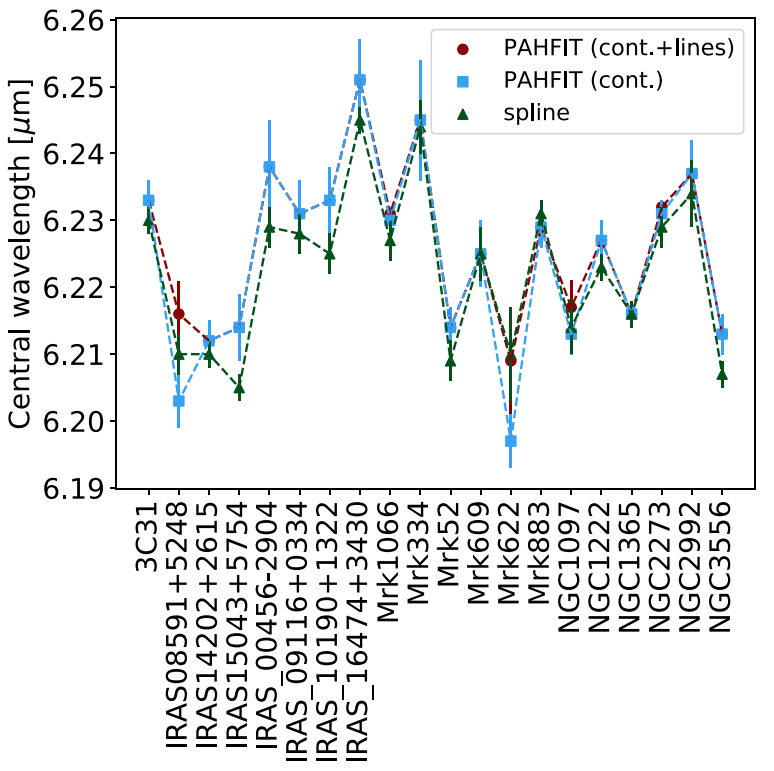

Figure B2. Results for the $6.2 \mu \mathrm{m}$ profile central wavelength depending on each decomposition method - (i) circle; (ii) square; and (iii) triangle.

Therefore, the greatest discrepancy is due to the program used. To better understand the results, the spline decomposition is preferable to the PAHFIT fit for our particular analysis because it already considers the PAH plateau of $5-10 \mu \mathrm{m}$ rather than diluting it in the Drude profiles of the PAH bands (Peeters et al. 2017). Moreover, this difference in the band intensity is crucial when considering other PAH bands such as the $7.7 \mu \mathrm{m}$ band, for instance. 


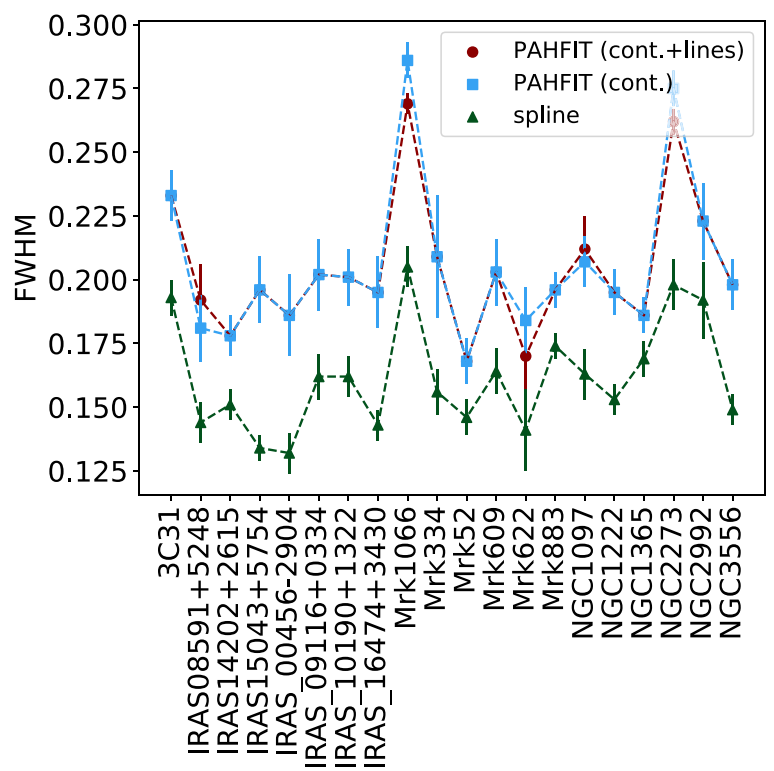

Figure B3. Results for the $6.2 \mu \mathrm{m}$ profile FWHM depending on each decomposition method - (i) circle; (ii) square; and (iii) triangle.

This paper has been typeset from a $\mathrm{T}_{\mathrm{E}} \mathrm{X} / \mathrm{LAT}_{\mathrm{E}} \mathrm{X}$ file prepared by the author. 
2.2 Artigo submetido sobre a região de 6 a $9 \mu \mathrm{m}$ em galáxias dominadas por starbursts 


\title{
Profile comparison of the $6-9 \mu \mathrm{m}$ polycyclic aromatic hydrocarbon bands in starburst-dominated galaxies
}

\author{
Carla M. Canelo, ${ }^{1 \star}$ Amâncio C. S. Friaça ${ }^{1}$, Dinalva A. Sales ${ }^{2}$, Miriani Pastoriza $^{3}$, \\ Karín Menéndez-Delmestre ${ }^{4}$ \\ ${ }^{1}$ Departamento de Astronomia, Instituto de Astronomia, Geofísica e Ciências Atmosféricas, Universidade de São Paulo, São Paulo, Brazil \\ ${ }^{2}$ Instituto de Matemática, Estatística e Física, Universidade Federal do Rio Grande, Rio Grande do Sul, Brazil \\ ${ }^{3}$ Departamento de Astronomia, Instituto de Física, Universidade Federal do Rio Grande do Sul, Rio Grande do Sul, Brazil \\ ${ }^{4}$ Observatório do Valongo, Universidade Federal do Rio de Janeiro, Ladeira Pedro Antônio 43, Rio de Janeiro, Brazil, $20080-090$
}

Accepted XXX. Received YYY; in original form ZZZ

\begin{abstract}
Polycyclic aromatic hydrocarbons (PAHs) are of great astrochemical and astrobiological interest due to their potential to form prebiotic molecules. In particular, their simplest units with $\mathrm{N}$ atoms included in the aromatic rings, denominated Polycyclic Aromatic Nitrogen Heterocycles (PANHs), are involved in the production of Nucleobases. Analyses of the PAH feature profiles, especially the $6.2,7.7$ and $8.6 \mu \mathrm{m}$ midinfrared (MIR) bands, could indicate their presence in astrophysical environments of galaxies. In this work, 126 predominantly starburst-dominated galaxies (including HII regions and Seyferts, for example), extracted from the Spitzer/IRS ATLAS project, have these bands fitted allowing their separation into the Peeters' A, B and C classes. The blueshift of the $6.2 \mu \mathrm{m}$ PAH emission band, typical for a class A object, was attributed to PANH molecules and seems to dominate this spectral feature in starburstdominated galaxies, suggesting a significant presence of these molecules. The $7.7 \mu \mathrm{m}$ complex is equally distributed in class A and B objects in our sample while $8.6 \mu \mathrm{m}$ band presents more class B sources. In our sample, 39 per cent of the galaxies were distributed into class A objects for both 6.2 and $7.7 \mu \mathrm{m}$ bands and only 18 per cent received the same A classification for the three bands. This result may not allow us to indirectly study the PANHs emission at $6.2 \mu \mathrm{m}$ by correlations of the bands, but it supports the complexity of PAH emissions in astrophysical environments.
\end{abstract}

Key words: galaxies: ISM - infrared: galaxies - ISM: molecules - astrochemistry astrobiology

\section{INTRODUCTION}

The main reservoir of molecular organic material in space is in the form of polycyclic aromatic hydrocarbons (PAHs) (Ehrenfreund et al. 2006). Their emission in the interstellar medium (ISM) belongs to a molecular class normally referred as the Aromatic Infrared Bands (AIB, Joblin et al. 1992), in which other classes of organics and inorganics contribute on a tiny scale to the emitting material (Allamandola et al. 1999). Due to their high luminosity, the AIBs dominate the mid-infrared (MIR) emission of many objects including those with high redshift (Papovich et al. 2006; Teplitz et al. 2007). PAHs can be responsible for up to 50 per cent of the MIR luminosity, with the major bands peaking at 3.3, 6.2, 7.7, 8.6, 11.3 and $12.7 \mu \mathrm{m}$ (Li 2004; Smith et al. 2007), and being observed in the ISM of galactical and

* E-mail: camcanelo@gmail.com extra-galactical environment (e.g. Sales et al. 2010, 2013; Ruschel-Dutra et al. 2014).

Together with other aromatic macromolecules, they are the most abundant class of molecular species that must have been transported to the planets by comets, meteorites and interplanetary dust deposition (Ehrenfreund et al. 2002). Because of their stable molecular structure, they have been delivered almost intact to planets such as Earth and Mars despite of have been produced in other parts of the Solar System or Galaxy. They are of great astrobiological interest due to their potential to form prebiotic molecules and to have played a fundamental role in the origins of life in the stages preceding the RNA World (PAH World model, Ehrenfreund et al. 2006).

For instance, the substitution of a carbon for a nitrogen atom creates a polycyclic aromatic nitrogen heterocycle (PANH), which can be a precursor for prebiotic nitrogen heterocycle molecules. Hudgins et al. (2005) suggested that 
a significant fraction of the nitrogen in the ISM is depleted into PANHs and, moreover, these molecules could be causing the shift in the position of the $6.2 \mu \mathrm{m}$ PAH band to sligthly shorter wavelengths.

Peeters et al. (2002) studied the profile variations among the PAH bands in several astrophysical objects. Considering the $6-9 \mu \mathrm{m}$ spectral region, their sample could be separated into three different classes - A, B and C - depending on the band peak positions. This region is composed by three main features - a band at $6.2 \mu \mathrm{m}$, a complex of overlapping bands at $7.7 \mu \mathrm{m}$ with two components at 7.6 and $7.8 \mu \mathrm{m}$, and a band at $8.6 \mu \mathrm{m}$ (Ricca et al. 2018). For the $6.2 \mu \mathrm{m}$ band, the profile A peaks at shorter wavelengths compared to $\mathrm{B}$ and $\mathrm{C}$ profiles. On the other hand, the classes $\mathrm{A}$ and $\mathrm{B}$ differ in the relative strength of the 7.6 and $7.8 \mu \mathrm{m}$ features, which seem to be shifted to $8.2 \mu \mathrm{m}$ for class C objects (Tielens 2008).

The PAH features are often present in star-forming systems, diminished and modified in high-intensity starbursts and, eventually, disappear in active galactic nuclei (AGN) systems (Yan et al. 2007). In spite of this, the $11.3 \mu \mathrm{m}$ PAH band can be observed in nuclear regions of galaxies as close as dozen parsecs from the AGN and for Seyfert-like AGN luminosities, suggesting a dusty material such as a nuclear tori or discs that allow the survival of PAH molecules in nuclear environments (Sales et al. 2013; Alonso-Herrero et al. 2014, 2016; Monfredini et al. 2019). Starburst spectra are dominated by strong emission of these features, not only in the continuum shape (Genzel \& Cesarsky 2000) but in the 5 $8 \mu \mathrm{m}$ spectral range with the $6.2 \mu \mathrm{m}$ band and the blue wing of the $7.7 \mu \mathrm{m}$ PAH complex (Brandl et al. 2006) as well. In fact, starburst galaxies and most ULIRGs (Ultra-Luminous Infrared Galaxies, Yan et al. 2005) present the MIR spectra dominated by dust grain emission and absorption features.

In general, the $6.2,7.7$ and $8.6 \mu \mathrm{m}$ band emissions arise from the contribution of ionised PAHs (e.g. Tielens 2008). Nevertheless, the variability of the PAH profiles (and their peak positions) in different astrophysical environments have been attributed, for example, to the local physical conditions and to the PAH molecules' size, charge, geometry and heterogeneity (e.g Draine \& Li 2001, 2007; Smith et al. 2007; Sales et al. 2013). Despite of displaying considerable diversity, the 6.2 and $7.7 \mu \mathrm{m}$ features are produced by $\mathrm{CC}$ vibration modes and are prominently evident even in relatively low-resolution data (Tielens 2008). Although these CC modes allow these bands to be connected to each other in some cases, mainly for class A (van Diedenhoven et al. 2004), the $8.6 \mu \mathrm{m}$ band $\mathrm{CH}$ vibration modes vary less and the profile variation may not be necessarily connected to that of the others (van Diedenhoven et al. 2004; Tielens 2008; Candian \& Sarre 2015). Despite this, the three bands are tightly correlated (Peeters et al. 2017).

In this sense, analyses of these profiles could reveal physical and chemical conditions of the molecules. The observed class A $6.2 \mu \mathrm{m}$ band, for example, has only been well reproduced by an inner carbon replaced by nitrogen within the aromatic rings (Hudgins et al. 2005). Canelo et al. (2018) analysed the $6.2 \mu \mathrm{m}$ band profile of 155 starburst-dominated galaxies with redshift $\leq 2.5$ and distributed 67 per cent of the objects into the class A, suggesting a dominance of the PAHN emission in this band. The vibration mode association, specially for the $7.7 \mu \mathrm{m}$ complex, could furnish another

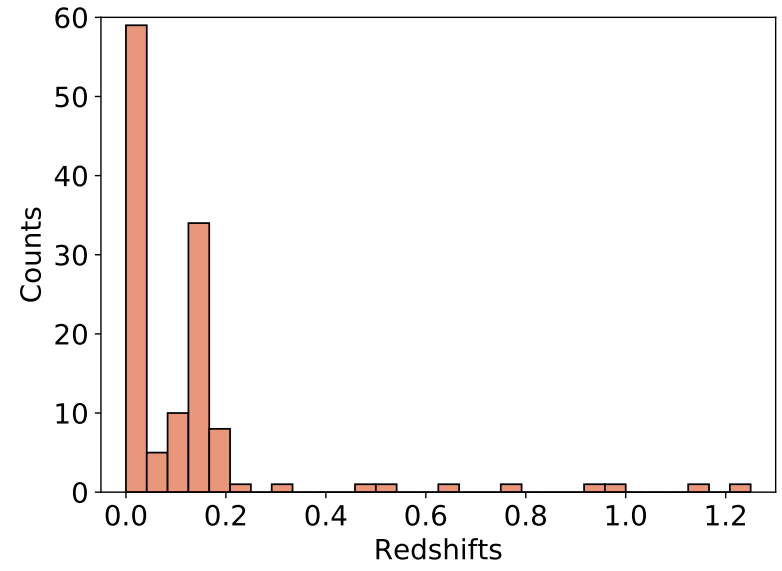

Figure 1. Histogram of the 126 redshifts of our galaxy's sample.

strategy for deriving the variations of the $6.2 \mu \mathrm{m}$ band in an indirect way by analysing if both features present the same classification for the same object. In addition, the advantage of this kind of study provides important insights to the behaviour of PAH molecules through the ISM galaxy evolution in the Universe.

With this in mind, we here analyse and classify the 7.7 and $8.6 \mu \mathrm{m}$ features of 126 galaxies observed with the Spitzer telescope according to the Peeters' classes, searching for a correlation with the previous classification of the $6.2 \mu \mathrm{m}$ band performed by Canelo et al. (2018). This paper is structured as follows: the selection of our sample is explained in Section 2 and the data analysis performed in the spectroscopic data is described in Section 3. Section 4 discusses the results and Section 5 presents the summary and conclusion.

\section{DATA SELECTION}

Starburst galaxies are the best targets since they carry different burst of young stellar population and, consequently, present strong $\mathrm{PAH}$ emission in the MIR spectral wavelengths, particularly in the $6-9 \mu \mathrm{m}$ region. Furthermore, in order to continue the analysis of Canelo et al. (2018), the same data sample was considered (hereafter, MIR_SB sample). It is a sub-sample originally extracted from the ATLAS MIR starburst-dominated galaxies sample of the Spitzer/IRS ATLAS project ${ }^{1}$ Hernán-Caballero \& Hatziminaoglou (2011).

The ATLAS project possesses spectra of several types of extragalactic objects, such as Seyfert, radiogalaxies and submillimeter galaxies. The limit set between AGN- and starburst-dominated sources was previously classified by Hernán-Caballero \& Hatziminaoglou (2011) and was based on the fraction of a PDR (photo-dissociation region) component at $\mathrm{r}_{P D R}=0.15$, corresponding to equivalent widths $(\mathrm{EW})$ of $\mathrm{EW}_{6.2}=0.2 \mu \mathrm{m}$ or $\mathrm{EW}_{11.3}=0.2 \mu \mathrm{m}$ as an alternative boundary.

The sources were observed in low resolution by the Infrared Spectrograph (IRS, Houck et al. 2004) of the Spitzer

${ }^{1}$ http://www.denebola.org/atlas/ 
Space Telescope (Werner et al. 2004) and their reduced spectra were extracted from Postscript figures uploaded to the arxiv.org preprint service by their authors. Most of the galaxies in the ATLAS were observed as point-like sources, including our sample. Although the data are not at the rest wavelength, the MIR_SB sample have already been corrected with best redshift values supplied by the ATLAS project, which were selected from the literature and checked with NED (NASA Extragalactic Database).

The $7-9 \mu \mathrm{m}$ region can present higher uncertainties than the $6.2 \mu \mathrm{m}$ band due to its complexity, such as the blending bands in the $7.7 \mu \mathrm{m}$ complex. For this reason, not all of the 155 objects from MIR_SB were used. Instead, 126 galaxies were studied in this work and their information is available in Table A1. The distribution of the redshifts can be seen in Fig. 1. For more details about the sample and the redshift values used here, see Canelo et al. (2018) and references therein.

\section{DATA ANALYSIS}

\subsection{Continuum subtraction}

The continuum emission has to be subtracted from the spectra before the fitting of the PAH features. One approach is to decompose the continuum with a spline and subtract it from the spectrum. Peeters et al. (2017) define two distinct spline methods according to the anchor points used in the process. The local spline is determined by anchor points at roughly $5.4,5.8,6.6,7.2,8.2,9.0,9.3,9.9,10.2,10.9,11.7$, $12.1,13.1,13.9,14.7$ and $15.0 \mu \mathrm{m}$. On the other hand, the global spline does not consider $8.2 \mu \mathrm{m}$ as an anchor point, leaving a broad emission feature to the $7-9 \mu \mathrm{m}$ region.

The overall conclusions on PAH intensity correlations for a large sample of objects are independent of the chosen decomposition approach (e.g. Smith et al. 2007; Galliano et al. 2008; Peeters et al. 2017). The fit of the $6.2 \mu \mathrm{m}$ is independent of the spline decomposition method. However, the chosen spline clearly influences the $7.7 \mu \mathrm{m}$ complex intensities (Peeters et al. 2017). This influence relies on the $8.2 \mu \mathrm{m}$ anchor point that have been previously called of broad emission feature and is located underneath the 7.7 and $8.6 \mu \mathrm{m}$ bands. It is prominent in post-AGB (Asymptotic Giant Branch) star spectra, although its remnant could be present in planetary nebula (Joblin et al. 2008, and references therein). This dust feature is not normally present in star-forming regions but can be very strong in $\mathrm{C}$ class objects, such as a few LINER (Low-Ionisation Nuclear Emission-line Region) galaxies (Vega et al. 2010).

Peeters et al. (2017) considered the $8.2 \mu \mathrm{m}$ dust emission as a bump feature in the local spline decomposition and also treated it as a PAH feature in the global spline decomposition, produced by very large PAH molecules with a number of carbon atoms varying from 100 to 150, PAH clusters or very small grains. However, other PAH decomposition methods (such as PAHFIT, Smith et al. 2007) do not consider this bump as a PAH feature, instead it is diluted in the wings of the Drude profiles of the PAH bands. This feature was also reported as a $\mathrm{C}_{60}^{+}$emission detected at a position close to the B star HD 200775 in the NGC7023 reflection nebula (Berné et al. 2013).
In this work, we decided to use previous widely method based on local spline decomposition to focus specifically on the 7.7 and $8.6 \mu \mathrm{m}$ PAH bands. This continuum approach allow us to study both bands separately by fitting the PAH features of each region independently of the other (Brandl et al. 2006). This procedure is not possible with the global spline because of the blending of the profiles and at least another PAH feature must be considered during the fitting. Four examples of this method are displayed in Fig. 2 in which is possible to see differences in the $8 \mu \mathrm{m}$ bump due to the $\mathrm{PAH}$ profiles of each galaxy.

Concerning the extinction, we have not performed a correction for our galaxies once they may present different extinction contributions or even low values. However, some sources do present higher silicate absorption as exemplified by NGC 3079 (Fig. 2). In such cases, the $8.6 \mu \mathrm{m}$ band fitting could be compromised if an extinction correction is not applied. One way to approach this issue is to compare the results, especially for this band, with the optical depth at $9.7 \mu \mathrm{m}\left(\tau_{9.7}\right)$ calculated by (Hernán-Caballero \& Hatziminaoglou 2011) whom performed an spectral decomposition by fitting the $5-15 \mu \mathrm{m}$ rest-frame range of the spectra using the Galactic Centre extinction law (Chiar \& Tielens 2006). Possible trends could indicate if the absence of an extinction correction influences the final results.

\subsection{Gaussian fit of the $7-9 \mu \mathrm{m}$ region}

To study the PAH profile of starburst-dominated galaxies, we applied the same procedure used for the $6.2 \mu \mathrm{m}$ band fitting (Canelo et al. 2018) in the 7.6, 7.8 and $8.6 \mu \mathrm{m}$ features. We constructed a PYTHON-based script to estimate their central wavelength, amplitude and full width at half maximum (FWHM) through the optimisation algorithms from the submodule scipy.optmize.curve_fit. The uncertainties were also derived using this tool with least-squares minimisation and the initial guesses were selected from Smith et al. (2007).

In order to standardise the procedure, we also used Gaussian profiles to fit the features (e.g. Peeters et al. 2017; Stock \& Peeters 2017; Canelo et al. 2018). The $\mathrm{H}_{2}$ emission line at $8.026 \mu \mathrm{m}$, when present, was also subtracted before the PAH fit. There is also the fainter component at $8.33 \mu \mathrm{m}$ (Peeters et al. 2002; Smith et al. 2007) that can be ignored from the analyses if not present (Peeters et al. 2017; Stock \& Peeters 2017). However, some sources of our sample possess this feature and the separation of the fit regions allows us to constrain the $8.6 \mu \mathrm{m}$ band to avoid the $8.33 \mu \mathrm{m}$ component.

Spectra with local spline decomposition can be separated into two fitting regions - the $7.7 \mu \mathrm{m}$ complex and the $8.6 \mu \mathrm{m}$ band. We used three Gaussian profiles to fit the 7.6, 7.8 and $8.6 \mu \mathrm{m}$ features, with the latter fitted independently of the others. The fit uncertainties of the complex are expected to be higher than those of the $8.6 \mu \mathrm{m}$ band due to the blending of the 7.6 and $7.8 \mu \mathrm{m}$ features. Stock \& Peeters (2017), for instance, firstly fitted this spectral region with free parameters in order to previously test their fixed-parameter Gaussian decomposition in the HII region W49A. In this situation, their source had its $7.7 \mu \mathrm{m}$ complex better fitted with just one component and they decided to constraint the peak positions and widths of the features to recover the 7.6 and $7.7 \mu \mathrm{m}$ features. Our goal is to better comprehend a possible variability in the profiles and, 

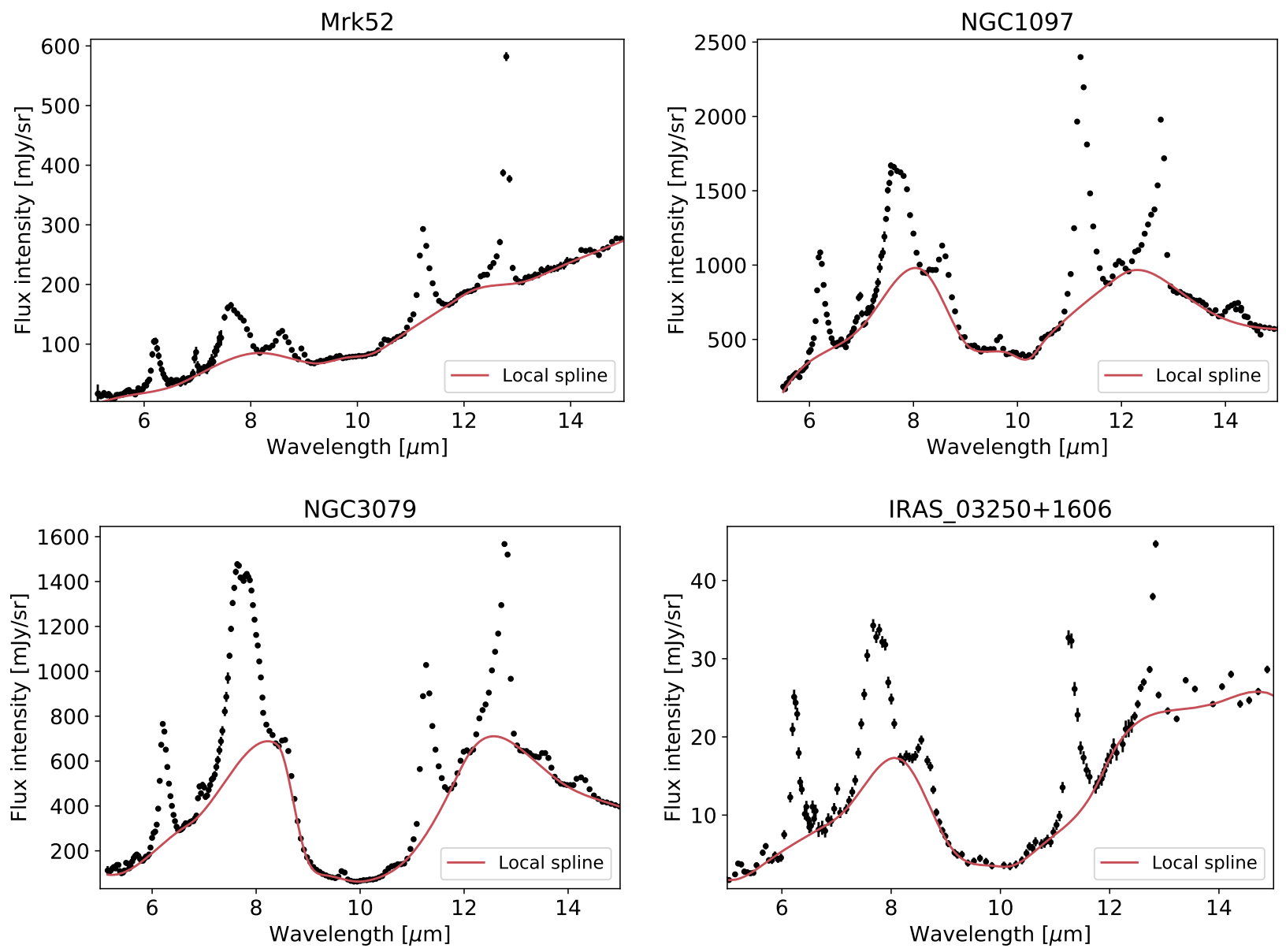

Figure 2. Local spline decomposition of the continuum emission represented by the red line for four objects. The data points are represented by the dots with the vertical error-bars as uncertainties.

therefore, we need to perform a free parameter fit. However, in order to avoid the blending of the features and broader profile's widths than expected by the previous works, we fixed the FWHM values according to Peeters et al. (2002) in $0.28 \mu \mathrm{m}$ to $7.6 \mu \mathrm{m}$ and $0.32 \mu \mathrm{m}$ to $7.8 \mu \mathrm{m}$ profiles, respectively.

For the comparison of the PAH features, we also calculated the flux intensities by integration of the fitted Gaussian profiles in the intervals of $6.1-6.35 \mu \mathrm{m}$ for the $6.2 \mu \mathrm{m}$ band; $7.2-8.2 \mu \mathrm{m}$ for the 7.6 and $7.8 \mu \mathrm{m}$ components; and 8.2 $-9 \mu \mathrm{m}$ for the $8.6 \mu \mathrm{m}$ band. The uncertainty of the fluxes were taken as the difference between of each integrated flux and those integrated flux obtained by adding the respective uncertainties to the fitted parameters.

The Gaussian fit results allow us to group the galaxies into the Peeters' classes according to the Table 1 . The classification system for the 6.2 and $8.6 \mu \mathrm{m}$ bands consists basically in the divergence of the central wavelength bands. The $7.7 \mu \mathrm{m}$ complex is comprised of both 7.6 and $7.8 \mu \mathrm{m}$ components and class identification relies on their relative strength. This was measured by the flux ratio of the features, hereafter as $\mathrm{F}_{7.6} / \mathrm{F}_{7.8}$. The classification of the complex is based on this bands ratio which will be described in the next sections and used in our analysis instead of the complex peak position's method. The ratio uncertainties
Table 1. Profile peak positions for each Peeters' classes (Peeters et al. 2002). $\mathrm{F}_{7.6} / \mathrm{F}_{7.8}$ represents the flux ratio between 7.6 and $7.8 \mu \mathrm{m}$ features and is used to classify the $7.7 \mu \mathrm{m}$ complex.

\begin{tabular}{cccc}
\hline Class & $6.2 \mu \mathrm{m}$ & $7.7 \mu \mathrm{m}$ & $8.6 \mu \mathrm{m}$ \\
\hline A & $<6.23$ & $\sim 7.6$ & $<8.6$ \\
& & $\left(\mathrm{~F}_{7.6} / \mathrm{F}_{7.8} \geq 1\right)$ & \\
\hline B & $6.23<\lambda<6.29$ & $\sim 7.8$ & $>8.6$ \\
& & $\left(\mathrm{~F}_{7.6} / \mathrm{F}_{7.8}<1\right)$ & \\
\hline C & $>6.29$ & $\sim 8.22$ & - \\
\hline
\end{tabular}

were obtained with error propagation derived from Gaussian equation for normally-distributed errors. The greater flux indicates the prominent feature of the complex. The decomposition of this complex can be very sensitive to the used method.

Another approach to be tested is to determine the barycenter of the complex by identifying at which wavelength the area under the band is half of the area of the entire complex (Sloan et al. 2007; Shannon \& Boersma 2019). To estimate the barycenter of the complex, the integration was performed for the sum of both Gaussian profiles and in the same range as described before for these features. 
The Peeters' classification for the sample obtained with this method is only one per cent different from the results of the previous approach. Therefore, we used the flux ratio method in our analysis.

\section{RESULTS AND DISCUSSION}

\subsection{The band profiles of starburst-dominated galaxies}

The $6.2 \mu \mathrm{m}$ fit results are presented and discussed in Canelo et al. (2018). They are also compared here with the statistics obtained for the other two bands. Table A2 contains all the fit results of the 7.7 and $8.6 \mu \mathrm{m}$ bands. Although the FWHM values for the 7.6 and $7.8 \mu \mathrm{m}$ features were maintained constant along the sources, they failed to adequately reproduce these features in ten galaxies. Smaller values for one or both features needed to be used to accomplish the fitting. They are shown in Table A2 together with the FWHM values for the $8.6 \mu \mathrm{m}$ band that were treated as free parameters for this band.

The same galaxies shown in the previous section with the local spline decomposition (Fig. 2) are now presented with the fitted PAH profiles in Fig. 3. A feature between 7.2 and $7.5 \mu \mathrm{m}$ that is not well reproduced by the fitting can be seen in the residual plots. It can be related to the fainter $7.42 \mu \mathrm{m}$ PAH emission that belongs to the $7.7 \mu \mathrm{m}$ complex (Smith et al. 2007). The objects Mrk 52 and NGC 1097 appear to have the $8.33 \mu \mathrm{m}$ band, as can be also seen in the residual values. Nevertheless, both features are faint and do not interfere with the analyses of the prominent PAH bands.

In general, the $7.7 \mu \mathrm{m}$ features present higher intensities than the $8.6 \mu \mathrm{m}$ feature. However, the relative intensities between them vary according to the object as can be seen in Fig. 3. The starburst galaxy Mrk 52 and NGC 1097 (starburst and Seyfert 1 galaxy) received the same "A" classification for the three bands $(6.2,7.7$ and $8.6 \mu \mathrm{m})$ and present prominent PAH features, despite of the predominance of the $7.6 \mu \mathrm{m}$ component. On the other hand, the starburst galaxy NGC 3079 received the classification of "A A B" for the bands, respectively. Finally, the ULIRG + LINER source IRAS_03250+1606 has its three bands classified as "B" object.

The $8.6 \mu \mathrm{m}$ band flux of NGC 3079 could be affected by the extinction that reduces the flux of the band. In comparison to the other galaxies shown if Fig. 3, this source presents the lowest relative flux intensity for the $8.6 \mu \mathrm{m}$ band and also the deepest silicate absorption at $9.7 \mu \mathrm{m}$. Indeed, if we compare the relative intensities of the $8.6 \mu \mathrm{m}$ band of the four presented objects, we note that IRAS_03250+1606 and NGC3079 possess lower values than the other two sources. This could also evince an strong correlation of band fluxes along the spectra. This well-known correlation in the relative band intensities is expected because of the similar vibration modes $(\mathrm{CC}$ and $\mathrm{CH}$ ) and can also reveal the properties of the emitting $\mathrm{PAH}$ population. In fact, variations in the intrinsic relative strength of the $\mathrm{CC}$ versus $\mathrm{CH}$ modes have been attributed to the effect of ionisation (Tielens 2008). For instance, the $6.2,7.7$ and $8.6 \mu \mathrm{m}$ bands are generally linked to ionised $\mathrm{PAH}$ in which $\mathrm{PAH}$ cations dominate the emission of the 6.2 and 7.6 features (Allamandola et al. 1999; Peeters et al. 2002; Galliano et al. 2008). On the other hand, the $7.8 \mu \mathrm{m}$ feature and the $8.6 \mu \mathrm{m}$ band may have greater contribution of neutral and anion PAHs (Ricca et al. 2012; Peeters et al. 2017).

The size distribution and structure of the PAH population can also contribute to the observed profile variations. Peeters et al. (2017, and references therein) attributed the $7.6 \mu \mathrm{m}$ emission to compact (and positively charged) PAH with 50-100 carbon atoms while the $7.8 \mu \mathrm{m}$ emission to very large PAH with 100-150 carbon atoms or PAH clusters with bay regions or modified duo $\mathrm{CH}$ groups. Finally, the $8.6 \mu \mathrm{m}$ emission can be attributed to very large, compact and symmetric PAHs with $96-150$ carbon atoms. Unfortunately, the relative intensities between the $6.2 \mu \mathrm{m}$ band and the $7.7 \mu \mathrm{m}$ complex do not track PAH size effectively due to this mixed distribution of sizes throughout this range (Maragkoudakis et al. 2018).

In order to verify the well-known correlations between the PAH profiles, Fig. 4 compares the flux ratios of $\mathrm{F}_{7.6} / \mathrm{F}_{6.2}$ and $\mathrm{F}_{8.6} / \mathrm{F}_{6.2}$. The band fluxes are available in Table A3. The different types of objects do not seem to influence the ratios. They are probably connected to physical conditions and the dominant PAH population of the sources. The correlation between these two bands is well established in a variety of environments and within extended sources. Peeters et al. (2017); Ricca et al. (2018) presented the ratios for reflection nebulae observations and computed values simulated for an excitation of $8 \mathrm{eV}$. Some of our results are similar, but we also obtained greater ratios specially for $\mathrm{F}_{7.6} / \mathrm{F}_{6.2}$. According to Ricca et al. (2018), the relative intensity of the 7.7 and $6.2 \mu \mathrm{m}$ bands is greater than 1 for PAHs with even number of carbons and less than 1 for odd-carbon PAHs, which we can conclude from our data (Fig. 4) that most of our galaxies may be dominated by even-carbon PAHs.

We can also see in Fig. 4 that ULIRGs are located in a region with lower $\mathrm{F}_{8.6} / \mathrm{F}_{6.2}$ ratios than the other types of galaxies. As a matter of fact, this could be related to the extinction that has not been corrected. To accomplish that, Fig. 5 shows the relative intensities of $\mathrm{F}_{8.6} / \mathrm{F}_{6.2}$ compared with the optical depths at $9.7 \mu \mathrm{m}\left(\tau_{9.7}\right)$. The ULIRGs present higher $\tau_{9.7}$ values than the other galaxies' types, which also correspond to lower $\mathrm{F}_{8.6} / \mathrm{F}_{6.2}$ ratio. Even than the $8.6 \mu$ m flux may be underestimated due to the optical depth strength, the extinction is not expected to explain the majority of the observed band ratio variations (Galliano et al. 2008). We therefore investigated the peak position and FWHM of the band and they do not seem to be influenced by the extinction, corroborating with Galliano et al. (2008) claims. We also analysed the spectral contribution of the PDR and AGN (Active Galactic Nuclei) components calculated by Hernán-Caballero \& Hatziminaoglou (2011) and our sample presented no correlations.

\subsection{Distribution into the Peeters' classes}

In spite of profile variations caused by differences in the astrophysical environments, the classes are normally linked to the type of ionisation source. Class A sources can be associated with interstellar material illuminated by a star, including HII regions, reflection nebulae, and the general ISM of the Milky Way and other galaxies. On the other hand, class 

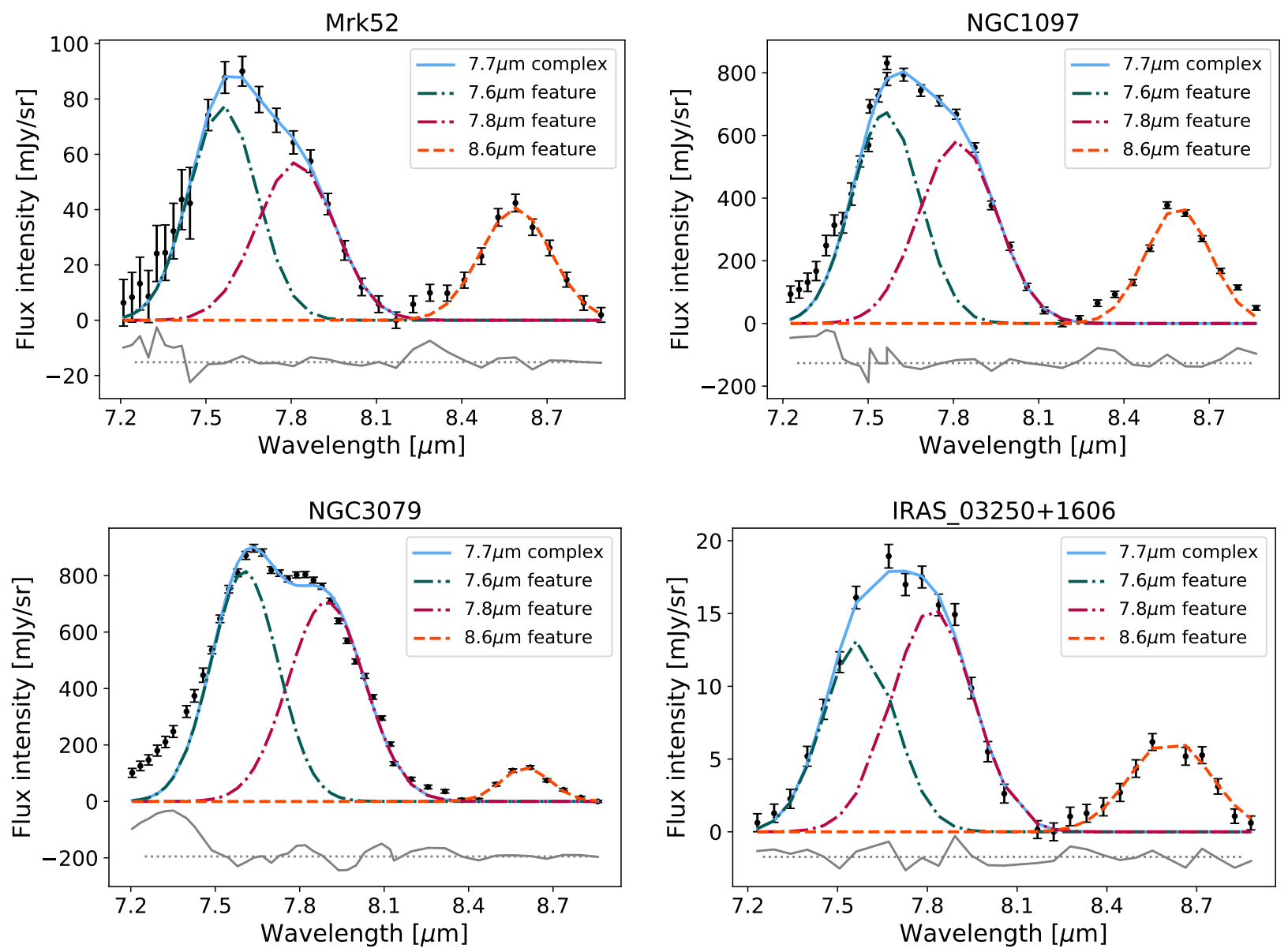

Figure 3. Gaussian fit results of four objects. The solid blue line represents the total fit for the $7.7 \mu$ m complex with two Gaussian components respectively identified in the label. The individual Gaussian of the $8.6 \mu \mathrm{m}$ band is also shown. The data points are represented by the dots with the vertical error-bars as uncertainties and the solid grey lines represent the fit residuals offset to better visualisation (dotted grey line).

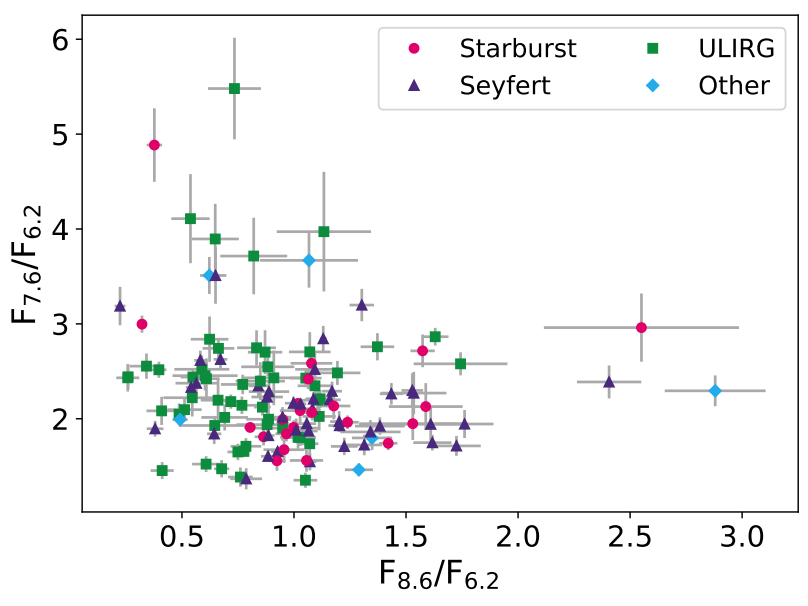

Figure 4. Comparison of the flux intensities obtained for each galaxy normalised for the $6.2 \mu \mathrm{m}$ band. Their types were divided into four main groups with data points represented by the symbols labelled in the legend. The uncertainties are displayed as grey error bars.

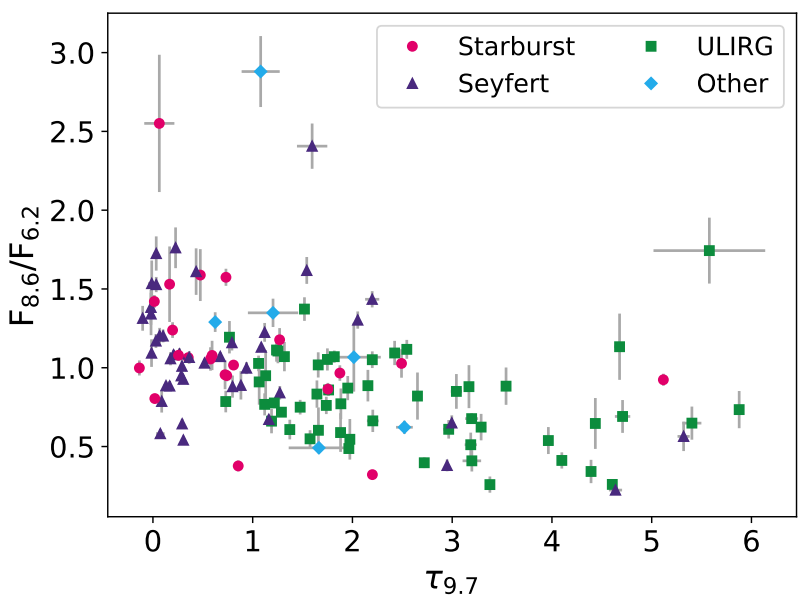

Figure 5. Comparison of the flux intensities of $\mathrm{F}_{8.6} / \mathrm{F}_{6.2}$ with the optical depths at $9.7 \mu \mathrm{m}$. The type of the sources are labelled in the legend. The uncertainties are displayed as grey error bars. 


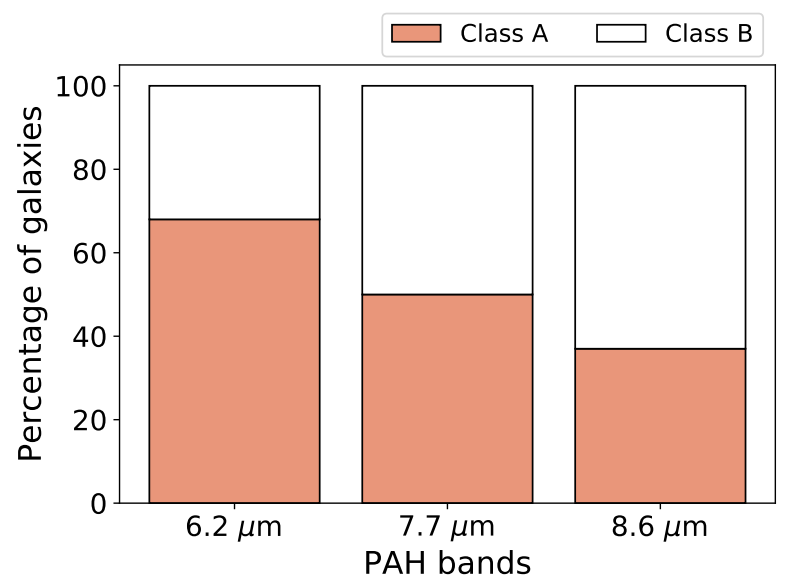

Figure 6. Histogram of the Peeters' classification for the 6.2, 7.7 and $8.6 \mu \mathrm{m}$ bands in percentage. In the case of the $6.2 \mu \mathrm{m}$, the 1 per cent of class $\mathrm{C}$ sources was add to the class B percentage.

Table 2. Profile distribution of the 126 studied galaxies into the Peeters' class.

\begin{tabular}{cccc}
\hline $\begin{array}{c}\text { Band } \\
(\mu \mathrm{m})\end{array}$ & $\begin{array}{c}\text { Class A } \\
(\text { per cent })\end{array}$ & $\begin{array}{c}\text { Class B } \\
(\text { per cent })\end{array}$ & $\begin{array}{c}\text { Class C } \\
(\text { per cent })\end{array}$ \\
\hline 6.2 & 68 & 31 & 1 \\
7.7 & 50 & 50 & - \\
8.6 & 37 & 63 & - \\
\hline
\end{tabular}

B objects can be associated with circumstellar material and include planetary nebula, a variety of post-AGB objects and Herbig AeBe stars. Finally, class C sources are limited to a few extreme carbon-rich post-AGB objects (Peeters et al. 2002; Tielens 2008).

In the case of our sample, the starburst-dominated emission seems to suppress the possible influence of different type classification of the galaxies. To complement the analysis, the type of the sources were divided into four main groups which comprise the following types: Starburst - starburst with contribution of HII region, Seyfert and LINER; Seyfert - Seyferts 1, intermediate, 2 and 3; ULIRG - ULIRGs and ULIRGs with contribution of HII region and LINER; and Other - infrared galaxy, Fanaroff-Riley galaxy, LINER, quasi-stellar object and submillimeter galaxy.

Table 2 and Fig. 6 summarise the classification of the 126 objects into Peeters' classes derived from the fits. The classifications of the whole sample are given in Table A4. Class A objects represent 68 per cent of the $6.2 \mu \mathrm{m}$ profile. As matter of fact, they are the most common objects in the Universe and embrace several astrophysical sources (Pino et al. 2008). The $7.7 \mu \mathrm{m}$ complex obtained 50 per cent of class $\mathrm{A}$ and $\mathrm{B}$ objects. Actually, the $\mathrm{F}_{7.6} / \mathrm{F}_{7.8}$ ratios varied around 1.0, which is the limit between the A and B classes. Apparently, fixed FHWM values furnished a balance in the class distribution of the objects. On the other hand, the $8.6 \mu \mathrm{m}$ band is 63 per cent represented by class B objects. Class $\mathrm{C}$ only appears for the $6.2 \mu \mathrm{m}$ profile representing 1 per cent.

From the total of our sample, 39 per cent of the galaxies were distributed into class A objects for both 6.2 and $7.7 \mu \mathrm{m}$
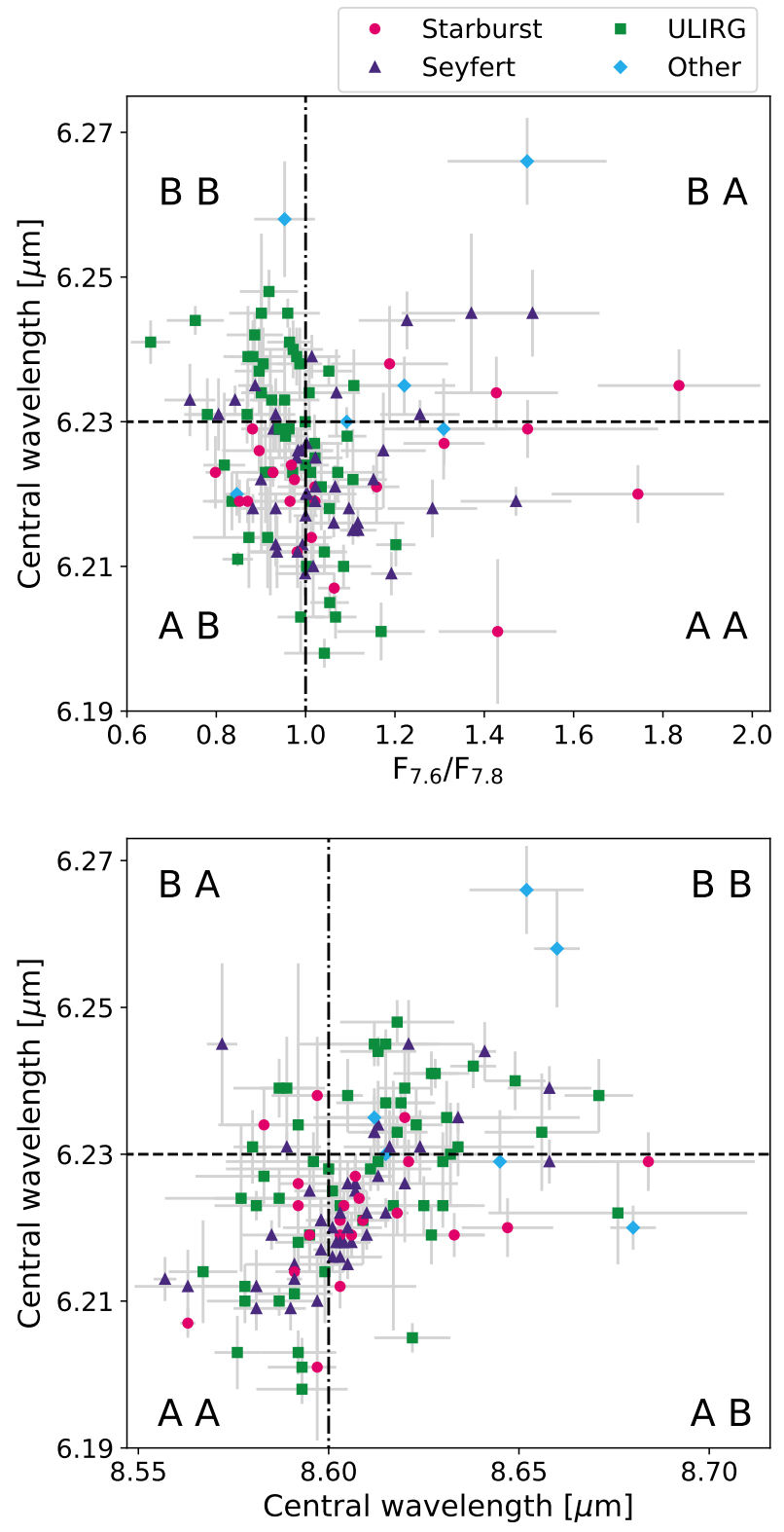

Figure 7. Relation between the flux ratio $\mathrm{F}_{7.6} / \mathrm{F}_{7.8}$ (top) and the peak position of the $8.6 \mu \mathrm{m}$ band (bottom) through the peak position of the $6.2 \mu \mathrm{m}$ band. Horizontal dashed lines represents the limits of the Peeters' classes which are also indicated by the letters in each quadrant of the figures. The first letter always corresponds to the $6.2 \mu \mathrm{m}$ band. The error bars are displayed in grey.

bands and 18 per cent maintained the same A classification for the $8.6 \mu \mathrm{m}$ band. This suggests an strong correlation between the 6.2 and $7.7 \mu \mathrm{m}$ features, as also the minor connection of the 6.2 and $8.6 \mu \mathrm{m}$ bands. Considering the B class, 20 per cent of the sources were distributed into this class for the first two bands and 17 per cent for all three bands. This fact could indicate that the correlation between the classes is stronger in class A objects as already discussed in van Diedenhoven et al. (2004). Nevertheless, this seems to be more restricted to the 6.2 and $7.7 \mu \mathrm{m}$ bands while 7.7 and $8.6 \mu \mathrm{m}$ bands may be more correlated for B objects. 

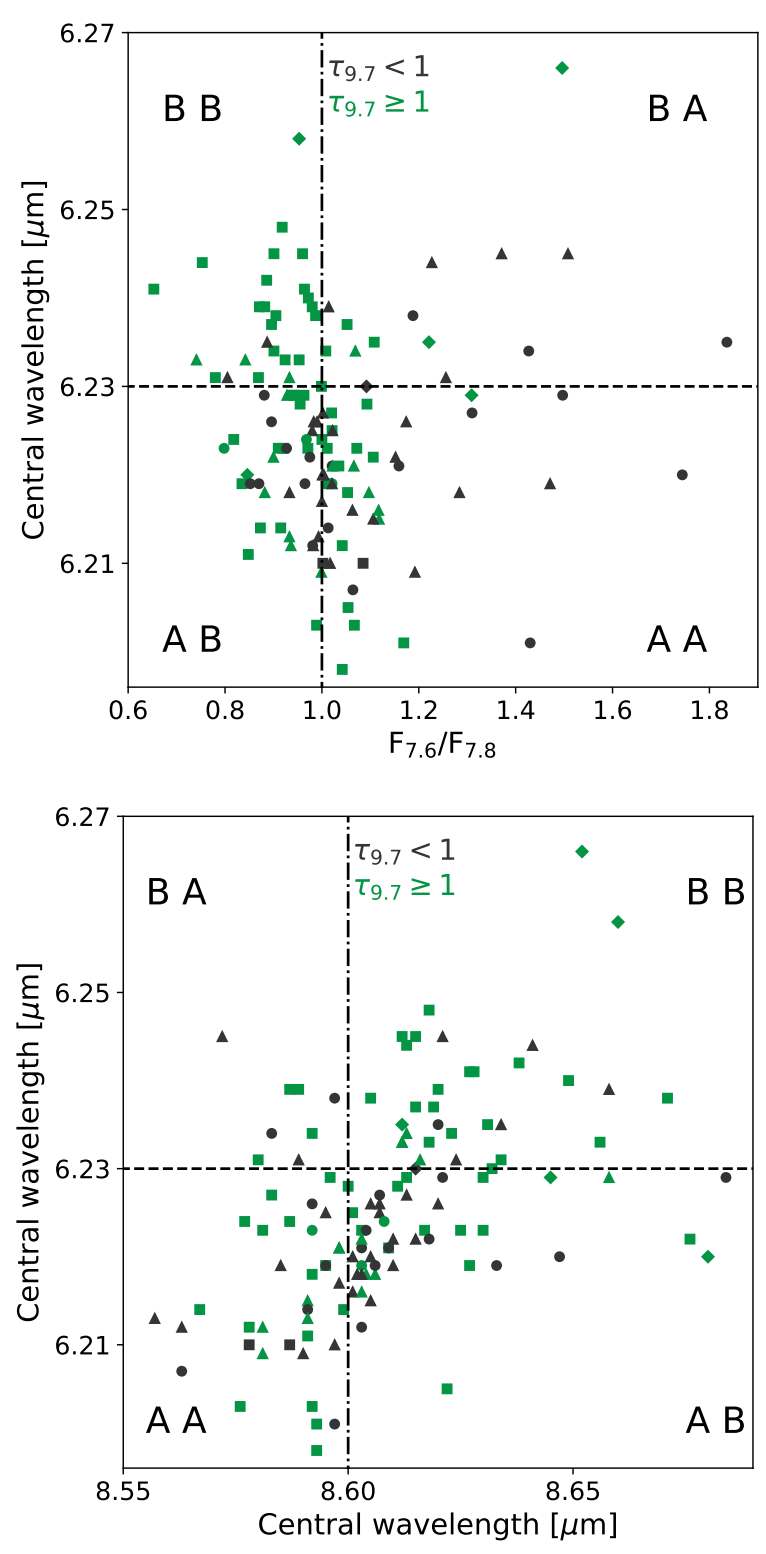

Figure 8. Relation between the flux ratio $\mathrm{F}_{7.6} / \mathrm{F}_{7.8}$ (top) and the peak position of the $8.6 \mu \mathrm{m}$ band (bottom) through the peak position of the $6.2 \mu \mathrm{m}$ band. Horizontal dashed lines represents the limits of the Peeters' classes which are also indicated by the letters in each quadrant of the figures. The first letter always corresponds to the $6.2 \mu \mathrm{m}$ band. The error bars are not displayed. The types of the galaxies are represented by circle - Starburst, triangle - Seyfert, square - ULIRG and diamond - Others. The colours represent different intervals of $\tau_{9.7}$ values.

The Fig.7 compares the Peeters' classification for the 6.2 and $7.7 \mu \mathrm{m}$ bands and shows the possible combinations for the classifications - "A A", "A B", "B A" and "B B", with the first letter always corresponding to the $6.2 \mu \mathrm{m}$ band. As can be reinforced by the graph, class A objects are more correlated once one can see more "A A" objects than "B B", although most of the sources (41 per cent) received different classification for these bands. It is also interesting to notice that, in such cases, "A B" objects are more abundant than "B A". The majority of starbursts and Seyferts are classified as
A objects for $6.2 \mu \mathrm{m}$ band while the other galaxy types are more sparsely distributed in the plot. Although the $7.7 \mu \mathrm{m}$ band is not necessarily connected to a possible PANH emission such as the $6.2 \mu \mathrm{m}$ band, galaxies equally classified as A objects for both bands have their classification reinforced.

The correlation between the 6.2 and $8.6 \mu \mathrm{m}$ bands is shown in Fig.7. ULIRGs are sparsely distributed along the plot but they dominated "A A" and "B B" sources. Starbursts and Seyferts are more represented by "A B" sources. We can note that fewer sources received the "B A" classification. Considering all three bands, the most common classification in our sample is "A A B" and "A B B", respectively to the $6.2,7.7$ and $8.6 \mu$ bands.

Stronger $7.8 \mu \mathrm{m}$ flux could arise from very large PAHs with multiple bay regions, irregular edges and modified duo $\mathrm{CH}$ groups (such as $\mathrm{N}$ substitution) in regions closest to the radiation sources (Peeters et al. 2017). However, this kind of PANH with nitrogen in the bay regions does not contribute to the class A $6.2 \mu \mathrm{m}$ band emission (Hudgins et al. 2005). Roughly 20 per cent of our sources were doubly classified as B objects for 6.2 and $7.7 \mu \mathrm{m}$ bands and they are better represented by ULIRGs. Class $\mathrm{C}$ does not have enough data for any interpretation. Although our sample is composed by galaxies, the gradual variation noticed by Peeters et al. (2002), in which lower $6.2 \mu \mathrm{m}$ central wavelengths can present higher flux ratios, is also recovered in our analyses.

In order to analyse if the silicate absorption influences the fitting and the classification, the band comparisons were also performed using the $\tau_{9.7}$ values available in the ATLAS. They were divided into two different groups for better visualisation: $\tau_{9.7}<1$, which corresponds to 40 per cent of our sample; and $\tau_{9.7} \geq 1$, representing 60 per cent of the objects. Figure 8 shows the results for the three bands. Most of galaxies with higher optical depths are ULIRGs, as already mentioned. Nevertheless, a correlation between the PAH classification and $\tau_{9.7} \geq 1$ can not be seen, specially for the $8.6 \mu \mathrm{m}$ band which should be more affected by the silicate absorption. Therefore, the silicate absorption does not interfere significantly to the fitting or to the PAH classification results.

The optical depth was also compared with the $\mathrm{F}_{70} / \mathrm{F}_{24}$ ratio and can be seen in Figure 9. The fluxes at 24 and $70 \mu \mathrm{m}$ were extracted directly from the ATLAS. As expected, higher values of $\tau_{9.7}$ are followed by higher $\mathrm{F}_{70} / \mathrm{F}_{24}$ ratio, indicating that colder regions have more dust absorption. Considering the galaxies that obtained the same classification for the three bands, it is possible to see that class "A A A" sources are located mainly at lower values of $\mathrm{F}_{70} / \mathrm{F}_{24}$ and $\tau_{9.7}$, in which the temperature is higher and there is less dust in the ISM. This could suggest that dust grains have also been processed into PAH molecules and even in heteroatomic PAHs, such as PANHs. On the other hand, class "B B B" sources are shifted to higher values of $\mathrm{F}_{70} / \mathrm{F}_{24}$ and $\tau_{9.7}$, suggesting environments with greater abundance of grains. In general, our results point out that galaxies with $\tau_{9.7}<1$ are Starbursts and Seyferts, in which this kind of source usually has higher temperature than ULIRGs.

Regarding to the evolution of aromatic species according to the object type, Shannon \& Boersma (2019) claim that exposed interstellar environments show class A profiles while class B profiles are observed from circumstellar environments. We also find an spread in the classification of 

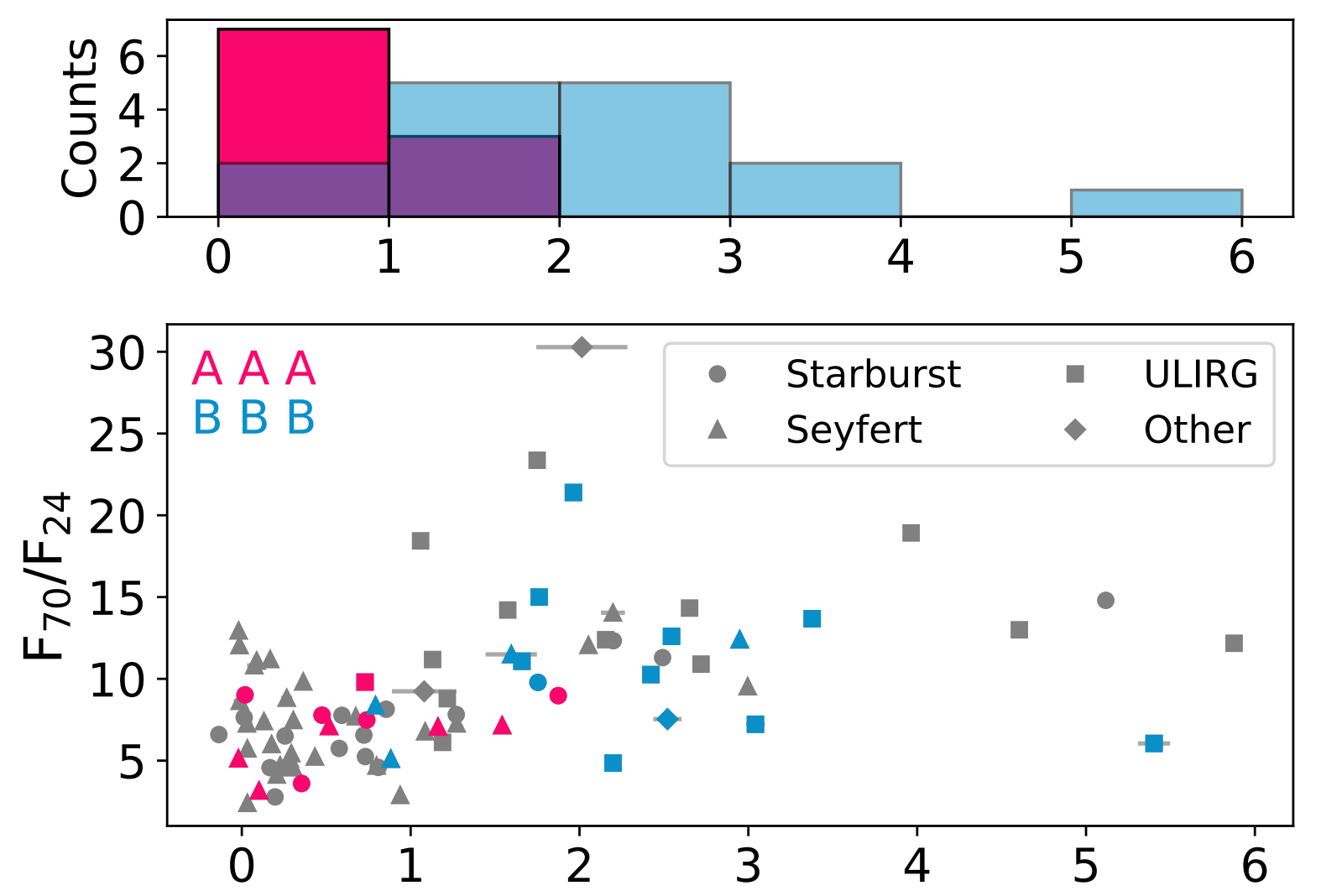

$\tau_{9.7}$

Figure 9. Comparison between $\mathrm{F}_{25} / \mathrm{F}_{20}$ ratio and $\tau_{9.7}$. The types of the galaxies are shown in the label. Galaxies that received the same "AAA" or "BBB" classification are indicated by the colours pink and blue, respectively. The upper panel shows a histogram of the "AAA" and "BBB" galaxies according to the $\tau_{9.7}$ values.

the $6.2,7.7$ and $8.6 \mu \mathrm{m}$ band profiles through the ionisation source of our sample. In addition, Starbursts and Seyferts were the type of galaxies that most varied the classification along the bands and they are concentrated in the lower region of the plot. This could also indicate different emitting PAH populations due to the physical conditions of exposed interstellar environments.

An overview of the results is shown in Fig. 10, which displays the distribution of the central wavelengths (for the 6.2 and $8.6 \mu \mathrm{m}$ bands) and the flux ratio (for the $7.7 \mu \mathrm{m}$ complex) in comparison of redshifts of the galaxies. It is possible to perceive the predominance of class A objects over class B objects for the $6.2 \mu \mathrm{m}$ band. The opposite occurs for the $8.6 \mu \mathrm{m}$ band. Again, the type of galaxies does not seem to interfere with the classification. Higher $\tau_{9.7}$ values occur mainly for ULIRGs but all types of galaxies have a dominance of B objects for the $8.6 \mu \mathrm{m}$ band. Instead, the redshift may play an important role in the results. For all three bands, the quantity of class B sources seems to increase to galaxies at higher redshifts, which may suggest a evolutionary timescale of the PAH population through the galaxies evolution in the Universe.

From the light of Astrochemistry, chemically young astrophysical sources might have reduced PAH abundances and PAH molecules are not as efficiently produced in low- metallicity environments because fewer carbon atoms are available in the ISM (Shivaei et al. 2017). As can be seen in Fig. 10, lower $\mathrm{F}_{7.6} / \mathrm{F}_{7.8}$ ratios are often for galaxies at redshifts higher than 0.01 indicating a greater predominance of the $7.8 \mu \mathrm{m}$ component respect to the $7.7 \mu \mathrm{m}$ complex. This feature has also been attributed to evaporating very small grains (eVSGs, Rapacioli et al. 2005; Berné et al. 2007), which may suggest a greater contribution of these molecular material to galaxies at higher redshifts.

\section{CONCLUSIONS}

We have analysed the MIR spectra of 126 starburstdominated galaxies, searching for the contribution of the Peeters' classes to the total sample. To date, the class A position of the $6.2 \mu \mathrm{m}$ band can only be attributed to PANHs, PAHs containing $\mathrm{N}$ atoms. Thus, the PAH feature profiles could indicate different PAH populations including the presence of nitrogen incorporated to the rings.

The class A of the $6.2 \mu \mathrm{m}$ PAH emission band seems to dominate this spectral feature in starburst-dominated galaxies, suggesting a significant presence of these molecules (Canelo et al. 2018). The fitted 7.7 and $8.6 \mu \mathrm{m}$ bands were also classified in classes A, B and C following Peeters et al. 


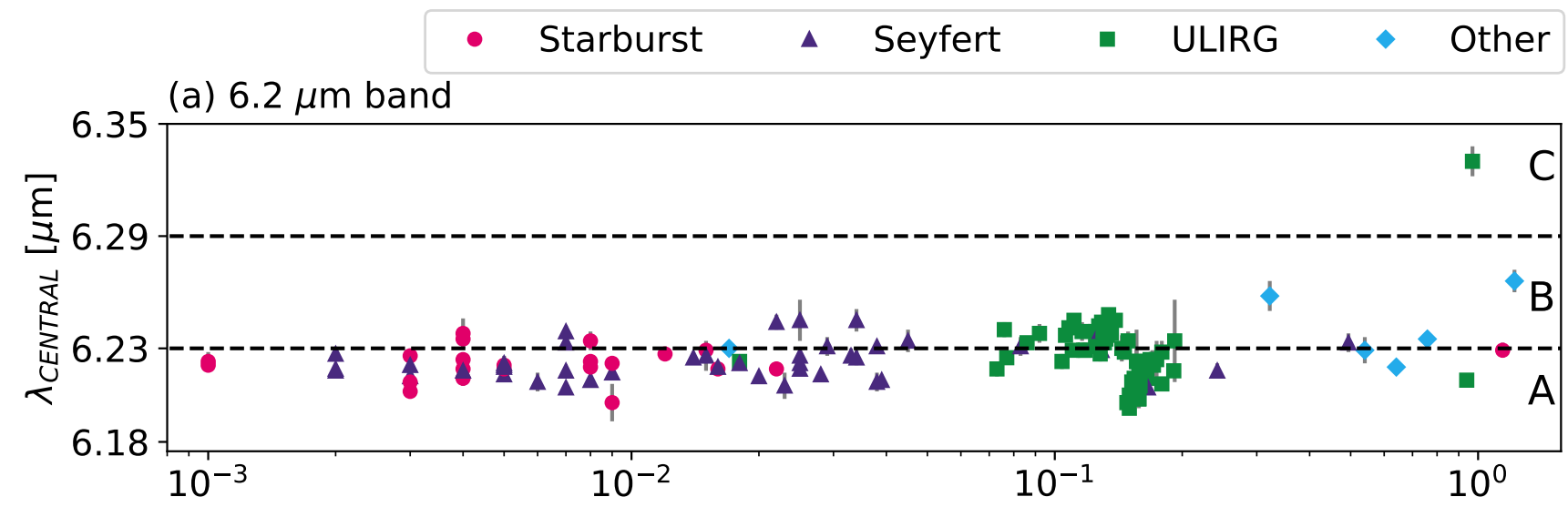

(b) $7.7 \mu \mathrm{m}$ complex

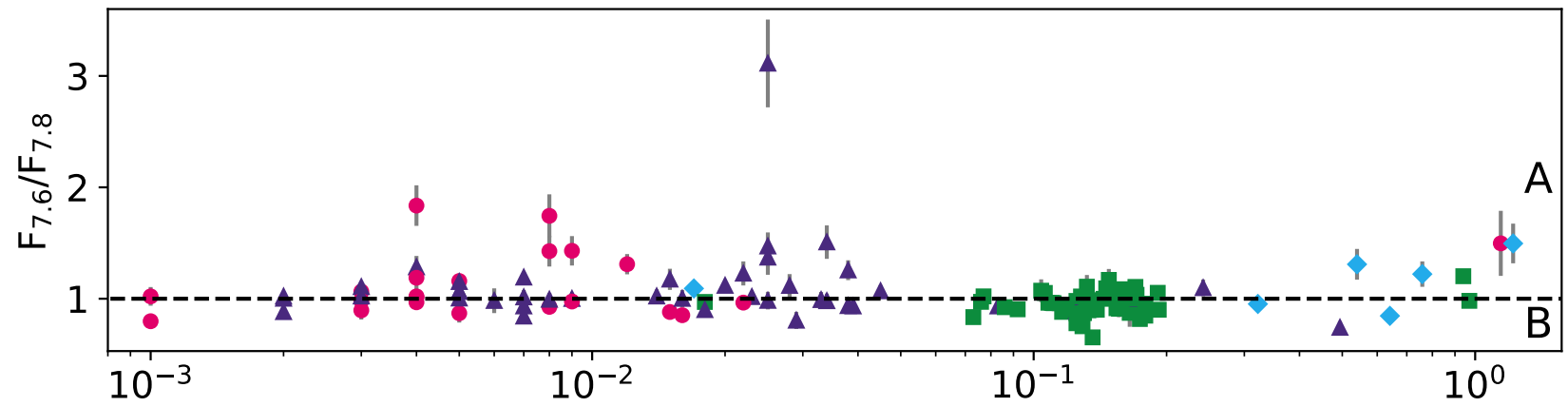

(c) $8.6 \mu \mathrm{m}$ band

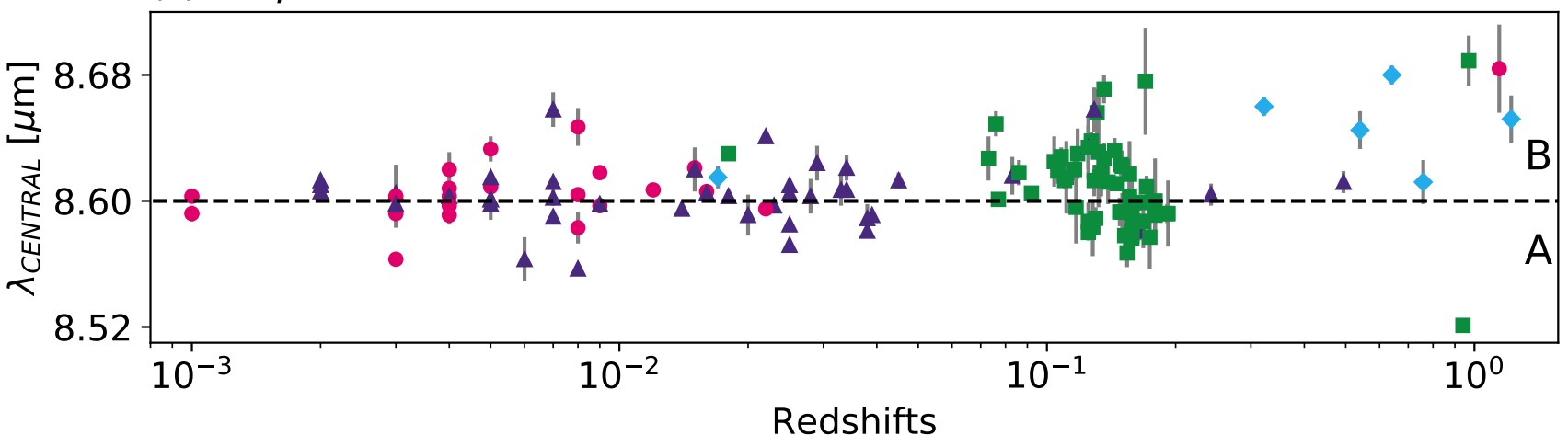

Figure 10. Distribution of the $6.2,7.7$ and $8.6 \mu \mathrm{m}$ bands, respectively, according to the galaxies redshift. The dashed lines are the limits among the Peeters' classes, indicated also by A, B or C letter. The redshift axis is in logarithmic scale. The type of the galaxies were divided into four main groups and their data points are represented in the plots by different symbols. The uncertainties are displayed as grey error bars.

(2002). The $7.7 \mu \mathrm{m}$ complex presented 50 per cent of class A objects while $8.6 \mu \mathrm{m}$ band presented 63 per cent of class B sources. Only the $6.2 \mu \mathrm{m}$ band has a class C profile with 1 per cent of our sample.

Considering the class A objects, 39 per cent of the galaxies were distributed into class A objects for both 6.2 and $7.7 \mu \mathrm{m}$ bands and only 18 per cent received the same A classification for all three bands. Although this result may not allow us to indirectly study the PANHs emission at $6.2 \mu \mathrm{m}$ with the other bands correlation, it supports the complexity of PAH emission. The differences in the profiles, even for the same source, arise from the astrophysical and chemical conditions of the environments, including all the molecular species that contribute differently for each band emission. For instance, according to Monfredini et al. (2019), a more sophisticated interplay between PAHs and dust grains should be present in order to circumvent molecular destruction in AGN circumnuclear medium.

Analysis of other types of objects also available in the ATLAS project, such as AGNs, could shed light on how the starburst-dominated emission of the sources is responsible for the majority of class A objects and could provide 
a broader overview of the PAH band behaviour in astrophysical environments. It would be also important to compare extended intervals of redshift, specially the highest, in order to better comprehend a possible PAH evolutionary timescale. Furthermore, our results could also indicate an aromatic evolution in the ISM of galaxies along to redshifts lower than 1.5 similar to the aromatic evolution in stellar lifecicle proposed by Shannon \& Boersma (2019).

The $\mathrm{PAH}$ profile variations that reproduce different Peeters' classification for the same source could be addressed with chemical evolution models taking into account differences in metallicity, star formation history and the nature of molecular clouds in the harboring galaxy, e.g. the chemodynamical model in Friaça \& Barbuy (2017). In addition, further computational calculations taken together with laboratory and observational measurements are needed to address issues about emitting PAH population, mainly in the conditions prevailing in active galaxies with high star formation and/or super-massive black hole.

\section{ACKNOWLEDGEMENTS}

We would like to thank Dr. Els Peeters (Dept. of Physics \& Astronomy, University of Western Ontario, SETI Institute) for the comments and discussions. CMC acknowledges the support of CNPq, Conselho Nacional de Desenvolvimento Científico e Tecnológico - Brazil, process number 141714/2016-6. This study was financed in part by the Coordenação de Aperfeiçoamento de Pessoal de Nível Superior - Brasil (CAPES) - Finance Code 001. KMD thanks the support of the Serrapilheira Institute as well as that of the Brazilian National Council for Scientific and Technological Development $(\mathrm{CNPq})$ and of the Fundação de Amparo à Pesquisa do Estado do Rio de Janeiro (FAPERJ), Brazil

\section{REFERENCES}

Allamandola L. J., Hudgins D. M., Sandford S. A., 1999, ApJ, 511, L115

Alonso-Herrero A., et al., 2014, MNRAS, 443, 2766

Alonso-Herrero A., et al., 2016, MNRAS, 455, 563

Berné O., et al., 2007, A\&A, 469, 575

Berné O., Mulas G., Joblin C., 2013, A\&A, 550, L4

Brandl B. R., et al., 2006, ApJ, 653, 1129

Buchanan C. L., Gallimore J. F., O'Dea C. P., Baum S. A., Axon D. J., Robinson A., Elitzur M., Elvis M., 2006, AJ, 132, 401

Candian A., Sarre P. J., 2015, MNRAS, 448, 2960

Canelo C. M., Friaça A. C. S., Sales D. A., Pastoriza M. G., Ruschel-Dutra D., 2018, MNRAS, 475, 3746

Chiar J. E., Tielens A. G. G. M., 2006, ApJ, 637, 774

Dasyra K. M., et al., 2009, ApJ, 701, 1123

Deo R. P., Crenshaw D. M., Kraemer S. B., Dietrich M., Elitzur M., Teplitz H., Turner T. J., 2007, ApJ, 671, 124

Draine B. T., Li A., 2001, in American Astronomical Society Meeting Abstracts. p. 1451

Draine B. T., Li A., 2007, ApJ, 657, 810

Ehrenfreund P., et al., 2002, Reports on Progress in Physics, 65, 1427

Ehrenfreund P., Rasmussen S., Cleaves J., Chen L., 2006, Astrobiology, 6, 490

Farrah D., Weedman D., Lonsdale C. J., Polletta M., RowanRobinson M., Houck J., Smith H. E., 2009, ApJ, 696, 2044

Friaça A. C. S., Barbuy B., 2017, A\&A, 598, A121
Galliano F., Madden S. C., Tielens A. G. G. M., Peeters E., Jones A. P., 2008, ApJ, 679, 310

Genzel R., Cesarsky C. J., 2000, ARA\&A, 38, 761

Hernán-Caballero A., Hatziminaoglou E., 2011, MNRAS, 414, 500

Hernán-Caballero A., et al., 2009, MNRAS, 395, 1695

Hiner K. D., Canalizo G., Lacy M., Sajina A., Armus L., Ridgway S., Storrie-Lombardi L., 2009, ApJ, 706, 508

Houck J. R., et al., 2004, ApJS, 154, 18

Hudgins D. M., Bauschlicher Jr. C. W., Allamandola L. J., 2005, ApJ, 632, 316

Imanishi M., 2009, ApJ, 694, 751

Imanishi M., Dudley C. C., Maiolino R., Maloney P. R., Nakagawa T., Risaliti G., 2007, ApJS, 171, 72

Imanishi M., Maiolino R., Nakagawa T., 2010, ApJ, 709, 801

Joblin C., Leger A., Martin P., 1992, ApJ, 393, L79

Joblin C., Szczerba R., Berné O., Szyszka C., 2008, A\&A, 490, 189

Leipski C., Antonucci R., Ogle P., Whysong D., 2009, ApJ, 701, 891

Li A., 2004, in Witt A. N., Clayton G. C., Draine B. T., eds, Astronomical Society of the Pacific Conference Series Vol. 309, Astrophysics of Dust. p. 417 (arXiv:astro-ph/0311066)

Maragkoudakis A., Ivkovich N., Peeters E., Stock D. J., Hemachandra D., Tielens A. G. G. M., 2018, MNRAS, 481, 5370

Monfredini T., et al., 2019, MNRAS, 488, 451

Murphy E. J., Chary R.-R., Alexander D. M., Dickinson M., Magnelli B., Morrison G., Pope A., Teplitz H. I., 2009, ApJ, 698, 1380

Papovich C., et al., 2006, ApJ, 640, 92

Peeters E., Hony S., Van Kerckhoven C., Tielens A. G. G. M., Allamandola L. J., Hudgins D. M., Bauschlicher C. W., 2002, A\&A, 390, 1089

Peeters E., Bauschlicher Jr. C. W., Allamandola L. J., Tielens A. G. G. M., Ricca A., Wolfire M. G., 2017, ApJ, 836, 198

Pino T., et al., 2008, A\&A, 490, 665

Pope A., et al., 2008, ApJ, 675, 1171

Rapacioli M., Joblin C., Boissel P., 2005, A\&A, 429, 193

Ricca A., Bauschlicher Jr. C. W., Boersma C., Tielens A. G. G. M., Allamandola L. J., 2012, ApJ, 754, 75

Ricca A., Bauschlicher Jr. C. W., Roser J. E., Peeters E., 2018, ApJ, 854, 115

Ruschel-Dutra D., Pastoriza M., Riffel R., Sales D. A., Winge C., 2014, MNRAS, 438, 3434

Sales D. A., Pastoriza M. G., Riffel R., 2010, ApJ, 725, 605

Sales D. A., Pastoriza M. G., Riffel R., Winge C., 2013, MNRAS, 429, 2634

Shannon M. J., Boersma C., 2019, ApJ, 871, 124

Shi Y., et al., 2006, ApJ, 653, 127

Shivaei I., et al., 2017, ApJ, 837, 157

Sloan G. C., et al., 2007, ApJ, 664, 1144

Smith J. D. T., et al., 2007, ApJ, 656, 770

Stock D. J., Peeters E., 2017, ApJ, 837, 129

Teplitz H. I., et al., 2007, ApJ, 659, 941

Tielens A. G. G. M., 2008, ARA\&A, 46, 289

Vega O., et al., 2010, ApJ, 721, 1090

Weedman D. W., Houck J. R., 2009, ApJ, 693, 370

Weedman D. W., et al., 2005, ApJ, 633, 706

Werner M. W., et al., 2004, ApJS, 154, 1

Wu Y., Charmandaris V., Huang J., Spinoglio L., Tommasin S., 2009, ApJ, 701, 658

Yan L., et al., 2005, ApJ, 628, 604

Yan L., et al., 2007, ApJ, 658, 778

van Diedenhoven B., Peeters E., Van Kerckhoven C., Hony S., Hudgins D. M., Allamandola L. J., Tielens A. G. G. M., 2004, ApJ, 611, 928 


\section{APPENDIX A: SOURCES - IDENTIFICATION AND DERIVED PROPERTIES}

Table A1: Sources and their respective informations extracted from the MIR_SB sample (Spitzer/IRS ATLAS, version 1.0) and Yan et al. (2007), including their ID, type, source reference, right ascension, declination and redshift. Acronyms: AGN - Active Galactic Nucleus, FR - Fanaroff-Riley galaxy, HII - HII region, IRgal - Infrared galaxy, LINER - Low-Ionization Nuclear Emission-line Region, QSO - Quasi-Stellar Object, SB - Starburst galaxy, SMG - Submillimeter Galaxy, Sy - Seyfert galaxy, ULIRG - Ultra-Luminous Infrared Galaxy.

\begin{tabular}{|c|c|c|c|c|c|}
\hline ID & Type & Reference & RA (hms) & Dec (dms) & $\mathrm{z}$ \\
\hline $3 \mathrm{C} 293$ & Sy3 & Leipski et al. (2009) & $13: 52: 17.80$ & $31: 26: 46.50$ & 0.045 \\
\hline 3C31 & FR-1 & Leipski et al. (2009) & 01:07:24.90 & $32: 24: 45.20$ & 0.017 \\
\hline $\mathrm{AGN} 15^{a}$ & LINER & Weedman \& Houck (2009) & $17: 18: 52.71$ & $59: 14: 32.00$ & 0.322 \\
\hline Arp220 & ULIRG & Imanishi et al. (2007) & $15: 34: 57.10$ & $23: 30: 11.00$ & 0.018 \\
\hline E12-G21 & Sy1 & Wu et al. (2009) & $00: 40: 47.80$ & $-79: 14: 27.00$ & 0.033 \\
\hline EIRS- $2^{a}$ & SB & Hernán-Caballero et al. (2009) & $16: 13: 49.94$ & $54: 26: 28.40$ & 1.143 \\
\hline GN26 & SMG & Pope et al. (2008) & $12: 36: 34.51$ & $62: 12: 40.90$ & 1.219 \\
\hline IC342 & SB & Brandl et al. (2006) & $03: 46: 48.51$ & $68: 05: 46.00$ & 0.001 \\
\hline IRAS02021-2103 & ULIRG & Imanishi et al. (2010) & 02:04:27.30 & $-20: 49: 41$ & 0.116 \\
\hline IRAS02480-3745 & ULIRG & Imanishi et al. (2010) & 02:50:01.70 & $-37: 32: 45$ & 0.165 \\
\hline IRAS03209-0806 & Sy1 & Imanishi et al. (2010) & $03: 23: 22.90$ & $-07: 56: 15$ & 0.166 \\
\hline IRAS05020-2941 & ULIRG & Imanishi et al. (2010) & 05:04:00.70 & $-29: 36: 55$ & 0.154 \\
\hline IRAS08591+5248 & ULIRG & Imanishi et al. (2010) & 09:02:47.50 & $52: 36: 30$ & 0.158 \\
\hline IRAS10594+3818 & ULIRG & Imanishi et al. (2010) & $11: 02: 14.00$ & $38: 02: 35$ & 0.158 \\
\hline IRAS12447+3721 & ULIRG & Imanishi et al. (2010) & $12: 47: 07.70$ & $37: 05: 37$ & 0.158 \\
\hline IRAS13106-0922 & ULIRG & Imanishi et al. (2010) & $13: 13: 14.80$ & $-09: 38: 00$ & 0.174 \\
\hline IRAS14121-0126 & ULIRG & Imanishi et al. (2010) & $14: 14: 45.50$ & $-01: 40: 55$ & 0.150 \\
\hline IRAS14197+0813 & ULIRG & Imanishi et al. (2010) & $14: 22: 11.60$ & $07: 59: 28$ & 0.131 \\
\hline IRAS14202+2615 & ULIRG & Imanishi et al. (2010) & $14: 22: 31.40$ & $26: 02: 05$ & 0.159 \\
\hline IRAS14485-2434 & ULIRG & Imanishi et al. (2010) & $14: 51: 23.80$ & $-24: 46: 30$ & 0.148 \\
\hline IRAS15043+5754 & ULIRG & Imanishi et al. (2010) & $15: 05: 39.50$ & $57: 43: 07$ & 0.150 \\
\hline IRAS21477+0502 & ULIRG & Imanishi et al. (2010) & $21: 50: 16.30$ & $05: 16: 03$ & 0.171 \\
\hline IRAS22088-1831 & ULIRG & Imanishi et al. (2010) & $22: 11: 33.80$ & $-18: 17: 06$ & 0.170 \\
\hline IRAS_00091-0738 & ULIRG + HII & Imanishi et al. (2007) & 00:11:43.30 & $-07: 22: 07.00$ & 0.118 \\
\hline IRAS_00456-2904 & $\mathrm{ULIRG}+\mathrm{HII}$ & Imanishi et al. (2007) & $00: 48: 06.80$ & $-28: 48: 19.00$ & 0.110 \\
\hline IRAS_01199-2307 & $\mathrm{ULIRG}+\mathrm{HII}$ & Imanishi (2009) & $01: 22: 20.90$ & $-22: 52: 07.00$ & 0.156 \\
\hline IRAS_01355-1814 & $\mathrm{ULIRG}+\mathrm{HII}$ & Imanishi (2009) & $01: 37: 57.40$ & $-17: 59: 21.00$ & 0.192 \\
\hline IRAS_01494-1845 & ULIRG & Imanishi (2009) & $01: 51: 51.40$ & $-18: 30: 46.00$ & 0.158 \\
\hline IRAS_02411+0353 & ULIRG + HII & Imanishi et al. (2007) & $02: 43: 46.10$ & 04:06:37.00 & 0.144 \\
\hline IRAS_03250+1606 & ULIRG + LINER & Imanishi et al. (2007) & $03: 27: 49.80$ & $16: 17: 00.00$ & 0.129 \\
\hline IRAS_03521+0028 & ULIRG + LINER & Imanishi (2009) & $03: 54: 42.20$ & 00:37:03.00 & 0.152 \\
\hline IRAS_08201+2801 & ULIRG + HII & Imanishi (2009) & $08: 23: 12.60$ & $27: 51: 40.00$ & 0.168 \\
\hline IRAS_09039+0503 & ULIRG + LINER & Imanishi et al. (2007) & 09:06:34.20 & 04:51:25.00 & 0.125 \\
\hline IRAS_09116+0334 & ULIRG + LINER & Imanishi et al. (2007) & 09:14:13.80 & 03:22:01.00 & 0.146 \\
\hline IRAS_09463+8141 & ULIRG + LINER & Imanishi (2009) & $09: 53: 00.50$ & $81: 27: 28.00$ & 0.156 \\
\hline IRAS_09539+0857 & Sy2 & Imanishi et al. (2007) & $09: 56: 34.30$ & 08:43:06.00 & 0.129 \\
\hline IRAS_10190+1322 & $\mathrm{ULIRG}+\mathrm{HII}$ & Imanishi et al. (2007) & $10: 21: 42.50$ & 13:06:54.00 & 0.077 \\
\hline
\end{tabular}


Continued from previous page

\begin{tabular}{|c|c|c|c|c|c|}
\hline ID & Type & Reference & RA (hms) & Dec (dms) & $\mathrm{z}$ \\
\hline IRAS_10485-1447 & ULIRG + LINER & Imanishi et al. (2007) & 10:51:03.10 & $-15: 03: 22.00$ & 0.133 \\
\hline IRAS_10494+4424 & ULIRG + LINER & Imanishi et al. (2007) & $10: 52: 23.50$ & $44: 08: 48.00$ & 0.092 \\
\hline IRAS_11130-2659 & ULIRG + LINER & Imanishi et al. (2007) & $11: 15: 31.60$ & $-27: 16: 23.00$ & 0.136 \\
\hline IRAS_11387+4116 & ULIRG + HII & Imanishi et al. (2007) & $11: 41: 22.00$ & $40: 59: 51.00$ & 0.149 \\
\hline IRAS_11506+1331 & $\mathrm{ULIRG}+\mathrm{HII}$ & Imanishi et al. (2007) & $11: 53: 14.20$ & $13: 14: 28.00$ & 0.127 \\
\hline IRAS_12112+0305 & ULIRG + LINER & Imanishi et al. (2007) & $12: 13: 46.00$ & 02:48:38.00 & 0.073 \\
\hline IRAS_13335-2612 & ULIRG + LINER & Imanishi et al. (2007) & $13: 36: 22.30$ & $-26: 27: 34.00$ & 0.125 \\
\hline IRAS_13469+5833 & ULIRG + HII & Imanishi (2009) & $13: 48: 40.20$ & $58: 18: 52.00$ & 0.158 \\
\hline IRAS_13509+0442 & ULIRG + HII & Imanishi et al. (2007) & $13: 53: 31.60$ & $04: 28: 05.00$ & 0.136 \\
\hline IRAS_13539+2920 & ULIRG + HII & Imanishi et al. (2007) & $13: 56: 10.00$ & $29: 05: 35.00$ & 0.108 \\
\hline IRAS_14060+2919 & $\mathrm{ULIRG}+\mathrm{HII}$ & Imanishi et al. (2007) & $14: 08: 19.00$ & 29:04:47.00 & 0.117 \\
\hline IRAS_14348-1447 & Sy1 & Imanishi et al. (2007) & $14: 37: 38.30$ & $-15: 00: 23.00$ & 0.083 \\
\hline IRAS_15206+3342 & Sy2 & Imanishi et al. (2007) & $15: 22: 38.00$ & $33: 31: 36.00$ & 0.125 \\
\hline IRAS_15225+2350 & ULIRG + HII & Imanishi et al. (2007) & $15: 24: 43.90$ & $23: 40: 10.00$ & 0.139 \\
\hline IRAS_16090-0139 & ULIRG + LINER & Imanishi et al. (2007) & $16: 11: 40.50$ & $-01: 47: 06.00$ & 0.134 \\
\hline IRAS_16300+1558 & Sy2 & Imanishi (2009) & $16: 32: 21.40$ & $15: 51: 46.00$ & 0.242 \\
\hline IRAS_16333+4630 & ULIRG + LINER & Imanishi (2009) & $16: 34: 52.60$ & $46: 24: 53.00$ & 0.191 \\
\hline IRAS_16474+3430 & ULIRG + HII & Imanishi et al. (2007) & $16: 49: 14.20$ & $34: 25: 10.00$ & 0.111 \\
\hline IRAS_16487+5447 & ULIRG + LINER & Imanishi et al. (2007) & $16: 49: 47.00$ & $54: 42: 35.00$ & 0.104 \\
\hline IRAS_17028+5817 & ULIRG + LINER & Imanishi et al. (2007) & $17: 03: 41.90$ & $58: 13$ & 0.106 \\
\hline IRAS_17068+4027 & ULIRG + HII & Imanishi (2009) & $17: 08: 31.90$ & $40: 23: 28.00$ & 0.179 \\
\hline IRAS_20414-1651 & ULIRG + HII & Imanishi et al. (2007) & $20: 44: 18.20$ & $-16: 40: 16.00$ & 0.086 \\
\hline IRAS_21208-0519 & $\mathrm{ULIRG}+\mathrm{HII}$ & Imanishi et al. (2007) & $21: 23: 29.10$ & $-05: 06: 56.00$ & 0.130 \\
\hline IRAS_21329-2346 & ULIRG + LINER & Imanishi et al. (2007) & $21: 35: 45.80$ & $-23: 32: 35.00$ & 0.125 \\
\hline IRAS_22206-2715 & ULIRG + HII & Imanishi et al. (2007) & $22: 23: 28.90$ & $-27: 00: 04.00$ & 0.132 \\
\hline IRAS_22491-1808 & ULIRG + HII & Imanishi et al. (2007) & $22: 51: 49.20$ & $-17: 52: 23.00$ & 0.076 \\
\hline IRAS_23129+2548 & ULIRG + LINER & Imanishi (2009) & $23: 15: 21.40$ & $26: 04: 32.00$ & 0.179 \\
\hline IRAS_23234+0946 & ULIRG + LINER & Imanishi et al. (2007) & $23: 25: 56.20$ & $10: 02: 49.00$ & 0.128 \\
\hline $\mathrm{M}+0-29-23$ & Sy 2 & Wu et al. (2009) & $11: 21:$ & $-02: 59: 03.00$ & 0.025 \\
\hline MIPS562 & IRgal & Dasyra et al. (2009) & $17: 12: 39.60$ & $58: 59: 55.10$ & 0.540 \\
\hline MIPS8040 & IRgal & Dasyra et al. (2009) & $17: 13: 12.00$ & $60: 08: 40.20$ & 0.759 \\
\hline Mrk52 & SB & Brandl et al. (2006) & $12: 25: 42.67$ & $00: 34: 20.40$ & 0.007 \\
\hline Mrk273 & Sy2 & Wu et al. (2009) & $13: 44: 42.10$ & $55: 53: 13.00$ & 0.038 \\
\hline Mrk334 & Sy1.8 & Deo et al. (2007) & 00:03:09.60 & $21: 57: 37.00$ & 0.022 \\
\hline Mrk471 & Sy1.8 & Deo et al. (2007) & $14: 22: 55.40$ & $32: 51: 03.00$ & 0.034 \\
\hline Mrk609 & Sy1.8 & Deo et al. (2007) & $03: 25: 25.30$ & $-06: 08: 38.00$ & 0.034 \\
\hline Mrk622 & Sy2 & Deo et al. (2007) & 08:07:41.00 & $39: 00: 15.00$ & 0.023 \\
\hline Mrk883 & Sy1.9 & Deo et al. (2007) & $16: 29: 52.90$ & $24: 26: 38.00$ & 0.038 \\
\hline Mrk938 & Sy2 & Wu et al. (2009) & $00: 11: 06.50$ & $-12: 06: 26.00$ & 0.020 \\
\hline Mrk1066 & Sy2 & Shi et al. (2006) & $02: 59: 58.60$ & $36: 49: 14.00$ & 0.012 \\
\hline Murphy22 & $\mathrm{SMG}$ & Murphy et al. (2009) & $12: 37: 34.52$ & $62: 17: 23.20$ & 0.641 \\
\hline NGC520 & $\mathrm{SB}+\mathrm{Sy} 1.8$ & Brandl et al. (2006) & $01: 24: 35.07$ & $03: 47: 32.7$ & 0.0071 \\
\hline NGC660 & SB & Brandl et al. (2006) & $01: 43: 02.45$ & $13: 38: 44.4$ & 0.0029 \\
\hline NGC1056 & Sy2 & Wu et al. (2009) & $02: 42: 48.30$ & $28: 34: 27.00$ & 0.005 \\
\hline NGC1097 & $\mathrm{SB}+\mathrm{Sy} 1$ & Wu et al. (2009) & $02: 46: 19.08$ & $-30: 16: 28.00$ & 0.004 \\
\hline
\end{tabular}


Continued from previous page

\begin{tabular}{|c|c|c|c|c|c|}
\hline ID & Type & Reference & RA (hms) & Dec (dms) & $\mathrm{z}$ \\
\hline NGC1143 & Sy2 & Wu et al. (2009) & $02: 55: 12.20$ & $-00: 11: 01.00$ & 0.029 \\
\hline NGC1222 & SB & Brandl et al. (2006) & 03:08:56.74 & $-02: 57: 18.50$ & 0.008 \\
\hline NGC1365 & $\mathrm{SB}+\mathrm{Sy} 1.8$ & Brandl et al. (2006) & $03: 33: 36.37$ & $-36: 08: 25.50$ & 0.005 \\
\hline NGC1566 & Sy 1.5 & Wu et al. (2009) & 04:20:00.40 & $-54: 56: 16.00$ & 0.005 \\
\hline NGC1614 & $\mathrm{SB}+\mathrm{HII}$ & Brandl et al. (2006) & $04: 33: 59.85$ & $-08: 34: 44.00$ & 0.015 \\
\hline NGC1667 & Sy2 & Wu et al. (2009) & $04: 48: 37.10$ & $-06: 19: 12.00$ & 0.015 \\
\hline NGC2146 & SB & Brandl et al. (2006) & $06: 18: 37.71$ & $78: 21: 25.30$ & 0.004 \\
\hline NGC2623 & $\mathrm{SB}$ & Brandl et al. (2006) & $08: 38: 24.08$ & $25: 45: 16.90$ & 0.018 \\
\hline NGC2992 & Sy1.9 & Wu et al. (2009) & 09:45:42.00 & $-14: 19: 35.00$ & 0.008 \\
\hline NGC3079 & Sy2 & Weedman et al. (2005) & 10:01:57.80 & $55: 40: 47.00$ & 0.004 \\
\hline NGC3227 & Sy1.5 & Wu et al. (2009) & $10: 23: 30.60$ & $19: 51: 54.00$ & 0.004 \\
\hline NGC3256 & SB & Brandl et al. (2006) & $10: 27: 51.27$ & $-43: 54: 13.80$ & 0.008 \\
\hline NGC3310 & $\mathrm{SB}+\mathrm{HII}$ & Brandl et al. (2006) & $10: 38: 45.96$ & $53: 30: 12.00$ & 0.005 \\
\hline NGC3511 & Sy1 & Wu et al. (2009) & $11: 03: 23.80$ & $-23: 05: 12.00$ & 0.004 \\
\hline NGC3556 & SB & Brandl et al. (2006) & $11: 11: 30.97$ & $55: 40: 26.80$ & 0.003 \\
\hline NGC3628 & $\mathrm{SB}+\mathrm{LINER}$ & Brandl et al. (2006) & $11: 20: 17.02$ & $13: 35: 22.20$ & 0.002 \\
\hline NGC3786 & Sy1.8 & Deo et al. (2007) & $11: 39: 42.50$ & $31: 54: 33.00$ & 0.009 \\
\hline NGC3982 & Sy1.9 & Wu et al. (2009) & $11: 56: 28.10$ & $55: 07: 31.00$ & 0.004 \\
\hline NGC4088 & $\mathrm{SB}$ & Brandl et al. (2006) & $12: 05: 34.19$ & $50: 32: 20.50$ & 0.003 \\
\hline NGC4194 & $\mathrm{SB}+\mathrm{HII}$ & Brandl et al. (2006) & 12:14:09.64 & $54: 31: 34.60$ & 0.009 \\
\hline NGC4388 & Sy2 & Wu et al. (2009) & $12: 25: 46.70$ & $12: 39: 44.00$ & 0.008 \\
\hline NGC4676 & SB & Brandl et al. (2006) & $12: 46: 10.10$ & $30: 43: 55.00$ & 0.022 \\
\hline NGC4818 & SB & Brandl et al. (2006) & $12: 56: 48.90$ & $-08: 31: 31.10$ & 0.002 \\
\hline NGC4945 & $\mathrm{SB}+\mathrm{Sy} 2$ & Brandl et al. (2006) & $13: 05: 27.48$ & $-49: 28: 05.60$ & 0.001 \\
\hline NGC5005 & Sy2 & Wu et al. (2009) & $13: 10: 56.20$ & $37: 03: 33.00$ & 0.003 \\
\hline NGC5033 & Sy1.8 & Wu et al. (2009) & $13: 13: 27.50$ & $36: 35: 38.00$ & 0.003 \\
\hline NGC5135 & Sy2 & Wu et al. (2009) & $13: 25: 44.00$ & $-29: 50: 01.00$ & 0.014 \\
\hline NGC5194 & Sy2 & Wu et al. (2009) & $13: 29: 52.70$ & $47: 11: 43.00$ & 0.002 \\
\hline NGC5256 & Sy2 & Wu et al. (2009) & $13: 38: 17.50$ & $48: 16: 37.00$ & 0.028 \\
\hline NGC5674 & Sy1.9 & Shi et al. (2006) & $14: 33: 52.20$ & $05: 27: 30.00$ & 0.025 \\
\hline NGC5953 & Sy2 & Buchanan et al. (2006) & $15: 34: 32.40$ & $15: 11: 38.00$ & 0.007 \\
\hline NGC6810 & Sy2 & Wu et al. (2009) & $19: 43: 34.40$ & $-58: 39: 21.00$ & 0.007 \\
\hline NGC7252 & SB & Brandl et al. (2006) & $22: 20: 44.77$ & $-24: 40: 41.80$ & 0.016 \\
\hline NGC7469 & Sy1.5 & Wu et al. (2009) & $23: 03: 15.60$ & $08: 52: 26.00$ & 0.016 \\
\hline NGC7496 & Sy2 & Buchanan et al. (2006) & $23: 09: 47.30$ & $-43: 25: 41.00$ & 0.006 \\
\hline NGC7582 & Sy2 & Wu et al. (2009) & $23: 18: 23.50$ & $-42: 22: 14.00$ & 0.005 \\
\hline NGC7590 & Sy2 & Wu et al. (2009) & $23: 18: 55.00$ & $-42: 14: 17.00$ & 0.005 \\
\hline NGC7714 & $\mathrm{SB}+\mathrm{HII}$ & Brandl et al. (2006) & $23: 36: 14.10$ & 02:09:18.60 & 0.009 \\
\hline SST172458.3+591545 & Sy1 & Hiner et al. (2009) & $17: 24: 58.30$ & $59: 15: 45$ & 0.494 \\
\hline SWIRE4_J103637.18+584217.0 $0^{a}$ & ULIRG & Farrah et al. (2009) & $10: 36: 37.18$ & $58: 42: 17.00$ & 0.970 \\
\hline SWIRE4_J104830.58+591810.2 $2^{a}$ & ULIRG & Farrah et al. (2009) & $10: 48: 30.58$ & $59: 18: 10.20$ & 0.940 \\
\hline UGC5101 & Sy1 & Wu et al. (2009) & $09: 35: 51.60$ & $61: 21: 11.00$ & 0.039 \\
\hline UGC7064 & Sy1.9 & Wu et al. (2009) & $12: 04: 43.30$ & $31: 10: 38.00$ & 0.025 \\
\hline UGC12138 & Sy1.8 & Deo et al. (2007) & $22: 40: 17.00$ & 08:03:14.00 & 0.025 \\
\hline
\end{tabular}

Table A2: Best-fit results for the 7.7 and $8.6 \mu \mathrm{m}$ bands (Section 3.2). A is the amplitude, $\lambda_{-} c$ is the central wavelength and FWHM is the full width at half maximum.

\begin{tabular}{ccccccc}
\hline Source & $\lambda \_c$ & Err & A & Err & FWHM & Err \\
\hline & \multicolumn{4}{c}{ Continued on next page } \\
\hline
\end{tabular}


Continued from previous page

\begin{tabular}{|c|c|c|c|c|c|c|}
\hline Source & $\begin{array}{c}\lambda_{-} c \\
{[\mu \mathrm{m}]}\end{array}$ & $\begin{array}{c}\text { Err } \\
{[\mu \mathrm{m}]}\end{array}$ & $\begin{array}{c}\text { Amplitude } \\
{[\mathrm{mJy} / \mathrm{sr}]}\end{array}$ & $\begin{array}{c}\text { Err } \\
{[\mathrm{mJy} / \mathrm{sr}]}\end{array}$ & FWHM & Err \\
\hline & {$[\mu \mathrm{m}]$} & {$[\mu \mathrm{m}]$} & {$[\mathrm{mJy} / \mathrm{sr}]$} & {$[\mathrm{mJy} / \mathrm{sr}]$} & & \\
\hline \multirow[t]{3}{*}{ 3C293 } & 7.609 & 0.006 & 1.476 & 0.051 & 0.280 & - \\
\hline & 7.862 & 0.007 & 1.389 & 0.051 & 0.320 & - \\
\hline & 8.613 & 0.005 & 0.529 & 0.021 & 0.261 & 0.013 \\
\hline \multirow[t]{3}{*}{ 3C31 } & 7.596 & 0.007 & 2.055 & 0.091 & 0.280 & - \\
\hline & 7.829 & 0.008 & 1.887 & 0.094 & 0.320 & - \\
\hline & 8.615 & 0.007 & 1.821 & 0.094 & 0.335 & 0.023 \\
\hline \multirow[t]{3}{*}{ AGN15 } & 7.645 & 0.012 & 1.292 & 0.070 & 0.280 & - \\
\hline & 7.913 & 0.015 & 1.380 & 0.071 & 0.320 & - \\
\hline & 8.660 & 0.006 & 0.229 & 0.015 & 0.200 & 0.017 \\
\hline \multirow[t]{3}{*}{ Arp220 } & 7.586 & 0.008 & 79.727 & 3.712 & 0.280 & - \\
\hline & 7.867 & 0.009 & 82.679 & 4.126 & 0.320 & - \\
\hline & 8.630 & 0.004 & 8.468 & 0.354 & 0.189 & 0.009 \\
\hline \multirow[t]{3}{*}{ E12-G21 } & 7.562 & 0.010 & 23.021 & 1.191 & 0.280 & - \\
\hline & 7.842 & 0.010 & 23.322 & 1.168 & 0.320 & - \\
\hline & 8.607 & 0.010 & 23.720 & 1.706 & 0.418 & 0.040 \\
\hline \multirow[t]{3}{*}{ EIRS-2 } & 7.587 & 0.016 & 0.520 & 0.059 & 0.280 & - \\
\hline & 7.837 & 0.033 & 0.349 & 0.057 & 0.320 & - \\
\hline & 8.684 & 0.028 & 0.456 & 0.103 & 0.348 & 0.095 \\
\hline \multirow[t]{3}{*}{ GN26 } & 7.671 & 0.015 & 0.509 & 0.037 & 0.280 & - \\
\hline & 7.950 & 0.028 & 0.352 & 0.040 & 0.320 & - \\
\hline & 8.652 & 0.015 & 0.148 & 0.030 & 0.154 & 0.036 \\
\hline \multirow[t]{3}{*}{ IC342 } & 7.583 & 0.009 & 104.704 & 6.068 & 0.280 & - \\
\hline & 7.812 & 0.009 & 102.705 & 6.013 & 0.320 & - \\
\hline & 8.603 & 0.003 & 60.704 & 1.439 & 0.283 & 0.009 \\
\hline \multirow[t]{3}{*}{ IRAS02021-2103 } & 7.596 & 0.009 & 3.860 & 0.216 & 0.280 & - \\
\hline & 7.850 & 0.010 & 4.395 & 0.220 & 0.320 & - \\
\hline & 8.620 & 0.007 & 3.024 & 0.158 & 0.353 & 0.024 \\
\hline \multirow[t]{3}{*}{ IRAS02480-3745 } & 7.543 & 0.019 & 3.994 & 0.423 & 0.280 & - \\
\hline & 7.816 & 0.020 & 4.577 & 0.444 & 0.320 & - \\
\hline & 8.599 & 0.005 & 2.536 & 0.072 & 0.438 & 0.016 \\
\hline \multirow[t]{3}{*}{ IRAS03209-0806 } & 7.545 & 0.007 & 4.066 & 0.180 & 0.280 & - \\
\hline & 7.808 & 0.009 & 4.070 & 0.207 & 0.320 & - \\
\hline & 8.581 & 0.006 & 2.926 & 0.122 & 0.340 & 0.019 \\
\hline \multirow[t]{3}{*}{ IRAS05020-2941 } & 7.539 & 0.008 & 6.636 & 0.263 & 0.280 & - \\
\hline & 7.816 & 0.008 & 7.253 & 0.283 & 0.320 & - \\
\hline & 8.567 & 0.009 & 0.867 & 0.102 & 0.161 & 0.023 \\
\hline \multirow[t]{3}{*}{ IRAS08591+5248 } & 7.546 & 0.006 & 5.273 & 0.202 & 0.280 & - \\
\hline & 7.804 & 0.008 & 4.858 & 0.204 & 0.320 & - \\
\hline & 8.578 & 0.010 & 2.532 & 0.204 & 0.272 & 0.027 \\
\hline \multirow[t]{3}{*}{ IRAS10594+3818 } & 7.546 & 0.006 & 8.331 & 0.241 & 0.280 & - \\
\hline & 7.807 & 0.006 & 7.806 & 0.265 & 0.320 & - \\
\hline & 8.592 & 0.010 & 2.981 & 0.257 & 0.251 & 0.026 \\
\hline IRAS12447+3721 & 7.529 & 0.008 & 4.583 & 0.202 & 0.280 & - \\
\hline
\end{tabular}


Continued from previous page

\begin{tabular}{|c|c|c|c|c|c|c|}
\hline Source & $\begin{array}{c}\lambda \_c \\
{[\mu \mathrm{m}]}\end{array}$ & $\begin{array}{c}\text { Err } \\
{[\mu \mathrm{m}]}\end{array}$ & $\begin{array}{c}\text { Amplitude } \\
{[\mathrm{mJy} / \mathrm{sr}]}\end{array}$ & $\begin{array}{c}\text { Err } \\
{[\mathrm{mJy} / \mathrm{sr}]}\end{array}$ & FWHM & Err \\
\hline & 7.805 & 0.009 & 4.522 & 0.213 & 0.320 & - \\
\hline & 8.595 & 0.018 & 1.067 & 0.219 & 0.183 & 0.043 \\
\hline \multirow[t]{3}{*}{ IRAS13106-0922 } & 7.503 & 0.005 & 1.828 & 0.076 & 0.200 & - \\
\hline & 7.774 & 0.007 & 2.234 & 0.089 & 0.250 & - \\
\hline & 8.577 & 0.020 & 0.831 & 0.122 & 0.296 & 0.053 \\
\hline \multirow[t]{3}{*}{ IRAS14121-0126 } & 7.532 & 0.010 & 7.445 & 0.447 & 0.280 & - \\
\hline & 7.787 & 0.011 & 7.130 & 0.443 & 0.290 & - \\
\hline & 8.593 & 0.012 & 2.251 & 0.226 & 0.238 & 0.028 \\
\hline \multirow[t]{3}{*}{ IRAS14197+0813 } & 7.579 & 0.010 & 2.879 & 0.173 & 0.280 & - \\
\hline & 7.857 & 0.012 & 3.036 & 0.172 & 0.320 & - \\
\hline & 8.656 & 0.015 & 1.077 & 0.134 & 0.241 & 0.036 \\
\hline \multirow[t]{3}{*}{ IRAS14202+2615 } & 7.537 & 0.006 & 8.790 & 0.377 & 0.280 & - \\
\hline & 7.786 & 0.008 & 8.760 & 0.383 & 0.320 & - \\
\hline & 8.587 & 0.010 & 4.030 & 0.338 & 0.249 & 0.025 \\
\hline \multirow[t]{3}{*}{ IRAS14485-2434 } & 7.562 & 0.009 & 5.425 & 0.295 & 0.280 & - \\
\hline & 7.812 & 0.012 & 4.647 & 0.301 & 0.320 & - \\
\hline & 8.593 & 0.009 & 2.148 & 0.150 & 0.293 & 0.027 \\
\hline \multirow[t]{3}{*}{ IRAS15043+5754 } & 7.556 & 0.005 & 5.617 & 0.151 & 0.280 & - \\
\hline & 7.824 & 0.006 & 5.335 & 0.161 & 0.320 & - \\
\hline & 8.622 & 0.010 & 1.262 & 0.123 & 0.213 & 0.024 \\
\hline \multirow[t]{3}{*}{ IRAS21477+0502 } & 7.544 & 0.007 & 3.502 & 0.141 & 0.280 & - \\
\hline & 7.806 & 0.009 & 3.383 & 0.148 & 0.320 & - \\
\hline & 8.609 & 0.007 & 1.765 & 0.087 & 0.310 & 0.020 \\
\hline \multirow[t]{3}{*}{ IRAS22088-1831 } & 7.506 & 0.005 & 2.276 & 0.062 & 0.280 & - \\
\hline & 7.783 & 0.007 & 2.050 & 0.065 & 0.320 & - \\
\hline & 8.676 & 0.034 & 0.764 & 0.246 & 0.310 & 0.131 \\
\hline \multirow[t]{3}{*}{ IRAS_00091-0738 } & 7.545 & 0.009 & 4.456 & 0.182 & 0.280 & - \\
\hline & 7.855 & 0.009 & 4.753 & 0.186 & 0.320 & - \\
\hline & 8.630 & 0.016 & 0.596 & 0.081 & 0.238 & 0.038 \\
\hline \multirow[t]{3}{*}{ IRAS_00456-2904 } & 7.587 & 0.006 & 9.276 & 0.354 & 0.280 & - \\
\hline & 7.830 & 0.007 & 9.656 & 0.348 & 0.320 & - \\
\hline & 8.613 & 0.005 & 5.298 & 0.202 & 0.299 & 0.014 \\
\hline \multirow[t]{3}{*}{ IRAS_01199-2307 } & 7.543 & 0.013 & 5.312 & 0.369 & 0.280 & - \\
\hline & 7.868 & 0.014 & 5.801 & 0.386 & 0.320 & - \\
\hline & 8.617 & 0.021 & 1.520 & 0.201 & 0.328 & 0.053 \\
\hline \multirow[t]{3}{*}{ IRAS_01355-1814 } & 7.540 & 0.007 & 1.762 & 0.063 & 0.280 & - \\
\hline & 7.815 & 0.007 & 1.957 & 0.069 & 0.320 & - \\
\hline & 8.592 & 0.021 & 0.605 & 0.090 & 0.311 & 0.058 \\
\hline \multirow[t]{3}{*}{ IRAS_01494-1845 } & 7.548 & 0.007 & 4.413 & 0.158 & 0.280 & - \\
\hline & 7.806 & 0.007 & 4.462 & 0.173 & 0.320 & - \\
\hline & 8.576 & 0.006 & 1.421 & 0.060 & 0.278 & 0.014 \\
\hline \multirow[t]{3}{*}{ IRAS_02411+0353 } & 7.586 & 0.008 & 8.158 & 0.377 & 0.280 & - \\
\hline & 7.837 & 0.009 & 8.190 & 0.395 & 0.320 & - \\
\hline & 8.632 & 0.008 & 2.455 & 0.195 & 0.239 & 0.025 \\
\hline
\end{tabular}


Continued from previous page

\begin{tabular}{|c|c|c|c|c|c|c|}
\hline Source & $\begin{array}{c}\lambda_{-} c \\
{[\mu \mathrm{m}]} \\
\end{array}$ & $\begin{array}{c}\text { Err } \\
{[\mu \mathrm{m}]}\end{array}$ & $\begin{array}{c}\text { Amplitude } \\
{[\mathrm{mJy} / \mathrm{sr}]}\end{array}$ & $\begin{array}{c}\text { Err } \\
{[\mathrm{mJy} / \mathrm{sr}]}\end{array}$ & FWHM & Err \\
\hline \multirow[t]{3}{*}{ IRAS_03250+1606 } & 7.572 & 0.010 & 3.918 & 0.276 & 0.280 & - \\
\hline & 7.817 & 0.009 & 5.214 & 0.248 & 0.320 & - \\
\hline & 8.613 & 0.010 & 2.157 & 0.164 & 0.319 & 0.037 \\
\hline \multirow[t]{3}{*}{ IRAS_03521+0028 } & 7.561 & 0.005 & 3.842 & 0.105 & 0.280 & - \\
\hline & 7.825 & 0.006 & 3.693 & 0.108 & 0.320 & - \\
\hline & 8.578 & 0.014 & 1.253 & 0.153 & 0.249 & 0.037 \\
\hline \multirow{3}{*}{ IRAS_08201+2801 } & 7.517 & 0.008 & 4.766 & 0.164 & 0.280 & - \\
\hline & 7.812 & 0.008 & 4.760 & 0.184 & 0.320 & - \\
\hline & 8.587 & 0.017 & 1.648 & 0.229 & 0.294 & 0.053 \\
\hline \multirow{3}{*}{ IRAS_09039+0503 } & 7.537 & 0.007 & 2.483 & 0.114 & 0.280 & - \\
\hline & 7.819 & 0.007 & 3.184 & 0.098 & 0.320 & - \\
\hline & 8.580 & 0.005 & 0.623 & 0.038 & 0.184 & 0.016 \\
\hline \multirow[t]{3}{*}{ IRAS_09116+0334 } & 7.579 & 0.004 & 6.008 & 0.148 & 0.280 & - \\
\hline & 7.828 & 0.005 & 5.512 & 0.170 & 0.320 & - \\
\hline & 8.611 & 0.004 & 1.977 & 0.061 & 0.248 & 0.010 \\
\hline \multirow[t]{3}{*}{ IRAS_09463+8141 } & 7.543 & 0.006 & 1.933 & 0.065 & 0.280 & - \\
\hline & 7.814 & 0.006 & 2.125 & 0.067 & 0.320 & - \\
\hline & 8.603 & 0.016 & 0.379 & 0.058 & 0.214 & 0.039 \\
\hline \multirow[t]{3}{*}{ IRAS_09539+0857 } & 7.569 & 0.007 & 2.455 & 0.098 & 0.280 & - \\
\hline & 7.854 & 0.008 & 2.657 & 0.085 & 0.320 & - \\
\hline & 8.658 & 0.014 & 0.583 & 0.094 & 0.185 & 0.036 \\
\hline \multirow[t]{3}{*}{ IRAS_10190+1322 } & 7.580 & 0.004 & 17.347 & 0.422 & 0.280 & - \\
\hline & 7.829 & 0.005 & 17.030 & 0.440 & 0.320 & - \\
\hline & 8.601 & 0.003 & 8.135 & 0.191 & 0.279 & 0.008 \\
\hline \multirow[t]{3}{*}{ IRAS_10485-1447 } & 7.586 & 0.008 & 2.106 & 0.093 & 0.280 & - \\
\hline & 7.841 & 0.008 & 2.359 & 0.102 & 0.320 & - \\
\hline & 8.615 & 0.013 & 0.596 & 0.070 & 0.237 & 0.034 \\
\hline \multirow{3}{*}{ IRAS_10494+4424 } & 7.597 & 0.007 & 10.368 & 0.436 & 0.280 & - \\
\hline & 7.862 & 0.007 & 11.528 & 0.426 & 0.320 & - \\
\hline & 8.605 & 0.003 & 2.466 & 0.063 & 0.220 & 0.007 \\
\hline \multirow[t]{3}{*}{ IRAS_11130-2659 } & 7.574 & 0.006 & 3.036 & 0.116 & 0.280 & - \\
\hline & 7.851 & 0.008 & 3.090 & 0.103 & 0.320 & - \\
\hline & 8.671 & 0.009 & 0.505 & 0.069 & 0.150 & 0.026 \\
\hline \multirow[t]{3}{*}{ IRAS_11387+4116 } & 7.578 & 0.008 & 2.877 & 0.151 & 0.280 & - \\
\hline & 7.828 & 0.010 & 2.859 & 0.142 & 0.320 & - \\
\hline & 8.623 & 0.007 & 1.586 & 0.074 & 0.320 & 0.019 \\
\hline \multirow{3}{*}{ IRAS_11506+1331 } & 7.600 & 0.007 & 5.329 & 0.302 & 0.280 & - \\
\hline & 7.849 & 0.009 & 6.044 & 0.262 & 0.320 & - \\
\hline & 8.638 & 0.006 & 2.454 & 0.110 & 0.291 & 0.016 \\
\hline \multirow[t]{3}{*}{ IRAS_12112+0305 } & 7.563 & 0.010 & 11.685 & 0.709 & 0.280 & - \\
\hline & 7.820 & 0.010 & 14.020 & 0.689 & 0.320 & - \\
\hline & 8.627 & 0.014 & 5.197 & 0.509 & 0.301 & 0.035 \\
\hline \multirow[t]{2}{*}{ IRAS_13335-2612 } & 7.576 & 0.009 & 4.742 & 0.241 & 0.280 & - \\
\hline & 7.816 & 0.010 & 4.848 & 0.258 & 0.320 & - \\
\hline
\end{tabular}


Continued from previous page

\begin{tabular}{|c|c|c|c|c|c|c|}
\hline Source & $\begin{array}{c}\lambda \_c \\
{[\mu \mathrm{m}]}\end{array}$ & $\begin{array}{c}\text { Err } \\
{[\mu \mathrm{m}]}\end{array}$ & $\begin{array}{c}\text { Amplitude } \\
{[\mathrm{mJy} / \mathrm{sr}]}\end{array}$ & $\begin{array}{c}\text { Err } \\
{[\mathrm{mJy} / \mathrm{sr}]}\end{array}$ & FWHM & Err \\
\hline \multirow{4}{*}{ IRAS_13469+5833 } & 8.587 & 0.012 & 1.892 & 0.191 & 0.251 & 0.031 \\
\hline & 7.533 & 0.007 & 3.098 & 0.100 & 0.280 & - \\
\hline & 7.822 & 0.008 & 3.065 & 0.109 & 0.320 & - \\
\hline & 8.581 & 0.011 & 0.747 & 0.078 & 0.209 & 0.025 \\
\hline \multirow{3}{*}{ IRAS_13509+0442 } & 7.548 & 0.009 & 4.509 & 0.261 & 0.280 & - \\
\hline & 7.808 & 0.007 & 6.905 & 0.244 & 0.320 & - \\
\hline & 8.627 & 0.010 & 2.543 & 0.189 & 0.287 & 0.028 \\
\hline \multirow[t]{2}{*}{ IRAS_13539+2920 } & 7.596 & 0.006 & 8.128 & 0.307 & 0.280 & - \\
\hline & 7.845 & 0.007 & 8.465 & 0.307 & 0.320 & - \\
\hline \multirow{3}{*}{ IRAS_14060+2919 } & 8.628 & 0.006 & 3.295 & 0.133 & 0.301 & 0.014 \\
\hline & 7.582 & 0.007 & 8.342 & 0.434 & 0.280 & - \\
\hline & 7.828 & 0.009 & 8.891 & 0.393 & 0.320 & - \\
\hline \multirow{3}{*}{ IRAS_14348-1447 } & 8.596 & 0.023 & 4.165 & 0.704 & 0.273 & 0.051 \\
\hline & 7.578 & 0.006 & 11.653 & 0.381 & 0.280 & - \\
\hline & 7.867 & 0.006 & 12.568 & 0.443 & 0.320 & - \\
\hline \multirow{3}{*}{ IRAS_15206+3342 } & 8.616 & 0.012 & 2.336 & 0.221 & 0.248 & 0.028 \\
\hline & 7.576 & 0.005 & 8.789 & 0.294 & 0.280 & - \\
\hline & 7.832 & 0.005 & 9.933 & 0.261 & 0.320 & - \\
\hline \multirow{3}{*}{ IRAS_15225+2350 } & 8.634 & 0.014 & 3.419 & 0.340 & 0.293 & 0.035 \\
\hline & 7.549 & 0.008 & 3.345 & 0.208 & 0.280 & - \\
\hline & 7.794 & 0.011 & 3.712 & 0.186 & 0.320 & - \\
\hline \multirow{4}{*}{ IRAS_16090-0139 } & 8.612 & 0.014 & 1.184 & 0.140 & 0.244 & 0.033 \\
\hline & 7.544 & 0.009 & 7.779 & 0.430 & 0.280 & - \\
\hline & 7.830 & 0.011 & 8.485 & 0.373 & 0.320 & - \\
\hline & 8.618 & 0.015 & 0.825 & 0.163 & 0.154 & 0.037 \\
\hline \multirow[t]{2}{*}{ IRAS_16300+1558 } & 7.558 & 0.008 & 3.354 & 0.157 & 0.280 & - \\
\hline & 7.855 & 0.010 & 3.071 & 0.156 & 0.320 & - \\
\hline \multirow{4}{*}{ IRAS_16333+4630 } & 8.604 & 0.007 & 0.235 & 0.023 & 0.155 & 0.017 \\
\hline & 7.560 & 0.007 & 4.921 & 0.196 & 0.280 & - \\
\hline & 7.805 & 0.008 & 4.677 & 0.209 & 0.320 & - \\
\hline & 8.592 & 0.006 & 2.448 & 0.109 & 0.294 & 0.016 \\
\hline \multirow[t]{2}{*}{ IRAS_16474+3430 } & 7.594 & 0.008 & 10.911 & 0.642 & 0.280 & - \\
\hline & 7.864 & 0.011 & 11.436 & 0.555 & 0.320 & - \\
\hline \multirow{4}{*}{ IRAS_16487+5447 } & 8.615 & 0.023 & 2.680 & 0.643 & 0.199 & 0.051 \\
\hline & 7.595 & 0.012 & 5.038 & 0.367 & 0.280 & - \\
\hline & 7.879 & 0.016 & 4.740 & 0.308 & 0.320 & - \\
\hline & 8.625 & 0.016 & 1.112 & 0.183 & 0.211 & 0.043 \\
\hline \multirow{3}{*}{ IRAS_17028+5817 } & 7.598 & 0.006 & 5.802 & 0.194 & 0.280 & - \\
\hline & 7.850 & 0.007 & 5.540 & 0.216 & 0.320 & - \\
\hline & 8.619 & 0.008 & 2.515 & 0.161 & 0.256 & 0.020 \\
\hline \multirow{3}{*}{ IRAS_17068+4027 } & 7.525 & 0.005 & 5.410 & 0.164 & 0.280 & - \\
\hline & 7.812 & 0.006 & 6.376 & 0.166 & 0.320 & - \\
\hline & 8.591 & 0.010 & 0.851 & 0.071 & 0.240 & 0.024 \\
\hline IRAS_20414-1651 & 7.604 & 0.006 & 5.359 & 0.179 & 0.280 & - \\
\hline
\end{tabular}


Continued from previous page

\begin{tabular}{|c|c|c|c|c|c|c|}
\hline Source & $\begin{array}{c}\lambda_{-} c \\
{[\mu \mathrm{m}]} \\
\end{array}$ & $\begin{array}{c}\text { Err } \\
{[\mu \mathrm{m}]}\end{array}$ & $\begin{array}{c}\text { Amplitude } \\
{[\mathrm{mJy} / \mathrm{sr}]}\end{array}$ & $\begin{array}{c}\text { Err } \\
{[\mathrm{mJy} / \mathrm{sr}]}\end{array}$ & FWHM & Err \\
\hline & 7.861 & 0.006 & 5.838 & 0.197 & 0.320 & - \\
\hline & 8.618 & 0.008 & 2.714 & 0.135 & 0.309 & 0.019 \\
\hline \multirow{3}{*}{ IRAS_21208-0519 } & 7.571 & 0.006 & 3.353 & 0.131 & 0.280 & - \\
\hline & 7.823 & 0.006 & 3.857 & 0.121 & 0.320 & - \\
\hline & 8.589 & 0.007 & 1.524 & 0.080 & 0.289 & 0.021 \\
\hline \multirow[t]{3}{*}{ IRAS_21329-2346 } & 7.558 & 0.010 & 3.087 & 0.184 & 0.280 & - \\
\hline & 7.829 & 0.011 & 3.559 & 0.174 & 0.320 & - \\
\hline & 8.634 & 0.020 & 0.680 & 0.091 & 0.315 & 0.051 \\
\hline \multirow[t]{3}{*}{ IRAS_22206-2715 } & 7.593 & 0.011 & 3.225 & 0.204 & 0.280 & - \\
\hline & 7.862 & 0.015 & 2.928 & 0.214 & 0.320 & - \\
\hline & 8.631 & 0.035 & 0.792 & 0.275 & 0.219 & 0.095 \\
\hline \multirow[t]{3}{*}{ IRAS_22491-1808 } & 7.597 & 0.010 & 9.626 & 0.479 & 0.280 & - \\
\hline & 7.864 & 0.011 & 9.967 & 0.491 & 0.320 & - \\
\hline & 8.649 & 0.008 & 4.496 & 0.239 & 0.297 & 0.018 \\
\hline \multirow[t]{3}{*}{ IRAS_23129+2548 } & 7.500 & 0.009 & 3.588 & 0.138 & 0.280 & - \\
\hline & 7.802 & 0.012 & 3.741 & 0.139 & 0.320 & - \\
\hline & 8.600 & 0.027 & 0.477 & 0.104 & 0.258 & 0.066 \\
\hline \multirow[t]{3}{*}{ IRAS_23234+0946 } & 7.566 & 0.007 & 3.015 & 0.139 & 0.280 & - \\
\hline & 7.837 & 0.010 & 2.966 & 0.123 & 0.320 & - \\
\hline & 8.583 & 0.018 & 0.735 & 0.109 & 0.249 & 0.039 \\
\hline \multirow[t]{3}{*}{$\mathrm{M}+0-29-23$} & 7.576 & 0.011 & 36.698 & 2.043 & 0.280 & - \\
\hline & 7.823 & 0.010 & 37.415 & 2.191 & 0.320 & - \\
\hline & 8.605 & 0.004 & 25.690 & 0.542 & 0.375 & 0.010 \\
\hline \multirow[t]{3}{*}{ MIPS562 } & 7.563 & 0.010 & 0.364 & 0.023 & 0.280 & - \\
\hline & 7.836 & 0.018 & 0.279 & 0.024 & 0.320 & - \\
\hline & 8.645 & 0.012 & 0.278 & 0.020 & 0.381 & 0.035 \\
\hline \multirow[t]{3}{*}{ MIPS8040 } & 7.593 & 0.010 & 0.388 & 0.022 & 0.230 & - \\
\hline & 7.823 & 0.014 & 0.318 & 0.024 & 0.250 & - \\
\hline & 8.612 & 0.014 & 0.495 & 0.040 & 0.391 & 0.041 \\
\hline \multirow[t]{3}{*}{ Mrk1066 } & 7.609 & 0.006 & 40.367 & 1.750 & 0.280 & - \\
\hline & 7.840 & 0.009 & 30.932 & 1.691 & 0.320 & - \\
\hline & 8.607 & 0.002 & 17.802 & 0.206 & 0.317 & 0.005 \\
\hline \multirow[t]{3}{*}{ Mrk273 } & 7.564 & 0.009 & 40.406 & 2.093 & 0.280 & - \\
\hline & 7.842 & 0.010 & 43.318 & 1.936 & 0.320 & - \\
\hline & 8.581 & 0.004 & 7.477 & 0.239 & 0.274 & 0.010 \\
\hline \multirow[t]{3}{*}{ Mrk334 } & 7.614 & 0.008 & 33.224 & 1.777 & 0.280 & - \\
\hline & 7.870 & 0.011 & 27.287 & 1.967 & 0.320 & - \\
\hline & 8.641 & 0.003 & 17.090 & 0.350 & 0.258 & 0.006 \\
\hline \multirow[t]{3}{*}{ Mrk471 } & 7.629 & 0.010 & 4.898 & 0.276 & 0.250 & - \\
\hline & 7.858 & 0.013 & 3.253 & 0.268 & 0.270 & - \\
\hline & 8.621 & 0.008 & 3.740 & 0.207 & 0.303 & 0.020 \\
\hline \multirow[t]{3}{*}{ Mrk52 } & 7.560 & 0.005 & 23.130 & 0.566 & 0.280 & - \\
\hline & 7.816 & 0.005 & 19.427 & 0.584 & 0.320 & - \\
\hline & 8.590 & 0.004 & 12.549 & 0.334 & 0.290 & 0.009 \\
\hline
\end{tabular}


Carla M. Canelo et al.

Continued from previous page

\begin{tabular}{|c|c|c|c|c|c|c|}
\hline Source & $\begin{array}{c}\lambda \_c \\
{[\mu \mathrm{m}]}\end{array}$ & $\begin{array}{c}\text { Err } \\
{[\mu \mathrm{m}]}\end{array}$ & $\begin{array}{c}\text { Amplitude } \\
{[\mathrm{mJy} / \mathrm{sr}]}\end{array}$ & $\begin{array}{c}\text { Err } \\
{[\mathrm{mJy} / \mathrm{sr}]}\end{array}$ & FWHM & Err \\
\hline \multirow[t]{3}{*}{ Mrk609 } & 7.568 & 0.008 & 19.511 & 0.959 & 0.280 & - \\
\hline & 7.810 & 0.008 & 19.931 & 0.921 & 0.320 & - \\
\hline & 8.607 & 0.003 & 11.906 & 0.260 & 0.290 & 0.008 \\
\hline \multirow[t]{3}{*}{ Mrk622 } & 7.570 & 0.003 & 4.544 & 0.089 & 0.280 & - \\
\hline & 7.814 & 0.003 & 4.473 & 0.085 & 0.320 & - \\
\hline & 8.597 & 0.001 & 4.121 & 0.024 & 0.308 & 0.002 \\
\hline \multirow[t]{3}{*}{ Mrk883 } & 7.615 & 0.008 & 3.883 & 0.177 & 0.280 & - \\
\hline & 7.872 & 0.011 & 3.115 & 0.174 & 0.320 & - \\
\hline & 8.589 & 0.009 & 3.653 & 0.225 & 0.393 & 0.032 \\
\hline \multirow[t]{3}{*}{ Mrk938 } & 7.575 & 0.005 & 71.080 & 1.698 & 0.280 & - \\
\hline & 7.829 & 0.005 & 63.744 & 1.777 & 0.320 & - \\
\hline & 8.591 & 0.013 & 28.211 & 2.436 & 0.341 & 0.038 \\
\hline \multirow[t]{3}{*}{ Murphy22 } & 7.557 & 0.005 & 0.243 & 0.006 & 0.280 & - \\
\hline & 7.813 & 0.005 & 0.287 & 0.007 & 0.320 & - \\
\hline & 8.680 & 0.006 & 0.060 & 0.004 & 0.181 & 0.013 \\
\hline \multirow[t]{3}{*}{ NGC1056 } & 7.578 & 0.005 & 57.289 & 1.833 & 0.280 & - \\
\hline & 7.822 & 0.005 & 49.525 & 1.535 & 0.320 & - \\
\hline & 8.609 & 0.003 & 27.352 & 0.671 & 0.276 & 0.008 \\
\hline \multirow[t]{3}{*}{ NGC1097 } & 7.562 & 0.006 & 200.588 & 6.072 & 0.280 & - \\
\hline & 7.815 & 0.006 & 198.217 & 6.865 & 0.320 & - \\
\hline & 8.591 & 0.005 & 105.209 & 4.280 & 0.266 & 0.014 \\
\hline \multirow[t]{3}{*}{ NGC1143 } & 7.599 & 0.009 & 28.956 & 2.125 & 0.230 & - \\
\hline & 7.818 & 0.011 & 36.043 & 2.218 & 0.320 & - \\
\hline & 8.624 & 0.011 & 16.668 & 1.404 & 0.260 & 0.026 \\
\hline \multirow[t]{3}{*}{ NGC1222 } & 7.569 & 0.003 & 47.627 & 0.752 & 0.280 & - \\
\hline & 7.827 & 0.003 & 51.489 & 0.744 & 0.320 & - \\
\hline & 8.604 & 0.003 & 20.060 & 0.522 & 0.256 & 0.009 \\
\hline \multirow[t]{3}{*}{ NGC1365 } & 7.594 & 0.005 & 65.868 & 1.911 & 0.280 & - \\
\hline & 7.845 & 0.006 & 62.239 & 1.884 & 0.320 & - \\
\hline & 8.601 & 0.006 & 34.774 & 1.408 & 0.308 & 0.015 \\
\hline \multirow[t]{3}{*}{ NGC1566 } & 7.551 & 0.013 & 42.257 & 2.998 & 0.280 & - \\
\hline & 7.810 & 0.012 & 48.595 & 3.188 & 0.320 & - \\
\hline & 8.633 & 0.008 & 28.550 & 1.884 & 0.242 & 0.019 \\
\hline \multirow[t]{3}{*}{ NGC1614 } & 7.586 & 0.003 & 115.857 & 2.451 & 0.280 & - \\
\hline & 7.834 & 0.003 & 131.889 & 2.446 & 0.320 & - \\
\hline & 8.621 & 0.004 & 60.610 & 2.121 & 0.274 & 0.013 \\
\hline \multirow[t]{3}{*}{ NGC1667 } & 7.599 & 0.012 & 37.811 & 1.921 & 0.250 & - \\
\hline & 7.840 & 0.010 & 32.235 & 2.023 & 0.270 & - \\
\hline & 8.620 & 0.014 & 27.326 & 2.670 & 0.314 & 0.040 \\
\hline \multirow[t]{3}{*}{ NGC2146 } & 7.574 & 0.003 & 686.774 & 12.550 & 0.280 & - \\
\hline & 7.823 & 0.003 & 711.171 & 12.760 & 0.320 & - \\
\hline & 8.608 & 0.003 & 323.184 & 7.528 & 0.278 & 0.009 \\
\hline \multirow[t]{3}{*}{ NGC2623 } & 7.569 & 0.004 & 38.780 & 0.986 & 0.280 & - \\
\hline & 7.828 & 0.004 & 43.187 & 0.983 & 0.320 & - \\
\hline & & & & \multicolumn{3}{|c|}{ Continued on next page } \\
\hline
\end{tabular}


Continued from previous page

\begin{tabular}{|c|c|c|c|c|c|c|}
\hline Source & $\begin{array}{c}\lambda \_c \\
{[\mu \mathrm{m}]}\end{array}$ & $\begin{array}{c}\text { Err } \\
{[\mu \mathrm{m}]}\end{array}$ & $\begin{array}{c}\text { Amplitude } \\
{[\mathrm{mJy} / \mathrm{sr}]}\end{array}$ & $\begin{array}{c}\text { Err } \\
{[\mathrm{mJy} / \mathrm{sr}]}\end{array}$ & FWHM & Err \\
\hline & 8.603 & 0.003 & 19.085 & 0.490 & 0.276 & 0.009 \\
\hline \multirow[t]{3}{*}{ NGC2992 } & 7.593 & 0.010 & 39.880 & 2.503 & 0.260 & - \\
\hline & 7.839 & 0.012 & 27.981 & 2.061 & 0.280 & - \\
\hline & 8.583 & 0.010 & 22.300 & 1.566 & 0.380 & 0.036 \\
\hline \multirow{3}{*}{ NGC3079 } & 7.604 & 0.005 & 242.957 & 5.842 & 0.280 & - \\
\hline & 7.895 & 0.004 & 240.912 & 5.482 & 0.320 & - \\
\hline & 8.603 & 0.006 & 26.067 & 1.548 & 0.202 & 0.014 \\
\hline \multirow[t]{3}{*}{ NGC3227 } & 7.626 & 0.008 & 42.024 & 2.025 & 0.280 & - \\
\hline & 7.858 & 0.010 & 32.927 & 2.050 & 0.320 & - \\
\hline & 8.603 & 0.005 & 14.706 & 0.568 & 0.270 & 0.014 \\
\hline \multirow[t]{3}{*}{ NGC3256 } & 7.529 & 0.007 & 259.479 & 10.963 & 0.280 & - \\
\hline & 7.772 & 0.007 & 260.903 & 10.754 & 0.320 & - \\
\hline & 8.557 & 0.003 & 164.412 & 4.133 & 0.294 & 0.009 \\
\hline \multirow[t]{3}{*}{ NGC3310 } & 7.570 & 0.004 & 60.432 & 1.034 & 0.280 & - \\
\hline & 7.828 & 0.003 & 52.564 & 1.047 & 0.320 & - \\
\hline & 8.615 & 0.003 & 33.882 & 0.870 & 0.299 & 0.010 \\
\hline \multirow[t]{3}{*}{ NGC3511 } & 7.560 & 0.011 & 34.944 & 2.846 & 0.280 & - \\
\hline & 7.817 & 0.013 & 29.460 & 2.213 & 0.320 & - \\
\hline & 8.597 & 0.012 & 19.952 & 1.956 & 0.293 & 0.039 \\
\hline \multirow[t]{3}{*}{ NGC3556 } & 7.536 & 0.005 & 41.938 & 0.926 & 0.280 & - \\
\hline & 7.781 & 0.005 & 39.352 & 0.992 & 0.320 & - \\
\hline & 8.563 & 0.002 & 21.871 & 0.398 & 0.273 & 0.006 \\
\hline \multirow[t]{3}{*}{ NGC3628 } & 7.587 & 0.006 & 190.166 & 5.939 & 0.280 & - \\
\hline & 7.852 & 0.005 & 216.568 & 5.793 & 0.320 & - \\
\hline & 8.606 & 0.005 & 42.298 & 1.734 & 0.248 & 0.013 \\
\hline \multirow[t]{3}{*}{ NGC3786 } & 7.597 & 0.009 & 9.380 & 0.519 & 0.280 & - \\
\hline & 7.827 & 0.012 & 6.579 & 0.494 & 0.320 & - \\
\hline & 8.597 & 0.003 & 7.020 & 0.129 & 0.321 & 0.007 \\
\hline \multirow[t]{3}{*}{ NGC3982 } & 7.626 & 0.010 & 37.580 & 2.074 & 0.270 & - \\
\hline & 7.881 & 0.014 & 20.560 & 1.737 & 0.290 & - \\
\hline & 8.620 & 0.011 & 18.546 & 1.639 & 0.303 & 0.036 \\
\hline \multirow[t]{3}{*}{ NGC4088 } & 7.568 & 0.003 & 25.365 & 0.333 & 0.280 & - \\
\hline & 7.831 & 0.003 & 22.982 & 0.335 & 0.320 & - \\
\hline & 8.605 & 0.004 & 15.835 & 0.559 & 0.291 & 0.014 \\
\hline \multirow[t]{3}{*}{ NGC4194 } & 7.566 & 0.004 & 144.466 & 2.692 & 0.280 & - \\
\hline & 7.819 & 0.003 & 144.735 & 2.762 & 0.320 & - \\
\hline & 8.598 & 0.002 & 70.183 & 1.421 & 0.267 & 0.007 \\
\hline \multirow[t]{3}{*}{ NGC4388 } & 7.623 & 0.010 & 41.598 & 2.680 & 0.200 & - \\
\hline & 7.857 & 0.013 & 23.857 & 2.145 & 0.220 & - \\
\hline & 8.647 & 0.012 & 17.404 & 1.550 & 0.298 & 0.033 \\
\hline \multirow[t]{3}{*}{ NGC4676 } & 7.574 & 0.004 & 21.664 & 0.533 & 0.280 & - \\
\hline & 7.820 & 0.004 & 22.481 & 0.527 & 0.320 & - \\
\hline & 8.595 & 0.003 & 10.331 & 0.228 & 0.268 & 0.007 \\
\hline NGC4818 & 7.574 & 0.004 & 95.029 & 1.730 & 0.280 & - \\
\hline
\end{tabular}


Continued from previous page

\begin{tabular}{|c|c|c|c|c|c|c|}
\hline Source & $\begin{array}{c}\lambda \_c \\
{[\mu \mathrm{m}]}\end{array}$ & $\begin{array}{c}\text { Err } \\
{[\mu \mathrm{m}]}\end{array}$ & $\begin{array}{c}\text { Amplitude } \\
{[\mathrm{mJy} / \mathrm{sr}]}\end{array}$ & $\begin{array}{c}\text { Err } \\
{[\mathrm{mJy} / \mathrm{sr}]}\end{array}$ & FWHM & Err \\
\hline & 7.828 & 0.003 & 93.243 & 1.778 & 0.320 & - \\
\hline & 8.610 & 0.003 & 43.817 & 1.208 & 0.277 & 0.010 \\
\hline \multirow[t]{3}{*}{ NGC4945 } & 7.591 & 0.005 & 540.767 & 11.888 & 0.280 & - \\
\hline & 7.871 & 0.003 & 682.926 & 11.540 & 0.320 & - \\
\hline & 8.592 & 0.005 & 41.699 & 2.099 & 0.186 & 0.011 \\
\hline \multirow[t]{3}{*}{ NGC5005 } & 7.576 & 0.012 & 31.393 & 2.634 & 0.280 & - \\
\hline & 7.844 & 0.012 & 32.113 & 2.029 & 0.320 & - \\
\hline & 8.603 & 0.020 & 24.783 & 4.406 & 0.341 & 0.077 \\
\hline \multirow[t]{3}{*}{ NGC5033 } & 7.566 & 0.012 & 143.764 & 9.371 & 0.280 & - \\
\hline & 7.815 & 0.012 & 160.569 & 10.610 & 0.320 & - \\
\hline & 8.592 & 0.005 & 85.285 & 3.280 & 0.288 & 0.015 \\
\hline \multirow[t]{3}{*}{ NGC5135 } & 7.571 & 0.008 & 80.748 & 3.409 & 0.280 & - \\
\hline & 7.821 & 0.007 & 79.128 & 3.316 & 0.320 & - \\
\hline & 8.595 & 0.004 & 46.048 & 1.324 & 0.305 & 0.011 \\
\hline \multirow[t]{3}{*}{ NGC5194 } & 7.582 & 0.007 & 197.194 & 6.663 & 0.280 & - \\
\hline & 7.832 & 0.007 & 197.376 & 7.532 & 0.320 & - \\
\hline & 8.613 & 0.004 & 108.669 & 3.556 & 0.267 & 0.011 \\
\hline \multirow[t]{3}{*}{ NGC520 } & 7.585 & 0.003 & 135.283 & 2.587 & 0.280 & - \\
\hline & 7.839 & 0.003 & 161.199 & 2.585 & 0.320 & - \\
\hline & 8.612 & 0.003 & 64.685 & 1.388 & 0.292 & 0.008 \\
\hline \multirow{3}{*}{ NGC5256 } & 7.605 & 0.011 & 44.059 & 2.673 & 0.280 & - \\
\hline & 7.842 & 0.012 & 39.599 & 2.859 & 0.320 & - \\
\hline & 8.603 & 0.011 & 19.156 & 1.528 & 0.318 & 0.033 \\
\hline \multirow[t]{3}{*}{ NGC5674 } & 7.587 & 0.010 & 4.133 & 0.194 & 0.280 & - \\
\hline & 7.854 & 0.014 & 2.823 & 0.203 & 0.320 & - \\
\hline & 8.585 & 0.002 & 1.645 & 0.021 & 0.297 & 0.005 \\
\hline \multirow[t]{3}{*}{ NGC5953 } & 7.629 & 0.007 & 23.785 & 1.060 & 0.280 & - \\
\hline & 7.863 & 0.008 & 23.604 & 1.088 & 0.320 & - \\
\hline & 8.658 & 0.011 & 17.179 & 1.465 & 0.301 & 0.033 \\
\hline \multirow[t]{3}{*}{ NGC660 } & 7.576 & 0.003 & 308.017 & 4.594 & 0.280 & - \\
\hline & 7.833 & 0.003 & 302.210 & 4.565 & 0.320 & - \\
\hline & 8.598 & 0.004 & 71.400 & 2.601 & 0.236 & 0.011 \\
\hline \multirow[t]{3}{*}{ NGC6810 } & 7.553 & 0.007 & 108.317 & 5.109 & 0.280 & - \\
\hline & 7.815 & 0.006 & 116.230 & 4.165 & 0.320 & - \\
\hline & 8.602 & 0.005 & 50.924 & 1.797 & 0.279 & 0.012 \\
\hline \multirow[t]{3}{*}{ NGC7252 } & 7.570 & 0.005 & 39.304 & 1.224 & 0.280 & - \\
\hline & 7.809 & 0.004 & 46.175 & 1.232 & 0.320 & - \\
\hline & 8.606 & 0.001 & 32.106 & 0.348 & 0.306 & 0.004 \\
\hline \multirow[t]{3}{*}{ NGC7469 } & 7.570 & 0.010 & 105.551 & 5.840 & 0.280 & - \\
\hline & 7.816 & 0.009 & 105.639 & 5.909 & 0.320 & - \\
\hline & 8.605 & 0.003 & 56.692 & 1.280 & 0.291 & 0.009 \\
\hline \multirow[t]{3}{*}{ NGC7496 } & 7.500 & 0.016 & 24.929 & 1.957 & 0.280 & - \\
\hline & 7.767 & 0.015 & 25.256 & 2.004 & 0.320 & - \\
\hline & 8.563 & 0.014 & 20.701 & 1.868 & 0.342 & 0.039 \\
\hline
\end{tabular}


Continued from previous page

\begin{tabular}{|c|c|c|c|c|c|c|}
\hline Source & $\begin{array}{c}\lambda \_c \\
{[\mu \mathrm{m}]}\end{array}$ & $\begin{array}{c}\text { Err } \\
{[\mu \mathrm{m}]}\end{array}$ & $\begin{array}{c}\text { Amplitude } \\
{[\mathrm{mJy} / \mathrm{sr}]}\end{array}$ & $\begin{array}{c}\text { Err } \\
{[\mathrm{mJy} / \mathrm{sr}]}\end{array}$ & FWHM & Err \\
\hline \multirow[t]{3}{*}{ NGC7582 } & 7.585 & 0.004 & 225.169 & 5.428 & 0.280 & - \\
\hline & 7.841 & 0.004 & 211.928 & 4.503 & 0.320 & - \\
\hline & 8.598 & 0.003 & 57.594 & 1.654 & 0.219 & 0.007 \\
\hline \multirow[t]{3}{*}{ NGC7590 } & 7.540 & 0.014 & 30.179 & 2.552 & 0.280 & - \\
\hline & 7.800 & 0.013 & 30.055 & 2.096 & 0.320 & - \\
\hline & 8.601 & 0.013 & 20.422 & 1.784 & 0.322 & 0.037 \\
\hline \multirow[t]{3}{*}{ NGC7714 } & 7.569 & 0.003 & 53.779 & 0.903 & 0.280 & - \\
\hline & 7.820 & 0.003 & 55.273 & 0.900 & 0.320 & - \\
\hline & 8.618 & 0.003 & 33.960 & 0.837 & 0.291 & 0.010 \\
\hline \multirow[t]{3}{*}{ SST172458.3+591545 } & 7.539 & 0.010 & 0.257 & 0.016 & 0.280 & - \\
\hline & 7.794 & 0.010 & 0.347 & 0.016 & 0.320 & - \\
\hline & 8.612 & 0.007 & 0.258 & 0.013 & 0.302 & 0.020 \\
\hline \multirow[t]{3}{*}{ SWIRE4_J103637.18+584217.0 } & 7.511 & 0.011 & 0.322 & 0.015 & 0.280 & - \\
\hline & 7.861 & 0.009 & 0.329 & 0.013 & 0.320 & - \\
\hline & 8.689 & 0.016 & 0.218 & 0.028 & 0.260 & 0.040 \\
\hline \multirow[t]{3}{*}{ SWIRE4_J104830.58+591810.2 } & 7.549 & 0.005 & 0.551 & 0.013 & 0.280 & - \\
\hline & 7.818 & 0.006 & 0.459 & 0.013 & 0.320 & — \\
\hline & 8.521 & 0.004 & 0.315 & 0.009 & 0.312 & 0.010 \\
\hline \multirow{3}{*}{ UGC12138 } & 7.638 & 0.007 & 7.901 & 0.356 & 0.250 & - \\
\hline & 7.895 & 0.015 & 2.540 & 0.302 & 0.210 & - \\
\hline & 8.610 & 0.004 & 3.219 & 0.100 & 0.257 & 0.010 \\
\hline \multirow[t]{3}{*}{ UGC5101 } & 7.570 & 0.005 & 30.797 & 0.844 & 0.280 & - \\
\hline & 7.819 & 0.005 & 33.047 & 0.845 & 0.320 & - \\
\hline & 8.591 & 0.002 & 15.750 & 0.271 & 0.309 & 0.007 \\
\hline \multirow[t]{3}{*}{ UGC7064 } & 7.611 & 0.015 & 21.836 & 1.693 & 0.280 & - \\
\hline & 7.903 & 0.025 & 16.155 & 1.490 & 0.320 & - \\
\hline & 8.572 & 0.004 & 14.587 & 0.466 & 0.331 & 0.014 \\
\hline
\end{tabular}

Table A3: Integrated flux intensities for the 6.2, 7.7 and $8.6 \mu \mathrm{m}$ PAH bands. The values are in $\mathrm{mJy} / \mathrm{sr}$.

\begin{tabular}{lcccccccc}
\hline Source & $\mathrm{F}_{6.2}$ & Err & $\mathrm{F}_{7.6}$ & Err & $\mathrm{F}_{7.8}$ & Err & $\mathrm{F}_{8.6}$ & Err \\
\hline 3C293 & 0.629 & 0.020 & 1.476 & 0.051 & 1.380 & 0.049 & 0.529 & 0.021 \\
3C31 & 1.405 & 0.014 & 2.054 & 0.091 & 1.881 & 0.092 & 1.812 & 0.086 \\
AGN15 & 0.368 & 0.005 & 1.292 & 0.070 & 1.356 & 0.062 & 0.229 & 0.015 \\
Arp220 & 32.624 & 0.751 & 79.680 & 3.720 & 82.089 & 3.973 & 8.468 & 0.354 \\
E12-G21 & 13.416 & 0.430 & 22.994 & 1.197 & 23.224 & 1.138 & 23.143 & 1.254 \\
EIRS-2 & 0.176 & 0.008 & 0.520 & 0.059 & 0.348 & 0.055 & 0.448 & 0.074 \\
GN26 & 0.139 & 0.004 & 0.509 & 0.037 & 0.340 & 0.032 & 0.148 & 0.030 \\
IC342 & 38.532 & 0.978 & 104.637 & 6.081 & 102.484 & 5.947 & 60.651 & 1.413 \\
IRAS02021-2103 & 2.853 & 0.055 & 3.858 & 0.216 & 4.373 & 0.213 & 2.999 & 0.139 \\
IRAS02480-3745 & 2.295 & 0.067 & 3.986 & 0.426 & 4.566 & 0.436 & 2.456 & 0.053 \\
IRAS03209-0806 & 2.375 & 0.071 & 4.058 & 0.181 & 4.062 & 0.205 & 2.908 & 0.112 \\
IRAS05020-2941 & 1.612 & 0.172 & 6.622 & 0.265 & 7.236 & 0.279 & 0.867 & 0.102 \\
IRAS08591+5248 & 2.119 & 0.071 & 5.263 & 0.203 & 4.849 & 0.202 & 2.530 & 0.201 \\
IRAS10594+3818 & 3.882 & 0.122 & 8.316 & 0.243 & 7.791 & 0.262 & 2.980 & 0.255 \\
IRAS12447+3721 & 1.812 & 0.066 & 4.570 & 0.204 & 4.514 & 0.211 & 1.067 & 0.219 \\
\hline & & & & & & Continued on next page \\
\hline
\end{tabular}


Continued from previous page

\begin{tabular}{|c|c|c|c|c|c|c|c|c|}
\hline Source & $\mathrm{F}_{6.2}$ & Err & $\mathrm{F}_{7.6}$ & Err & $\mathrm{F}_{7.8}$ & Err & $\mathrm{F}_{8.6}$ & Err \\
\hline IRAS13106-0922 & 0.943 & 0.061 & 1.828 & 0.076 & 2.234 & 0.089 & 0.830 & 0.117 \\
\hline IRAS14121-0126 & 2.702 & 0.077 & 7.426 & 0.451 & 7.127 & 0.442 & 2.251 & 0.225 \\
\hline IRAS14197+0813 & 1.183 & 0.121 & 2.877 & 0.173 & 3.018 & 0.166 & 1.077 & 0.131 \\
\hline IRAS14202+2615 & 5.129 & 0.132 & 8.770 & 0.379 & 8.750 & 0.380 & 4.029 & 0.336 \\
\hline IRAS14485-2434 & 2.004 & 0.109 & 5.419 & 0.296 & 4.637 & 0.297 & 2.145 & 0.145 \\
\hline IRAS15043+5754 & 2.299 & 0.052 & 5.609 & 0.152 & 5.320 & 0.158 & 1.262 & 0.123 \\
\hline IRAS21477+0502 & 1.592 & 0.083 & 3.495 & 0.142 & 3.377 & 0.146 & 1.761 & 0.083 \\
\hline IRAS22088-1831 & 1.174 & 0.048 & 2.265 & 0.063 & 2.048 & 0.065 & 0.759 & 0.187 \\
\hline IRAS_00091-0738 & 0.812 & 0.072 & 4.448 & 0.184 & 4.727 & 0.179 & 0.596 & 0.080 \\
\hline IRAS_00456-2904 & 5.148 & 0.159 & 9.271 & 0.355 & 9.625 & 0.341 & 5.289 & 0.195 \\
\hline IRAS_01199-2307 & 1.335 & 0.190 & 5.302 & 0.372 & 5.759 & 0.369 & 1.513 & 0.180 \\
\hline IRAS_01355-1814 & 0.873 & 0.050 & 1.758 & 0.064 & 1.952 & 0.068 & 0.603 & 0.084 \\
\hline IRAS_01494-1845 & 1.631 & 0.127 & 4.405 & 0.159 & 4.454 & 0.171 & 1.420 & 0.059 \\
\hline IRAS_02411+0353 & 3.713 & 0.319 & 8.153 & 0.378 & 8.159 & 0.387 & 2.455 & 0.194 \\
\hline IRAS_03250+1606 & 2.825 & 0.054 & 3.915 & 0.277 & 5.201 & 0.244 & 2.150 & 0.150 \\
\hline IRAS_03521+0028 & 1.625 & 0.055 & 3.837 & 0.105 & 3.682 & 0.106 & 1.253 & 0.152 \\
\hline IRAS_08201+2801 & 1.864 & 0.032 & 4.748 & 0.167 & 4.750 & 0.181 & 1.646 & 0.219 \\
\hline IRAS_09039+0503 & 1.022 & 0.077 & 2.477 & 0.115 & 3.176 & 0.096 & 0.623 & 0.038 \\
\hline IRAS_09116+0334 & 2.753 & 0.066 & 6.004 & 0.148 & 5.495 & 0.167 & 1.977 & 0.061 \\
\hline IRAS_09463+8141 & 0.926 & 0.058 & 1.929 & 0.065 & 2.120 & 0.066 & 0.379 & 0.058 \\
\hline IRAS_09539+0857 & 1.033 & 0.036 & 2.453 & 0.098 & 2.643 & 0.082 & 0.583 & 0.094 \\
\hline IRAS_10190+1322 & 10.469 & 0.252 & 17.335 & 0.423 & 16.976 & 0.432 & 8.129 & 0.188 \\
\hline IRAS_10485-1447 & 1.448 & 0.061 & 2.105 & 0.093 & 2.349 & 0.100 & 0.596 & 0.070 \\
\hline IRAS_10494+4424 & 5.061 & 0.182 & 10.364 & 0.437 & 11.454 & 0.411 & 2.466 & 0.063 \\
\hline IRAS_11130-2659 & 0.779 & 0.068 & 3.033 & 0.116 & 3.074 & 0.099 & 0.505 & 0.069 \\
\hline IRAS_11387+4116 & 1.420 & 0.071 & 2.875 & 0.151 & 2.850 & 0.139 & 1.580 & 0.069 \\
\hline IRAS_11506+1331 & 3.617 & 0.093 & 5.327 & 0.302 & 6.014 & 0.254 & 2.449 & 0.106 \\
\hline IRAS_12112+0305 & 5.851 & 0.377 & 11.672 & 0.712 & 13.984 & 0.678 & 5.186 & 0.481 \\
\hline IRAS_13335-2612 & 3.113 & 0.065 & 4.738 & 0.242 & 4.837 & 0.254 & 1.892 & 0.190 \\
\hline IRAS_13469+5833 & 1.127 & 0.025 & 3.090 & 0.101 & 3.057 & 0.107 & 0.747 & 0.078 \\
\hline IRAS_13509+0442 & 2.495 & 0.080 & 4.501 & 0.262 & 6.891 & 0.241 & 2.540 & 0.182 \\
\hline IRAS_13539+2920 & 3.828 & 0.068 & 8.124 & 0.307 & 8.427 & 0.299 & 3.288 & 0.128 \\
\hline IRAS_14060+2919 & 4.385 & 0.204 & 8.337 & 0.435 & 8.863 & 0.385 & 4.163 & 0.687 \\
\hline IRAS_14348-1447 & 6.145 & 0.179 & 11.644 & 0.382 & 12.478 & 0.428 & 2.336 & 0.219 \\
\hline IRAS_15206+3342 & 3.842 & 0.142 & 8.782 & 0.295 & 9.899 & 0.256 & 3.413 & 0.322 \\
\hline IRAS_15225+2350 & 1.393 & 0.077 & 3.339 & 0.209 & 3.707 & 0.184 & 1.184 & 0.139 \\
\hline IRAS_16090-0139 & 3.193 & 0.066 & 7.764 & 0.433 & 8.458 & 0.364 & 0.825 & 0.163 \\
\hline IRAS_16300+1558 & 1.051 & 0.045 & 3.350 & 0.158 & 3.054 & 0.151 & 0.235 & 0.023 \\
\hline IRAS_16333+4630 & 1.782 & 0.059 & 4.915 & 0.197 & 4.668 & 0.207 & 2.445 & 0.106 \\
\hline IRAS_16474+3430 & 4.444 & 0.105 & 10.906 & 0.643 & 11.359 & 0.531 & 2.680 & 0.642 \\
\hline IRAS_16487+5447 & 1.356 & 0.109 & 5.036 & 0.368 & 4.697 & 0.288 & 1.112 & 0.182 \\
\hline IRAS_17028+5817 & 2.388 & 0.050 & 5.800 & 0.194 & 5.512 & 0.210 & 2.514 & 0.159 \\
\hline IRAS_17068+4027 & 2.143 & 0.029 & 5.393 & 0.166 & 6.362 & 0.164 & 0.851 & 0.071 \\
\hline IRAS_20414-1651 & 2.424 & 0.062 & 5.357 & 0.179 & 5.801 & 0.191 & 2.707 & 0.128 \\
\hline IRAS_21208-0519 & 2.030 & 0.071 & 3.350 & 0.131 & 3.846 & 0.119 & 1.522 & 0.078 \\
\hline
\end{tabular}


Continued from previous page

\begin{tabular}{|c|c|c|c|c|c|c|c|c|}
\hline Source & $\mathrm{F}_{6.2}$ & Err & $\mathrm{F}_{7.6}$ & Err & $\mathrm{F}_{7.8}$ & Err & $\mathrm{F}_{8.6}$ & Err \\
\hline IRAS_21329-2346 & 1.086 & 0.064 & 3.083 & 0.185 & 3.548 & 0.170 & 0.677 & 0.082 \\
\hline IRAS_22206-2715 & 1.451 & 0.091 & 3.223 & 0.204 & 2.909 & 0.205 & 0.792 & 0.180 \\
\hline IRAS_22491-1808 & 4.099 & 0.201 & 9.622 & 0.480 & 9.900 & 0.470 & 4.483 & 0.226 \\
\hline IRAS_23129+2548 & 1.396 & 0.048 & 3.567 & 0.141 & 3.735 & 0.137 & 0.477 & 0.102 \\
\hline IRAS_23234+0946 & 1.437 & 0.044 & 3.012 & 0.139 & 2.955 & 0.120 & 0.735 & 0.108 \\
\hline $\mathrm{M}+0-29-23$ & 23.694 & 0.463 & 36.669 & 2.050 & 37.311 & 2.157 & 25.380 & 0.469 \\
\hline MIPS562 & 0.202 & 0.006 & 0.364 & 0.023 & 0.278 & 0.023 & 0.273 & 0.016 \\
\hline MIPS8040 & 0.169 & 0.007 & 0.388 & 0.022 & 0.318 & 0.024 & 0.487 & 0.032 \\
\hline Mrk1066 & 16.681 & 0.170 & 40.355 & 1.752 & 30.807 & 1.656 & 17.749 & 0.196 \\
\hline Mrk273 & 11.498 & 0.772 & 40.361 & 2.101 & 43.135 & 1.882 & 7.472 & 0.236 \\
\hline Mrk334 & 15.784 & 0.345 & 33.216 & 1.779 & 27.080 & 1.897 & 17.081 & 0.346 \\
\hline Mrk471 & 2.841 & 0.079 & 4.898 & 0.276 & 3.248 & 0.265 & 3.732 & 0.198 \\
\hline Mrk52 & 11.855 & 0.348 & 23.101 & 0.569 & 19.381 & 0.577 & 12.534 & 0.328 \\
\hline Mrk609 & 9.895 & 0.259 & 19.492 & 0.962 & 19.890 & 0.910 & 11.892 & 0.254 \\
\hline Mrk622 & 2.335 & 0.171 & 4.540 & 0.089 & 4.463 & 0.084 & 4.112 & 0.023 \\
\hline Mrk883 & 2.221 & 0.030 & 3.882 & 0.177 & 3.090 & 0.166 & 3.592 & 0.183 \\
\hline Mrk938 & 31.859 & 0.420 & 71.023 & 1.705 & 63.542 & 1.747 & 28.046 & 2.203 \\
\hline Murphy22 & 0.122 & 0.002 & 0.243 & 0.006 & 0.286 & 0.007 & 0.060 & 0.004 \\
\hline NGC1056 & 31.629 & 1.230 & 57.247 & 1.838 & 49.391 & 1.514 & 27.334 & 0.662 \\
\hline NGC1097 & 108.832 & 3.385 & 200.354 & 6.102 & 197.760 & 6.780 & 105.165 & 4.239 \\
\hline NGC1143 & 21.178 & 0.757 & 28.955 & 2.125 & 35.954 & 2.186 & 16.661 & 1.383 \\
\hline NGC1222 & 24.930 & 0.501 & 47.581 & 0.755 & 51.333 & 0.731 & 20.055 & 0.519 \\
\hline NGC1365 & 29.932 & 0.526 & 65.838 & 1.914 & 61.959 & 1.836 & 34.697 & 1.356 \\
\hline NGC1566 & 27.039 & 0.695 & 42.190 & 3.015 & 48.495 & 3.148 & 28.545 & 1.870 \\
\hline NGC1614 & 60.651 & 2.021 & 115.789 & 2.455 & 131.422 & 2.405 & 60.567 & 2.083 \\
\hline NGC1667 & 20.313 & 0.866 & 37.808 & 1.922 & 32.208 & 2.011 & 27.244 & 2.470 \\
\hline NGC2146 & 317.573 & 6.400 & 686.204 & 12.588 & 709.202 & 12.585 & 322.950 & 7.405 \\
\hline NGC2623 & 17.545 & 0.381 & 38.743 & 0.989 & 43.053 & 0.967 & 19.073 & 0.483 \\
\hline NGC2992 & 18.680 & 0.392 & 39.873 & 2.505 & 27.947 & 2.044 & 21.995 & 1.307 \\
\hline NGC3079 & 81.037 & 1.474 & 242.874 & 5.852 & 237.924 & 5.173 & 26.067 & 1.548 \\
\hline NGC3227 & 22.826 & 0.716 & 42.017 & 2.026 & 32.732 & 1.990 & 14.699 & 0.561 \\
\hline NGC3256 & 114.344 & 3.002 & 258.745 & 11.059 & 260.686 & 10.703 & 164.031 & 4.027 \\
\hline NGC3310 & 36.486 & 0.683 & 60.376 & 1.039 & 52.401 & 1.032 & 33.823 & 0.842 \\
\hline NGC3511 & 20.851 & 0.365 & 34.901 & 2.855 & 29.389 & 2.182 & 19.926 & 1.881 \\
\hline NGC3556 & 20.248 & 0.450 & 41.839 & 0.936 & 39.311 & 0.986 & 21.850 & 0.393 \\
\hline NGC3628 & 72.661 & 1.699 & 190.058 & 5.954 & 215.437 & 5.634 & 42.292 & 1.726 \\
\hline NGC3786 & 4.405 & 0.450 & 9.376 & 0.520 & 6.559 & 0.486 & 6.996 & 0.123 \\
\hline NGC3982 & 18.016 & 0.503 & 37.576 & 2.075 & 20.461 & 1.688 & 18.507 & 1.546 \\
\hline NGC4088 & 13.143 & 0.300 & 25.340 & 0.335 & 22.906 & 0.329 & 15.816 & 0.544 \\
\hline NGC4194 & 79.169 & 1.812 & 144.315 & 2.706 & 144.369 & 2.729 & 70.154 & 1.408 \\
\hline NGC4388 & 16.098 & 0.367 & 41.598 & 2.680 & 23.854 & 2.143 & 17.354 & 1.444 \\
\hline NGC4676 & 10.867 & 0.339 & 21.646 & 0.535 & 22.423 & 0.520 & 10.326 & 0.226 \\
\hline NGC4818 & 43.885 & 1.182 & 94.950 & 1.737 & 92.954 & 1.752 & 43.786 & 1.189 \\
\hline NGC4945 & 110.651 & 8.425 & 540.494 & 11.921 & 677.641 & 11.116 & 41.699 & 2.099 \\
\hline NGC5005 & 16.103 & 0.413 & 31.368 & 2.640 & 31.972 & 1.976 & 24.640 & 3.806 \\
\hline
\end{tabular}


Carla M. Canelo et al.

Continued from previous page

\begin{tabular}{lcccccccc}
\hline Source & $\mathrm{F}_{6.2}$ & Err & $\mathrm{F}_{7.6}$ & Err & $\mathrm{F}_{7.8}$ & Err & $\mathrm{F}_{8.6}$ & Err \\
\hline NGC5033 & 92.216 & 1.578 & 143.614 & 9.408 & 160.198 & 10.462 & 85.191 & 3.207 \\
NGC5135 & 43.126 & 1.105 & 80.675 & 3.422 & 78.919 & 3.270 & 45.955 & 1.281 \\
NGC5194 & 122.836 & 1.882 & 197.064 & 6.684 & 196.708 & 7.391 & 108.619 & 3.516 \\
NGC520 & 62.739 & 0.799 & 135.202 & 2.592 & 160.562 & 2.531 & 64.599 & 1.353 \\
NGC5256 & 17.473 & 0.495 & 44.044 & 2.677 & 39.432 & 2.795 & 19.097 & 1.431 \\
NGC5674 & 1.452 & 0.006 & 4.131 & 0.195 & 2.808 & 0.196 & 1.642 & 0.021 \\
NGC5953 & 12.370 & 0.216 & 23.781 & 1.061 & 23.449 & 1.052 & 17.112 & 1.340 \\
NGC660 & 132.024 & 3.039 & 307.776 & 4.611 & 301.164 & 4.477 & 71.395 & 2.596 \\
NGC6810 & 53.632 & 1.206 & 108.155 & 5.131 & 115.962 & 4.115 & 50.886 & 1.770 \\
NGC7252 & 22.552 & 0.604 & 39.267 & 1.228 & 46.082 & 1.220 & 32.038 & 0.337 \\
NGC7469 & 56.084 & 1.871 & 105.453 & 5.861 & 105.390 & 5.835 & 56.623 & 1.249 \\
NGC7496 & 12.760 & 0.505 & 24.784 & 1.996 & 25.237 & 1.994 & 20.545 & 1.700 \\
NGC7582 & 85.696 & 3.036 & 225.033 & 5.440 & 211.054 & 4.404 & 57.593 & 1.653 \\
NGC7590 & 13.263 & 0.658 & 30.115 & 2.568 & 30.006 & 2.074 & 20.352 & 1.655 \\
NGC7714 & 27.370 & 0.884 & 53.728 & 0.906 & 55.130 & 0.887 & 33.914 & 0.813 \\
SST172458.3+591545 & 0.107 & 0.004 & 0.256 & 0.016 & 0.347 & 0.016 & 0.258 & 0.012 \\
SWIRE4_J103637.18+584217.0 & 0.124 & 0.001 & 0.321 & 0.015 & 0.327 & 0.012 & 0.217 & 0.026 \\
SWIRE4_J104830.58+591810.2 & 0.192 & 0.004 & 0.550 & 0.013 & 0.458 & 0.013 & 0.313 & 0.009 \\
UGC12138 & 2.470 & 0.070 & 7.901 & 0.356 & 2.539 & 0.301 & 3.218 & 0.099 \\
UGC5101 & 13.432 & 0.455 & 30.768 & 0.847 & 32.963 & 0.833 & 15.713 & 0.261 \\
UGC7064 & 9.497 & 0.091 & 21.830 & 1.695 & 15.922 & 1.323 & 14.511 & 0.436 \\
\hline
\end{tabular}


Table A4: Distribution of the galaxies into the Peeters' classes for three PAH bands. The classification for the $6.2 \mu \mathrm{m}$ band was extracted from Canelo et al. (2018).

\begin{tabular}{|c|c|c|c|}
\hline Source & $\begin{array}{c}6.2 \mu \mathrm{m} \\
\text { Class }\end{array}$ & $\begin{array}{c}7.7 \mu \mathrm{m} \\
\text { Class }\end{array}$ & $\begin{array}{c}8.6 \mu \mathrm{m} \\
\text { Class }\end{array}$ \\
\hline $3 \mathrm{C} 293$ & $\mathrm{~B}$ & $\mathrm{~A}$ & $\mathrm{~B}$ \\
\hline 3C31 & $\mathrm{A}$ & $\mathrm{A}$ & B \\
\hline AGN15 & $\mathrm{B}$ & B & B \\
\hline Arp220 & $\mathrm{A}$ & B & $\mathrm{B}$ \\
\hline E12-G21 & $\mathrm{A}$ & B & B \\
\hline EIRS-2 & $\mathrm{A}$ & $\mathrm{A}$ & $\mathrm{B}$ \\
\hline GN26 & B & $\mathrm{A}$ & $\mathrm{B}$ \\
\hline IC342 & $\mathrm{A}$ & $\mathrm{A}$ & $\mathrm{B}$ \\
\hline IRAS02021-2103 & $\mathrm{B}$ & B & B \\
\hline IRAS02480-3745 & $\mathrm{A}$ & B & $\mathrm{A}$ \\
\hline IRAS03209-0806 & $\mathrm{A}$ & $\mathrm{B}$ & $\mathrm{A}$ \\
\hline IRAS05020-2941 & $\mathrm{A}$ & $\mathrm{B}$ & $\mathrm{A}$ \\
\hline IRAS08591+5248 & $\mathrm{A}$ & $\mathrm{A}$ & $\mathrm{A}$ \\
\hline IRAS10594+3818 & $\mathrm{A}$ & $\mathrm{A}$ & $\mathrm{A}$ \\
\hline IRAS12447+3721 & $\mathrm{A}$ & $\mathrm{A}$ & $\mathrm{A}$ \\
\hline IRAS13106-0922 & $\mathrm{A}$ & B & $\mathrm{A}$ \\
\hline IRAS14121-0126 & $\mathrm{A}$ & $\mathrm{A}$ & $\mathrm{A}$ \\
\hline IRAS14197+0813 & B & B & $\mathrm{B}$ \\
\hline IRAS14202+2615 & $\mathrm{A}$ & $\mathrm{A}$ & $\mathrm{A}$ \\
\hline IRAS14485-2434 & $\mathrm{A}$ & $\mathrm{A}$ & $\mathrm{A}$ \\
\hline IRAS15043+5754 & $\mathrm{A}$ & $\mathrm{A}$ & $\mathrm{B}$ \\
\hline IRAS21477+0502 & $\mathrm{A}$ & $\mathrm{A}$ & $\mathrm{B}$ \\
\hline IRAS22088-1831 & $\mathrm{A}$ & $\mathrm{A}$ & B \\
\hline IRAS_00091-0738 & $\mathrm{A}$ & B & $\mathrm{B}$ \\
\hline IRAS_00456-2904 & $\mathrm{A}$ & B & $\mathrm{B}$ \\
\hline IRAS_01199-2307 & $\mathrm{A}$ & $\mathrm{B}$ & $\mathrm{B}$ \\
\hline IRAS_01355-1814 & $\mathrm{B}$ & B & $\mathrm{A}$ \\
\hline IRAS_01494-1845 & $\mathrm{A}$ & $\mathrm{B}$ & $\mathrm{A}$ \\
\hline IRAS_02411+0353 & $\mathrm{A}$ & $\mathrm{B}$ & $\mathrm{B}$ \\
\hline IRAS_03250+1606 & $\mathrm{B}$ & $\mathrm{B}$ & $\mathrm{B}$ \\
\hline IRAS_03521+0028 & $\mathrm{A}$ & $\mathrm{A}$ & $\mathrm{A}$ \\
\hline IRAS_08201+2801 & $\mathrm{A}$ & $\mathrm{A}$ & $\mathrm{A}$ \\
\hline IRAS_09039+0503 & $\mathrm{B}$ & $\mathrm{B}$ & $\mathrm{A}$ \\
\hline IRAS_09116+0334 & $\mathrm{A}$ & $\mathrm{A}$ & $\mathrm{B}$ \\
\hline IRAS_09463+8141 & $\mathrm{A}$ & B & $\mathrm{B}$ \\
\hline IRAS_09539+0857 & $\mathrm{A}$ & B & B \\
\hline IRAS_10190+1322 & $\mathrm{A}$ & $\mathrm{A}$ & $\mathrm{B}$ \\
\hline IRAS_10485-1447 & $\mathrm{B}$ & $\mathrm{B}$ & $\mathrm{B}$ \\
\hline \multicolumn{4}{|c|}{ Continued on next page } \\
\hline
\end{tabular}

Continued from previous page

\begin{tabular}{|c|c|c|c|}
\hline Source & $\begin{array}{c}6.2 \mu \mathrm{m} \\
\text { Class }\end{array}$ & $\begin{array}{c}7.7 \mu \mathrm{m} \\
\text { Class }\end{array}$ & $\begin{array}{c}8.6 \mu \mathrm{m} \\
\text { Class }\end{array}$ \\
\hline IRAS_10494+4424 & B & B & B \\
\hline IRAS_11130-2659 & B & B & B \\
\hline IRAS_11387+4116 & B & A & B \\
\hline IRAS_11506+1331 & B & B & B \\
\hline IRAS_12112+0305 & A & B & $\mathrm{B}$ \\
\hline IRAS_13335-2612 & B & $\mathrm{B}$ & $\mathrm{A}$ \\
\hline IRAS_13469+5833 & A & A & A \\
\hline IRAS_13509+0442 & B & B & B \\
\hline IRAS_13539+2920 & B & B & $\mathrm{B}$ \\
\hline IRAS_14060+2919 & A & B & $\mathrm{A}$ \\
\hline IRAS_14348-1447 & B & B & B \\
\hline IRAS_15206+3342 & B & B & B \\
\hline IRAS_15225+2350 & B & B & $\mathrm{B}$ \\
\hline IRAS_16090-0139 & B & B & B \\
\hline IRAS_16300+1558 & $\mathrm{A}$ & $\mathrm{A}$ & B \\
\hline IRAS_16333+4630 & A & $\mathrm{A}$ & $\mathrm{A}$ \\
\hline IRAS_16474+3430 & B & B & $\mathrm{B}$ \\
\hline IRAS_16487+5447 & A & $\mathrm{A}$ & $\mathrm{B}$ \\
\hline IRAS_17028+5817 & B & A & $\mathrm{B}$ \\
\hline IRAS_17068+4027 & A & $\mathrm{B}$ & $\mathrm{A}$ \\
\hline IRAS_20414-1651 & B & B & B \\
\hline IRAS_21208-0519 & B & B & $\mathrm{A}$ \\
\hline IRAS_21329-2346 & B & B & B \\
\hline IRAS_22206-2715 & B & $\mathrm{A}$ & B \\
\hline IRAS_22491-1808 & B & B & B \\
\hline IRAS_23129+2548 & A & B & $\mathrm{A}$ \\
\hline IRAS_23234+0946 & $\mathrm{A}$ & $\mathrm{A}$ & $\mathrm{A}$ \\
\hline$M+0-29-23$ & $\mathrm{~A}$ & $\mathrm{~B}$ & $\mathrm{~B}$ \\
\hline MIPS562 & $\mathrm{A}$ & $\mathrm{A}$ & $\mathrm{B}$ \\
\hline MIPS8040 & B & $\mathrm{A}$ & B \\
\hline Mrk1066 & $\mathrm{A}$ & $\mathrm{A}$ & B \\
\hline Mrk273 & $\mathrm{A}$ & B & $\mathrm{A}$ \\
\hline Mrk334 & B & $\mathrm{A}$ & B \\
\hline Mrk471 & B & $\mathrm{A}$ & B \\
\hline Mrk52 & $\mathrm{A}$ & $\mathrm{A}$ & $\mathrm{A}$ \\
\hline Mrk609 & $\mathrm{A}$ & $\mathrm{B}$ & $\mathrm{B}$ \\
\hline Mrk622 & $\mathrm{A}$ & $\mathrm{A}$ & $\mathrm{A}$ \\
\hline Mrk883 & $\mathrm{B}$ & $\mathrm{A}$ & $\mathrm{A}$ \\
\hline Mrk938 & $\mathrm{A}$ & $\mathrm{A}$ & $\mathrm{A}$ \\
\hline Murphy22 & $\mathrm{A}$ & $\mathrm{B}$ & $\mathrm{B}$ \\
\hline NGC1056 & $\mathrm{A}$ & $\mathrm{A}$ & B \\
\hline NGC1097 & $\mathrm{A}$ & $\mathrm{A}$ & $\mathrm{A}$ \\
\hline NGC1143 & B & $\mathrm{B}$ & $\mathrm{B}$ \\
\hline NGC1222 & A & $\mathrm{B}$ & B \\
\hline
\end{tabular}


Carla M. Canelo et al.

Continued from previous page

\begin{tabular}{|c|c|c|c|}
\hline Source & $\begin{array}{c}6.2 \mu \mathrm{m} \\
\text { Class }\end{array}$ & $\begin{array}{l}7.7 \mu \mathrm{m} \\
\text { Class }\end{array}$ & $\begin{array}{l}8.6 \mu \mathrm{m} \\
\text { Class }\end{array}$ \\
\hline NGC1365 & $\mathrm{A}$ & $\mathrm{A}$ & B \\
\hline NGC1566 & A & B & B \\
\hline NGC1614 & A & B & B \\
\hline NGC1667 & A & A & B \\
\hline NGC2146 & A & B & B \\
\hline NGC2623 & A & B & B \\
\hline NGC2992 & B & A & $\mathrm{A}$ \\
\hline NGC3079 & A & A & B \\
\hline NGC3227 & A & $\mathrm{A}$ & B \\
\hline NGC3256 & A & B & A \\
\hline NGC3310 & A & A & B \\
\hline NGC3511 & B & A & A \\
\hline NGC3556 & A & A & A \\
\hline NGC3628 & $\mathrm{A}$ & B & B \\
\hline NGC3786 & A & A & A \\
\hline NGC3982 & B & $\mathrm{A}$ & B \\
\hline NGC4088 & $\mathrm{A}$ & A & B \\
\hline NGC4194 & A & $\mathrm{A}$ & $\mathrm{A}$ \\
\hline NGC4388 & A & A & B \\
\hline NGC4676 & A & B & A \\
\hline NGC4818 & A & A & B \\
\hline NGC4945 & A & B & A \\
\hline NGC5005 & A & B & B \\
\hline NGC5033 & A & B & A \\
\hline NGC5135 & A & $\mathrm{A}$ & $\mathrm{A}$ \\
\hline NGC5194 & $\mathrm{A}$ & A & B \\
\hline NGC520 & B & B & B \\
\hline NGC5256 & $\mathrm{A}$ & A & B \\
\hline NGC5674 & A & A & A \\
\hline NGC5953 & B & A & B \\
\hline NGC660 & A & A & A \\
\hline NGC6810 & $\mathrm{A}$ & B & B \\
\hline NGC7252 & A & B & B \\
\hline NGC7469 & $\mathrm{A}$ & $\mathrm{A}$ & B \\
\hline NGC7496 & A & B & A \\
\hline NGC7582 & A & A & A \\
\hline NGC7590 & A & $\mathrm{A}$ & B \\
\hline NGC7714 & A & B & B \\
\hline $\begin{array}{l}\text { SST172458.3 } \\
+591545\end{array}$ & B & B & B \\
\hline $\begin{array}{l}\text { SWIRE4_J103637.18 } \\
+584217.0\end{array}$ & $\mathrm{C}$ & B & B \\
\hline $\begin{array}{l}\text { SWIRE4_J104830.58 } \\
+591810.2\end{array}$ & $\mathrm{~A}$ & A & $\mathrm{A}$ \\
\hline
\end{tabular}

Continued from previous page

\begin{tabular}{lccc}
\hline Source & $\begin{array}{c}6.2 \mu \mathrm{m} \\
\text { Class }\end{array}$ & $\begin{array}{c}7.7 \mu \mathrm{m} \\
\text { Class }\end{array}$ & $\begin{array}{c}8.6 \mu \mathrm{m} \\
\text { Class }\end{array}$ \\
\hline UGC12138 & $\mathrm{A}$ & $\mathrm{A}$ & $\mathrm{B}$ \\
UGC5101 & $\mathrm{A}$ & $\mathrm{B}$ & $\mathrm{A}$ \\
UGC7064 & $\mathrm{B}$ & $\mathrm{A}$ & $\mathrm{A}$ \\
\hline
\end{tabular}

This paper has been typeset from a $\mathrm{T}_{\mathrm{E}} \mathrm{X} / \mathrm{L}_{\mathrm{A}} \mathrm{T}_{\mathrm{E}} \mathrm{X}$ file prepared by the author. 
Capítulo 3

\section{PARTE II - Moléculas de interesse biótico no sub/milimétrico}

\subsection{Objetos galácticos estudados e o código NAUTILUS}

No capítulo anterior, a complexidade molecular do Universo foi discutida do ponto de vista extragaláctico, com as análises das bandas dos PAHs em galáxias com emissão dominada por starburst. Nas próximas seções, serão apresentados estudos de percursores dessa classe molecular, além de outras COMs que estão presentes no meio interestelar e possuem potencial prebiótico.

Os objetos astrofísicos estudados também estão em escalas e distâncias menores, fazendo parte da fase fria molecular do ISM da Galáxia. Essa fase consiste em uma estrutura hierárquica de nuvens, desde os complexos de nuvens moleculares gigantes aos pequenos núcleos gravitacionalmente instáveis, onde o colapso espontâneo ou desencadeado das partes mais densas das nuvens inicia o processo de formação estrelar (Frieswijk, 2008).

A Tabela 3.1 mostra um panorama das características gerais de alguns desses ambientes de acordo com seus tamanhos, densidades, massas e temperaturas médias. Como discutido em Frieswijk (2008), as nuvens moleculares (MC, na sigla em inglês) representam a maioria do gás molecular frio na Galáxia e podem variar em diversos tamanhos, desde nuvens pequenas como as nuvens escuras (nuvens observadas em extinção no óptico, normalmente associadas a regiões de formação de estrelas de massa baixa/intermediária) até chegar em nuvens moleculares gigantes (relacionadas à formação de estrelas massivas ou aglomerados estelares). Por outro lado, as nuvens frias infravermelhas são opticamente espessas em um comprimento de onda de cerca de $10 \mu \mathrm{m}$, sendo identificadas como escuras em relação à emissão de fundo infravermelha galáctica (Butler e Tan, 2009), além de também estarem 
Tabela 3.1 - Visão geral das propriedades globais de alguns ambientes de nuvens moleculares. MC e IRDC são siglas, em inglês, para nuvem molecular e nuvem fria infravermelha, respectivamente. Informações extraídas da tabela original de Frieswijk (2008).

\begin{tabular}{l|c|c|c|c} 
& \multicolumn{2}{|c}{ Nuvens } & \multicolumn{2}{c}{ Núcleos (pré-) } \\
& MC $^{a}$ & IRDC & estelar & cluster $^{b}$ \\
\hline \hline Tamanho (pc) & $20-100$ & $1-10$ & 0.1 & $1[0.02]$ \\
Densidade $\left(\mathbf{c m}^{-3}\right)$ & $>200$ & $>10^{4}$ & $10^{4}-10^{5}$ & $10^{6}-10^{7}$ \\
Massa $\left(\mathbf{M}_{\odot}\right)$ & $10^{4}-10^{6}$ & $100-10^{4}$ & $0.5-5$ & $10-1000$ \\
Temperatura (K) & $10-30$ & $<25$ & $<15$ & $<25$ \\
\hline
\end{tabular}

${ }^{a}$ Valores variando de pequenas nuvens escuras a nuvens moleculares gigantes. ${ }^{b} \mathrm{O}$ tamanho entre colchetes, bem como a densidade, representam valores para núcleos pré-estelares individuais em um cluster.

ligadas à formação de estrelas massivas.

Em relação aos núcleos, já gravitacionalmente instáveis, os pré-estelares são nuvens moleculares pequenas onde uma única estrela ou sistema estelar forma-se, enquanto os massivos pré-clusters irão originar aglomerados estelares pequenos ou estrelas de alta massa (Frieswijk, 2008). Nos clusters com formação de estrelas massivas, o excesso de momento angular de objetos proto-estelares individuais impulsiona jatos e outflows para o seus envelopes, formando os núcleos moleculares quentes, cujas regiões centrais podem chegar a uma temperatura de alguns $100 \mathrm{~K}$ (Frieswijk, 2008).

A seguir, será apresentada uma introdução dos objetos galácticos utilizados nesse trabalho. Também será realizada uma descrição do código astroquímico NAUtilus, uma importante abordagem usada para simular as abundâncias de algumas moléculas detectadas nessas fontes.

\subsubsection{O núcleo molecular quente G331.512-0.103}

G331.512-0.103, doravante G331, é um núcleo molecular quente com uma velocidade sistêmica aproximada de $90 \mathrm{~km} \mathrm{~s}^{-1}$, localizado na direção à condensação de poeira mais brilhante e maciça da região de formação estelar de G331.5-0.1, no braço espiral de Norma, a uma distância heliocêntrica de cerca de $7.5 \mathrm{kpc}$. G331 apresenta um dos outflows mais brilhantes e energéticos observados na Galáxia, com uma massa em torno de $25 \mathrm{M}_{\odot}$ para cada lobo e de $40 \mathrm{M}_{\odot}$ para seu núcleo (Hervías-Caimapo et al., 2019). A Figura 3.1 mostra três mapas de emissão integrada dessa região obtidos com o ALMA.

Essa fonte também apresenta um momento de cerca de $2.4 \times 10^{3} \mathrm{M}_{\odot} \mathrm{km} \mathrm{s} \mathrm{s}^{-1}$ e uma 


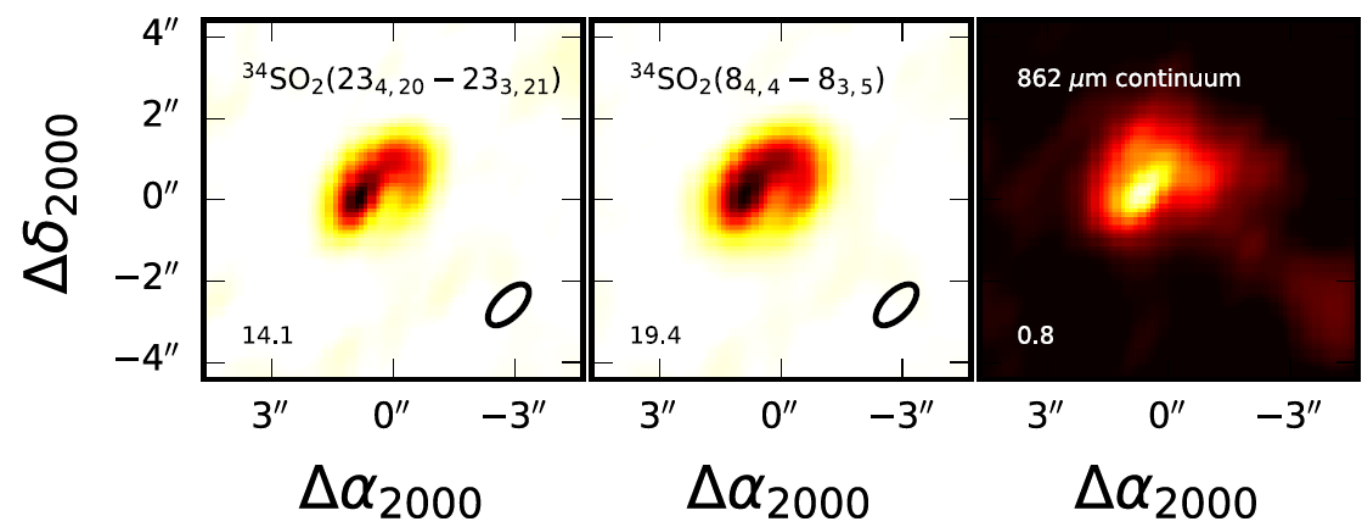

Figura 3.1: Mapas de emissão integrada na velocidade sistêmica de $\mathrm{SO}_{2}$ e do contínuo em $862 \mu \mathrm{m}$. A intensidade integrada de pico é mostrada em cada painel em unidades de Jybeam ${ }^{-1}$. A posição em (0"0,0.'0) corresponde a $16^{h} 12^{m} 09 .{ }^{s} 99-51^{\circ} 28^{\prime} 37$. .'75. Imagens retiradas de Hervías-Caimapo et al. (2019).

energia cinética de $1.4 \times 10^{48}$ erg (Bronfman et al., 2008). Tais valores são esperados em fluxos provenientes de objetos estelares jovens e maciços com luminosidades bolométricas da ordem de $\mathrm{L}_{b o l} \sim 1 \times 10^{5} L_{\odot}$. Observações obtidas com a banda 7 do ALMA revelaram uma densidade média de hidrogênio no núcleo de $5 \times 10^{6} \mathrm{~cm}^{-3}$ e uma temperatura de $70 \mathrm{~K}$ (Hervías-Caimapo et al., 2019), aproximadamente.

Por outro lado, linhas de emissão de diferentes moléculas observadas em G331 têm revelado temperaturas diferentes. Observações do $\mathrm{CH}_{3} \mathrm{OH}$ e $\mathrm{CH}_{3} \mathrm{CN}$ indicaram temperaturas em torno de 74 e $141 \mathrm{~K}$ (Mendoza et al., 2018), respectivamente. Temperaturas derivadas a partir do $\mathrm{HC}_{3} \mathrm{~N}$ variaram entre 80 e $90 \mathrm{~K}$ (Duronea et al., 2019). E linhas de $\mathrm{SiO}$ e $\mathrm{SO}_{2}$ indicaram uma região ainda mais densa com temperaturas de até $220 \mathrm{~K}$ (Hervías-Caimapo et al., 2019). Esse fato sugere que G331 possa ter uma estrutura em forma de conchas em expansão, conforme esquematizado na Figura 3.2.

\subsubsection{A nuvem fria infravermelha IRDC-C9 Main}

Desde as primeiras observações com o observatório ALMA, uma química nova e importante de nuvens frias infravermelhas foi revelada. Essas fontes são compostas de gás denso em baixa temperatura (alguns Kelvin) e condensações de poeira (Butler e Tan, 2009). Embora muitas não possuam um núcleo estelar, elas podem se tornar berçários de estrelas massivas (de Wit et al., 2005; Battersby et al., 2014). Transições moleculares no intervalo milimétrico, especialmente a linha de $\mathrm{N}_{2} \mathrm{D}^{+}(3-2)$, têm sido usadas para identificar e caracterizar estas fontes (Crapsi et al., 2005; Tan et al., 2013; Kong et al., 2017).

A presença de COMs em núcleos pré-estelares é bem estabelecida na literatura (e.g., 


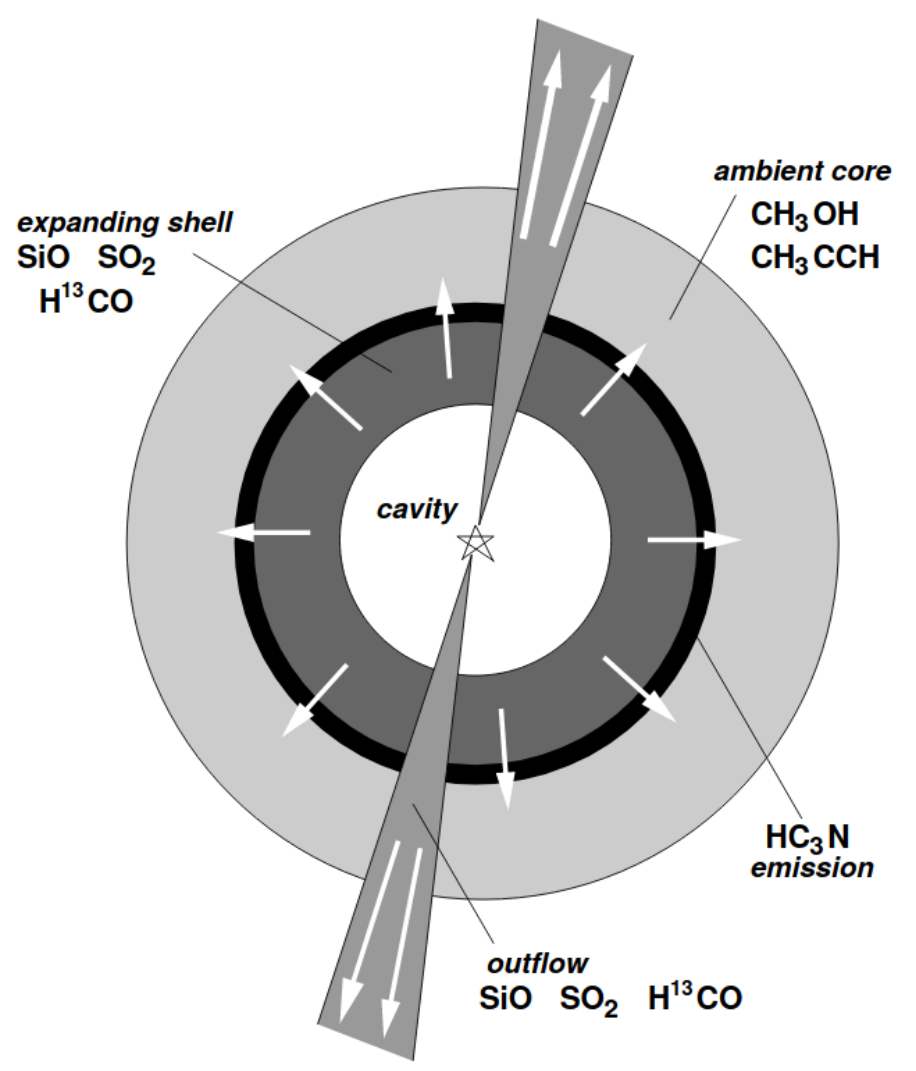

Figura 3.2: Esboço do modelo simples proposto para explicar a emissão de moléculas detectadas em G331, baseado nos modelos de Merello et al. (2013) e Hervías-Caimapo et al. (2019). Imagem retirada de Duronea et al. (2019).

Ellder et al., 1980; Blake et al., 1987; Ceccarelli et al., 1998; Kuan et al., 2004; Bottinelli et al., 2004; Arce et al., 2008; Jaber et al., 2014; Brouillet et al., 2015; ALMA Partnership et al., 2015; Jørgensen et al., 2016). E diferentes modelos foram construídos para explicar suas rotas de formação em ambas as fases gasosa e sólida (Brown et al., 1988; Charnley et al., 1992; Hasegawa e Herbst, 1993; Horn et al., 2004; Garrod e Herbst, 2006; Ruaud et al., 2015, entre outros). Com a detecção de COMs em núcleos frios nos últimos anos, um processo não termal para a formação dessas moléculas foi sugerido (por exemplo, Bacmann e Faure, 2016). O estudo desses ambientes astrofísicos permite aprofundar os conhecimentos de suas propriedades físicas e químicas.

Beaklini et al. (2020) investigaram observações do arquivo do ALMA Banda 6 de 231 GHz da região da nuvem escura infravermelha C9 realizadas por Kong et al. (2017), com foco na fonte brilhante que foi chamada de IRDC-C9 Main. Neste objeto, foi reportada a existência de duas subestruturas no mapa do contínuo (Figura 3.3): um ponto compacto e brilhante com grande diversidade química chamado de núcleo, e uma região mais fraca e 


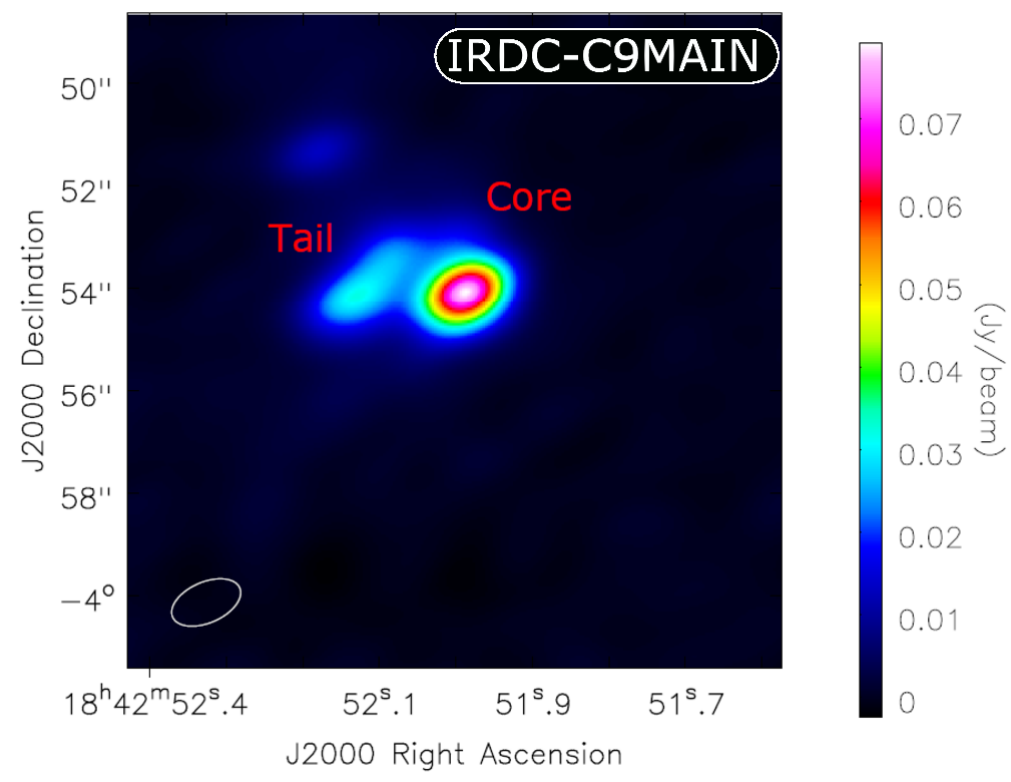

Figura 3.3: Mapa do contínuo de IRDC-C9 Main, mostrando a emissão de um núcleo com uma cauda nas redondezas. O fluxo é apresentado em escala de cor nas unidades Jy/beam. O beam resolvido é de $1.4 \times 0.7$ arcsec. Imagem retirada de Beaklini et al. (2020).

estendida chamada de cauda.

No núcleo, foram identificadas linhas das moléculas OCS (19-18), ${ }^{13} \mathrm{CS}(5-4)$ e $\mathrm{CH}_{3} \mathrm{CH}_{2} \mathrm{CN}$, diversas linhas de $\mathrm{CH}_{3} \mathrm{CHO}$ e a emissão de $k$-ladder de ${ }^{13} \mathrm{CH}_{3} \mathrm{CN}$. Na cauda, somente as linhas de OCS (19-18) e ${ }^{13} \mathrm{CS}$ (5-4) foram detectadas. O espectro de cada região pode ser visto na Figura 3.4. A densidade volumétrica esperada para essa fonte é $\mathrm{n}\left(\mathrm{H}_{2}\right) \approx 4.6 \times 10^{-6}$ $\mathrm{cm}^{-2}$ (Kong et al., 2017).

Também foram reportadas duas regiões de temperaturas: enquanto o diagrama rotacional de $\mathrm{CH}_{3} \mathrm{CHO}$ indica uma temperatura de $25 \mathrm{~K}$, o diagrama rotacional de ${ }^{13} \mathrm{CH}_{3} \mathrm{CN}$ indica uma fase mais quente em uma temperatura de cerca de $450 \mathrm{~K}$ (Beaklini et al., 2020). De acordo com as análises de Beaklini et al. (2020), a emissão do contínuo no núcleo é mais brilhante do que na cauda, e forneceu uma estimativa para a massa de cada região - $57 \mathrm{M} \odot$ para o núcleo e $28 \mathrm{M} \odot$ para a cauda. Uma explicação é que o núcleo poderia abrigar uma protoestrela. Inclusive, a diferença entre as velocidades e FWHM das linhas observadas em cada região também sugere um outflow fraco no núcleo.

Nesse sentido, o núcleo pode estar em um estágio evolutivo avançado, em que o processo de contração da sua região mais interna já está na fase de aquecimento e possibilita a evaporação de algumas moléculas. Por outro lado, a cauda ainda se encontra em um estágio inicial. Essas suposições podem ser corroboradas pelo fato de que a linha do OCS 


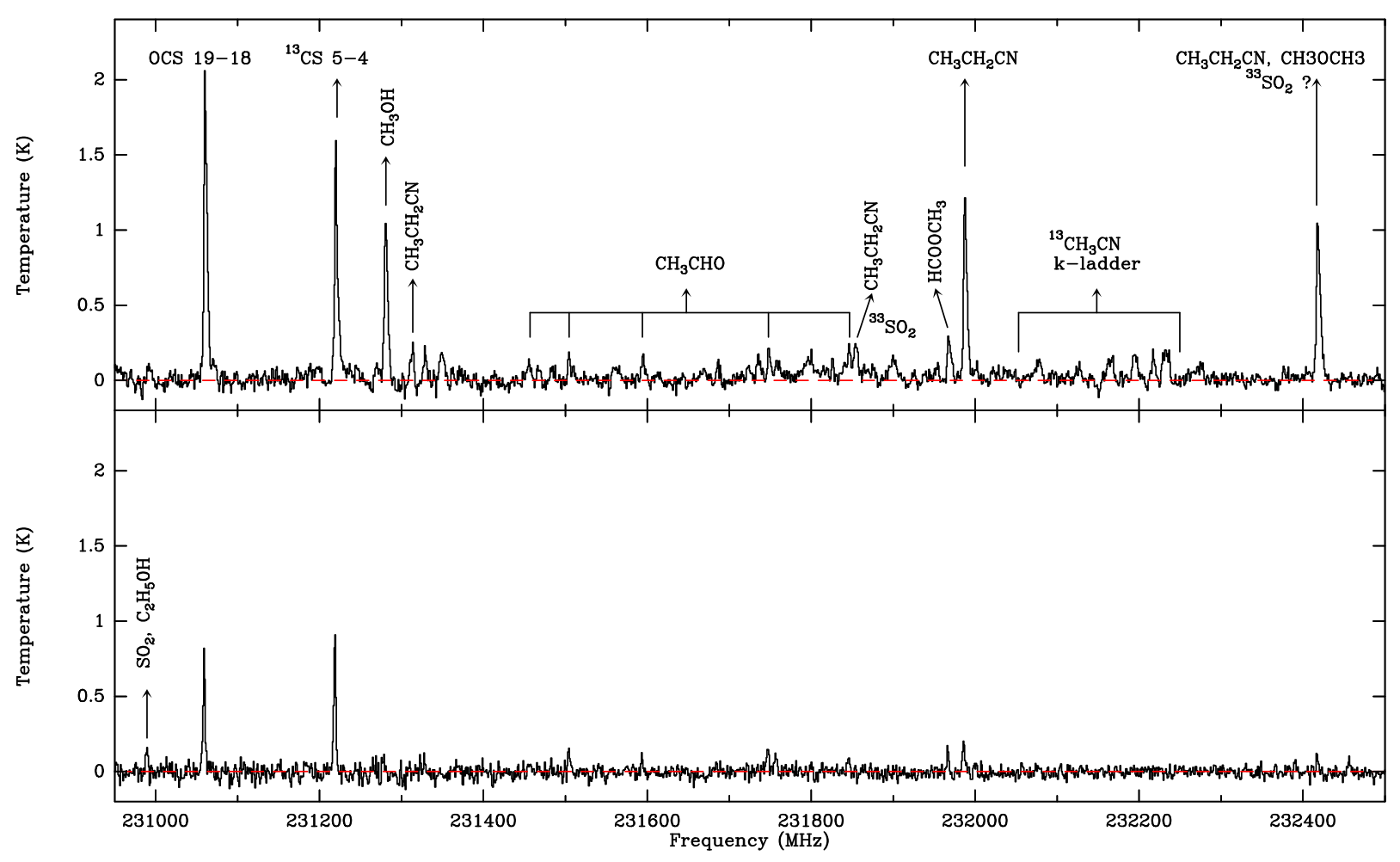

Figura 3.4: Espectros do núcleo (superior) e cauda (inferior) de IRDC-C9 Main mostrando a identificação das linhas mais intensas. Linhas de base espectrais foram subtraídas nos segmentos sem linhas; as linhas tracejadas vermelhas indicam a linha de base padrão. Imagem retirada de Beaklini et al. (2020).

é mais intensa no núcleo do que na cauda, uma vez que ela é normalmente encontrada em gás ao redor de fontes recém-formadas.

\subsubsection{Modelagem química com NAUTILUS}

Uma possível abordagem para melhor entender a química do ISM é modelar os ambientes astrofísicos - tanto a parte física como a química. Modelos fornecem informações sobre os possíveis caminhos químicos que podem estar presentes nesses ambientes, além de considerarem diversos parâmetros físicos específicos de cada objeto. Essa metodologia, apresentada a seguir, é importante para complementar as observações que serão discutidas nas próximas seções.

Dos diversos códigos disponíveis para realizar essas simulações, foi usado o código NAUTILUs (Ruaud et al., 2016). Ele é uma ferramenta útil para entender as reações químicas que ocorrem em núcleos moleculares quentes e frios (Semenov et al., 2010; Reboussin et al., 2014; Ruaud et al., 2015, 2016). O modelo simulado pelo código prevê a evolução temporal das abundâncias químicas para um determinado conjunto de parâmetros físicos e químicos.

Para a química do estado sólido, ele considera o manto e a superfície de grãos como 
quimicamente ativos, seguindo o formalismo de Hasegawa e Herbst (1993) e os resultados experimentais de Ghesquière et al. (2015). Ele ainda pode executar uma simulação química trifásica (gás, manto do grão e superfície) dependente do tempo, incluindo reações químicas em ambas as fases gasosa e sólida (Ruaud et al., 2015).

A química dos grãos também considera a fotodissociação direta por fótons (normalmente a padrão para os modelos de fotoionização) juntamente com a fotodissociação induzida por fótons UV secundários (Prasad e Tarafdar, 1983), que são processos eficazes na superfície e manto dos grãos. Além disso, a competição entre reação, difusão e evaporação, como sugerida por Chang et al. (2007) e Garrod e Pauly (2011), também foi implementada no código. As energias de difusão de cada espécie são calculadas como uma fração de suas energias de ligação na proporção indicada por Vidal e Wakelam (2018) - 0.4 para a superfície e 0.8 para o manto, como sugerido por trabalhos teóricos e experimentais (para maiores detalhes, ver Ruaud et al., 2016; Vidal e Wakelam, 2018).

As abundâncias elementares necessárias para o início da modelagem foram baseadas nos estudos Vidal e Wakelam (2018) e são mostradas na Tabela 3.2. Elas representam um caso de abundância intermediária de metais, no qual todas as abundâncias são iguais a um caso de abundância baixa de metais (como as abundâncias das modelagens de Wakelam et al., 2006; Wakelam e Herbst, 2008, por exemplo), exceto que a quantidade do elemento enxofre é aumentada para $1.5 \times 10^{-5}$ em comparação com $\mathrm{H}_{2}$ (Wakelam et al., 2006).

Tabela 3.2 - Abundâncias iniciais assumidas para o modelo do NAUTILus, extraídas de Vidal e Wakelam (2018). Abundâncias dadas no formato $a(b)$ representando $a \times 10^{b}$. Referências: 1 . Wakelam e Herbst (2008); 2. Jenkins (2009); 3. Hincelin et al. (2011); 4. abundância baixa de metais de Graedel et al. (1982); 5. Neufeld et al. (2005).

\begin{tabular}{lcclcc}
\hline Elemento & $\mathbf{n}_{i} / \mathbf{n}_{H}$ & Ref. & Elemento & $\mathbf{n}_{i} / \mathbf{n}_{H}$ & Ref. \\
\hline $\mathrm{H}_{2}$ & 0.5 & & $\mathrm{He}$ & $9.0(-2)$ & 1 \\
$\mathrm{~N}$ & $6.2(-5)$ & 2 & $\mathrm{O}$ & $2.4(-4)$ & 3 \\
$\mathrm{C}^{+}$ & $1.7(-4)$ & 2 & $\mathrm{~S}^{+}$ & $1.5(-5)$ & 2 \\
$\mathrm{Fe}^{+}$ & $3.0(-9)$ & 4 & $\mathrm{Si}^{+}$ & $8.0(-9)$ & 4 \\
$\mathrm{Na}^{+}$ & $2.0(-9)$ & 4 & $\mathrm{Mg}^{+}$ & $7.0(-9)$ & 4 \\
$\mathrm{Cl}^{+}$ & $1.0(-9)$ & 4 & $\mathrm{P}^{+}$ & $2.0(-10)$ & 4 \\
$\mathrm{~F}$ & $6.7(-9)$ & 5 & & & \\
\hline
\end{tabular}

A rede completa de reações usadas pelo NAUTILUs é apresentada no catálogo KIDA ${ }^{1}$

\footnotetext{
${ }^{1}$ http://kida.obs.u-bordeaux1.fr/
} 
(KInetic Database for Astrochemistry, Wakelam et al., 2015). Todas as simulações realizadas nesse trabalho são zero dimensionais, ou seja, as condições físicas como temperatura do gás e densidade total são consideradas uniformes dentro da nuvem e através do tempo da simulação. Uma evolução estrutural para cada objeto também não foi considerada nos modelos. Esses são os modelos mais simples disponíveis no NAUTILUS, e já revelam resultados interessantes.

\subsubsection{Distância de desacordo}

A escala de tempo simulada pelo NAuTiLus está relacionada com a idade química do objeto. Nesse sentido, o tempo inicial (zero) não é , necessariamente, a idade de formação da fonte. Ele está ligado com o início das reações químicas. Dessa forma, o modelo permite uma estimativa dessa idade química ao se comparar as abundâncias modeladas com as observadas.

Para se obter uma aproximação mais refinada, especialmente nos casos em que as espécies moleculares não são bem reproduzidas pelo modelo, Wakelam et al. (2006) sugeriram um parâmetro chamado distância de desacordo $D$. Ele pode ser definido como a soma das distâncias entre os valores observados e modelados das abundâncias de todas as espécies observadas, como descrito pela Equação 3.1.

$$
D=\frac{1}{N_{o b s}} \sum\left|\log \left(n_{o b s, i}\right)-\log \left(n_{i, t}\right)\right|
$$

onde $\mathrm{N}_{o b s}$ é o número de espécies observadas, $\mathrm{n}_{o b s, i}$ a abundância observada da espécie $\mathrm{i}$ e $\mathrm{n}_{i, t}$ a abundância modelada da espécie i a cada tempo t. O valor mínimo de $\mathrm{D}$ corresponde à mínima diferença em magnitude entre os valores modelados e observados das abundâncias das espécies. Consequentemente, o tempo em que D mínimo ocorre pode ser considerado como a idade química estimada do objeto de acordo com o modelo.

\subsection{Análises Observacionais de G331.512-0.103}

Pelos trabalhos que vem sendo feitos a respeito de G331, fica claro que existe uma importante complexidade química nessa região. Para melhor se estudar esse objeto, especialmente do ponto de vista astroquímico, foram utilizadas as observações realizadas com o APEX de Bronfman et al. (2008), Mendoza et al. (2018) e Mendoza et al. in prep. APEX 
ou Atacama Pathfinder Experiment ${ }^{2}$ é uma antena submilimétrica de $12 \mathrm{~m}$ localizada em San Pedro de Atacama, Chile. Os dados foram obtidos pelos instrumentos APEX-1 e APEX-2 nas frequências de 213-275 GHz e 267-378 GHz, respectivamente.

Através do software CLASS/GILDAS ${ }^{3}$, produzido para analisar dados de radiotelescópios, uma identificação preliminar de linhas com temperaturas de antena menores do que $1 \mathrm{~K}$ foi realizada. Espécies moleculares mais complexas (maiores e com mais heterogeneização, por exemplo) tendem a ser menos abundantes no ISM, ou por causa da dificuldade de produção ou pela maior facilidade de fragmentação. Por esse motivo, é nesse regime de baixa temperatura de antena que pode ser encontrada emissão de várias COMs. Um exemplo do uso do CLASS é mostrado na Figura 3.5.

Os prováveis comprimentos de onda centrais de cada linha são obtidos diretamente pelo software. Em seguida, são comparados com os de diversas moléculas das bases de dados Splatalogue ${ }^{4}, \mathrm{CDMS}^{5}$ (Cologne Database for Molecular Spectroscopy, Endres et al., 2016) e JPL $^{6}$ (Jet Propulsion Laboratory Catalog, Pickett et al., 1998), que contêm as frequências e informações teóricas e laboratoriais de espécies moleculares.

Para avaliar quais dessas espécies são responsáveis por cada emissão, foram levados em conta fatores como as energias dos níveis envolvidos nas transições, os valores dos coeficientes de Einstein $\left(\mathrm{A}_{i, j}\right)$ e energia do nível superior $\left(\mathrm{E}_{u p}\right)$, disponíveis nos catálogos. Preferiram-se transições com os menores níveis de energia por serem, normalmente, as mais prováveis. Da mesma forma, valores de $\mathrm{E}_{u p}$ maiores que $350 \mathrm{~K}$, que é a temperatura máxima estimada para G331, também não foram considerados. Afinal, essa é uma temperatura relativamente baixa para que transições altamente energéticas ocorram nessa fonte.

Por fim, como muitas das espécies contidas nos catálogos procedem somente de cálculos teóricos e experimentos, ainda foi considerado o catálogo Lovas/NIST ${ }^{7}$ (Recommended Rest Frequencies for Observed Interstellar Molecular Microwave Transitions, Lovas, 2004). Por conter, apenas, moléculas já observadas anteriormente no ISM, foi dada preferência às moléculas presentes no Lovas/NIST como opção final para identificar a linha espectral.

A Tabela 3.3 abaixo apresenta um levantamento das possíveis linhas de emissão pre-

\footnotetext{
2 http://www.apex-telescope.org/

3 https://www.iram.fr/IRAMFR/GILDAS/

4 https://www.cv.nrao.edu/php/splat/

5 https://www.astro.uni-koeln.de/cdms/catalog

6 https://spec.jpl.nasa.gov/

7 http://physics.nist.gov/cgi-bin/micro/table5/start.pl
} 
$0 ; 0$ G331.512-0.1 C2H5CN 30-29 AP-H301-X20-0:22-MAR-2016 R:10-APR-2017

RA: $16: 12: 10.10$ DEC: $-51: 28: 38.1$ Eq 2000.0 Rad. $0.0^{\circ}$ Offs: $+0.1+0.0$

Unknown tau: 0.214 Tsys: 326. Time: 64. min El: 0.0

$N: 52429$ I0: $26215.4 \quad$ VO: $-90.00 \quad$ DV: $-6.5362 \mathrm{E}-02$ LSR

Fo: $\quad 349800.000 \quad$ Df: $7.6264 \mathrm{E}-02 \mathrm{Fi}: 337804.648$

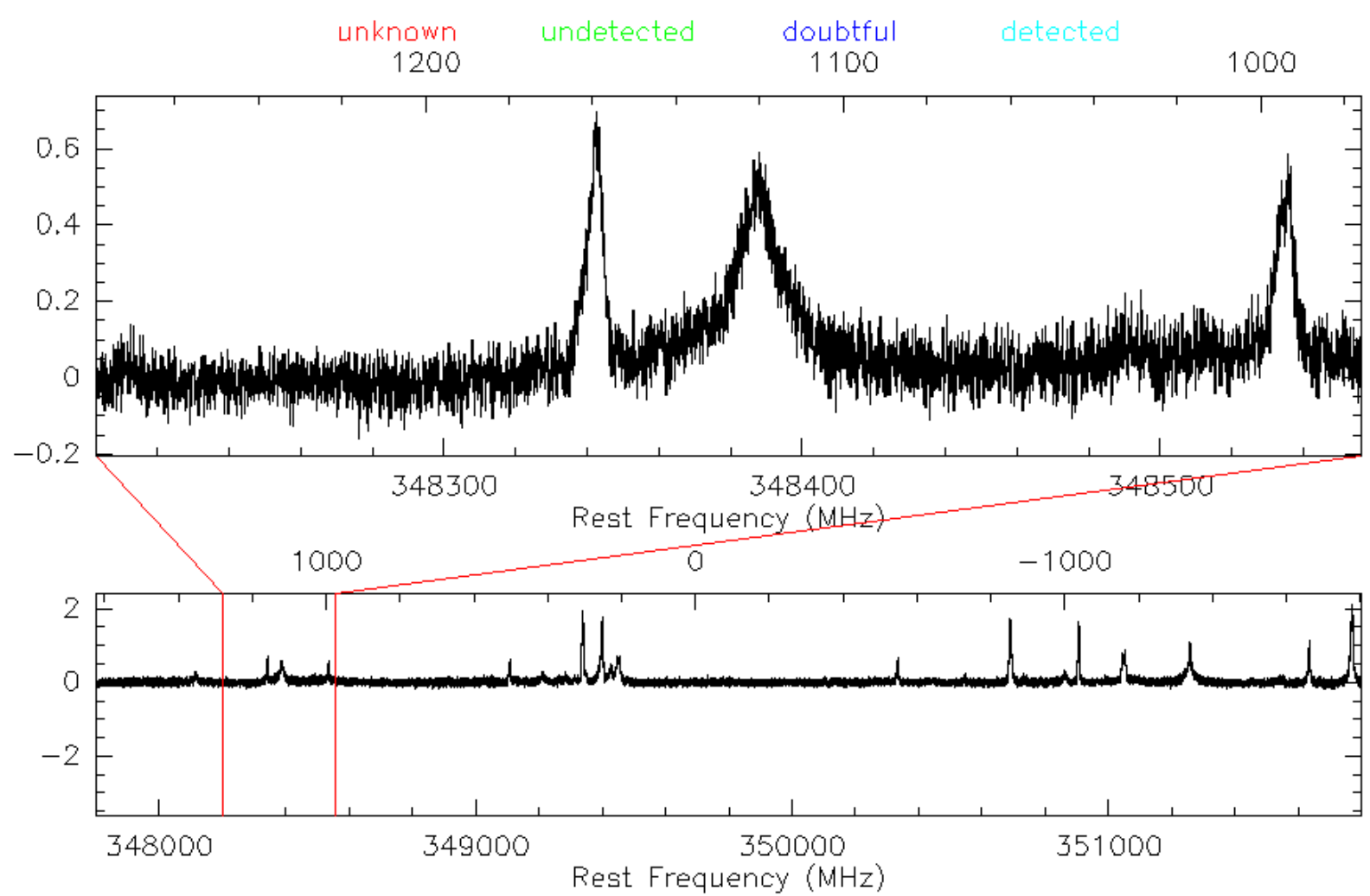

Figura 3.5: Uma das bandas espectrais observadas do objeto G331 com frequência de 347.8 a $351.8 \mathrm{GHz}$. O gráfico superior mostra um aumento em relação à banda inteira (gráfico inferior) com três linhas de temperaturas menores que $1 \mathrm{~K}$ visíveis. O eixo x está em $\mathrm{MHz}$ e o eixo y em $\mathrm{K}$. A imagem foi obtida pelo software CLASS/GILDAS.

sentes em G331 observadas com o APEX-2. A temperatura de antena $\left(\mathrm{T}_{a}\right)$ é obtida com o CLASS e os demais dados são extraídos dos catálogos. Juntamente com a possível espécie identificada, são exibidos alguns contaminantes que podem contribuir com cada emissão. As linhas mais fracas também podem ser mais largas, compreendendo mais de uma espécie molecular. Em último caso, elas também podem ser apenas um ruído instrumental.

Por todas essas razões, vale reforçar que essa é uma identificação preliminar. Ainda assim, a quantidade de linhas obtidas somente pelo APEX-2 reforça a relevância desse objeto no estudo de COMs e impulsiona novas observações que auxiliem em uma maior compreensão de G331. Esse tipo de trabalho é de extrema importância para se ter uma visão geral da química de um ambiente astrofísico. Ele também ajuda a identificar as emissões mais promissoras que devem revelar as condições físicas e químicas do objeto 
com maior resolução.

Dentre as moléculas previamente identificadas em G331, destacam-se possíveis emissões de COMs e de moléculas prebióticas, como $\mathrm{CH}_{3} \mathrm{OCH}_{3}, \mathrm{CH}_{3} \mathrm{OCHO}, \mathrm{C}_{2} \mathrm{H}_{5} \mathrm{CN}$ e $\mathrm{C}_{3} \mathrm{H}_{7} \mathrm{CN}$, por exemplo. Além delas, uma molécula simples cujo valor prebiótico já foi discutido - o HNCO - apresentou várias linhas de emissão. A formamida, que pode estar correlacionada com o HNCO e também é uma molécula fundamental para a química prebiótica, como discutido no Capítulo 1, não possui emissões intensas o suficiente para serem analisadas nesses dados.

Após essa identificação preliminar, a espécie escolhida para ser amplamente analisada foi o HNCO. Além de possuir mais de 40 transições observadas, muitas linhas também estão bem resolvidas. Esses dois fatores juntos permitem um trabalho mais aprimorado por causa da quantidade de dados. Os resultados derivados e complementados com simulações do NAUtilus são discutidos nas seções 3.2.1 e 3.2.1.2. 


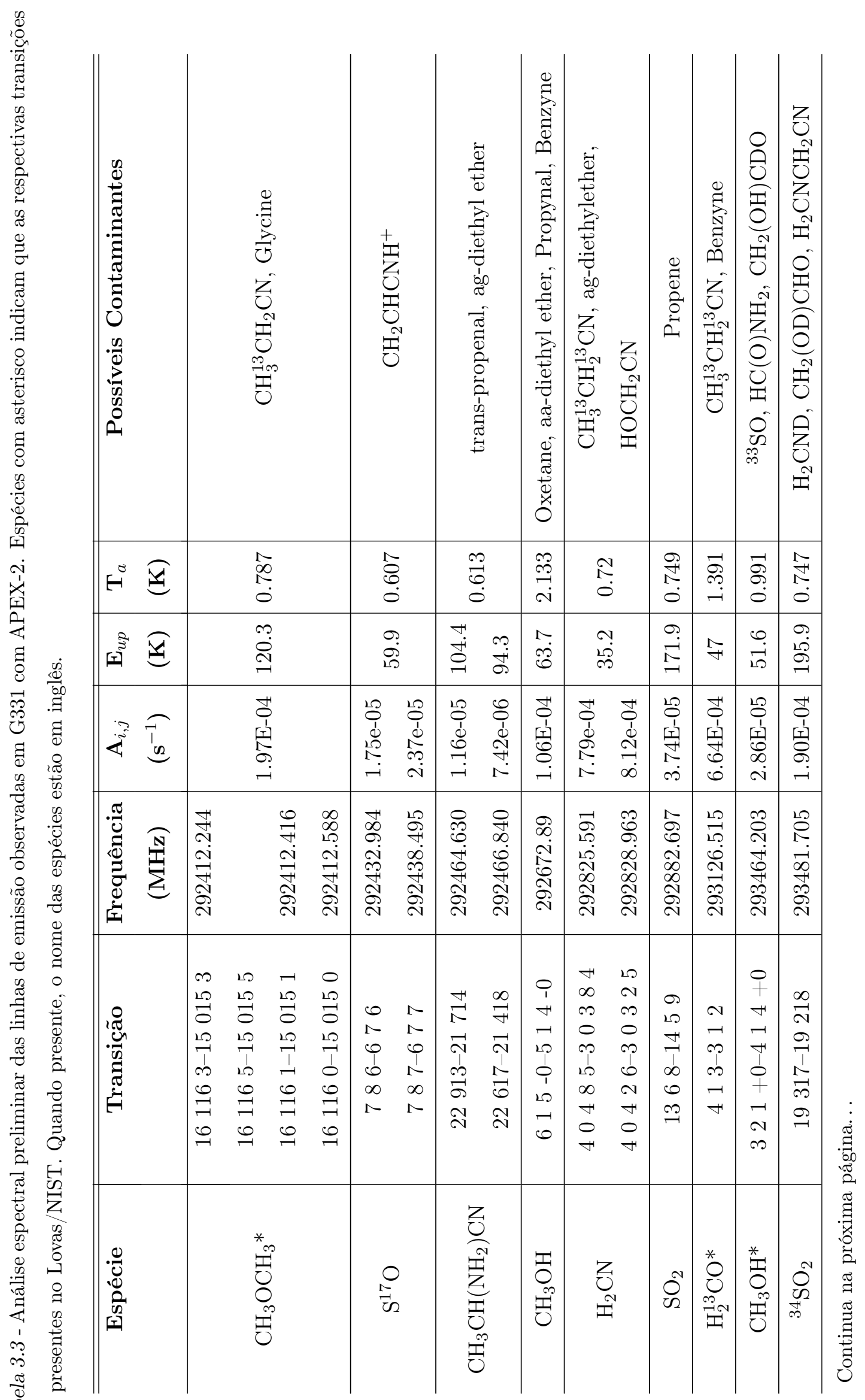




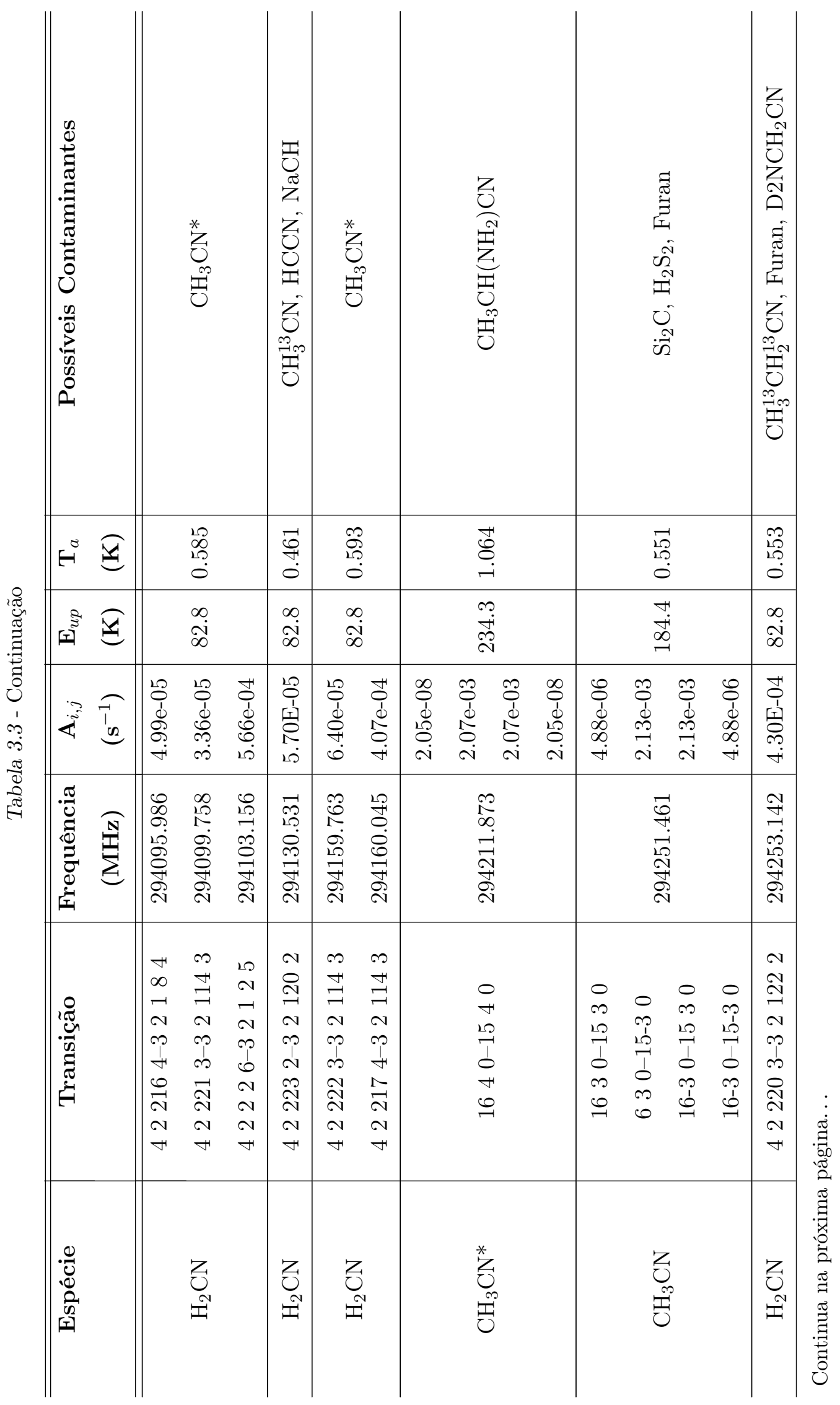




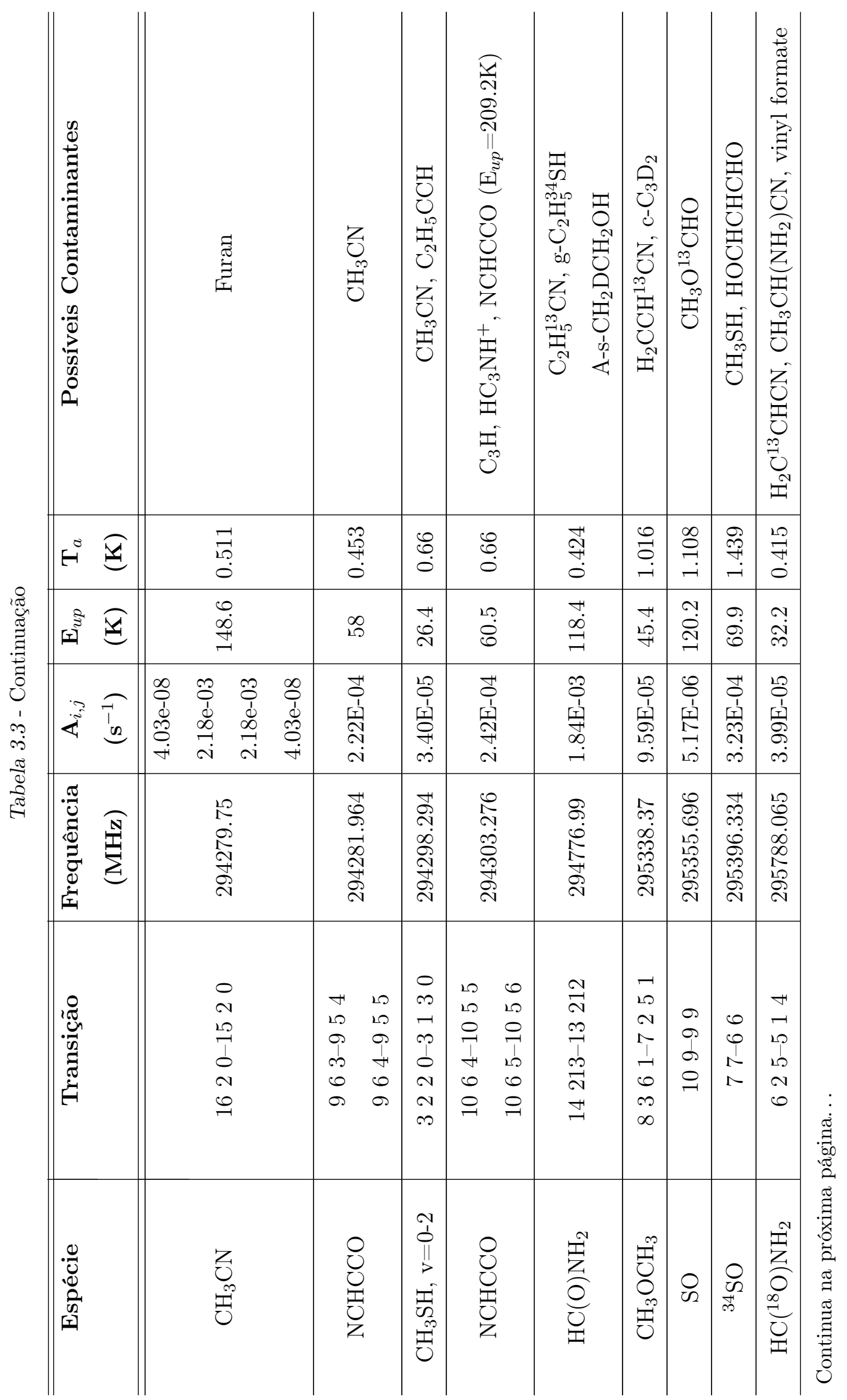




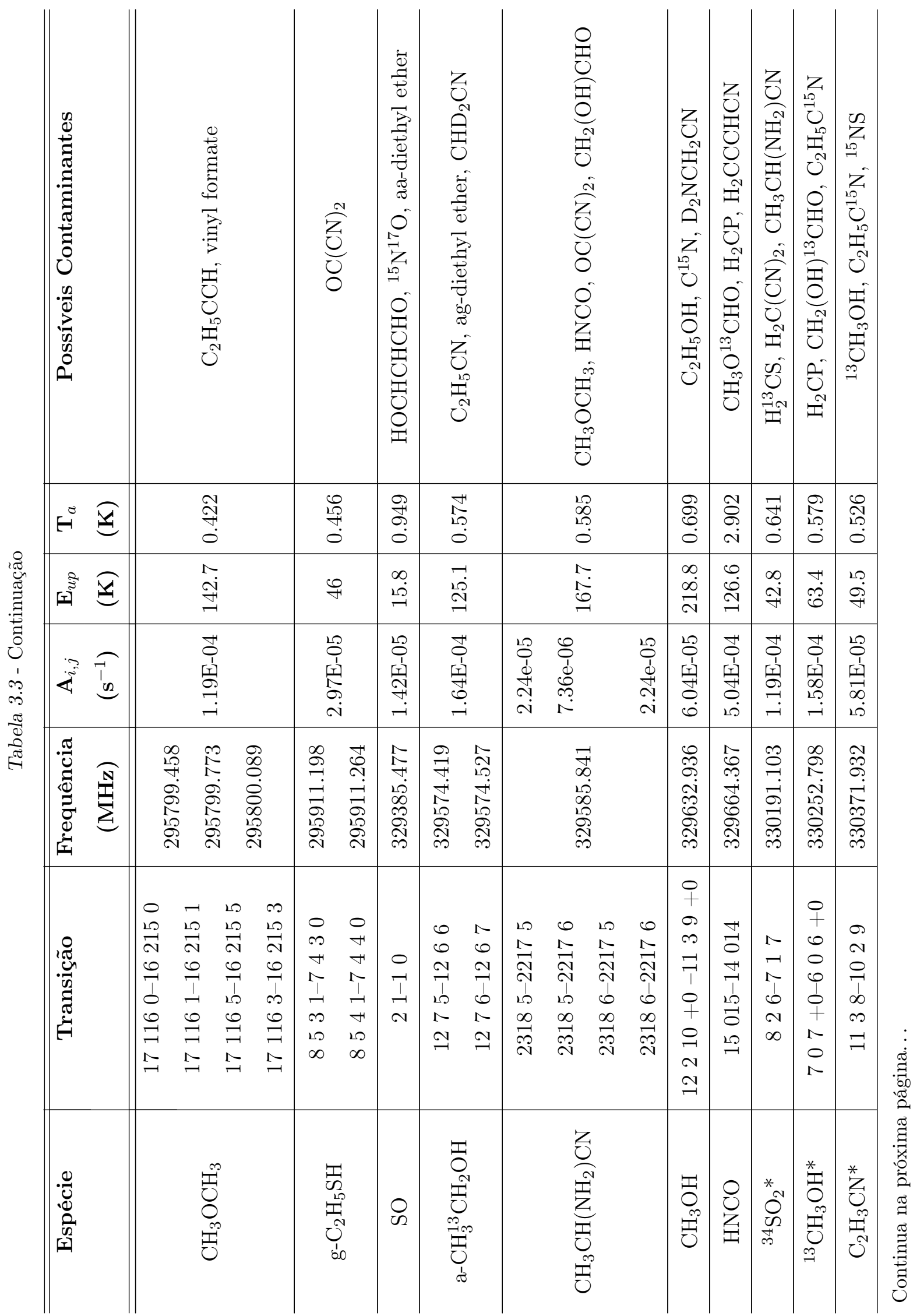




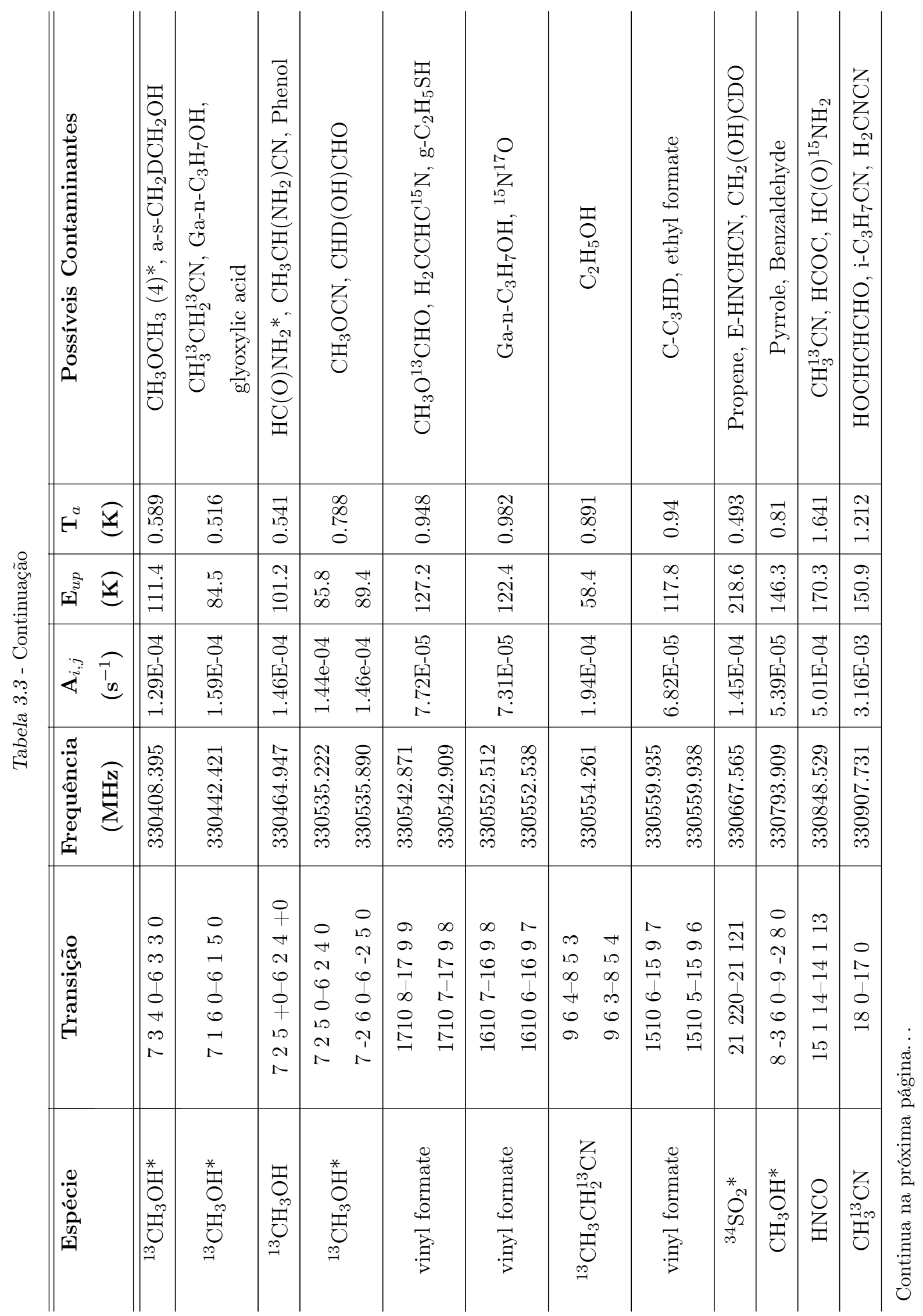




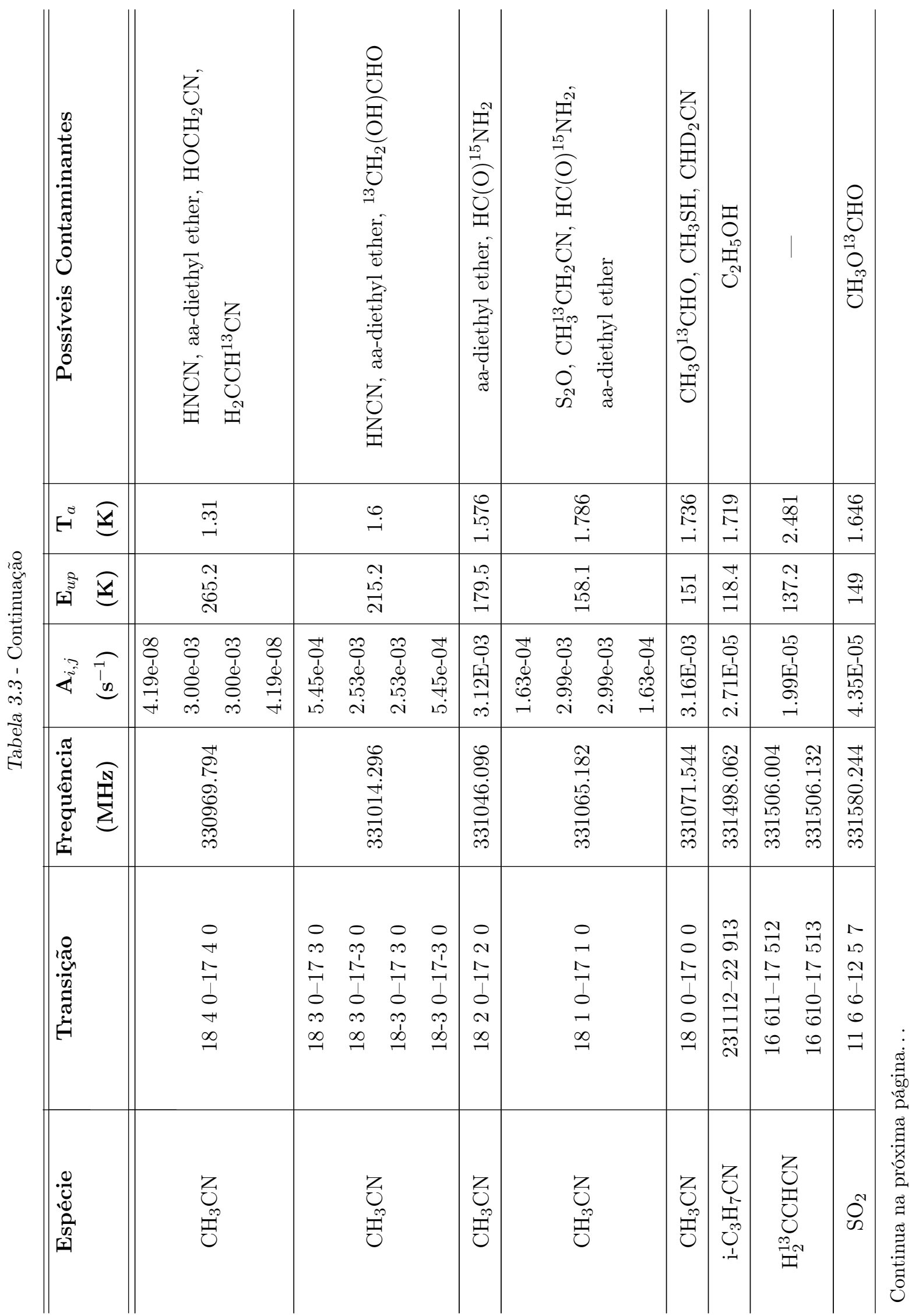




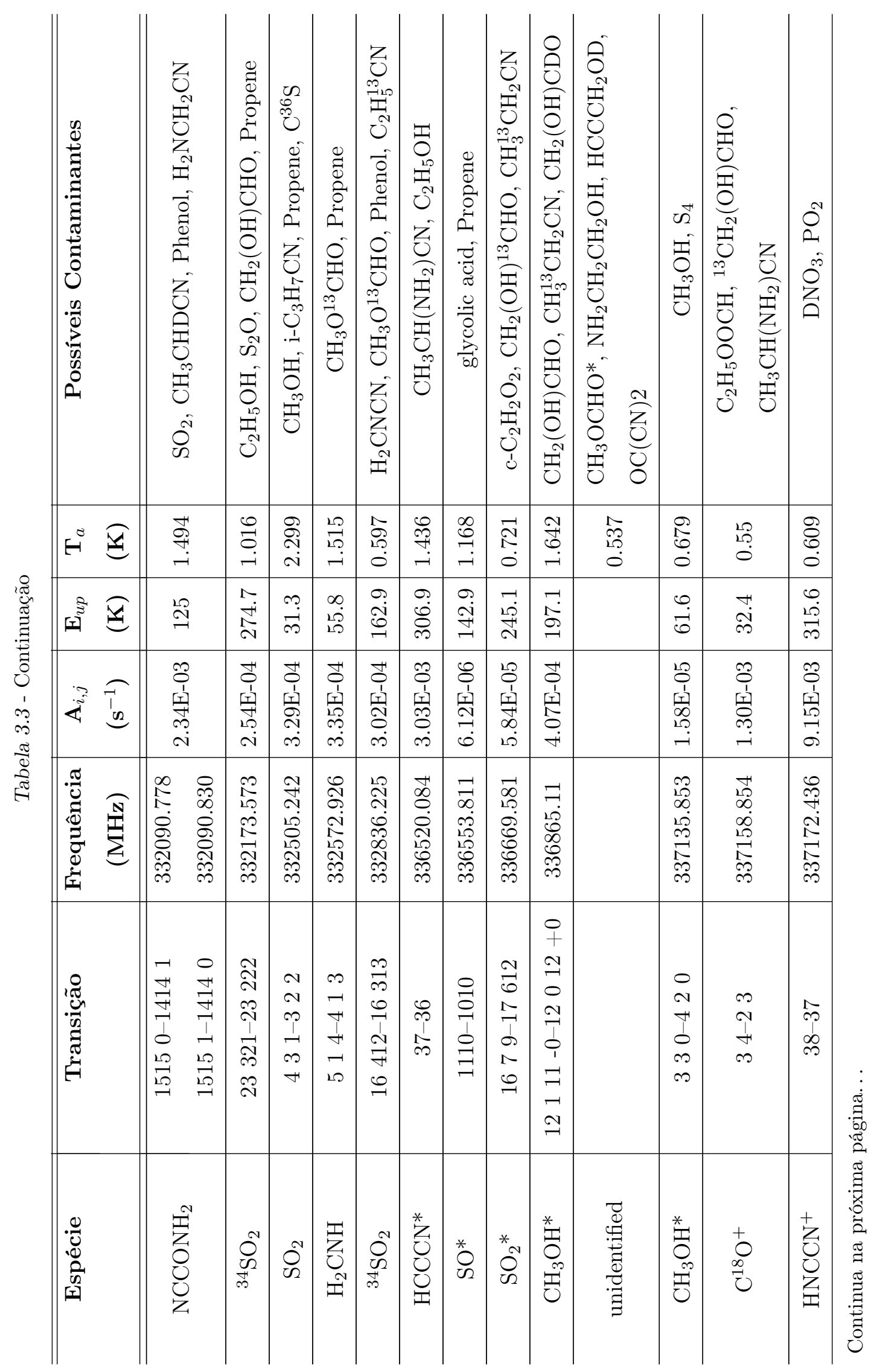




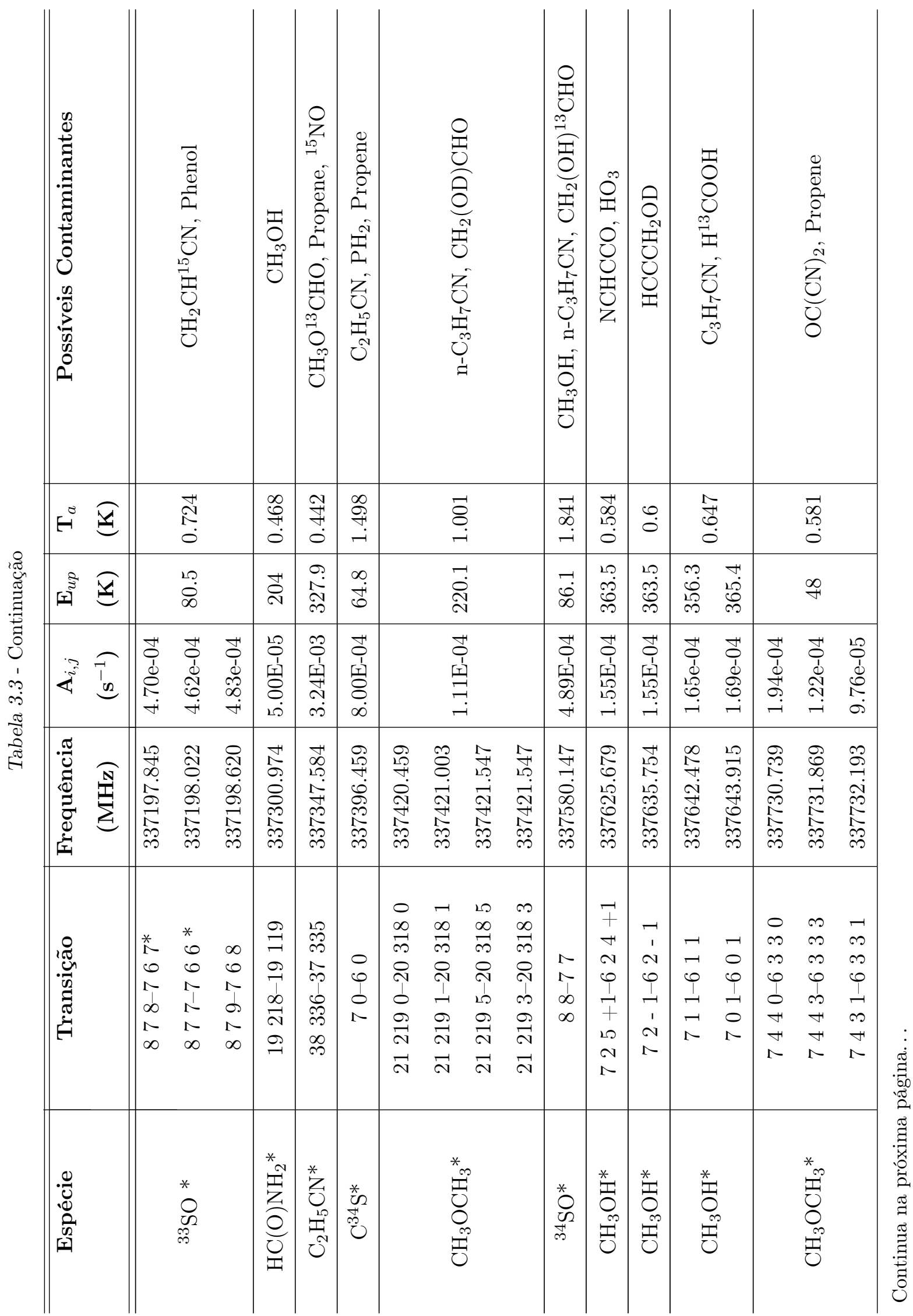




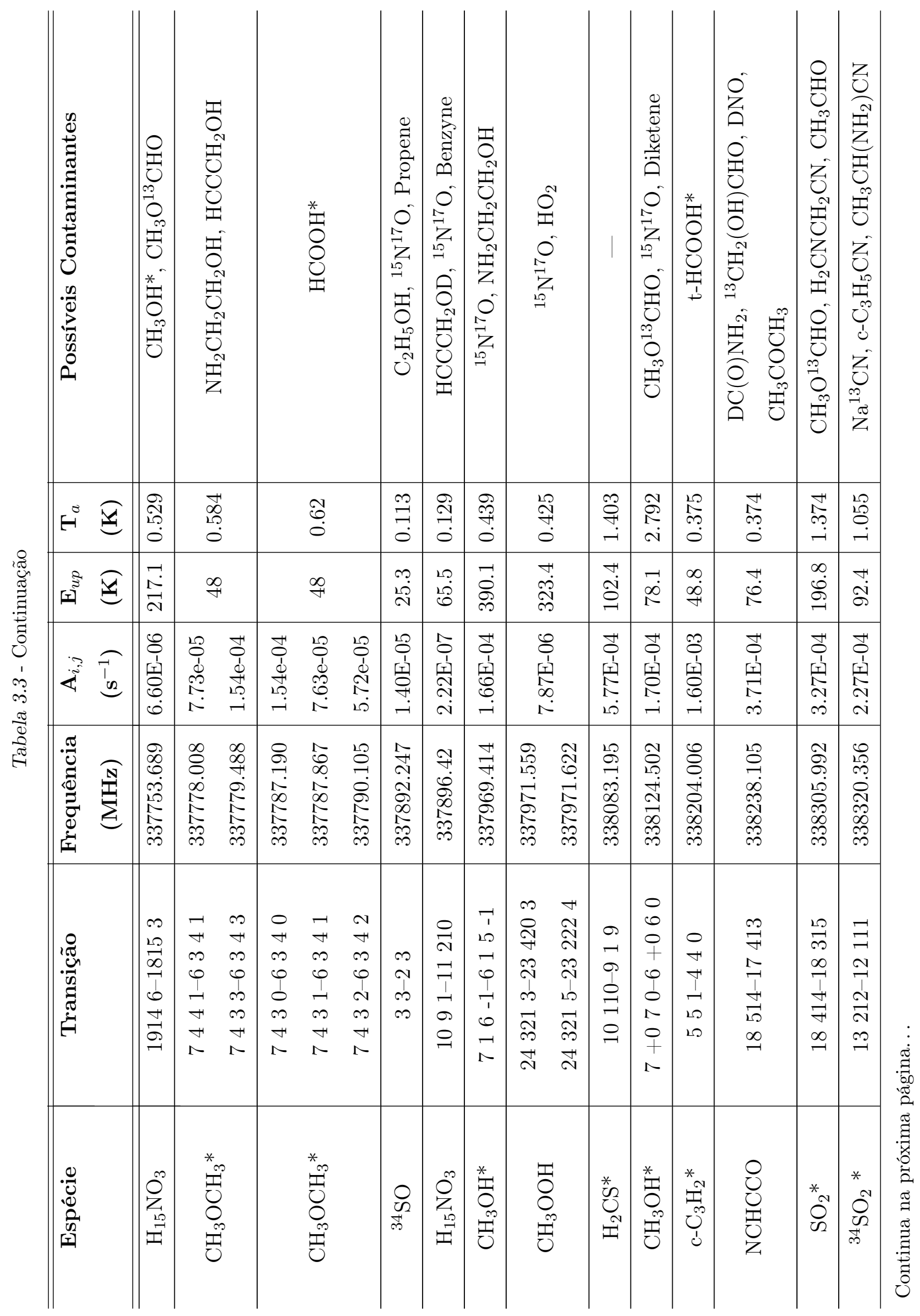




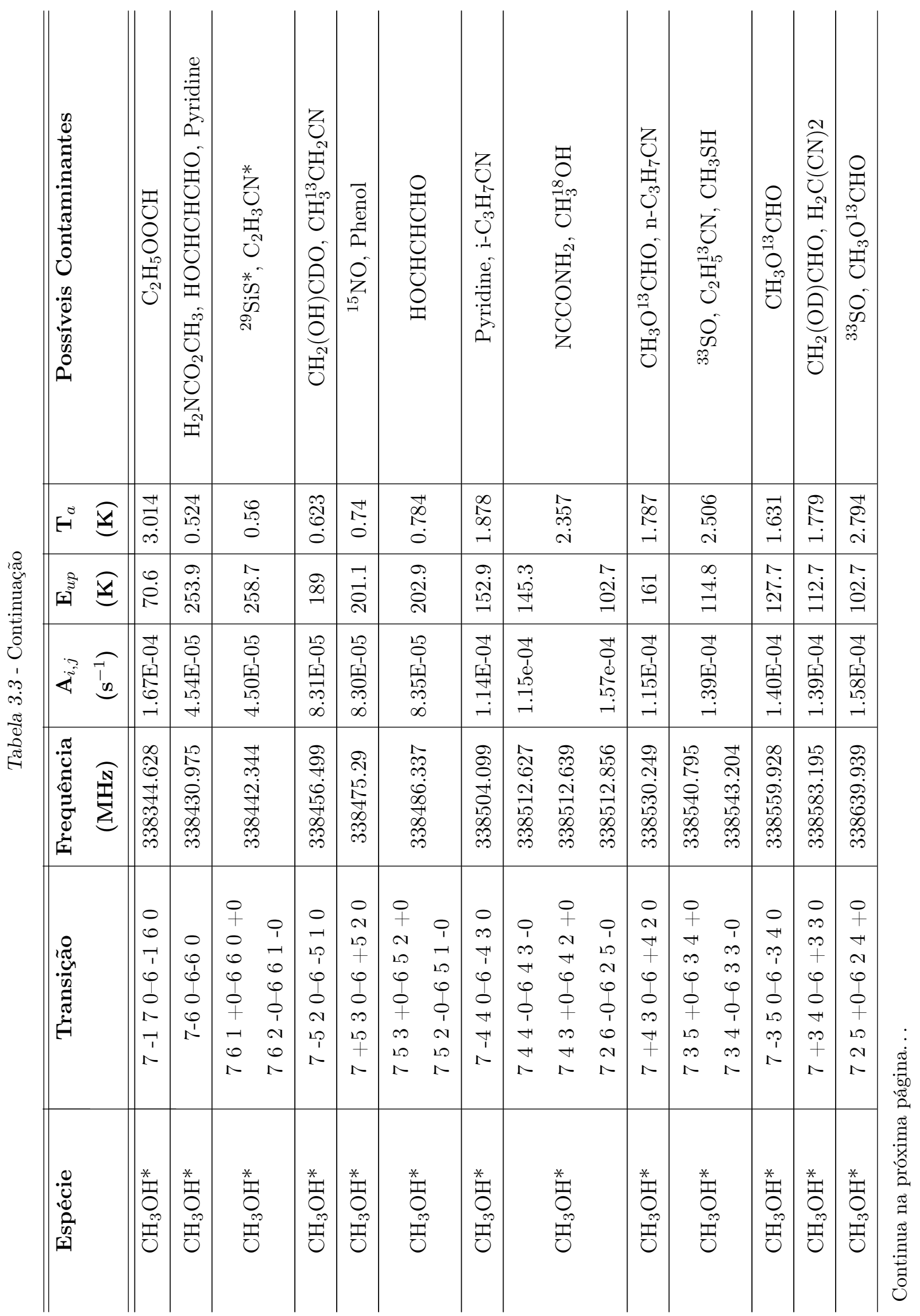




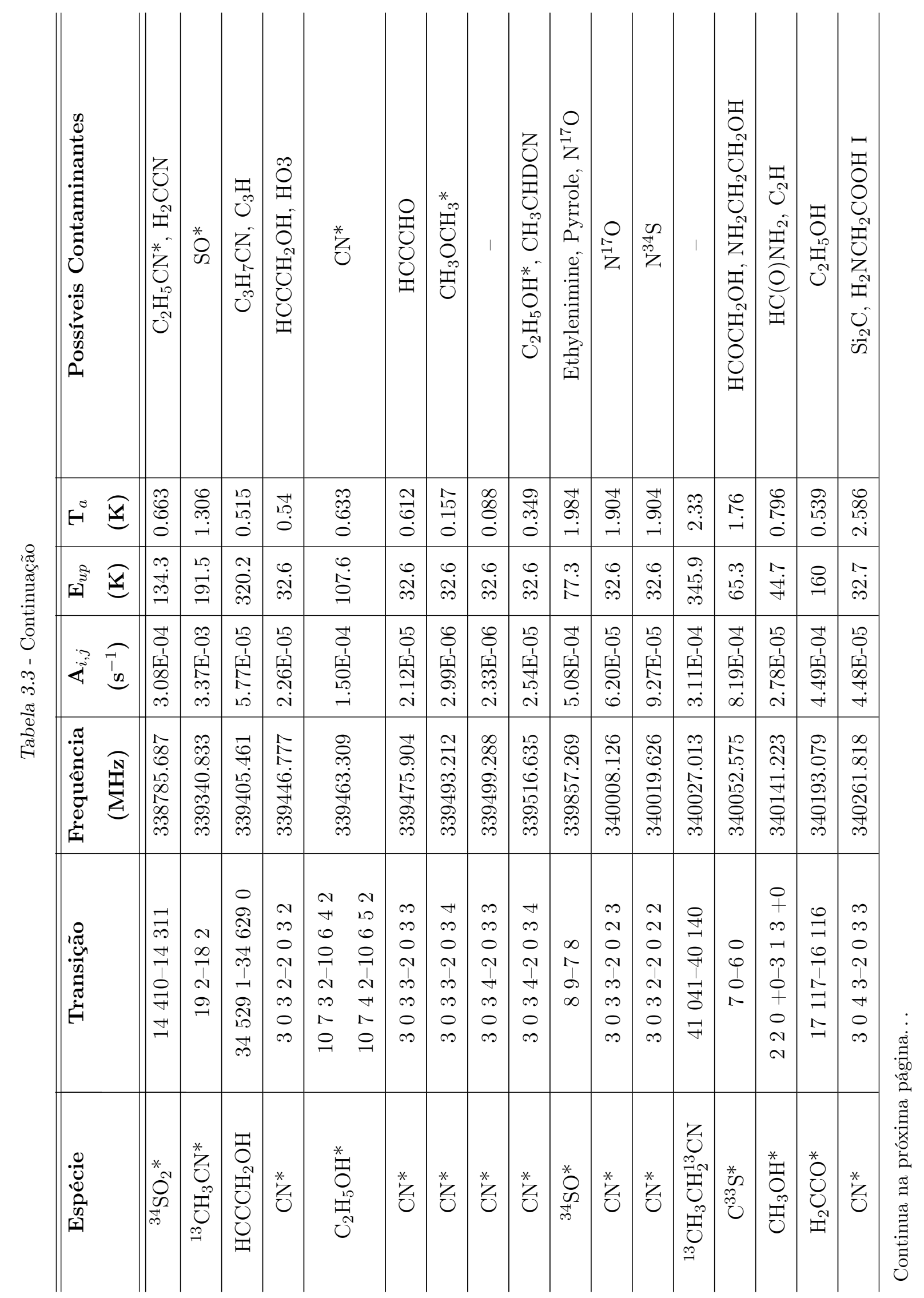




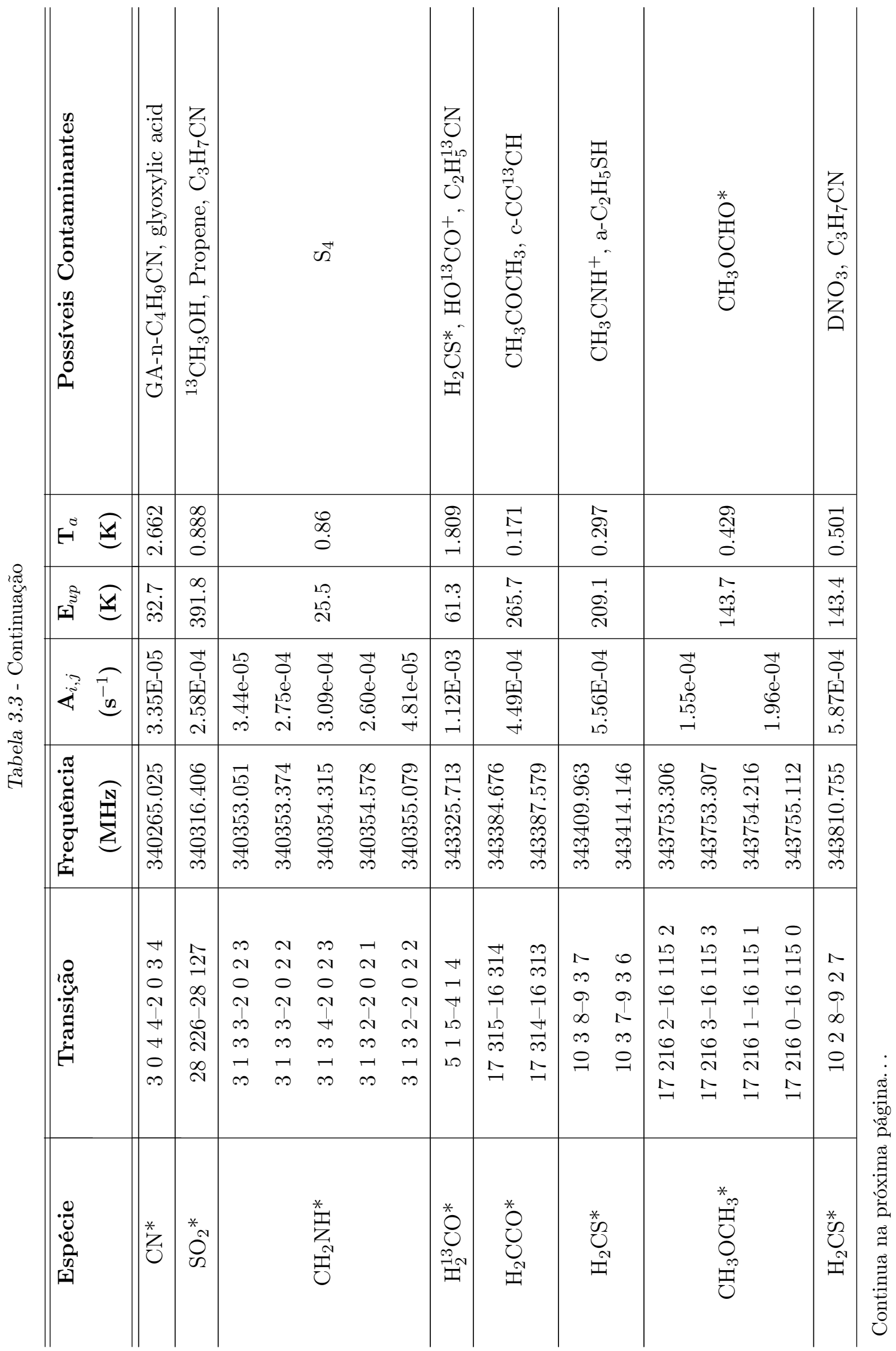




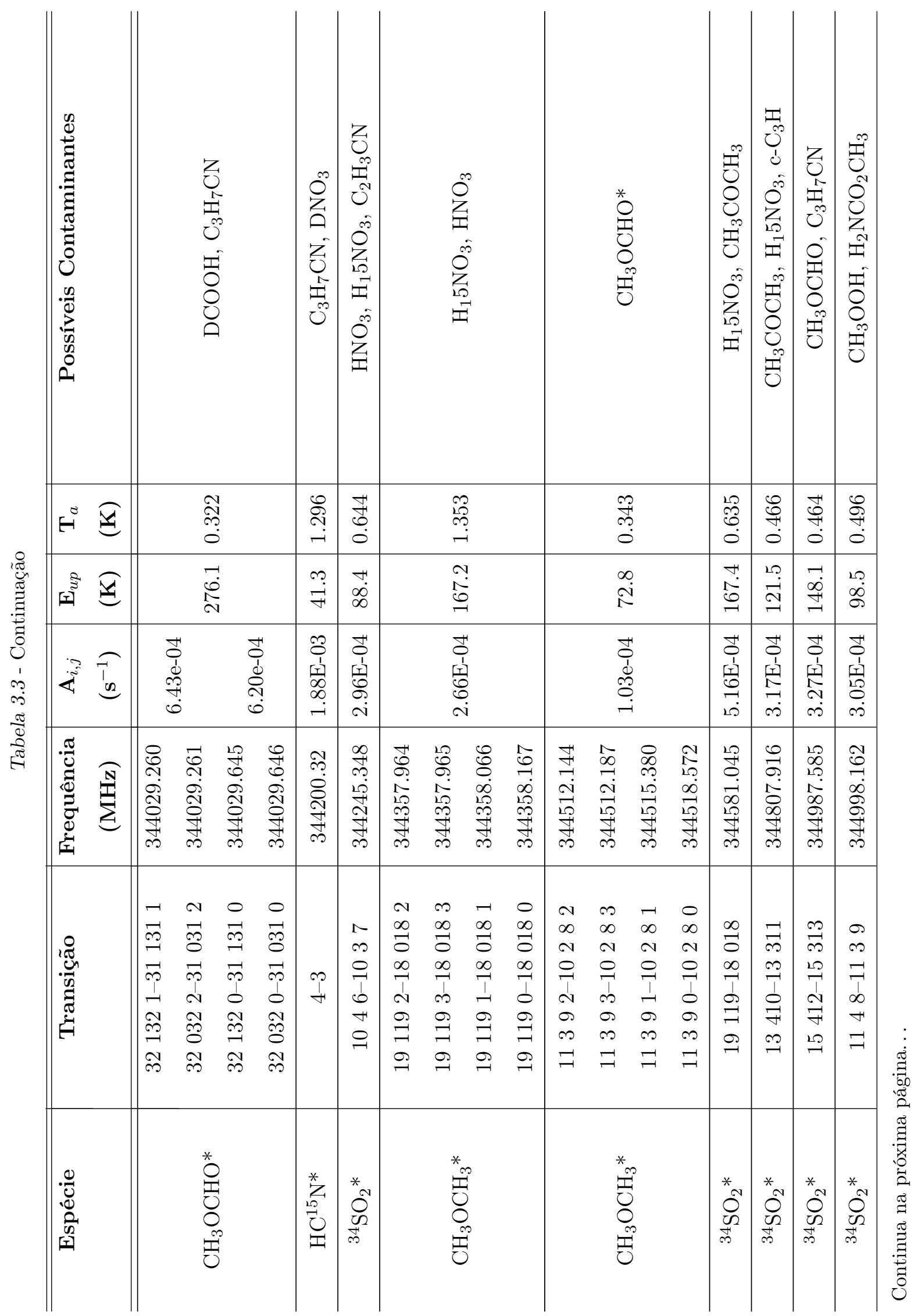




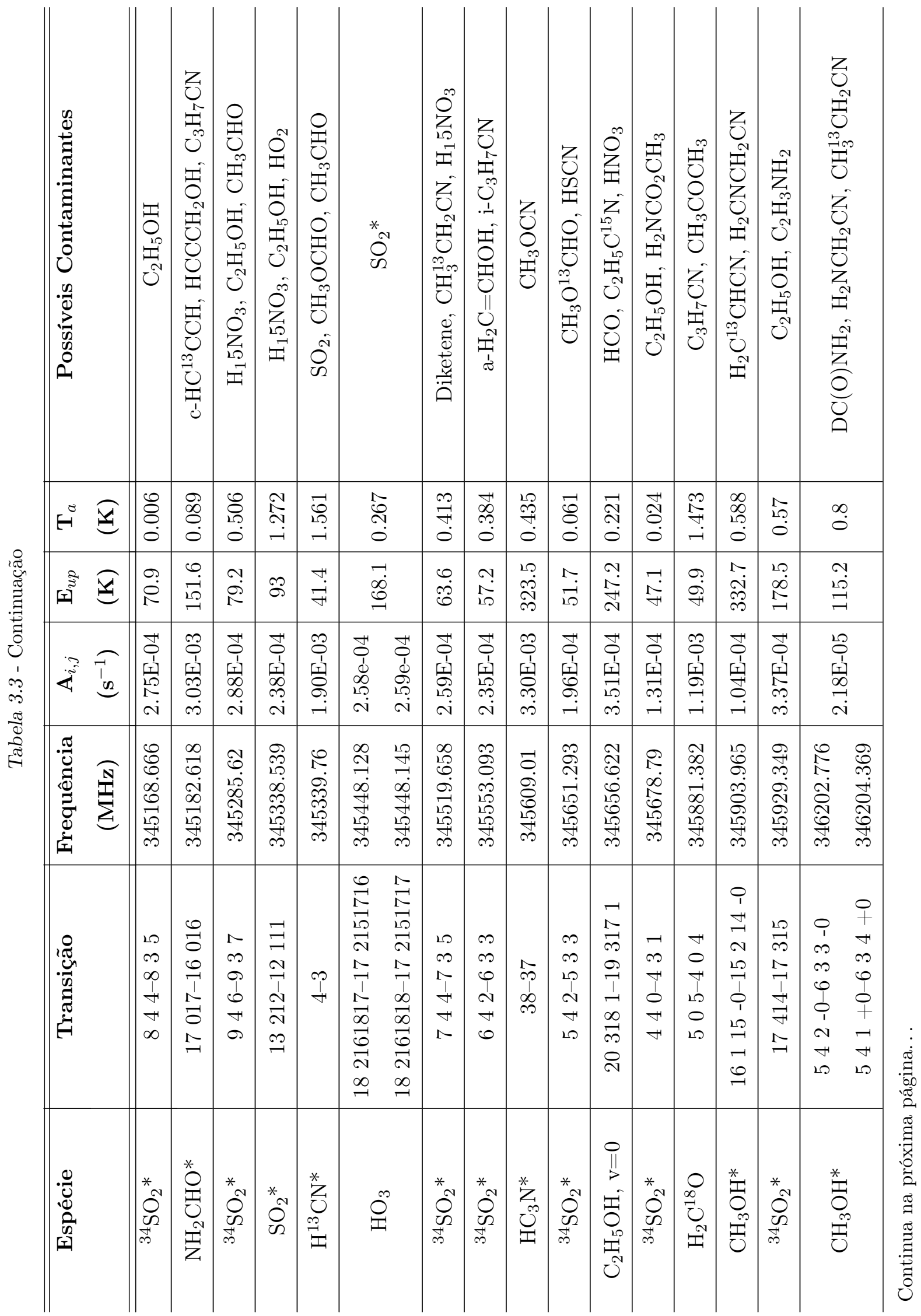




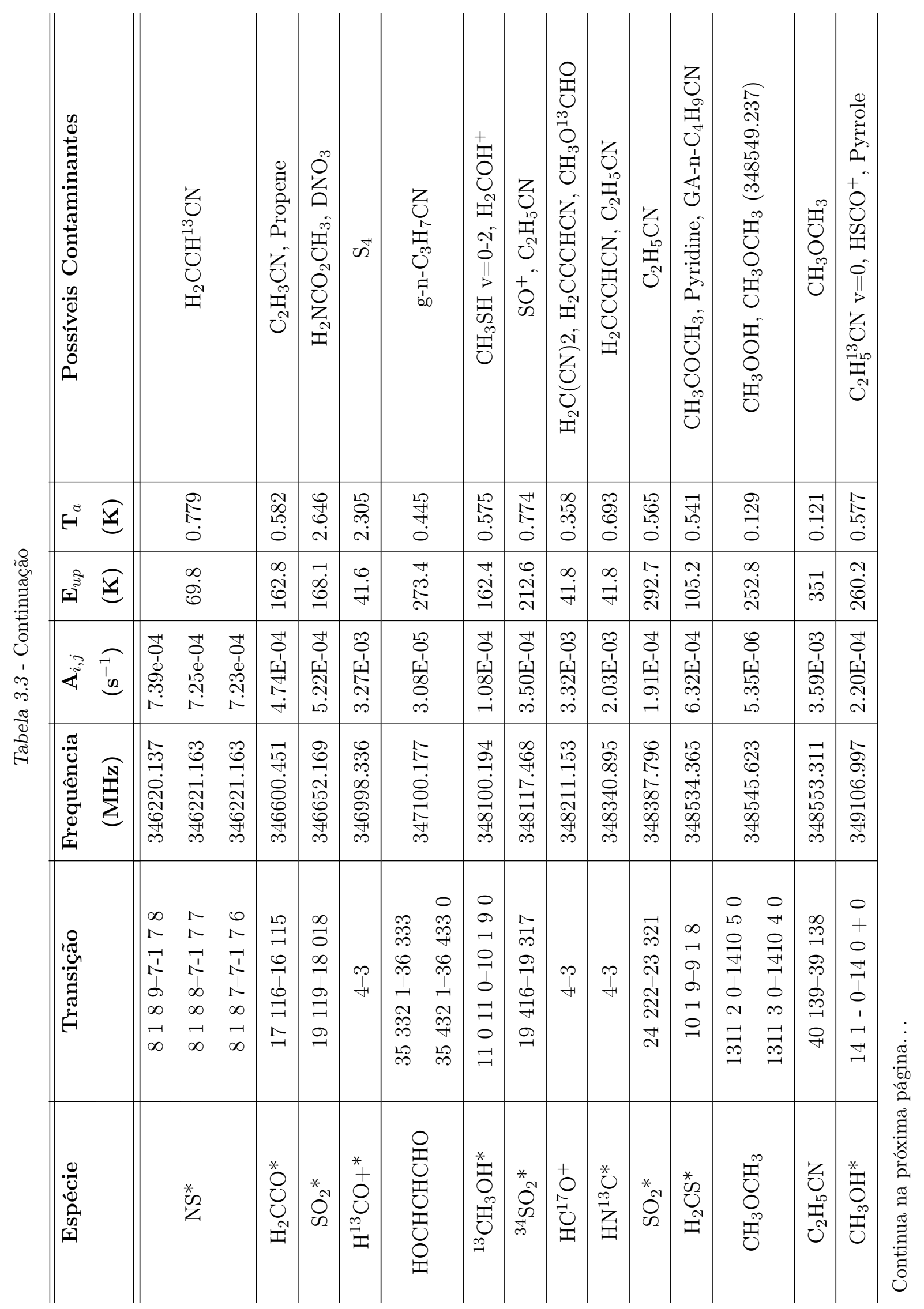




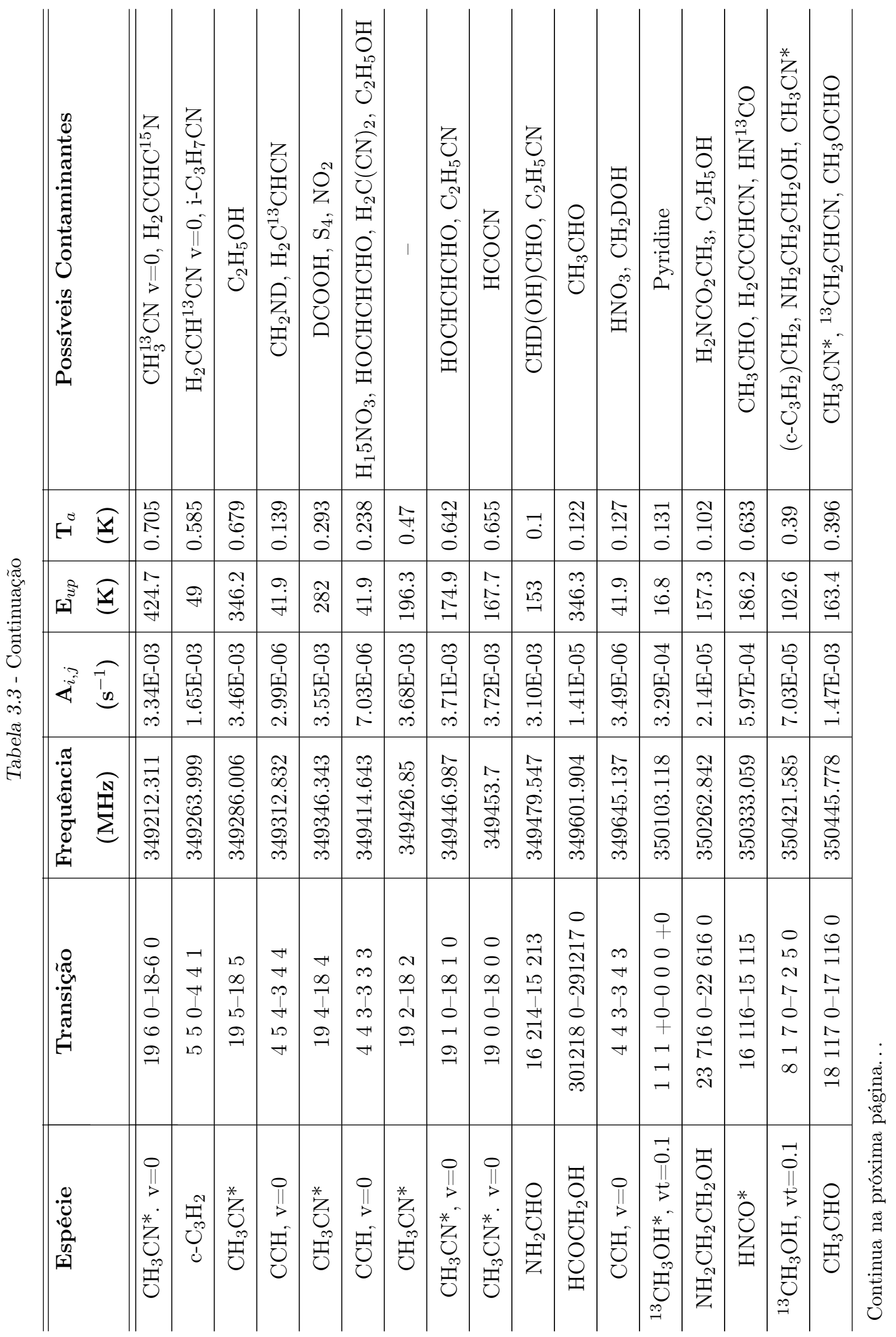




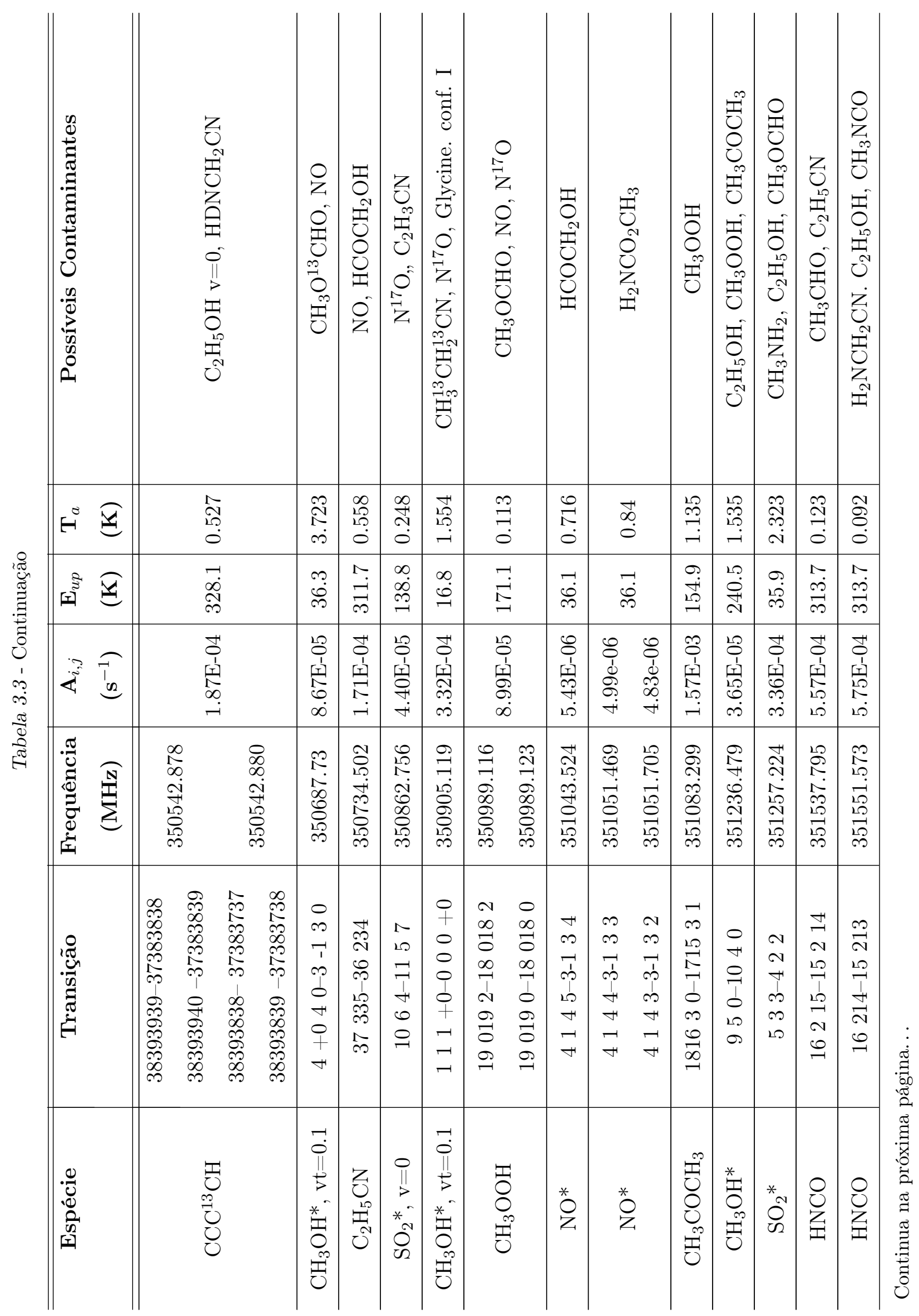




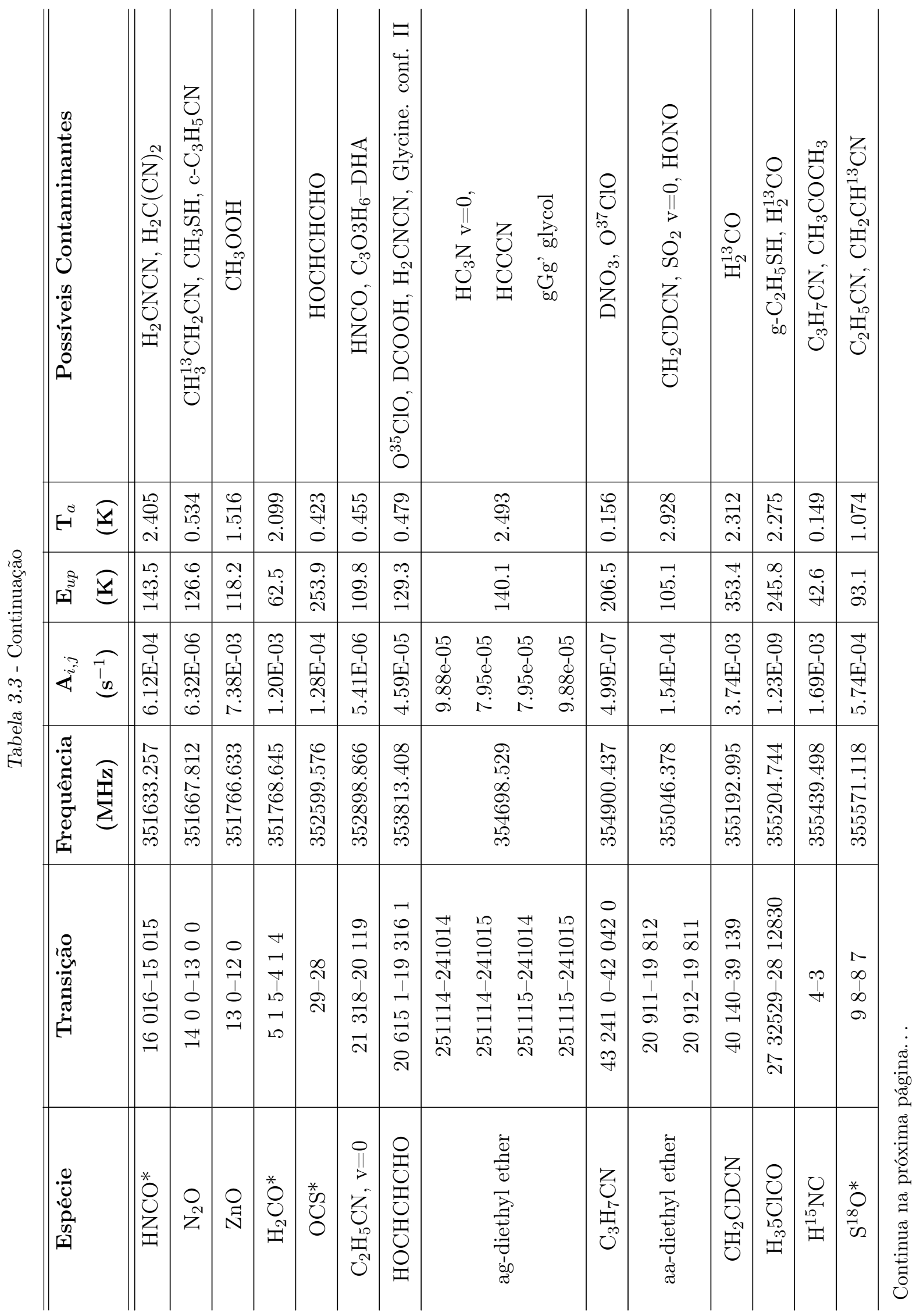




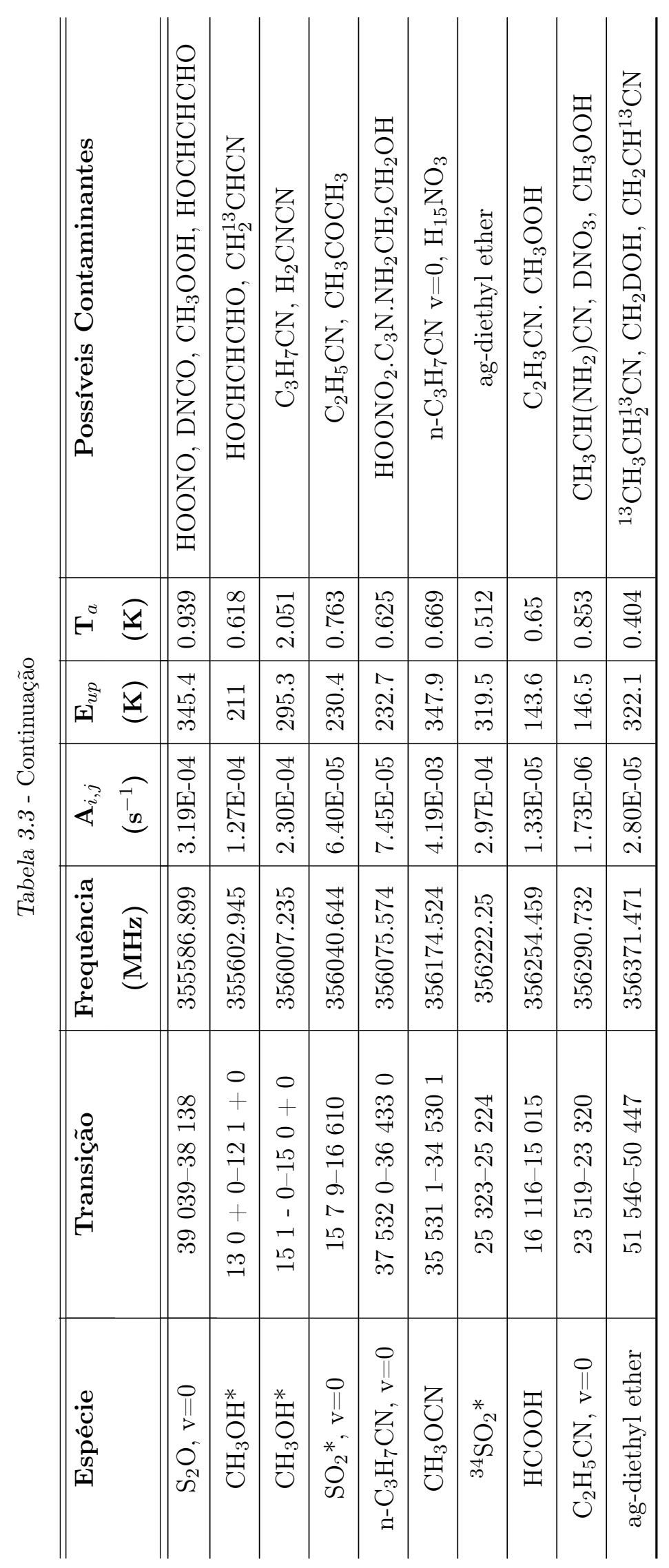




\subsubsection{HNCO em G331: Detecção e Modelagem Química}

Os níveis rotacionais do HNCO podem ser indicados pelos três números quânticos $J_{K_{a}, K_{c}}$, em que $J$ é o momento angular total, e $K_{a}$ e $K_{c}$ representam a projeção de $J$ no eixo de simetria para os casos particulares de prolato e oblato do superior simétrico, respectivamente (e.g. Hocking et al. 1974; Niedenhoff et al. 1995; Zinchenko et al. 2000). Como o HNCO possui uma simetria superior, seus níveis de energia também podem ser representados por um conjunto de escadas com diferentes valores de $K_{a}$.

Graças à alta resolução espectral e à sensibilidade dos dados coletados, diferentes níveis de transição de HNCO foram identificados em G331 e estão apresentados na Tabela 3.4, junto com a referência do Lovas/NIST quando disponível. As 42 transições estão dispostas de acordo com $K_{a}$. As intensidades das linhas variam entre 300 e $1200 \mathrm{mK}$. As suas respectivas áreas, FWHM e velocidades foram calculadas a partir de um ajuste Gaussiano realizado com o pacote CLASS após o ajuste de uma linha de base. Uma exemplificação desse ajuste para duas linhas é mostrada na Figura 3.6. Pode-se perceber que elas estão bem centradas na velocidade sistêmica de G331, corroborando suas detecções.
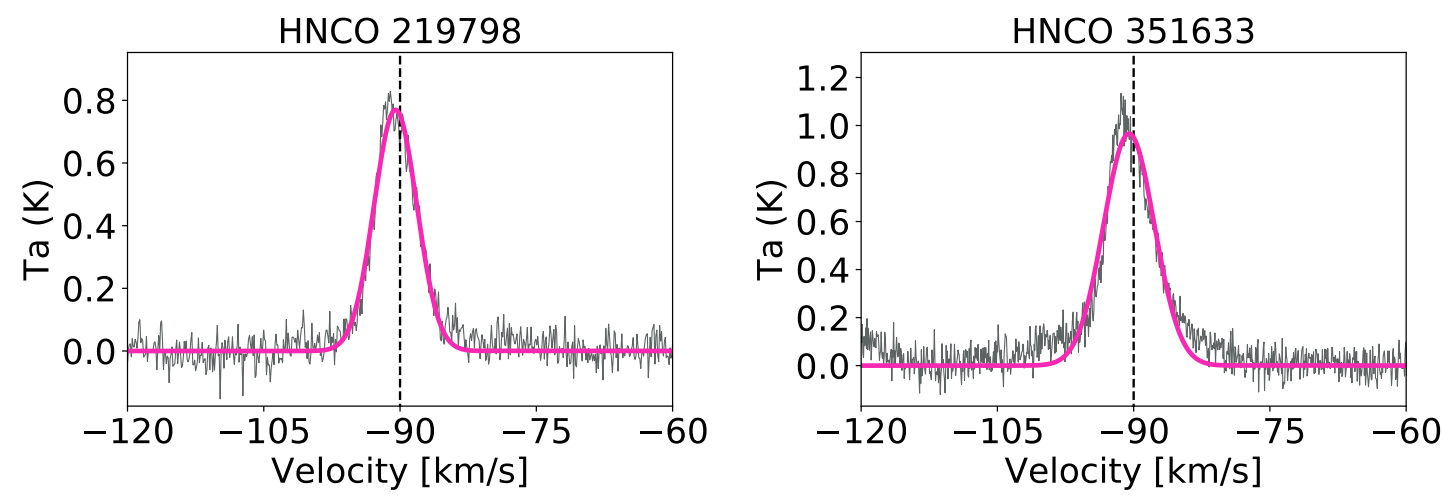

Figura 3.6: Ajuste Gaussiano das linhas de HNCO com frequências em 219798.274 e 351633.257 MHz, realizado com o pacote CLASS e apresentado em rosa. As intensidades das linhas são dadas em temperatura de antena e o eixo x está em velocidade, com cada emissão calibrada por sua frequência de repouso. A linha tracejada preta mostra a velocidade sistêmica de G331.

Todas as transições do HNCO observadas em G331 estão exibidas nas Figuras 3.7, 3.8 e 3.9 para os valores de $K_{a}$ de 0,1 e 2 , respectivamente. As linhas tracejadas verticais indicam as frequências de repouso de cada transição. Emissões dos níveis de $K_{a}=0$ apresentam as linhas mais intensas, enquanto as menos intensas e sobrepostas estão relacionadas com as transições de $K_{a}=2$. No caso de algumas linhas, outras características podem ser vistas nos espectros e podem indicar emissões de outras moléculas. 
Tabela 3.4 - Linhas de HNCO detectadas em G331. As transições estão apresentadas de acordo com os números $k$-ladder $k=0, k=1$ and $k=2$. O fluxo integrado $\left(\mathrm{K} \mathrm{km} \mathrm{s}^{-1}\right)$, a largura da linha $\left(\mathrm{FWHM}, \mathrm{km} \mathrm{s}^{-1}\right)$ e a posição da linha $\left(V_{l s r}, \mathrm{~km} \mathrm{~s}^{-1}\right)$ de cada transição foram estimados por ajuste gaussiano.

\begin{tabular}{|c|c|c|c|c|c|c|c|}
\hline $\begin{array}{l}\text { Frequência } \\
(\mathrm{MHz})\end{array}$ & $\begin{array}{l}\text { Transição } \\
\left(J_{k_{a}}, k_{c}\right)_{u}-\left(J_{k_{a}, k_{c}}\right)_{l}\end{array}$ & $\begin{array}{c}A_{u l} \\
\left(10^{-5} \mathrm{~s}^{-1}\right)\end{array}$ & $\begin{array}{l}E_{u} \\
(\mathrm{~K})\end{array}$ & $\begin{array}{c}\text { Flux } \\
\left(\mathrm{K} \mathrm{km} \mathrm{s}^{-1}\right)\end{array}$ & $\begin{array}{c}\text { FWHM } \\
\left(\mathrm{km} \mathrm{s}^{-1}\right)\end{array}$ & $\begin{array}{c}V_{l s r} \\
\left(\mathrm{~km} \mathrm{~s}^{-1}\right)\end{array}$ & $\begin{array}{c}\text { NIST } \\
\text { referência }\end{array}$ \\
\hline 175843.695 & $808-707$ & 7.43 & 37.98 & $3.71 \pm 0.03$ & $5.91 \pm 0.05$ & $-90.23 \pm 0.02$ & - \\
\hline 197821.461 & $909-808$ & 10.70 & 47.47 & $3.63 \pm 0.03$ & $5.86 \pm 0.07$ & $-90.36 \pm 0.03$ & - \\
\hline 219798.274 & $10010-909$ & 14.70 & 58.02 & $4.53 \pm 0.05$ & $5.53 \pm 0.07$ & $-90.50 \pm 0.03$ & {$[1]$} \\
\hline 241774.032 & $11011-10010$ & 19.60 & 69.62 & $5.35 \pm 0.33$ & $5.71 \pm 0.44$ & $-90.47 \pm 0.17$ & [3] \\
\hline 263748.625 & $12012-11011$ & 25.60 & 82.28 & $4.86 \pm 0.08$ & $5.95 \pm 0.12$ & $-90.66 \pm 0.04$ & [1] \\
\hline 285721.951 & $13013-12012$ & 32.60 & 95.99 & $7.90 \pm 0.10$ & $6.70 \pm 0.10$ & $-90.76 \pm 0.04$ & - \\
\hline 307693.905 & $14014-13013$ & 40.90 & 110.76 & $7.24 \pm 0.03$ & $6.07 \pm 0.03$ & $-90.56 \pm 0.01$ & - \\
\hline 329664.367 & $15015-14014$ & 50.40 & 126.58 & $8.02 \pm 0.07$ & $6.42 \pm 0.08$ & $-90.64 \pm 0.03$ & - \\
\hline 351633.257 & $16016-15015$ & 61.30 & 143.46 & $6.79 \pm 0.06$ & $6.60 \pm 0.08$ & $-90.51 \pm 0.03$ & [7] \\
\hline 175189.027 & $818-717$ & 7.16 & 81.11 & $1.01 \pm 0.01$ & $5.08 \pm 0.07$ & $-90.93 \pm 0.03$ & - \\
\hline 176472.191 & $817-716$ & 7.31 & 81.39 & $0.76 \pm 0.01$ & $4.60 \pm 0.10$ & $-90.97 \pm 0.03$ & - \\
\hline 197085.416 & $919-818$ & 10.30 & 90.57 & $0.68 \pm 0.01$ & $4.42 \pm 0.08$ & $-90.99 \pm 0.03$ & - \\
\hline 198528.881 & $918-817$ & 10.50 & 90.92 & $1.01 \pm 0.01$ & $5.60 \pm 0.10$ & $-90.85 \pm 0.04$ & - \\
\hline 218981.009 & $10110-919$ & 14.20 & 101.08 & $1.75 \pm 0.05$ & $5.73 \pm 0.21$ & $-90.67 \pm 0.07$ & {$[1]$} \\
\hline 220584.751 & $1019-918$ & 14.50 & 101.50 & $1.73 \pm 0.06$ & $6.29 \pm 0.31$ & $-90.79 \pm 0.10$ & {$[2]$} \\
\hline 240875.727 & $11111-10110$ & 19.00 & 112.64 & $1.56 \pm 0.03$ & $5.48 \pm 0.12$ & $-90.91 \pm 0.05$ & [3] \\
\hline 242639.704 & $11110-1019$ & 19.50 & 113.15 & $1.74 \pm 0.04$ & $5.52 \pm 0.13$ & $-90.67 \pm 0.05$ & [3] \\
\hline 262769.477 & $12112-11111$ & 24.80 & 125.25 & $1.80 \pm 0.10$ & $5.80 \pm 0.50$ & $-90.80 \pm 0.20$ & - \\
\hline 264693.655 & $12111-11110$ & 25.40 & 125.85 & $1.59 \pm 0.04$ & $5.21 \pm 0.19$ & $-90.85 \pm 0.07$ & [4] \\
\hline 284662.172 & $13113-12112$ & 31.70 & 138.91 & $2.13 \pm 0.03$ & $5.30 \pm 0.10$ & $-90.86 \pm 0.04$ & - \\
\hline 286746.514 & $13112-12111$ & 32.40 & 139.61 & $2.53 \pm 0.02$ & $5.53 \pm 0.06$ & $-90.91 \pm 0.02$ & - \\
\hline 306553.733 & $14114-13113$ & 39.70 & 153.62 & $2.13 \pm 0.02$ & $5.91 \pm 0.07$ & $-90.86 \pm 0.02$ & - \\
\hline 308798.184 & $14113-13112$ & 40.60 & 154.43 & $2.74 \pm 0.02$ & $5.83 \pm 0.07$ & $-90.86 \pm 0.02$ & - \\
\hline 330848.569 & $15114-14113$ & 50.10 & 170.31 & $4.64 \pm 0.05$ & $7.40 \pm 0.10$ & $-90.56 \pm 0.04$ & {$[5]$} \\
\hline 350333.059 & $16116-15115$ & 59.70 & 186.20 & $3.24 \pm 0.04$ & $5.84 \pm 0.09$ & $-90.81 \pm 0.03$ & [6] \\
\hline 352897.581 & $16115-15114$ & 61.00 & 187.25 & $2.55 \pm 0.03$ & $5.25 \pm 0.08$ & $-90.81 \pm 0.03$ & - \\
\hline 175791.267 & $827-726$ & 6.65 & 208.25 & $0.37 \pm 0.01$ & $7.60 \pm 0.30$ & $-92.20 \pm 0.10$ & - \\
\hline 175792.957 & $826-725$ & 6.65 & 208.25 & $0.41 \pm 0.03$ & $7.90 \pm 0.60$ & $-89.40 \pm 0.30$ & - \\
\hline 197762.939 & $928-827$ & 9.66 & 217.74 & $0.51 \pm 0.02$ & $9.10 \pm 0.40$ & $-92.50 \pm 0.20$ & - \\
\hline 197765.372 & $927-826$ & 9.66 & 217.74 & $0.52 \pm 0.05$ & $9.20 \pm 0.40$ & $-88.8 \pm 0.10$ & - \\
\hline $219733.850^{\dagger}$ & $1029-928$ & 13.50 & 228.29 & - & - & - & [3] \\
\hline $219737.193^{\dagger}$ & $1028-927$ & 13.50 & 228.29 & - & - & - & [3] \\
\hline $241703.852^{a}$ & $11210-1029$ & 18.10 & 239.89 & - & - & - & - \\
\hline 241708.312 & $1129-1028$ & 18.10 & 239.89 & $0.67 \pm 0.04$ & $4.90 \pm 0.40$ & $-91.20 \pm 0.10$ & - \\
\hline 285640.923 & $13212-12211$ & 30.40 & 266.25 & $0.63 \pm 0.05$ & $6.40 \pm 0.60$ & $-91.60 \pm 0.20$ & - \\
\hline 285648.301 & $13211-12210$ & 30.40 & 266.25 & $0.53 \pm 0.04$ & $6.20 \pm 0.60$ & $-91.30 \pm 0.1$ & - \\
\hline 307607.799 & $14213-13212$ & 38.20 & 281.01 & $0.64 \pm 0.02$ & $7.70 \pm 0.40$ & $-91.70 \pm 0.1$ & - \\
\hline 307617.020 & $14212-13211$ & 38.20 & 281.01 & $0.47 \pm 0.02$ & $6.10 \pm 0.30$ & $-90.70 \pm 0.10$ & - \\
\hline 329573.452 & $15214-14213$ & 47.20 & 296.83 & $0.57 \pm 0.05$ & $5.50 \pm 0.60$ & $-91.30 \pm 0.20$ & - \\
\hline 329584.800 & $15213-14212$ & 47.20 & 296.83 & $0.64 \pm 0.08$ & $7.00 \pm 1.00$ & $-90.8 \pm 0.30$ & - \\
\hline 351537.795 & $16215-15214$ & 57.50 & 313.70 & $0.72 \pm 0.07$ & $5.80 \pm 0.70$ & $-91.60 \pm 0.20$ & - \\
\hline 351551.573 & $16214-15213$ & 57.50 & 313.70 & $0.46 \pm 0.04$ & $5.70 \pm 0.70$ & $-90.80 \pm 0.20$ & - \\
\hline
\end{tabular}

Notas: ${ }^{\dagger}$ linhas fracas e não-resolvidas. ${ }^{a}$ Linha afetada por provável emissão dominante de $\mathrm{CH}_{3} \mathrm{OH}$. Referências: [1] Armstrong e Loren (1984); [2] Loren e Mundy (1984); [3] Sutton et al. (1985); [4] Greaves e White (1991); [5] Sutton et al. (1991); [6] MacDonald et al. (1996); [7] Jewell et al. (1989). 

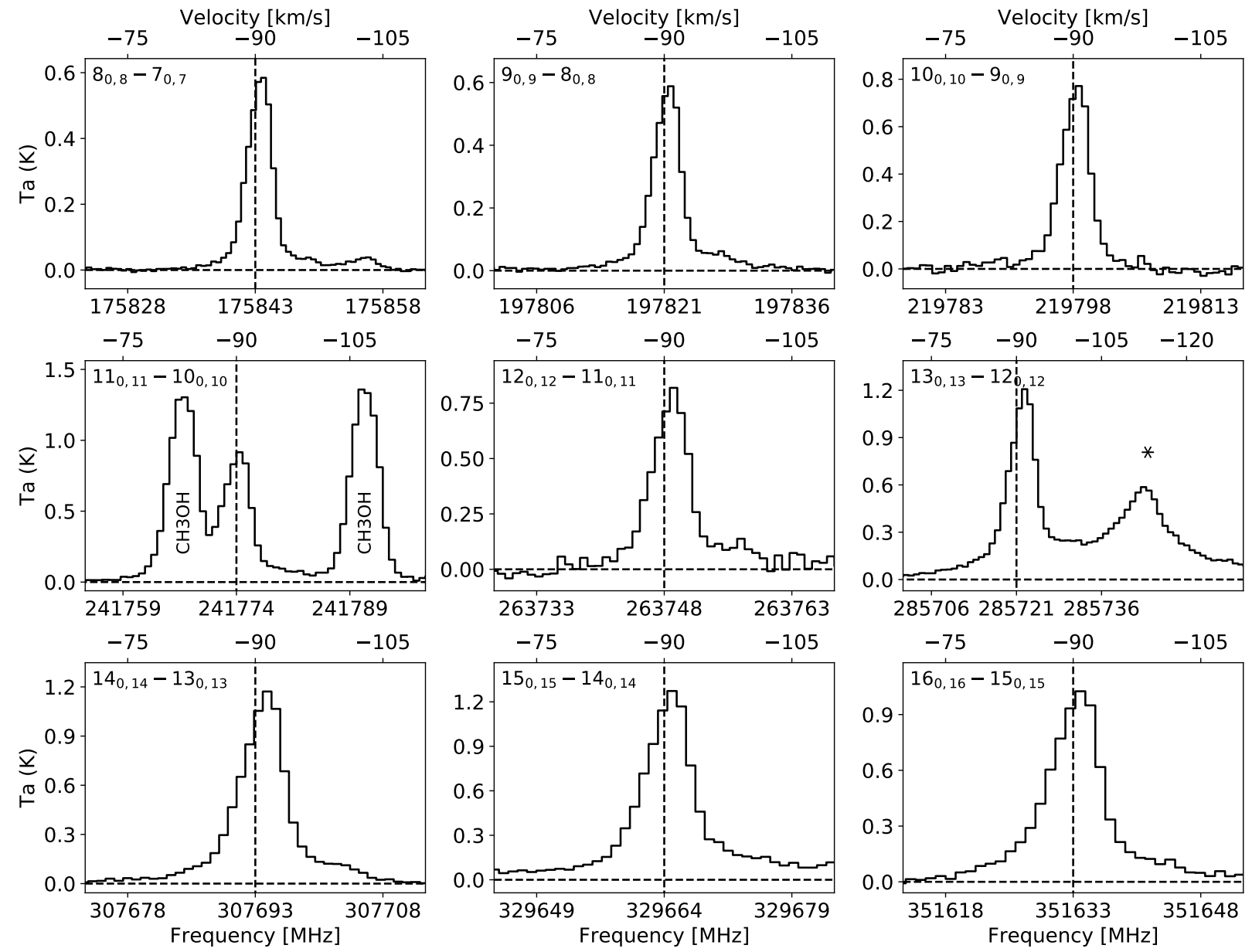

Figura 3.7: Linhas de emissão do $\mathrm{HNCO}$ para $K_{a}=0$ com uma resolução de $1 \mathrm{~km} / \mathrm{s}$. As intensidades das linhas são dadas em temperatura de antena e os eixos x estão em frequência (inferior) e velocidade (superior). As transições estão indicadas em cada gráfico e as linhas verticais tracejadas representam a velocidade sistêmica de G331. As linhas tracejadas horizontais mostram a linha de base.

Na Figura 3.7, a transição do HNCO de 241774.032 MHz está cercada por duas emissões indicadas no gráfico que devem ser, provavelmente, de $\mathrm{CH}_{3} \mathrm{OH}$. Essa molécula também foi detectada por Loren e Mundy (1984) na Nuvem Molecular de Orion 1 (OMC-1) e suas frequências e transições são $241767.247 \mathrm{MHz}\left(5_{-1,5}-4_{-1,4}\right.$ E) e $241791.367 \mathrm{MHz}\left(5_{0,5}-\right.$ $4_{0,4} \mathrm{~A}+$ ). Outra transição de HNCO, em $285721.951 \mathrm{MHz}$, também apresenta uma emissão vizinha que está representada por um asterisco no gráfico. Essa emissão larga não possui referência no Lovas/NIST. Uma identificação preliminar feita no CDMS, da mesma forma como realizado anteriormente, sugere que essa emissão deve ser de $\mathrm{SO}_{2}$ em $285743.589 \mathrm{MHz}$ $\left(17_{3,15}-17_{2,16}\right)$. Alguns possíveis contaminantes poderiam ser, por exemplo, $\mathrm{HCCCH}_{2} \mathrm{NH}_{2}$, $\mathrm{OC}(\mathrm{CN})_{2}$ e $\mathrm{H}_{2} \mathrm{CNCH}_{2} \mathrm{CN}$, embora não seja esperada a deteç̧ão de tais moléculas no ISM devido à sua maior complexidade. 

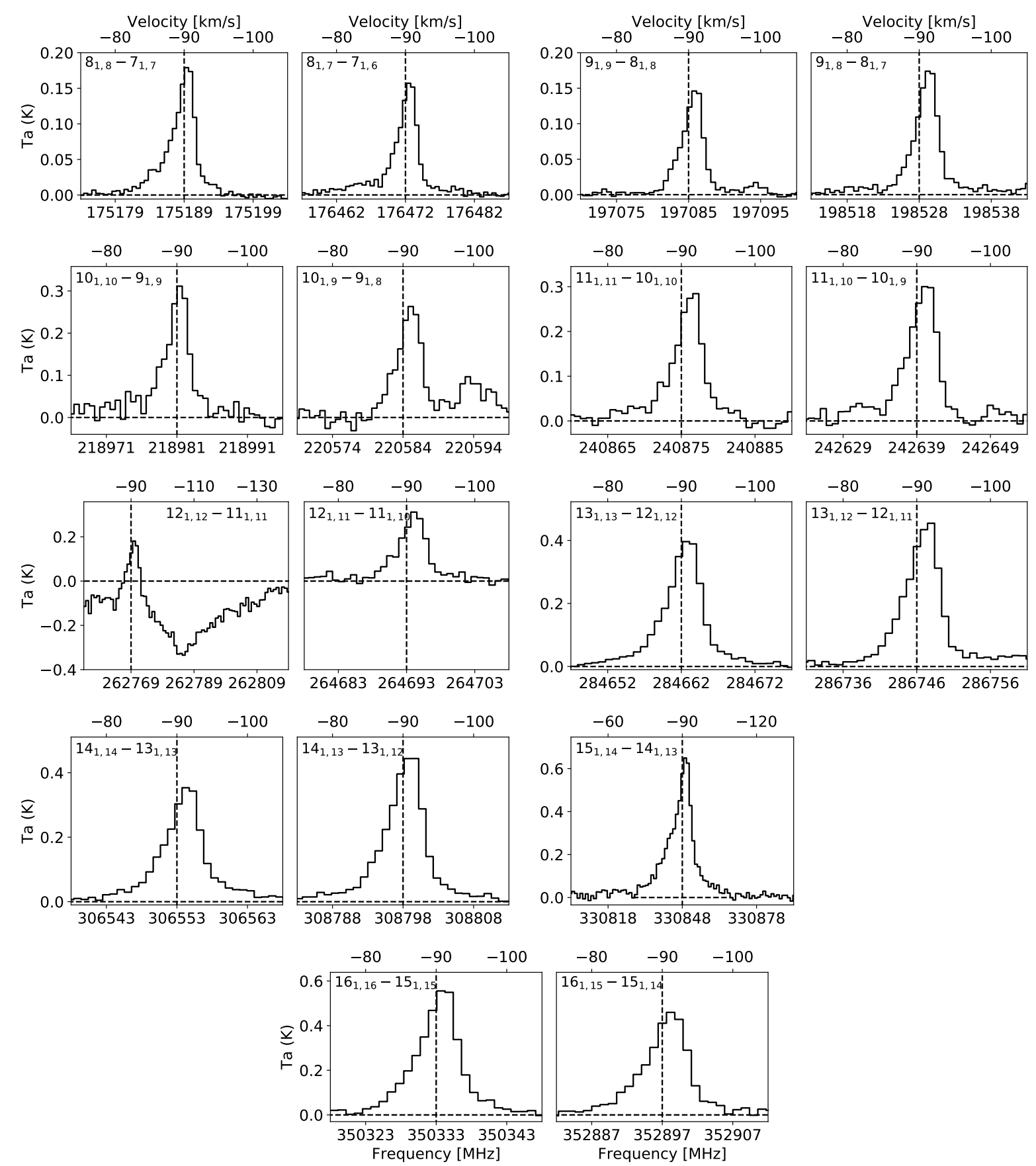

Figura 3.8: Linhas de emissão do HNCO para $K_{a}=1 \mathrm{com}$ uma resolução de $1 \mathrm{~km} / \mathrm{s}$. As intensidades das linhas são dadas em temperatura de antena e os eixos x estão em frequência (inferior) e velocidade (superior). As transições estão indicadas em cada gráfico e as linhas verticais tracejadas representam a velocidade sistêmica de G331. As linhas tracejadas horizontais mostram a linha de base. 

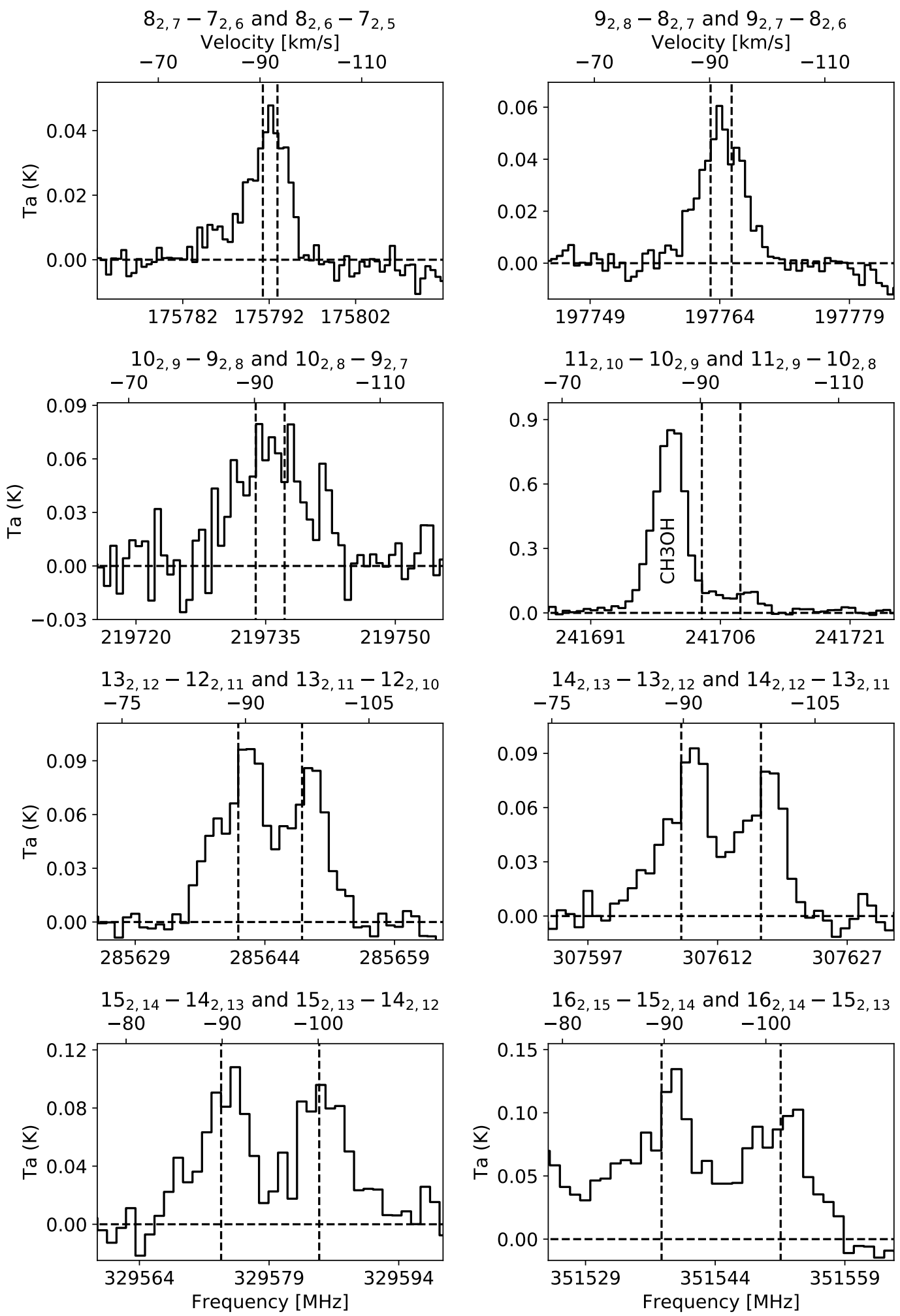

Figura 3.9: Linhas de emissão do HNCO para $K_{a}=2$ com uma resolução de $1 \mathrm{~km} / \mathrm{s}$. As intensidades das linhas são dadas em temperatura de antena e os eixos x estão em frequência (inferior) e velocidade (superior). As transições estão indicadas em cada gráfico e as linhas verticais tracejadas representam a velocidade sistêmica de G331 (centradas nas primeiras transições de cada gráfico). As linhas tracejadas horizontais mostram a linha de base. 

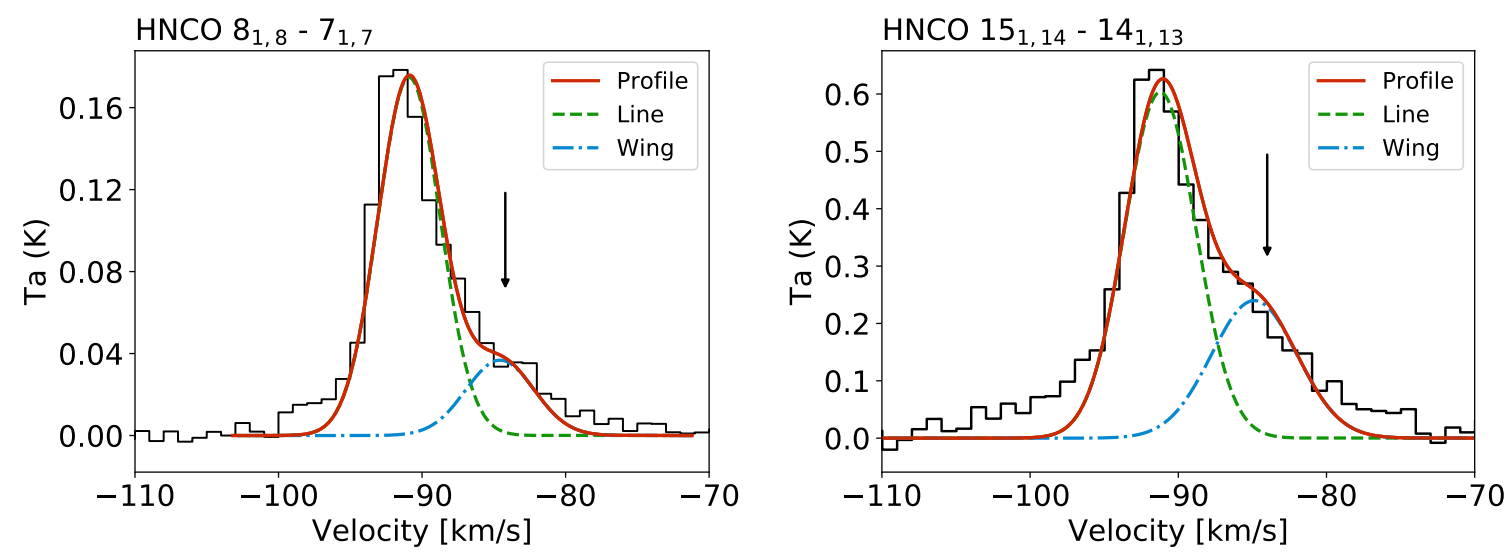

Figura 3.10: Emissões de HNCO em 175189.027 (esquerda) e $330848.569 \mathrm{MHz}$ (direita), com uma resolução de $1 \mathrm{~km} \mathrm{~s}^{-1}$. As respectivas transições estão escritas no título de cada gráfico. Os ajustes Gaussianos mostram o perfil total de cada linha (em vermelho) juntamente com a contribuição da emissão do HNCO (em verde, representada como Line) e de uma assimetria/característica secundária (em azul, descrita como Wing e indicada pela seta).

Já na Figura 3.8, no gráfico da linha de HNCO em $220584.751 \mathrm{MHz}$, também está presente outra emissão em uma frequência maior que pode ser $\mathrm{CH}_{3} \mathrm{CN}$. Essa transição também foi observada em OMC-1 (transição $12_{6}-11_{6}$, Loren e Mundy, 1984). Nessa figura, também se pode notar um perfil de absorção ao lado da emissão de HNCO em 262769.477 MHz que prejudica toda a análise dessa região espectral. Tal absorção deve corresponder a vapor d'água e já foi abordada em estudos sobre transparência atmosférica em Chajnantor ${ }^{8}$.

Ainda na mesma figura, as linhas de HNCO em 175189.027 e $330848.569 \mathrm{MHz}$ podem estar contaminadas pela emissão de outras moléculas. As possíveis espécies contaminantes do primeiro caso poderiam ser HCOCN em $175184.4265 \mathrm{MHz}\left(30_{1,29}-30_{0,30}\right)$ e $\mathrm{CH}_{2} \mathrm{ND}$ em 175185.023 MHz $\left(3_{0,3}-2_{0,2}\right)$. Em relação à segunda emissão de HNCO, o HCOCN também poderia estar contribuindo em $330845.855 \mathrm{MHz}\left(10_{4,6}-11_{3,9}\right)$. Além dele, outra possibilidade seria o $\mathrm{CH}_{3} \mathrm{OCH}_{3}$ com até quatro emissões nas frequências $330844.866 \mathrm{MHz}$ $\left(1711_{6,3}-1812_{6,3}\right), 330845.900 \mathrm{MHz}\left(1711_{6,5}-1812_{7,5}\right.$ e $\left.1711_{7,5}-1812_{6,5}\right)$ e $330846.933 \mathrm{MHz}$ $\left(1711_{7,3}-1812_{7,3}\right)$.

Ambas transições podem ser melhor visualizadas na Figura 3.10, que apresenta um ajuste Gaussiano do perfil de cada linha supondo-se duas emissões moleculares diferentes. Nos dois gráficos, o HNCO corresponde à emissão mais intensa (denominada como "Line"), que domina o fluxo da linha em cerca de 80\%. As emissões mais fracas ("Wing")

\footnotetext{
8 https://almascience.eso.org/about-alma/atmosphere-model
} 


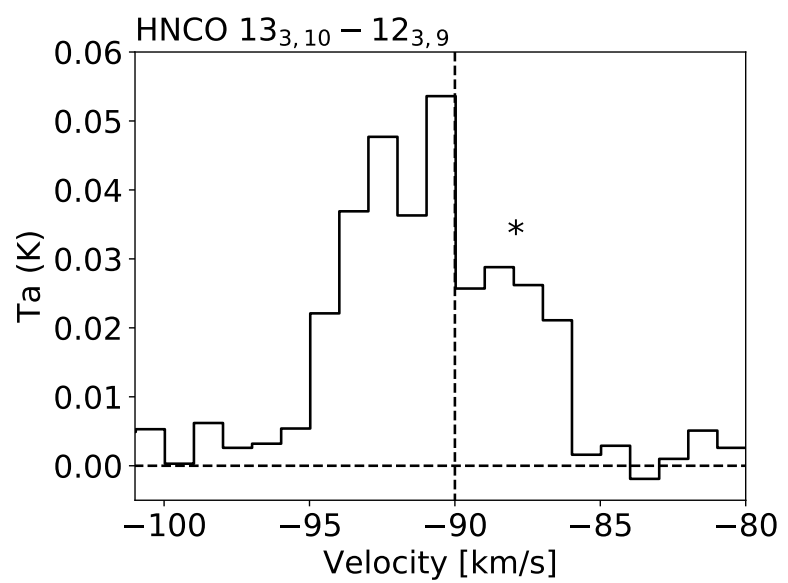

Figura 3.11: Emissão de HNCO $\left(13_{3,10}\right.$ - 12 $\left.2_{3,9}\right)$ em $285541.575 \mathrm{MHz}$, suavizada a uma resolução de $1 \mathrm{~km} \mathrm{~s}^{-1}$. As linhas tracejadas horizontal e vertical representam a linha de base e a velocidade da fonte, respectivamente. Uma emissão fraca próxima à linha de HNCO está indicada por um asterisco.

podem estar relacionadas aos possíveis contaminantes. Contudo, outro fator poderia estar influenciando os perfis dessas emissões de HNCO. Essa cauda em velocidades maiores, mais evidente no caso da linha em 330848.569 MHz, também pode ser causada pelo outflow. Esse alargamento das asas da linha é bem usual em um outflow e já foi observado em outras emissões moleculares em G331 (e.g. Duronea et al., 2019; Hervías-Caimapo et al., 2019). Uma explicação é o aumento na velocidade de expansão do outflow com a distância do centro da fonte (Velilla Prieto et al., 2015).

Por fim, no caso das transições com $K_{a}=2$, elas apresentam linhas bem menos intensas e seus dubletos estão sobrepostos. Nesse cenário, linhas contaminantes podem ser, simplesmente, efeitos observacionais de ruído, ao invés de emissões moleculares. A única exceção é a emissão dominante de $\mathrm{CH}_{3} \mathrm{OH}$ (Figura 3.9), em que estão presentes as transições de HNCO em 241703.852 e 241708.312 MHz. A transição do $\mathrm{CH}_{3} \mathrm{OH}$ ocorre na frequência de 241700.168 MHz $\left(5_{0,5}-4_{0,4} \mathrm{E}\right)$, já detectada em OMC-1 (Loren e Mundy, 1984).

Uma busca por transições do HNCO com $K_{a}=3$ também foi realizada, embora elas estejam relacionas a condições físicas de alta excitação, que não são esperadas em G331. Uma possível evidência desse caso pode ser vista na linha marginal de HNCO em 285541.575 MHz $\left(13_{3,10}-12_{3,9}\right.$, Figura 3.11). Essa linha está no limiar da velocidade de G331 e possui outra emissão contaminante. Tal emissão, que também não consta no Lovas/NIST (ainda não foi observada no ISM), poderia ser de $\mathrm{CH}_{3} \mathrm{OCN}$ em 285539.098 MHz, DNCO em 285539.531 MHz ou acetona $\left(\mathrm{CH}_{3}\right)_{2} \mathrm{CO}$ em 285542.349 MHz. 


\subsubsection{Diagrama rotacional e propriedades derivadas}

Após a identificação das emissões do $\mathrm{HNCO}$, foram construídos diagramas rotacionais para estimar as condições físicas traçadas por essa molécula em G331. Esse tipo de técnica, amplamente descrita e discutida em Goldsmith e Langer (1999), é uma importante ferramenta para se inferir propriedades de fontes astrofísicas a partir de emissões de linha moleculares. Uma breve apresentação dessa abordagem será feita a seguir.

Diagrama rotacionais consistem, basicamente, de um gráfico da densidade de coluna pelo peso estatístico de uma série níveis de energia molecular, em função de sua energia acima do estado fundamental. Caso o equilíbrio termodinâmico local (LTE, em inglês) possa ser aplicado, as populações dos níveis podem ser representadas pela distribuição de Boltzmann, de forma que o diagrama rotacional possa ser descrito pela Equação 3.2. Além disso, se as linhas de emissão também puderem ser consideradas opticamente finas, a função graficada é uma linha reta com uma inclinação de $1 / \mathrm{T}_{\text {rot }}$. Dessa forma, pode-se derivar a temperatura rotacional da molécula, que deve ser igual à temperatura cinética do gás em condições LTE.

$$
\ln \left(\frac{N_{u, t h i n}}{g_{u}}\right)=\ln \left(\frac{3 k_{B} W}{8 \pi^{3} \nu S_{u l} \mu^{2}}\right)=\ln \left(\frac{N}{Z}\right)-\frac{E_{u}}{k_{B} T_{\text {rot }}}
$$

onde $N_{u}$ é a densidade de coluna do nível superior, $g_{u}$ a degenerescência do nível superior, $k_{B}$ é a constante de Boltzmann, $W$ a intensidade integrada na velocidade da transição (Equação 3.3, em que $\mathrm{T}_{a}$ é a temperatura de antena), $\nu$ a frequência de repouso da linha, $\mathrm{S}_{u l}$ a força da linha da transição, $\mu$ o momento de dipolo da transição, $Z$ a função de partição e $E_{u}$ a energia do nível superior da transição.

$$
W=\int T_{a} d V
$$

Para se construir um diagrama rotacional, também pode ser preciso levar em consideração um fator de diluição do feixe associado a uma região emissora pontual. Essa correção pode ser realizada com a adição do termo $\ln \left(\Delta \Omega_{a} / \Delta \Omega_{s}\right)$ no lado direito da Equação 3.2, de acordo com (Goldsmith e Langer, 1999), onde $\Delta \Omega_{a}$ é o ângulo sólido da antena e $\Delta \Omega_{s}$ é o ângulo sólido da antena da fonte. No caso de G331, um tamanho médio de 5 arcsec foi assumido, baseado no trabalho de Duronea et al. (2019).

Por fim, no caso de transições opticamente espessas, a correção da profundidade óptica 

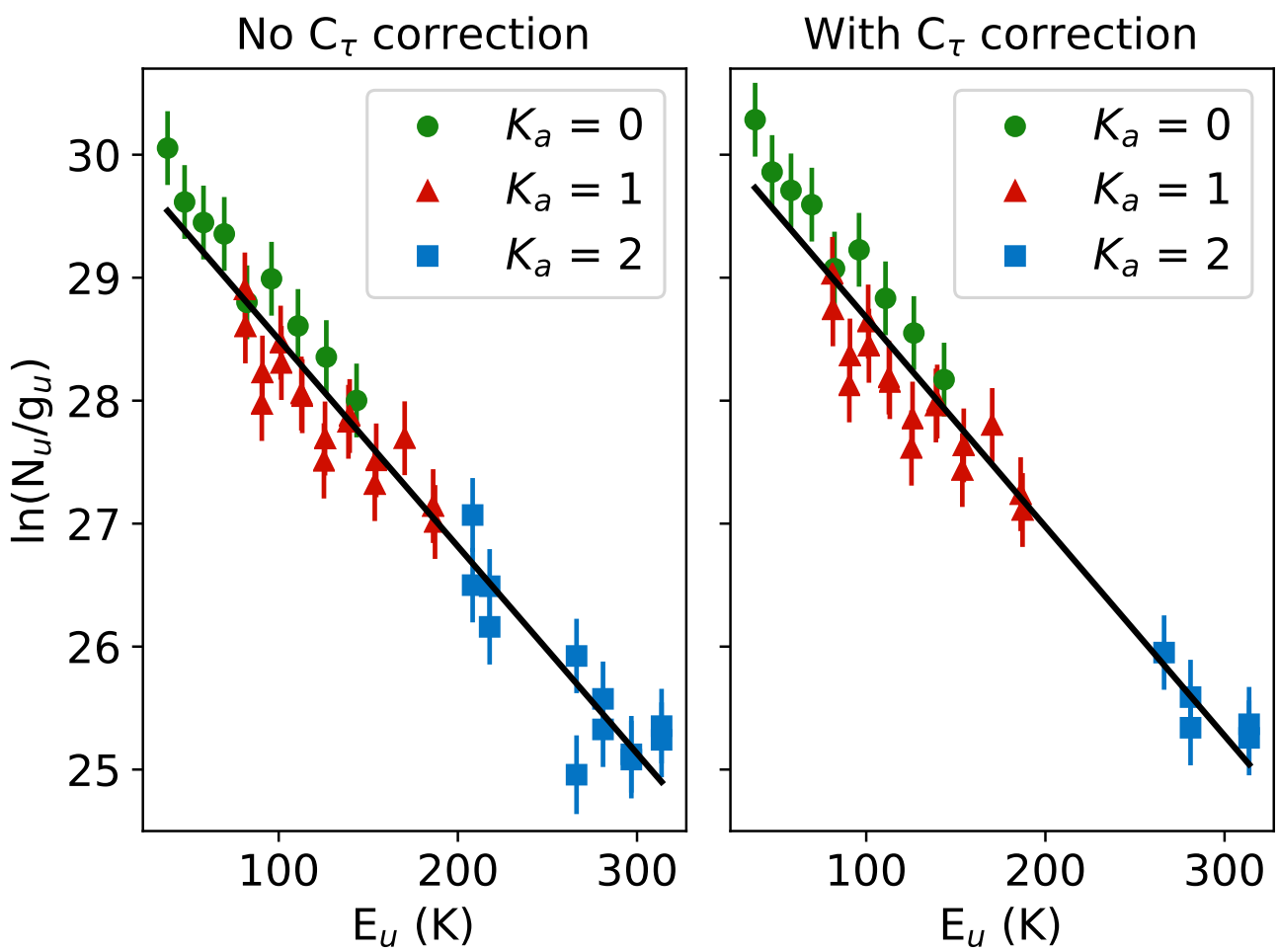

Figura 3.12: Diagramas rotacionas de HNCO para uma correção da diluição do feixe de 5 arcsec. O segundo gráfico também mostra as linhas com a correção de opacidade. Linhas que apresentam emissões contaminantes ou sobrepostas foram dispensadas. O melhor ajuste linear para cada caso resultou em, respectivamente, $T_{e x}=59.4 \pm 2.3 \mathrm{~K} \mathrm{e} N=(3.1 \pm 0.4) \times 10^{15} \mathrm{~cm}^{-2}$; e $T_{e x}=58.8 \pm 2.7 \mathrm{~K}$ e $N=(3.7 \pm$ $0.5) \times 10^{15} \mathrm{~cm}^{-2}$. Os diferentes valores de $K_{a}$ também estão indicados.

pode ser feita com o fator de correção (Equação 3.4) de acordo com a Equação 3.5 (como derivado em Goldsmith e Langer, 1999). Os diagramas rotacionais para um tamanho da fonte de 5 arcsec, com e sem a correção de opacidade, podem ser vistos na Figura 3.12.

$$
C_{\tau}=\frac{\tau}{1-e^{-\tau}}
$$

onde $\tau$ é a profundidade óptica.

$$
\frac{N_{u, t h i c k}}{g_{u}}=\frac{N_{u, t h i n}}{g_{u}} C_{\tau}
$$

De acordo com o melhor ajuste linear para os diagramas, a densidade de coluna e temperatura de excitação derivadas foram de $N(\mathrm{HNCO})=(3.1 \pm 0.4) \times 10^{15} \mathrm{~cm}^{-2} \mathrm{e}$ $T_{e x}=59.4 \pm 2.3 \mathrm{~K}$ para o diagrama sem correção de opacidade, e de $N(\mathrm{HNCO})=(3.7 \pm$ $0.5) \times 10^{15} \mathrm{~cm}^{-2}$ e $T_{e x}=58.8 \pm 2.7 \mathrm{~K}$ para o diagrama com a correção. Os valores de $\chi^{2}$ reduzidos de cada ajuste foram de 1.12 e 1.21, respectivamente. Além de ambos os ajustes revelarem resultados semelhantes, essas temperaturas de cerca de $60 \mathrm{~K}$ sugerem que as 
moléculas de HNCO devem estar concentradas nas regiões mais externas e frias de G331, de acordo com a estrutura prevista desse objeto (Merello et al., 2013; Hervías-Caimapo et al., 2019; Duronea et al., 2019).

Churchwell et al. (1986) realizaram observações do $\operatorname{HNCO}\left(K_{a}=0,1\right.$ e 2) na nuvem molecular gigante de Sagittarius B2 (Sgr B2) e indicaram que transições com $\mathrm{E}_{u}>40 \mathrm{~K}$ requerem uma temperatura de excitação aproximada de $70 \mathrm{~K}$. Além disso, também foi demonstrado nesse trabalho que processos radiativos, ao invés de colisionais, são responsáveis pela excitação de HNCO em Sgr B2. Nesse sentido, o HNCO seria um bom indicador do campo de radiação infravermelho distante, mas não das propriedades do gás, como densidade e temperatura cinética. A única transição em comum com as mostradas aqui é $10_{0,10}-9_{0,9}$, as demais do artigo estão em frequências menores (Churchwell et al., 1986). Nesse ponto de vista, de acordo com a temperatura de excitação similar de cerca de $60 \mathrm{~K}$ obtida através dos diagramas rotacionais, a mesma tendência parece ser mantida em níveis de transição de HNCO mais altos, indicando que processos radiativos também podem dominar a excitação de moléculas de HNCO em G331.

A partir dessas densidades de coluna, é possível obter uma estimativa da abundância do $\mathrm{HNCO}$ em relação ao $\mathrm{H}_{2}$ pela Equação 3.6. Para tanto, a densidade de coluna de $\mathrm{H}_{2}$ precisa ser conhecida. Os valores mais recentes dessa grandeza derivados para G331 são $N\left(\mathrm{H}_{2}\right)=9.7 \times 10^{23} \mathrm{~cm}^{-2}$, calculado a partir da razão de abundâncias de $\mathrm{H}^{13} \mathrm{CO}^{+} / \mathrm{H}_{2}$ de Orion $\mathrm{KL}$, e $N\left(\mathrm{H}_{2}\right)=2.7 \times 10^{23} \mathrm{~cm}^{-2}$, obtido através da densidade superficial derivada da emissão do contínuo em 1.2 mm de G331 (Duronea et al., 2019, e referências nele contidas).

$$
[H N C O]=N(H N C O) / N\left(H_{2}\right)
$$

onde $N(\mathrm{HNCO})$ e $N\left(\mathrm{H}_{2}\right)$ são as respectivas densidades de coluna de $\mathrm{HNCO}$ e $\mathrm{H}_{2}$.

Com bases nesses valores, a abundância relativa de HNCO pode estar compreendida em um intervalo de $3.2 \times 10^{-9}$ a $1.4 \times 10^{-8}$. Essa alta variação de abundâncias está diretamente relacionada com as estimativas de $N\left(\mathrm{H}_{2}\right)$, uma vez que os valores de $N(\mathrm{HNCO})$ encontrados com os diagramas rotacionais são muito próximos. Valores de $\mathrm{N}\left(\mathrm{H}_{2}\right)$ mais precisos são necessários para melhores estimativas das densidades de coluna. Além disso, também é possível perceber a dificuldade desse procedimento, uma vez que a molécula de $\mathrm{H}_{2}$ não é observada nessa região espectral (por não possuir momento de dipolo permanente) e relações indiretas devem ser utilizadas para se obter sua densidade de coluna. 
HNCO columns densities

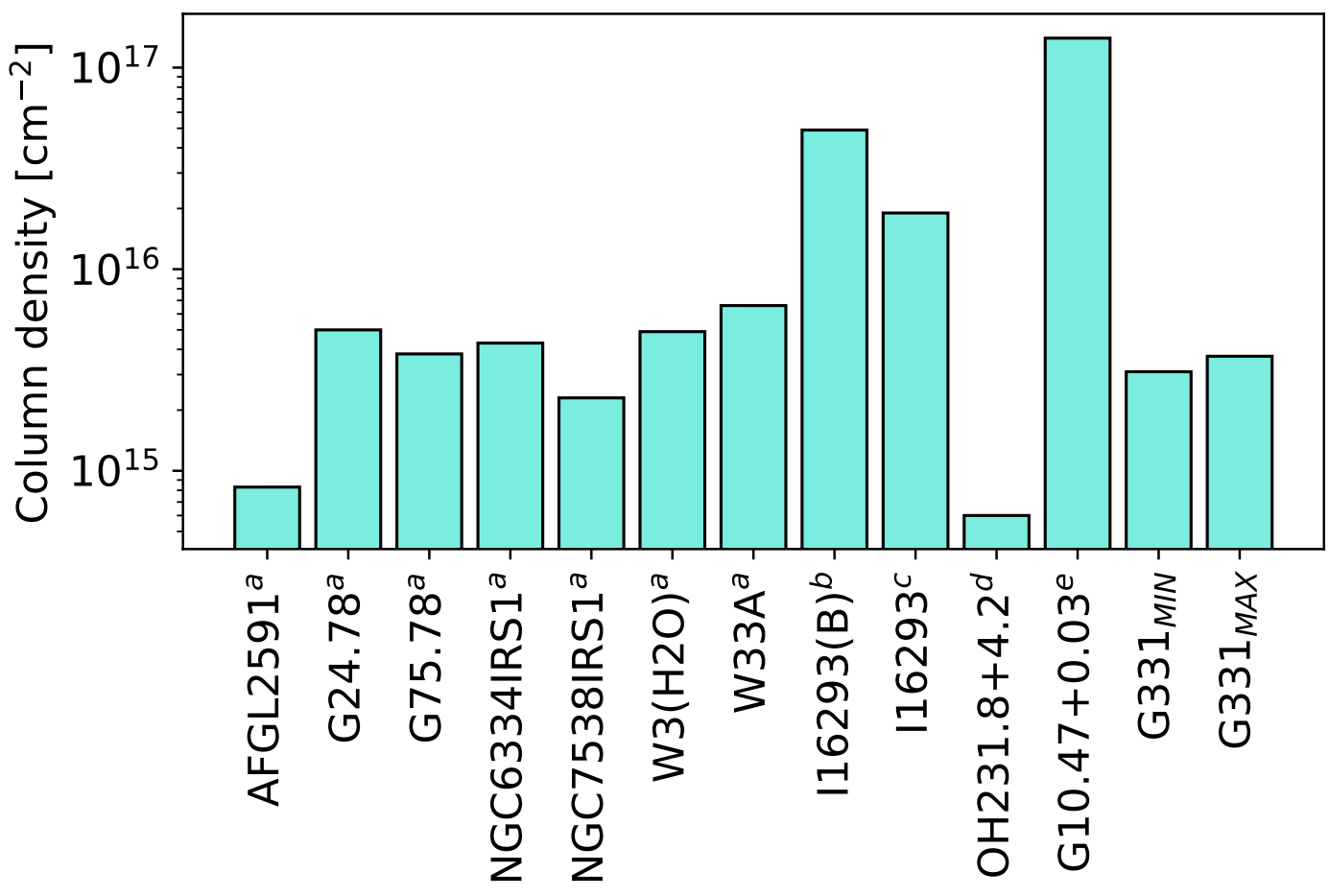

HNCO abundances

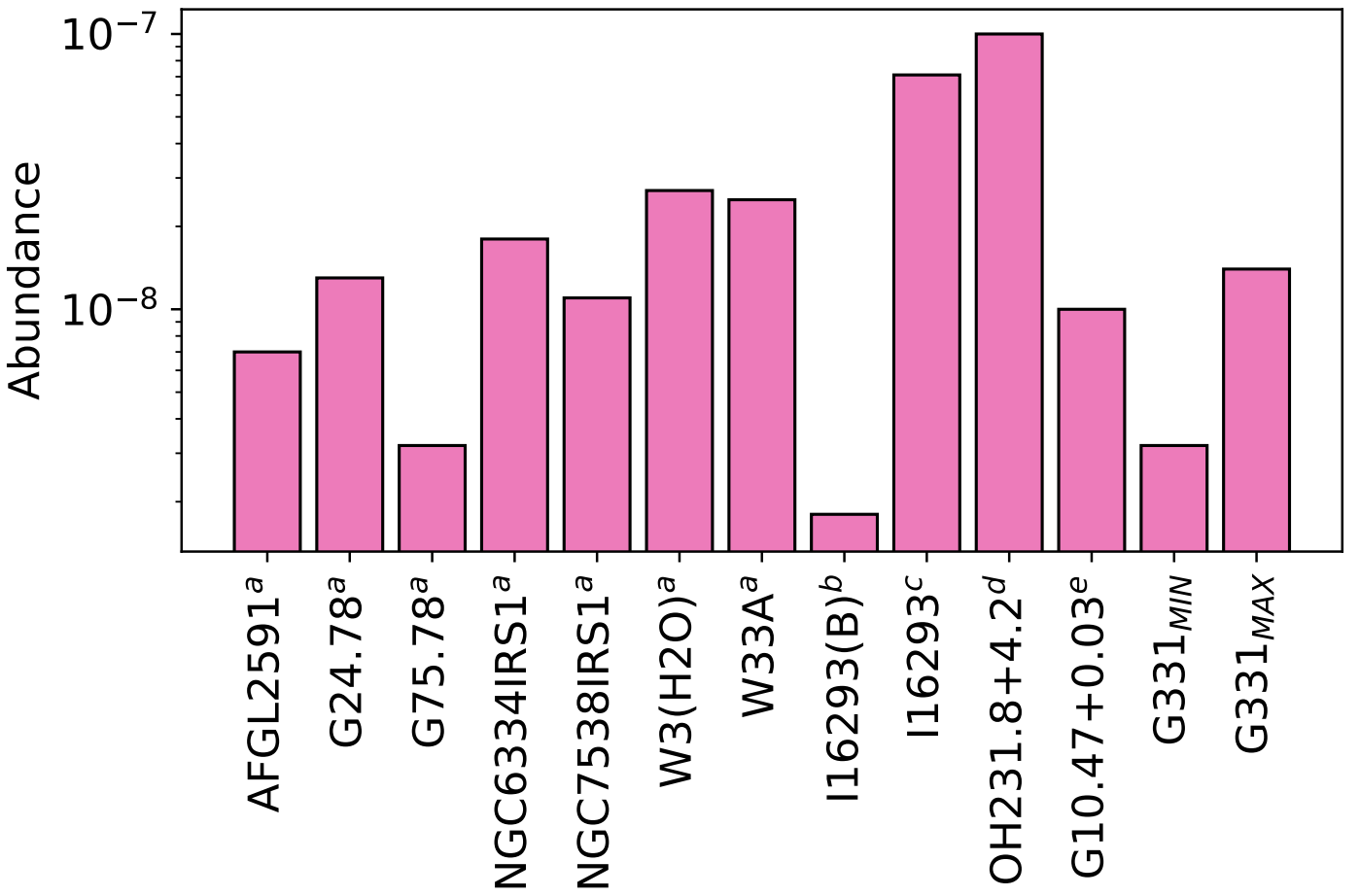

Figura 3.13: Histogramas das densidades de coluna de HNCO (superior) e suas abundâncias relativas ao $\mathrm{H}_{2}$ (inferior) em diversos objetos: ${ }^{a}$ Bisschop et al. (2007), ${ }^{b}$ IRAS 16293-2422 (B, Martín-Doménech et al., 2017), ' IRAS 16293-2422 (hot corino, Hernández-Gómez et al., 2019), ' Velilla Prieto et al. (2015), ${ }^{e}$ Gorai et al. (2020). Os valores máximos (G331 ${ }_{M A X}$ ) e mínimos (G331 $1_{M I N}$ ) obtidos para G331 também estão inclusos. 
A Figura 3.13 exibe histogramas das densidades de coluna e abundâncias de HNCO para várias fontes, juntamente com os resultados obtidos aqui para comparação. Esses objetos são YSOs (sigla em inglês para young stellar objects) de alta massa em estágios de núcleo quente (AFGL 2591, G24.78, G75.78, NGC 6334 IRS1, NGC 7538 IRS1, W3(H2O) e W 33A, Bisschop et al., 2007); protoestrela de baixa massa IRAS 16293-2422, na fonte compacta B (Martín-Doménech et al., 2017) e na região do hot corino (Hernández-Gómez et al., 2019); envelope circunstelar rico em oxigênio em torno de uma estrela evoluída de massa intermediária (OH231.8+4.2, Velilla Prieto et al., 2015); e núcleo molecular quente (G10.47+0.03, Gorai et al., 2020).

Em relação às densidades de coluna de HNCO, pode-se perceber que G331 e a maioria dos YSOs de Bisschop et al. (2007) apresentaram valores bem semelhantes. Isso pode ser explicado pelo estado evolutivo similar desses objetos, com protoestrelas embebidas em núcleos moleculares quentes. As duas fontes relacionadas com IRAS 16293-2422 possuem densidades de coluna um pouco maiores, que podem estar ligadas às regiões mais internas e densas desse objeto (e.g. hot corino). Nos envelopes mais frios, as densidades de coluna podem ser até quatro ordens de grandeza menores (Hernández-Gómez et al., 2019).

O histograma das abundâncias revelou uma maior variação entre todas as fontes. Essa questão pode ser influenciada pela determinação prévia de $\mathrm{N}\left(\mathrm{H}_{2}\right)$ de cada objeto, cujo efeito também pode ser visto diretamente em G331 ${ }_{M I N}$ e G331 MAX. As abundâncias de HNCO do objeto G75.78 e de G331 $1_{\text {MIN }}$ são muito parecidas, enquanto a de G331 ${ }_{\text {MAX }}$ se aproxima mais dos outros YSOs.

Vale notar que G10.47+0.03 apresentou uma densidade de coluna muito maior do que G331, porém a abundância de HNCO nessa fonte está no intervalo calculado para G331. Por sua vez, OH231.8+4.2, que é o objeto mais evoluído dessa amostra, mostrou os resultados mais extremos e inversos - a menor densidade de coluna e a maior abundância de HNCO. Apesar desses casos peculiares, os valores de densidade de coluna e abundância de HNCO em G331 estão de acordo com os derivados na maioria dos núcleos moleculares quentes mostrados nos histogramas.

Para concluir, a discussão sobre a detecção das linhas de HNCO, possíveis contaminantes e os resultados apresentados nessa tese estão sendo preparados para serem publicados em Canelo et al. in prep. Novas abordagens ainda estão sendo estudadas para complementar o trabalho que vem sendo realizado em G331. Dentre elas, pode-se destacar os 
modelos LTE necessários para se obter os espectros sintéticos das linhas. Essas análises complementares também deverão ser incluídas em Canelo et al. in prep.

\subsubsection{Simulações químicas com Nautilus}

Por causa dos diferentes regimes de temperatura e densidade de G331, algumas simulações foram realizadas para modelar uma visão geral das abundâncias do HNCO nessas condições físicas. Nas primeiras as simulações, foram utilizados valores de 10 mag para a extinção visual, típico de uma nuvem escura, além de $1.3 \times 10^{-17} \mathrm{~s}^{-1}$ para a taxa de ionização de raios cósmicos $(\zeta$ ), como usado normalmente em simulações do NAUTILUs (e.g. Wakelam et al., 2006; Wakelam e Herbst, 2008; Hincelin et al., 2011; Vidal e Wakelam, 2018).

As densidades do núcleo foram consideradas de $1 \times 10^{6}, 5 \times 10^{6}, 1 \times 10^{7}$ e $1 \times 10^{8} \mathrm{~cm}^{-3}$, como esperado para núcleos quentes. Para cada um desses valores, as temperaturas de 50, 100, 150 e $200 \mathrm{~K}$ foram utilizadas. As abundâncias do HNCO para esses cenários são apresentadas na Figura 3.14. As temperaturas do gás e da poeira foram consideradas iguais.

Uma característica geral das simulações é a baixa abundância dessa molécula para as temperaturas de 100, 150 e $200 \mathrm{~K}$ em comparação com $50 \mathrm{~K}$. Os modelos para valores de temperatura de $200 \mathrm{~K}$ e os modelos a uma densidade de $1 \times 10^{8} \mathrm{~cm}^{-3}$ podem ser considerados limites superiores das condições físicas de G331 e respectivas possíveis abundâncias do HNCO sob tais condições mais extremas. Portanto, os cenários mais promissores são aqueles com densidades de $5 \times 10^{6}$ e $1 \times 10^{7} \mathrm{~cm}^{-3}$ para uma temperatura de $50 \mathrm{~K}$.

Uma possível razão para essas pequenas abundâncias nos modelos com altas temperaturas são as reações de formação usadas pelo código. Em temperaturas mais baixas, o código usa duas reações de formação: $\mathrm{H}+\mathrm{OCN} \rightarrow \mathrm{HNCO}$ na fase sólida e $\mathrm{HNCO}(\mathrm{s}) \rightarrow$ HNCO (g). Por outro lado, para temperaturas mais altas, o código considera apenas a última reação. A química do $\mathrm{HNCO}$ em tais condições astrofísicas ainda é uma questão em aberto.

A reação $\mathrm{NH}+\mathrm{CO} \rightarrow \mathrm{HNCO}$, normalmente proposta em estudos espectroscópicos para explicar a formação de HNCO em misturas análogas de gelo interestelar processadas por próton ou radiação UV, pode ser a principal via de produção dessa molécula (Fedoseev et al., 2015). Essa reação, por exemplo, não está disponível no banco de dados usado pelo 


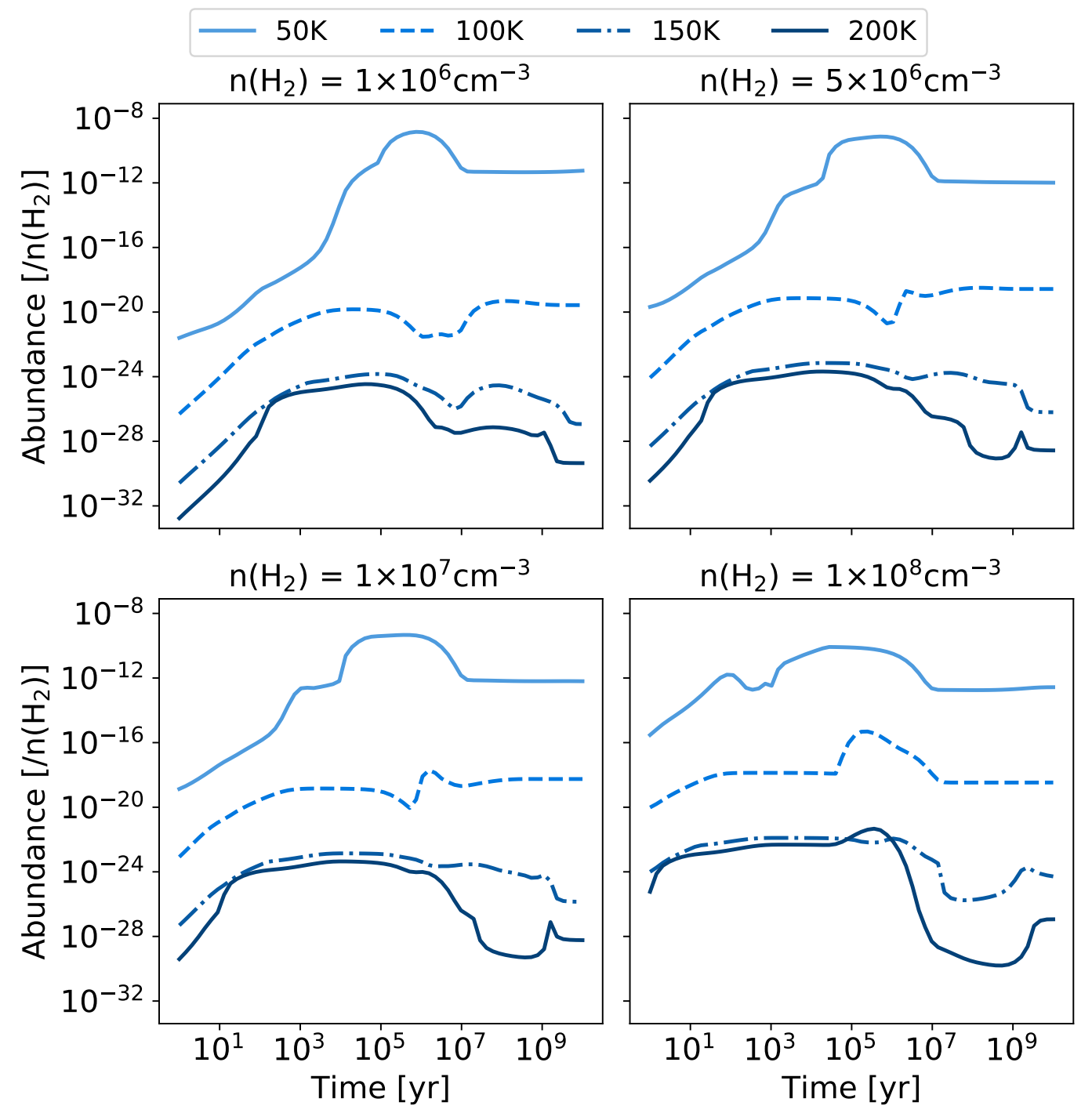

Figura 3.14: Simulações químicas realizadas para o $\mathrm{HNCO}$ em diferentes regimes de densidades e temperaturas, como descrito no título e legenda dos gráficos. As temperaturas do gás e da poeira foram consideradas iguais em todas as simulações.

código. Logo, outros intervalos de temperatura podem apresentar valores de abundância muito mais baixos se outras reações não forem consideradas na rede química, especialmente em uma simulação zero dimensional.

A partir desses primeiros modelos, novas simulações foram realizadas com uma densidade de $1 \times 10^{7} \mathrm{~cm}^{-3}$ e valores de temperatura mais próximos aos obtidos pelo diagrama rotacional - 55, 60 e 65 K. Elas são apresentadas na Figura 3.15. É interessante notar que, para esse valor de densidade, o modelo de $55 \mathrm{~K}$ apresenta as maiores abundâncias, inclusive em comparação com o modelo de $50 \mathrm{~K}$. Valores entre 70 e $100 \mathrm{~K}$ também foram considerados. As abundâncias máximas não passam de $10^{-11}$ e tendem a diminuir com o 
aumento da temperatura. Por esse motivo, esses modelos não foram apresentados aqui.

A taxa de ionização de raios cósmicos padrão é de $1.3 \times 10^{-17} \mathrm{~s}^{-1}$. Contudo, para analisar a influência desse parâmetro nos modelos, dois valores limites foram também simulados nas mesmas condições de temperatura e densidade. As simulações com o limite inferior de $1.3 \times 10^{-16} \mathrm{~s}^{-1}$ e com o limite superior de $1.3 \times 10^{-18} \mathrm{~s}^{-1}$ são mostradas na mesma Figura 3.15 .

A principal diferença entre os modelos com diferentes $\zeta$ é a largura dos picos de intensidade da abundância e, consequentemente, a posição do valor máximo. Essa posição do máximo está diretamente relacionada à idade química estimada. Nesse sentido, a taxa de ionização de raios cósmicos parece interferir no balanceamento das reações químicas e no equilíbrio químico.

Quanto maior a taxa, mais rapidamente o pico da abundância e o equilíbrio químico das reações ocorrem. =Isso acontece porque taxa maiores induzem mais reações químicas (como esperado, por ser uma fonte de energia mais significativa), que acabam durando um menor período de tempo. As abundâncias máximas também são menores. No caso de $60 \mathrm{~K}$, as abundâncias máximas obtidas foram de 1.86, 4.19 e $5.48 \times 10^{-8}$, para valores de $\zeta$ em ordem decrescente. Para $55 \mathrm{~K}$, as abundâncias chegam até $1.07 \times 10^{-7}$ e, para $65 \mathrm{~K}$, as abundâncias máximas não passam de $1.34 \times 10^{-9}$.

Tendo em vista que as abundâncias obtidas pelos diagramas rotacionais podem ser de $3.2 \times 10^{-9}$ a $1.4 \times 10^{-8}$, os modelos com $60 \mathrm{~K}$ podem ser considerados como os que melhor conseguem retratar todo esse intervalo. Eles também concordam com as temperaturas derivadas dos diagramas, que são da ordem de $60 \mathrm{~K}$. Embora as abundâncias máximas modeladas sejam um pouco maiores que $1.4 \times 10^{-8}$, as simulações com $55 \mathrm{~K}$ parecem sobrestimar a abundância de HNCO em G331. Já as simulações com $65 \mathrm{~K}$ tendem a subestimá-las.

Com o melhor cenário escolhido, pode-se estimar a idade química de G331 pelo intervalo de abundâncias calculado. Como somente o HNCO foi estudado nesse trabalho, não há outras moléculas para que se possa construir o gráfico da distância de desacordo. Então, a estimativa da idade química de G331 foi realizada comparando-se diretamente as abundâncias dos diagramas rotacionais com as simuladas.

Considerando $\zeta=1.3 \times 10^{-17} \mathrm{~s}^{-1}$, a idade química pode ser de $3 \times 10^{4-6}$ anos com abundância modelada máxima em torno de $10^{5}$ anos. Além disso, os modelos dos outros 

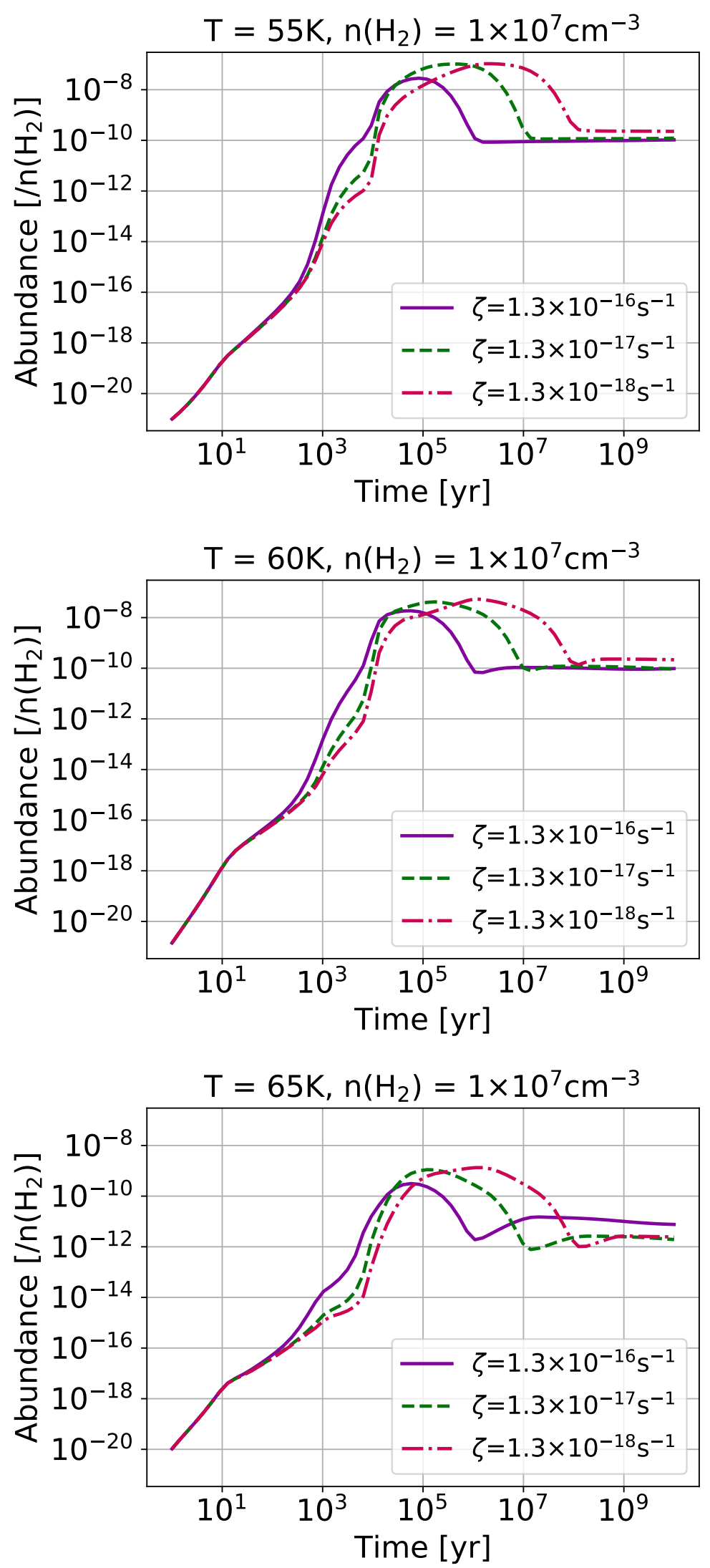

Figura 3.15: Melhores ajustes da evolução temporal das abundâncias de HNCO simuladas com NAUTILUS. Foi assumida uma densidade de $10^{7} \mathrm{~cm}^{-3}$ e a taxa de ionização de raios cósmicos $(\zeta)$ foi variada em $1.3 \times 10^{-16}, 1.3 \times 10^{-17}$ e $1.3 \times 10^{-18} \mathrm{~s}^{-1}$. 
dois valores de $\zeta$ se cruzam por volta de $1.4 \times 10^{-8}$ em $10^{5}$ anos, indicando que essa idade química pode ser bem plausível para G331 com base nesses modelos. Aliás, esse padrão de idades se mantém igual nos modelos de 65 e $67 \mathrm{~K}$, ainda que eles não representem adequadamente as abundâncias do HNCO.

Vale ressaltar que, apesar dessa simulação ser razoavelmente simples, ela já permite reproduzir alguns dados observacionais de G331 a partir do HNCO. Para aprimorar as simulações, será necessário um modelo de evolução estrutural de G331 em conjunto com um modelo de evolução temporal das condições físicas. Nesse cenário, é possível que outras reações de formação e destruição de moléculas precisem também ser incluídas na rede de reações do NAUTiLus para que as abundâncias possam ser reproduzidas.

\subsection{Simulações químicas com NAUTilus para IRDC-C9 Main}

Em relação à química dessas regiões frias, as abundâncias de $\mathrm{CH}_{3} \mathrm{OH}$ e OCS nesses ambientes indicam que reações de formação na superfície de grãos podem ser essenciais para a formação de COMs (Garrod et al., 2007; Loison et al., 2012). Em temperaturas baixas, a química nos mantos dos grãos de gelo podem ser mais eficientes e mais ricas do que na fase gasosa, através de diferentes mecanismos que podem influenciar a formação molecular (Garrod e Herbst, 2006; Garrod et al., 2008; Vasyunina et al., 2012; Chang e Herbst, 2016; Chen et al., 2018; Kalvāns, 2018). Por também considerar reações químicas em grãos e suas superfícies, o NAUTiLus é uma ferramenta relevante para se estudar IRDC-C9 Main. Com ela, pôde-se verificar as abundâncias e a idade química desse objeto, conforme descrito na seção 3.1.3.

Foram estudadas as moléculas identificadas que constam na base de dados do código: CS, OCS, $\mathrm{CH}_{3} \mathrm{CHO}$ e $\mathrm{CH}_{3} \mathrm{OCHO}$. Para se comparar os resultados com os valores observados, foram utilizadas as abundâncias de OCS, ${ }^{13} \mathrm{CS}$ e $\mathrm{CH}_{3} \mathrm{CHO}$ derivadas a partir das respectivas densidades de coluna observadas (Tabela 3.5, Beaklini et al., 2020). A molécula $\mathrm{CH}_{3} \mathrm{OCHO}$ só possui uma linha e, portanto, o diagrama rotacional do qual sua densidade de coluna é estimada não pôde ser construído. Como o código não considera o isotopotólogo ${ }^{13} \mathrm{CS}$, foi usada uma razão de $\mathrm{C} /{ }^{13} \mathrm{C} \sim 45$ para se estimar a abundância a partir da molécula isotópica mais abundante correspondente (Li et al., 2015).

Em relação à densidade desse objeto, foi assumido um valor de $4.6 \times 10^{4} \mathrm{~cm}^{-3}$. Ambas 
Tabela 3.5 - Abundâncias moleculares relativas a $\mathrm{n}\left(\mathrm{H}_{2}\right)$ derivadas para IRDC-C9 MAIN (Beaklini et al., 2020).

\begin{tabular}{cccc}
\hline Região & OCS & ${ }^{13} \mathrm{CS}$ & $\mathrm{CH}_{3} \mathrm{CHO}$ \\
\hline Núcleo & $3 \times 10^{-8}$ & $9 \times 10^{-9}$ & $8 \times 10^{-9}$ \\
Cauda & $2 \times 10^{-8}$ & $8 \times 10^{-9}$ & - \\
\hline
\end{tabular}

as temperaturas do gás e da poeira foram consideradas iguais a $15 \mathrm{~K}$. Tais valores são inferiores aos derivados da observação porque representam uma região menos densa e mais fria de IRDC-C9 Main em que não há o processo de contração e fase de aquecimento. Dessa maneira, esse modelo representa as condições iniciais de objeto, anteriores à contração. Também foram assumidos os valores típicos de $1.3 \times 10^{-17} \mathrm{~s}^{-1}$ para a taxa de ionização de raios cósmicos e de 10 mag para a extinção visual.

Os resultados do modelo apresentam abundâncias semelhantes às observadas, como mostrado na Figura 3.16. De acordo com a distância do desacordo, essa fonte tem, pelo menos, $10^{5}$ anos, que seria o tempo necessário para que essas abundâncias pudessem ser detectadas. A idade química estimada por este método é de $1.7 \times 10^{5}$ anos. O código NAUTILUS também fornece as reações de formação e destruição que contribuem com as abundâncias modeladas ao longo do tempo. O diagrama dessas reações para as moléculas CS e OCS é apresentado na Figura 3.17. Essas moléculas foram escolhidas para se fazer o diagrama por poderem estar quimicamente relacionadas. As setas pretas representam os processos de formação enquanto as vermelhas são as reações de destruição. Como esperado, as duas moléculas estão conetadas por uma via dupla de reação de formação e destruição. Todos esses resultados estão publicados em Beaklini et al. (2020).

\subsubsection{Outra abordagem com NAuTILus}

Além dos resultados apresentados anteriormente, que se referem a um estágio inicial de IRDC-C9 Main, diversos testes e simulações foram realizados na tentativa de se reproduzir os valores observacionais de densidade e temperatura. A densidade utilizada foi de $4.6 \times 10^{-6} \mathrm{~cm}^{-2}$ e foi considerado um intervalo de temperaturas de 10 a $40 \mathrm{~K}$, ao invés de simular um cenário inicial da fonte, como publicado em (Beaklini et al., 2020).

Os valores de $10 \mathrm{mag}$ para a extinção visual e de $1.3 \times 10^{-17} \mathrm{~s}^{-1}$ para a taxa de ionização de raios cósmicos foram mantidos. A razão $\mathrm{C} /{ }^{13} \mathrm{C} \sim 45$ também foi empregada quando necessária. Como a região da cauda apresenta muito menos linhas de emissão, 

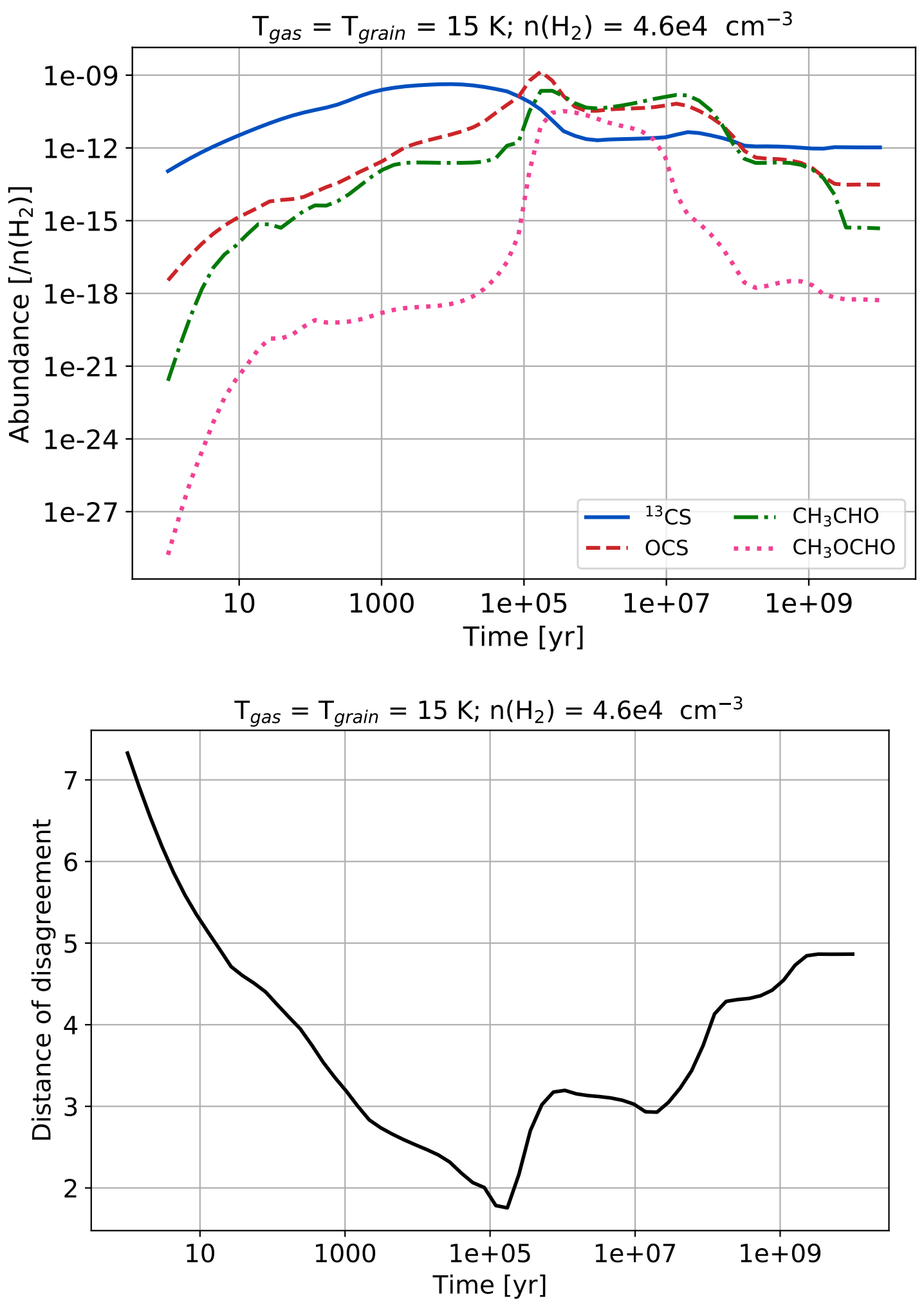

Figura 3.16: Esquerda: Evolução temporal das abundâncias simuladas com o NAUTiLus. Foram assumidas $\mathrm{C} /{ }^{13} \mathrm{C}=45$, temperaturas do gás e dos grãos de $15 \mathrm{~K}$, e densidade de $4 \times 10^{4} \mathrm{~cm}^{-3}$. Direita: Distância do desacordo (Wakelam et al., 2006), onde o mínimo indica uma estimativa da idade química de $1.7 \times 10^{5}$ anos. Gráficos publicados em Beaklini et al. (2020). 


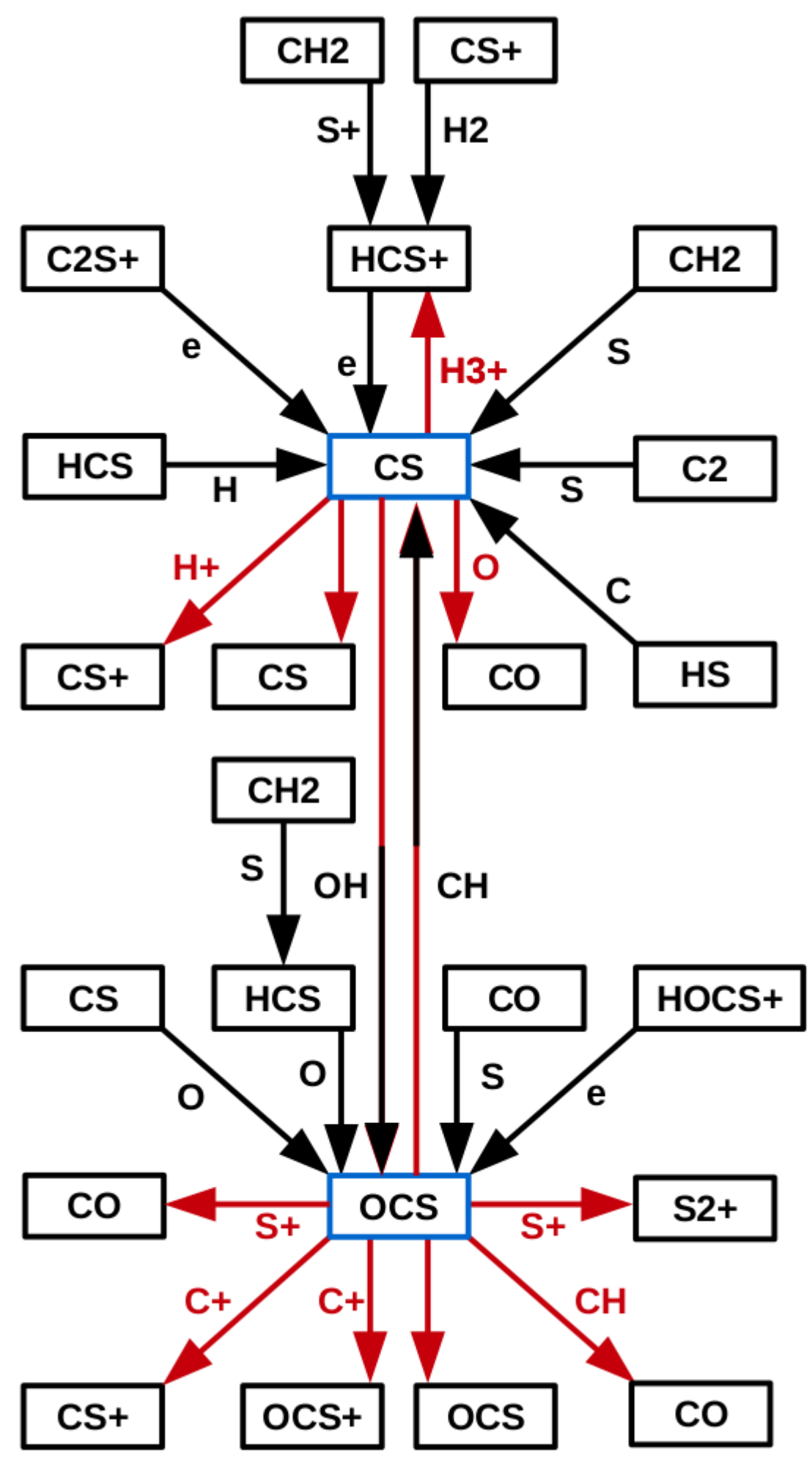

Figura 3.17: Rede química de CS e OCS com os caminhos de formação e destruição de moléculas portadoras de S para um modelo com temperatura do gás e do grão de $15 \mathrm{~K}$. As setas pretas representam as reações de formação e as setas vermelhas, as de destruição. Algumas outras reações contribuem muito pouco para a formação e destruição de CS e OCS na escala de tempo entre $10^{3}$ e $10^{5}$ anos. Imagem publicada em Beaklini et al. (2020). 
somente o núcleo foi considerado. A distância de desacordo e o diagrama de reações não foram construídos para esses testes.

Nesses modelos, também foi testada uma adaptação nos resultados para que fossem apresentados em densidade de coluna ao invés de abundância. Esse procedimento pode ser necessário, por exemplo, em situações em que não há informações suficientes para se derivar as abundâncias, como a falta da densidade volumétrica do objeto $-\mathrm{n}\left(\mathrm{H}_{2}\right)$. As abundâncias locais derivadas pelo NAUTILUs foram convertidas para densidades de coluna através da Equação 3.7, disponível na documentação do código (Ruaud et al., 2016).

$$
N_{i}=\frac{A_{v}}{5.34 \times 10^{-22}} X_{i}
$$

onde $\mathrm{N}_{i}$ é a densidade de coluna da espécie $\mathrm{em} \mathrm{cm}^{-2}, \mathrm{~A}_{v}$ é a extinção visual, $5.34 \times 10^{-22}$ é o fator de conversão da extinção visual para a densidade de coluna total de $\mathrm{H} \mathrm{em} \mathrm{cm}^{-2}$ (Wagenblast e Hartquist, 1989) e $\mathrm{X}_{i}$ é a abundância local das espécies.

Normalmente, os modelos consideram que as temperaturas do gás e da poeira são iguais ao longo da simulação, como feito anteriormente. Contudo, os grãos de poeira possuem uma distribuição grande de tamanhos, levando também a uma distribuição grande de temperaturas. Em muitas circunstâncias, o gás molecular é aquecido por pequenos grãos de grafite cuja temperatura pode ser consideravelmente maior que os grãos de silicato cuja emissão domina o espectro do infravermelho distante (Kruegel e Walmsley, 1984). Isso pode fazer com que a temperatura do gás pareça maior que a temperatura da poeira.

Já foram observadas diferenças entre as temperaturas de gás e poeira em alguns aglomerados galácticos relatados por Merello et al. (2019). De fato, Martel et al. (2012) investigaram os efeitos do aquecimento e do resfriamento de poeira e gás em simulações de aglomerados de estrelas, mostrando que, embora as temperaturas de gás e poeira sejam semelhantes ao acoplamento colisional gás-poeira em ambientes de alta densidade, a temperatura da poeira é mais baixa que a temperatura do gás nos momentos iniciais dos objetos.

No caso de IRDC-C9 Main, um ponto utilizado para que os modelos representassem melhor os dados observados foi se considerar a temperatura do gás superior à da poeira. Para a temperatura do gás $\left(\mathrm{T}_{\text {gas }}\right)$, foram simulados cenários de 10 a $40 \mathrm{~K}$, como já dito. Infelizmente, ainda não existe um modelo que possa inferir a temperatura da poeira $\left(\mathrm{T}_{\text {grain }}\right)$ a partir da temperatura do gás. Desse modo, foram escolhidos valores desde que fossem 
menores do que a temperatura do gás para cada situação.

Por enquanto, não há discussões conclusivas sobre essa questão na literatura. Logo, essa suposição pode ser questionada, especialmente em altas densidades em que ambas as temperaturas estão acopladas. Além disso, essa fonte não deve estar nos seus momentos iniciais, como discutido na seção anterior em que a idade química obtida com o NAUTILUS foi de cerca de $10^{5}$ anos. Entretanto, ela é necessária para melhor reproduzir as densidades de coluna encontradas nesse objeto.

A Figura 3.18 mostra os resultados para o modelo melhor ajustado, com as temperaturas de $\mathrm{T}_{\text {gas }}=25 \mathrm{~K}$ e $\mathrm{T}_{\text {grain }}=11.5 \mathrm{~K}$. Estas são as temperaturas que controlam as reações químicas, bem como a taxa de evaporação das moléculas. Nota-se que essa temperatura cinética do gás é consistente com a temperatura de rotação obtida para $\mathrm{CH}_{3} \mathrm{CHO}$ em Beaklini et al. (2020) na região do núcleo do objeto.

Por outro lado, a escala de tempo na qual as densidades de coluna são maiores e mais próximas aos valores observados é de $10^{3}$ a $10^{4}$ anos, sugerindo uma idade química bem menor do que a obtida em Beaklini et al. (2020). Ainda assim, a inversão nas densidades de coluna de OCS e ${ }^{1} 3 \mathrm{CS}$, com OCS se tornando mais dominante após $10^{3}$ anos, também pode sugerir diferentes estágios evolutivos (químicos) dessa fonte.

Como discutido na seção anterior, a linha de emissão de OCS é mais intensa do que a de CS no núcleo, enquanto o oposto acontece na cauda. Segundo esse novo modelo, CS é mais abundante no começo das interações químicas, levantando a hipótese de que processos químicos diferentes podem estar ocorrendo em cada região. Essa análise é complementar à suposição de que o núcleo esteja em uma fase de aquecimento e contração, na qual mais moléculas já estejam na fase gasosa. Nesse sentido, a química na cauda pode estar mais concentrada na fase de grãos.

Em relação à maioria das densidades de coluna, elas ainda são bem menores do que as obtidas pelas observações (apresentadas na figura). Os valores máximos simulados nesse cenário são uma ordem de grandeza menor para o OCS e duas ordens de grandeza menores para as outras COMs com oxigênio. Portanto, elas não estão de acordo com as observações, o que pode sugerir que algumas reações podem não estar ocorrendo nas taxas esperadas.

Na verdade, como apontado por Bacmann e Faure (2016), pode haver grandes incertezas nas taxas de coeficiente de algumas reações do NAUtilus. Após uma análise cuidadosa das reações, foram identificados os processos que parecem estar destruindo as moléculas 

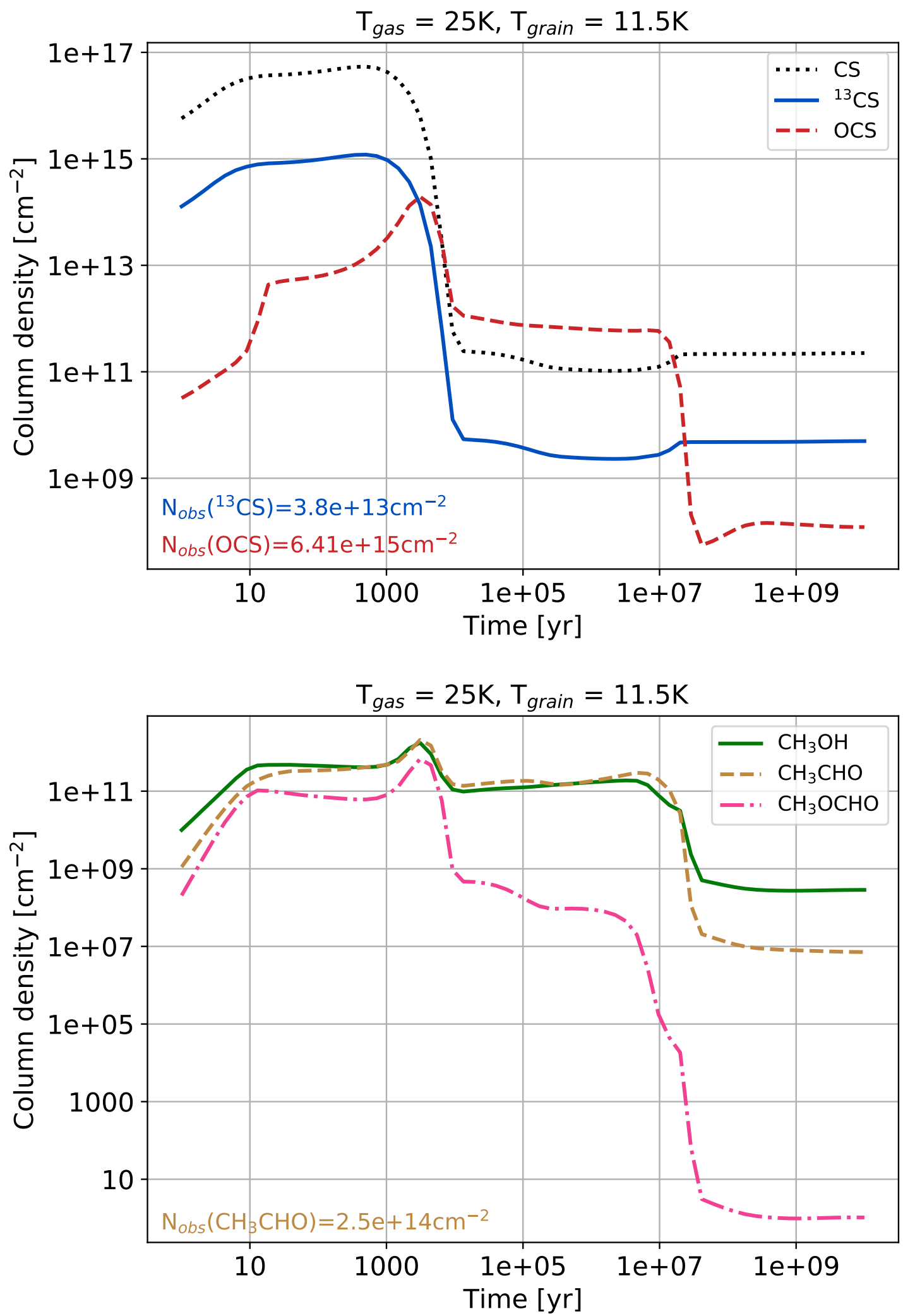

Figura 3.18: Evolução temporal das densidade de coluna moleculares simuladas com NAUtiLus com a rede química completa. Foi assumida a razão de $\mathrm{C} /{ }^{13} \mathrm{C}=45$, e temperaturas do gás e da poeira de $25 \mathrm{~K}$ e $11.5 \mathrm{~K}$, respectivamente. Superior: Moléculas com enxofre; Inferior: COMs com oxigênio. Os valores observados estão indicados nas figuras. 
em excesso. Alguns, inclusive, não estão na base de dados UMIST Database for Astrochemistry 9 (McElroy et al., 2013). Outros apresentam incertezas sobre as taxas de coeficiente que podem superestimar os efeitos dessas reações. Eles são listados abaixo.

$$
\begin{gathered}
\mathrm{C}+\mathrm{OCS} \rightarrow \mathrm{CO}+\mathrm{CS} \\
\mathrm{CH}_{3} \mathrm{OH}+\mathrm{C} \rightarrow \mathrm{CH}_{3}+\mathrm{HCO} \\
\mathrm{HCOOCH}_{3}+\mathrm{C} \rightarrow 2 \mathrm{CO}+\mathrm{H}+\mathrm{CH} \\
\mathrm{C}+\mathrm{CH}_{3} \mathrm{CHO} \rightarrow \mathrm{C}_{2} \mathrm{H}_{4}+\mathrm{CO}
\end{gathered}
$$

A taxa de coeficiente pode ser uma questão crítica para a química do OCS (Loison et al., 2012), o que pode comprometer a real importância da reação 3.8 no ISM. Em relação à química das COMs, é preciso ter cuidado com os processos que envolvem carbono atômico como reagente (3.9 e 3.10), pois requerem uma grande abundância de carbono atômico neutro na fase gasosa. Em particular, a reação entre $\mathrm{CH}_{3} \mathrm{OH}$ e $\mathrm{C}(3 \mathrm{P})$ - átomo de carbono tripleto - discutida em Shannon et al. (2014) já foi estudada em simulações do NAUTiLuS para uma nuvem densa. Os autores observaram a mesma diminuição da abundância de $\mathrm{CH}_{3} \mathrm{OH}$ devido à incorporação dessa reação no código, assim como apresentado acima.

As simulações em que as reações 3.8 a 3.11 não foram incluídas na rede do código revelaram valores compatíveis com as densidades da coluna observadas. A evolução da densidade da coluna de todas as espécies ao longo do tempo permaneceu praticamente a mesma para intervalos maiores do que $10^{4}$ anos. Esses novos modelos são apresentados na Figura 3.19.

Isso pode indicar que os processos de destruição retirados da rede química do código comprometem a formação inicial dessas moléculas, impedindo que elas alcancem as abundâncias observadas em IRDC-C9 Main. Com a retirada da reação 3.8, era esperado que a curva da densidade de coluna de OCS aumentasse enquanto a curva de CS (e $\left.{ }^{13} \mathrm{CS}\right)$ diminuísse. Contudo, a segunda curva não sofre alteração, questionando a validade dessa reação.

\footnotetext{
${ }^{9}$ http://udfa.ajmarkwick.net/index.php
} 

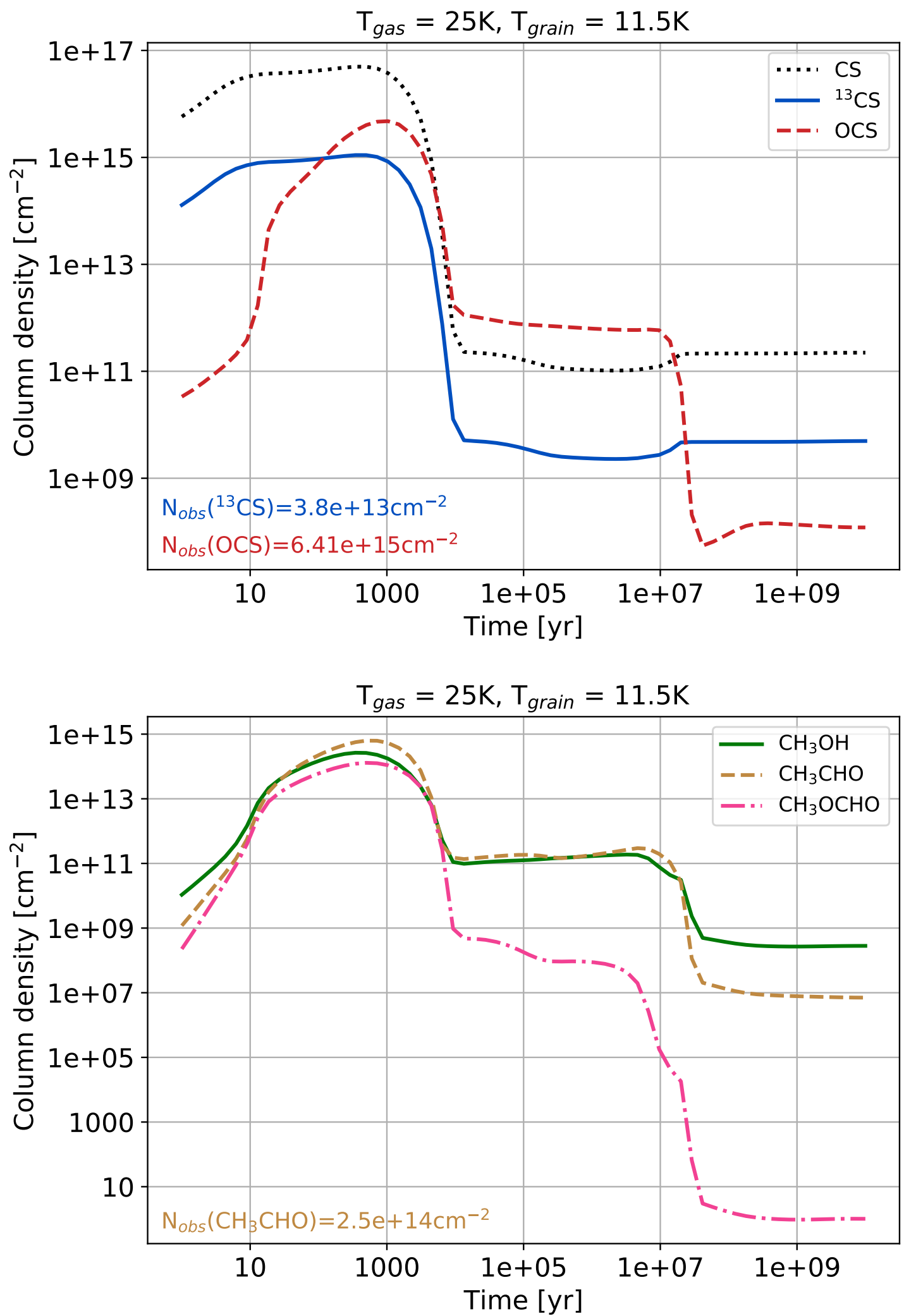

Figura 3.19: Evolução temporal das densidade de coluna moleculares simuladas com NAUTiLus com a rede química alterada. Foi assumida a razão de $\mathrm{C} /{ }^{13} \mathrm{C}=45$, e temperaturas do gás e da poeira de $25 \mathrm{~K}$ e $11.5 \mathrm{~K}$, respectivamente. Superior: Moléculas com enxofre; Inferior: COMs com oxigênio. Os valores observados estão indicados nas figuras. 
Embora as densidades de colunas sejam afetadas com a modificação da rede química do código, algumas conclusões gerais são similares para os dois casos. Em torno de $10^{4}$ anos, as densidades de coluna de todas as moléculas geralmente diminuem e tendem a um estágio de equilíbrio que é alcançado antes de $10^{8}$ anos. A evolução temporal das densidades de coluna de $\mathrm{CH}_{3} \mathrm{OH}$ e $\mathrm{CH}_{3} \mathrm{OCHO}$, que não possuem valores observacionais, são muito similares com a de $\mathrm{CH}_{3} \mathrm{CHO}$ até $10^{4}$ anos. Após esse tempo, $\mathrm{CH}_{3} \mathrm{OCHO}$ se torna bem menos abundante do que as outras duas moléculas.

Esses testes mostram a importância de comparar diferentes bases de dados. O ideal seria complementar a rede química com mais as reações (como de formação) ao invés de retirar reações, como foi necessário com os processos de destruição citados acima. Ainda assim, pode-se perceber que novos estudos com cálculos teóricos e experimentos laboratoriais continuam sendo fundamentais para se expandir os conhecimentos sobre as reações de formação e destruição das moléculas em condições do ISM.

\subsubsection{Simulação para $T=450 \mathrm{~K}$}

Um último cenário foi testado com o objetivo de explicar a emissão do k-ladder do ${ }^{13} \mathrm{CH}_{3} \mathrm{CN}$ com uma temperatura de $450 \mathrm{~K}$. As temperaturas do gás e da poeira foram consideradas bem acopladas devido, principalmente, ao valor elevado da temperatura (Garrod, 2013). Como ${ }^{13} \mathrm{CH}_{3} \mathrm{CN}$ é a única molécula observada com temperatura tão alta, as outras moléculas não foram analisadas. Em vez disso, os precursores básicos de $\mathrm{CH}_{3} \mathrm{CN}-\mathrm{HCN}$, HNC e $\mathrm{C}_{2} \mathrm{H}_{3}$ - também foram simulados e os resultados são mostrados na Figura 3.20. A emissão das moléculas precursoras não aparece nos espectros de IRDC-C9 Main porque elas não possuem linhas de emissão na faixa de frequência observada.

Toda a rede química do código foi utilizada. A densidade da fonte considerada para a simulação também foi de $4.6 \times 10^{-6} \mathrm{~cm}^{-2}$, assim como os valores de 10 mag para a extinção visual e de $1.3 \times 10^{-17} \mathrm{~s}^{-1}$ para a taxa de ionização de raios cósmicos. Novamente, a razão $\mathrm{C} /{ }^{13} \mathrm{C} \sim 45$ também foi empregada quando necessária.

O gráfico mostra que o HCN apresenta uma densidade de coluna quase constante ao longo do tempo. Ele também é a molécula mais abundante, seguido pelo HNC durante a maior parte do tempo. Por outro lado, a densidade da coluna de $\mathrm{C}_{2} \mathrm{H}_{3}$ diminui com o tempo, provavelmente devido ao seu papel na formação de moléculas mais complexas. A densidade de coluna de ${ }^{13} \mathrm{CH}_{3} \mathrm{CN}$ aumenta duas ordens de grandeza em um intervalo 


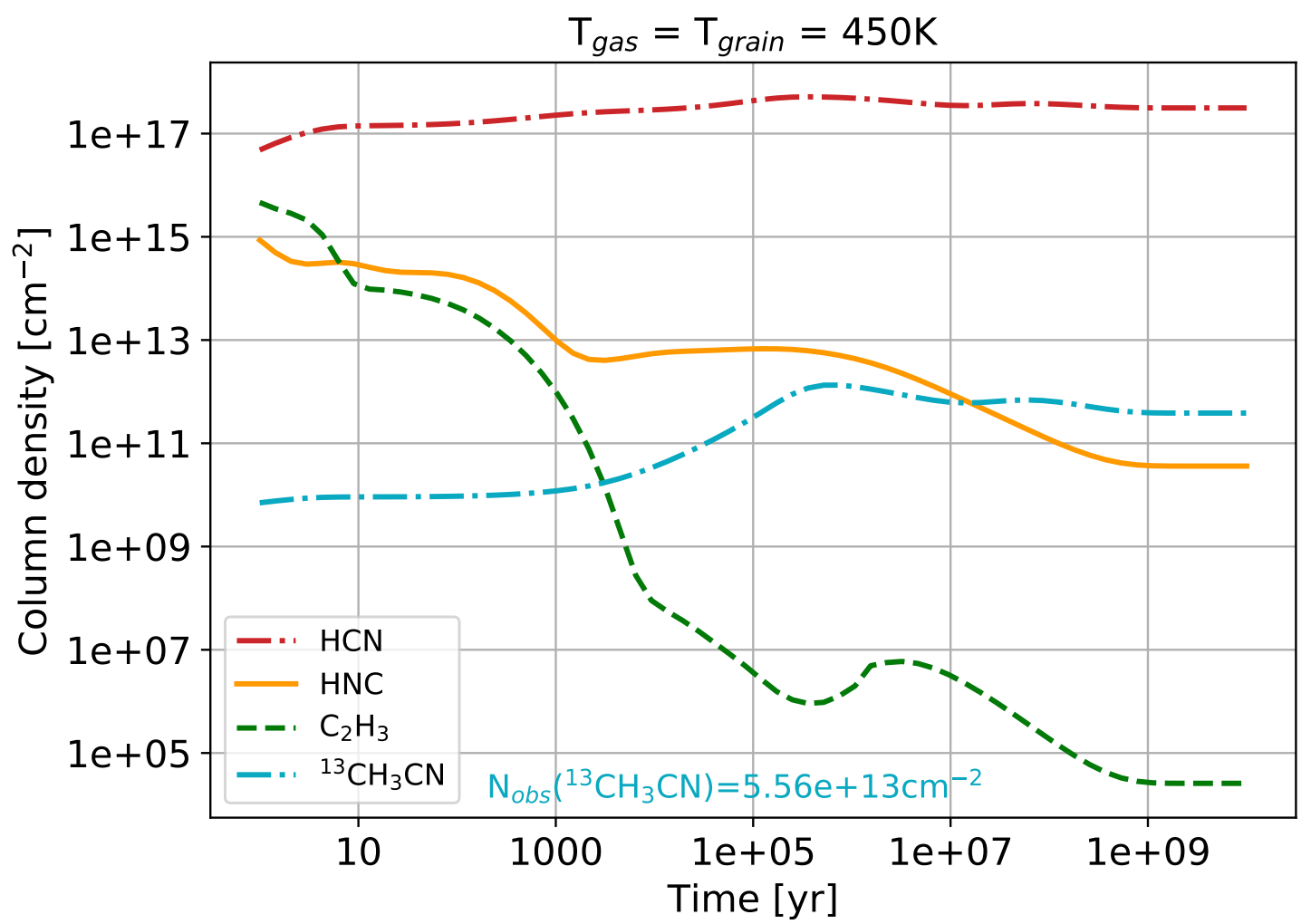

Figura 3.20: Evolução temporal das densidade de coluna moleculares simuladas com NAUtiLus para $\mathrm{HCN}, \mathrm{HNC}, \mathrm{C}_{2} \mathrm{H}_{3}$ e ${ }^{13} \mathrm{CH}_{3} \mathrm{CN}$ e para uma temperatura de $450 \mathrm{~K}$ para o gás e a poeira. Foi assumida a razão de $\mathrm{C} /{ }^{13} \mathrm{C}=45$ quando necessário.

entre $10^{3}$ e $10^{5}$ anos, permanecendo quase constante posteriormente. Seu valor máximo é de $1.3 \times 10^{12} \mathrm{~cm}^{-2}$, uma ordem de grandeza inferior ao valor calculado por Beaklini et al. (2020). Embora não tenha sido possível obter valores semelhantes, esse máximo ocorre depois de $10^{5}$ anos, mesma idade química sugerida em Beaklini et al. (2020). Assim, esse modelo reafirma que essa região está em um estágio evolutivo mais avançado com uma possível protoestrela.

A Figura 3.21 mostra o diagrama de reação com os precursores de $\mathrm{CH}_{3} \mathrm{CN}$. Nele, estão os principais caminhos de formação e destruição considerados pelo código para calcular as densidades das colunas. Pode-se perceber que tanto os produtos como os reagentes estão sendo formados e destruídos. Esse equilíbrio entre as reações pode estar levando às densidades de coluna quase constantes observadas no modelo para $\mathrm{HCN}$ e $\mathrm{CH}_{3} \mathrm{CN}$.

Como os parâmetros físicos são mantidos constantes durante toda a simulação, o modelo assume que a fonte já está em $450 \mathrm{~K}$ nos estágios inciais. Claramente, esse fato não procede, porque uma protoestrela precisa começar a se formar no interior da nuvem para 


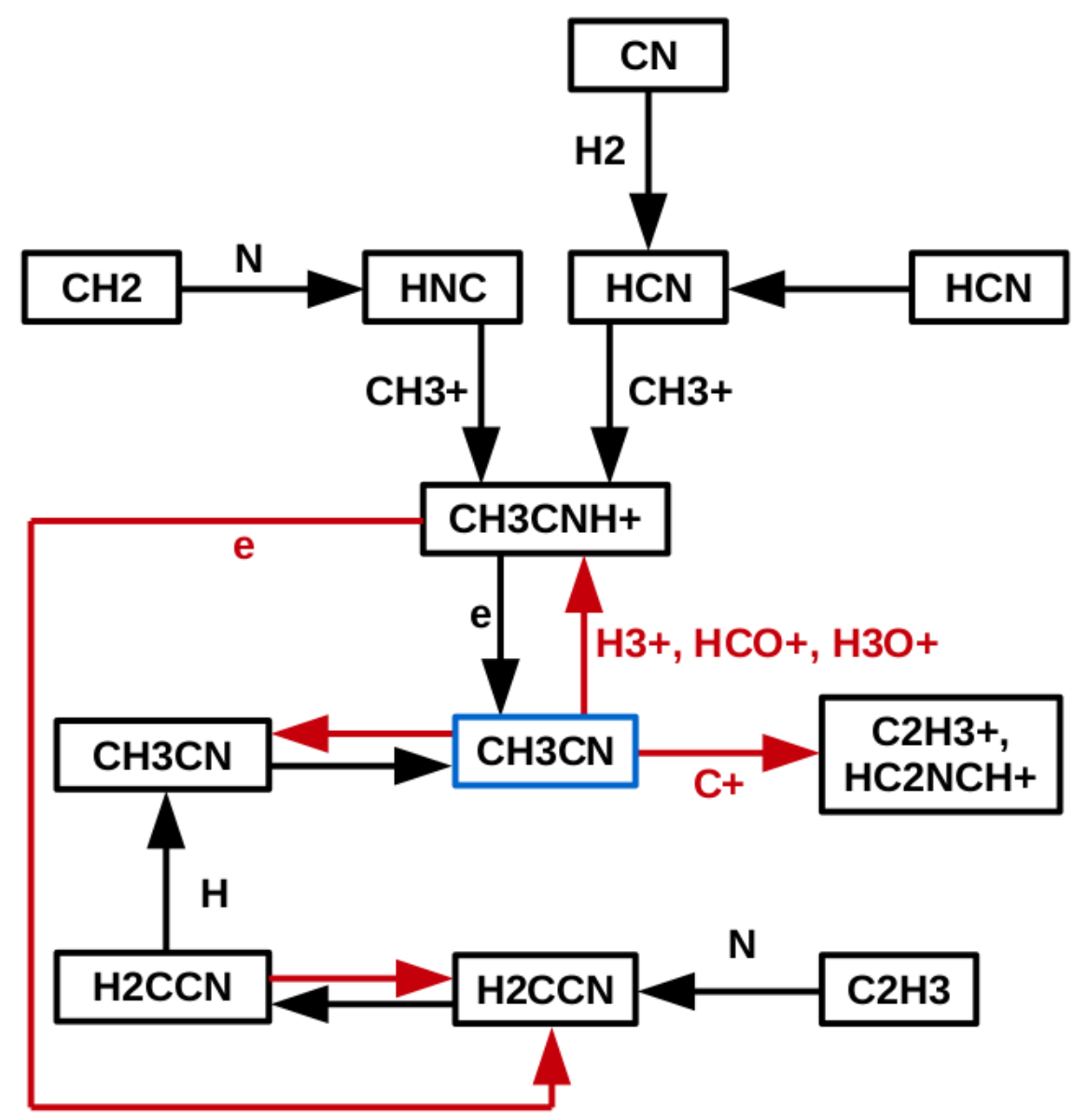

Figura 3.21: Principal rede química com os caminhos de formação e destruição de $\mathrm{CH}_{3} \mathrm{CN}$ para um modelo com temperatura do gás e do grão de $450 \mathrm{~K}$. As setas pretas representam as reações de formação e as setas vermelhas representam as de destruição. A escala de tempo desta rede é de $10^{3}$ a $10^{5}$ anos.

que a temperatura do gás (e da poeira) aumente. E também foram detectadas moléculas a uma temperatura bem inferior. Apesar disso, esse modelo já se aproxima dos resultados obtidos em (Beaklini et al., 2020) em relação ao ${ }^{1} 3 \mathrm{CH}_{3} \mathrm{CN}$ e sugere uma idade química compatível com a obtida para as demais moléculas. Simulações mais detalhadas com evolução estrutural são o próximo passo para melhorar a modelagem desse ambiente, especialmente do seu núcleo em contração. 
Capítulo 4

\section{Conclusão}

Nessa tese, uma abordagem em faixas espectrais distintas foi utilizada para estudar a complexidade molecular no Universo, através de observações no infravermelho e no sub/milimétrico. Através dela, foi possível estudar diferentes pontos de vistas da química prebiótica em ambientes astrofísicos. Mesmo sendo trabalhos diferentes, todas essas pesquisas estão concentradas na distribuição de espécies prebióticas no Universo. Desde as COMs no ISM da Galáxia aos PAHs e PANHs em fontes extragalácticas, esses resultados são complementares e realçam diferentes estágios químicos e evolutivos presentes nessa gama de objetos.

Primeiramente, centenas de galáxias foram examinadas com o objetivo de analisar variações nos perfis de emissão das bandas de PAH de 6.2, 7.7 e $8.6 \mu \mathrm{m}$. Essas galáxias com emissão dominada por starburst foram extraídas do projeto Spitzer/IRS ATLAS (HernánCaballero e Hatziminaoglou, 2011). A emissão do contínuo foi ajustada por splines e os perfis foram ajustados por Gaussianas. Em seguida, as bandas de cada galáxia foram distribuídas nas classes de Peeters (Peeters et al., 2002). Essa é a primeira vez que um estudo de PAHs (em conjunto com as classes de Peeters) é realizado com estatística robusta para um grande número de fontes extragalácticas com uma ampla variedade de redshifts.

Os resultados obtidos em Canelo et al. (2018, submitted) indicam a predominância da classe A para a banda de $6.2 \mu \mathrm{m}$, sugerindo uma contribuição significativa de PAHNs para essa emissão. A classe B se torna mais relevante para o complexo de $7.7 \mu \mathrm{m}$ e domina as variações dos perfis de $8.6 \mu \mathrm{m}$. Uma correlação entre as bandas de 6.2 e $7.7 \mu \mathrm{m}$ pode ser notada, especialmente para a classe A, como previsto por causa do mesmo modo vibracional C-C (e.g. van Diedenhoven et al., 2004).

Somente $18 \%$ de 126 galáxias obtiveram a mesma classificação "A" para as três bandas 
de PAHs, impossibilitando um estudo indireto dos PANHs a partir das outras bandas de PAH. Por outro lado, a distribuição das variações de cada perfil de PAH ao longo do redshift dessas galáxias (até z $<1.5$ ) pode sugerir uma possível escala de tempo evolutiva de PAHs, similar à evolução aromática no ciclo de vida estelar proposta por Shannon e Boersma (2019).

A segunda perspectiva abordada foi o estudo de COMs e precursores de PAHs, que foram observados em comprimentos de onda de rádio. Ao contrário do trabalho anterior, essa pesquisa estuda moléculas pequenas em relação aos PAHs mas que, assim como eles, também podem formar moléculas prebióticas mais complexas como as Nucleobases. Dois objetos foram observados e analisados com a ajuda do código astroquímico NAUTILUS, que permite modelar as abundâncias e evolução química de núcleos moleculares quentes e frios através de reações nas fases gasosas e grãos.

O núcleo quente de G331.512-0.103 (G331) foi observado com o APEX nas frequências de 160-355 GHz. Uma identificação preliminar das linhas de emissão presentes nessa fonte indicou uma grande complexidade química com emissão de várias COMs. Dentre elas, o HNCO apresentou quase 40 transições e foi analisado mais profundamente. A formamida, que pode estar quimicamente ligada ao $\mathrm{HNCO}$, não foi encontrada nesse intervalo de frequência observado.

De acordo com os resultados dos diagramas rotacionais com e sem correção de opacidade, as densidades de coluna do HNCO e suas temperaturas de excitação são de $N(\mathrm{HNCO})$ $=3.1 \pm 0.4 \times 10^{15} \mathrm{~cm}^{-2}$ e $T_{e x}=59.4 \pm 2.3 \mathrm{~K}$ e de $N(\mathrm{HNCO})=3.7 \pm 0.5 \times 10^{15} \mathrm{~cm}^{-2}$ e $T_{e x}=58.8 \pm 2.7 \mathrm{~K}$, respectivamente. A partir de duas estimativas da densidade de $\mathrm{H}_{2}$, também foi estipulado um intervalo de abundância de HNCO de $3.2 \times 10^{-9}$ a $1.4 \times 10^{-8}$. Esses resultados de densidade de coluna e abundância para essa molécula estão de acordo com valores apresentados para outros núcleos moleculares quentes (e.g. Bisschop et al., 2007).

Com uma temperatura derivada de cerca de $60 \mathrm{~K}$, as moléculas de HNCO devem se concentrar em cascas mais externas de G331, onde as temperaturas são menores. As melhores simulações químicas com o NAUTILus também estão nesse regime de temperatura e revelaram uma idade química por volta de $3 \times 10^{4-6}$ anos, com um possível valor médio de $10^{5}$ anos.

O núcleo infravermelho frio IRDC-C9 Main, com dados extraídos no ALMA, também foi 
analisado. O modelo para as abundâncias de ${ }^{13} \mathrm{CS}$, OCS, $\mathrm{CH}_{3} \mathrm{CHO}$ e $\mathrm{CH}_{3} \mathrm{OCHO}$ realizado pelo NAutilus sugere uma idade química de $10^{5}$ anos para essa fonte. Outras simulações também foram discutidas, nas quais foram feitas considerações como o acoplamento entre a temperatura do gás e da poeira e também da rede química utilizada pelo código.

Por fim, essa tese mostra uma ciência de fronteira sobre Astroquímica e Astrobiologia, assim como reforça a importância de múltiplas abordagens para a melhor compreensão do Universo.

\subsection{Próximas pesquisas e perspectivas}

\subsubsection{PAHs em fontes extragalácticas}

Expandir esse estudo para outras bandas de PAHs e, principalmente, para outros tipos de objetos como AGNs pode revelar novas informações sobre as populações de PAHs e sua relação com diferentes objetos astrofísicos, e até mesmo contribuir para aumentar o entendimento da evolução de galáxias no contexto cosmológico.

Um próximo passo é abranger a banda de PAH de $11.2 \mu \mathrm{m}$, que é importante para a determinação do tamanhos dos PAHs (Ricca et al., 2018). Além disso, ela também pode ser observada em regiões até algumas dezenas de parsecs próximas de regiões centrais de AGNs, o que sugere um material de poeira como um toro nuclear ou disco que permita a sobrevivência de PAHs nesses ambientes nucleares de AGNs (Sales et al., 2010, 2013; Alonso-Herrero et al., 2014, 2016; Monfredini et al., 2019).

Tendo esses fatores em vista, o trabalho realizado aqui pode embasar pesquisas futuras que devem ocorrer com a nova geração de telescópios. Em especial, o telescópio espacial James Webb (JWST) permitirá observar galáxias a redshifts mais elevados e com maior resolução no MIR, o que será indispensável para uma análise mais aprofundada dessas questões (Stiavelli et al., 2009).

Em relação à banda de $6.2 \mu \mathrm{m}$, o JWST deve conseguir obter observações em alta resolução até um redshift de cerca de 3.6. Isso será de extrema importância, uma vez que, atualmente, PAHs são usados para se derivar redshifts de galáxias distantes, como é o caso de $\mathrm{z} \sim 2.5$ em Canelo et al. (2018). Além da resolução já ser baixa nesses casos, incertezas na medição do redshift podem comprometer a classificação de Peeters para as bandas de PAH. 


\subsubsection{Observação e modelagem de COMs}

As moléculas previamente identificadas em G331 revelam uma fonte com muito potencial químico a ser estudado. As linhas do HNCO possibilitam um vislumbre da quantidade de informações que outras observações desse objeto podem trazer. A contínua familiarização de G331 pode abrir caminhos para a busca por moléculas maiores e mais complexas. As espécies $\mathrm{C}_{2} \mathrm{H}_{5} \mathrm{CN}$ e $\mathrm{C}_{3} \mathrm{H}_{7} \mathrm{CN}$, que estão na relação de linhas potencialmente identificadas nesse trabalho, são um exemplo de moléculas que podem ser estudadas no futuro em G331.

Observações realizadas pelo ALMA já permitiram, por exemplo, a construção de mapas de emissão integrada de alta resolução de várias moléculas (Hervías-Caimapo et al., 2019). Isso é só uma amostra do potencial esperado desse interferômetro, que pode fornecer dados importantes para o estudo de N-heterociclos e seus precursores.

Em relação às simulações químicas, incorporar uma evolução estrutural aos modelos do NAuTilus será um próximo passo de extrema importância. Nessa situação, a densidade, extinção visual, temperatura do gás e até a temperatura da poeira podem evoluir ao longo do tempo da simulação. Isso pode permitir uma maior proximidade dos modelos com as condições físicas do objeto estudado, melhorando a interpretação da química no ambiente.

As diferentes abordagens apresentadas, quando usadas em conjunto, possibilitam a visão de várias facetas da química e física de moléculas prebióticas no Universo - desde objetos mais próximos às galáxias mais distantes. Portanto, esse estudo pode ajudar a responder perguntas fundamentais que serão reveladas pelo JWST e que já estão sendo reveladas pelo ALMA. 


\section{Referências Bibliográficas}

ALMA Partnership Brogan C. L., Pérez L. M., Hunter T. R., Dent W. R. F., Hales A. S., Hills R. E., Corder S., Fomalont E. B., Vlahakis C., Asaki Y., Barkats D., Hirota A., Hodge J. A., et al. The 2014 ALMA Long Baseline Campaign: First Results from High Angular Resolution Observations toward the HL Tau Region, ApJ, 2015, vol. 808, p. L3

Alonso-Herrero A., Esquej P., Roche P. F., Ramos Almeida C., González-Martín O., Packham C., Levenson N. A., Mason R. E., Hernán-Caballero A., Pereira-Santaella M., Alvarez C., et al. A mid-infrared spectroscopic atlas of local active galactic nuclei on sub-arcsecond resolution using GTC/CanariCam, MNRAS, 2016, vol. 455, p. 563

Alonso-Herrero A., Ramos Almeida C., Esquej P., Roche P. F., Hernán-Caballero A., Hönig S. F., González-Martín O., Aretxaga I., Mason R. E., Packham C., et al. Nuclear 11.3 $\mu \mathrm{m}$ PAH emission in local active galactic nuclei, MNRAS, 2014, vol. 443, p. 2766

Altwegg K., Balsiger H., Bar-Nun A., Berthelier J. J., Bieler A., Bochsler P., Briois C., Calmonte U., Combi M. R., Cottin H., De Keyser J., Dhooghe F., Fiethe B., Fuselier S. A., et al. Prebiotic chemicals-amino acid and phosphorus-in the coma of comet 67P/Churyumov-Gerasimenko, Science Advances, 2016, vol. 2, p. e1600285

Altwegg K., Balsiger H., Hänni N., Rubin M., Schuhmann M., Schroeder I., Sémon T., Wampfler S., Berthelier J.-J., Briois C., Combi M., Gombosi T. I., Cottin H., De Keyser J., Dhooghe F., Fiethe B., Fuselier S. A., Evidence of ammonium salts in comet 67P as explanation for the nitrogen depletion in cometary comae, Nature Astronomy, 2020, p. 3 
Andrews H., Boersma C., Werner M. W., Livingston J., Allamandola L. J., Tielens A. G. G. M., PAH Emission at the Bright Locations of PDRs: the grandPAH Hypothesis, ApJ, 2015, vol. 807, p. 99

Arce H. G., Santiago-García J., Jørgensen J. K., Tafalla M., Bachiller R., Complex Molecules in the L1157 Molecular Outflow, ApJ, 2008, vol. 681, p. L21

Armstrong T., Loren R. B., MWO spectral line detections from 128 to 357 GHz 1979-1984., Tech. Rep. AST, 1984, vol. 8116403-1

Bacmann A., Faure A., The origin of gas-phase $\mathrm{HCO}$ and $\mathrm{CH}_{3} \mathrm{O}$ radicals in prestellar cores, A\&A, 2016, vol. 587, p. A130

Battersby C., Ginsburg A., Bally J., Longmore S., Dunham M., Darling J., The Onset of Massive Star Formation: The Evolution of Temperature and Density Structure in an Infrared Dark Cloud, ApJ, 2014, vol. 787, p. 113

Beaklini P. P. B., Mendoza E., Canelo C. M., Aleman I., Merello M., Kong S., Navarete F., Janot-Pacheco E., Abraham Z., Lépine J. R. D., de Almeida A. A., Friaça A. C. S., Sulphur-bearing and complex organic molecules in an infrared cold core, MNRAS, 2020, vol. 491 , p. 427

Berné O., Montillaud J., Joblin C., Top-down formation of fullerenes in the interstellar medium, A\&A, 2015, vol. 577, p. A133

Bisschop S. E., Jørgensen J. K., van Dishoeck E., Wachter E. B. M., , A\&A, 2007, vol. 566, p. L5

Bisschop S. E., Schilke P., Belloche A., et al., A\&A, 2013, vol. 552, p. 19p

Biver N., Bockelée-Morvan D., Debout V., et al., A\&A, 2014, vol. 566, p. L5

Blake G. A., Sutton E. C., Masson C. R., Phillips T. G., Molecular abundances in OMC-1 - The chemical composition of interstellar molecular clouds and the influence of massive star formation, ApJ, 1987, vol. 315, p. 621

Blumberg B. S., The NASA Astrobiology Institute: Early History and Organization, Astrobiology, 2003, vol. 3, p. 463 
Bottinelli S., Ceccarelli C., Lefloch B., Williams J. P., Castets A., Caux E., Cazaux S., Maret S., Parise B., Tielens A. G. G. M., Complex Molecules in the Hot Core of the Low-Mass Protostar NGC 1333 IRAS 4A, ApJ, 2004, vol. 615, p. 354

Bronfman L., Garay G., Merello M., Mardones D., May J., Brooks K. J., Nyman L.-A., Güsten R., Discovery of an Extremely High Velocity, Massive, and Compact Molecular Outflow in Norma, ApJ, 2008, vol. 672, p. 391

Brouillet N., Despois D., Lu X.-H., Baudry A., Cernicharo J., Bockelée-Morvan D., Crovisier J., Biver N., Antifreeze in the hot core of Orion. First detection of ethylene glycol in Orion-KL, A\&A, 2015, vol. 576, p. A129

Brown P. D., Charnley S. B., Millar T. J., A model of the chemistry in hot molecular cores, MNRAS, 1988, vol. 231, p. 409

Butler M. J., Tan J. C., Mid-Infrared Extinction Mapping of Infrared Dark Clouds: Probing the Initial Conditions for Massive Stars and Star Clusters, ApJ, 2009, vol. 696, p. 484

Canelo C. M., O Mundo Aromático - dos PAHs no meio interestelar às condições bióticas, IAG-USP, 2016, Dissertação de Mestrado

Canelo C. M., Friaça A. C. S., Sales D. A., Pastoriza M. G., Menéndez-Delmestre K., Profile comparison of the $6-9 \mu \mathrm{m}$ polycyclic aromatic hydrocarbon bands in starburstdominated galaxies, (submitted), 2021

Canelo C. M., Friaça A. C. S., Sales D. A., Pastoriza M. G., Ruschel-Dutra D., Variations in the $6.2 \mu \mathrm{m}$ emission profile in starburst-dominated galaxies: a signature of polycyclic aromatic nitrogen heterocycles (PANHs)?, MNRAS, 2018, vol. 475, p. 3746

Ceccarelli C., Caux E., White G. J., Molinari S., Furniss I., Liseau R., Nisini B., Saraceno P., Spinoglio L., Wolfire M., The far infrared line spectrum of the protostar IRAS 162932422, A\&A, 1998, vol. 331, p. 372

Chang Q., Cuppen H. M., Herbst E., Gas-grain chemistry in cold interstellar cloud cores with a microscopic Monte Carlo approach to surface chemistry, A\&A, 2007, vol. 469, p. 973 
Chang Q., Herbst E., Unified Microscopic-Macroscopic Monte Carlo Simulations of Complex Organic Molecule Chemistry in Cold Cores, ApJ, 2016, vol. 819, p. 145

Charnley S. B., Kuan Y.-J., Huang H.-C., Botta O., Butner H. M., Cox N., Despois D., Ehrenfreund P., Kisiel Z., Lee Y.-Y., Markwick A. J., Peeters Z., Rodgers S. D., Astronomical searches for nitrogen heterocycles, Advances in Space Research, 2005, vol. 36 , p. 137

Charnley S. B., Tielens A. G. G. M., Millar T. J., On the molecular complexity of the hot cores in Orion A - Grain surface chemistry as 'The last refuge of the scoundrel', ApJ, 1992, vol. 399, p. L71

Chen L.-F., Chang Q., Xi H.-W., Effect of stochastic grain heating on cold dense clouds chemistry, MNRAS, 2018, vol. 479, p. 2988

Churchwell E., Wood D., Myers P. C., Myers R. V., The Excitation, Abundance, and Distribution of HNCO in Sagittarius B2, ApJ, 1986, vol. 305, p. 405

Coelho L. d. S., CAMINHOS PARA A FORMAÇÃO DE MOLÉCULAS ORGÂNICAS PREBIÓTICAS NO MEIO INTERESTELAR, IAG-USP, 2018, Tese de Doutorado

Coutens A., Jørgensen J. K., van der Wiel M. H. D., et al., A\&A, 2016, vol. 590, p. L6

Crapsi A., Caselli P., Walmsley C. M., Myers P. C., Tafalla M., Lee C. W., Bourke T. L., Probing the Evolutionary Status of Starless Cores through $\mathrm{N}_{2} \mathrm{H}^{+}$and $\mathrm{N}_{2} \mathrm{D}^{+}$Observations, ApJ, 2005, vol. 619, p. 379

Dalgarno A., A serendipitous journey., ARA\&A, 2008, vol. 46, p. 1

de Wit W. J., Testi L., Palla F., Zinnecker H., The origin of massive O-type field stars: II. Field O stars as runaways, A\&A, 2005, vol. 437, p. 247

Dobrijevic M., Hébrard E., Loison J. C., Hickson K. M., Coupling of oxygen, nitrogen, and hydrocarbon species in the photochemistry of Titan's atmosphere, Icarus, 2014, vol. 228, p. 324

Duronea N. U., Bronfman L., Mendoza E., Merello M., Finger R., Reyes N., HervíasCaimapo C., Faure A., Cappa C. E., Arnal E. M., Lépine J. R. D., Kleiner I., Nyman 
L. A., Cyanoacetylene in the outflow/hot molecular core G331.512-0.103, MNRAS, 2019, vol. 489 , p. 1519

Dutra D. R., O ambiente circum-nuclear em galáxias ativas: formação estelar, toro de poeira e cinemática do gás., UFRGS, 2015, Tese de Doutorado

Ehrenfreund P., Irvine W., Becker L., Blank J., Brucato J. R., Colangeli L., Derenne S., Despois D., Dutrey A., Fraaije H., Lazcano A., Owen T., Robert F., International Space Science Institute ISSI-Team Astrophysical and astrochemical insights into the origin of life, Reports on Progress in Physics, 2002, vol. 65, p. 1427

Ehrenfreund P., Rasmussen S., Cleaves J., Chen L., Experimentally Tracing the Key Steps in the Origin of Life: The Aromatic World, Astrobiology, 2006, vol. 6, p. 490

Ellder J., Friberg P., Hjalmarson A., Hoglund B., Johansson L. E. B., Olofsson H., Rydbeck G., Rydbeck O. E. H., Guelin M., Irvine W. M., On methyl formate, methane, and deuterated ammonia in Orion A, ApJ, 1980, vol. 242, p. L93

Endres C. P., Schlemmer S., Schilke P., Stutzki J., Müller H. S. P., The Cologne Database for Molecular Spectroscopy, CDMS, in the Virtual Atomic and Molecular Data Centre, VAMDC, Journal of Molecular Spectroscopy, 2016, vol. 327, p. 95

Fedoseev G., Ioppolo S., Zhao D., Lamberts T., Linnartz H., Low-temperature surface formation of $\mathrm{NH}_{3}$ and $\mathrm{HNCO}$ : hydrogenation of nitrogen atoms in CO-rich interstellar ice analogues, MNRAS, 2015, vol. 446, p. 439

Ferus M., Nesvorný D., Šponer J., Kubelík P., Michalčíková R., Shestivská V., Šponer J. E., Civiš S., High-energy chemistry of formamide: A unified mechanism of nucleobase formation, Proceedings of the National Academy of Science, 2015, vol. 112, p. 657

Frieswijk W., Early stages of clustered star formation-massive dark clouds throughout the Galaxy-, 2008, Tese de Doutorado

Garrod R. T., , ApJ, 2013, vol. 765, p. 60

Garrod R. T., Herbst E., Formation of methyl formate and other organic species in the warm-up phase of hot molecular cores, A\&A, 2006, vol. 457, p. 927 
Garrod R. T., Pauly T., On the Formation of $\mathrm{CO}_{2}$ and Other Interstellar Ices, ApJ, 2011, vol. 735 , p. 15

Garrod R. T., Wakelam V., Herbst E., Non-thermal desorption from interstellar dust grains via exothermic surface reactions, A\&A, 2007, vol. 467, p. 1103

Garrod R. T., Widicus Weaver S. L., Herbst E., Complex Chemistry in Star-forming Regions: An Expanded Gas-Grain Warm-up Chemical Model, ApJ, 2008, vol. 682, p. 283

Ghesquière P., Mineva T., Talbi D., Theulé P., Noble J. A., Chiavassa T., Diffusion of molecules in the bulk of a low density amorphous ice from molecular dynamics simulations, Physical Chemistry Chemical Physics (Incorporating Faraday Transactions), 2015, vol. 17 , p. 11455

Goldsmith P. F., Langer W. D., , ApJ, 1999, vol. 517, p. 209

Gorai P., Bhat B., Sil M., Mondal S. K., Ghosh R., Chakrabarti S. K., Das A., Identification of pre-biotic molecules containing Peptide-like bond in a hot molecular core, G10.47+0.03, arXiv e-prints, 2020, p. arXiv:2003.09188

Graedel T. E., Langer W. D., Frerking M. A., The kinetic chemistry of dense interstellar clouds, ApJS, 1982, vol. 48, p. 321

Greaves J. S., White G. J., A 257-273 GHz spectral survey of the OMC 1 cloud core., A\&AS, 1991, vol. 91, p. 237

Guimarães M. C., Efeitos da presença de poeira na emissão de galáxias a altos e baixos redshifts, IAG-USP, 2006, Dissertação de Mestrado

Hasegawa T. I., Herbst E., Three-Phase Chemical Models of Dense Interstellar Clouds Gas Dust Particle Mantles and Dust Particle Surfaces, MNRAS, 1993, vol. 263, p. 589

Haupa K. A., Tarczay G., Lee Y.-P., Hydrogen Abstraction/Addition Tunneling Reactions Elucidate the Interstellar $\mathrm{H} 2 \mathrm{NCHO} / \mathrm{HNCO}$ Ratio and $\mathrm{H} 2$ Formation, Journal of the American Chemical Society, 2019, vol. 141, p. 11614

Herbst E., van Dishoeck E. F., Complex Organic Interstellar Molecules, ARA\&A, 2009, vol. 47, p. 427 
Hernán-Caballero A., Hatziminaoglou E., An atlas of mid-infrared spectra of star-forming and active galaxies, MNRAS, 2011, vol. 414, p. 500

Hernández-Gómez A., Sahnoun E., Caux E., Wiesenfeld L., Loinard L., Bottinelli S., Hammami K., Menten K. M., Modelling the abundance structure of isocyanic acid (HNCO) towards the low-mass solar type protostar IRAS 16293-2422, MNRAS, 2019, vol. 483, p. 2014

Hervías-Caimapo C., Merello M., Bronfman L., Åke-Nyman L., Garay G., Lo N., Evans Neal J. I., López-Calderón C., Mendoza E., ALMA Observations of the Massive Molecular Outflow G331.512-0.103. II. Physical Properties, Kinematics, and Geometry Modeling, ApJ, 2019, vol. 872, p. 200

Hincelin U., Wakelam V., Hersant F., Guilloteau S., Loison J. C., Honvault P., Troe J., Oxygen depletion in dense molecular clouds: a clue to a low $\mathrm{O}_{2}$ abundance?, A\&A, 2011, vol. 530 , p. A61

Hocking W. H., Gerry M. C. L., Winnewisser G., The Dipole Moment of Isocyanic Acid, HNCO, and its Astrophysical Consequences, ApJ, 1974, vol. 187, p. L89

Horn A., Møllendal H., Sekiguchi O., Uggerud E., Roberts H., Herbst E., Viggiano A. A., Fridgen T. D., The Gas-Phase Formation of Methyl Formate in Hot Molecular Cores, ApJ, 2004, vol. 611, p. 605

Houck J. R., Roellig T. L., van Cleve J., Forrest W. J., Herter T., Lawrence C. R., Matthews K., Reitsema H. J., Soifer B. T., Watson D. M., Weedman D., Huisjen M., Troeltzsch J., Barry D. J., Bernard-Salas J., Blacken C. E., Brandl B. R., et al. The Infrared Spectrograph (IRS) on the Spitzer Space Telescope., The Astrophysical Journal Supplement Series, 2004, vol. 154, p. Issue 1, 18

Hudgins D. M., Allamandola L. J., The spacing of the interestellar 6.2 and 7.7 micron emission features as an indicator of polycyclic aromatic hydrocarbon size., ApJ, 1999, vol. 513, p. L69

Hudgins D. M., Bauschlicher Jr. C. W., Allamandola L. J., Variations in the Peak Position of the $6.2 \mu \mathrm{m}$ Interstellar Emission Feature: A Tracer of $\mathrm{N}$ in the Interstellar Polycyclic Aromatic Hydrocarbon Population, ApJ, 2005, vol. 632, p. 316 
Jaber A. A., Ceccarelli C., Kahane C., Caux E., The Census of Complex Organic Molecules in the Solar-type Protostar IRAS16293-2422, ApJ, 2014, vol. 791, p. 29

Jenkins E. B., A Unified Representation of Gas-Phase Element Depletions in the Interstellar Medium, ApJ, 2009, vol. 700, p. 1299

Jewell P. R., Hollis J. M., Lovas F. J., Snyder L. E., Millimeter- and Submillimeter-Wave Surveys of Orion A Emission Lines in the Ranges 200.7-202.3, 203.7-205.3, and 330-360 GHz, ApJS, 1989, vol. 70, p. 833

Joblin C., Leger A., Martin P., Contribution of polycyclic aromatic hydrocarbon molecules to the interstellar extinction curve, ApJ, 1992, vol. 393, p. L79

Jørgensen J. K., van der Wiel M. H. D., Coutens A., Lykke J. M., Müller H. S. P., van Dishoeck E. F., Calcutt H., Bjerkeli P., Bourke T. L., Drozdovskaya M. N., et al. The ALMA Protostellar Interferometric Line Survey (PILS). First results from an unbiased submillimeter wavelength line survey of the Class 0 protostellar binary IRAS 16293-2422 with ALMA, A\&A, 2016, vol. 595, p. A117

Joyce G. F., The antiquity of RNA-based evolution, Nature, 2002, vol. 418, p. 214

Kalvāns J., The efficiency of photodissociation for molecules in interstellar ices, MNRAS, 2018, vol. 478, p. 2753

Kong S., Tan J. C., Caselli P., Fontani F., Liu M., Butler M. J., A Hunt for Massive Starless Cores, ApJ, 2017, vol. 834, p. 193

Kruegel E., Walmsley C. M., Dust and gas temperatures in dense molecular clouds, A\&A, 1984, vol. 130, p. 5

Kuan Y., Yan C., Charnley S. B., et al., MNRAS, 2003, vol. 345, p. 650

Kuan Y.-J., Huang H.-C., Charnley S. B., Hirano N., Takakuwa S., Wilner D. J., Liu S.-Y., Ohashi N., Bourke T. L., Qi C., Zhang Q., Organic Molecules in Low-Mass Protostellar Hot Cores: Submillimeter Imaging of IRAS 16293-2422, ApJ, 2004, vol. 616, p. L27

Li A., Interaction of Nanoparticles with Radiation. In Astrophysics of Dust, vol. 309 of Astronomical Society of the Pacific Conference Series, 2004, p. 417 
Li J., Wang J., Zhu Q., Zhang J., Li D., Sulfur-bearing Molecules in Massive Star-forming Regions: Observations of OCS, CS, $\mathrm{H}_{2} \mathrm{~S}$, and SO, ApJ, 2015, vol. 802, p. 40

Ligterink N. F. W., Terwisscha van Scheltinga J., Taquet V., Jørgensen J. K., Cazaux S., van Dishoeck E. F., Linnartz H., The formation of peptide-like molecules on interstellar dust grains, MNRAS, 2018, vol. 480, p. 3628

Loison J.-C., Halvick P., Bergeat A., Hickson K. M., Wakelam V., Review of OCS gas-phase reactions in dark cloud chemical models, MNRAS, 2012, vol. 421, p. 1476

López-Sepulcre A., Balucani N., Ceccarelli C., Codella C., Dulieu F., Theulé P., Interstellar Formamide (NH2CHO), a Key Prebiotic Precursor, ACS Earth and Space Chemistry, 2019, vol. 3, p. 2122

Loren R. B., Mundy L. G., The methyl cyanide hot and warm cores in Orion : statistical equilibrium excitation models of a symmetric-top molecule., ApJ, 1984, vol. 286, p. 232

Lovas F. J., NIST Recommended Rest Frequencies for Observed Interstellar Molecular Microwave Transitions-2002 Revision, Journal of Physical and Chemical Reference Data, 2004, vol. 33, p. 177

MacDonald G. H., Gibb A. G., Habing R. J., Millar T. J., A 330-360 GHz spectral survey of G 34.3+0.15. I. Data and physical analysis., A\&AS, 1996, vol. 119, p. 333

Majumdar L., Gorai P., Das A., et al., arXiv:1511.04343v1, 2015

Martel H., Urban A., Evans II N. J., Fragmentation and Evolution of Molecular Clouds. III. The Effect of Dust and Gas Energetics, ApJ, 2012, vol. 757, p. 59

Martín-Doménech R., Rivilla V. M., Jiménez-Serra I., Quénard D., Testi L., MartínPintado J., Detection of methyl isocyanate $\left(\mathrm{CH}_{3} \mathrm{NCO}\right)$ in a solar-type protostar, MNRAS, 2017, vol. 469, p. 2230

McElroy D., Walsh C., Markwick A. J., Cordiner M. A., Smith K., Millar T. J., The UMIST database for astrochemistry 2012, A\&A, 2013, vol. 550, p. A36

McGuire B. A., Burkhardt A. M., Kalenskii S., Shingledecker C. N., Remijan A. J., Herbst E., McCarthy M. C., Detection of the aromatic molecule benzonitrile $\left(\mathrm{c}-\mathrm{C}_{6} \mathrm{H}_{5} \mathrm{CN}\right)$ in the interstellar medium, Science, 2018, vol. 359, p. 202 
Mendoza E., Almeida G. C., Andrade D. P. P., et al., MNRAS, 2013, vol. 433, p. 3440

Mendoza E., Bronfman L., Duronea N. U., et al., ApJ, 2018, vol. 853, p. 152

Mendoza E., Lefloch B., López-Sepulcre A., Ceccarelli C., Codella C., Boechat-Roberty H. M., Bachiller R., Molecules with a peptide link in protostellar shocks: a comprehensive study of L1157, MNRAS, 2014, vol. 445, p. 151

Merello M., Bronfman L., Garay G., Lo N., Evans Neal J. I., Nyman L.-Å., Cortés J. R., Cunningham M. R., ALMA Observations of the Massive Molecular Outflow G331.5120.103, ApJ, 2013, vol. 774, p. L7

Merello M., Molinari S., Rygl K. L. J., Evans N. J., Elia D., Schisano E., Traficante A., Shirley Y., Svoboda B., Goldsmith P. F., Thermal balance and comparison of gas and dust properties of dense clumps in the Hi-GAL survey, MNRAS, 2019, vol. 483, p. 5355

Monfredini T., Quitián-Lara H. M., Fantuzzi F., Wolff W., Mendoza E., Lago A. F., Sales D. A., Pastoriza M. G., Boechat-Roberty H. M., Destruction and multiple ionization of PAHs by X-rays in circumnuclear regions of AGNs, MNRAS, 2019, vol. 488, p. 451

Neufeld D. A., Wolfire M. G., Schilke P., The Chemistry of Fluorine-bearing Molecules in Diffuse and Dense Interstellar Gas Clouds, ApJ, 2005, vol. 628, p. 260

Niedenhoff M., Yamada K. M. T., Belov S. P., Winnewisser G., Pure Rotational Spectra of HNCO in the Far Infrared: Ground State Analysis, Journal of Molecular Spectroscopy, 1995, vol. 174 , p. 151

Noble J. A., Theule P., Congiu E., Dulieu F., Bonnin M., Bassas A., Duvernay F., Danger G., Chiavassa T., Hydrogenation at low temperatures does not always lead to saturation: the case of HNCO, A\&A, 2015, vol. 576, p. A91

Palmer M. Y., Cordiner M. A., Nixon C. A., el al., Sci. Adv., 2017, vol. 3, p. e1700022

Papovich C., Moustakas L. A., Dickinson M., Le Floc'h E., Rieke G. H., Daddi E., Alexander D. M., Bauer F., Brandt W. N., Dahlen T., Egami E., Eisenhardt P., Elbaz D., Ferguson H. C., Giavalisco M., Lucas R. A., et al. Spitzer Observations of Massive, Red Galaxies at High Redshift, ApJ, 2006, vol. 640, p. 92 
Parker D. S. N., Kaiser R. I., , Chem. Soc. Rev., 2017, vol. 46, p. 452

Parker D. S. N., Kaiser R. I., Kostko O., Troy T. P., Ahmed M., Mebel A. M., Tielens A. G. G. M., Gas Phase Synthesis of (Iso)Quinoline and Its Role in the Formation of Nucleobases in the Interstellar Medium, ApJ, 2015, vol. 803, p. 53

Peeters E., Bauschlicher Charles W. J., Allamand ola L. J., Tielens A. G. G. M., Ricca A. r., Wolfire M. G., The PAH Emission Characteristics of the Reflection Nebula NGC 2023, ApJ, 2017, vol. 836, p. 198

Peeters E., Hony S., Van Kerckhoven C., Tielens A. G. G. M., Allamandola L. J., Hudgins D. M., Bauschlicher C. W., The rich 6 to $9 \mu \mathrm{m}$ spectrum of interstellar PAHs., A\&A, 2002, vol. 390, p. 1089

Peeters Z., Botta O., Charnley S. B., Kisiel Z., Kuan Y. J., Ehrenfreund P., Formation and photostability of N-heterocycles in space. I. The effect of nitrogen on the photostability of small aromatic molecules, A\&A, 2005, vol. 433, p. 583

Pickett H. M., Poynter R. L., Cohen E. A., Delitsky M. L., Pearson J. C., Müller H. S. P., Submillimeter, millimeter and microwave spectral line catalog., J. Quant. Spec. Radiat. Transf., 1998, vol. 60, p. 883

Prasad S. S., Tarafdar S. P., UV radiation field inside dense clouds - Its possible existence and chemical implications, ApJ, 1983, vol. 267, p. 603

Reboussin L., Wakelam V., Guilloteau S., Hersant F., Grain-surface reactions in molecular clouds: the effect of cosmic rays and quantum tunnelling, MNRAS, 2014, vol. 440, p. 3557

Ricca A., Bauschlicher Jr. C. W., Roser J. E., Peeters E., Polycyclic Aromatic Hydrocarbons with Straight Edges and the 7.6/6.2 and 8.6/6.2 Intensity Ratios in Reflection Nebulae, ApJ, 2018, vol. 854, p. 115

Riechers D. A., Pope A., Daddi E., Armus L., Carilli C. L., Walter F., Hodge J., Chary R.-R., Morrison G. E., Dickinson M., Dannerbauer H., Elbaz D., Polycyclic Aromatic Hydrocarbon and Mid-Infrared Continuum Emission in a $\mathrm{z}>4$ Submillimeter Galaxy, ApJ, 2014, vol. 786, p. 31 
Ruaud M., Loison J. C., Hickson K. M., Gratier P., Hersant F., Wakelam V., Modelling complex organic molecules in dense regions: Eley-Rideal and complex induced reaction, MNRAS, 2015, vol. 447, p. 4004

Ruaud M., Wakelam V., Hersant F., Gas and grain chemical composition in cold cores as predicted by the Nautilus three-phase model, MNRAS, 2016, vol. 459, p. 3756

Rubin R. H., Swenson G. W., Solomon Jr. R. C., Flygare H. L., , ApJ, 1971, vol. 169, p. L39

Ruschel-Dutra D., Pastoriza M., Riffel R., Sales D. A., Winge C., A mid-IR comparative analysis of the Seyfert galaxies NGC 7213 and NGC 1386, MNRAS, 2014, vol. 438, p. 3434

Saladino R., et al., PNAS, 2015, vol. 112, p. 2746

Sales D. A., Pastoriza M. G., Riffel R., Polycyclic Aromatic Hydrocarbon and Emission Line Ratios in Active Galactic Nuclei and Starburst Galaxies, ApJ, 2010, vol. 725, p. 605

Sales D. A., Pastoriza M. G., Riffel R., Winge C., Polycyclic aromatic hydrocarbon in the central region of the Seyfert 2 galaxy NGC 1808, MNRAS, 2013, vol. 429, p. 2634

Sanders D. B., Soifer B. T., Elias J. H., Madore B. F., Matthews K., Neugebauer G., Scoville N. Z., Ultraluminous Infrared Galaxies and the Origin of Quasars, ApJ, 1988, vol. 325 , p. 74

Sandström H., Rahm M., Can polarity-inverted membranes self-assemble on Titan?, Science Advances, 2020, vol. 6, p. eaax0272

Schneider P., Extragalactic Astronomy and Cosmology. Springer Heidelberg New York, 2006

Schwell M., Jochims H., et al., Chemical Physics, 2008, vol. 353, p. 145

Semenov D., Hersant F., Wakelam V., Dutrey A., Chapillon E., Guilloteau S., Henning T., Launhardt R., Piétu V., Schreyer K., Chemistry in disks. IV. Benchmarking gas-grain chemical models with surface reactions, A\&A, 2010, vol. 522, p. A42 
Shannon M. J., Boersma C., Examining the Class B to A Shift of the $7.7 \mu \mathrm{m}$ PAH Band with the NASA Ames PAH IR Spectroscopic Database, ApJ, 2019, vol. 871, p. 124

Shannon R. J., Cossou C., Loison J.-C., Caubet P., Balucani N., Seakins P. W., Wakelam V., Hickson K. M., The fast $\mathrm{C}(3 \mathrm{P})+\mathrm{CH} 3 \mathrm{OH}$ reaction as an efficient loss process for gas-phase interstellar methanol, RSC Adv., 2014, vol. 4, p. 26342

Siana B., Smail I., Swinbank A. M., Richard J., Teplitz H. I., Coppin K. E. K., Ellis R. S., Stark D. P., Kneib J.-P., Edge A. C., Detection of Far-Infrared and Polycyclic Aromatic Hydrocarbon Emission from the Cosmic Eye: Probing the Dust and Star Formation of Lyman Break Galaxies, ApJ, 2009, vol. 698, p. 1273

Simon M. N., Simon M., Search for Interstellar Acrylonitrile, Pyrimidine, and Pyridine, ApJ, 1973, vol. 184, p. 757

Snyder L. E., Buhl D., , ApJ, 1972, vol. 177, p. 619

Stevenson J., Lunine J., Clancy P., Membrane alternatives in worlds without oxygen: Creation of an azotosome, Science Advances, 2015, vol. 1, p. 1400067

Stiavelli M., Mather J., Clampin M., Doyon R., Flanagan K., Franx M., Gardner J., Greenhouse M., Hammel H., Hutchings J., Jakobsen P., Lilly S., McCaughrean M., et al. First light and reionization : open questions in the post-JWST era. In astro2010: The Astronomy and Astrophysics Decadal Survey, vol. 2010, 2009, p. 287

Sutton E. C., Blake G. A., Masson C. R., Phillips T. G., Molecular line survey of Orion A from 215 to 247 GHz., ApJS, 1985, vol. 58, p. 341

Sutton E. C., Jaminet P. A., Danchi W. C., Blake G. A., Molecular Line Survey of Sagittarius B2(M) from 330 to $355 \mathrm{GHz}$ and Comparison with Sagittarius B2(N), ApJS, 1991, vol. 77 , p. 255

Tan J. C., Kong S., Butler M. J., Caselli P., Fontani F., The Dynamics of Massive Starless Cores with ALMA, ApJ, 2013, vol. 779, p. 96

Teplitz H. I., Desai V., Armus L., Chary R., Marshall J. A., Colbert J. W., Frayer D. T., Pope A., Blain A., Spoon H. W. W., Charmandaris V., Scott D., Measuring PAH 
Emission in Ultradeep Spitzer IRS Spectroscopy of High-Redshift IR-Luminous Galaxies, ApJ, 2007, vol. 659, p. 941

Tielens A. G. G. M., The Physics and Chemistry of the Interstellar Medium. Cambridge University Press, 2005, 510 p.

Tielens A. G. G. M., Interstellar Polycyclic Aromatic Hydrocarbon Molecules, ARA\&A, 2008, vol. 46 , p. 289

Tielens A. G. G. M., The molecular universe, Reviews of Modern Physics, 2013, vol. 85, p. 1021

Urry C. M., Padovani P., Unified Schemes for Radio-Loud Active Galactic Nuclei, PASP, 1995, vol. 107, p. 803

van Diedenhoven B., Peeters E., Van Kerckhoven C., Hony S., Hudgins D. M., Allamandola L. J., Tielens A. G. G. M., The Profiles of the 3-12 Micron Polycyclic Aromatic Hydrocarbon Features, ApJ, 2004, vol. 611, p. 928

Vasyunina T., Vasyunin A. I., Herbst E., Linz H., Chemical Modeling of Infrared Dark Clouds: The Role of Surface Chemistry, ApJ, 2012, vol. 751, p. 105

Velilla Prieto L., Sánchez Contreras C., Cernicharo J., Agúndez M., Quintana-Lacaci G., Alcolea J., Bujarrabal V., Herpin F., Menten K. M., Wyrowski F., New N-bearing species towards $\mathrm{OH} 231.8+4.2$. $\mathrm{HNCO}, \mathrm{HNCS}, \mathrm{HC}_{3} \mathrm{~N}$, and NO, A\&A, 2015, vol. 575, p. A84

Vidal T. H. G., Wakelam V., A new look at sulphur chemistry in hot cores and corinos, MNRAS, 2018, vol. 474, p. 5575

Wagenblast R., Hartquist T. W., Non-equilibrium level populations of molecular hydrogen. II - Models of the Zeta OPH cloud, MNRAS, 1989, vol. 237, p. 1019

Wakelam V., Herbst E., Polycyclic Aromatic Hydrocarbons in Dense Cloud Chemistry, ApJ, 2008, vol. 680, p. 371

Wakelam V., Herbst E., Selsis F., The effect of uncertainties on chemical models of dark clouds, A\&A, 2006, vol. 451, p. 551 
Wakelam V., Loison J.-C., Herbst E., Pavone B., Bergeat A., Béroff K., Chabot M., Faure A., Galli D., Geppert W. D., Gerlich D., et al. The 2014 KIDA Network for Interstellar Chemistry, ApJS, 2015, vol. 217, p. 20

Werner M. W., Roellig T. L., Low F. J., Rieke G. H., Rieke M., Hoffmann W. F., Young E., Houck J. R., Brandl B., Fazio G. G., Hora J. L., Gehrz R. D., Helou G., Soifer B. T., et al. The Spitzer Space Telescope Mission., The Astrophysical Journal Supplement Series, 2004, vol. 154, p. Issue 1, 1

Yan L., Chary R., Armus L., Teplitz H., Helou G., Frayer D., Fadda D., Surace J., Choi P., Spitzer Detection of Polycyclic Aromatic Hydrocarbon and Silicate Dust Features in the Mid-Infrared Spectra of z 2 Ultraluminous Infrared Galaxies, ApJ, 2005, vol. 628, p. 604

Yan L., Sajina A., Fadda D., et al., ApJ, 2007, vol. 658, p. 778

Zinchenko I., Henkel C., Mao R. Q., HNCO in massive galactic dense cores, A\&A, 2000, vol. 361 , p. 1079 
Apêndice 



\section{Apêndice A}

\section{Figuras dos ajustes das bandas de PAH}

Abaixo estão apresentadas todas as figuras referentes aos ajustes do contínuo e das bandas de 6.2, 7.7 e $8.6 \mu \mathrm{m}$ das galáxias com emissão dominada por starburst, que foram discutidos no Capítulo 2. A Tabela A.1 mostra as figuras das 29 galáxias que tiveram somente a banda de $6.2 \mu \mathrm{m}$ ajustada (Canelo et al., 2018). Nesses casos, o contínuo foi ajustado com o spline global. Em seguida, as outras 126 galáxias com os ajustes das três bandas de PAH podem ser vistas na Tabela A.2 (Canelo et al., 2018, submitted). Nessas situações, o contínuo foi ajustado com o spline local. Vale ressaltar, novamente, que a escolha entre os dois tipos de spline não influencia o ajuste e resultados obtidos para a banda de $6.2 \mu \mathrm{m}$.

Tabela A.1 - Imagens dos ajustes do contínuo e bandas de $6.2 \mu \mathrm{m}$ das 29 galáxias com emissão dominada por starburst (Canelo et al., 2018) e que não tiveram as bandas de 7.7 e $8.6 \mu \mathrm{m}$ ajustadas .
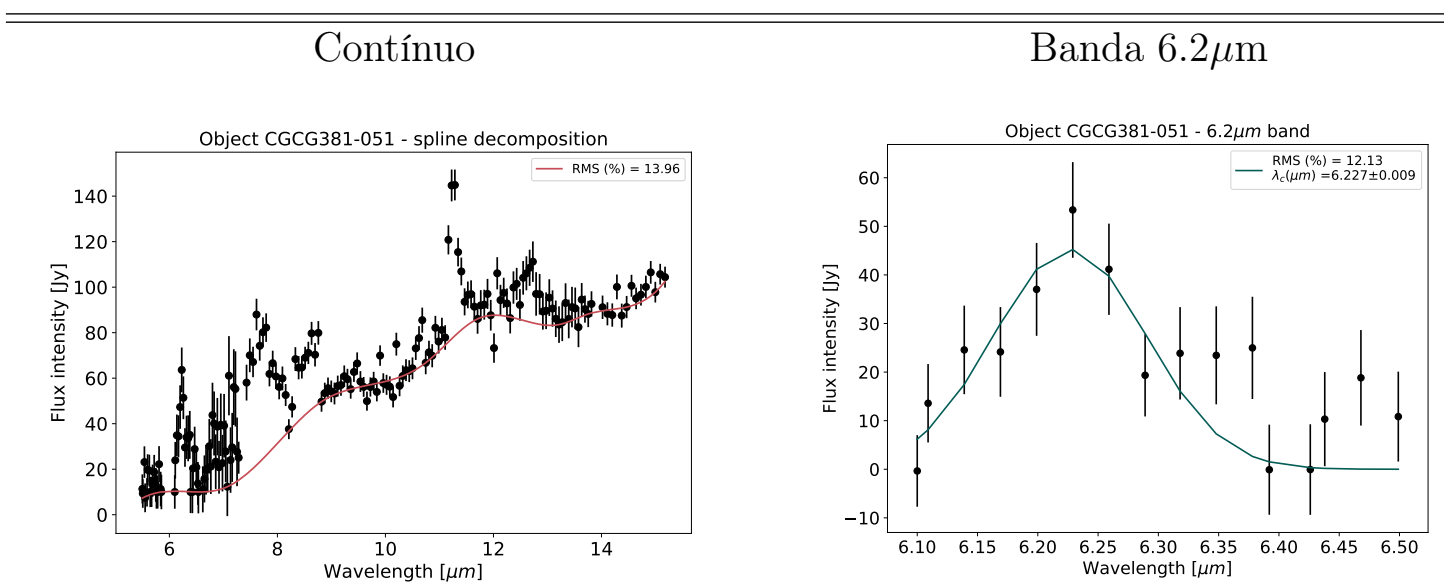

Continua na próxima página... 
Tabela A.1 - Continuação

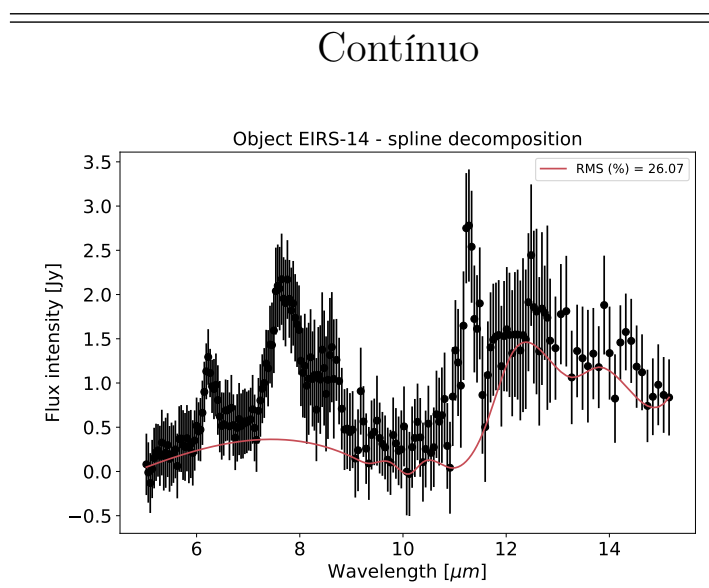

Banda $6.2 \mu \mathrm{m}$
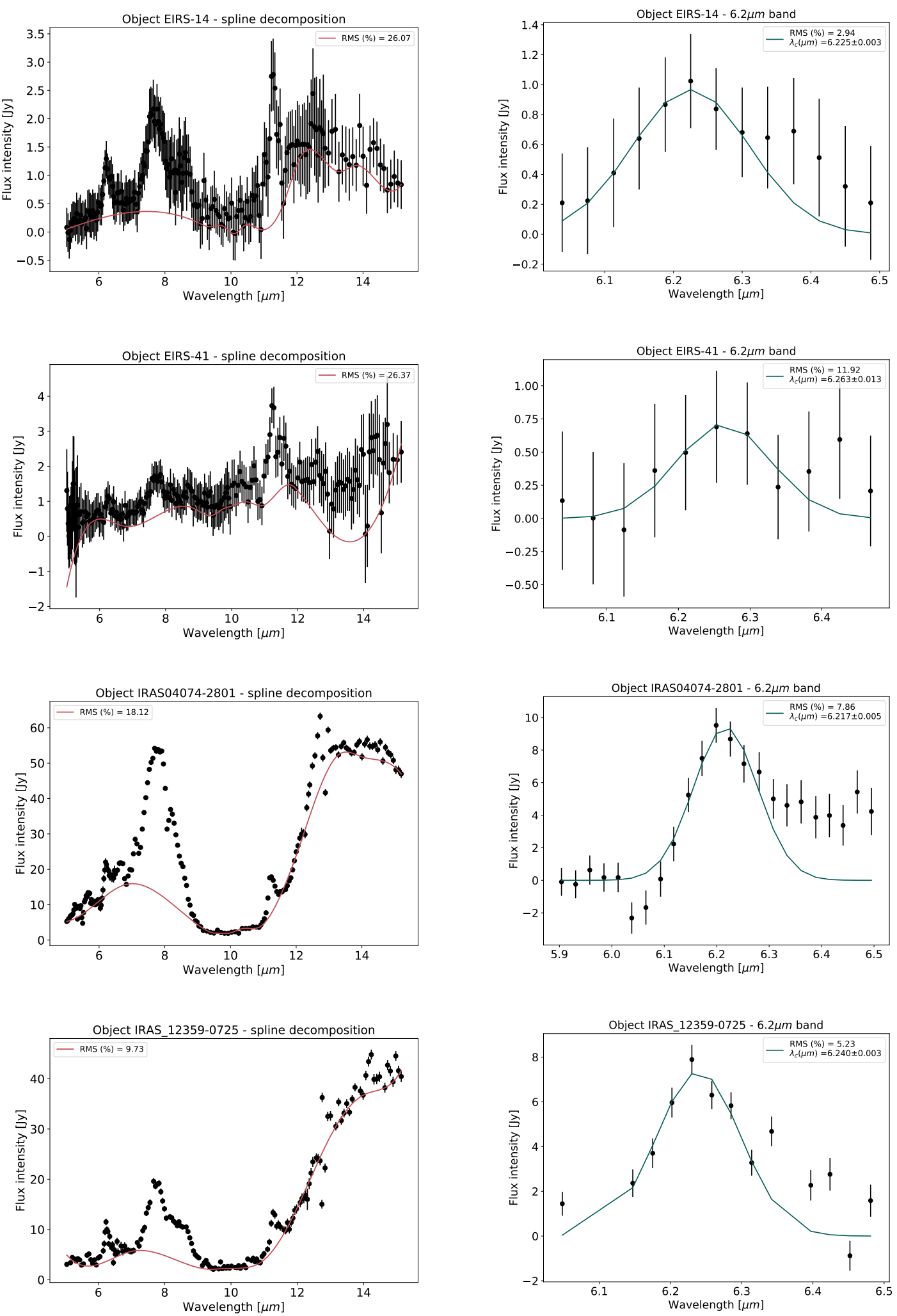

Continua na próxima página... 
Tabela A.1 - Continuação

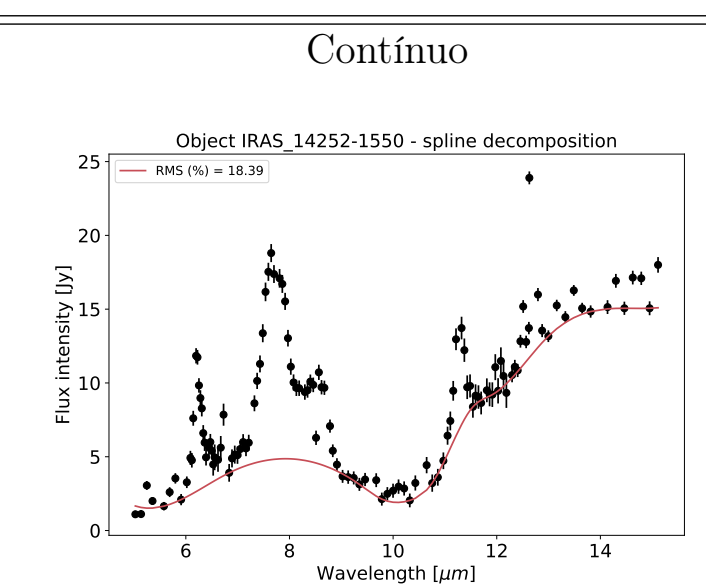

Banda $6.2 \mu \mathrm{m}$
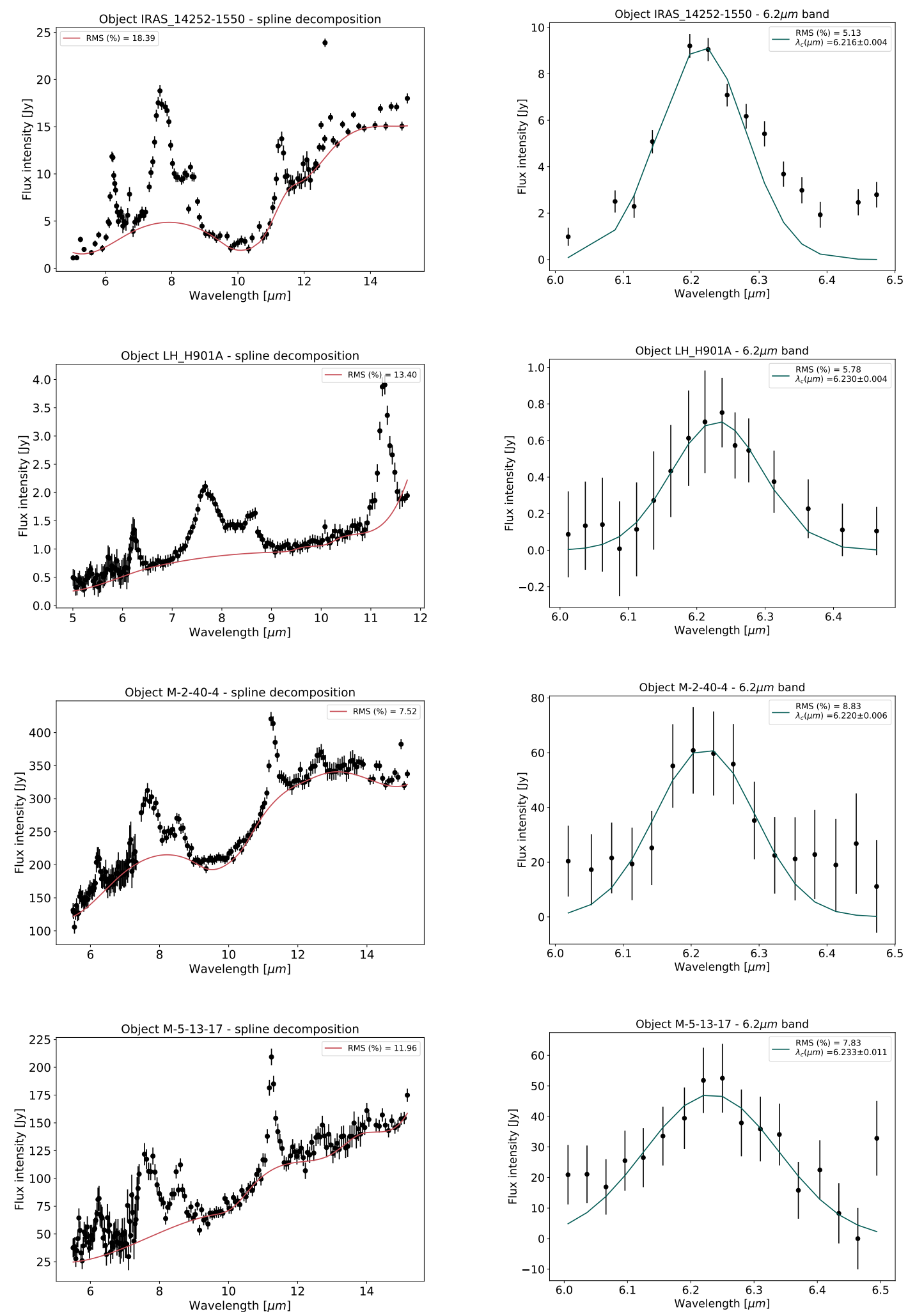

Continua na próxima página... 
Tabela A.1 - Continuação

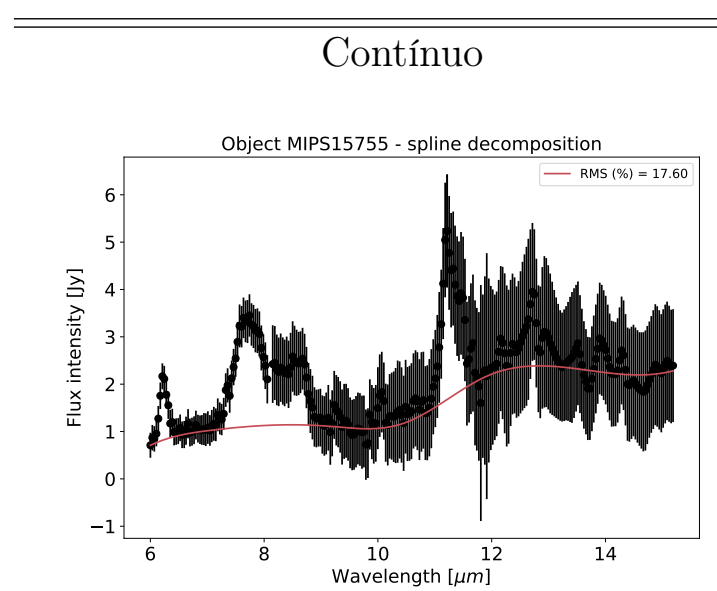

Banda $6.2 \mu \mathrm{m}$
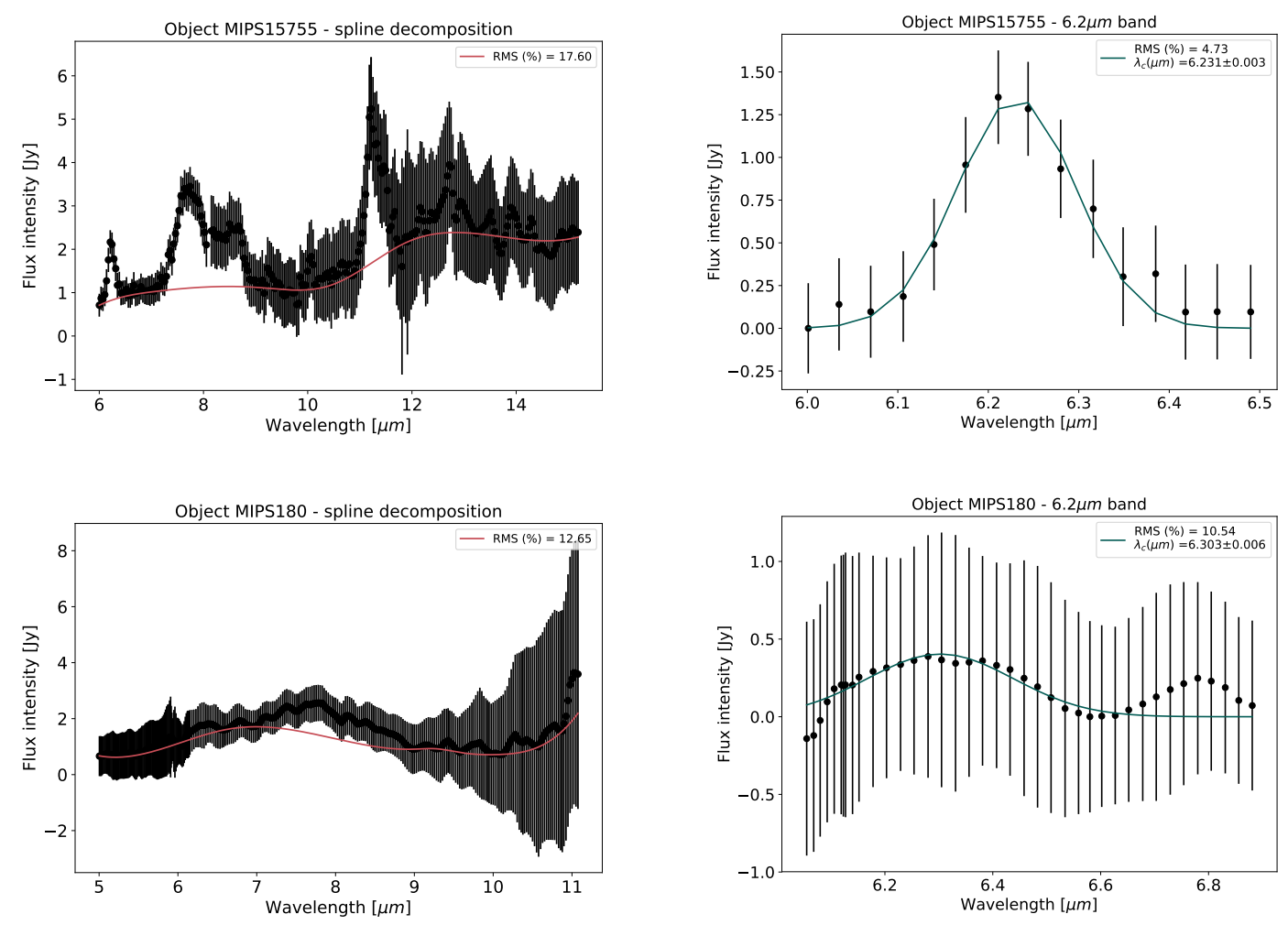

Object MIPS22307 - spline decomposition
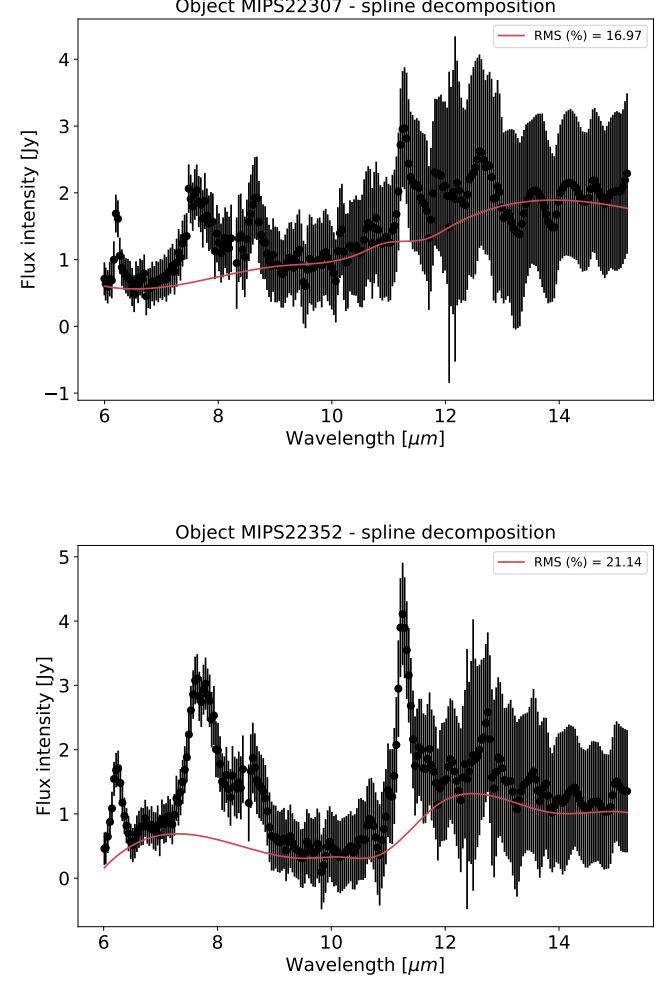
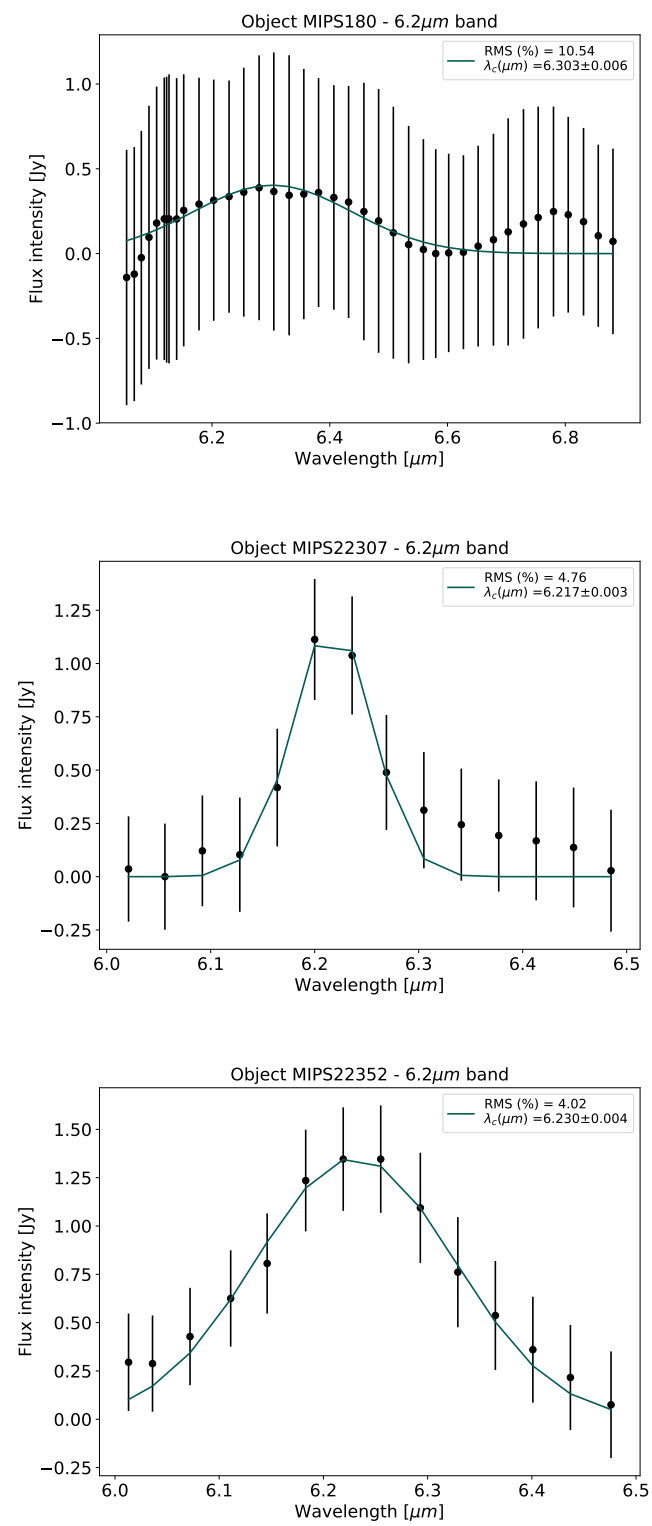

Continua na próxima página... 
Tabela A.1 - Continuação

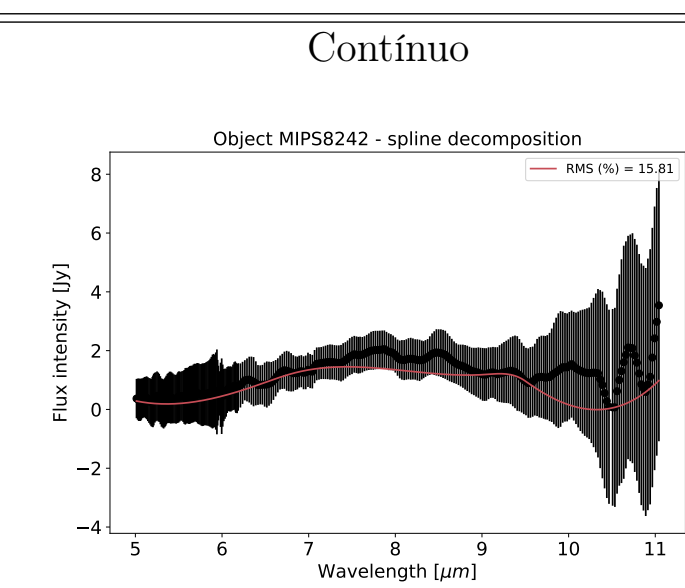

Banda $6.2 \mu \mathrm{m}$
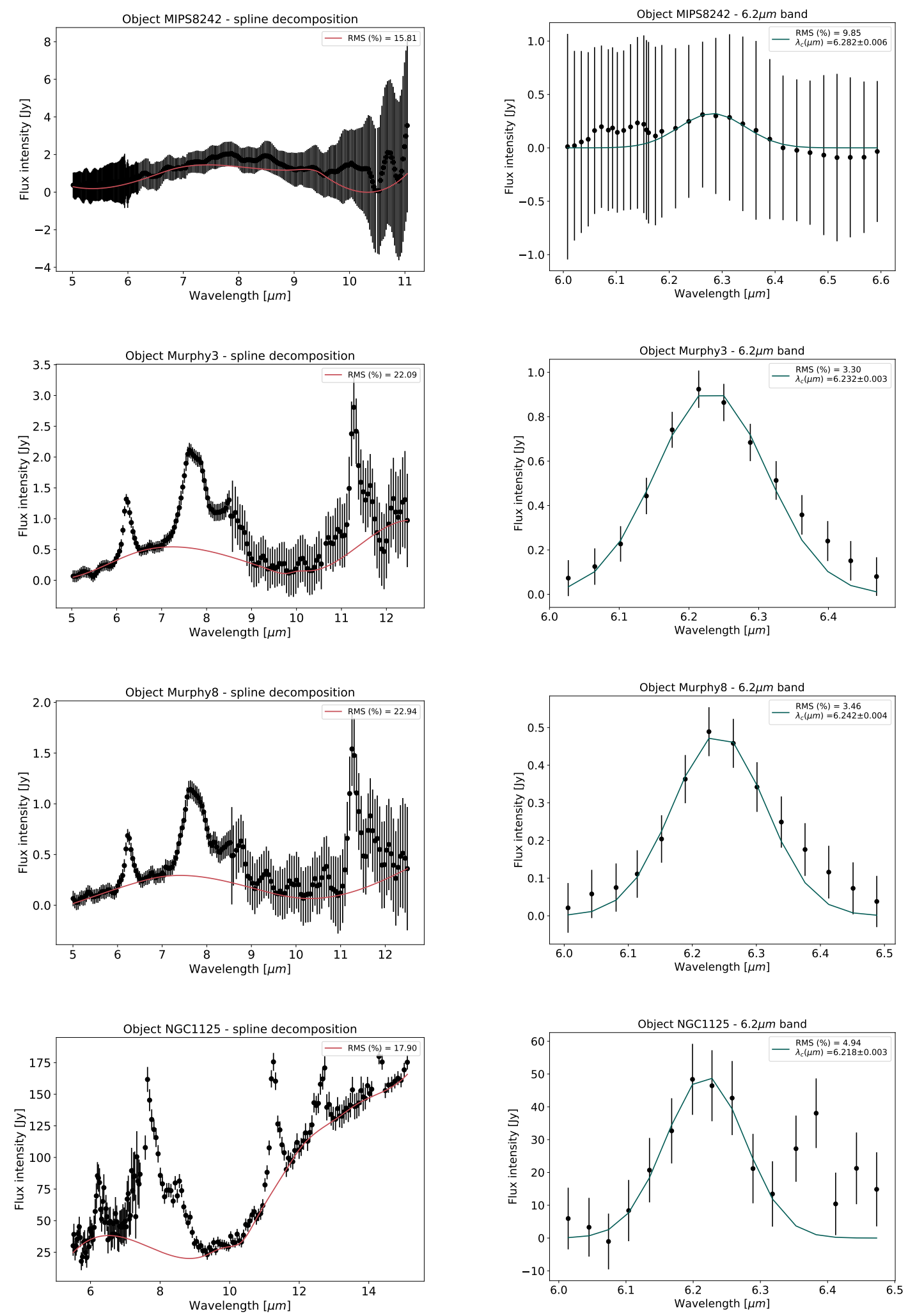

Continua na próxima página... 
Tabela A.1 - Continuação

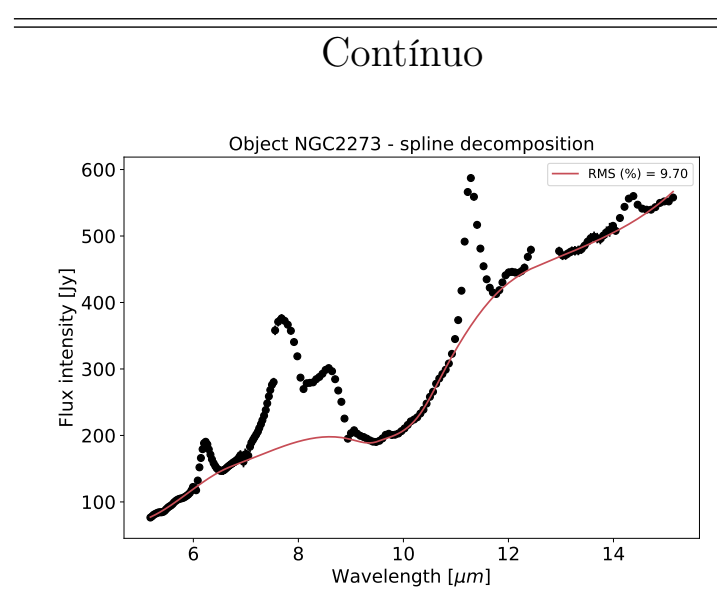

Banda $6.2 \mu \mathrm{m}$
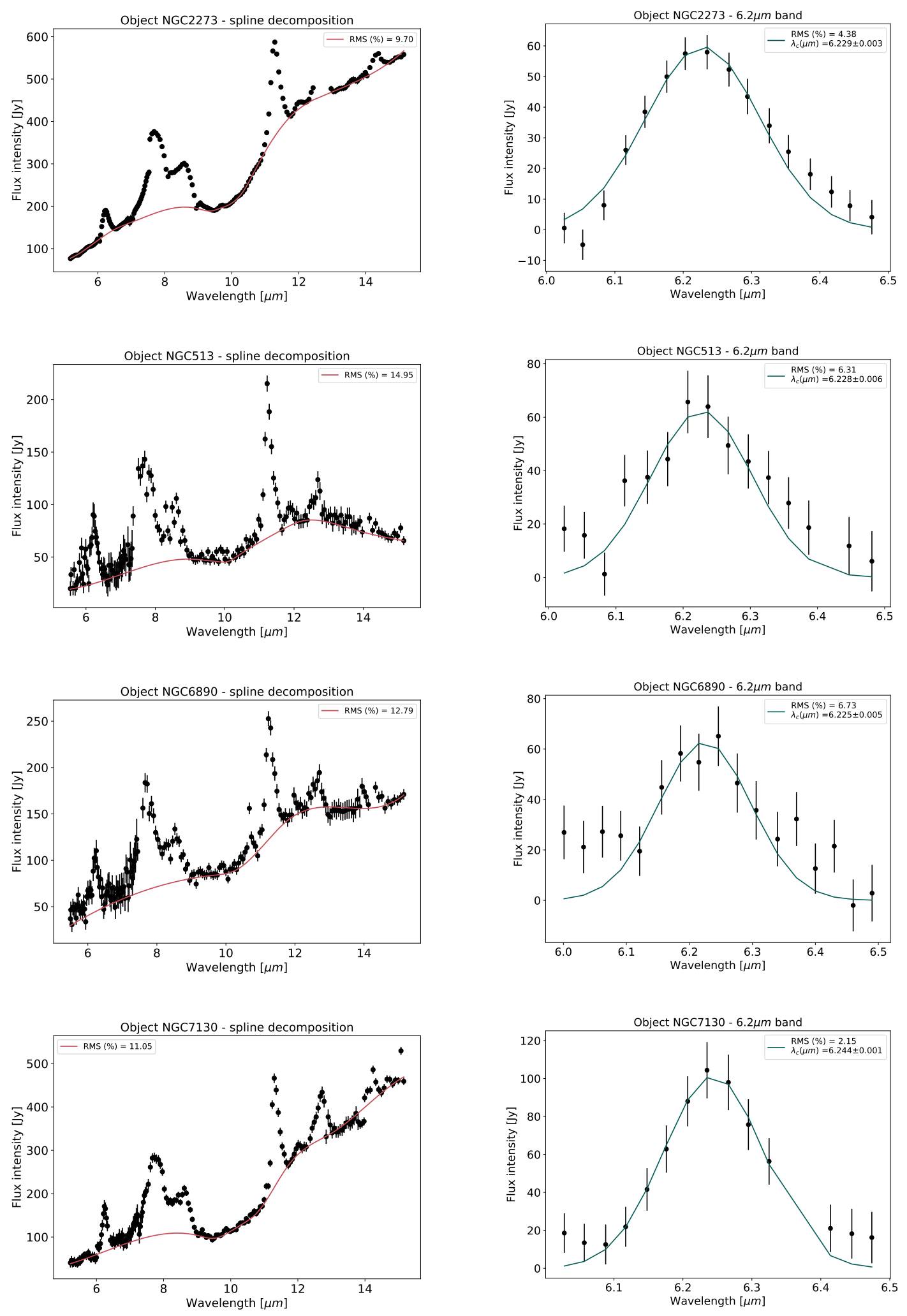

Continua na próxima página... 
Tabela A.1 - Continuação

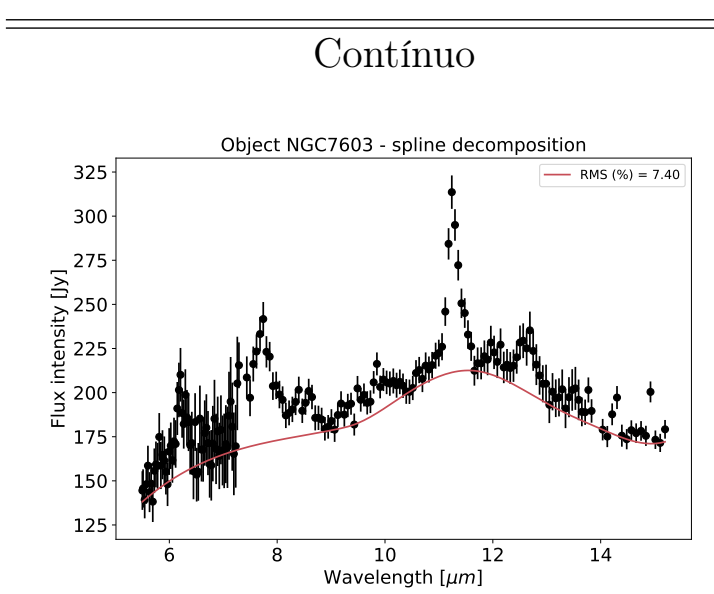

Banda $6.2 \mu \mathrm{m}$
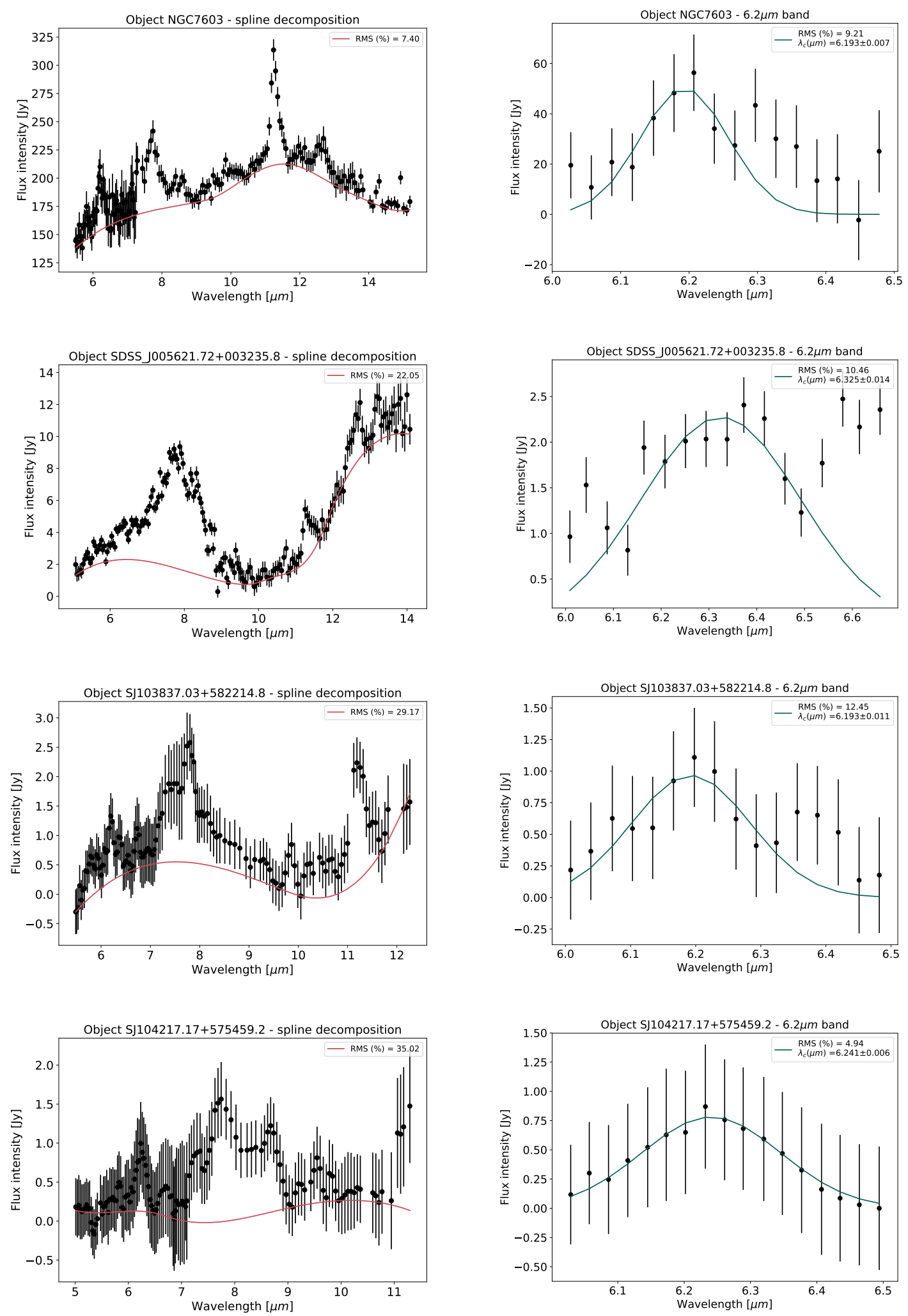

Continua na próxima página... 
Tabela A.1 - Continuação

\section{Contínuo}
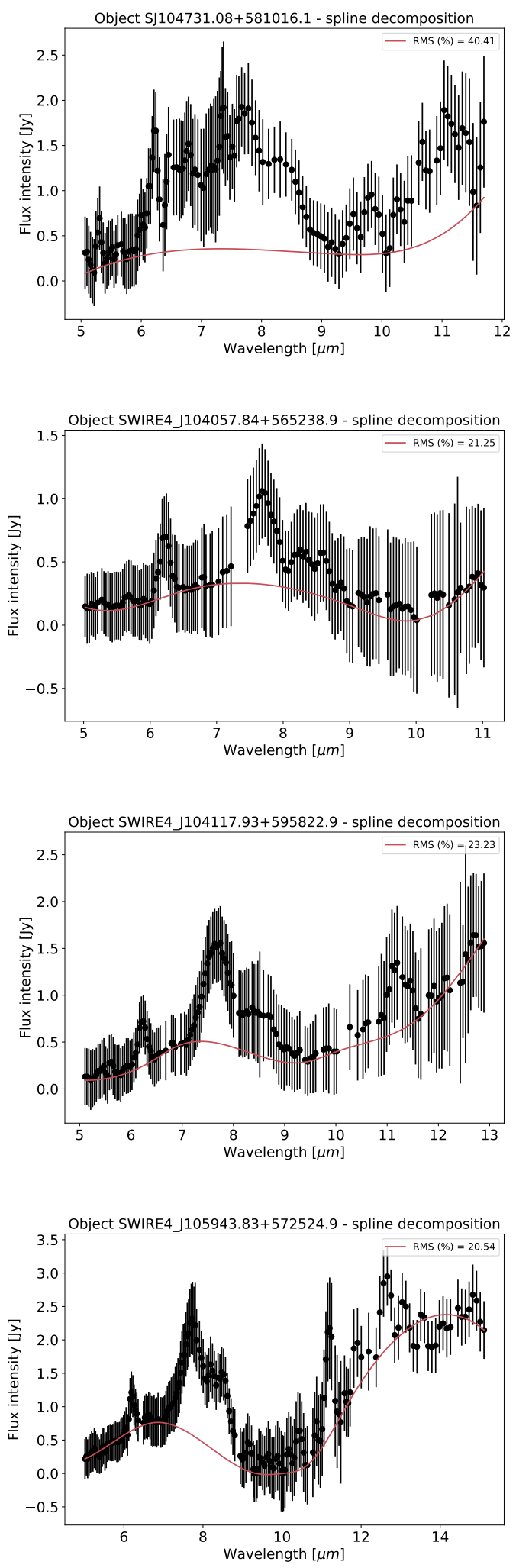

Banda $6.2 \mu \mathrm{m}$
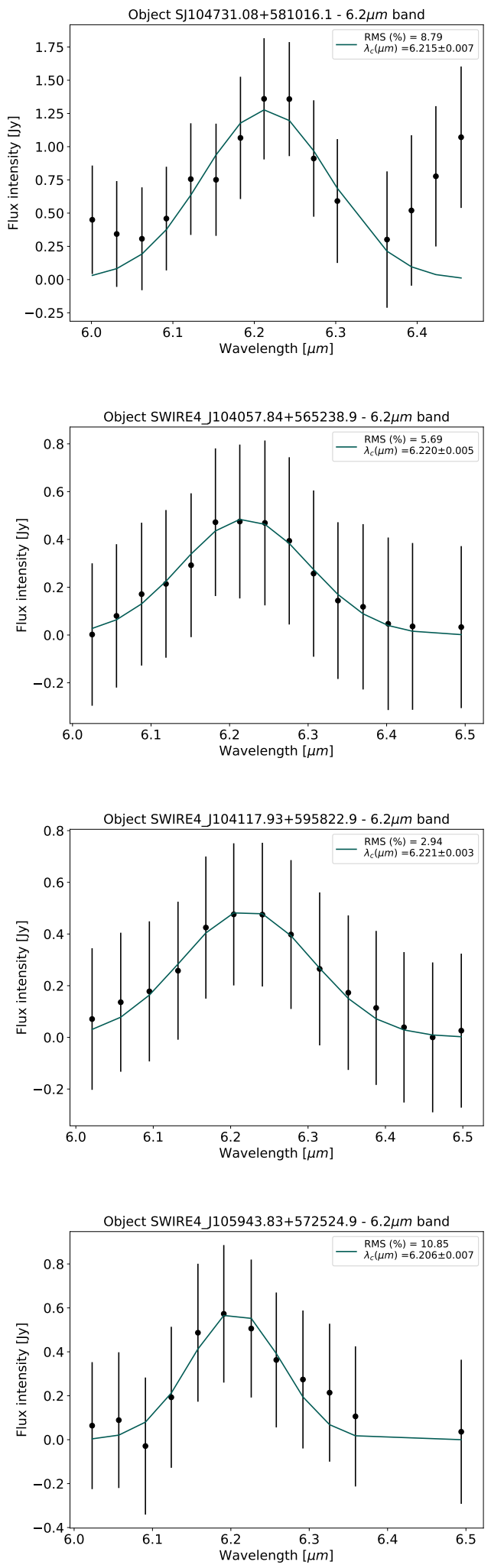
Tabela A.2 - Imagens dos ajustes do contínuo e bandas de 6.2, 7.7 e $8.6 \mu \mathrm{m}$ das 126 galáxias com emissão dominada por starburst (Canelo et al., 2018, submitted).
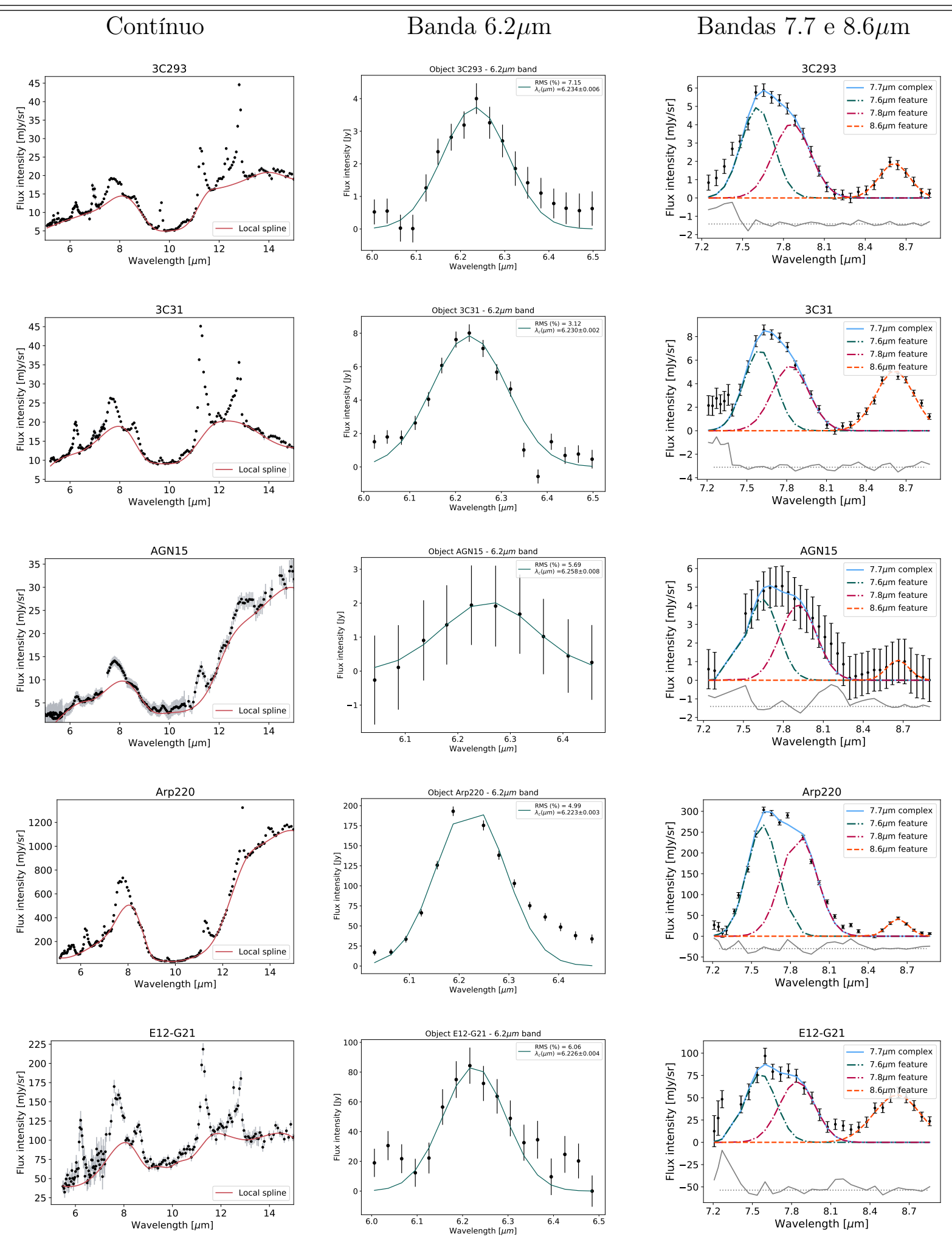

Continua na próxima página... 
Tabela A.2 - Continuação

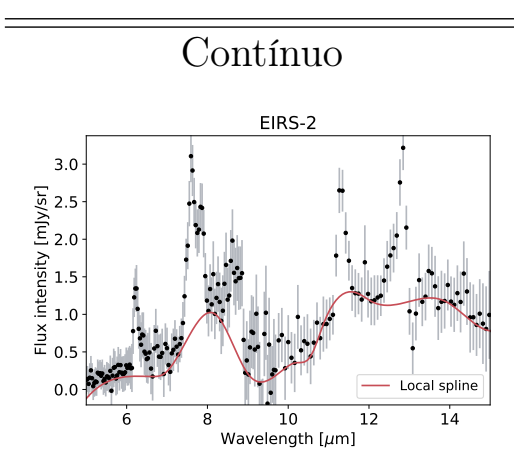

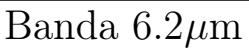
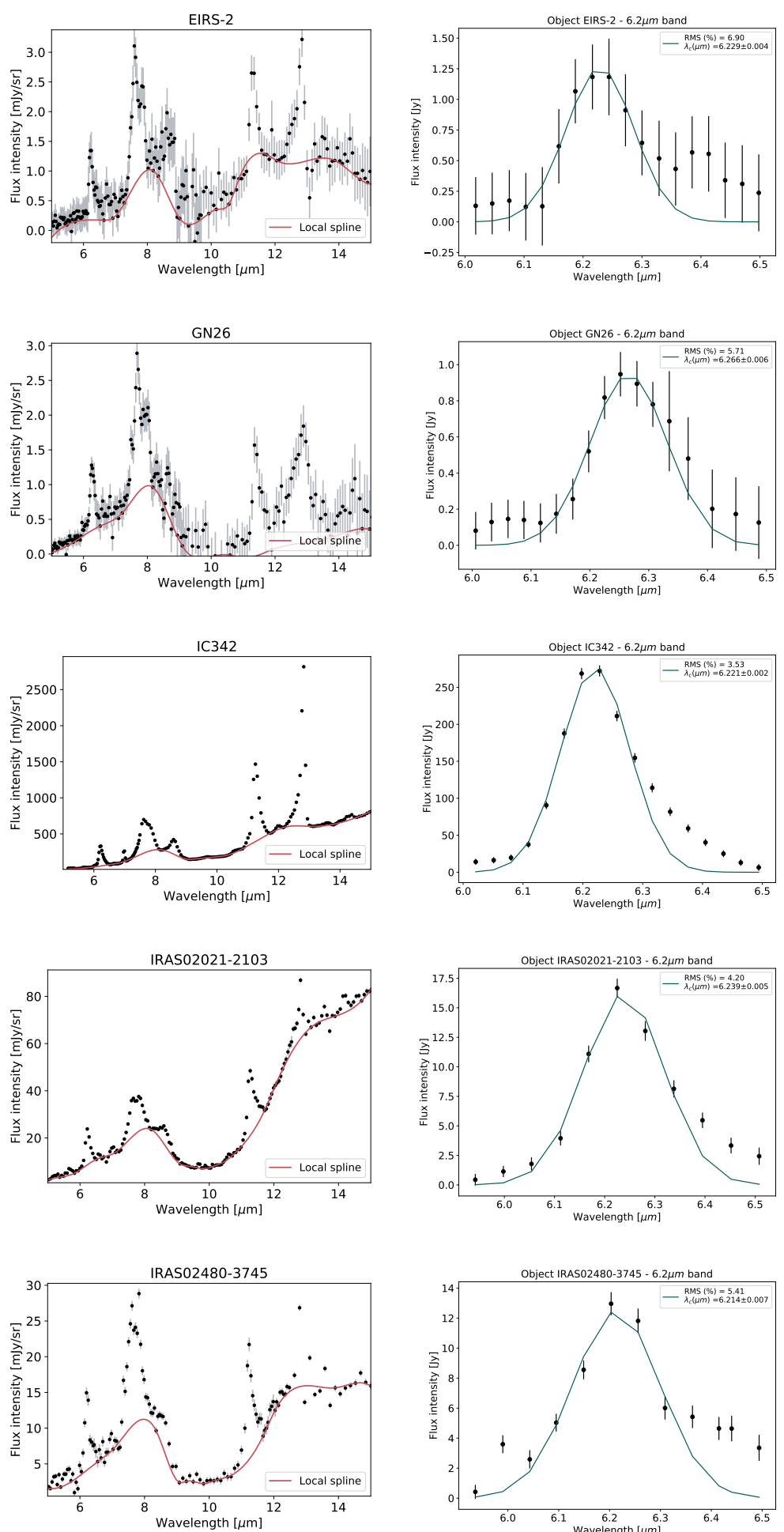
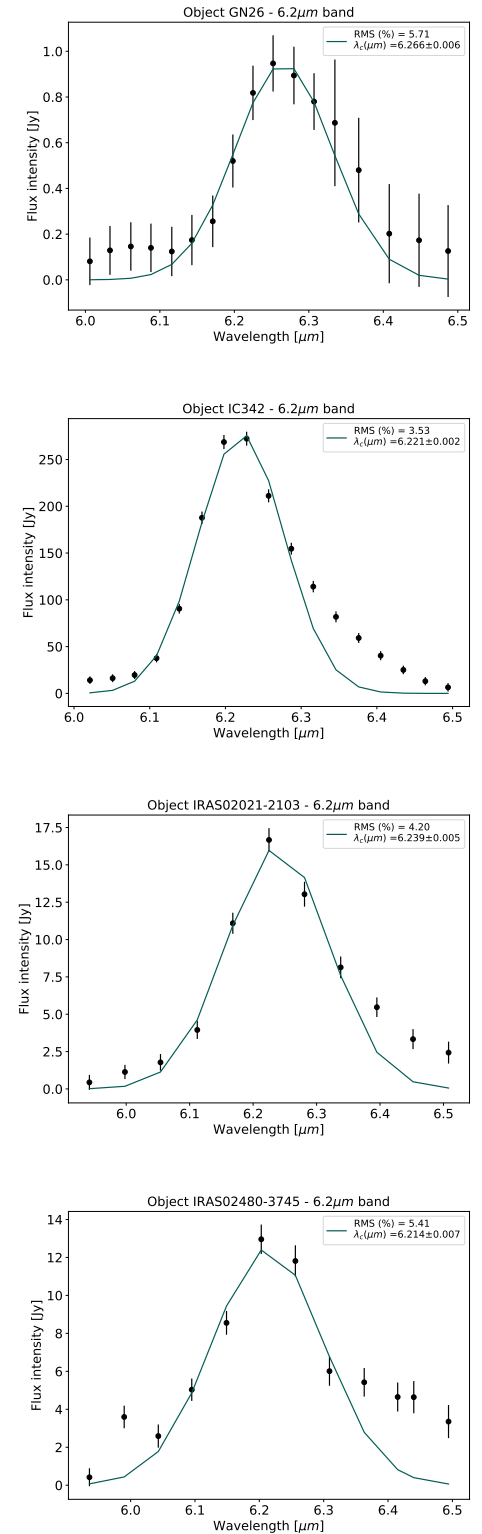

Bandas 7.7 e $8.6 \mu \mathrm{m}$
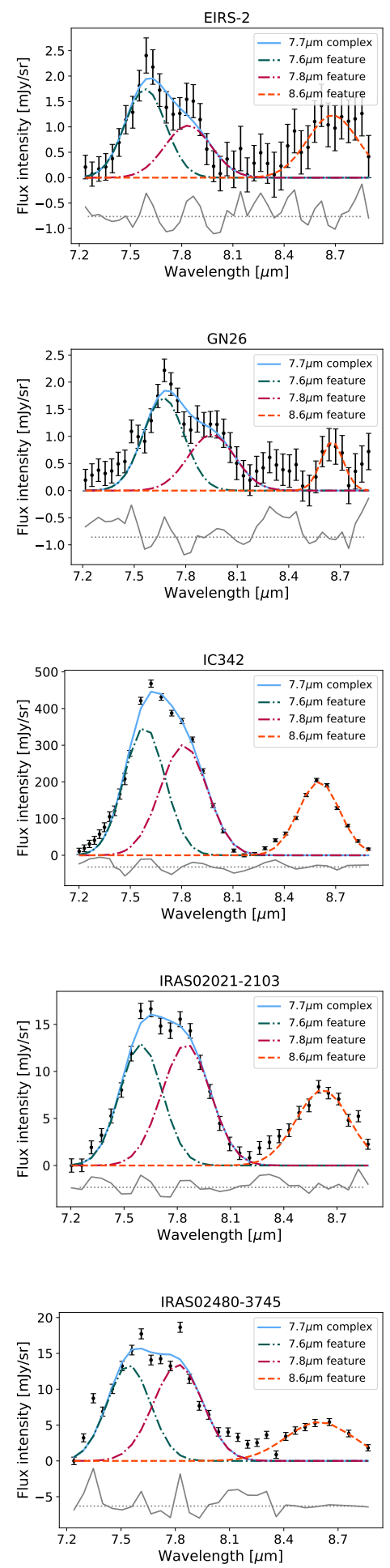

Continua na próxima página... 
Tabela A.2 - Continuação
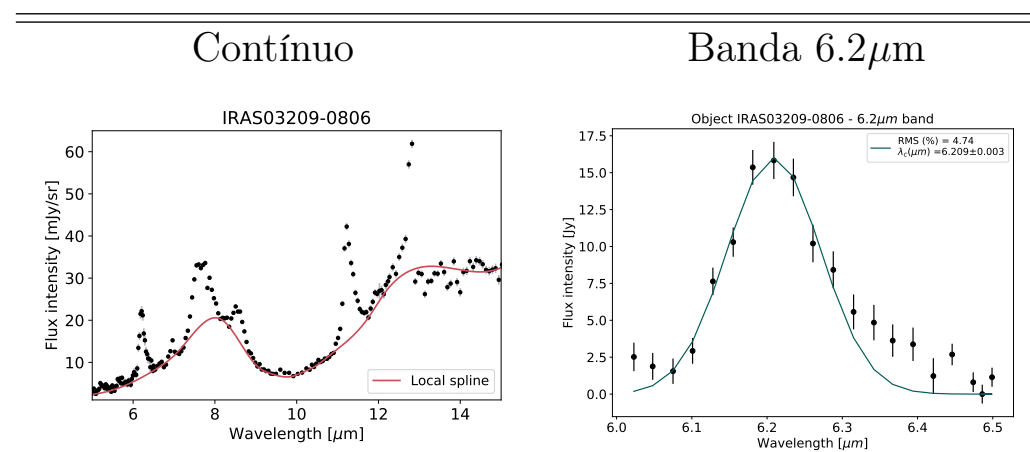

Bandas 7.7 e $8.6 \mu \mathrm{m}$
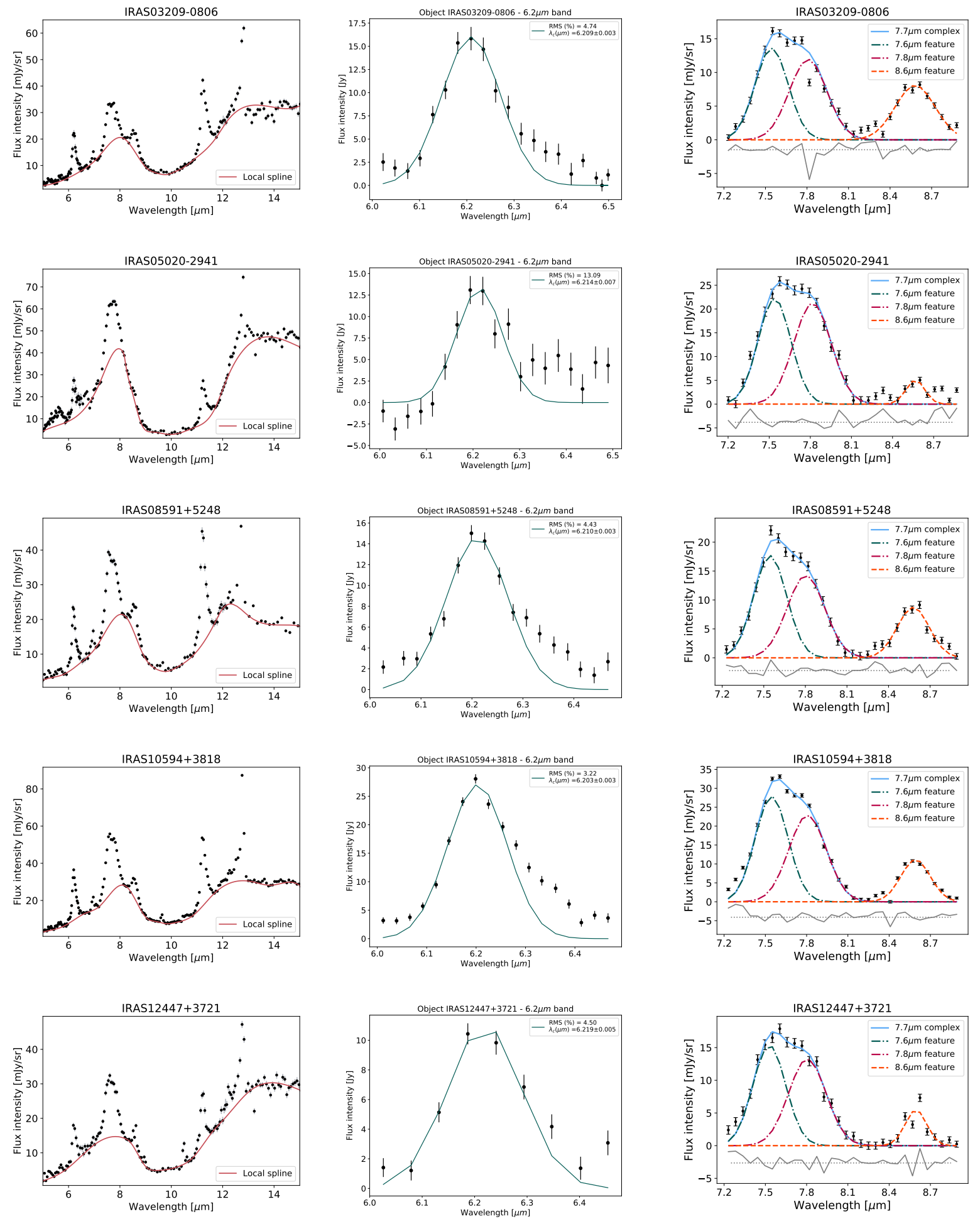

Continua na próxima página... 
Tabela A.2 - Continuação
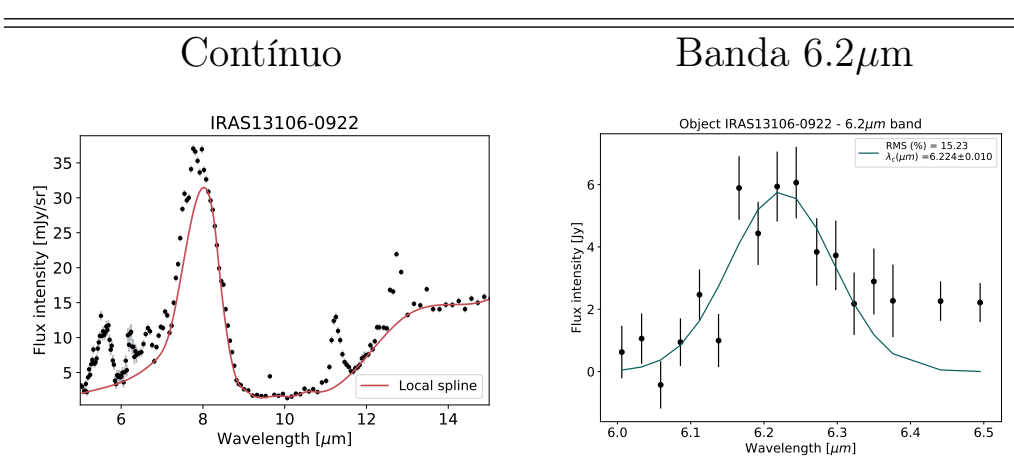

Bandas 7.7 e $8.6 \mu \mathrm{m}$
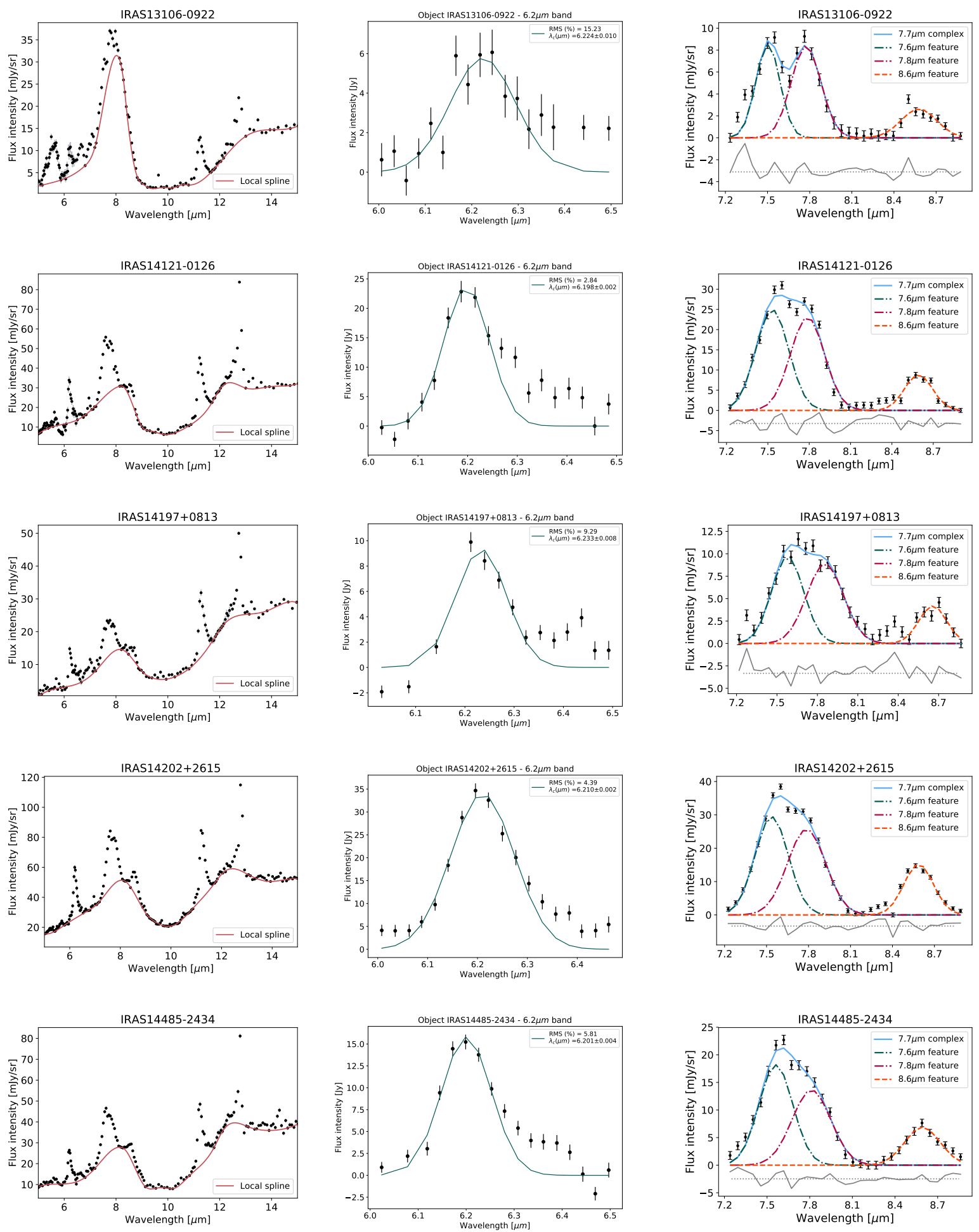

Continua na próxima página... 
Tabela A.2 - Continuação
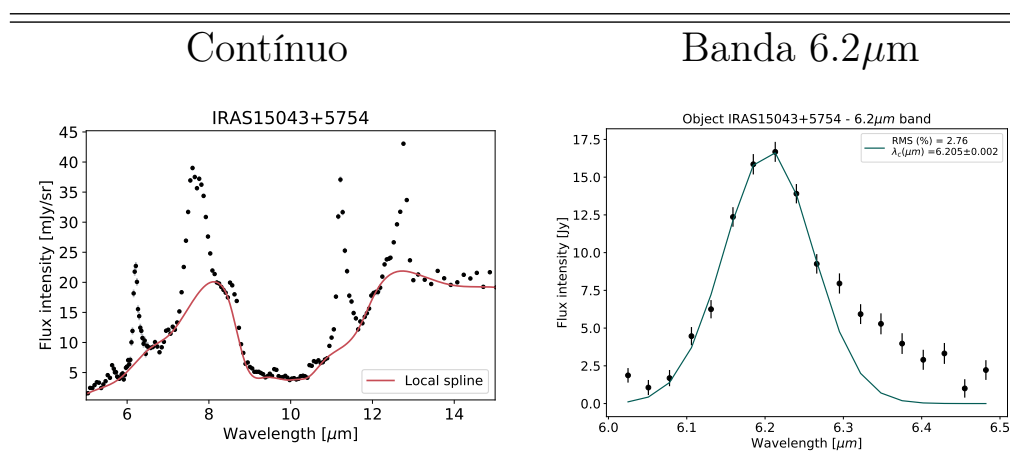

Bandas 7.7 e $8.6 \mu \mathrm{m}$
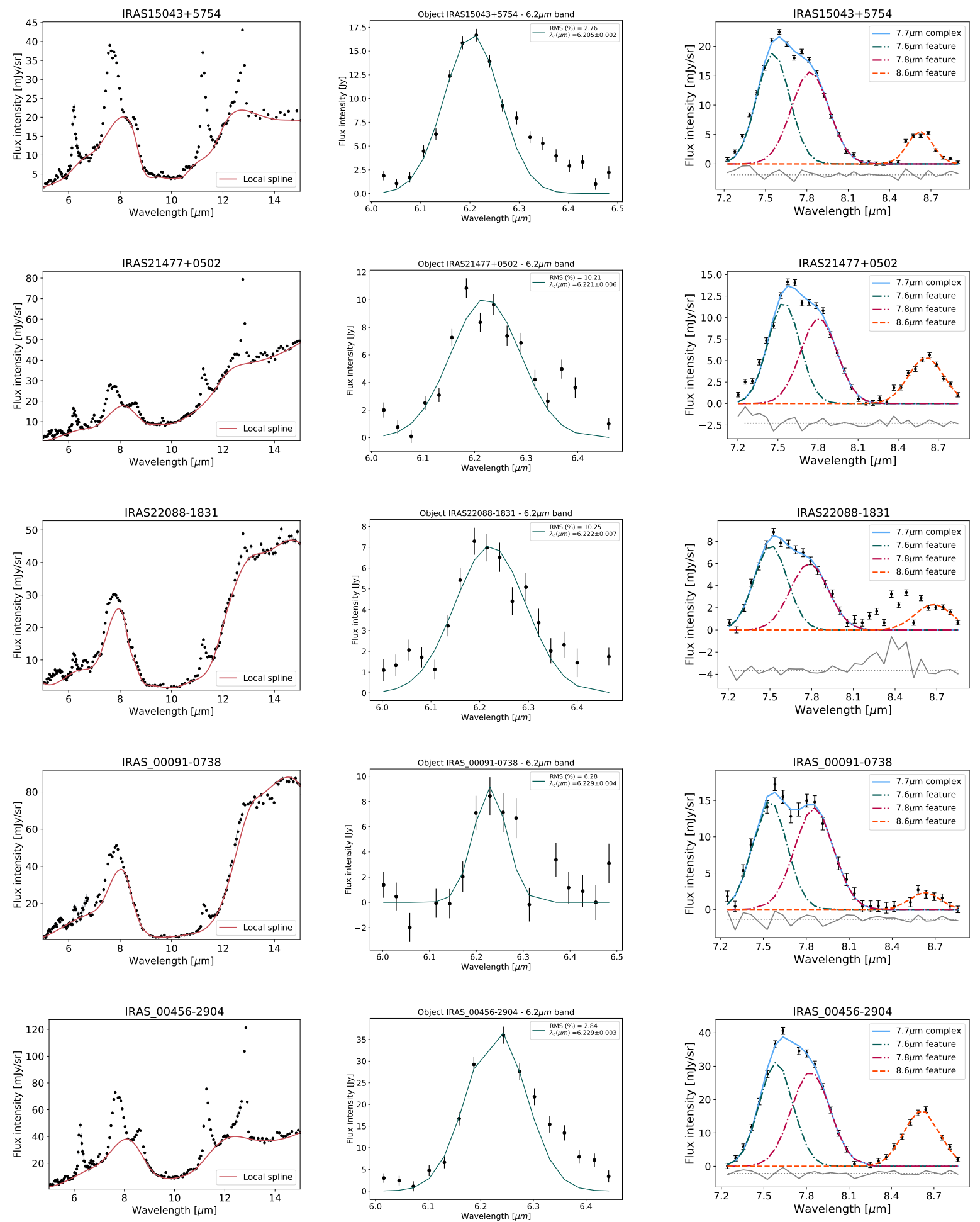

Continua na próxima página... 
Tabela A.2 - Continuação
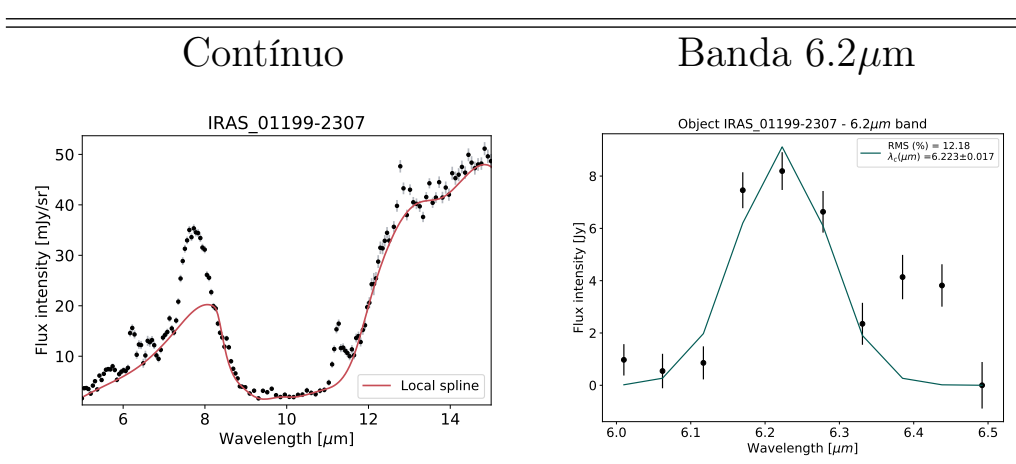

Bandas 7.7 e $8.6 \mu \mathrm{m}$
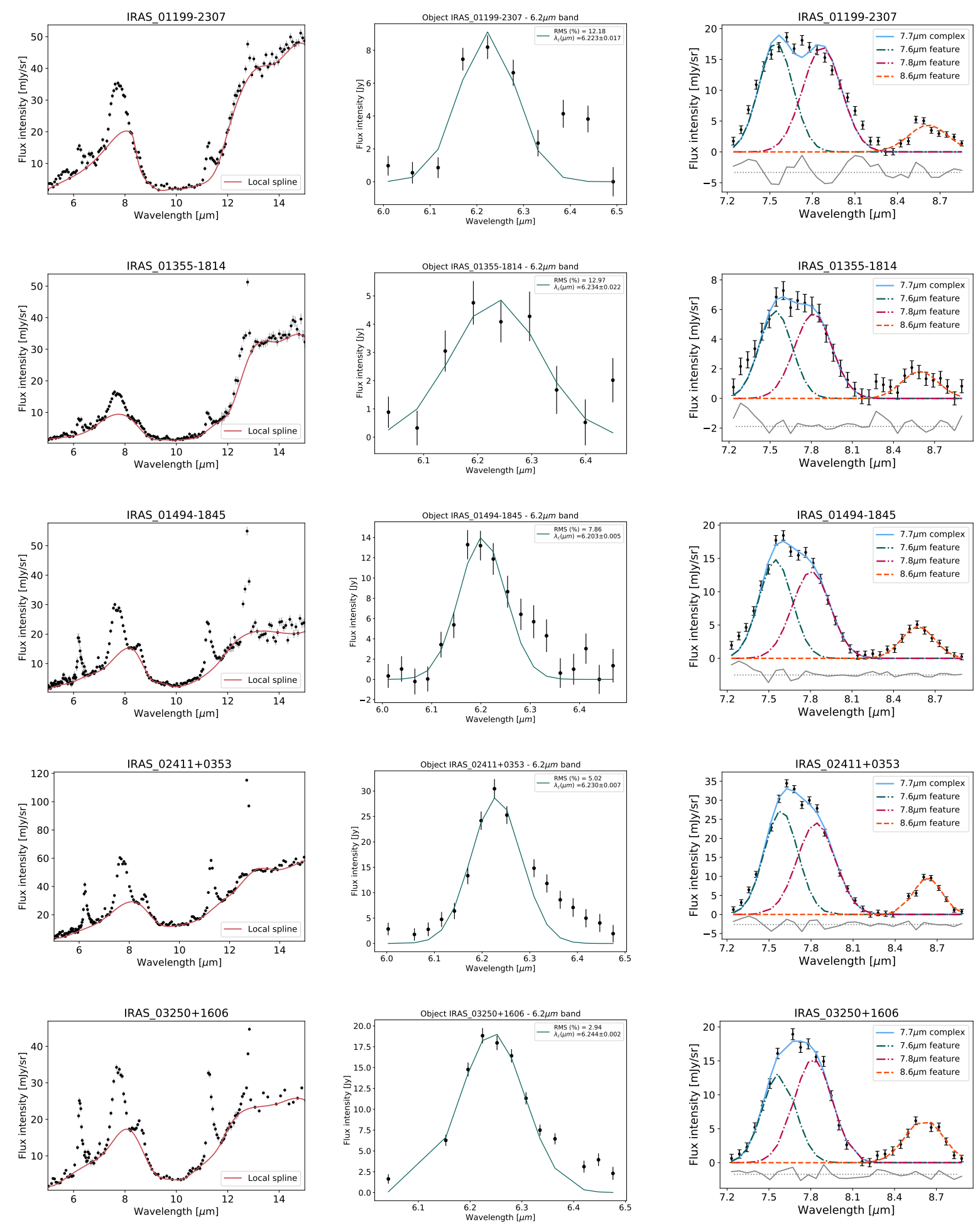

Continua na próxima página... 
Tabela A.2 - Continuação
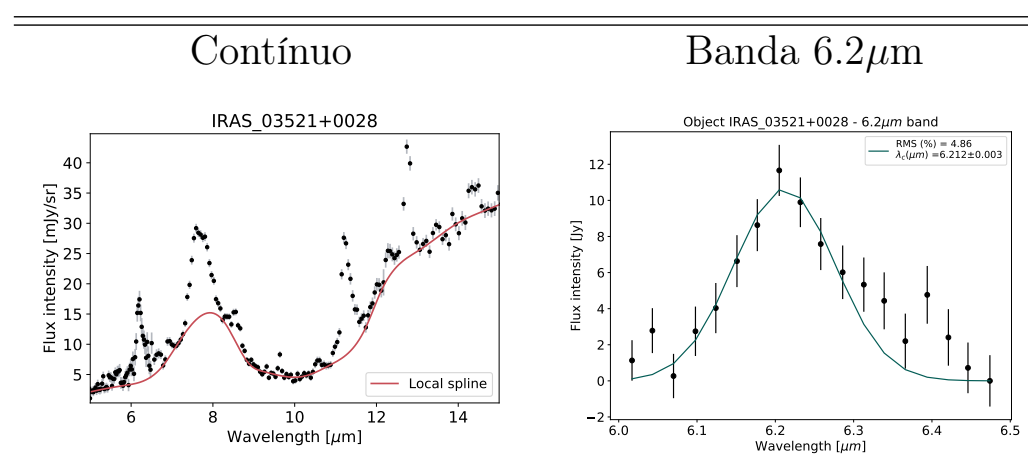

Bandas 7.7 e $8.6 \mu \mathrm{m}$
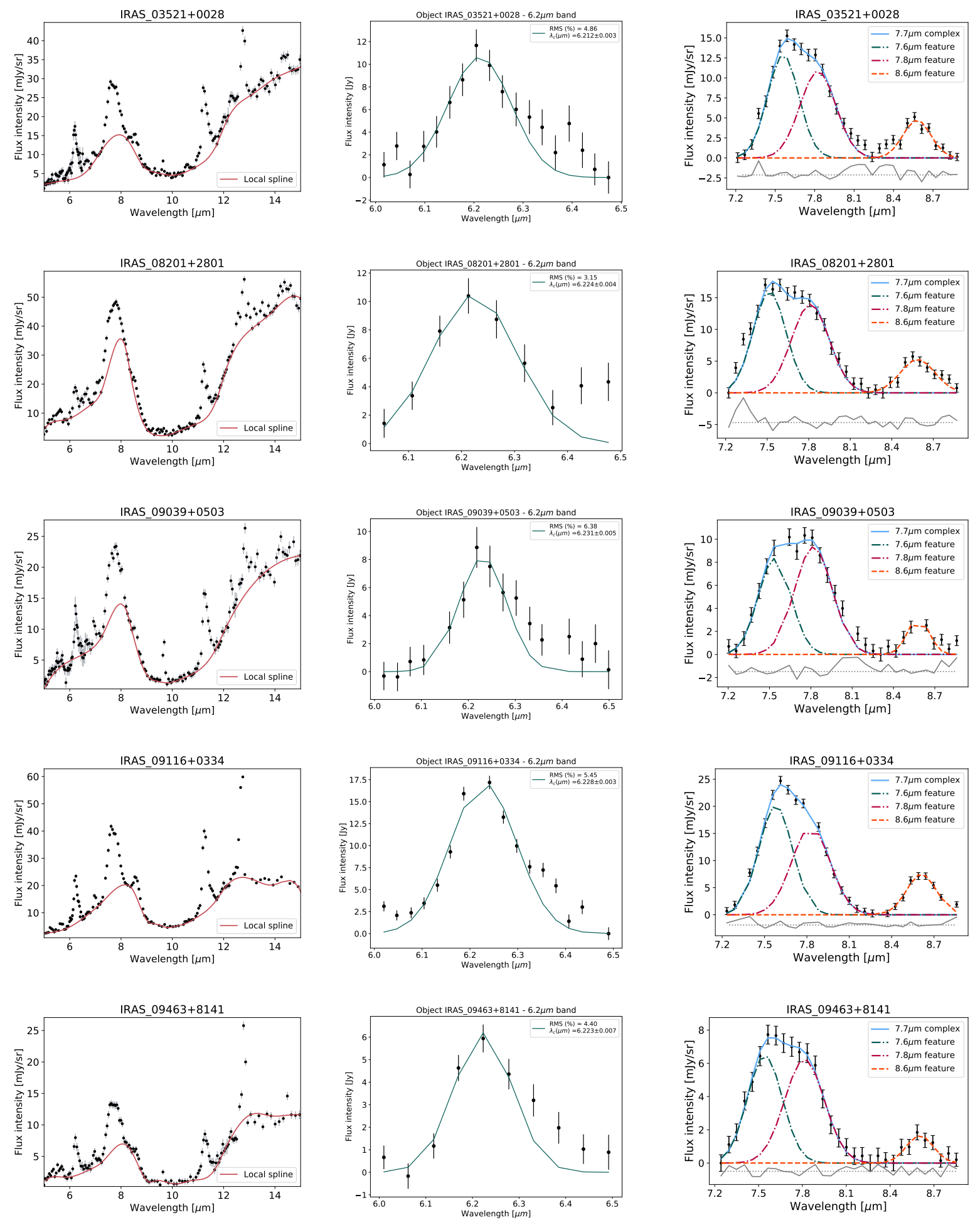

Continua na próxima página... 
Tabela A.2 - Continuação

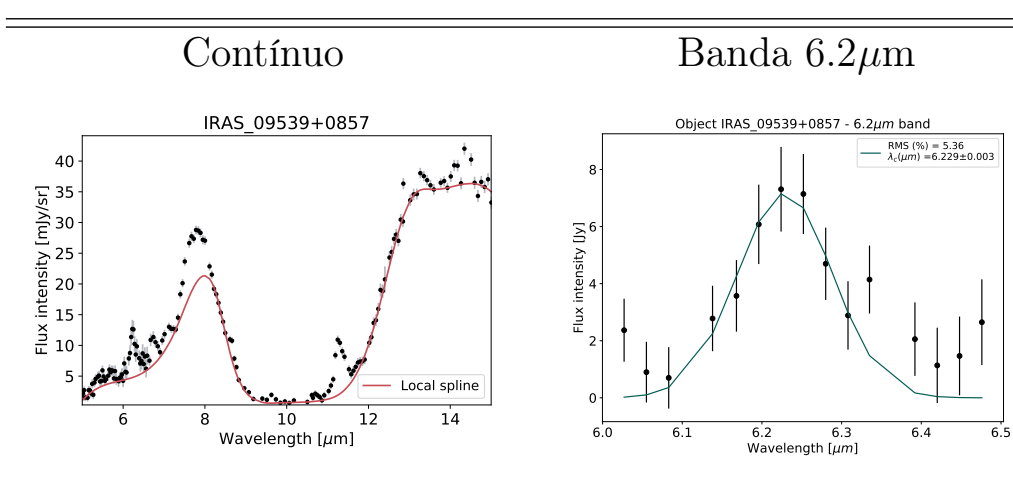

Bandas 7.7 e $8.6 \mu \mathrm{m}$
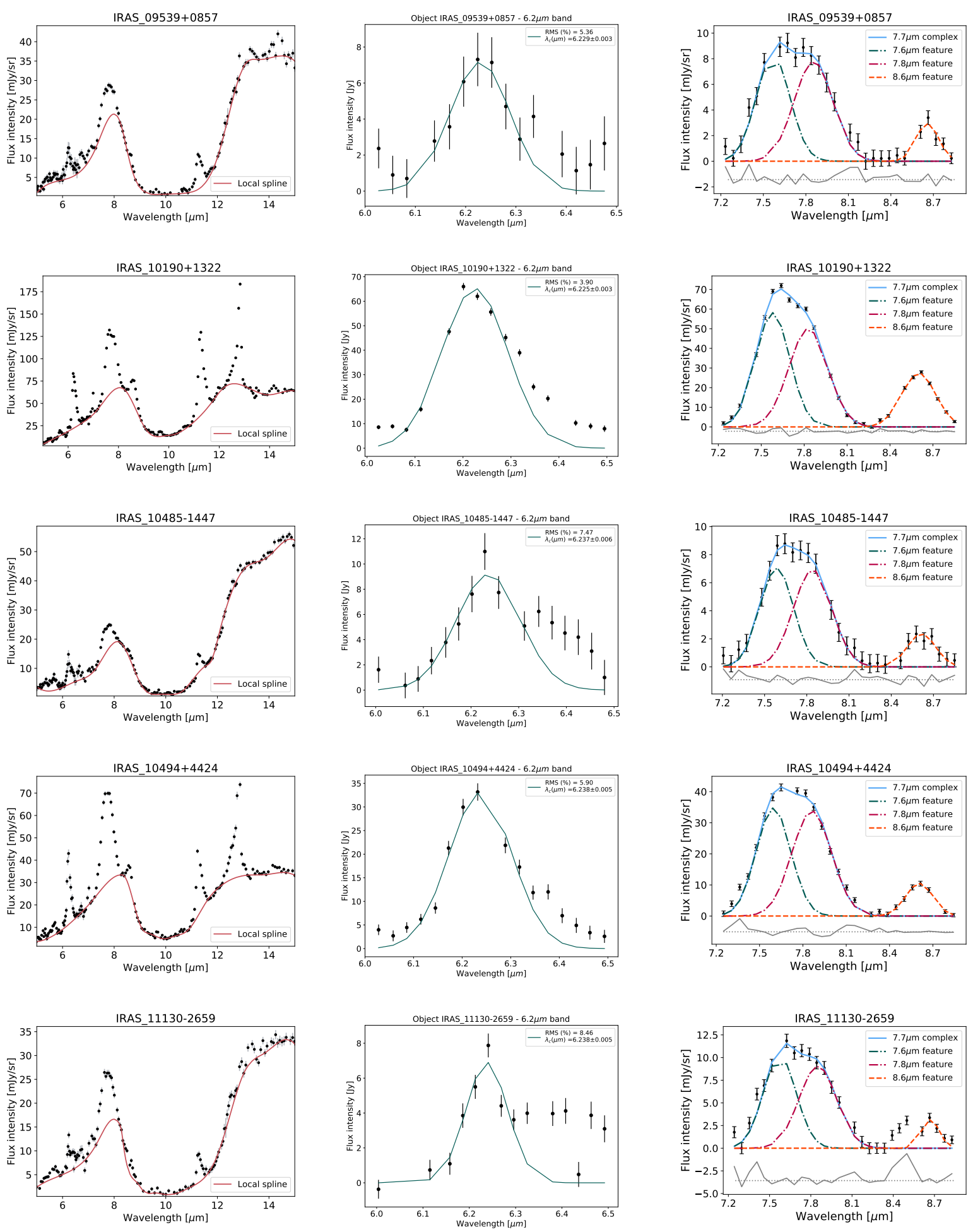

Continua na próxima página... 
Tabela A.2 - Continuação

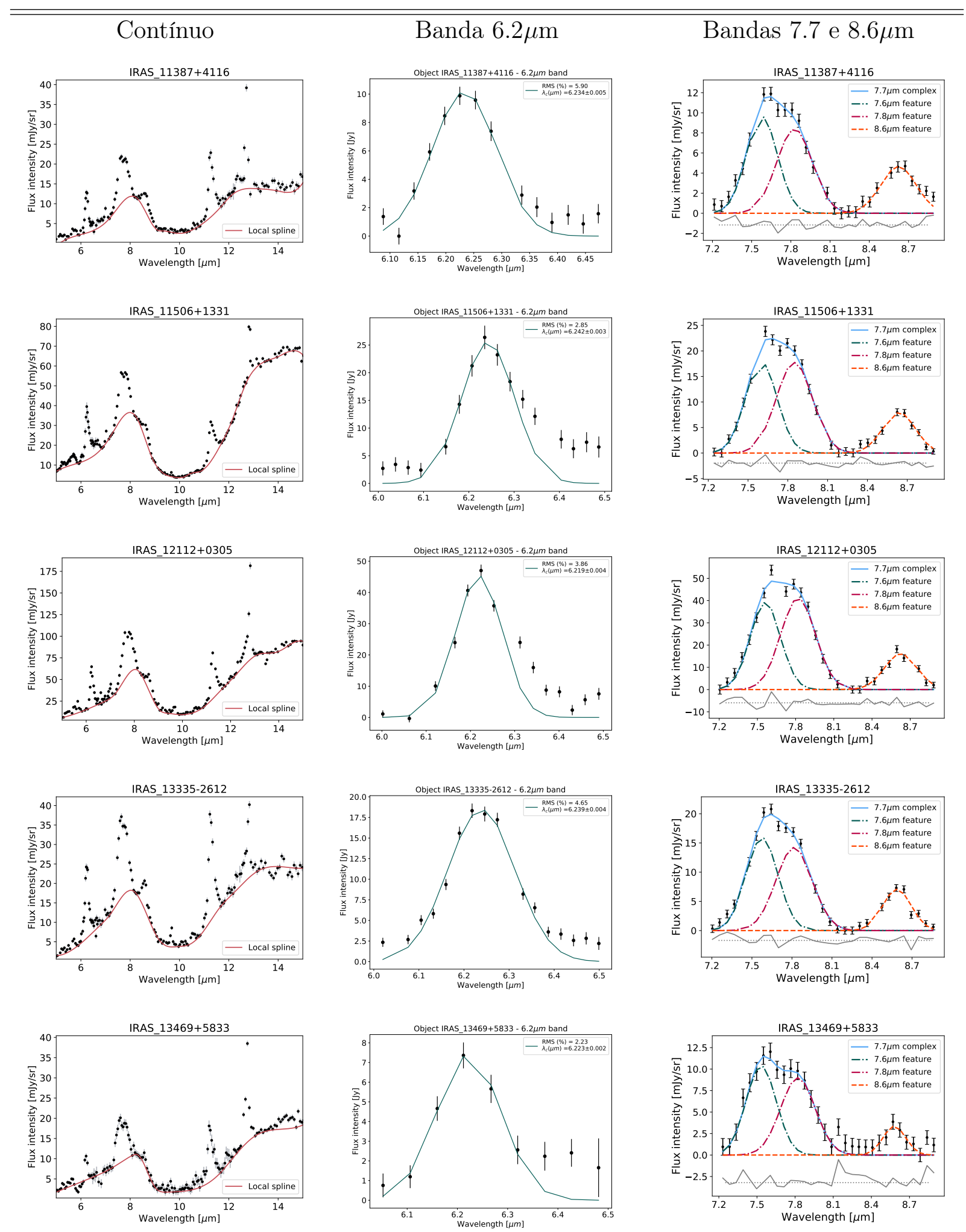

Continua na próxima página... 
Tabela A.2 - Continuação

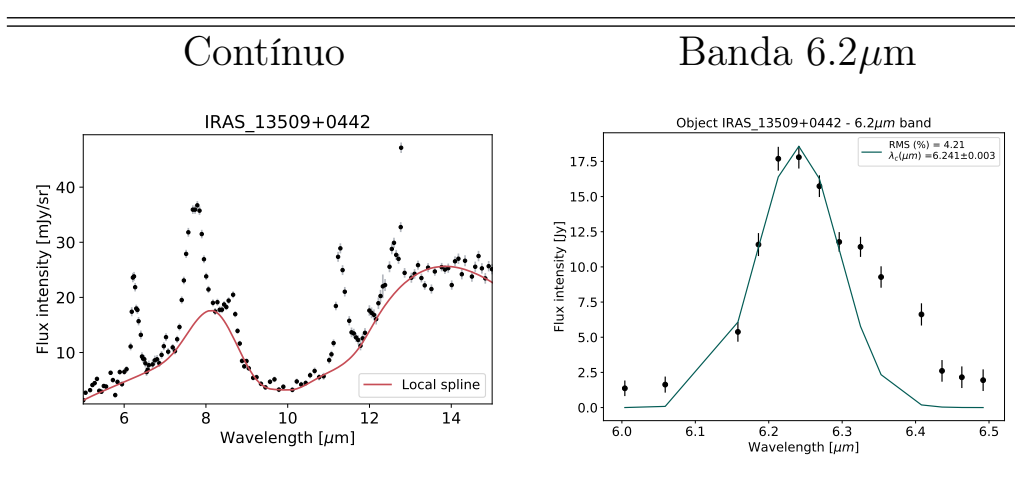

Bandas 7.7 e $8.6 \mu \mathrm{m}$
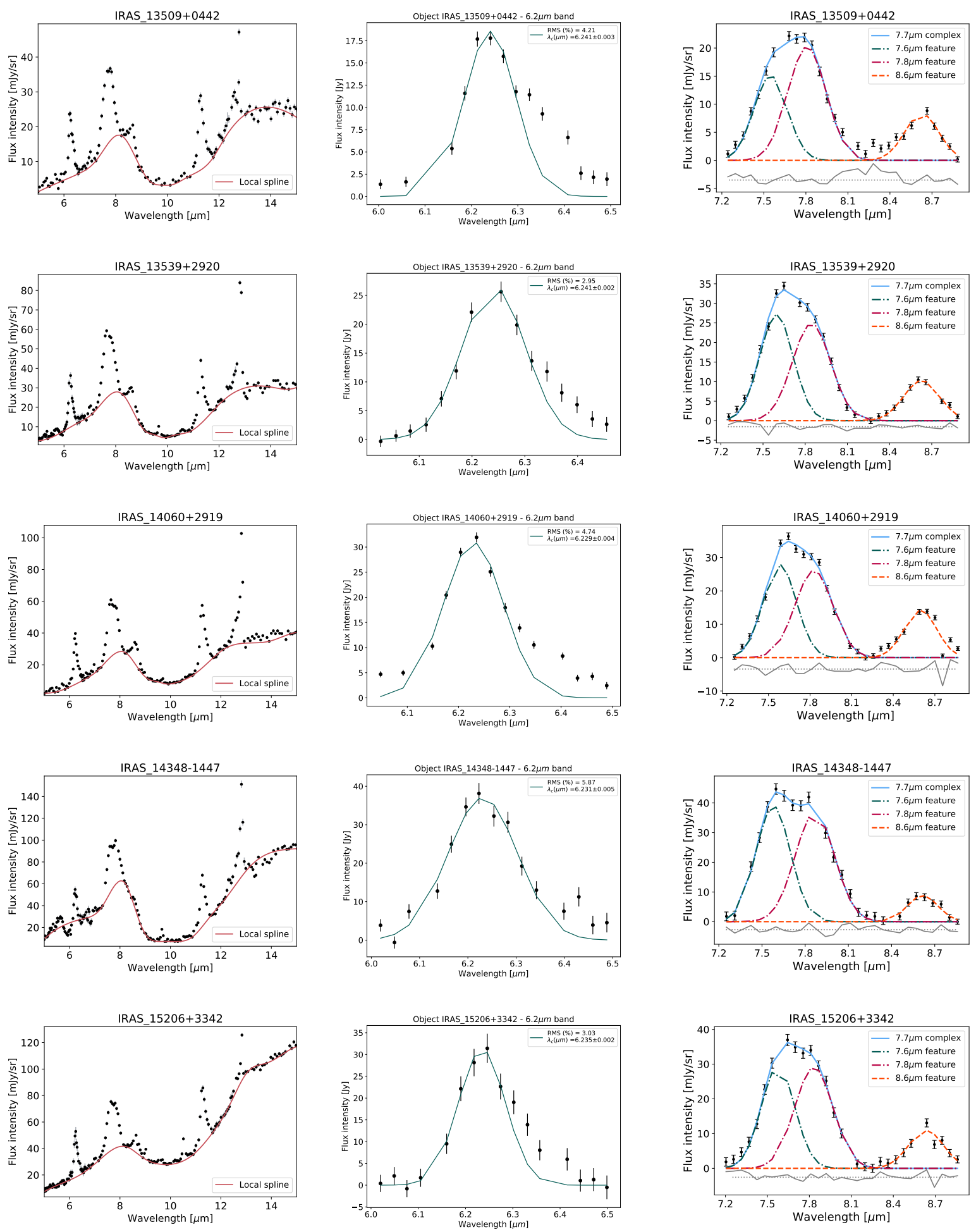

Continua na próxima página... 
Tabela A.2 - Continuação
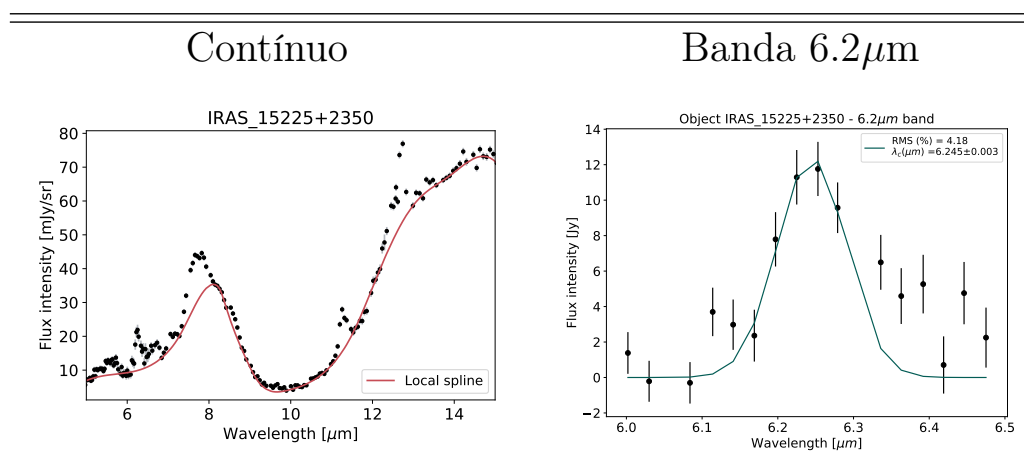

Bandas 7.7 e $8.6 \mu \mathrm{m}$
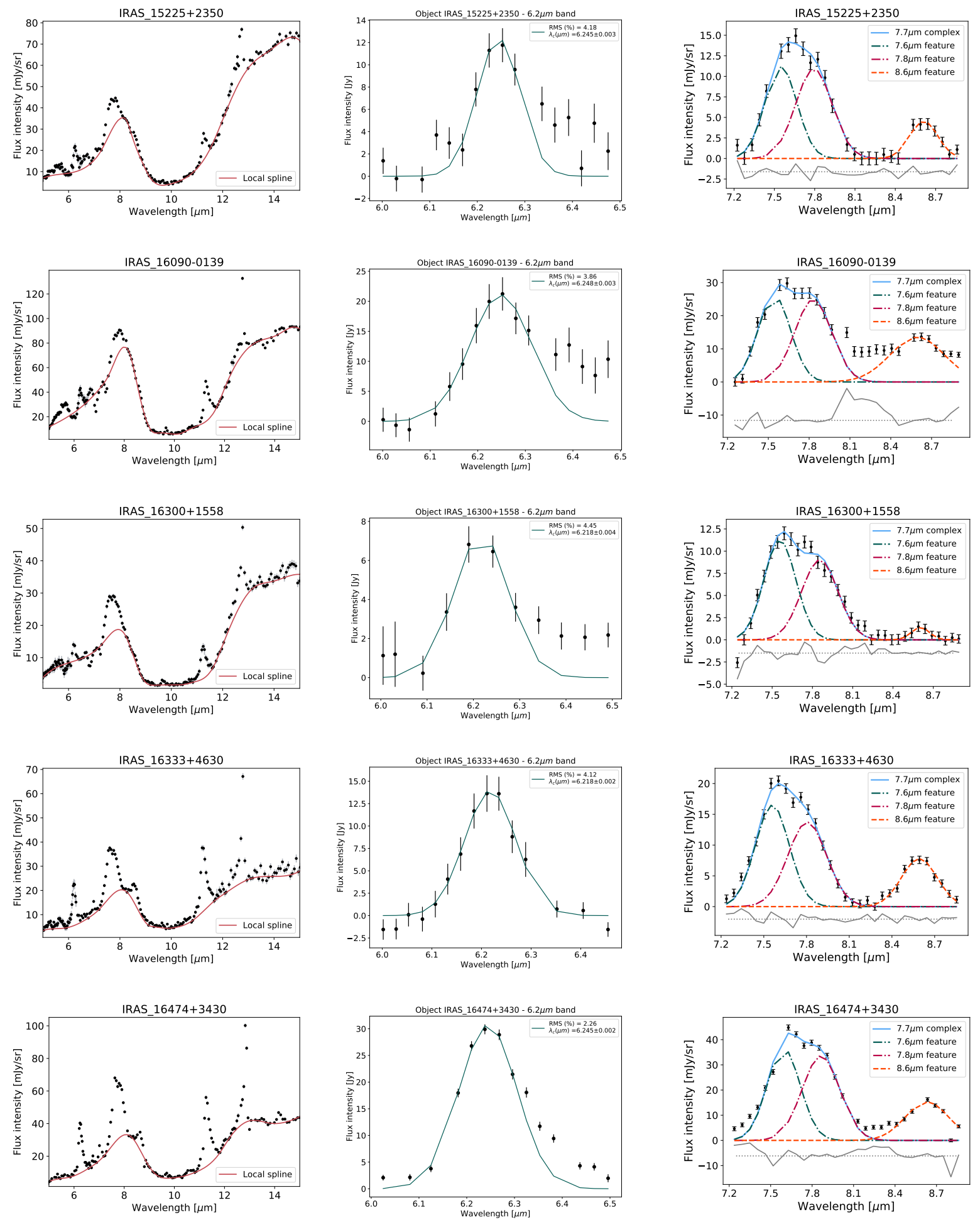

Continua na próxima página... 
Tabela A.2 - Continuação
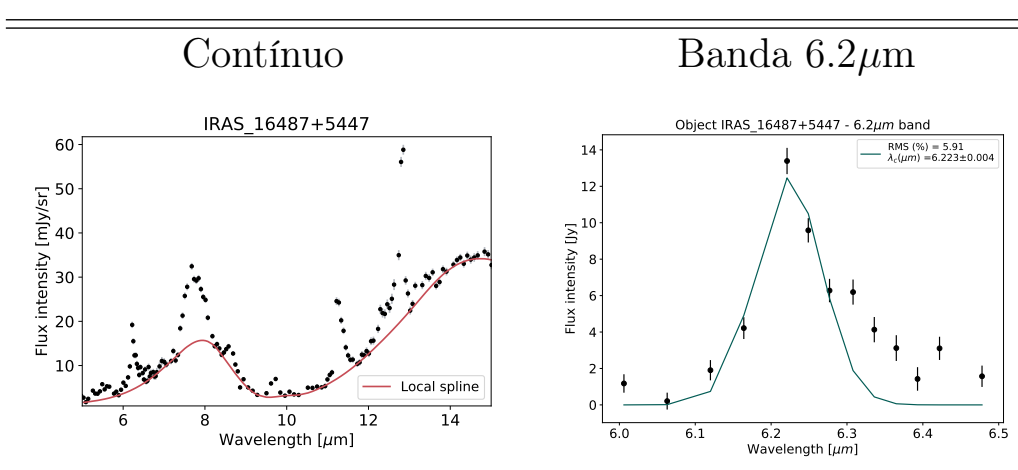

Bandas 7.7 e $8.6 \mu \mathrm{m}$
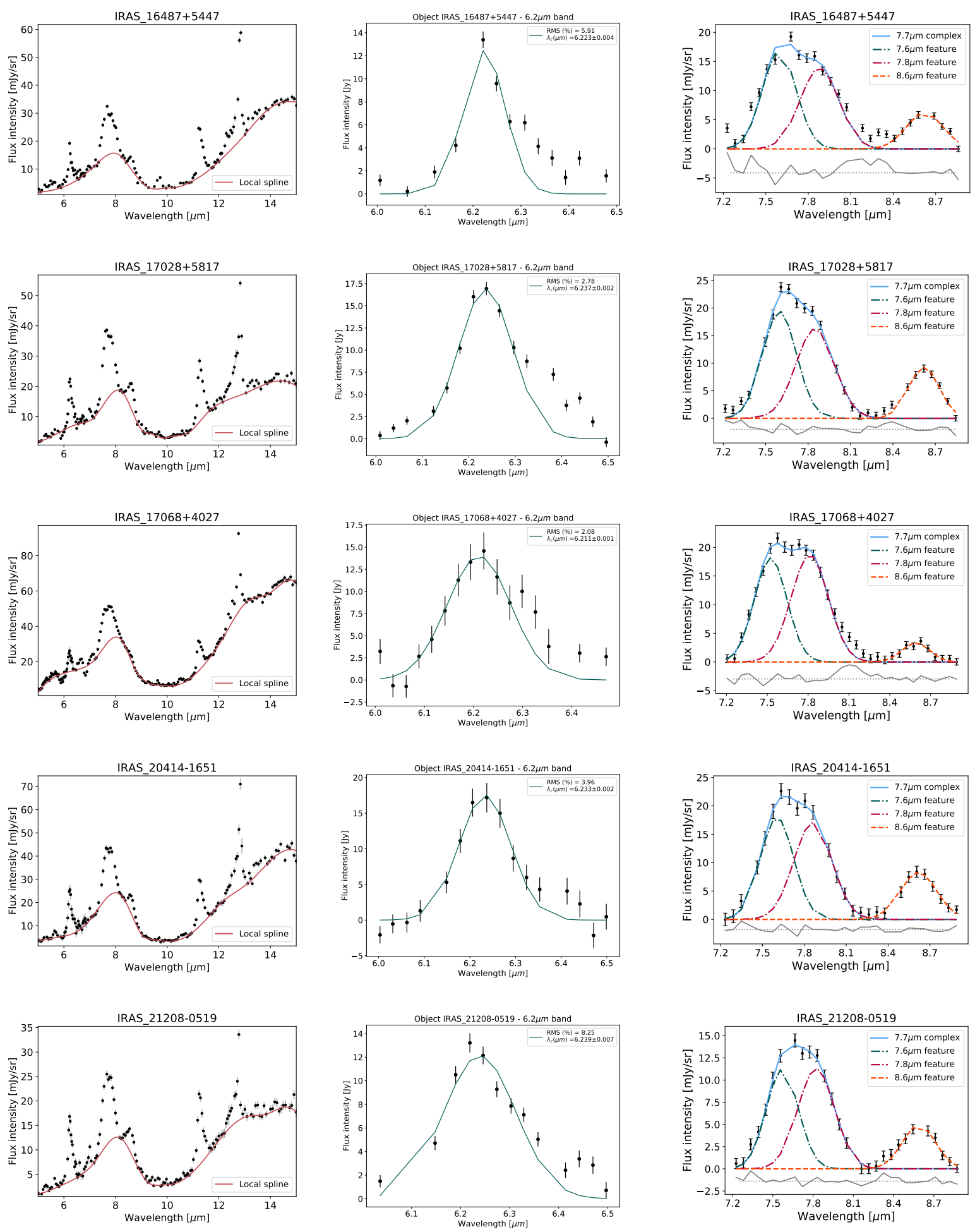

Continua na próxima página... 
Tabela A.2 - Continuação
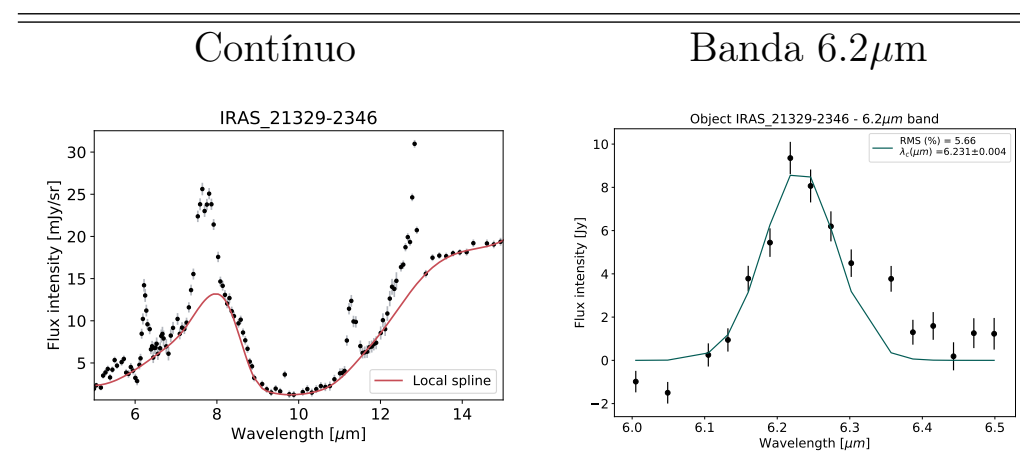

\section{Bandas 7.7 e $8.6 \mu \mathrm{m}$}
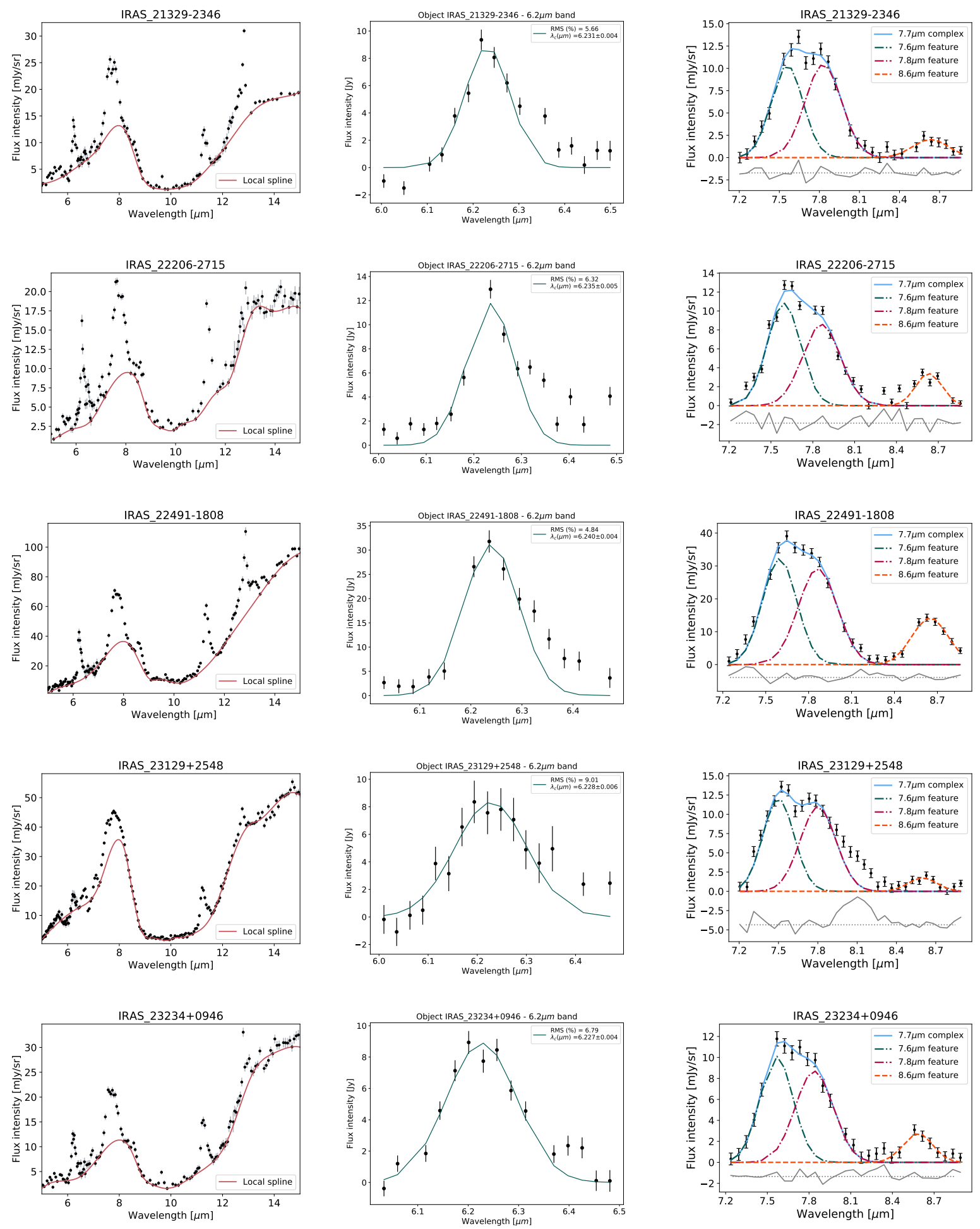

Continua na próxima página... 
Tabela A.2 - Continuação
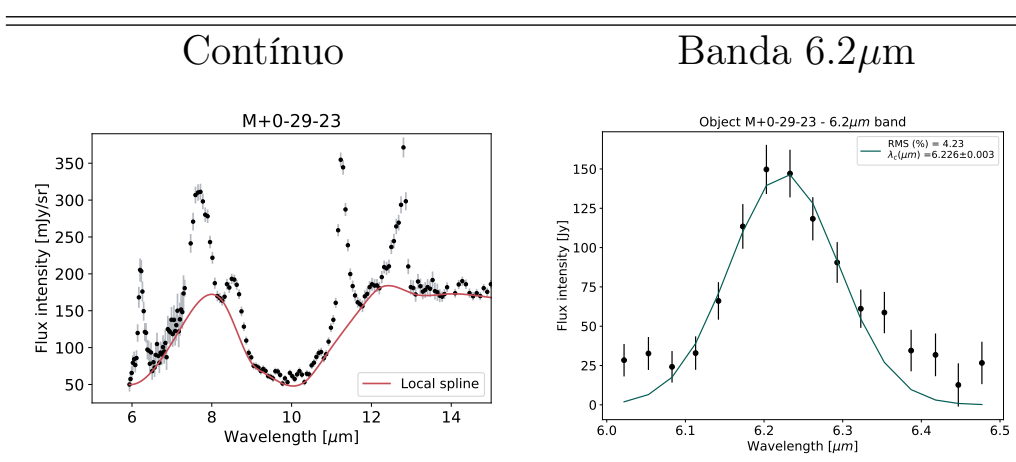

Bandas 7.7 e $8.6 \mu \mathrm{m}$
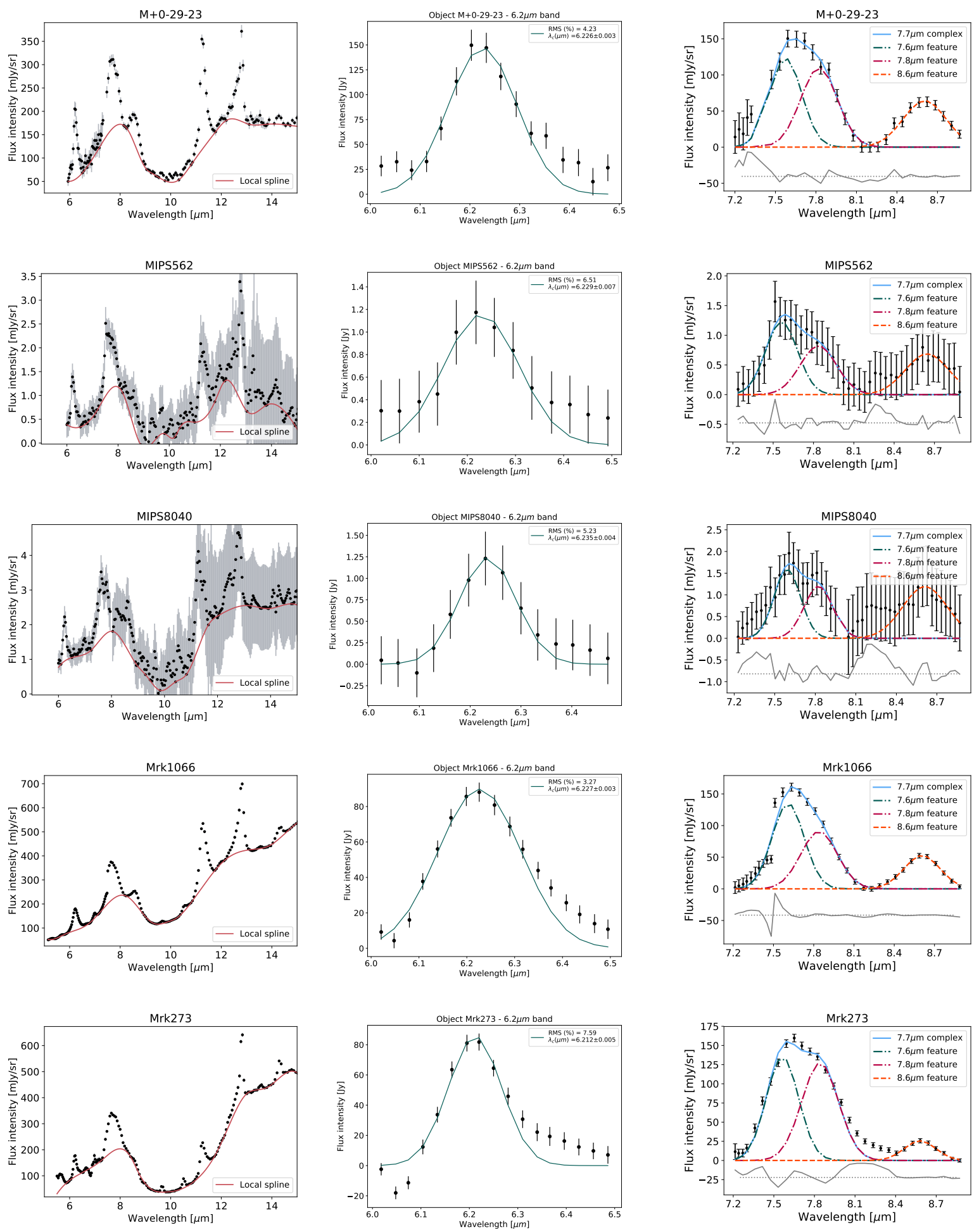

Continua na próxima página...

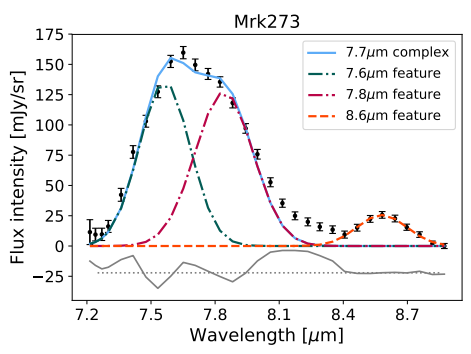


Tabela A.2 - Continuação

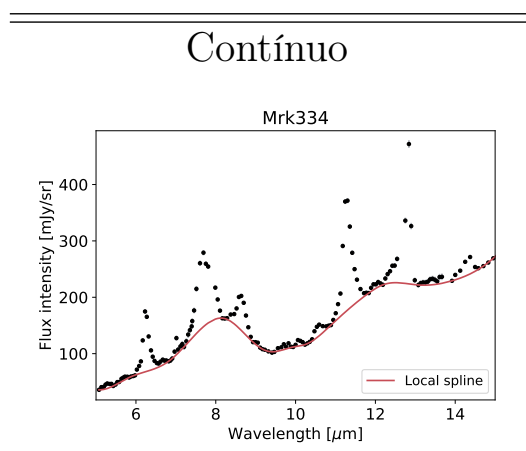

\section{Banda $6.2 \mu \mathrm{m}$}
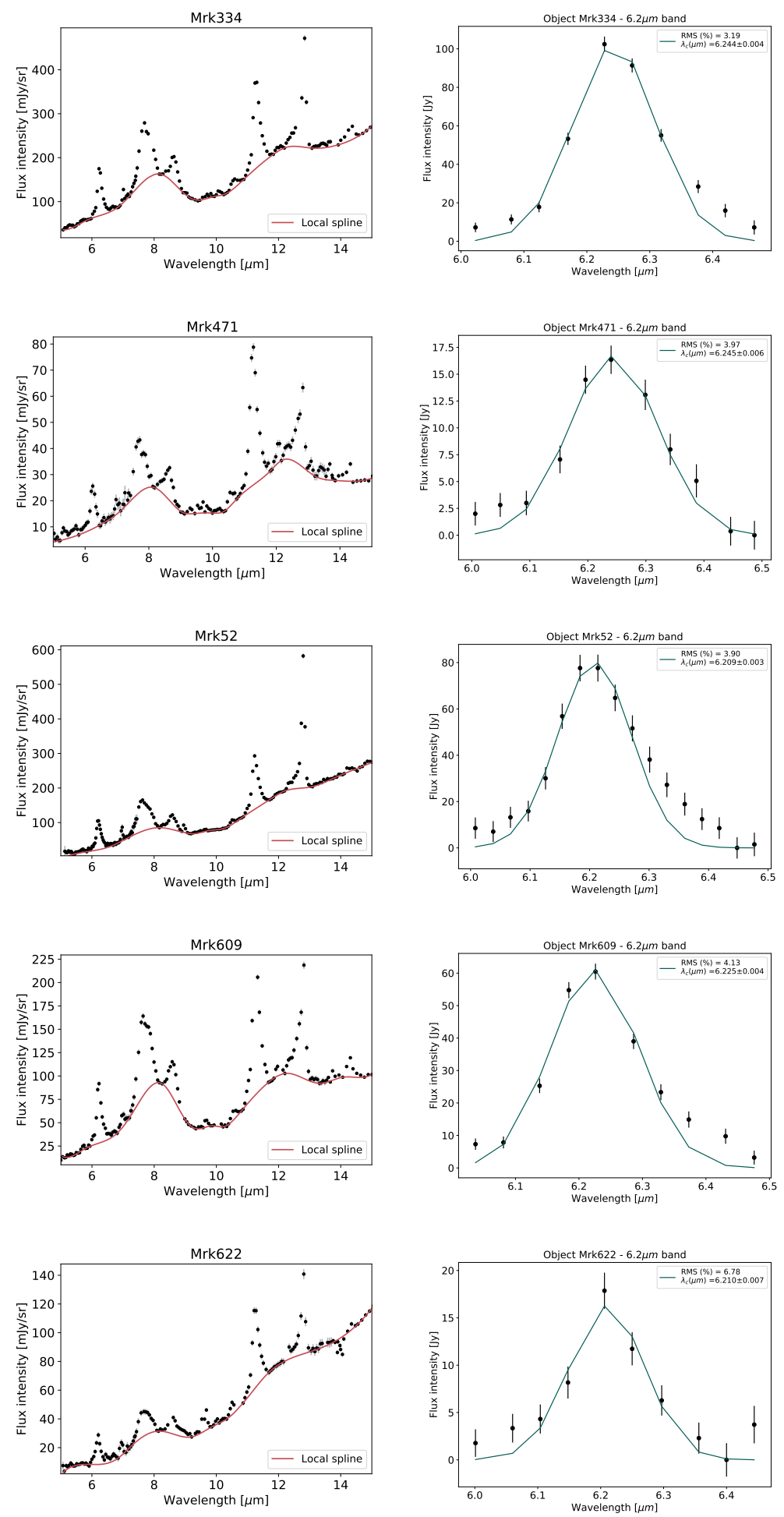

Bandas 7.7 e $8.6 \mu \mathrm{m}$
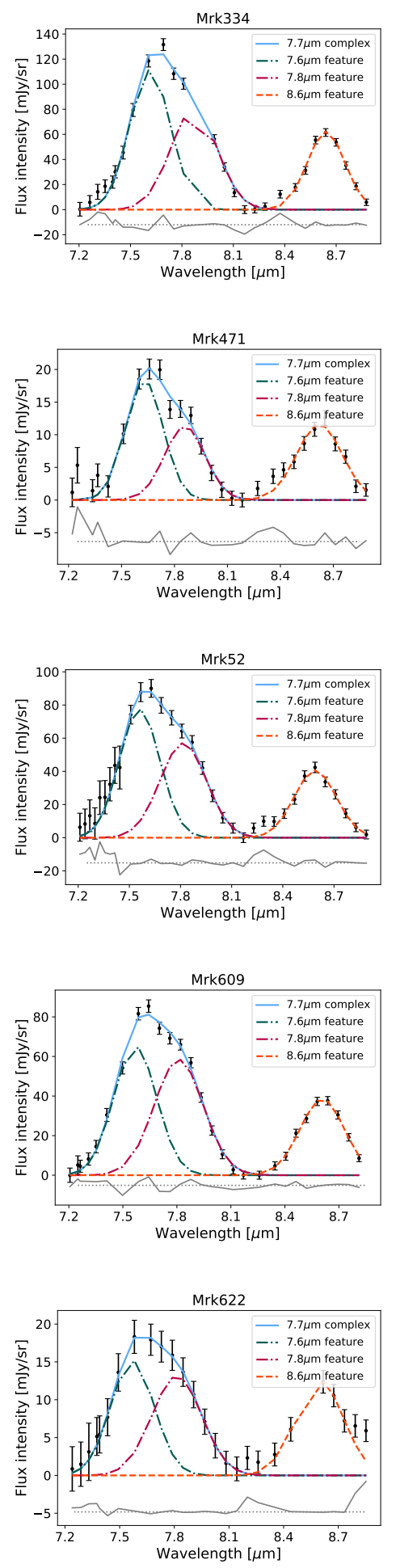

Continua na próxima página... 
Tabela A.2 - Continuação

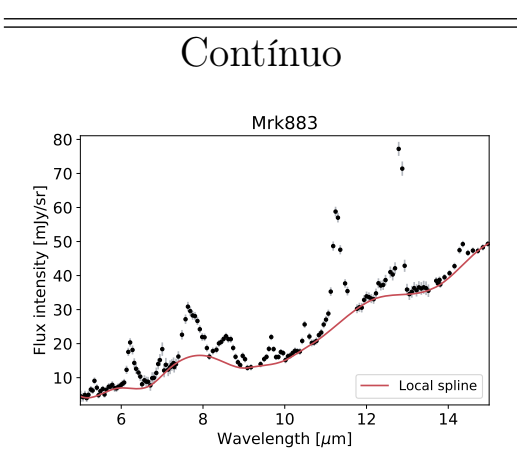

\section{Banda $6.2 \mu \mathrm{m}$}
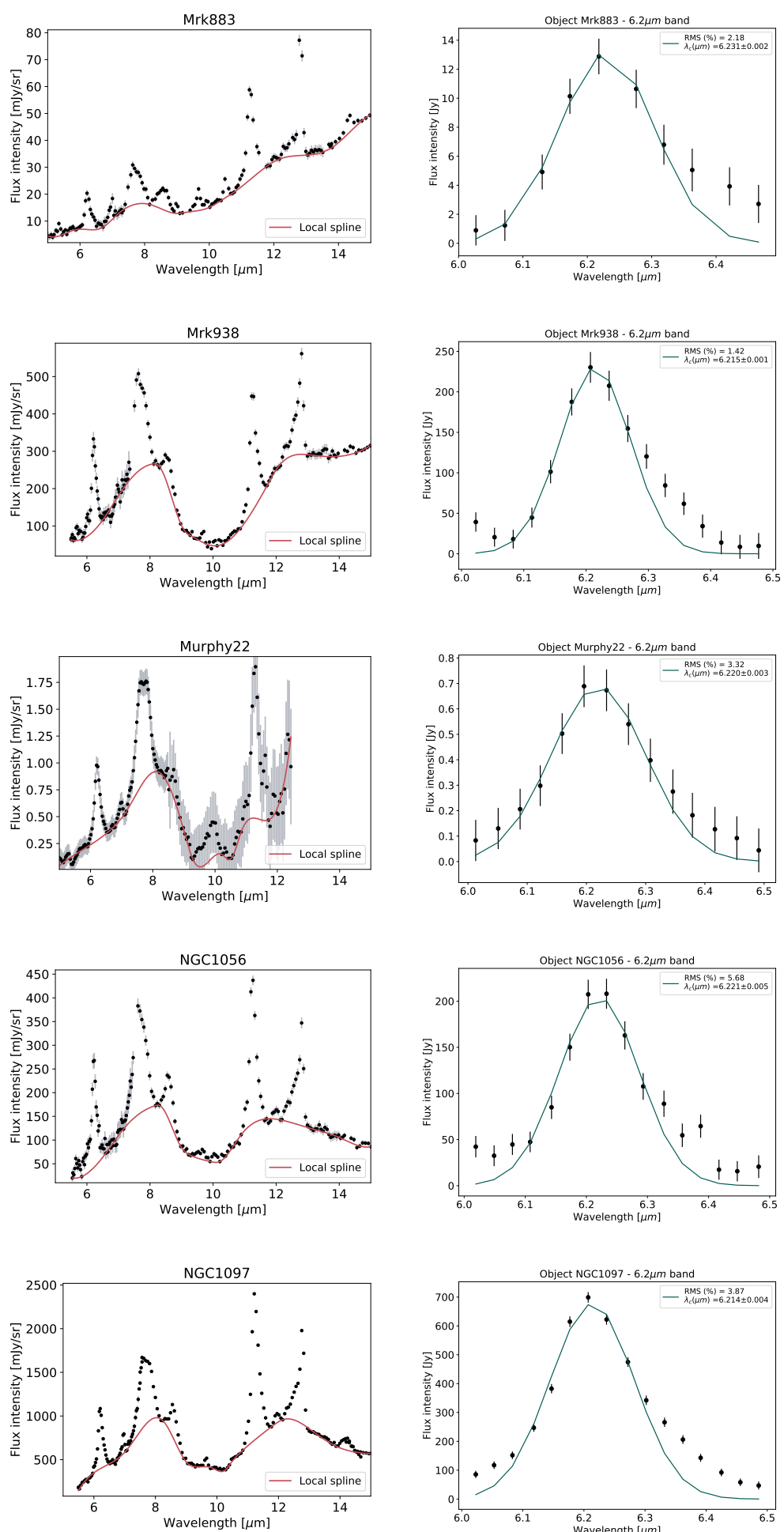
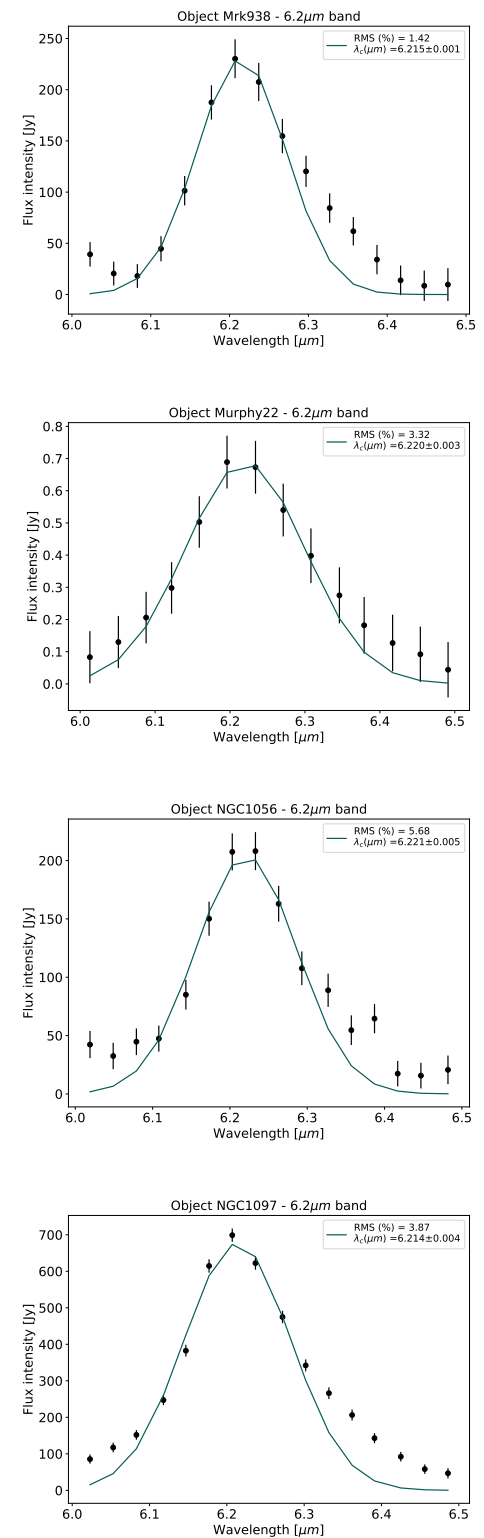

Bandas 7.7 e $8.6 \mu \mathrm{m}$
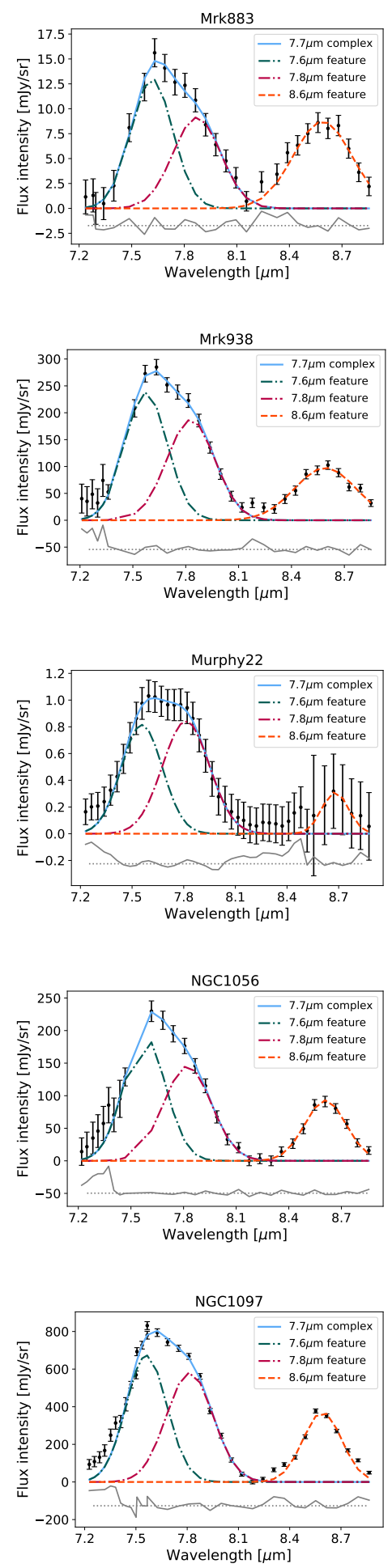

Continua na próxima página... 
Tabela A.2 - Continuação
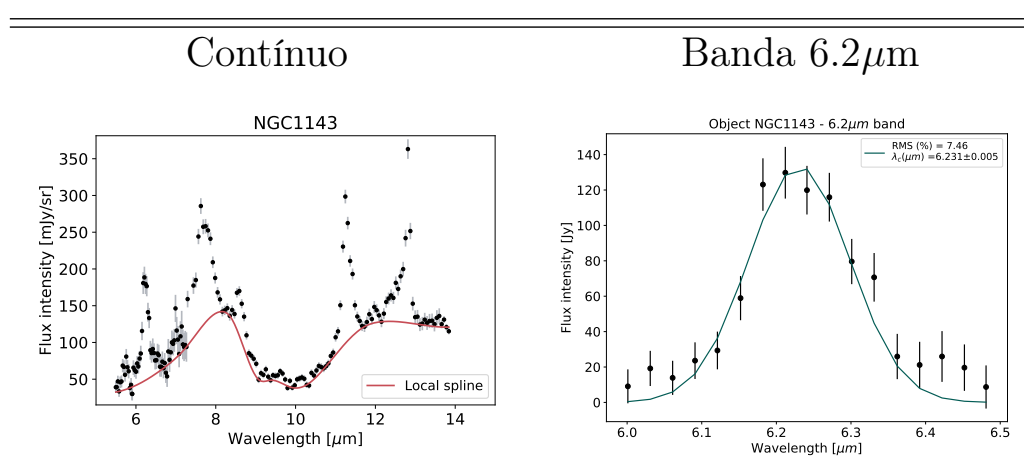

Bandas 7.7 e $8.6 \mu \mathrm{m}$
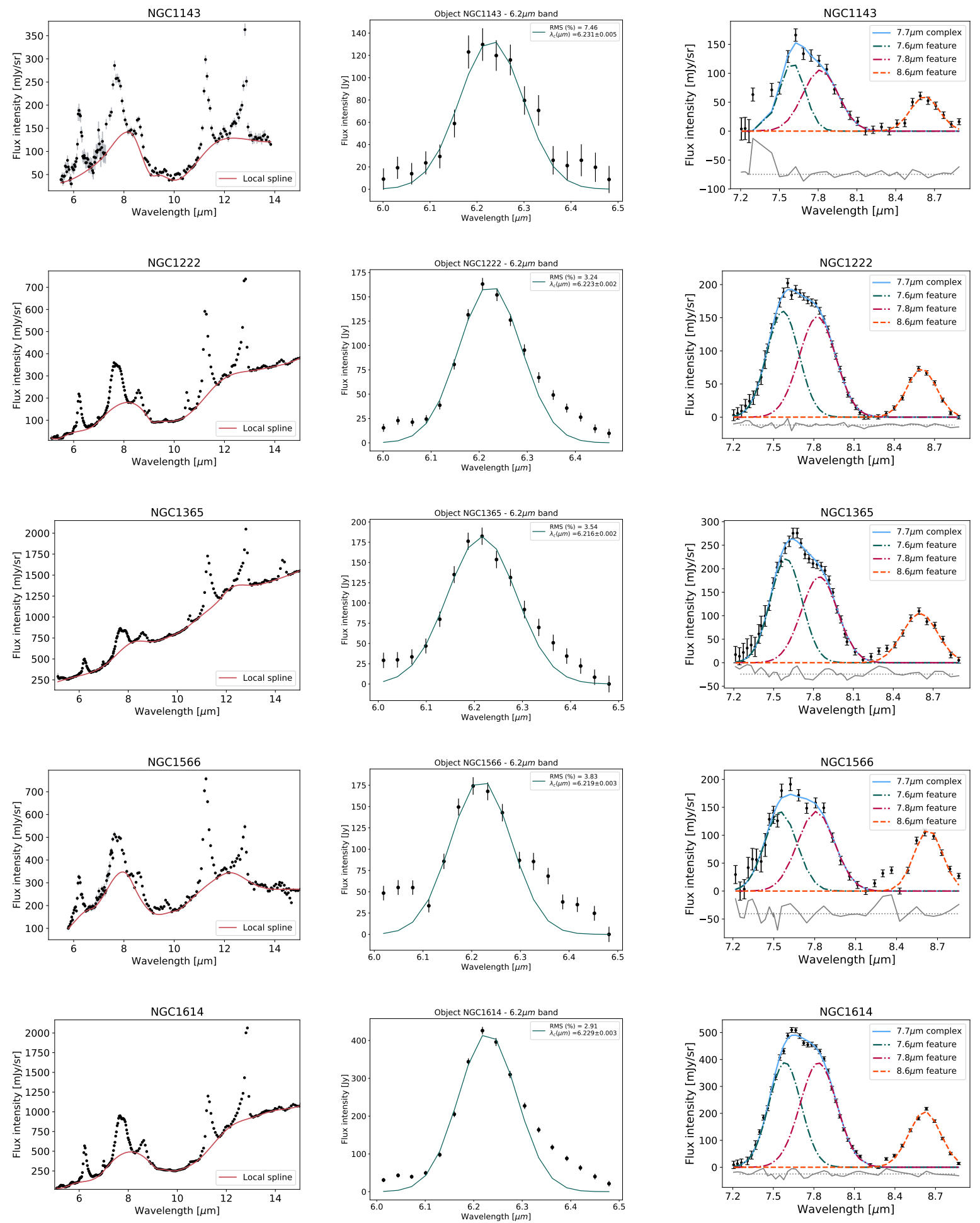

Continua na próxima página...

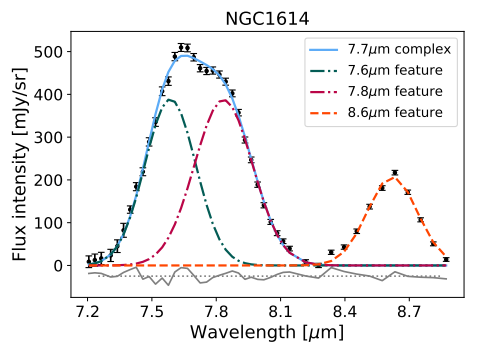


Tabela A.2 - Continuação
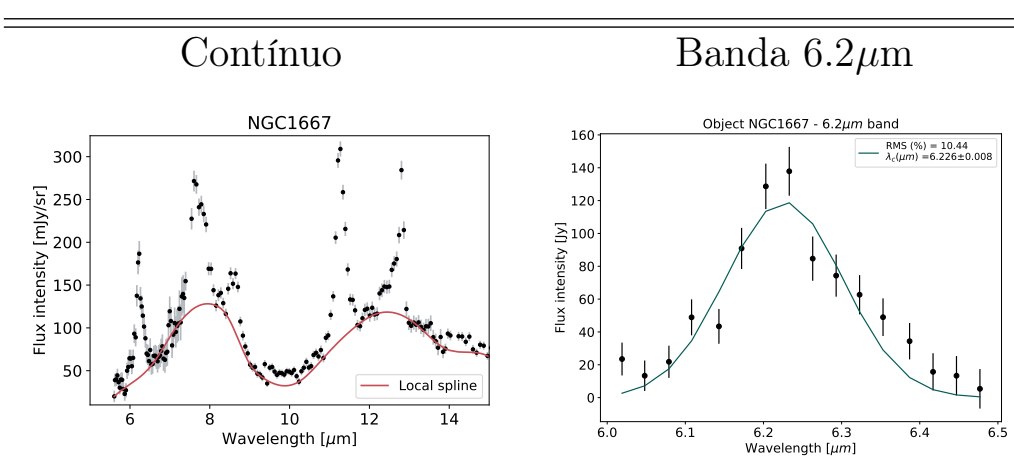

Bandas 7.7 e $8.6 \mu \mathrm{m}$
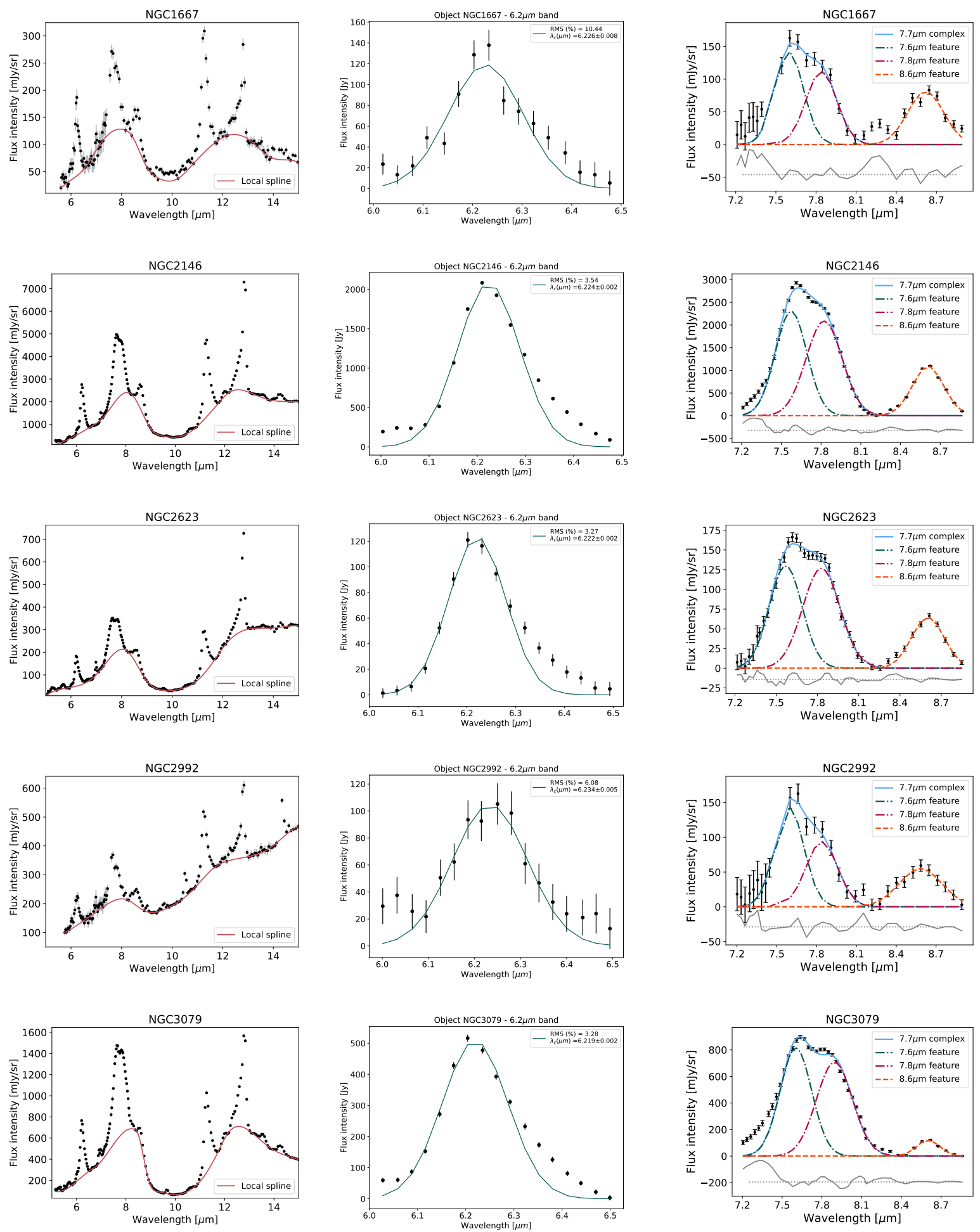

Continua na próxima página...

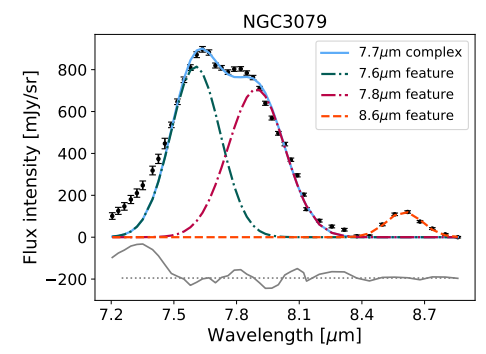


Tabela A.2 - Continuação
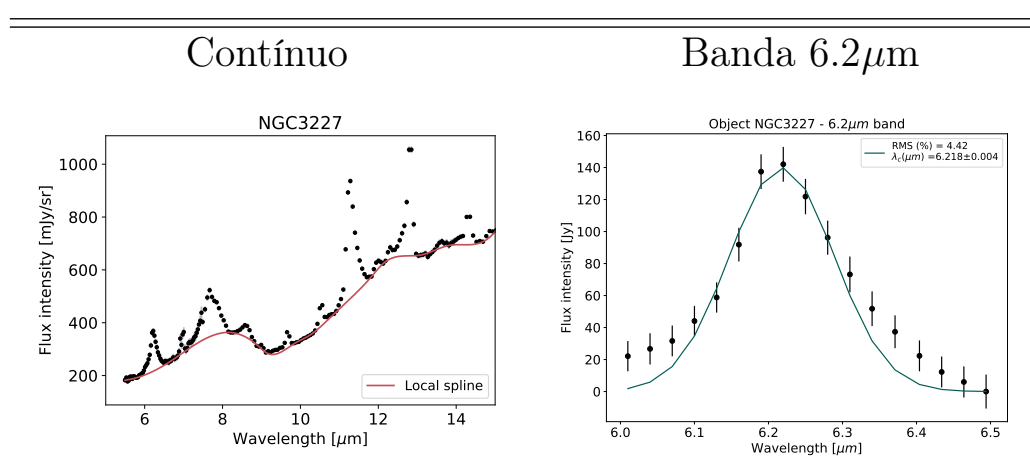

Bandas 7.7 e $8.6 \mu \mathrm{m}$
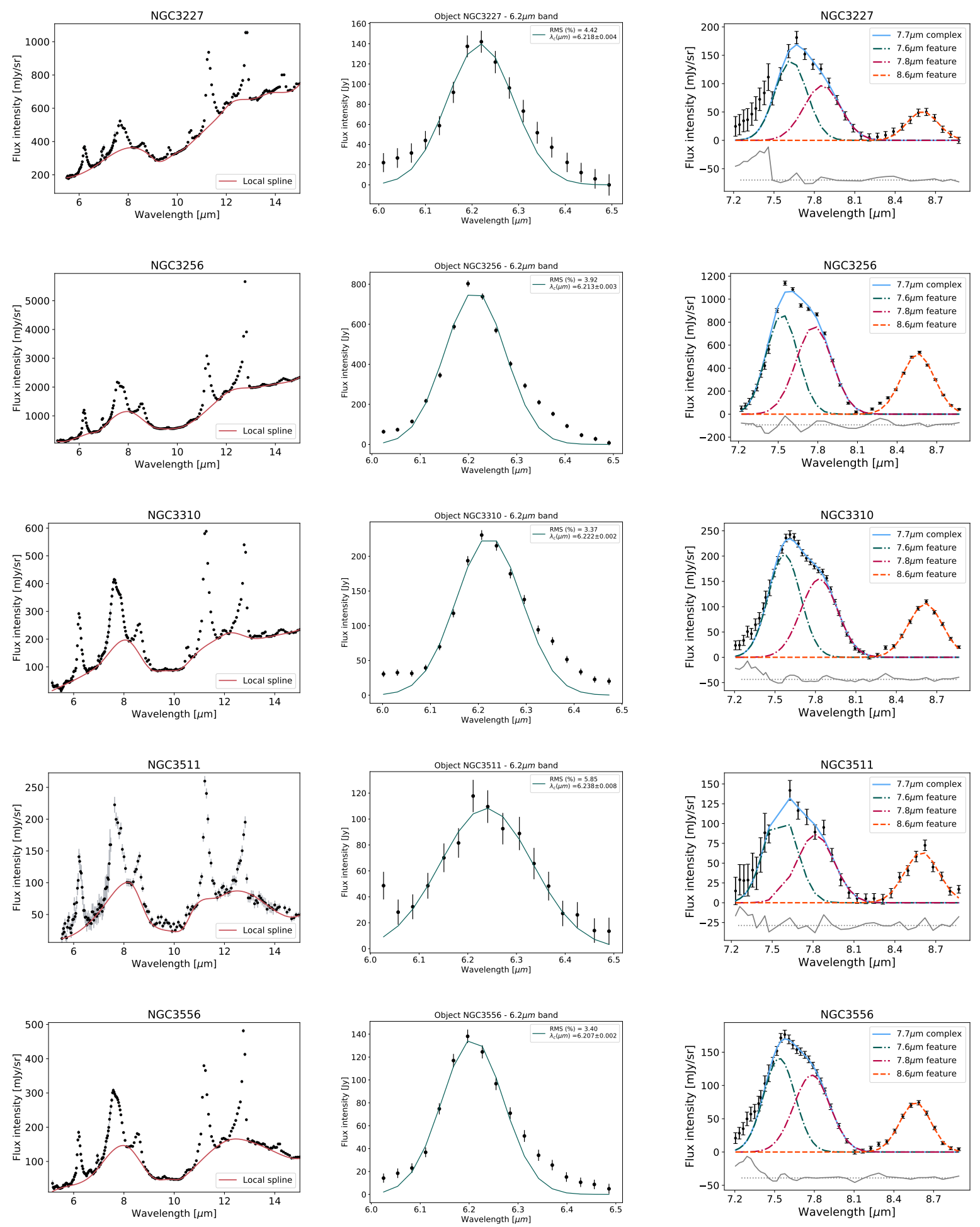

Continua na próxima página... 
Tabela A.2 - Continuação

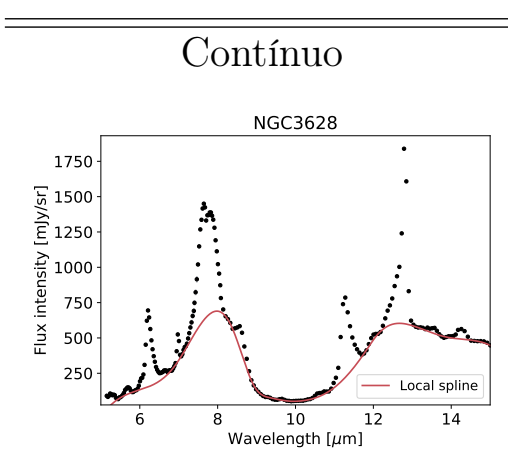

Banda $6.2 \mu \mathrm{m}$
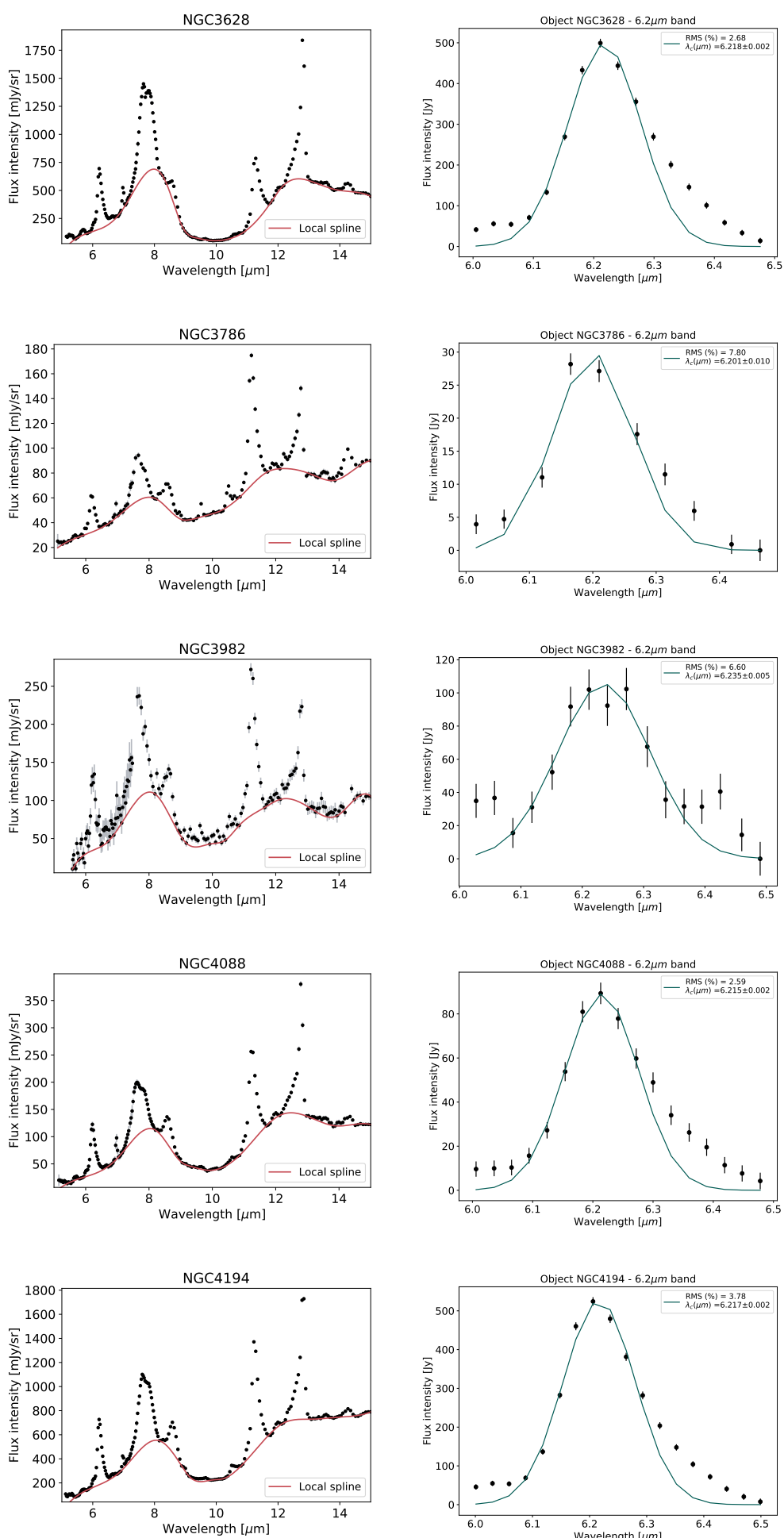
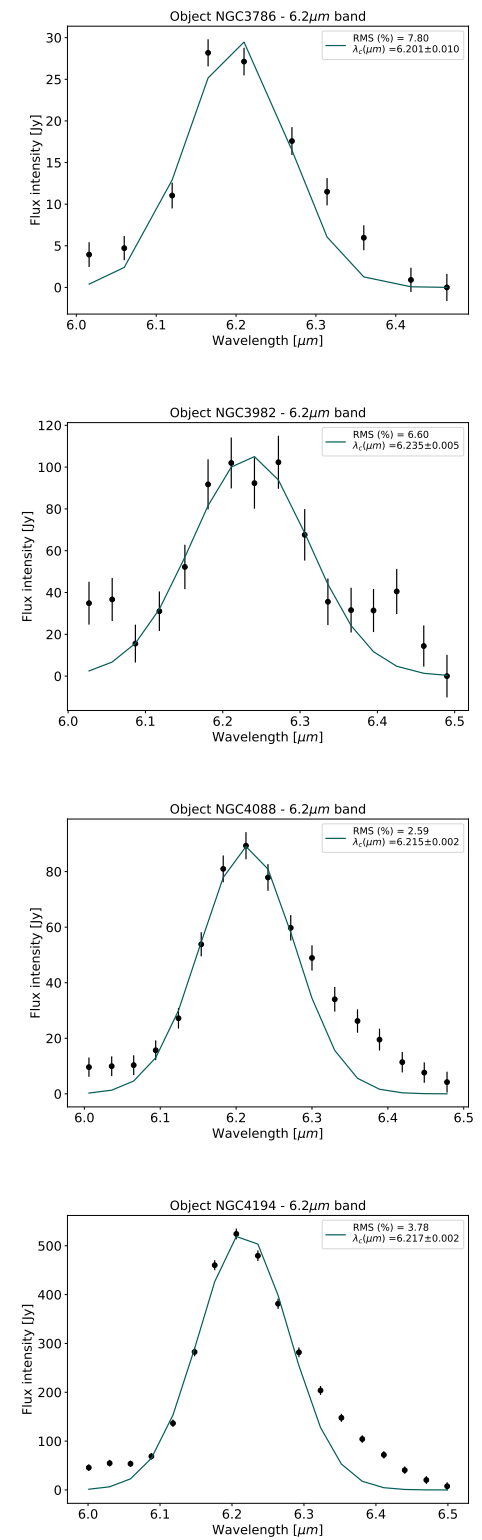

Bandas 7.7 e $8.6 \mu \mathrm{m}$
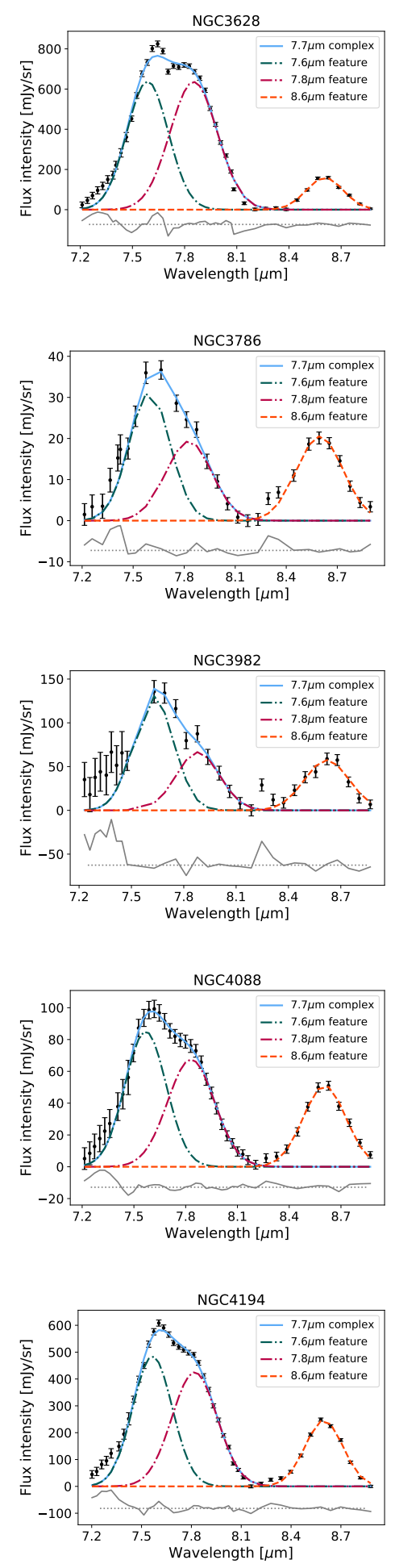

Continua na próxima página... 
Tabela A.2 - Continuação
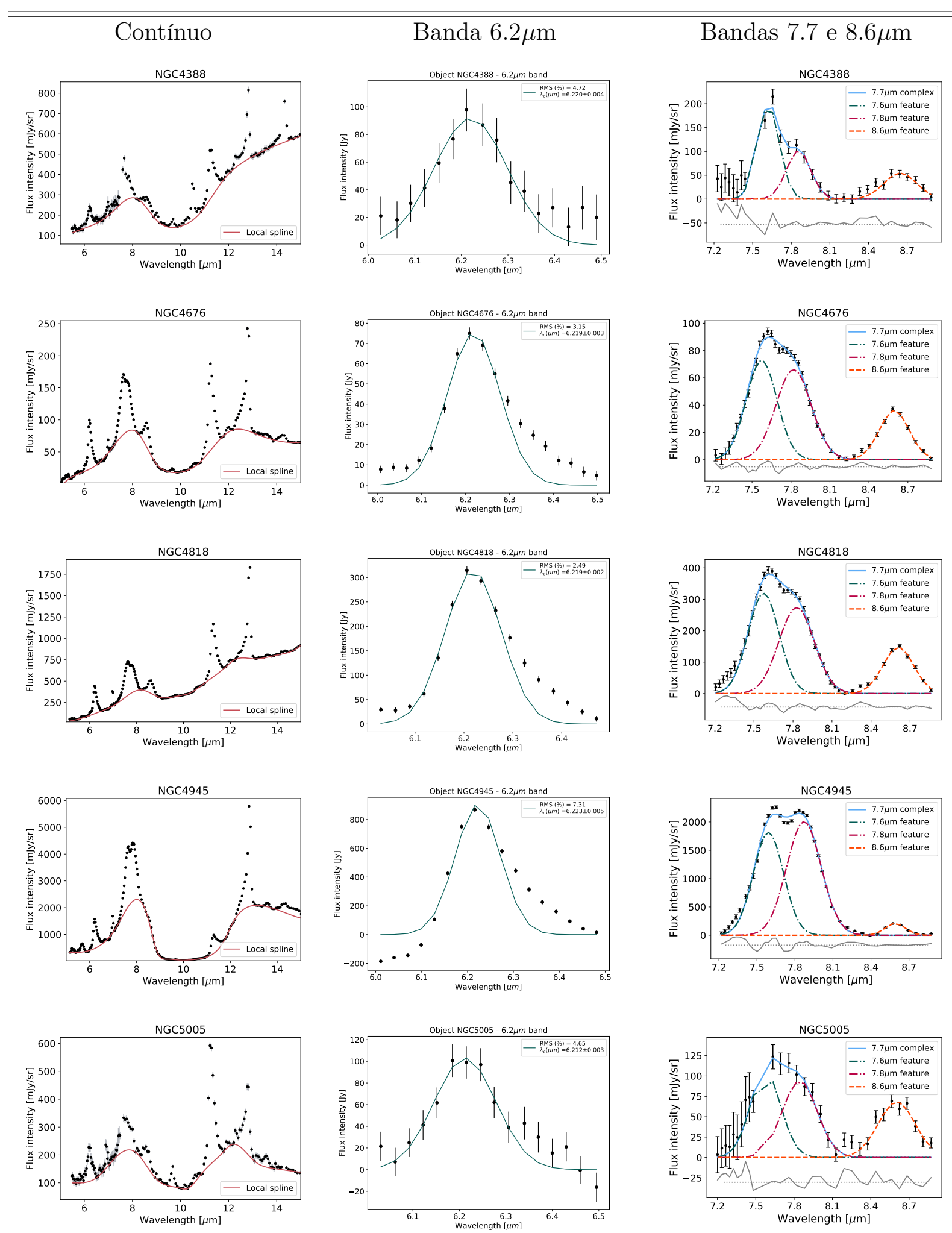

Continua na próxima página... 
Tabela A.2 - Continuação
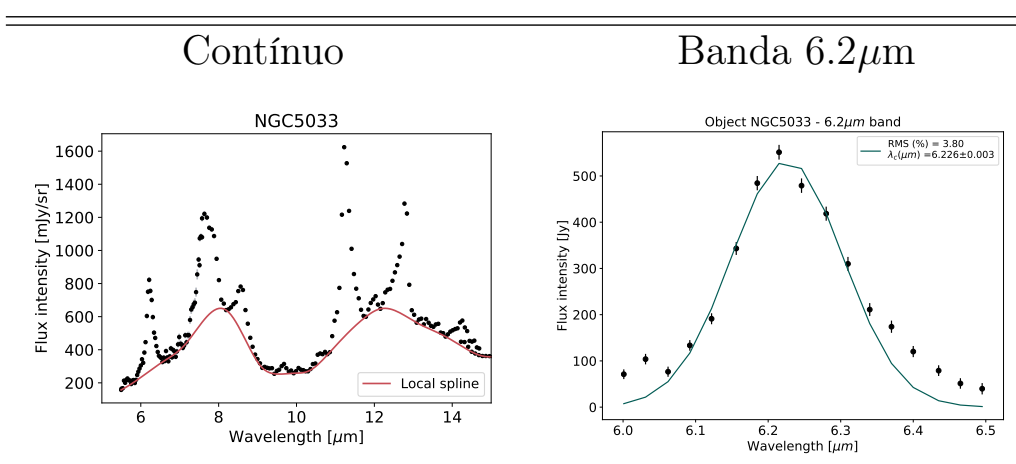

Bandas 7.7 e $8.6 \mu \mathrm{m}$
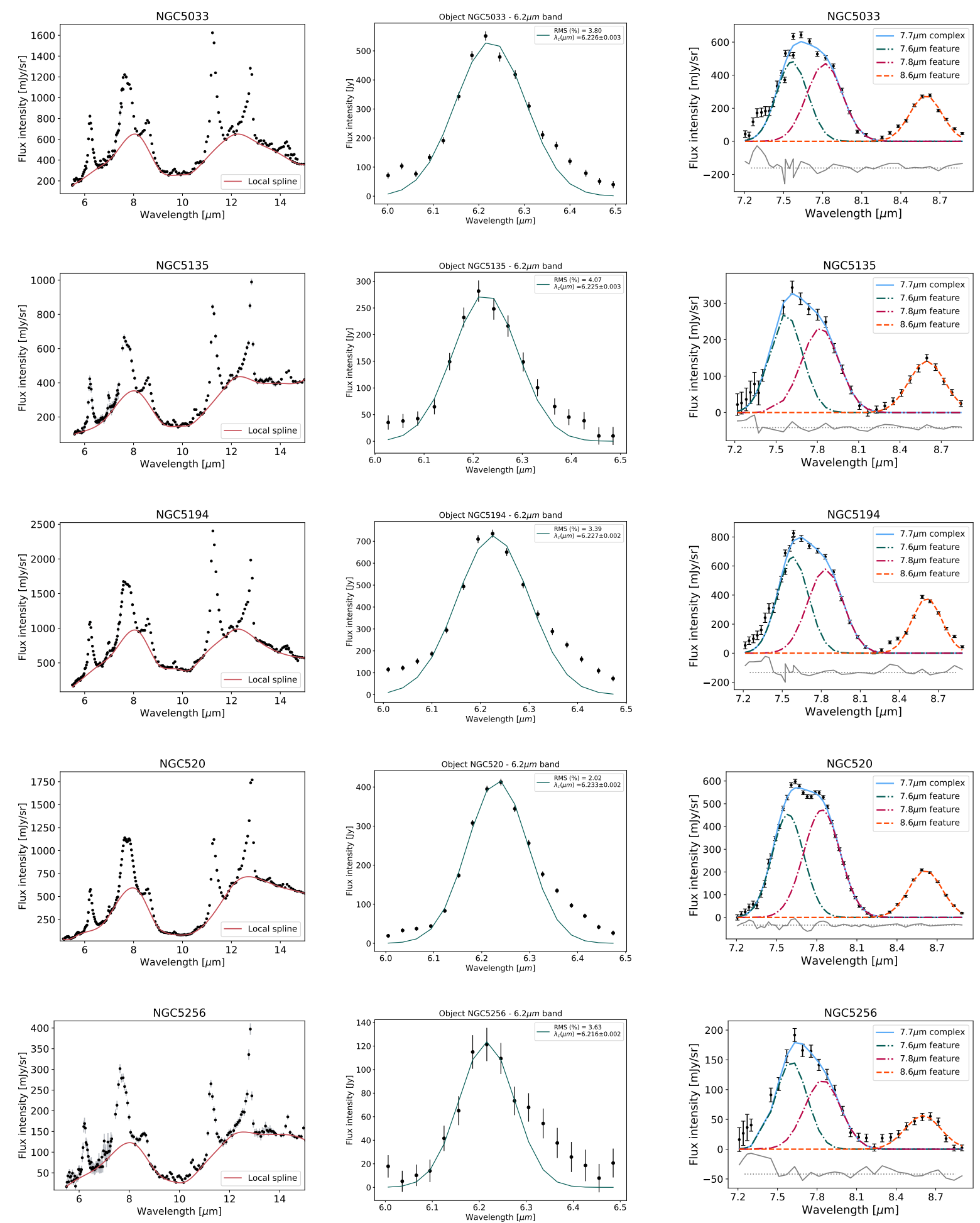

Continua na próxima página...

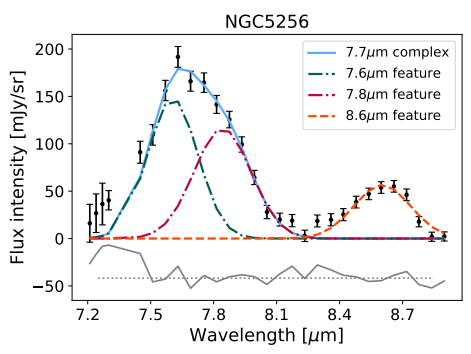


Tabela A.2 - Continuação

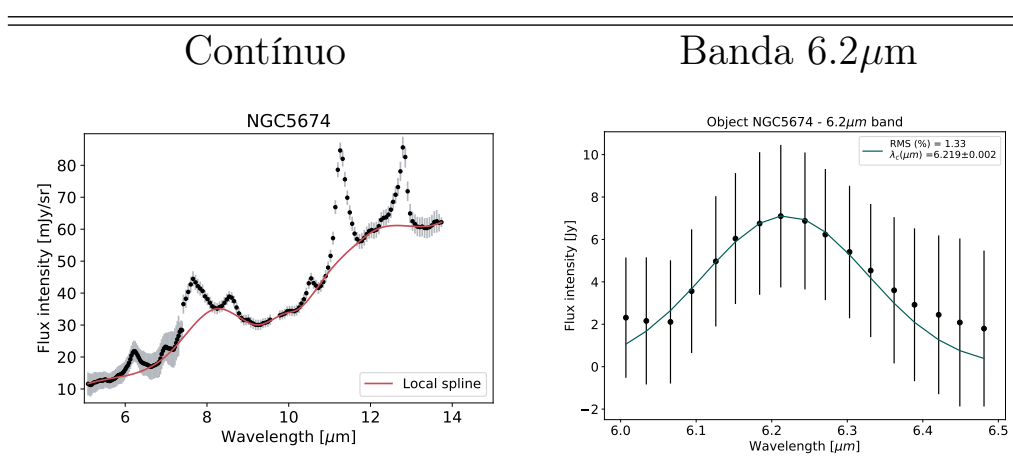

\section{Bandas 7.7 e $8.6 \mu \mathrm{m}$}
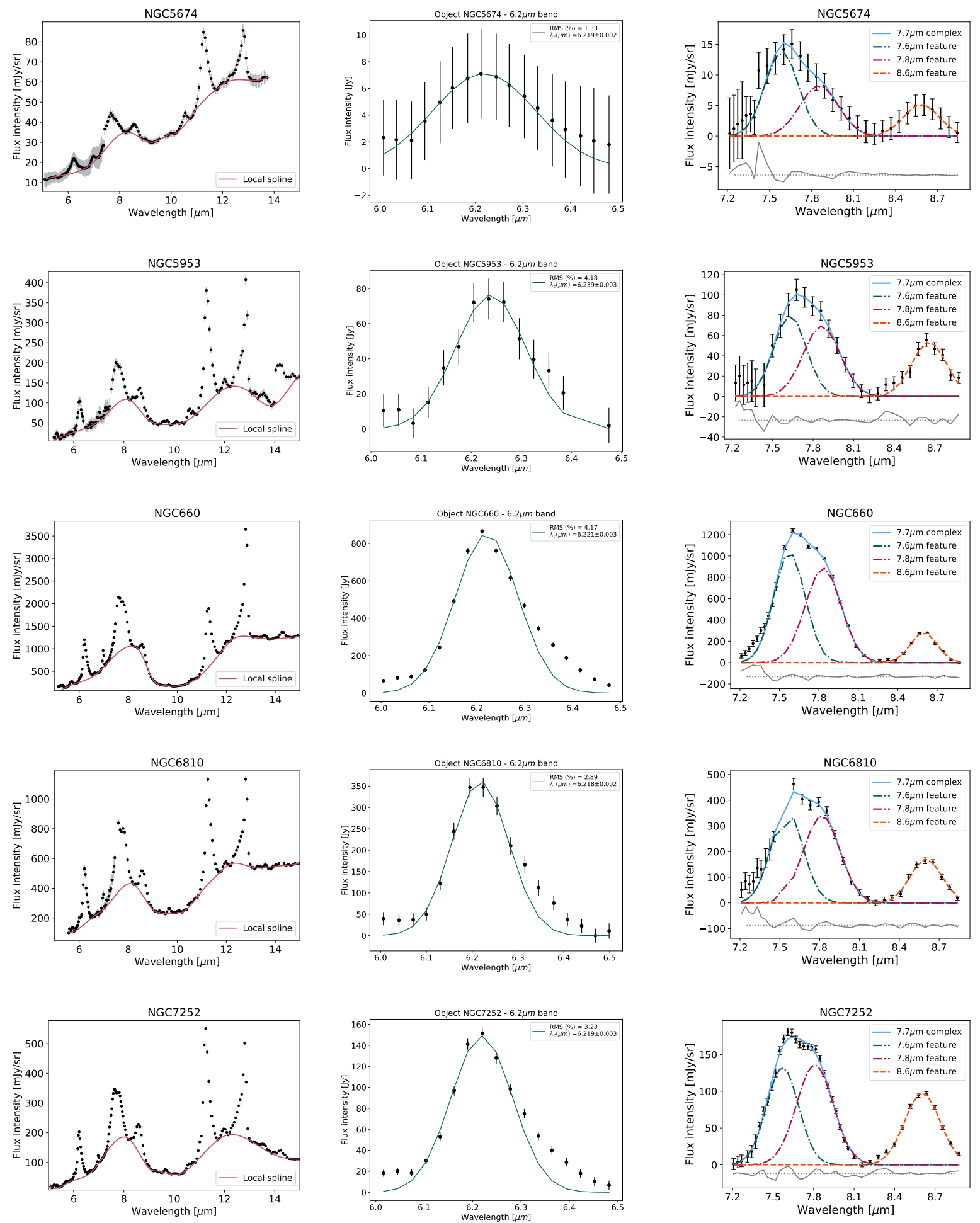

Continua na próxima página... 
Tabela A.2 - Continuação
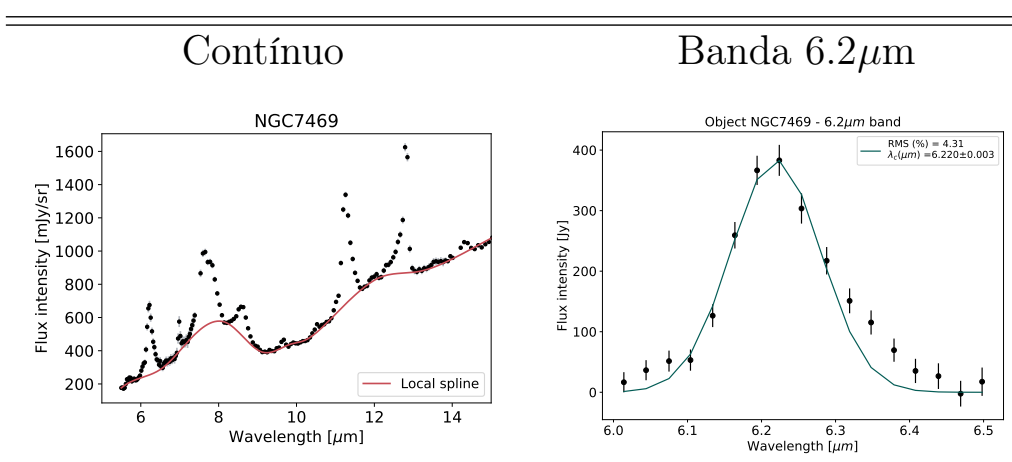

Bandas 7.7 e $8.6 \mu \mathrm{m}$
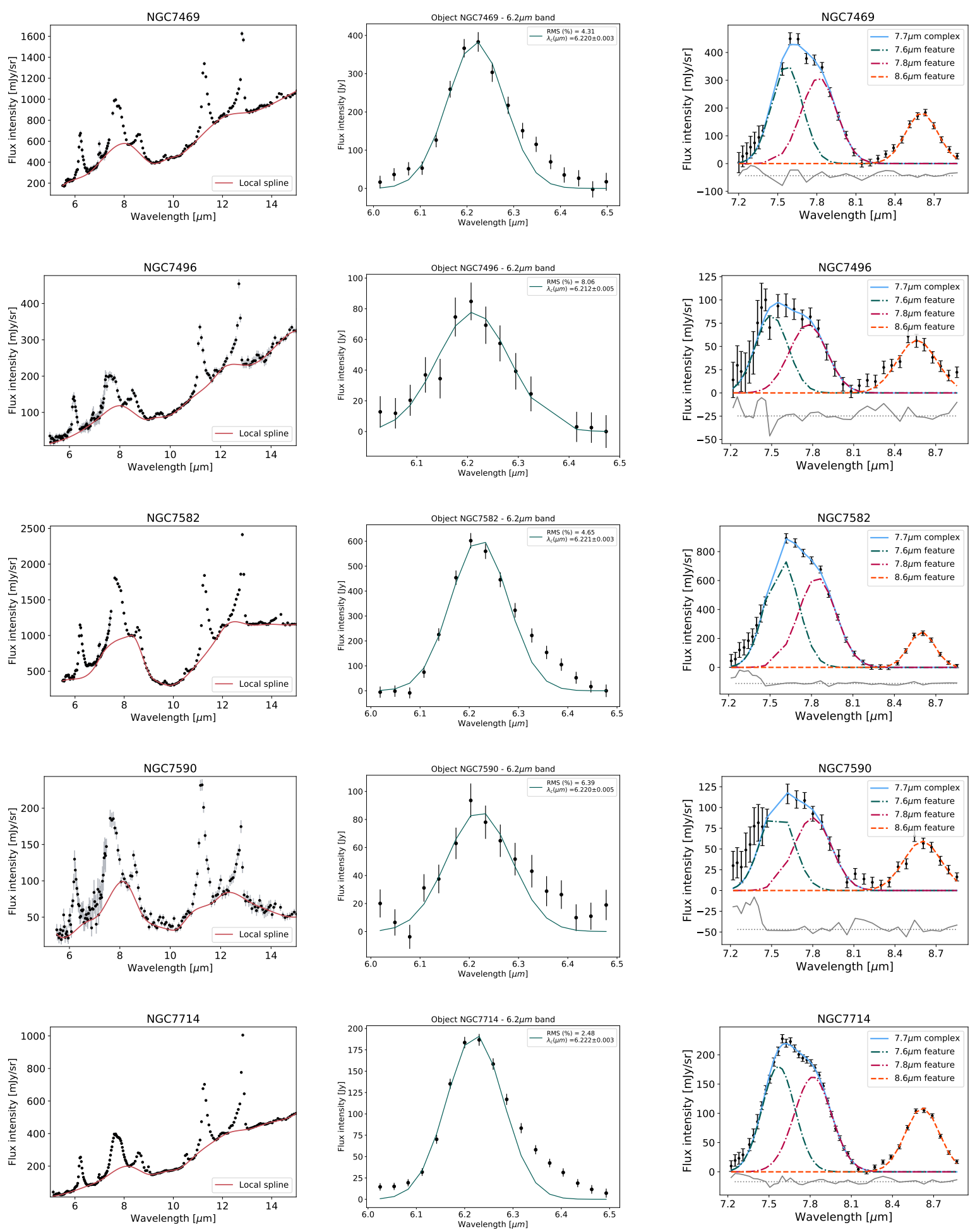

Continua na próxima página... 
Tabela A.2 - Continuação
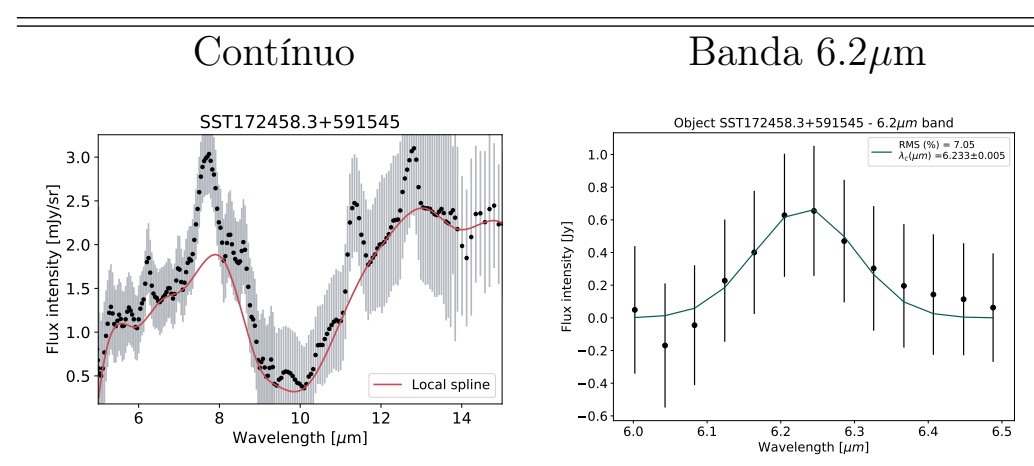

Bandas 7.7 e $8.6 \mu \mathrm{m}$
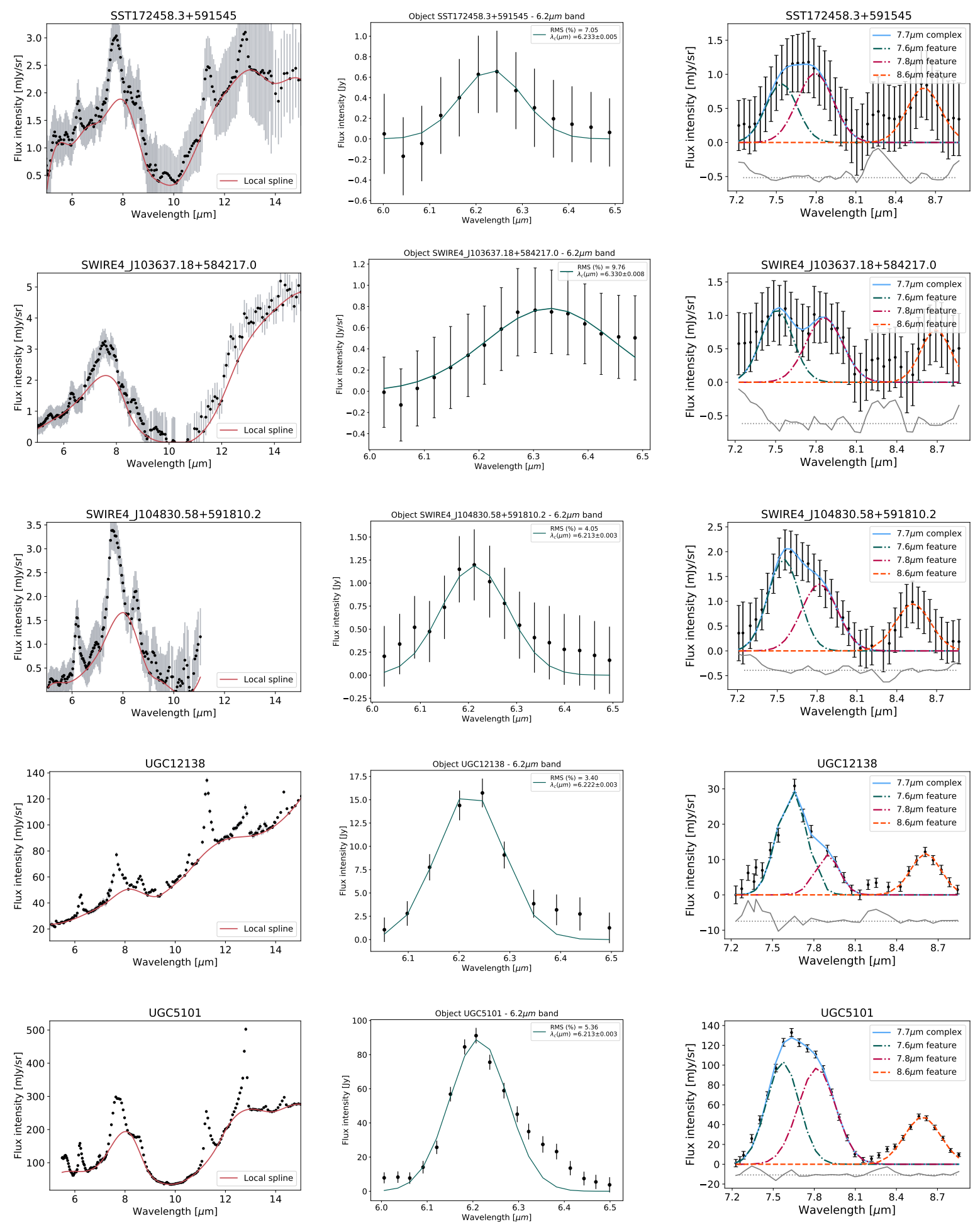

Continua na próxima página...

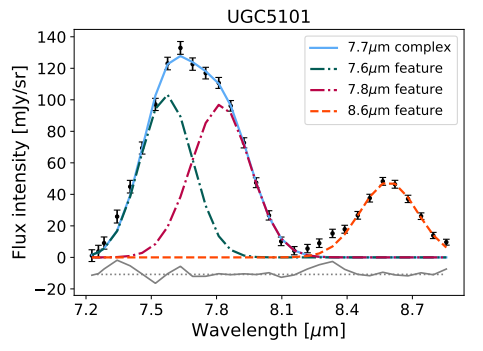


Tabela A.2 - Continuação

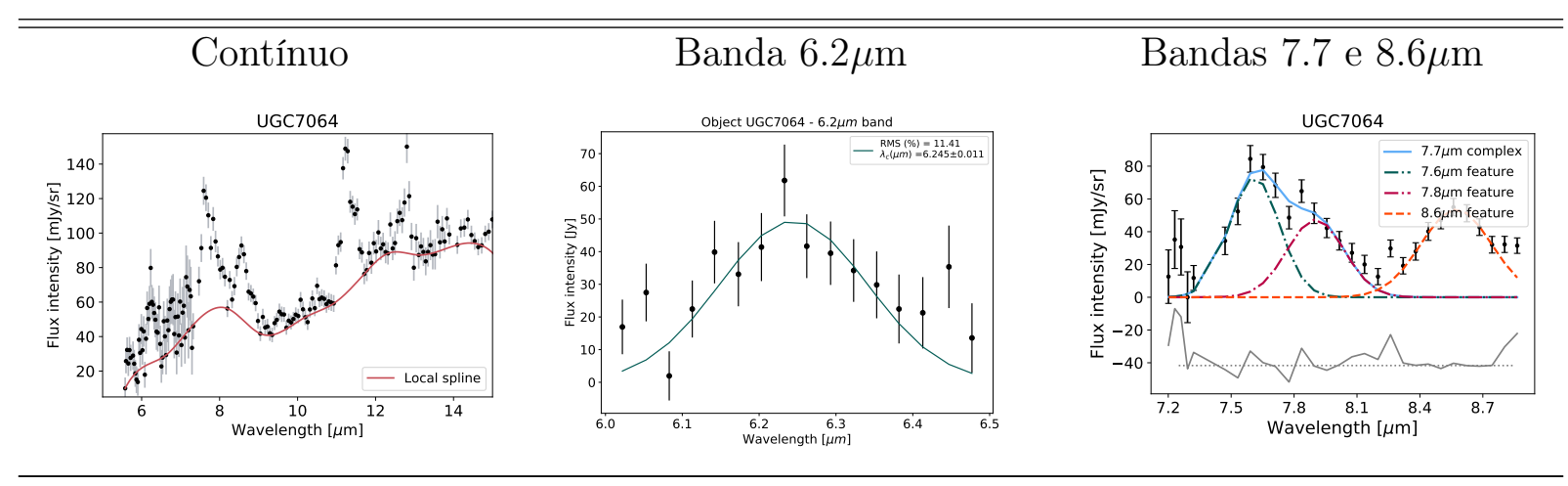

